Supporting Information to:

\title{
Chemical targeting of voltage sensitive dyes to specific cells and molecules in the brain
}

\author{
Tomas Fiala ${ }^{1}, \|$, Jihang Wang ${ }^{1, \|,}$, Matthew Dunn${ }^{1}$, Peter Šebej ${ }^{1,12}$, Se Joon Choi ${ }^{4}$, Ekeoma C. \\ Nwadibia ${ }^{1}$, Eva Fialova ${ }^{1}$, Diana M. Martinez, ${ }^{4}$ Claire E. Cheetham ${ }^{7}$, Keri J. Fogle ${ }^{8,9}$, Michael \\ J. Palladino ${ }^{8,9}$, Zachary Freyberg ${ }^{10,11}$, David Sulzer ${ }^{3,4,5,6 *}$ and Dalibor Sames ${ }^{1,2 *}$ \\ ${ }^{1}$ Department of Chemistry, Columbia University, New York, NY, USA. ${ }^{2}$ NeuroTechnology Center at Columbia
} University, New York, NY, USA ${ }^{3}$ Department of Neurology, Columbia University Irving Medical Center, New York, NY, USA. ${ }^{4}$ Department of Psychiatry, Columbia University Irving Medical Center, New York, NY, USA. ${ }^{5}$ Department of Pharmacology, Columbia University Irving Medical Center, New York, NY, USA. ${ }^{6}$ Department of Molecular Therapeutics, New York Psychiatric Institute, New York, NY, USA. ${ }^{7}$ Department of Neurobiology, University of Pittsburgh, Pittsburgh, PA, USA. ${ }^{8}$ Department of Pharmacology \& Chemical Biology, University of Pittsburgh, Pittsburgh, PA, USA. ${ }^{9}$ Pittsburgh Institute of Neurodegenerative Diseases (PIND), University of Pittsburgh, Pittsburgh, PA, USA. ${ }^{10}$ Department of Psychiatry, University of Pittsburgh, Pittsburgh, PA, USA.

${ }^{11}$ Department of Cell Biology, University of Pittsburgh, Pittsburgh, PA, USA. ${ }^{12}$ Current address: RECETOX, Faculty of Science, Masaryk University, Brno, Czech Republic. *Correspondence should be addressed to D. Sames (ds584@columbia.edu) or D. Sulzer (ds43@cumc.columbia.edu). "T.F. and J.W. contributed equally to this work. 


\section{Table of Contents}

Supplementary Figures Referenced in the Main Text ........................................................... 3

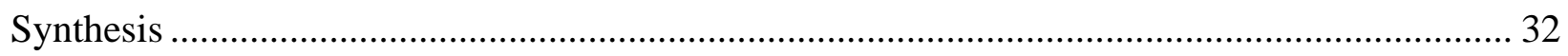

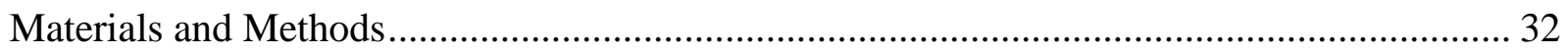

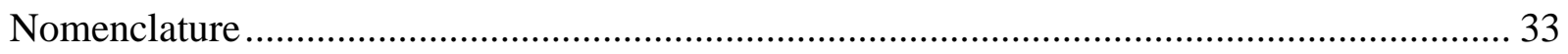

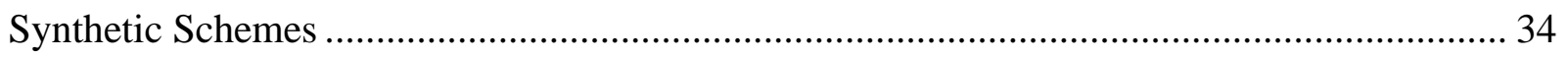

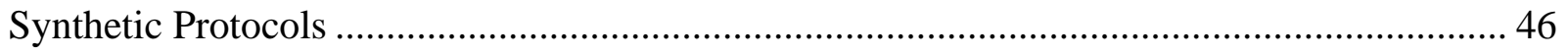

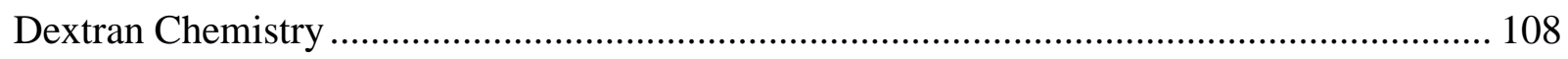

Biological Characterizations ............................................................................................ 128

Preparation and Storage of Stock Solutions................................................................... 128

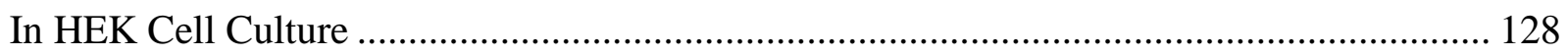

In Acute Murine Brain Slice .................................................................................... 132

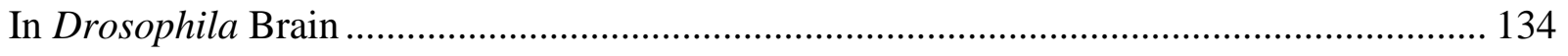

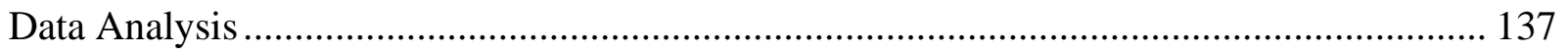

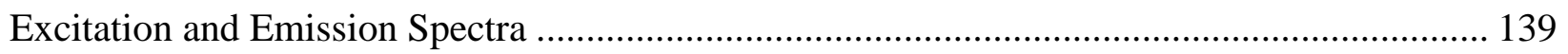

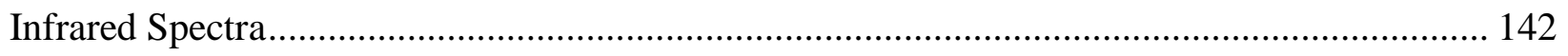

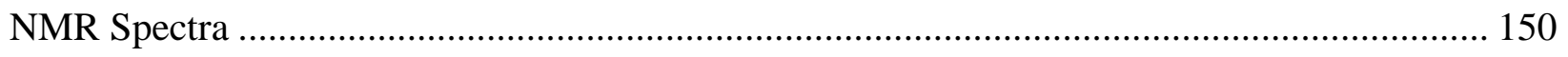

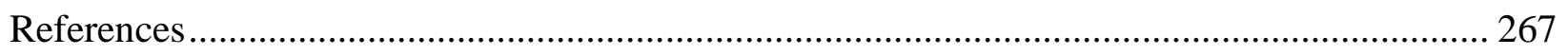




\section{Supplementary Figures Referenced in the Main Text}

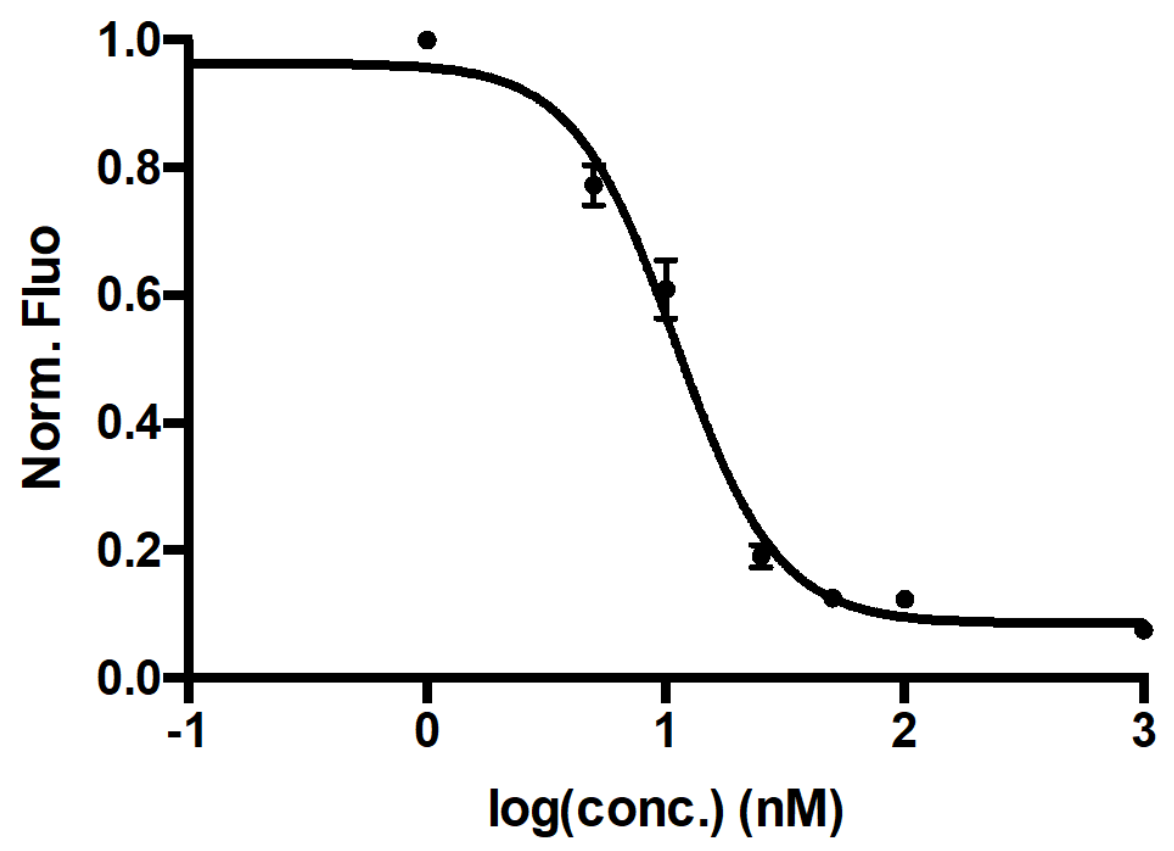

Figure S1. Determination of $I C_{50}$ of dichloropane derivative 5 to hDAT with APP+ uptake. Non-linear regression (1:1 binding model) showing the dose-dependent inhibition of APP+ uptake by dichloropane derivative 5 in hDAT-HEK cells, normalized to the maximum fluorescence intensity of each experiment (see Biological Characterizations section for details). Error bars are \pm SEM for $n=3$ experiments, 6 replicates per independent experiment (error bars of some data points are too small to display). 


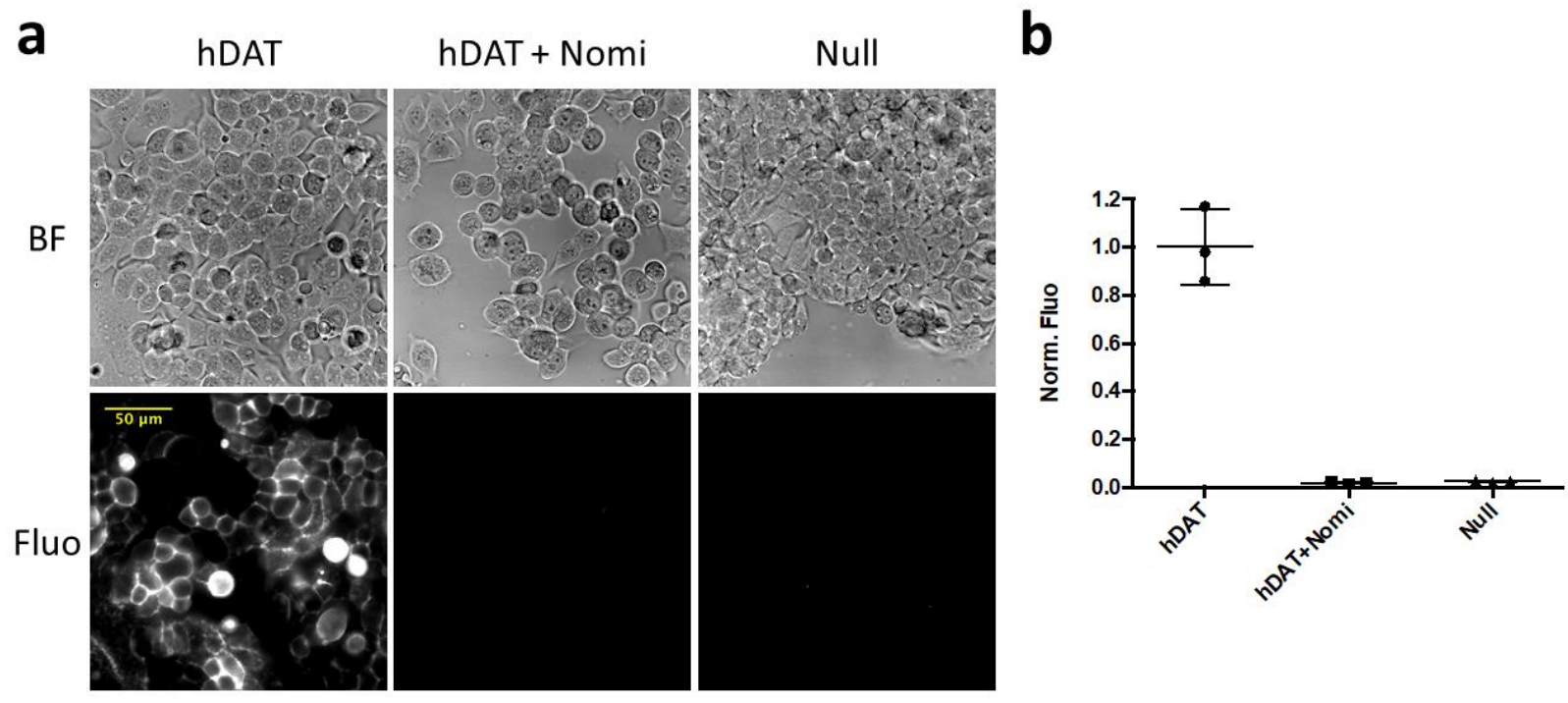

Figure S2. Labeling specificity of control probe 6 in hDAT-HEK cells. (a) Brightfield (BF, top row) and corresponding fluorescence (Fluo, bottom row) images of HEK cells labeled with 6 (1 nM). Labeling was compared in hDAT-HEK cells (left column), hDAT-HEK cells pre-incubated with nomifensine ( $2 \mu \mathrm{M}$; middle column), and null-transfected HEK cells (right column). All fluorescence images were equally contrasted. Excitation filter: $580 \mathrm{~nm}$ (40 nm bandpass), $610 \mathrm{~nm}$ dichroic, emission filter: $660 \mathrm{~nm}$ (50 nm bandpass). (b) Quantification of labeling specificity following the same procedure as that for the VoLDeMo probes, normalized to the mean fluorescence intensity of the hDAT-transfected, non-inhibited condition (see

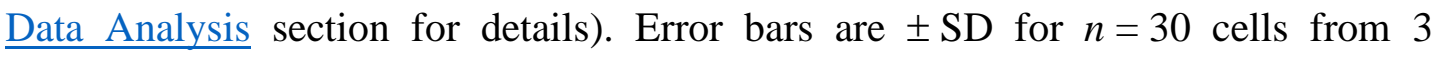
experiments. From two-tailed unpaired t-tests: $53.5 \pm 6.8$ fold decrease in labeling, $n=30$ cells from 3 experiments, $P=0.0004$ for hDAT+Nomi vs. hDAT; and $39.1 \pm 4.3$ fold decrease in labeling, $n=30$ cells from 3 experiments, $P=0.0004$ for Null vs. hDAT. 


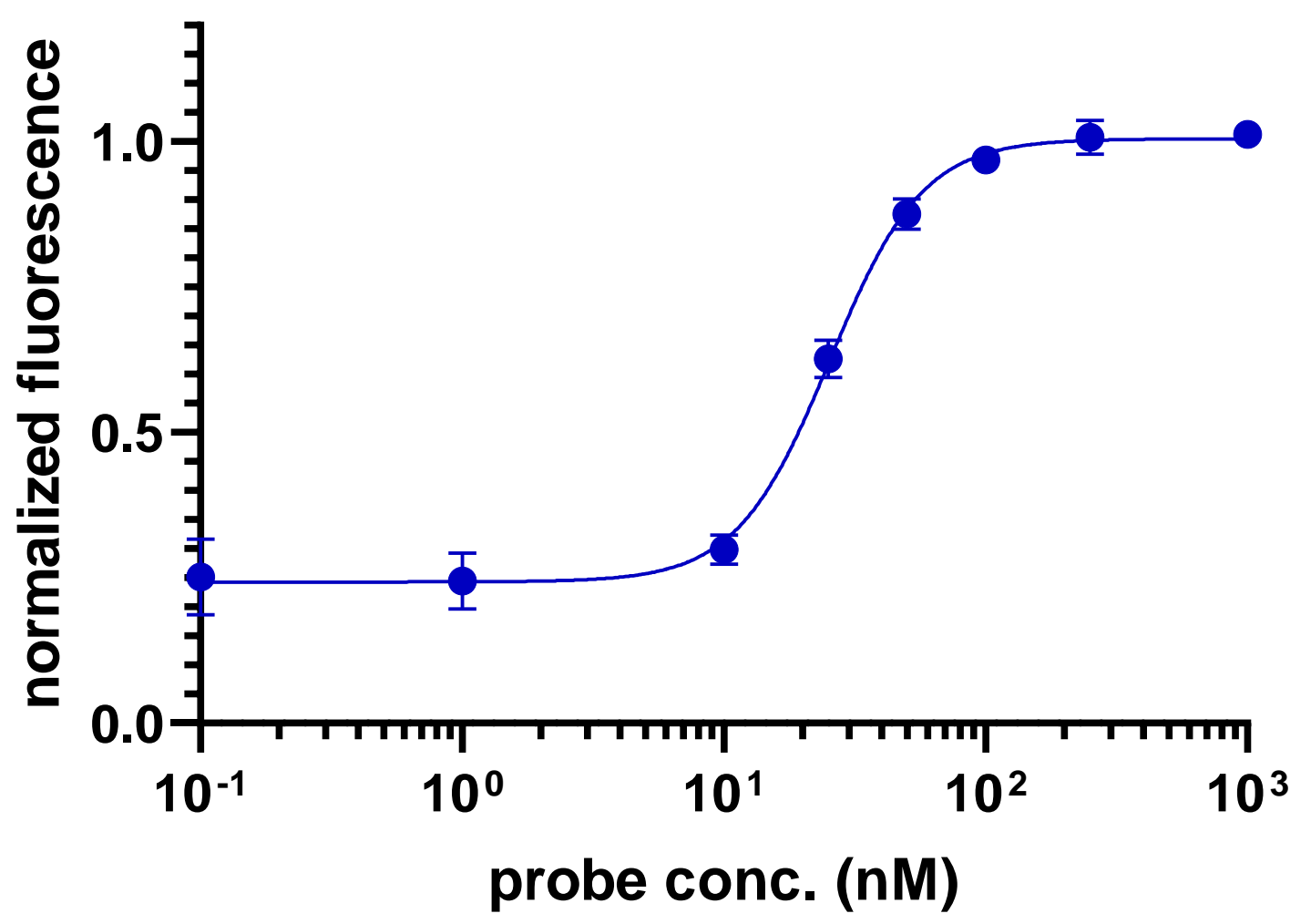

Figure S3. Determination of $I C_{50}$ of rhodamine-labeled dichloropane 6 to hDAT with APP+ uptake. Non-linear regression (1:1 binding model) showing the dosedependent inhibition of APP+ uptake by probe 6 in hDAT-HEK cells (see Biological

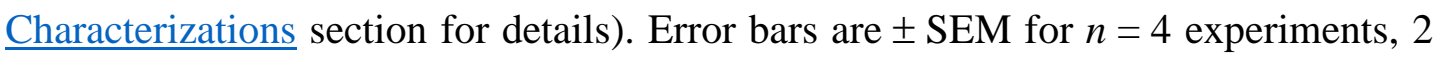
replicates per independent experiment (error bars of some data points are too small to display). 

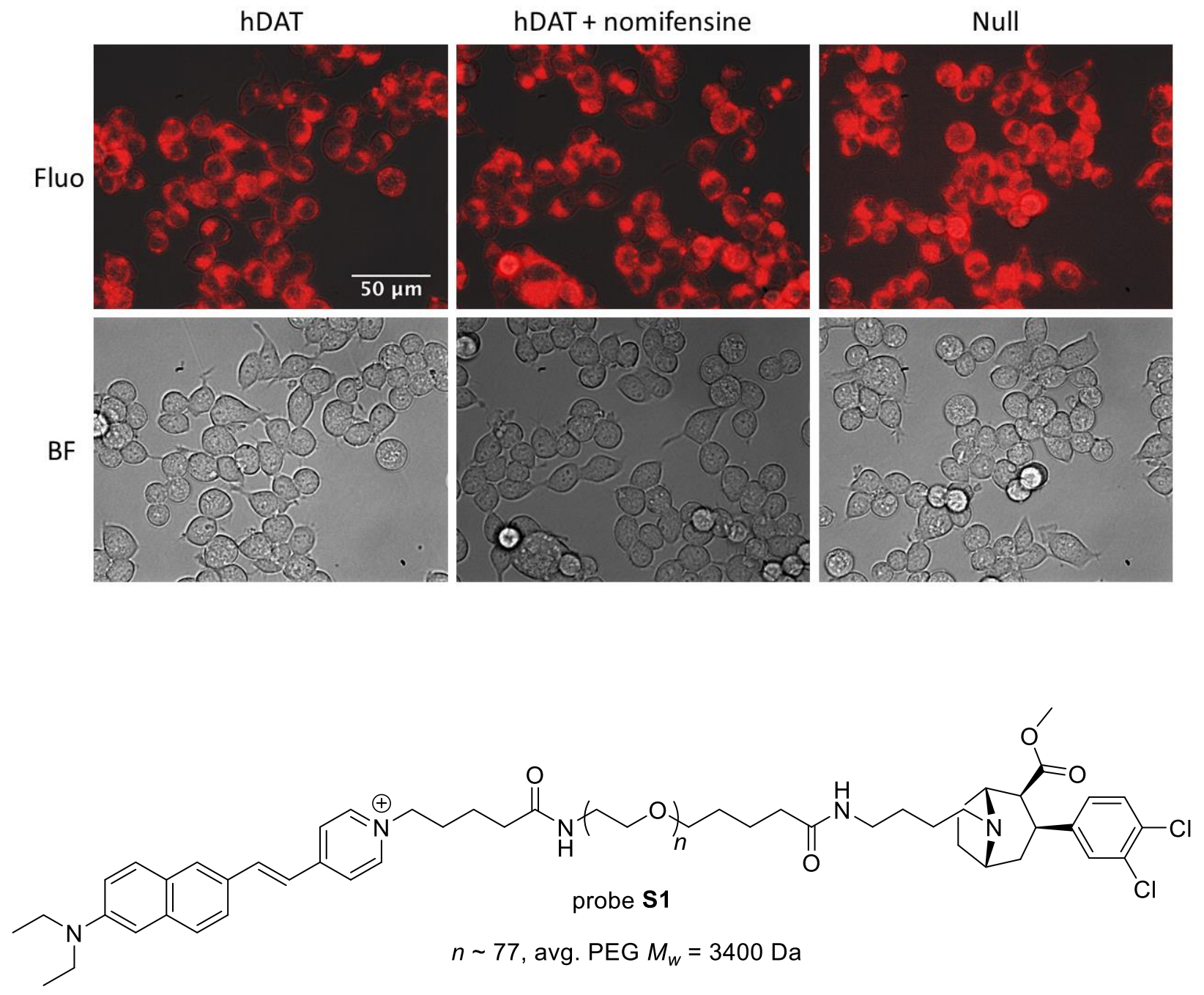

Figure S4. Dichloropane-PEG(3400)-di-2-ANEP (S1) labeling in HEK cells. Representative epifluorescence (Fluo) and brightfield (BF) images showing the labeling of dichloropane-PEG(3400)-di-2-ANEP (S1, $100 \mathrm{nM}$ ) in HEK cells following the same procedure as that for di-2-ANEP-VoLDeMo (12; see Fig. 4a). Labeling was compared in hDAT-HEK cells (left), hDAT-HEK cells pre-incubated with the hDAT inhibitor nomifensine ${ }^{1}(2 \mu \mathrm{M}$; middle), and null-transfected HEK cells (right). All fluorescence images are equally contrasted. PEG-based constructs carrying VSDs showed intracellular labeling independent of functional hDAT, in contrast to the dextran-based probes (see Fig. 4). Excitation filter: $480 \mathrm{~nm}$ (40 nm bandpass), $570 \mathrm{~nm}$ dichroic, emission filter: $590 \mathrm{~nm}$ (longpass). 


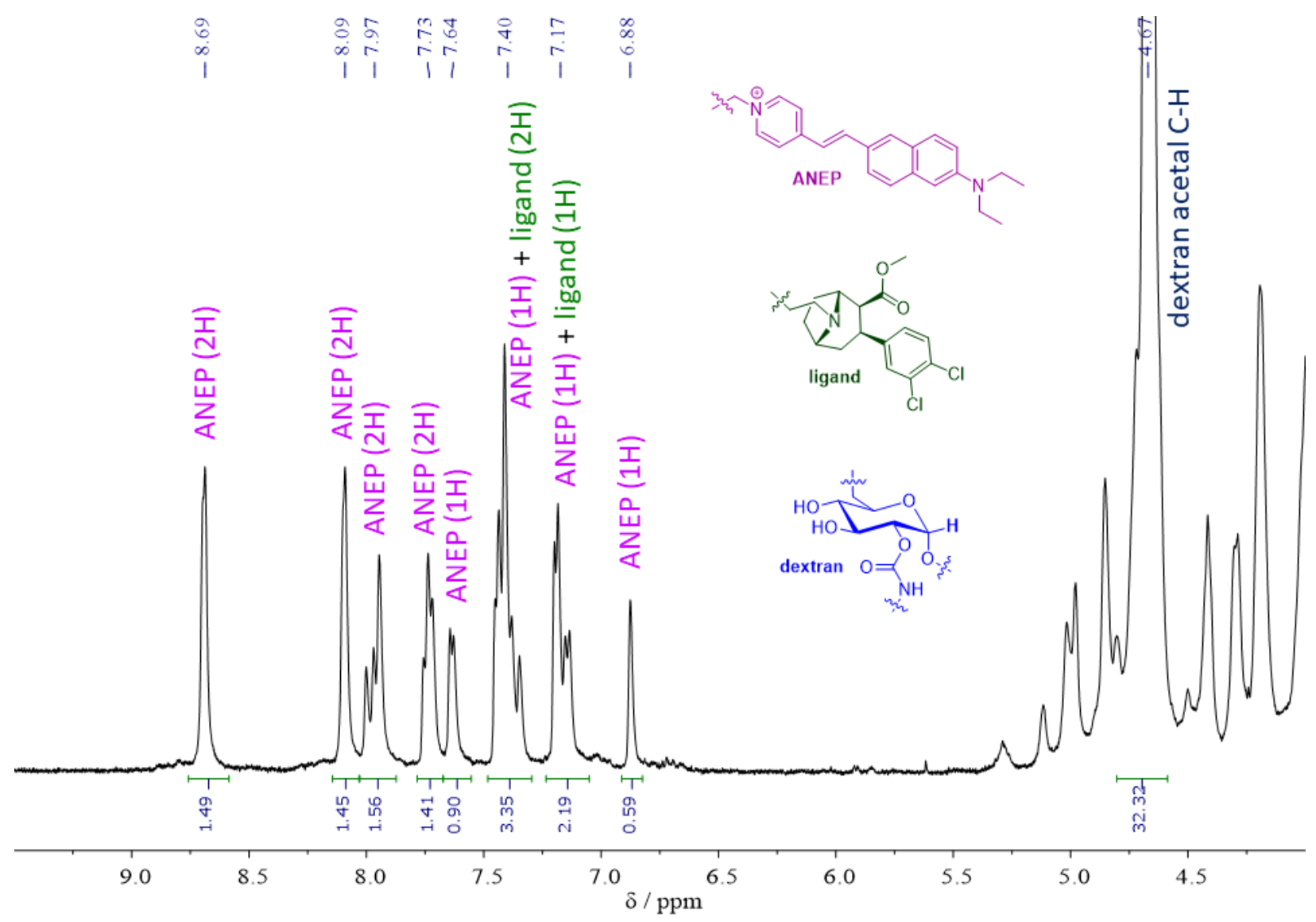

Figure S5. ${ }^{1} \mathrm{H}$ NMR $\left(500 \mathrm{MHz}\right.$; DMSO- $d_{6} / \mathrm{D}_{2} \mathrm{O}$ 5:1, v/v) characterization of di-2-ANEP-VoLDeMo (12). Relevant region of the spectrum shown; full spectrum is in Fig. S147. Coverage of the dextran carrier with ANEP units (0.74 ANEP/polymer) was obtained from the normalized average integration values of ANEP signals at $\delta$ $6.88,7.64,7.73,7.79,8.09$ and $8.69 \mathrm{ppm}$. Similarly, the coverage with dichloropane ligand units (1.38 ligand/polymer) was obtained from the 7.17 and $7.40 \mathrm{ppm}$ signals after subtraction of the average integration values of the ANEP units. See Dextran Chemistry section for a detailed discussion. 


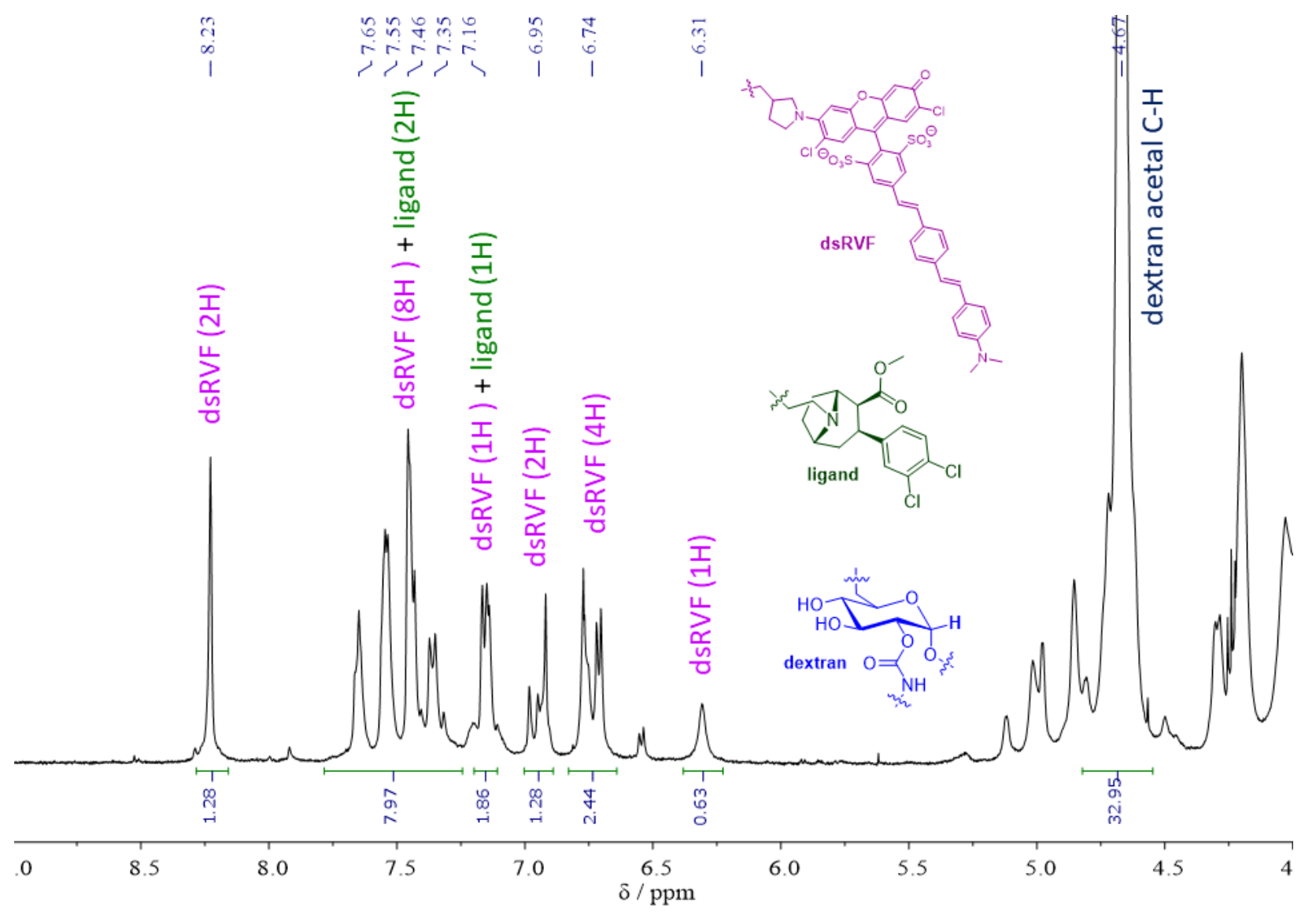

Figure S6. ${ }^{1} \mathrm{H}$ NMR $\left(500 \mathrm{MHz}\right.$; DMSO- $\left.d_{6} / \mathrm{D}_{2} \mathrm{O} 5: 1, v / v\right)$ characterization of dsRVF5-VoLDeMo (13) - sample 1. Relevant region of the spectrum shown; full spectrum is in Fig. S148. Coverage of the dextran carrier with dsRVF5 units ( $0.63 \mathrm{dsRVF5/polymer)} \mathrm{was} \mathrm{obtained} \mathrm{from} \mathrm{the} \mathrm{normalized} \mathrm{average} \mathrm{integration} \mathrm{values}$ of dsRVF5 signals at $\delta 6.31,6.74,6.95$ and $8.23 \mathrm{ppm}$. Similarly, the coverage with dichloropane ligand units (1.39 ligand/pol.) was obtained from the 7.16 and $7.35-7.65 \mathrm{ppm}$ signals after subtraction of the average integration values of the dsRVF5 units. See Dextran Chemistry section for a detailed discussion. 


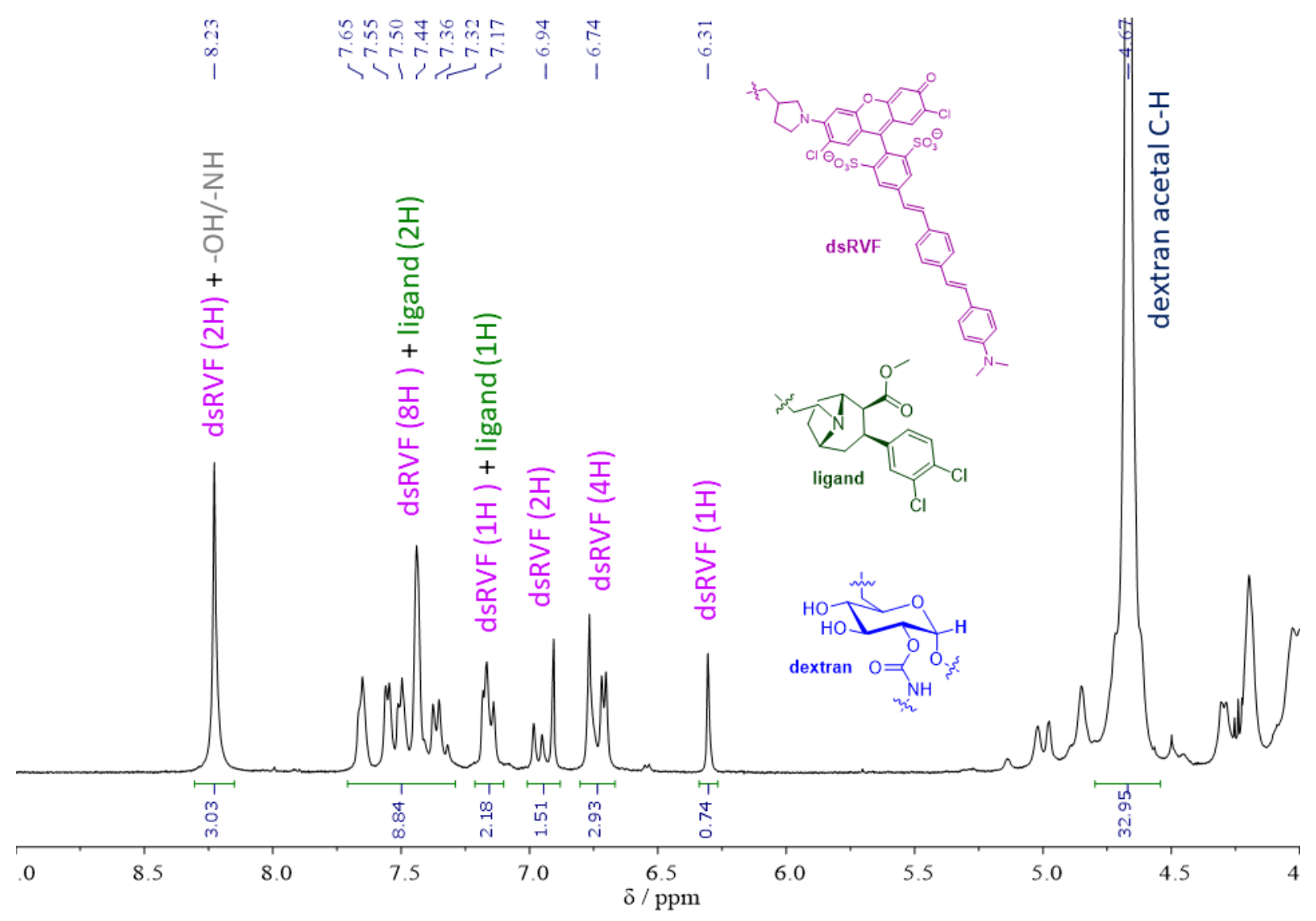

Figure S7. ${ }^{1} \mathrm{H}$ NMR $\left(500 \mathrm{MHz}\right.$; DMSO- $\left.d_{6} / \mathrm{D}_{2} \mathrm{O} 5: 1, v / v\right)$ characterization of dsRVF5-VoLDeMo (13) - sample 2. Relevant region of the spectrum shown; full spectrum is in Fig. S149. Coverage of the dextran carrier with dsRVF5 units ( 0.74 dsRVF5/polymer) was obtained from the normalized average integration values of dsRVF5 signals at $\delta 6.31,6.74$ and 6.94. Similarly, the coverage with dichloropane ligand units (1.45 ligand/pol.) was obtained from the 7.17 and $7.32-7.65$ ppm signals after subtraction of the average integration values of the dsRVF5 units. See Dextran Chemistry section for a detailed discussion. 
a

$\mathrm{D}_{2} \mathrm{O}$
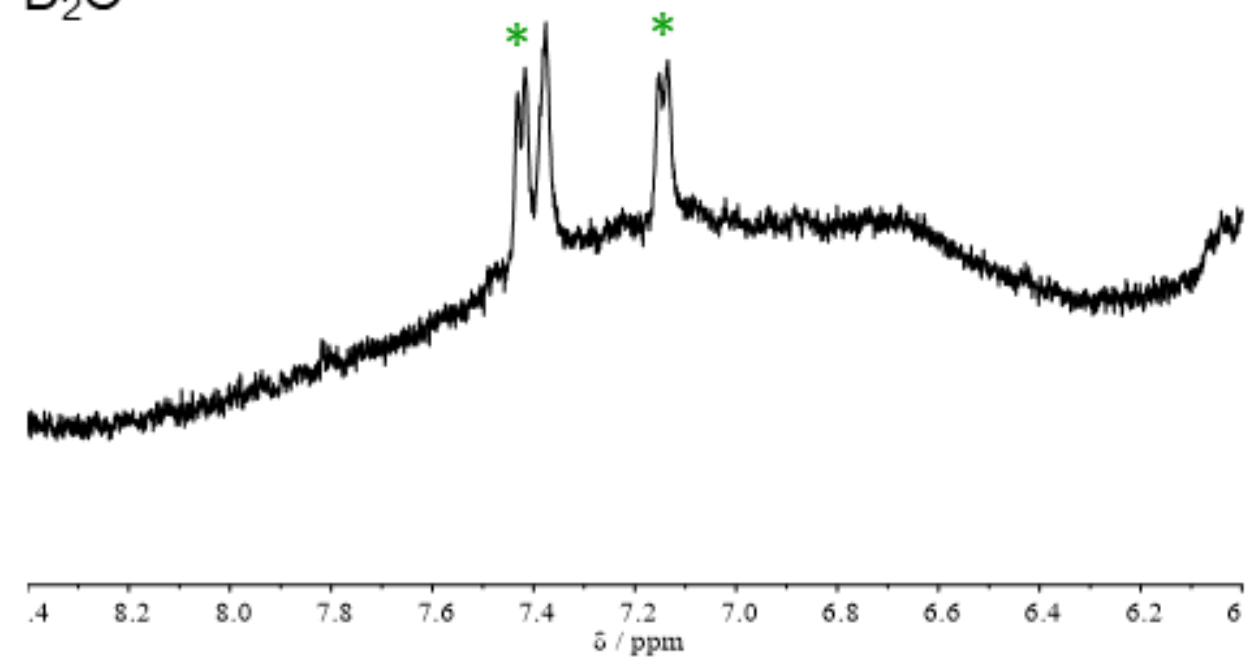

b

DMSO-d 6

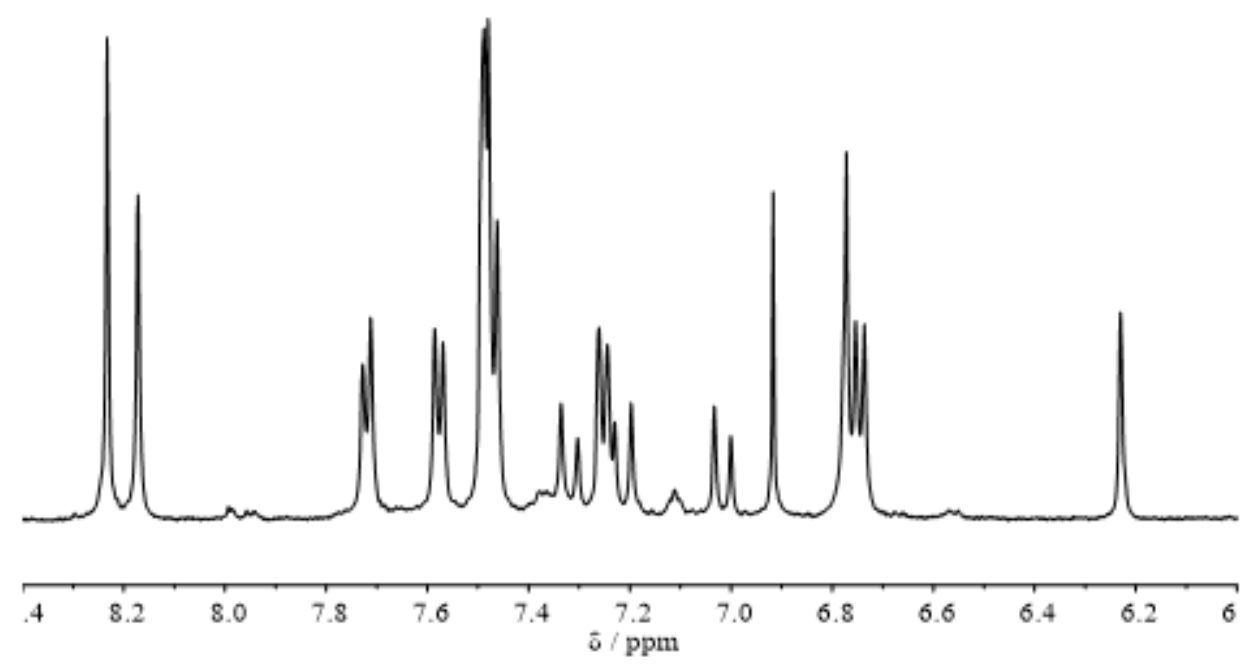

Figure S8. (a) ${ }^{1} \mathrm{H}$ NMR (500 MHz, $\left.\mathrm{D}_{2} \mathrm{O}\right)$ of dsRVF5-VoLDeMo (13), zoomed in aromatic region $(\delta 6.0-8.4 \mathrm{ppm})$. Broadened peaks reflect restrained conformational freedom of the lipophilic VSD which is shielded by the dextran carrier. Green asterisks - signals of the dichloropane ligand which are significantly sharper, reflecting more conformational freedom of the ligand. (b) ${ }^{1} \mathrm{H}$ NMR $\left(500 \mathrm{MHz}, \mathrm{DMSO}-d_{6}\right)$ of dsRVF5-VoLDeMo (13), zoomed in aromatic region $(\delta 6.0-8.4 \mathrm{ppm})$. Sharp peaks indicate high conformational flexibility of the construct in the more solubilizing environment of DMSO. 

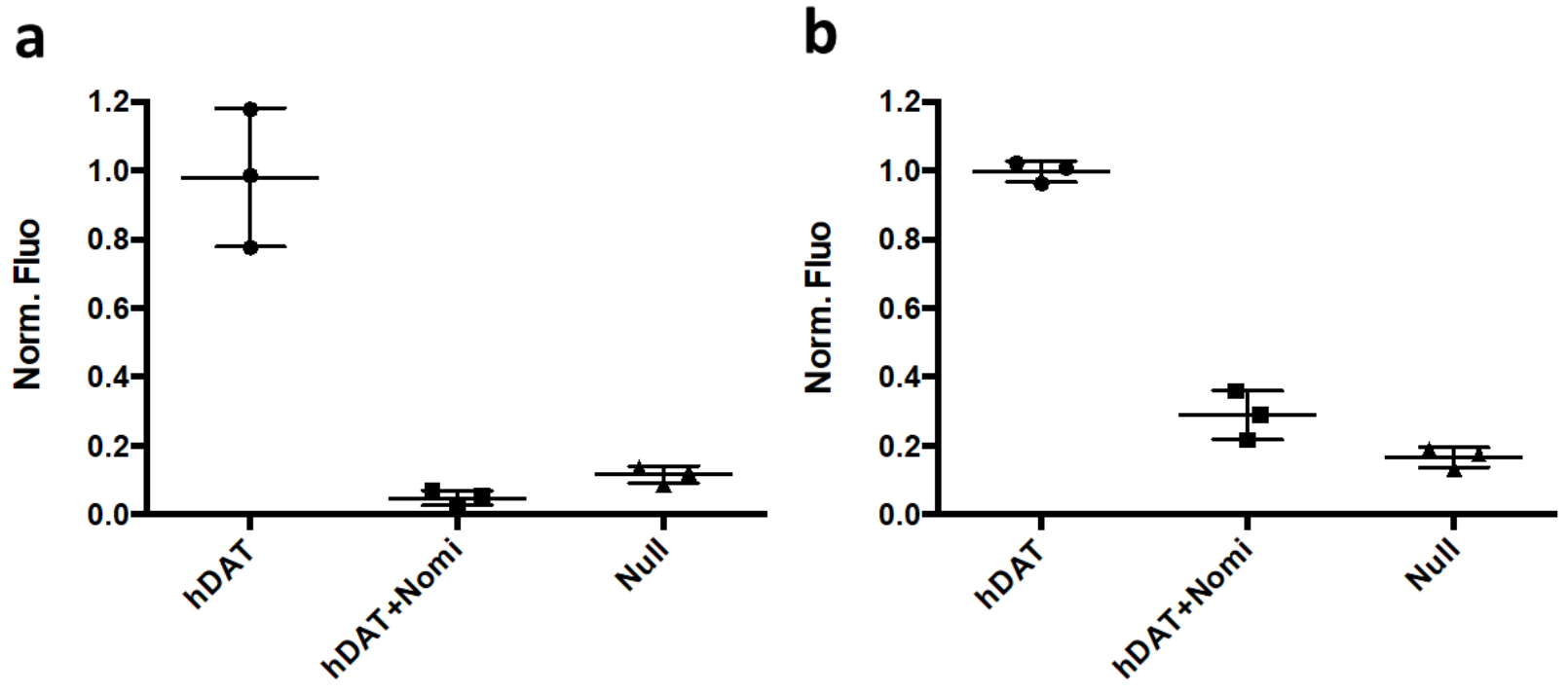

Figure S9. Quantification of VoLDeMo labeling in HEK cells. Mean fluorescence intensity of randomly selected cell bodies $(n=3,10$ ROIs per $n$; see Data Analysis section for details) stained with di-2-ANEP-VoLDeMo (12, $100 \mathrm{nM}$ dextran/74 nM VSD, panel (a) or dsRVF5-VoLDeMo (13, $100 \mathrm{nM}$ dextran/63 nM VSD, panel (b), normalized to the mean of the hDAT-transfected, non-inhibited condition (left panel in Fig. $4 a$ or $d$ ). Error bars are \pm SD for $n=30$ cells from 3 experiments. All from twotailed unpaired t-test: (a) $20.2 \pm 5.7$ fold decrease in labeling, $n=30$ cells from 3 experiments, $P=0.0013$ for hDAT+Nomi vs. hDAT; and $8.4 \pm 1.5$ fold decrease in labeling, $n=30$ cells from 3 experiments, $P=0.0018$ for Null vs. hDAT; (b) $3.4 \pm 0.5$ fold decrease in labeling, $n=30$ cells from 3 experiments, $P<0.0001$ for hDAT+Nomi vs. hDAT; and $6.0 \pm 0.6$ fold decrease in labeling, $n=30$ cells from 3 experiments, $P<0.0001$ for Null vs. hDAT). 

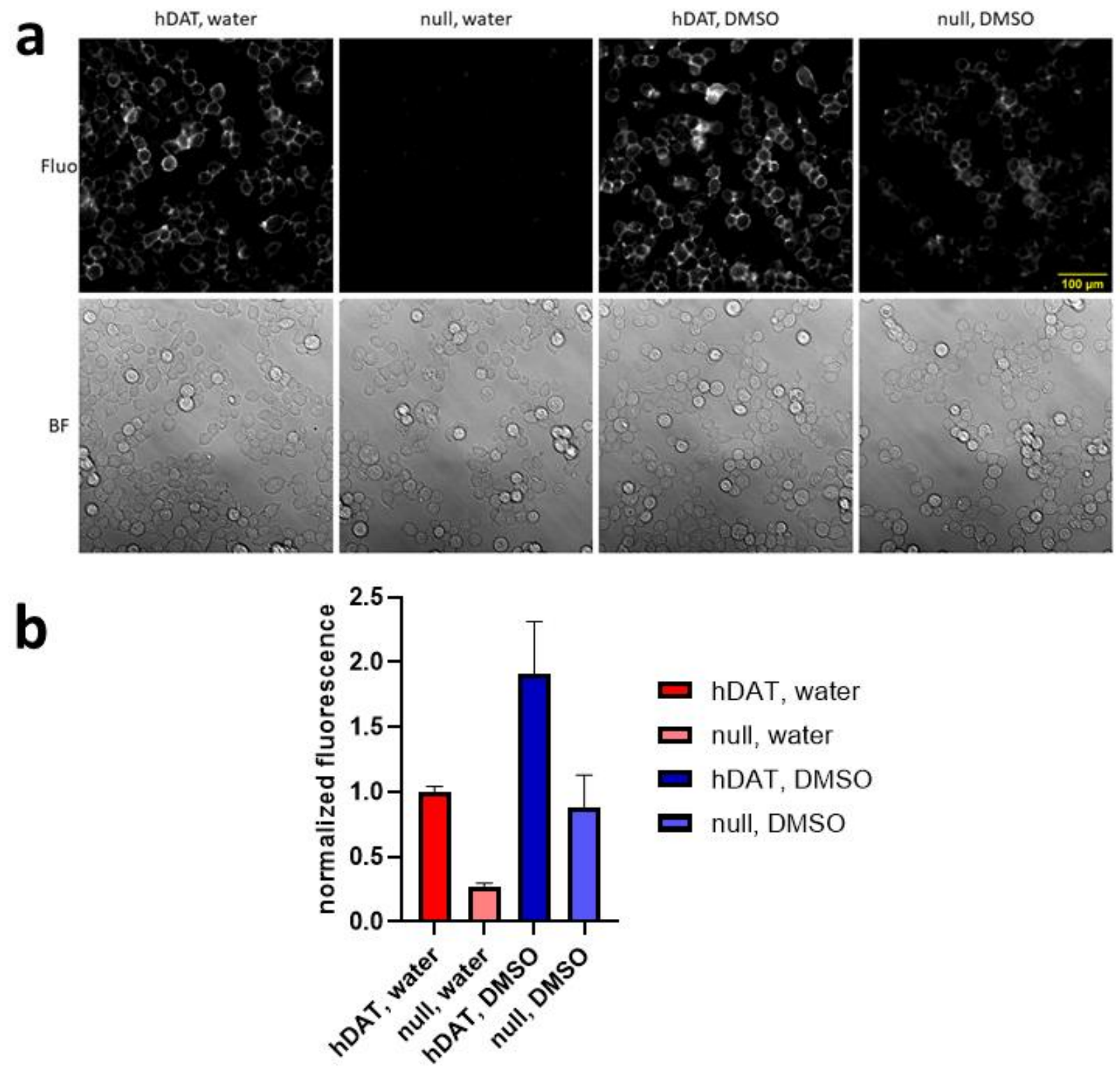

Figure S10. HEK cell labeling by dsRVF5-VoLDeMo using water and DMSO stock solutions. (a) Fluorescence (Fluo, top row) and corresponding brightfield (BF, bottom row) images of HEK cells labeled with dsRVF5-VoLDeMo (13) probes (68 nM dextran/50 nM VSD). Labeling was compared in hDAT-HEK cells and nulltransfected HEK cells; and with probe $\mathbf{1 3}$ added as a solution in water or DMSO (68 $\mu \mathrm{M}$ dextran/50 $\mu \mathrm{M} \mathrm{VSD}$ ). All fluorescence images are equally contrasted. (b) Mean fluorescence intensity of randomly selected cell bodies ( $n=3,5$ ROIs per $n$; see Data Analysis section for details) stained with dsRVF5-VoLDeMo (13) as described in (a), normalized to the mean of the hDAT, water condition (left panel in a). Error bars are \pm SEM for $n=3$ experiments, 5 cells per $n$. 


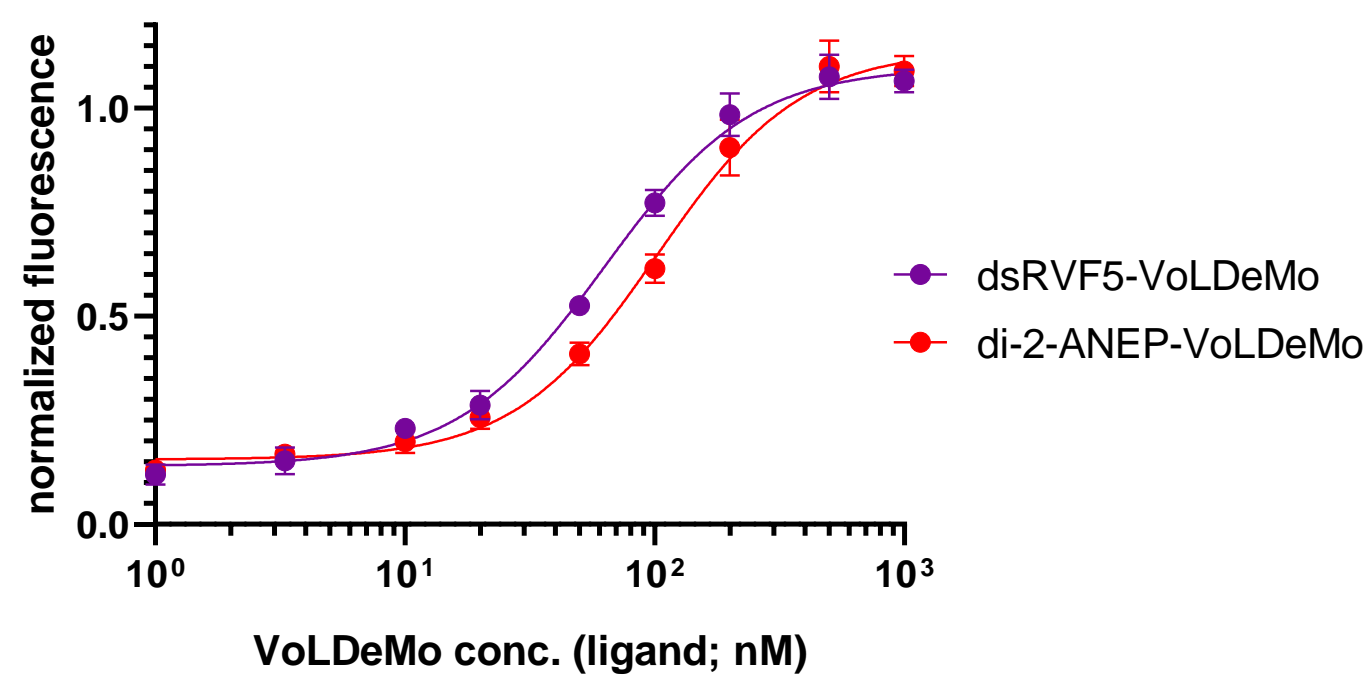

Figure S11. Determination of $I C_{50}$ of VoLDeMo probes 12 and 13 to hDAT with APP+ uptake. Non-linear regression (1:1 binding model) showing the dose-dependent inhibition of APP+ uptake by di-2-ANEP-VoLDeMo (12; red) and dsRVF5-VoLDeMo (13; purple) in hDAT-HEK cells (see Biological Characterizations section for details). Concentration values on the $x$-axis are normalized to the dichloropane ligand on VoLDeMo. Error bars are \pm SEM for $n=4$ experiments, 2 replicates per independent experiment (error bars of some data points are too small to display). 

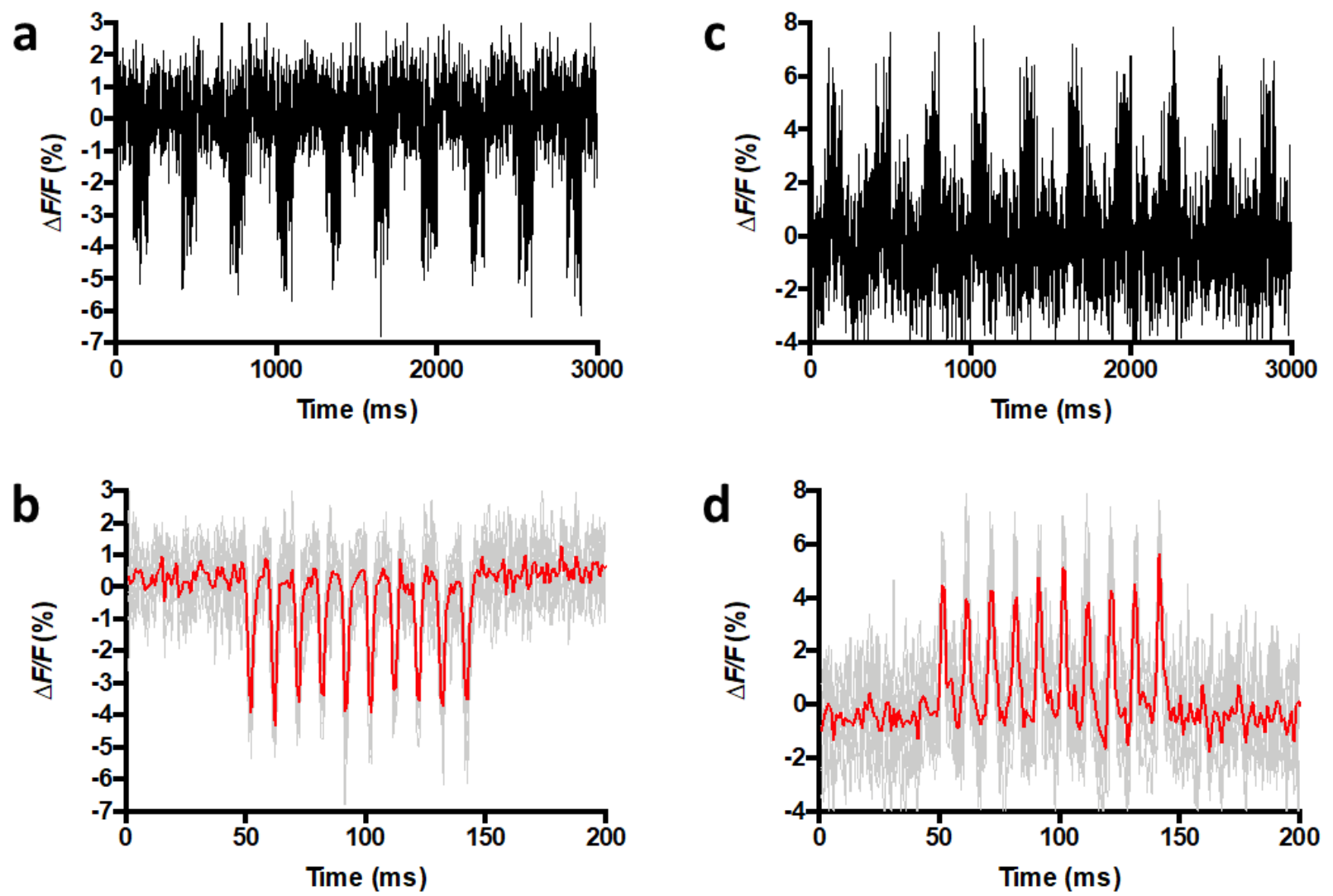

Figure S12. Whole-cell voltage clamp of hDAT-HEK cells with simulated trains of spiking events. Cells were held at $-60 \mathrm{mV}$ and subjected to bursts of ten individual voltage spikes of $2 \mathrm{~ms}$ length and $100 \mathrm{mV}$ magnitude. The ten voltage spikes within one burst were applied at $100 \mathrm{~Hz}$ in frequency. The fluorescence data were corrected for photobleaching and the mean fluorescence intensity converted to percent $\Delta F / F$ (see Data Analysis section for details). Raw single-trial imaging traces of di-2-ANEP-VoLDeMo $\quad(\mathbf{1 2}, 100 \mathrm{~nm}$ dextran/74 nM VSD, panel (a) and dsRVF5-VoLDeMo (13, $100 \mathrm{~nm}$ dextran/63 nM VSD, panel (c), respectively. Ten simulated bursts within (a) and (c) were overlaid to give (b) and (d) respectively, including $50 \mathrm{~ms}$ before and after the stimulation period. The traces of ten individual bursts are shown in gray, and their average is represented by the red trace. 

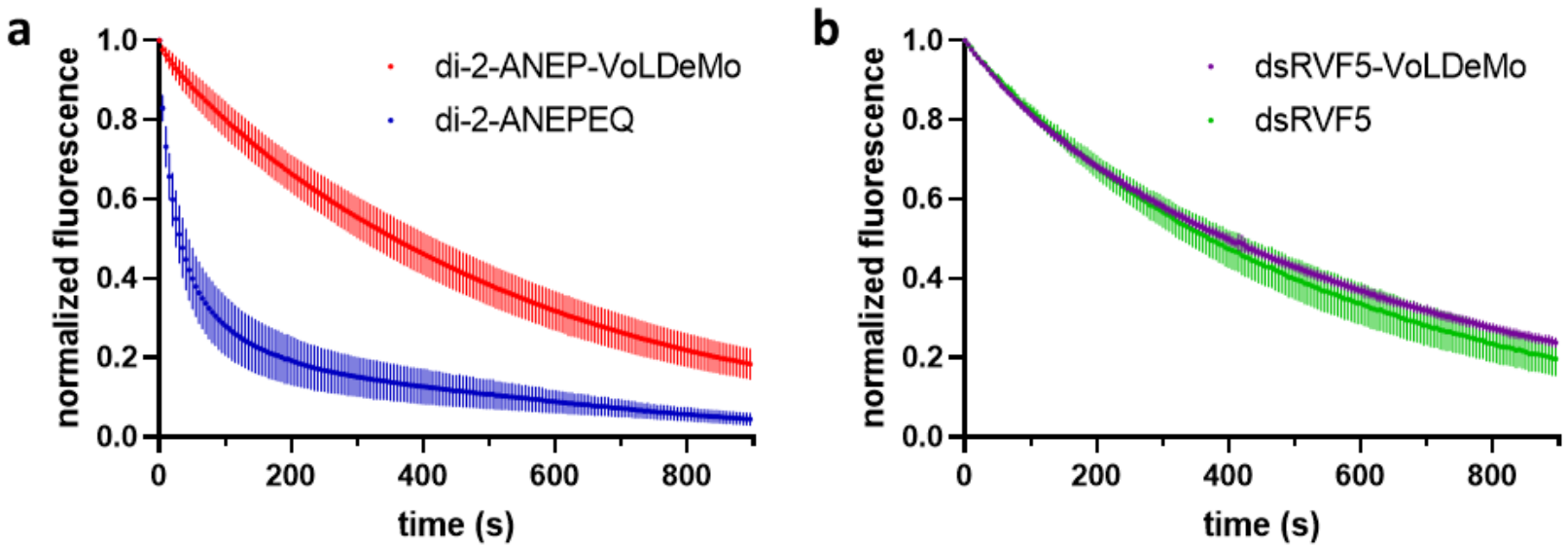

Figure S13. Comparison of photostability of untethered VSDs 1 and 2, and the respective VoLDeMo probes 12 and 13 in hDAT-HEK cells. hDAT-HEK cells were loaded with $500 \mathrm{~nm}$ untethered probes $\mathbf{1}$ or $\mathbf{2}$, or $100 \mathrm{nM}$ (with respect to dextran) VoLDeMo probes 12 or 13 and then continuously illuminated (15 min) with a xenon lamp using a $450 / 50 \mathrm{~nm}$ excitation filter (di-2-ANEP probes 1 and 12) or $540 / 25 \mathrm{~nm}$ excitation filter (dsRVF5 probes $\mathbf{2}$ and 13). Fluorescence images (emission filters: $580 / 40 \mathrm{~nm}$ for 1 and $\mathbf{1 2} ; 605 / 55 \mathrm{~nm}$ for $\mathbf{2}$ and 13) were collected at $5 \mathrm{~s}$ intervals. The normalized fluorescence intensities were plotted vs time. Error bars are \pm SD for $n=4$ independent experiments. 
a
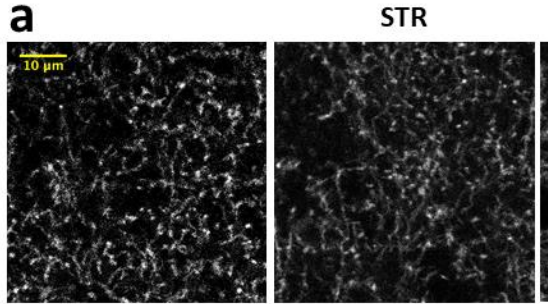

di-2-ANEP-VoLDeMo

b

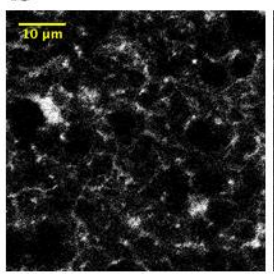

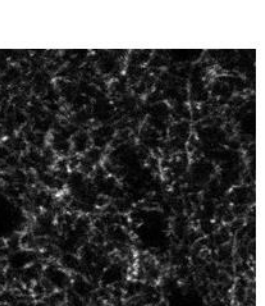

(

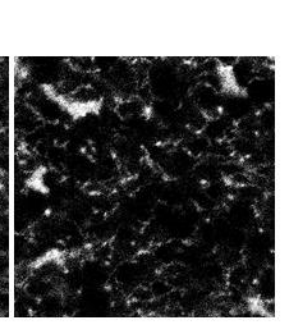

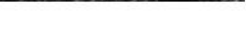

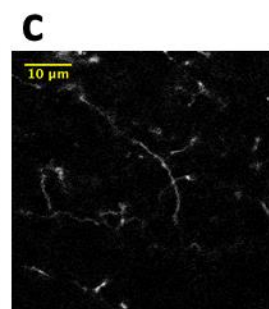

di-2-ANEP-VoLDeMo

d
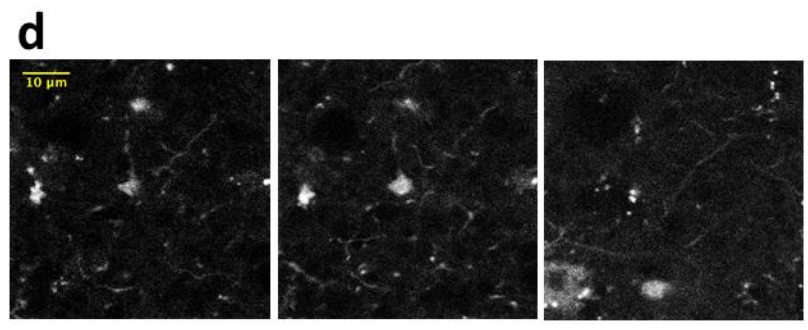

dsRVF5-VoLDeMo

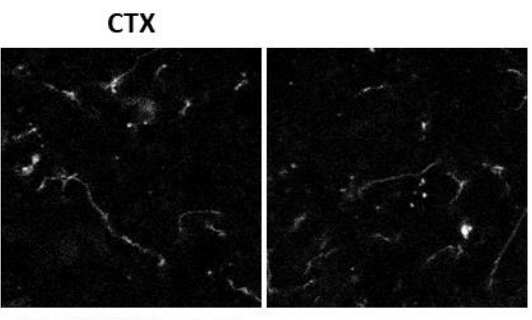

dsRVF5-VoLDeMo

Figure S14. Additional representative images of VoLDeMo labeling of catecholamine axons in acute murine brain slices. Images were obtained following the same procedure as that used for Fig. 5. Images in the same lettered panel were three replicates acquired under the same conditions. Each image was contrasted individually. (a) Striatal slices labeled with di-2-ANEP-VoLDeMo (12). (b) Striatal slices labeled with dsRVF5-VoLDeMo (13). (c) Cortical slices labeled with di-2-ANEP-VoLDeMo (12). (d) Cortical slices labeled with dsRVF5-VoLDeMo (13). 


\section{a}

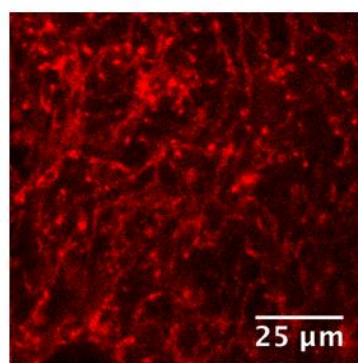

di-2-ANEP-VoLDeMo

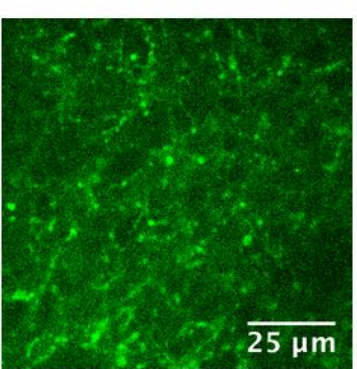

FFN102

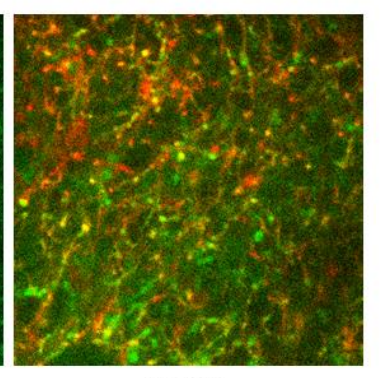

merge b

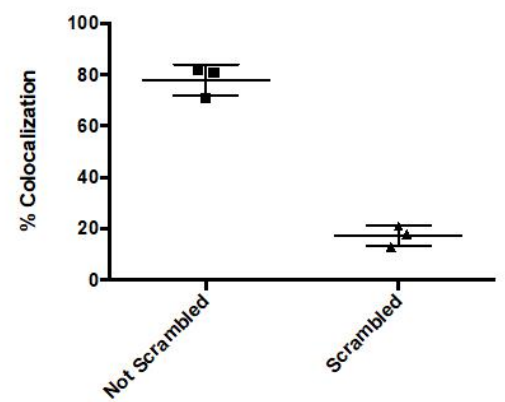

Figure S15. Colocalization of di-2-ANEP-VoLDeMo labeling with that of FFN102. (a) Representative images showing colocalization of di-2-ANEP-VoLDeMo (12, $100 \mathrm{nM}$ dextran/74 nM VSD, $30 \mathrm{~min}, 15 \mu \mathrm{m}$ depth from tissue surface) and FFN102 ( $2 \mu \mathrm{M}, 30 \mathrm{~min})$, a known marker of dopaminergic synapses ${ }^{2}$ in mouse striatal slice (see Fig. 5a). Left: di-2-ANEP-VoLDeMo (12) channel (exc. $920 \mathrm{~nm}$, em. 570-610 nm); middle: FFN102 channel (exc. $760 \mathrm{~nm}$, em. 435-485 nm); right: overlaid image of the two channels. (b) Quantification of di-2-ANEP-VoLDeMo (12) colocalization with FFN102. The "Not Scrambled" column represents the percentage of di-2-ANEP-VoLDeMo (12) signal that colocalized with FFN102 puncta from the same field of view. The "Scrambled" column represents analogical colocalization of randomly selected di-2-ANEP-VoLDeMo (12) and FFN102 figures from different fields of view (see Data Analysis section for details). Error bars are \pm SD for $n=45$ images from 9 slices from 3 animals. The "Not Scrambled" images gave higher level of colocalization $(78.0 \% \pm 3.51 \%)$ than the "Scrambled" control $(17.3 \% \pm 2.33 \%$, $\mathrm{P}<0.0001$, two-tailed unpaired t-test). 


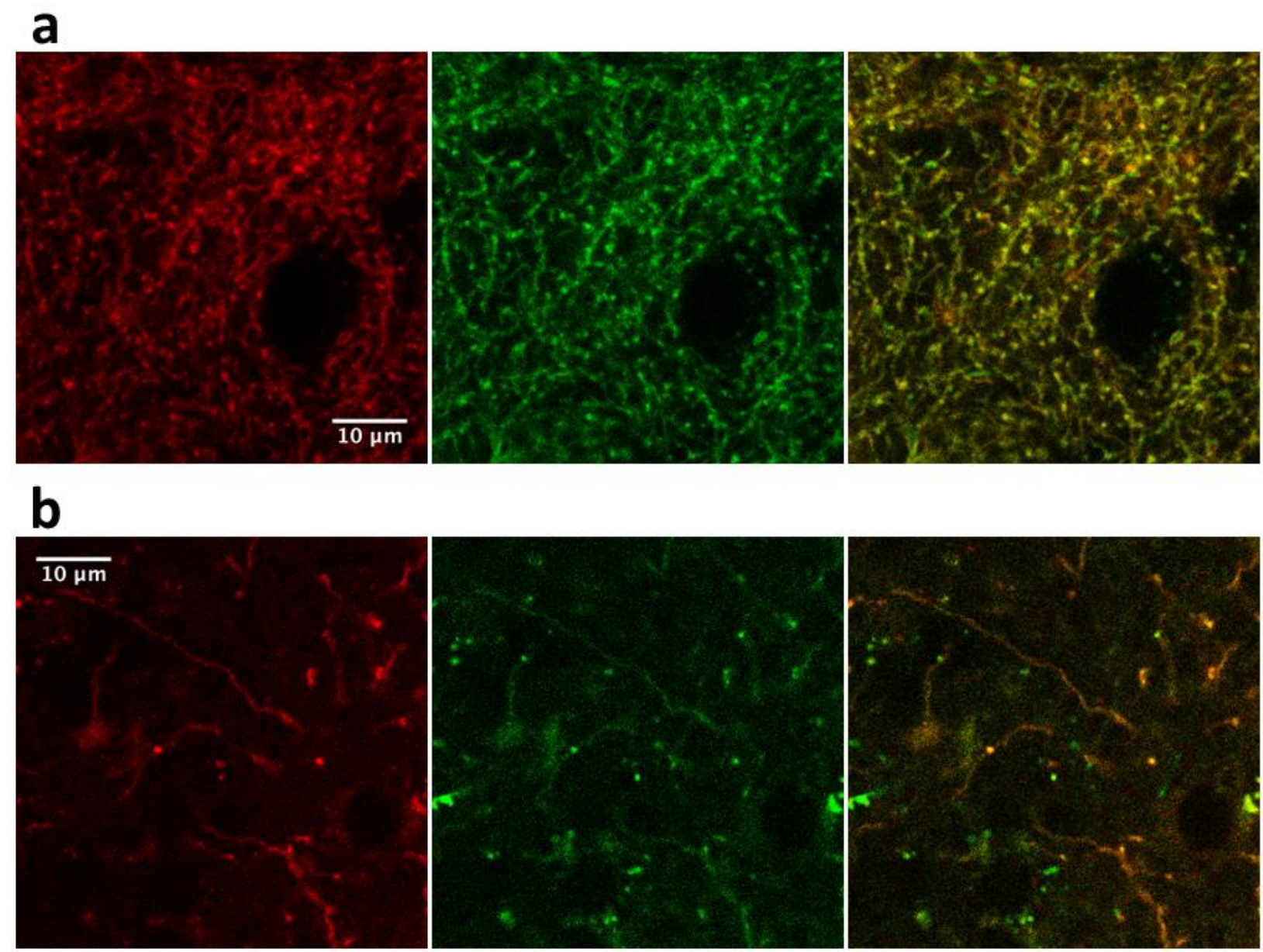

Figure S16. Colocalization of di-2-ANEP-VoLDeMo labeling with that of THGFP. Representative images showing colocalization of di-2-ANEP-VoLDeMo (12, $100 \mathrm{nM}$ dextran/74 nM VSD, $30 \mathrm{~min}, 15 \mu \mathrm{m}$ depth from tissue surface) and TH-driven GFP in mouse striatum (a; see Fig. 5a) or cortex (b; see Fig. 5d). Left: di-2-ANEP-VoLDeMo (12) channel (exc. 920 nm, em. 570-610 nm); middle: GFP channel (exc. $760 \mathrm{~nm}$, em. 440-500 nm); right: overlaid image of the two channels. 

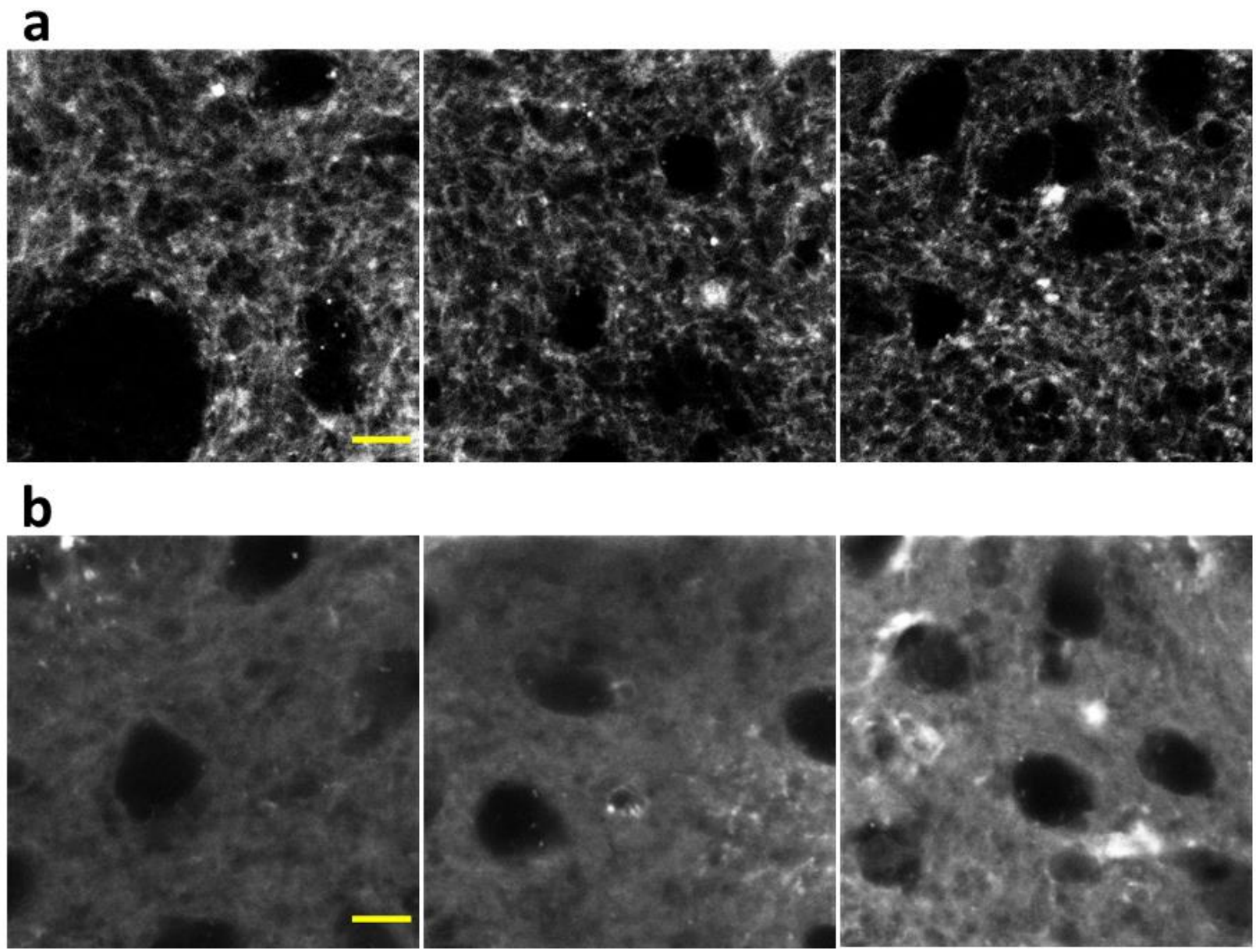

Figure S17. Representative two-photon microscopy images of striatal slices labeled with dsRVF5-VoLDeMo (13; $676 \mathrm{~nm}$ dextran/500 nM VSD, $30 \mathrm{~min}, 35 \mu \mathrm{m}$ depth from the tissue surface). (a) Without addition of an inhibitor. (b) Slices were pre- and co-incubated with the DAT/NET blocker nomifensine $(2 \mu \mathrm{M})$. Probes were excited at $820 \mathrm{~nm}$ and emission collected at 570-610 $\mathrm{nm}$. Scale bars are $50 \mu \mathrm{m}$. 
a

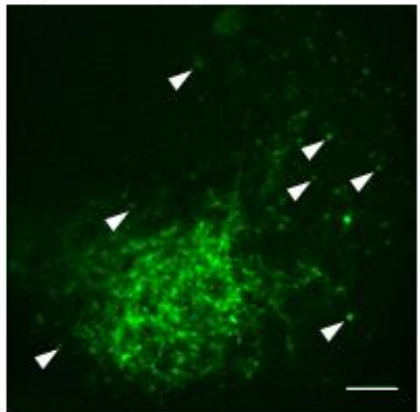

ArcLight

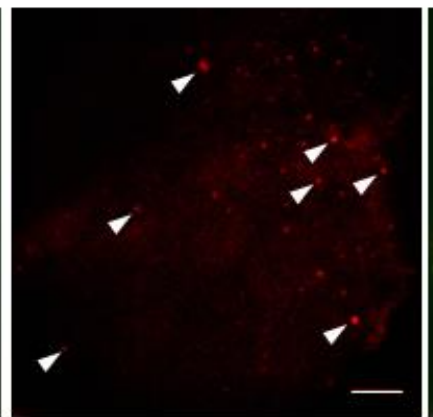

di-2-ANEP-VoLDeMo

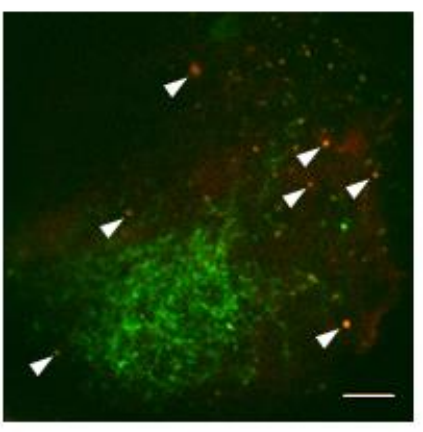

merge

b
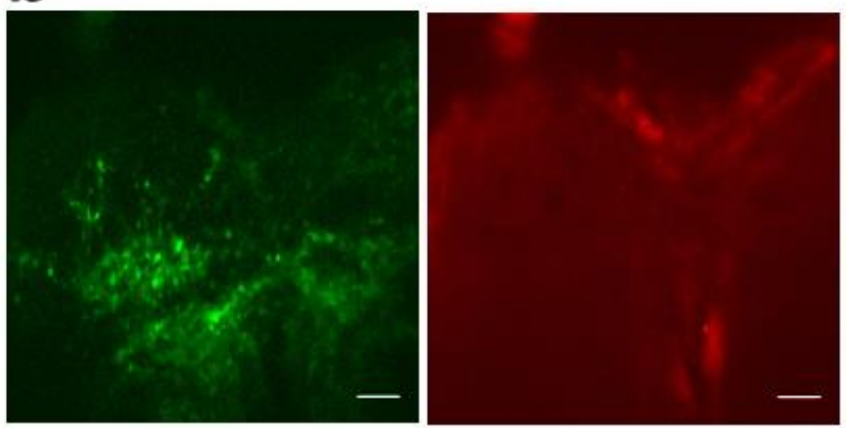

di-2-ANEP-VoLDeMo + $\mathbf{5}$

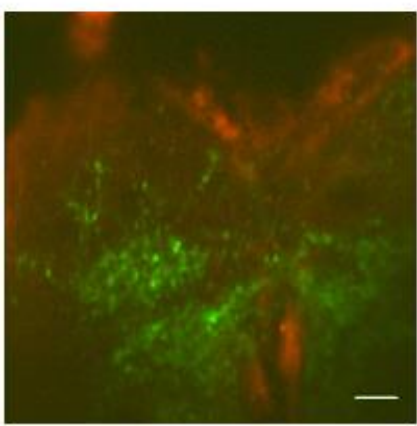

ArcLight

C

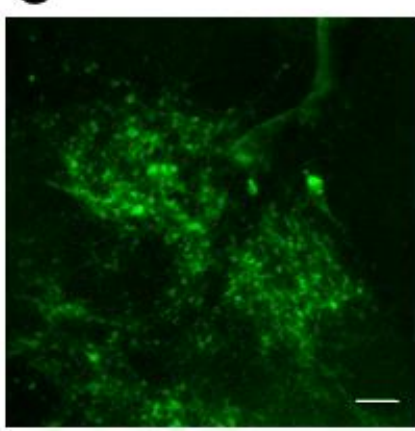

ArcLight

d

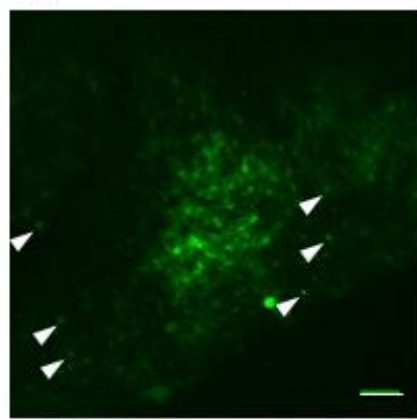

ArcLight

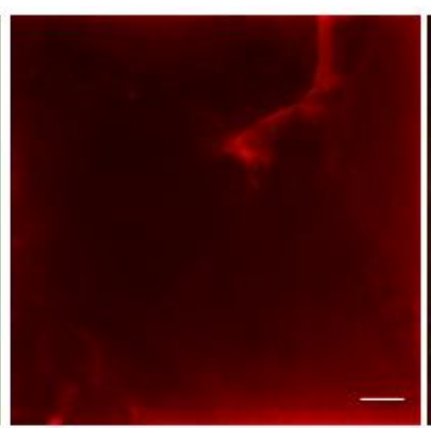

di-2-ANEPEQ (1)

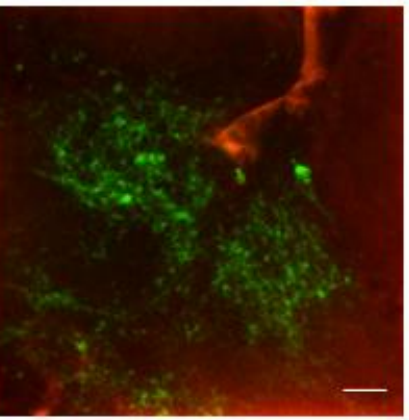

merge

merge 
Figure S18. Targeting of VoLDeMo probes in whole Drosophila brains. All panels: Excitation wavelength: $920 \mathrm{~nm}$. ArcLight (left, emission collected at 500-550 nm); the respective synthetic VSD (middle, emission collected at 572-642 $\mathrm{nm}$ ); merged left and middle images (right). All images are zoomed in to the suboesophageal ganglion (SOG). The scale bars are $10 \mu \mathrm{m}$. (a) A TH-driven ArcLight-expressing fly brain incubated with di-2-ANEP-VoLDeMo (12, $100 \mathrm{nM}$ dextran/74 nM VSD, $30 \mathrm{~min})$ resulted in punctate staining with partial overlap with ArcLight-labeled dopamine axonal varicosities (representative image; $n=4$ brains). Examples of co-localizing puncta are highlighted with white arrowheads. (b) A TH-driven ArcLight fly brain incubated with di-2-ANEP-VoLDeMo (12, $100 \mathrm{~nm}$ dextran/74 nM VSD, $30 \mathrm{~min})$ and pre- and co-incubated with dichloropane derivative $\mathbf{5}$ (targeting ligand of the construct, $2 \mu \mathrm{M}, 30 \mathrm{~min}$ pre-incubation) as a competing ligand resulted only in background staining without any punctate character or overlap with ArcLight localized to dopamine terminals (representative image; $n=3$ brains). (c) A TH-driven ArcLight fly brain incubated with untethered di-2-ANEPEQ $(1,100 \mathrm{nM}, 30 \mathrm{~min})$ resulted in nonspecific staining without any punctate character or overlap with ArcLight (representative image; $n=3$ brains). (d) A TH-driven ArcLight fly brain incubated with dsRVF5-VoLDeMo (13, $100 \mathrm{nM}$ dextran/63 nM VSD, $30 \mathrm{~min})$ resulted in punctate staining with partial overlap with ArcLight (representative image; $n=3$ brains). Examples of co-localizing puncta are highlighted with white arrowheads. 

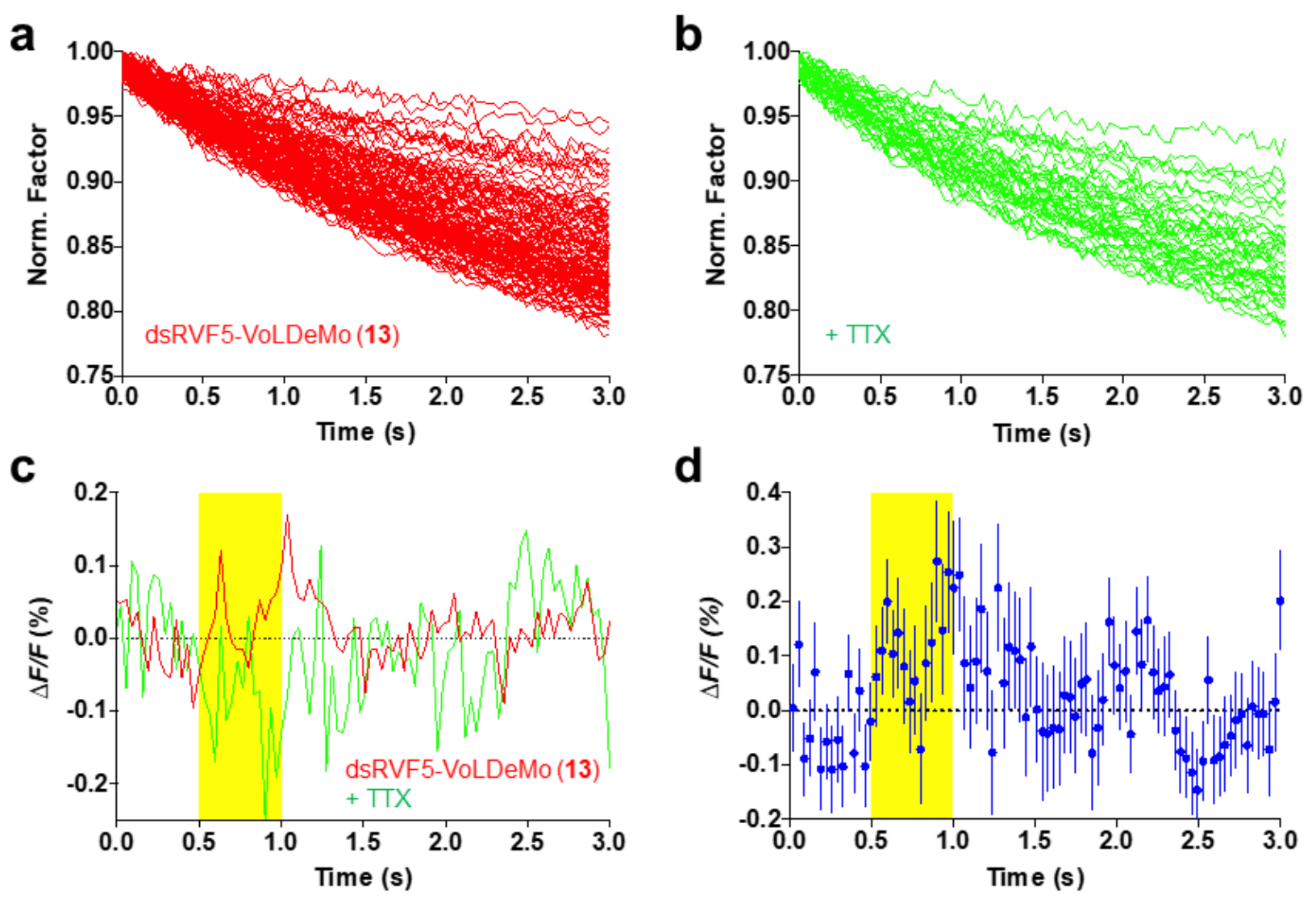

Figure S19. Two-photon fluorescence traces recorded by spiral scanning at $30 \mathrm{~Hz}$ from striatal slices labeled with dsRVF5-VoLDeMo $(13 ; 676 \mathrm{nM}$ dextran/500 nM VSD, $30 \mathrm{~min}$ ) with electric stimulation ( 25 pulses at $50 \mathrm{~Hz}$, period highlighted in yellow), with and without TTX (1 $\mu \mathrm{M})$ inhibition. The probe was excited at $820 \mathrm{~nm}$ and emission collected at 570-610 nm. (a, b) Normalized raw traces obtained for dsRVF5-VoLDeMo (a, $n=100)$ and dsRVF5-VoLDeMo + TTX inhibition (b, $n=50$ ). (c) Averaged traces after bleaching correction (two phase decay function; GraphPad Prism 8 software, San Diego, CA) was applied to each individual raw trace from panels (a) and (b) using $t=0.0$ to $0.5 \mathrm{~s}$ and 2.0 to $3.5 \mathrm{~s}$. (d) Subtracted traces from panel (c): dsRVF5-VoLDeMo (red in c) minus dsRVF5-VoLDeMo + TTX inhibition (green in c). This trace corresponds to Fig. $6 c$ in the manuscript with an expanded $x$-axis and without applying a box filter. 

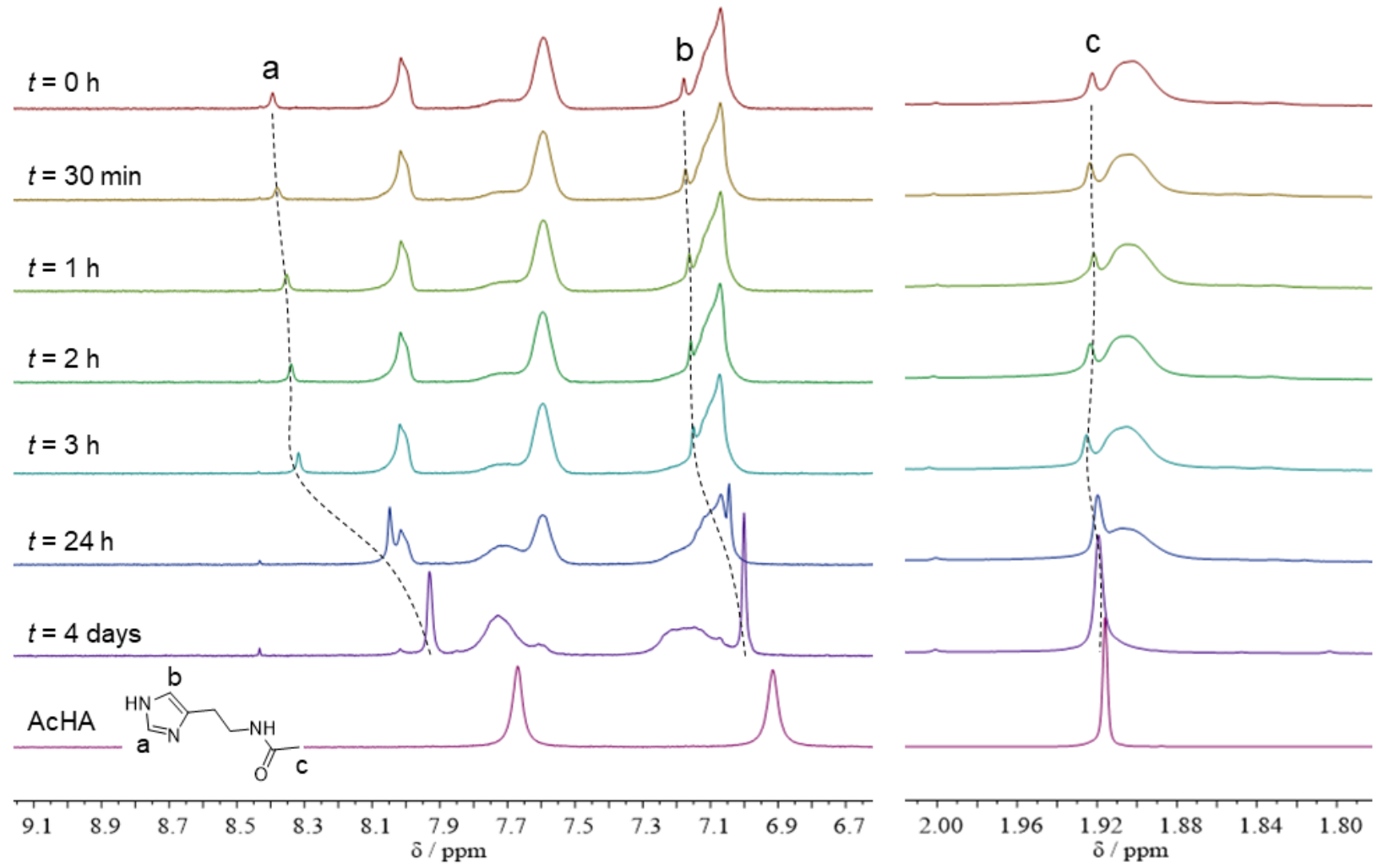

Figure S20. ${ }^{1} \mathrm{H}$ NMR $\left(500 \mathrm{MHz} ; \mathrm{D}_{2} \mathrm{O} ; c=11 \mathrm{mM}\right)$ monitoring of the stability of $17 \mathrm{in}$ $\mathrm{D}_{2} \mathrm{O}$ (diagnostic regions $\delta 1.8$ to $2.0 \mathrm{ppm}$ and $\delta 6.7$ to $9.1 \mathrm{ppm}$ ). The first spectrum $(t=0 \mathrm{~h})$ was taken $\sim 10$ min after dissolution in $\mathrm{D}_{2} \mathrm{O}$. Dotted lines are a visual aid indicating the gradually forming decomposition product $-\omega-N$-acetylhistamine (AcHA).

Table S1. Percent AcHA formation at individual time points.

$\begin{array}{cc}\text { time (h) } & \text { \% } \mathbf{A c H A}^{\mathrm{a}} \\ 0.0 & 14.0^{\mathrm{b}} \\ 0.5 & 14.2 \\ 1.0 & 15.8 \\ 2.0 & 15.0 \\ 3.0 & 16.3 \\ 24.0 & 39.5 \\ 96.0 & \sim 100\end{array}$

\footnotetext{
${ }^{\mathrm{a}}$ Determined from the integration values of signal $c$ and the overall acetyl group integral in Fig. S2O.

${ }^{\mathrm{b}}$ A significant amount of AcHA was already present at $t=0$. Some decomposition likely occurred in the solid state before dissolving the sample in water.
} 

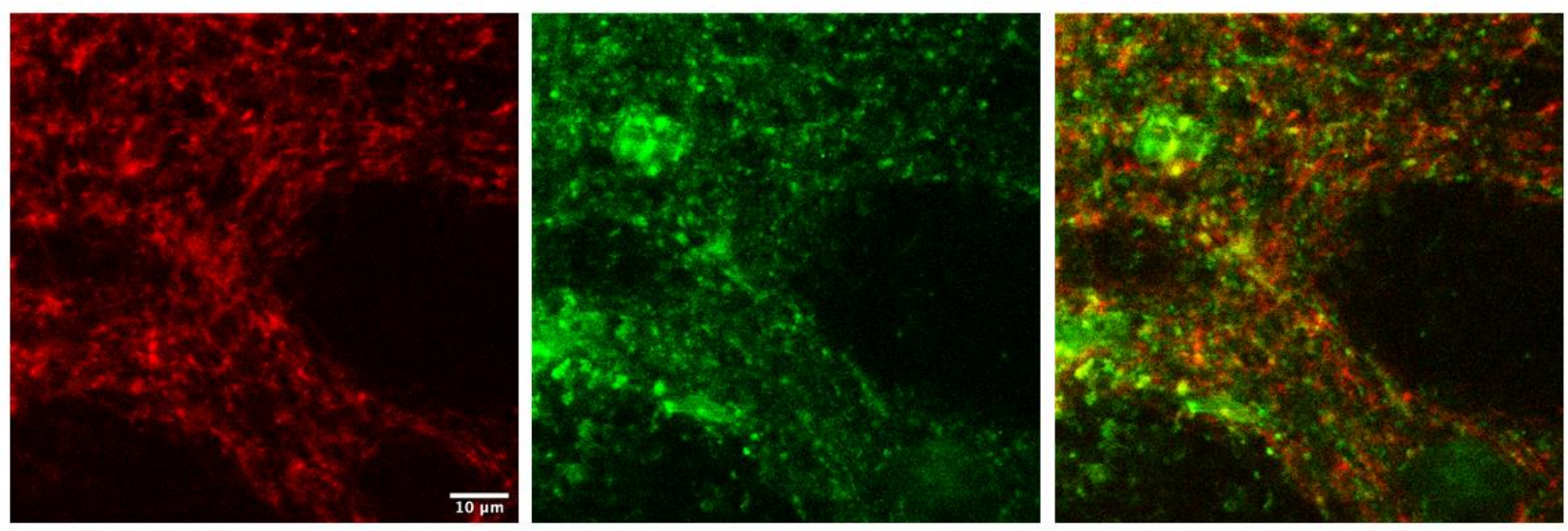

Figure S21. Dual-labeling of striatal slices with di-2-ANEP-VoLDeMo and CAM2(Fl). Representative images showing labeling of di-2-ANEP-VoLDeMo (12, $100 \mathrm{nM}$ dextran/74 nM VSD, 30 min, $15 \mu \mathrm{m}$ depth from tissue surface) and CAM2(Fl) ${ }^{3}$ $(1 \mu \mathrm{M}, 60 \mathrm{~min})$ in mouse striatum. Left: di-2-ANEP-VoLDeMo (12) channel (exc. $920 \mathrm{~nm}$, em. 570-610 nm); middle: CAM2(Fl) channel (exc. $760 \mathrm{~nm}$, em. 440$500 \mathrm{~nm}$ ); right: overlaid image of the two channels. 


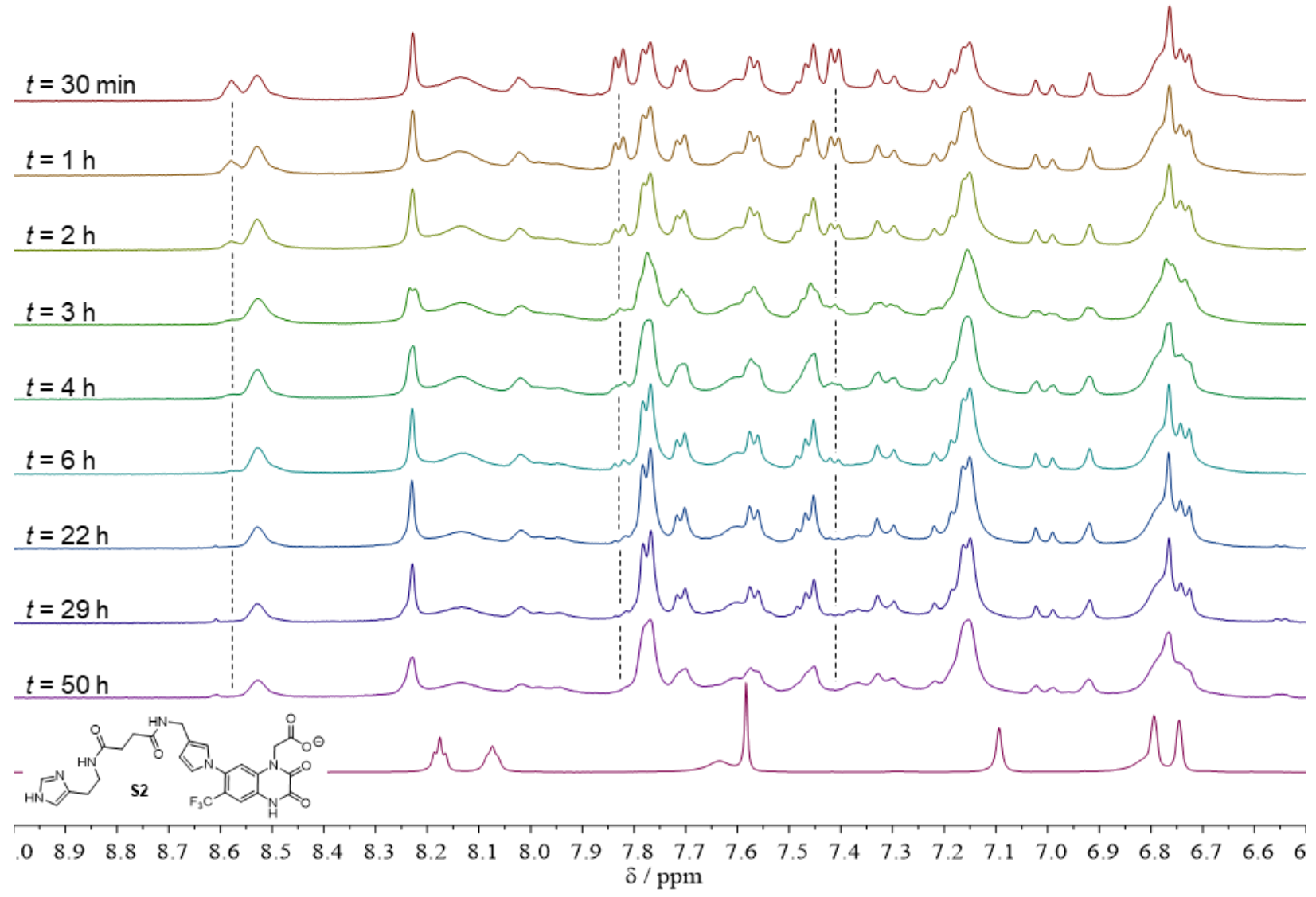

Figure S22. ${ }^{1} \mathrm{H}$ NMR (500 MHz; DMSO- $\left.d_{6}\right)$ monitoring of dsRVF5-VoLDeCAM (21) synthesis (closeup of the aromatic region $\delta 6.5$ to $9.0 \mathrm{ppm}$ ). Dotted lines are visual aids highlighting the gradual decrease in intensity of selected starting material signals. The bottom spectrum represents PFQX derivative S2, the expected product of acyl imidazole decomposition. We did not observe signs of such decomposition. 


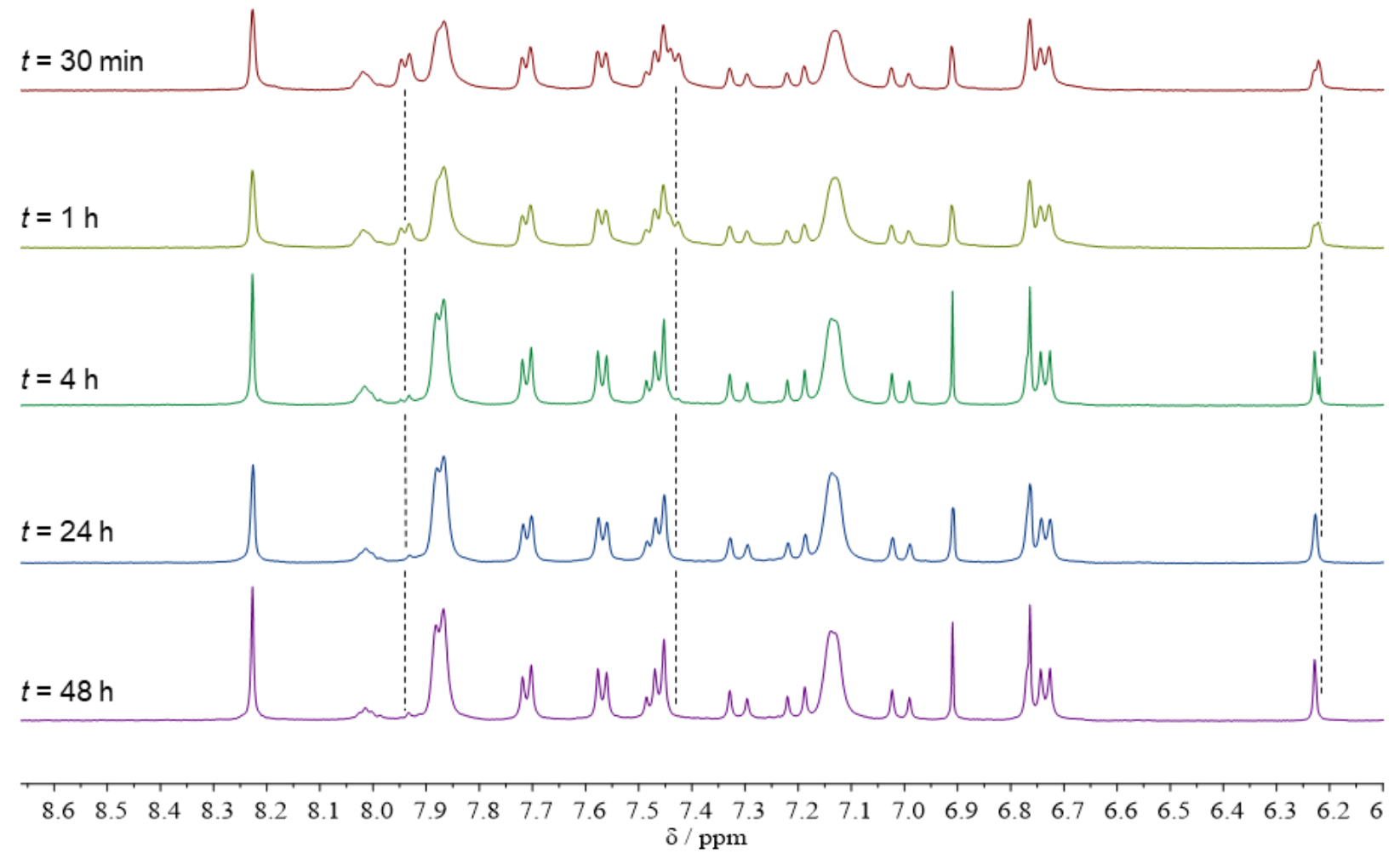

Figure S23. ${ }^{1} \mathrm{H}$ NMR $\left(500 \mathrm{MHz}\right.$; DMSO- $\left.d_{6}\right)$ monitoring of the synthesis of control probe 22 (closeup of the aromatic region 6.1 to $8.7 \mathrm{ppm}$ ). Dotted lines are visual aids highlighting the gradual decrease in intensity of selected starting material signals. 


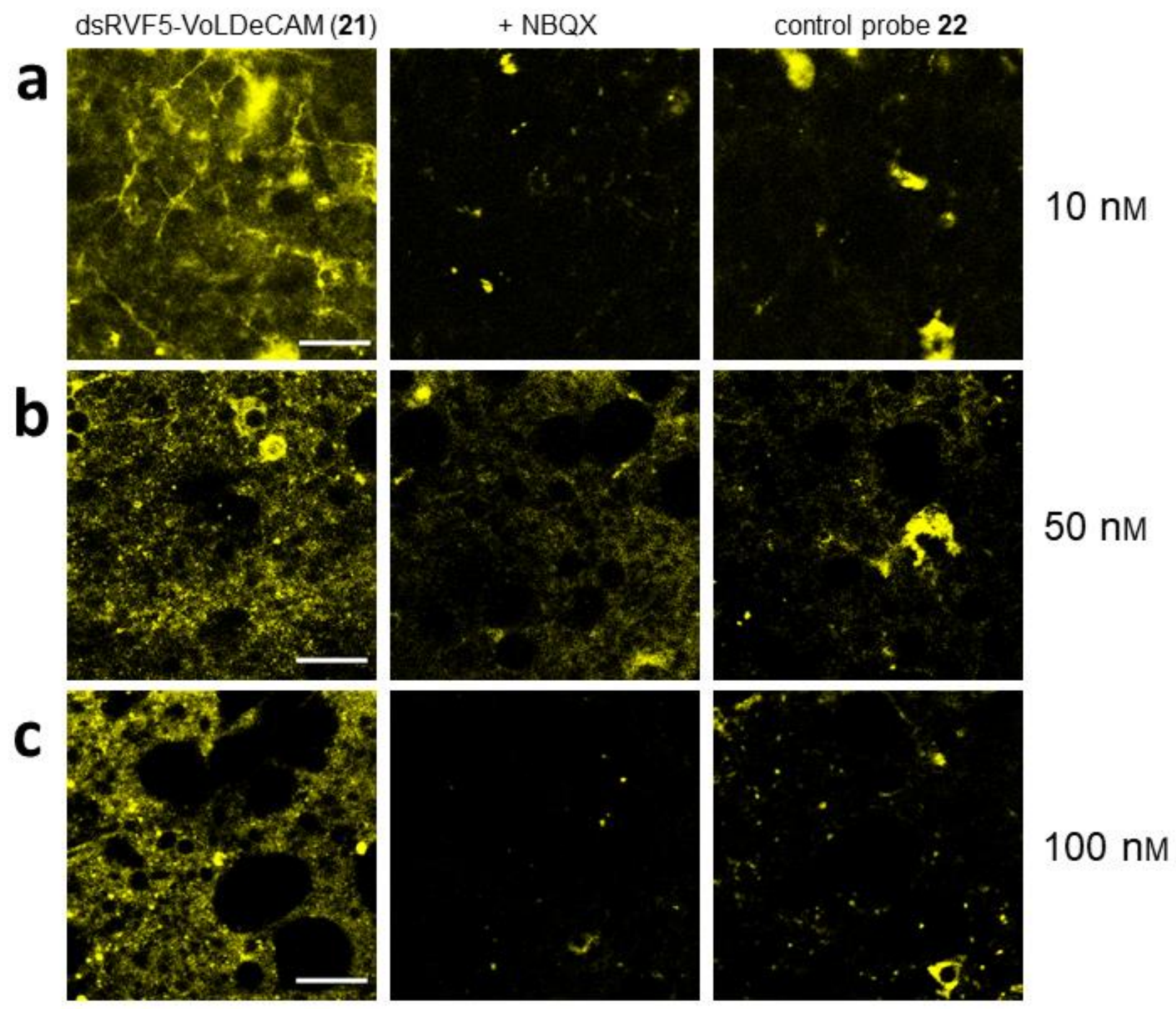

Figure S24. Representative two-photon microscopy images of cortical slices labeled with dsRVF5-VoLDeCAM (21) or control probe 22 using: (a) $14 \mathrm{~nm}$ dextran/10 nM VSD; (b) $71 \mathrm{~nm}$ dextran/50 nM VSD; and (c) $143 \mathrm{~nm}$ dextran/100 nM VSD. Left: slice incubated with the targeted dsRVF5-VoLDeCAM (21, $2 \mathrm{~h} ; 30 \mu \mathrm{m}$ depth); middle: an analogous experiment with pre- and co-incubation with the AMPAR blocker NBQX $(10 \mu \mathrm{M})$; right: the corresponding slice incubated with the untargeted control probe 22 ( $2 \mathrm{~h} ; 30 \mu \mathrm{m}$ depth). Images within the same panel were contrasted equally. The probes were excited at $820 \mathrm{~nm}$ and emission collected at $570-610 \mathrm{~nm}$. Scale bars are $20 \mu \mathrm{m}$. 

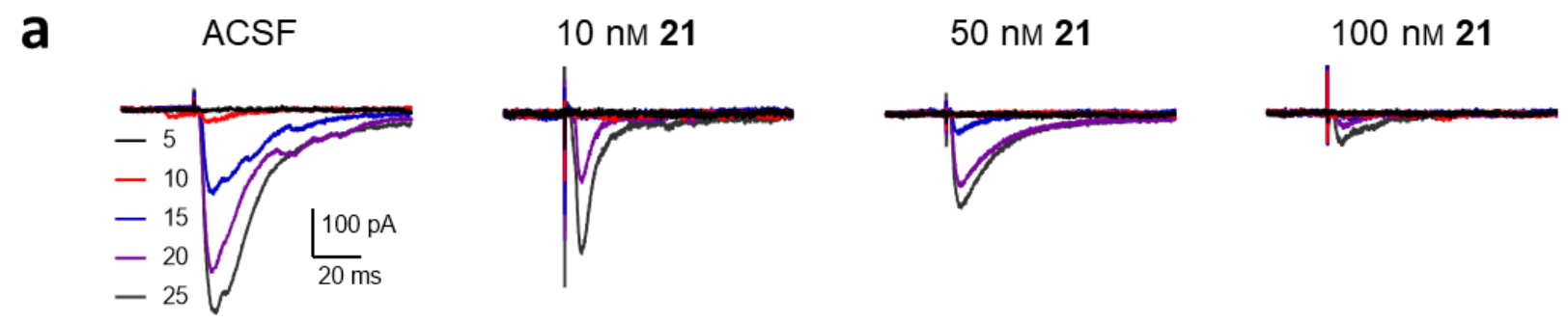

b

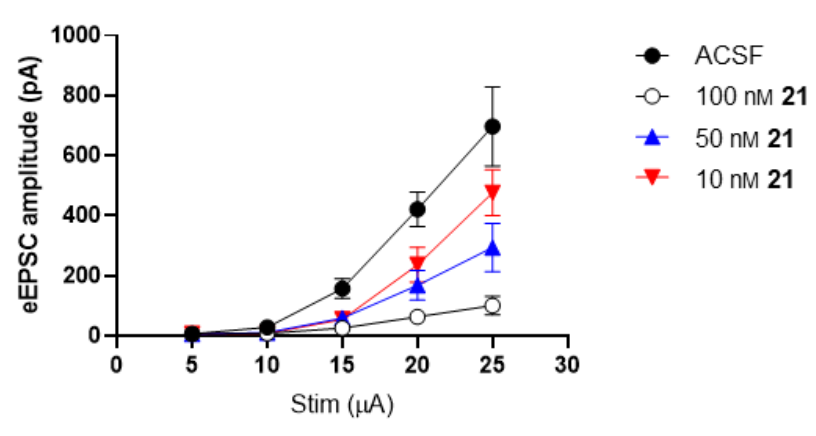

Figure S25. dsRVF5-VoLDeCAM (21) inhibits electrical stimulation driven EPSCs in a dose dependent manner. Cortical slices were incubated with dsRVF5-VoLDeCAM (21; $2 \mathrm{~h})$. Layer V pyramidal neurons in the prefrontal cortex were patched and electrical stimuli were delivered near the recorded neuron $(\sim 100 \mu \mathrm{m})$. (a) Representative traces show eEPSC changes by dsRVF5-VoLDeCAM (21) at 10, 50 and $100 \mathrm{nM}$ (concentration with respect to VSD) compared to untreated control (ACSF). (b) Dependence of mean eEPSC amplitude on stimulation intensity. $100 \mathrm{nM} 21$ almost abolishes EPSCs (86\%,n=6), while $50 \mathrm{~nm}$ and $10 \mathrm{nM} 21$ inhibits EPSCs by $58 \%(n=8)$ and $32 \%(n=6)$ respectively with respect to untreated control (ACSF, $n=8$ ). Data represent mean \pm SEM. 
a

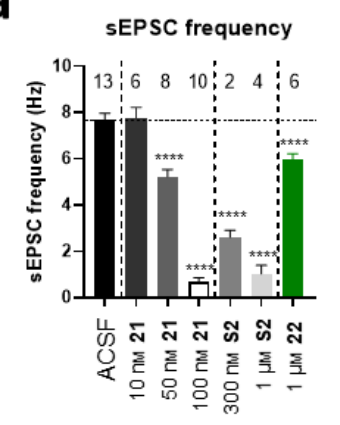

C

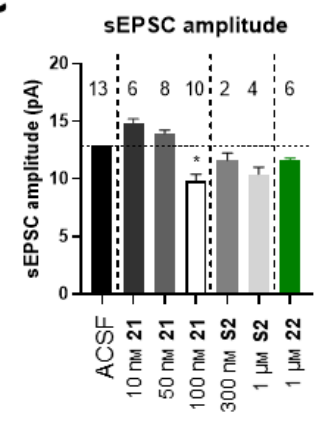

e

ACSF

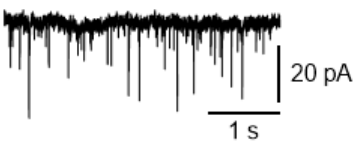<smiles>C=CC(C)C</smiles>

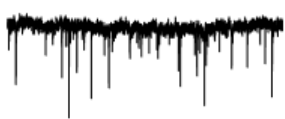

f b

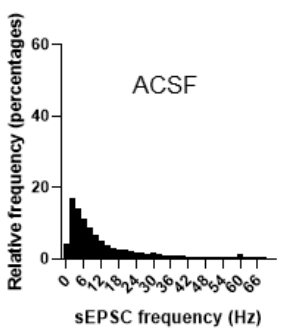

d
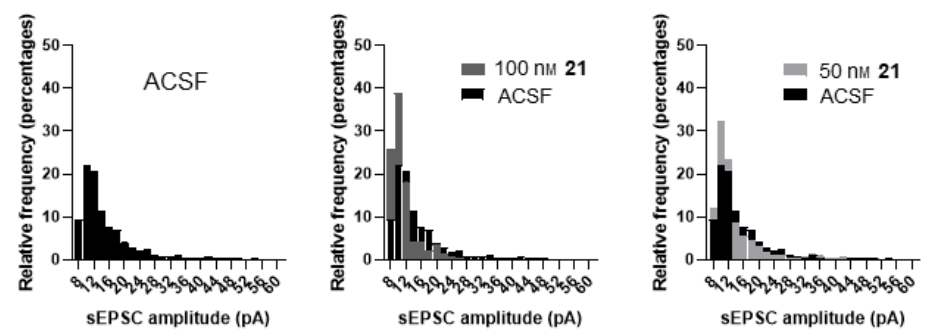

$10 \mathrm{~nm} 21$

$50 \mathrm{~nm} 21$

100 nм 21
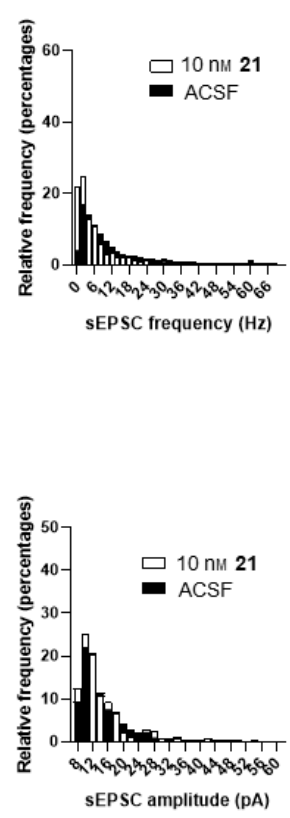

SEPSC amplitude (PA)

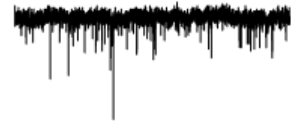

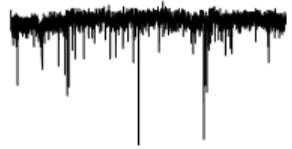

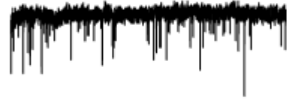

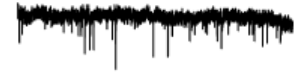
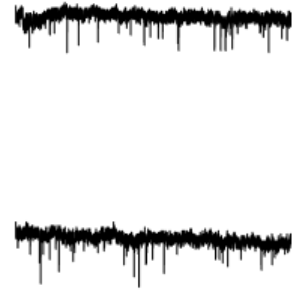
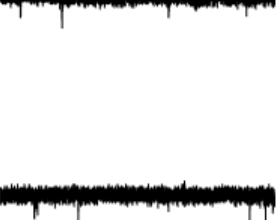<smiles>CCN[NH2+]C([O-])Cn1c(=O)c(=O)[nH]c2cc(C(F)(F)F)c(-n3ccc(CNC(=O)CCC(=O)NCCc4c[nH]cn4)c3)cc21</smiles> 
Figure S26. dsRVF5-VoLDeCAM (21) inhibits sEPSCs in a dose dependent manner. Cortical slices were incubated with dsRVF5-VoLDeCAM (21), PFQX derivative $\mathbf{S} 2$ or control probe $\mathbf{2 2}$ (all 2 h). Layer V pyramidal neurons in the prefrontal cortex were patched and sEPSCs were recorded. (a, c) Median sEPSC frequency (a) or amplitude (c) recorded for untreated control (ACSF) or after treatment with dsRVF5-VoLDeCAM (21) at 10, 50 and $100 \mathrm{nM}$ (concentration with respect to VSD), PFQX derivative S2 (300 nM and $1 \mu \mathrm{M})$ or control probe $22(1 \mu \mathrm{M})$. Number of repeats for each condition $(n)$ is indicated above each respective bar in the graph. Asterisks above graphs represent statistical significance of the difference from untreated control (ACSF): no asterisk $P>0.05$; $* P<0.05$; **** $P<0.0001$. Error bars represent the 95\% confidence interval. (b, d) sEPCS frequency (b) or amplitude (d) histograms recorded for untreated control (ACSF) and after treatment with dsRVF5-VoLDeCAM (21) at 10, 50 and $100 \mathrm{nM}$ (concentration with respect to VSD). (e) Representative sEPSC traces recorded in the experiment summarized in panels (a) through (d). Concentrations of dsRVF5-VoLDeCAM (21) are given with respect to the VSD. (f) Structure of PFQX derivative $\mathbf{S 2}$, the product of cleavage of the acyl imidazole linker in dsRVF5-VoLDeMo (21), also used as a control ligand in the experiments in panels (a) through $(\mathbf{d})$. 

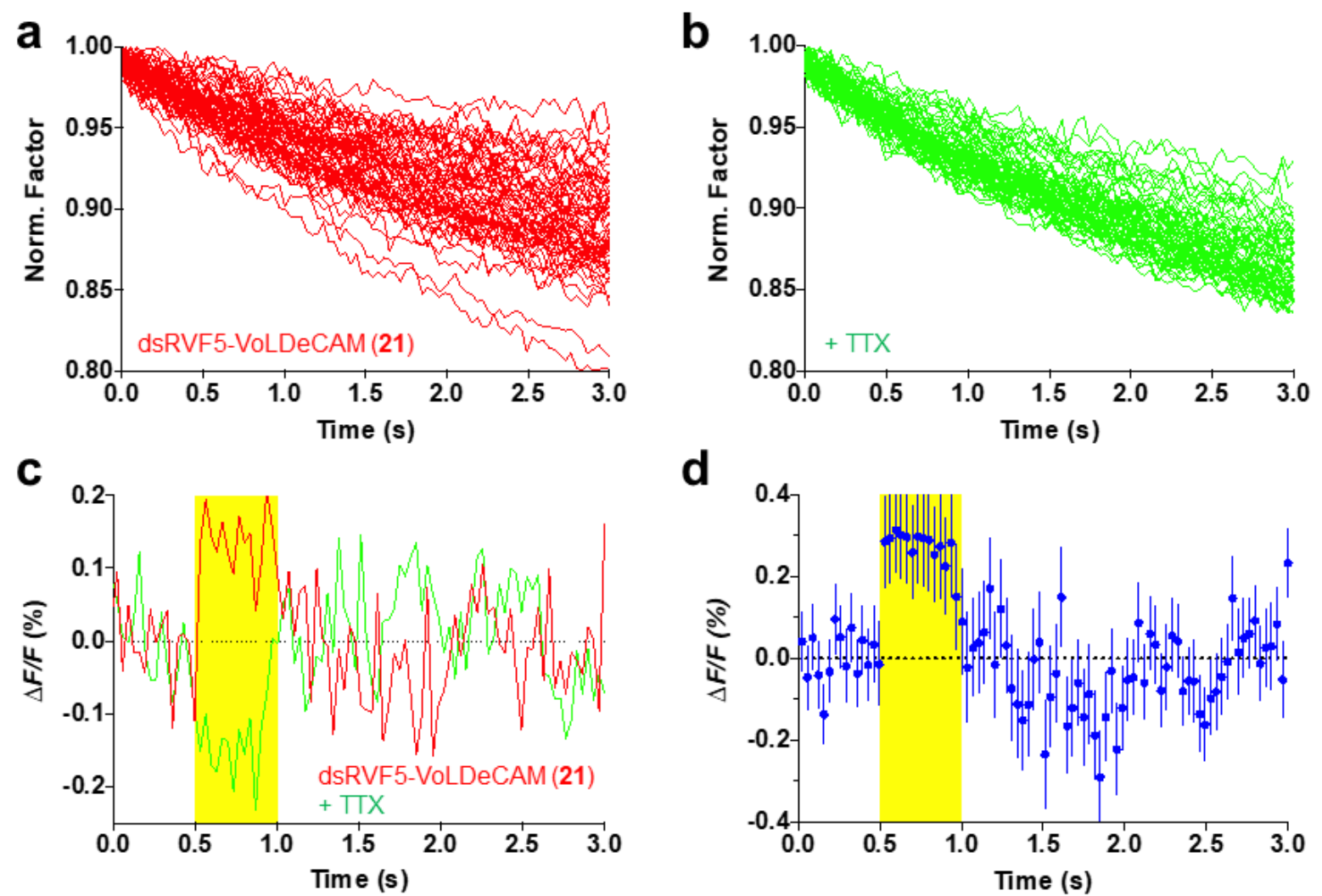

Figure S27. Two-photon fluorescence traces recorded by spiral scanning at $30 \mathrm{~Hz}$ from cortical slices labeled with dsRVF5-VoLDeCAM (21; $143 \mathrm{nM}$ dextran/100 nM VSD, $2 \mathrm{~h}$ ) with electric stimulation ( 25 pulses at $50 \mathrm{~Hz}$, period highlighted in yellow), without and with TTX $(1 \mu \mathrm{M})$ inhibition. The probe was excited at $820 \mathrm{~nm}$ and emission collected at 570-610 nm. (a, b) Normalized raw traces obtained for dsRVF5-VoLDeCAM (a, $n=50)$ and dsRVF5-VoLDeCAM + TTX inhibition (b, $n=50$ ). (c) Averaged traces after bleaching correction (two phase decay function; GraphPad Prism 8 software, San Diego, CA) was applied to each individual raw trace from panels (a) and (b) using $t=0.0$ to $0.5 \mathrm{~s}$ and 2.0 to $3.5 \mathrm{~s}$. (d) Subtracted traces from panel (c): dsRVF5-VoLDeCAM (red in c) minus dsRVF5-VoLDeCAM + TTX inhibition (green in c). This trace corresponds to Fig. $9 c$ in the manuscript with an expanded $x$-axis and without applying a box filter. 


\section{Synthesis}

\section{Materials and Methods}

Reagents, solvents and glassware. All reagents and solvents (including anhydrous solvents) were obtained from commercial sources and were used without purification unless stated otherwise. Synthetic steps were performed under ambient atmosphere unless stated otherwise. Synthetic and purification procedures and analyses were performed at $20^{\circ} \mathrm{C}$ unless stated otherwise. All glassware was oven-dried prior to use when water- and/or air-sensitive reagents were used.

Thin layer and column chromatography. Thin-layer chromatography (TLC) was performed on glass or polyethylene plates coated with silica gel (60 A pore diameter, $200 \mu \mathrm{m}$ layer thickness, 8.0-12.0 $\mu \mathrm{m}$ particle size, SiliCycle); or polyester plates coated with alumina (200 $\mu \mathrm{m}$ layer thickness, < $60 \mu \mathrm{m}$ particle size, Sigma-Aldrich); or glass plates coated with reverse phase (RP) $\mathrm{C}_{18}$-modified silica gel $\left(0.74-0.84 \mathrm{~mL} \mathrm{~g}^{-1}\right.$ pore volume, $200-270 \mu \mathrm{m}$ layer thickness, $9.5-11.5 \mu \mathrm{m}$ particle size, EMD Millipore). All TLC plates contained a fluorescent indicator and were visualized by UV light $\left(\lambda_{\mathrm{exc}}=254\right.$ or $366 \mathrm{~nm}$ ) or by staining in an iodine chamber, or with acidic ninhydrin solution, basic $\mathrm{KMnO}_{4}$ solution or methanolic $\mathrm{H}_{2} \mathrm{SO}_{4}$ solutions prepared according to standard procedures. All column chromatography purification procedures were performed on columns packed with silica (60 Å pore diameter, 40-60 $\mu \mathrm{m}$ particle size, SiliCycle), basic alumina

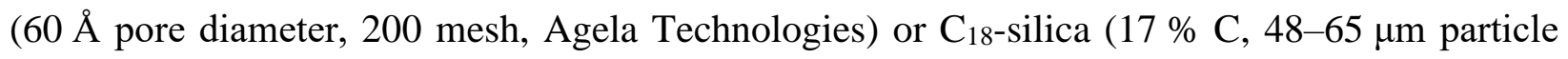
size, Acros Organics).

NMR. NMR spectra were recorded on 400 (equipped with a BBI or TBO probe) or 500 (equipped with a $\mathrm{H} / \mathrm{C} / \mathrm{N}$ or BBFOplus probe) $\mathrm{MHz}$ spectrometers at $27^{\circ} \mathrm{C}$ in acetone- $d_{6}$, acetonitrile- $d_{3}$, chloroform- $d$, dimethylsulfoxide- $d_{6}$, methanol- $d_{4}$, water- $d_{2}$, or their mixtures. The signals in ${ }^{1} \mathrm{H}$ and ${ }^{13} \mathrm{C}$ NMR spectra were referenced to tetramethylsilane (TMS) or to the residual peak of the (major) solvent as reported previously ${ }^{4}$ except for $\mathrm{D}_{2} \mathrm{O}$, while those of the ${ }^{1} \mathrm{H}$ and ${ }^{13} \mathrm{C}$ NMR spectra in $\mathrm{D}_{2} \mathrm{O}$ and ${ }^{19} \mathrm{~F}$ NMR in all solvents were unreferenced. The multiplicities of signals are reported as singlet $(\mathrm{s})$, doublet $(\mathrm{d})$, triplet $(\mathrm{t})$, quartet $(\mathrm{q})$, pentet $(\mathrm{p})$, multiplet $(\mathrm{m})$, broad (br) or a combination of these. 
Samples of all dextran constructs were co-evaporated with $\mathrm{D}_{2} \mathrm{O}$ prior to NMR experiments to reduce the intensity of the otherwise overwhelming $-\mathrm{OH}$ signals. To achieve more accurate integration of minor signals (linkers, ligands, VSDs) attached to dextran, 512 scans were acquired for all samples ( $5 \mathrm{mg}$ in $500 \mu \mathrm{L}$ solvent, $500 \mathrm{MHz}$ instrument with a H/C/N probe). Manual phase correction and ablative baseline correction (5 points, high enough number of passes to avoid artificial lowering of high intensity signals) were applied.

MS. Low-resolution mass spectra (LRMS) were recorded on an Advion expression-L CMS quadrupole mass spectrometer. The samples were loaded via flow injection, a Plate Express TLC plate reader system or an atmospheric solids analysis probe (ASAP) and ionized by atmospheric pressure chemical ionization (APCI) or electrospray ionization (ESI). High-resolution mass spectrometry (HRMS) experiments were carried out by Dr. Brandon Fowler on a Waters XEVO G2XS QToF mass spectrometer equipped with a UPC2 SFC inlet and ionized by ESI or APCI.

FTIR. Infrared (IR) spectra of neat solid or liquid samples were obtained on a Perkin Elmer FT-IR Spectrum Two spectrometer in the attenuated total reflectance (ATR) sampling mode on a diamond crystal.

Melting point. Melting points of crystalline solids were determined in open-end capillary tubes using a non-calibrated DigiMelt MPA160 melting point apparatus.

Polarimetry. Polarimetric experiments were performed on a Jasco P-2000 automatic polarimeter in methanolic solution $\left(c \sim 10 \mathrm{mg} \mathrm{mL}^{-1}\right)$ in $50 \mathrm{~mm}$ cuvettes using a sodium lamp as a light source $\left(\lambda_{\mathrm{em}}=589 \mathrm{~nm}\right)$.

\section{Nomenclature}

IUPAC names are used for small molecules with short comprehensible names. For molecules with longer IUPAC names, semi-trivial or trivial nomenclature (vide infra) is used, or the molecules are exclusively referred to using bold numbering.

The short-code nomenclature for electrochromic hemicyanine VSDs has been described previously. ${ }^{5}$ Hemicyanine di-2-ANEPEQ (1) refers to diethylamino (di-2-A) naphthyl (N) ethene 
(E) pyridinium (P) ethylene (E) quaternary ammonium (Q). The clickable di-2-ANEPB-BCN (9) and its precursor di-2-ANEPB-NHBoc (S18) contain a butylene (B) linker and are terminated with (endo-bicyclo[6.1.0]non-4-yn-9-yl)methyl (BCN; the exact carbamate linkage is omitted for simplicity) or NHBoc groups respectively.

The short-code nomenclature for PeT-based VSDs has been introduced by Miller et al. ${ }^{6,7}$ dsRVF5 refers to the disulfonated (ds) version of Rhodol VoltageFluor-5 (RVF5). For the clickable dsRVF5-BCN (10), dsRVF5-N 3 (18) and their precursor dsRVF5-NHBoc (S26), BCN and NHBoc reflect terminal functional handles of the molecules but not necessarily direct attachment of these functional groups to the dye core.

(endo-bicyclo[6.1.0]non-4-yn-9-yl)methyl succinimidyl carbonate (S8) is referred to as $\mathrm{BCN}-\mathrm{OSu}$ as established (see e.g. refs $^{8,9}$ ), although the technically correct trivial code should be $(\mathrm{BCN}-\mathrm{O})(\mathrm{SuO}) \mathrm{CO}$. Analogously, the respective 4-nitrophenyl carbonate (14) is referred to as BCN-OpNP.

\section{Synthetic Schemes}

$\mathrm{N}$-(3-azidopropyl)-O-(4-nitrophenyl) carbamate (7) for functionalization of dextran was prepared in two steps (Scheme S1).
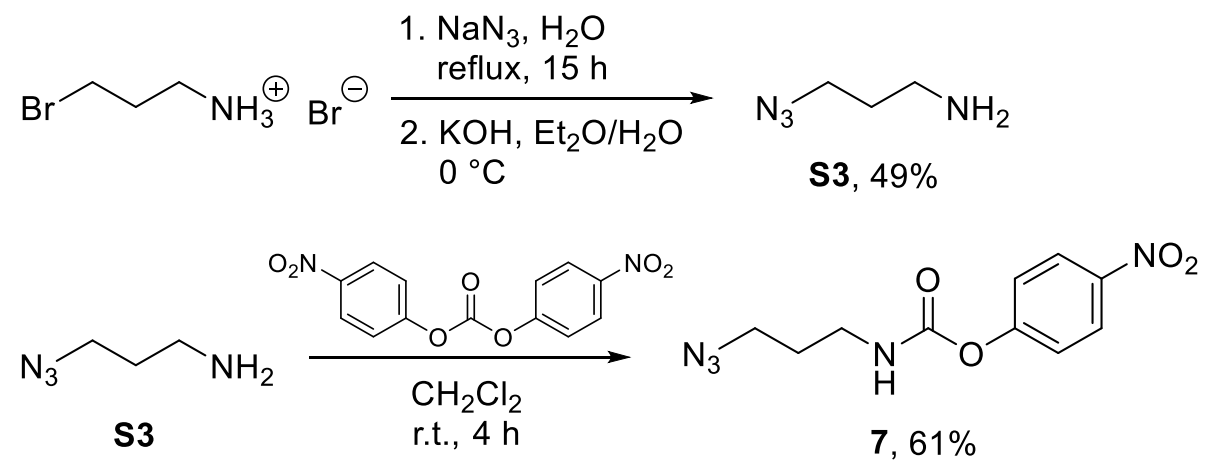

Scheme S1. Two step synthesis of $N$-(3-azidopropyl)- $O$-(4-nitrophenyl) carbamate (7), a reagent used for preparation of azido-group functionalized dextran. 
The succinimidyl carbonate (S8) of (endo-bicyclo[6.1.0]non-4-yn-9-yl)methanol (S7) for strainpromoted azide-alkyne cycloaddition (SP-AAC) click chemistry was prepared as described previously (Scheme S2). ${ }^{8,10}$

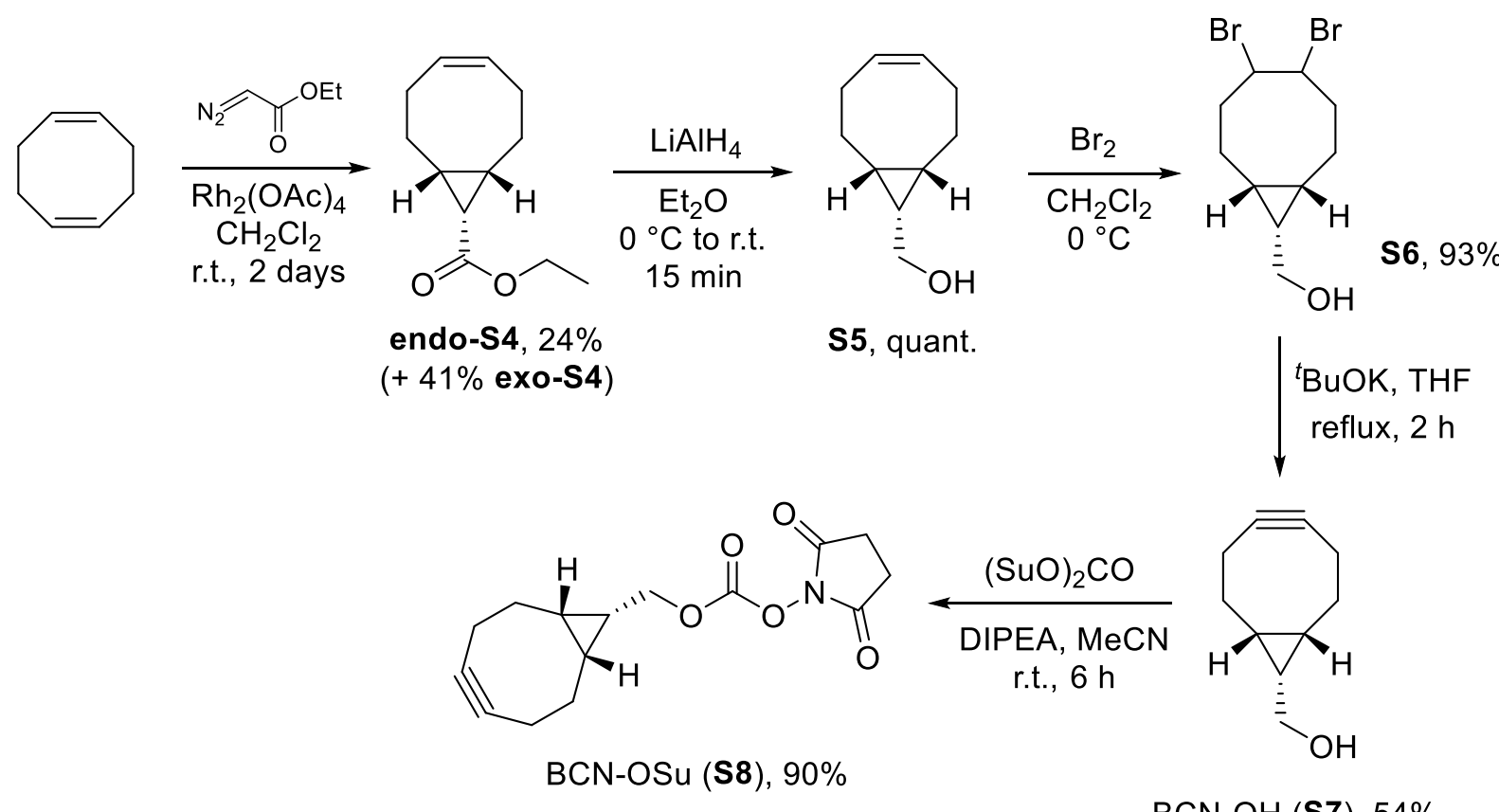

Scheme S2. Synthesis of BCN-OSu (S8), an activated carbonate for attachment of the clickable (endo-bicyclo[6.1.0]non-4-yn-9-yl)methyl (BCN) group via SP-AAC.

The di-2-ANEP voltage sensitive dye (VSD) equipped with a clickable BCN moiety (di-2-ANEPB-BCN, 9) was prepared by a Knoevenagel condensation of the corresponding 4-picolinium salt (S11) and 6-(diethylamino)-2-naphthaldehyde (S13), followed by Boc deprotection and BCN-group attachment (Scheme S3c). Compounds S11 and S13 were prepared from commercial starting materials in 3 and 2 steps, respectively (Scheme S3 $a$ and $b$ respectively). The activated $N$-hydroxysuccinimidyl (NHS) ester of di-2-ANEP (di-2-ANEPB-CO $2 \mathrm{Su}, \mathbf{S 1 7}$ ) was prepared by an alternative route from intermediate $\mathbf{S 1 2}$ via Heck coupling followed by $\mathrm{S}_{\mathrm{N}} 2$ attachment of the linker and elaboration of the carboxylic acid functional group (Scheme $S 3 b$ ). 


\section{a}

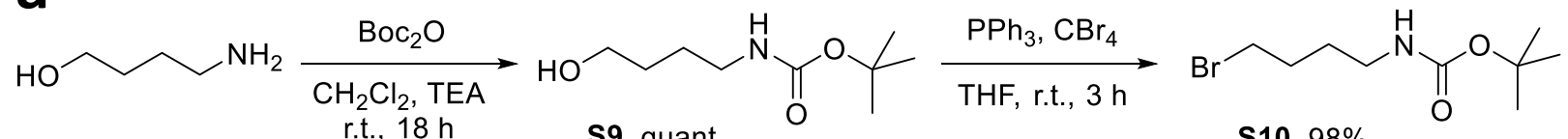

r.t., $18 \mathrm{~h}$

S9, quant.

S10, $98 \%$

b<smiles>CCNc1ccc2cc(C=O)ccc2c1</smiles>

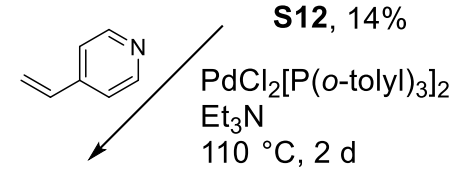<smiles>CCNc1ccc2cc(/C=C/c3ccncc3)ccc2c1</smiles><smiles>[R]OC(=O)CCCC[n+]1ccc(/C=C/c2ccc3cc(NCC)ccc3c2)cc1</smiles>

$\mathrm{R}=\mathrm{H}: \mathbf{S} 16,96 \%$

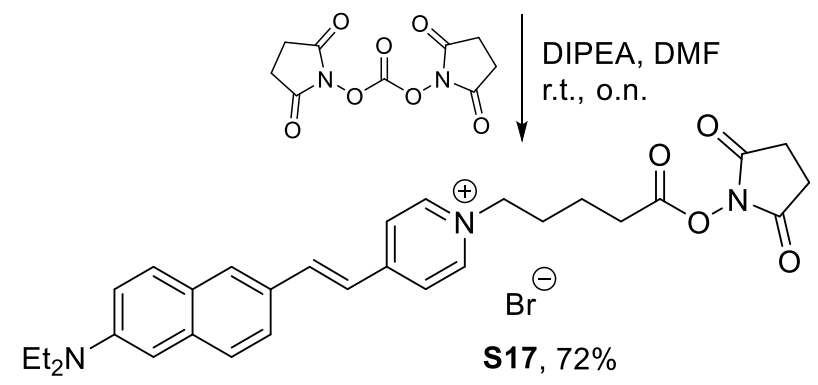

C<smiles>Cc1cc[n+](CCCCNC(=O)OC(C)(C)C)cc1</smiles><smiles>CCNc1ccc2cc(C=O)ccc2c1</smiles>

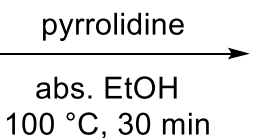
$100{ }^{\circ} \mathrm{C}, 30 \mathrm{~min}$

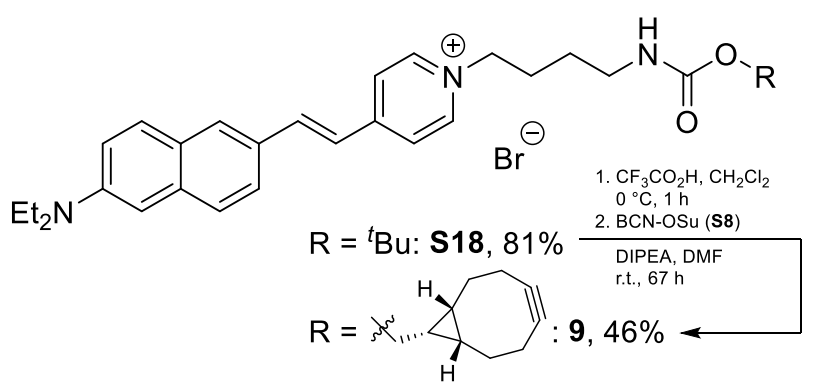


Scheme S3. Synthesis of functionalized di-2-ANEP VSDs. (a) Synthesis of linkerfunctionalized pyridinium intermediate S11. (b) Synthesis of naphthaldehyde intermediate S13 and NHS ester-functionalized di-2-ANEPB-CO $\mathrm{CO}_{2} \mathrm{Su}$ (S17). (c) Synthesis of the clickable di-2-ANEPB-BCN (9).

(E)-N,N-dimethyl-4-(4-vinylstyryl)aniline (S21), the $\pi$-wire portion of dsRVF5 VSDs 2,9 and 18 used in this study was prepared in 3 steps according to Miller et al. ${ }^{11}$ (Scheme S4).
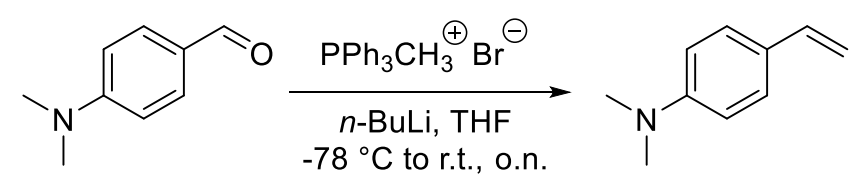

$\mathbf{S 1 9}, 95 \%$
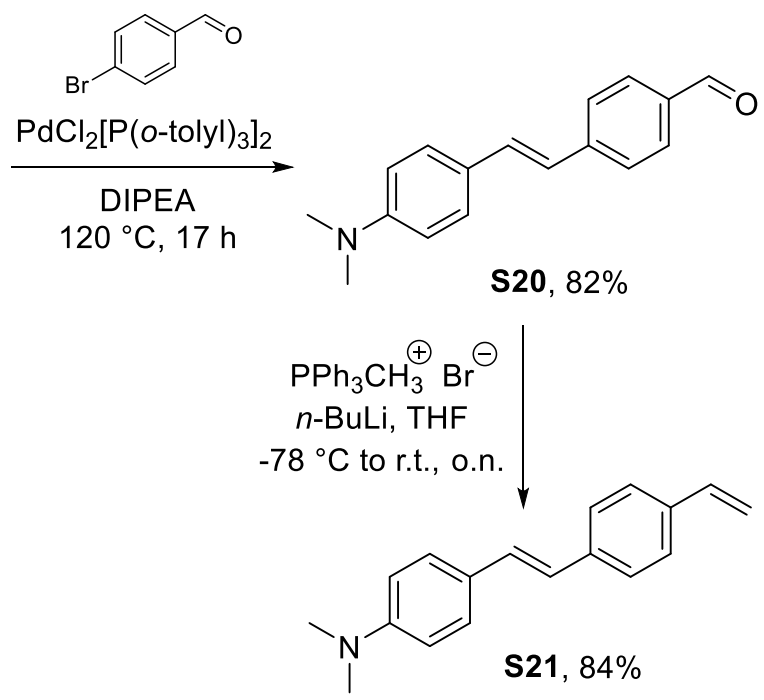

Scheme S4. Synthesis of (E)- $N, N$-dimethyl-4-(4-vinylstyryl)aniline (S21), the $\pi$-wire portion of PeT-based dsRVF5 voltage sensitive dyes. 
dsRVF5 VSD (2) and its clickable analog 10 were prepared as follows: Disulfodichlorofluorescein S23 was prepared in two steps according to Kulkarni et al. ${ }^{7}$ (Scheme S5). Rhodol chromophores S24 and S25 were formed by triflation of $\mathbf{S 2 3}$ followed by nucleophilic aromatic substitution with pyrrolidine or ( $N$-Boc-aminomethyl)pyrrolidine respectively. Subsequently, $\pi$-wire S21 was attached via Heck coupling providing non-clickable dsRVF5 2 and the NHBoc-containing version S26. The clickable dsRVF5 10 was obtained from S26 after acid-mediated Boc-deprotection followed by BCN attachment (Scheme S5).

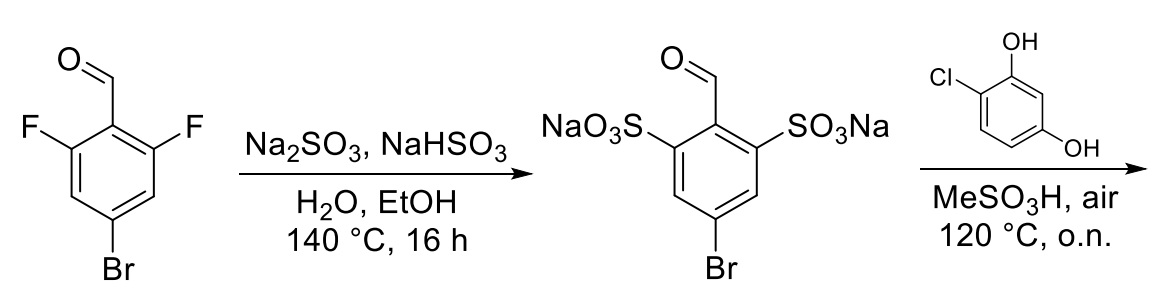

S22, $93 \%$

( $80 \%$ purity)

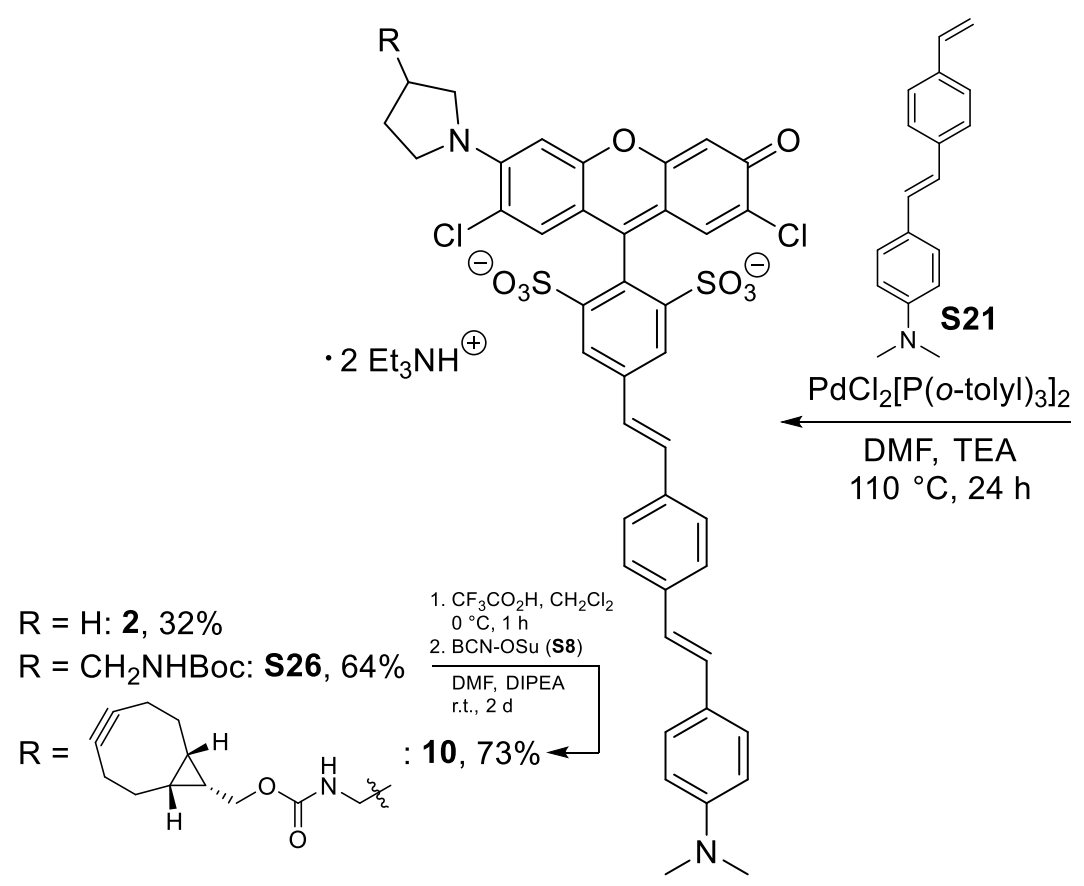<smiles>O=c1cc2oc3cc(O)c(Cl)cc3c(-c3c(S(=O)(=O)O)cc(Br)cc3S(=O)(=O)O)c-2cc1Cl</smiles>

S23, $31 \%$

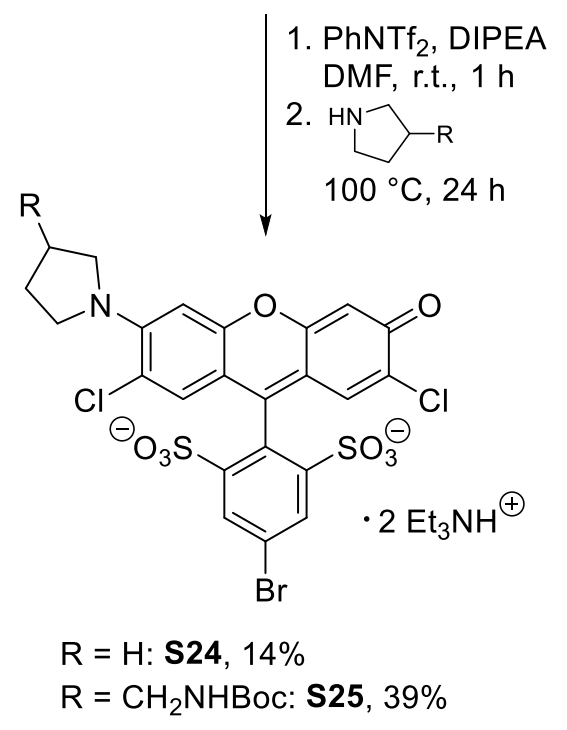

Scheme S5. Synthesis of the PeT-based dsRVF5 VSDs 2 and $\mathbf{1 0 .}$

Dichloropane ligand 11 containing a clickable BCN group connected through a short linker and allowing SP-AAC was prepared from tropinone in 9 -steps in a $0.9 \%$ overall yield (incl. $9 \%$ yield 
of the chiral resolution step) (Scheme S6). In the first step, tropinone enolate was nucleophilically added to dimethylcarbonate yielding racemic methyl carboxylate $( \pm)-\mathbf{S 2 7}$. Chiral resolution of $( \pm)$-S27 by preferential crystallization of a $(+)$-tartarate salt provided $(R)$-S27 in $\sim 9 \%$ yield and $>90 \%$ ee after three crystallization cycles on a 10 -g scale. The obtained $(R)$-S27 was transformed into a triflate ester $\mathbf{S 2 8}$ allowing installation of a 3,4-dichlorophenyl ring under Suzuki conditions. Reduction with $\mathrm{SmI}_{2}$ and chromatographic purification yielded 3. The $\mathrm{N}-\mathrm{CH}_{3}$ bridge was demethylated, followed by installation of linker S10 providing $\mathbf{5}$. Clickable ligand $\mathbf{1 1}$ was obtained after Boc deprotection and attachment of the strained BCN group.

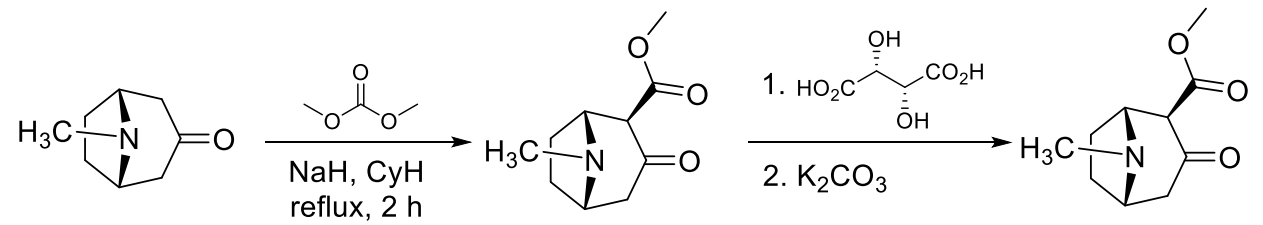

$\mathbf{S 2 7}+$ enantiomer $68 \%$

S27, $\sim 9 \%$ yield $>90 \%$ ee

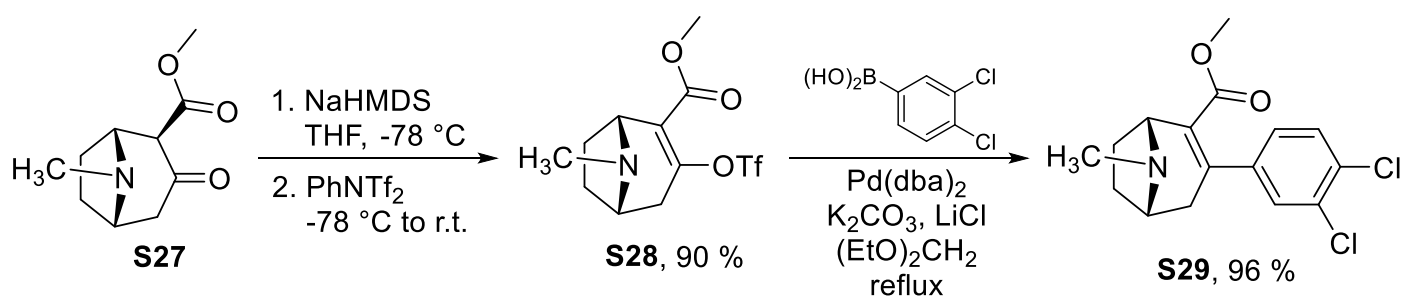

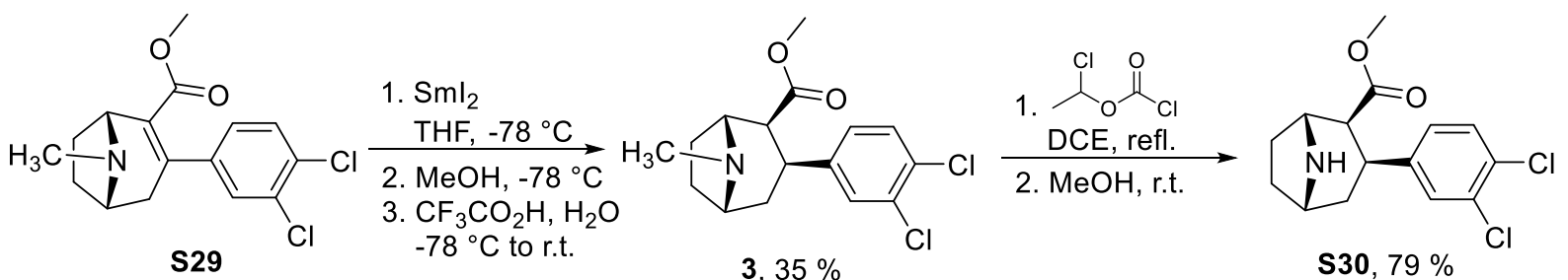<smiles>COC(=O)C1C2CCC(N2)[C@H]1c1ccc(Cl)c(Cl)c1</smiles>

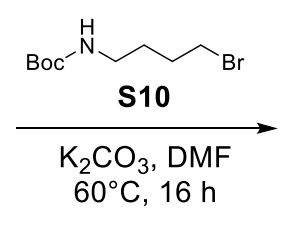
$60^{\circ} \mathrm{C}, 16 \mathrm{~h}$

S30

$3,35 \%$

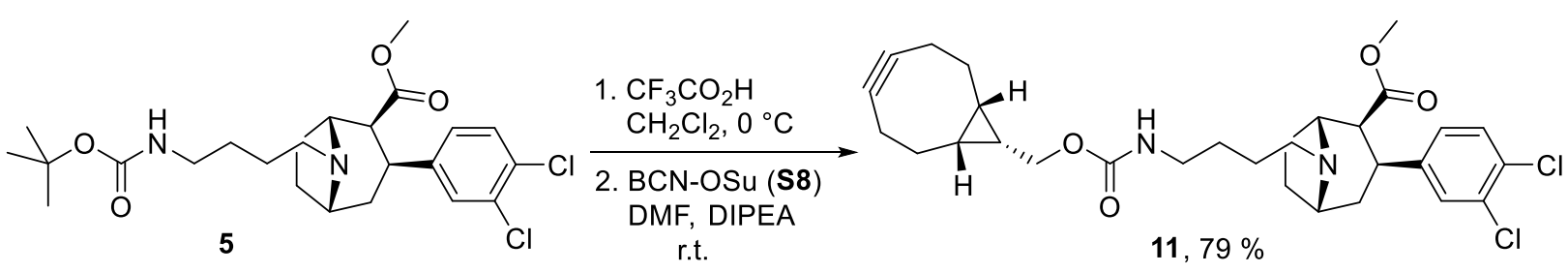

Scheme S6. Synthesis of clickable dichloropane ligand 11. 
Dichloropane-rhodamine red-X probe 6 was prepared from dichloropane-NHBoc (5) by acid mediated deprotection followed by amidation with commercial rhodamine red-X $N$-hydroxysuccinimidyl ester (Thermo Fisher Scientific; Scheme S7).

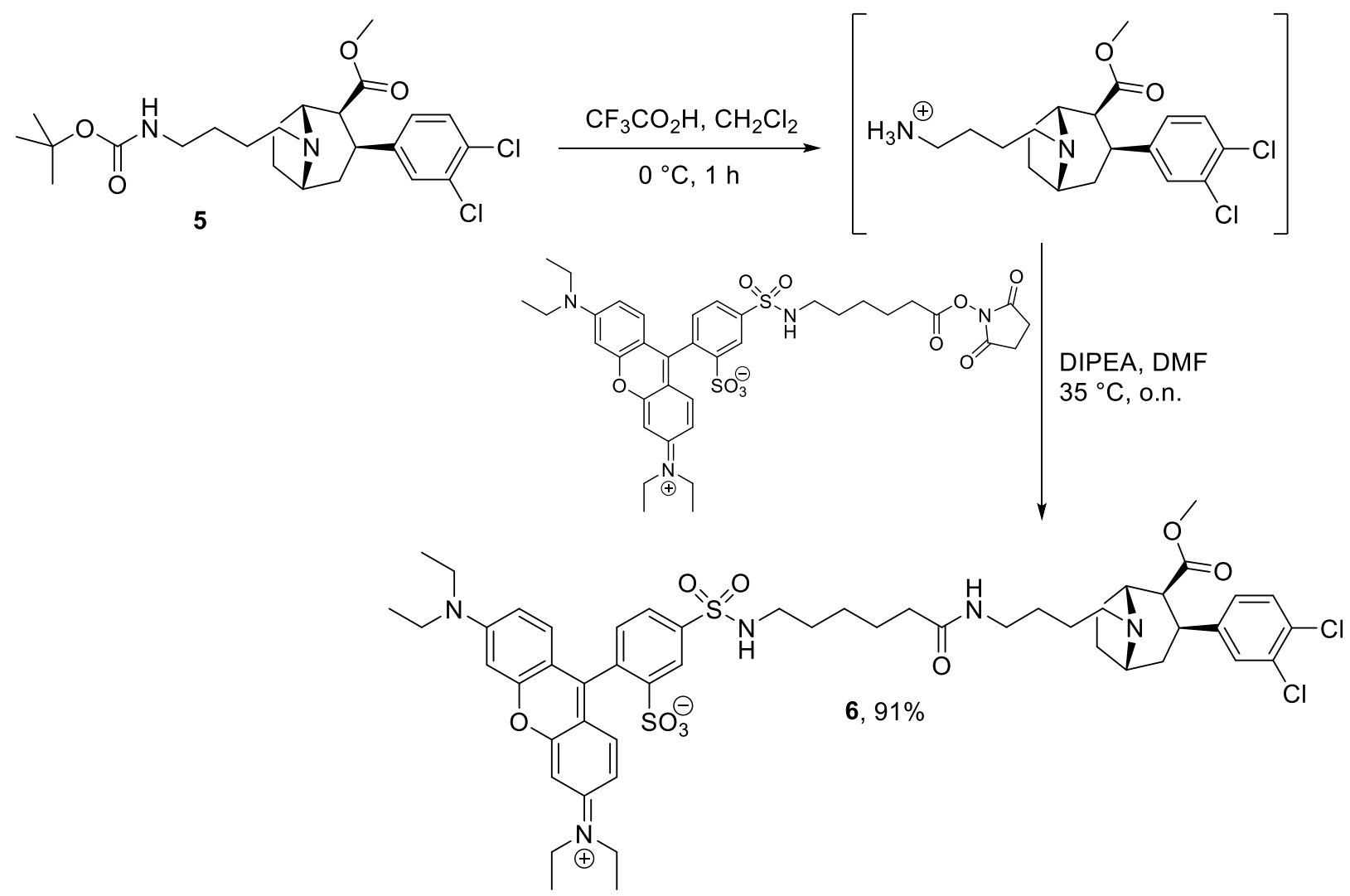

Scheme S7. Synthesis of dichloropane attached through a short linker to rhodamine red-X - control probe $\mathbf{6}$.

The probe connecting the dichloropane ligand with the di-2-ANEP VSD through a PEG(3400) linker (avg. $M_{w}=3400 \mathrm{Da}$ ) (dichloropane-PEG(3400)-di-2-ANEP, S1) was prepared by attaching the respective functionalities to a heterobifunctional BocNH-PEG(3400)-SVA (SVA = succinimidyl valerate) by a sequence of deprotection-amidation steps (Scheme S8). The Boc group of dichloropane-NHBoc (5) was deprotected by an acid-mediated reaction, followed by amidation with heterobifunctional BocNH-PEG(3400)-SVA. The obtained BocNH-PEG(3400)- 
dichloropane (S31) was further deprotected and amidated with di-2-ANEPB- $\mathrm{CO}_{2} \mathrm{Su}(\mathbf{S 1 7})$ to give the final dichloropane-PEG(3400)-di-2-ANEP VSD-construct S1 in 44\% overall yield (over 4 steps).

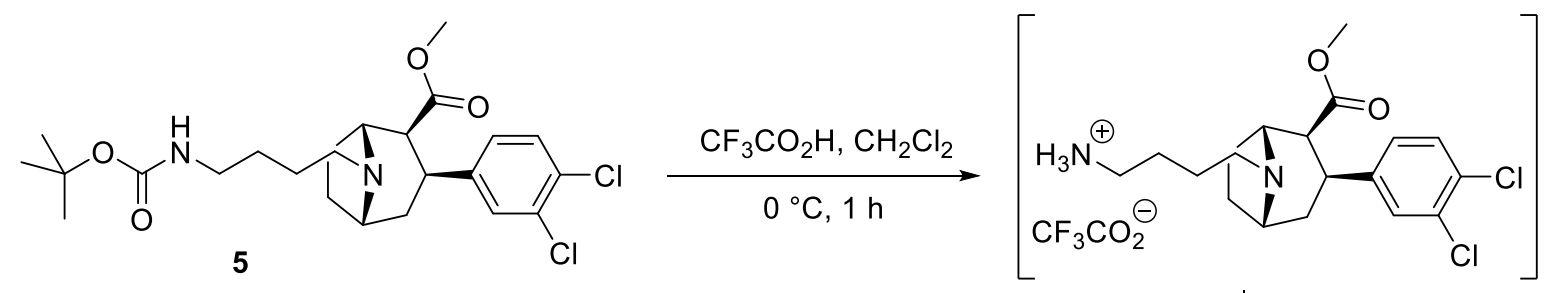

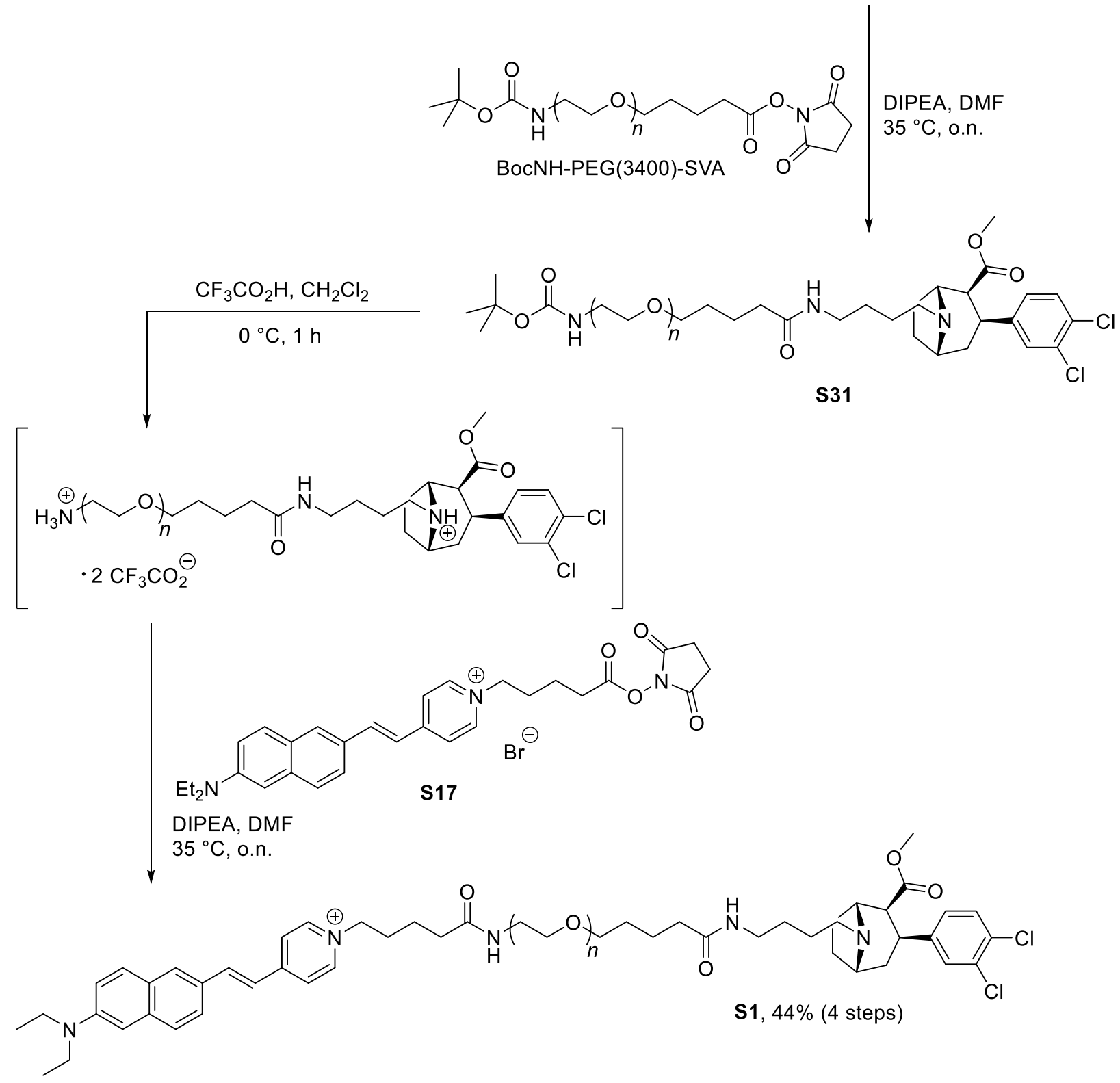

Scheme S8. Synthesis of dichloropane-PEG(3400)-di-2-ANEP construct (S1). 
The 4-nitrophenyl $\mathrm{BCN}$ carbonate $(\mathrm{BCN}-\mathrm{OpNP}, 14)$ for functionalization of dextran with $\mathrm{BCN}$ groups was prepared as described previously (Scheme S9). ${ }^{10}$

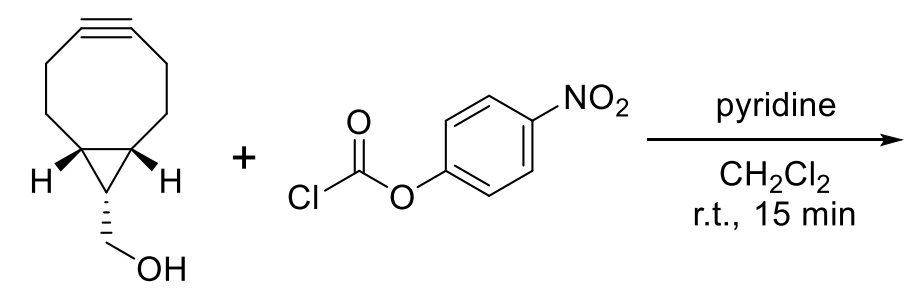

$\mathrm{BCN}-\mathrm{OH}(\mathbf{S 7})$<smiles>O=C(OC[C@H]1C[C@H]2CCC#CCC[C@@H]12)Oc1ccc([N+](=O)[O-])cc1</smiles>

BCN-OpNP (14)

$85 \%$

Scheme S9. Synthesis of BCN-OpNP (14).

Clickable imidazolyl carbamate 16 was prepared from 4-(bromomethyl)benzoic acid in five steps as shown in Scheme S10.

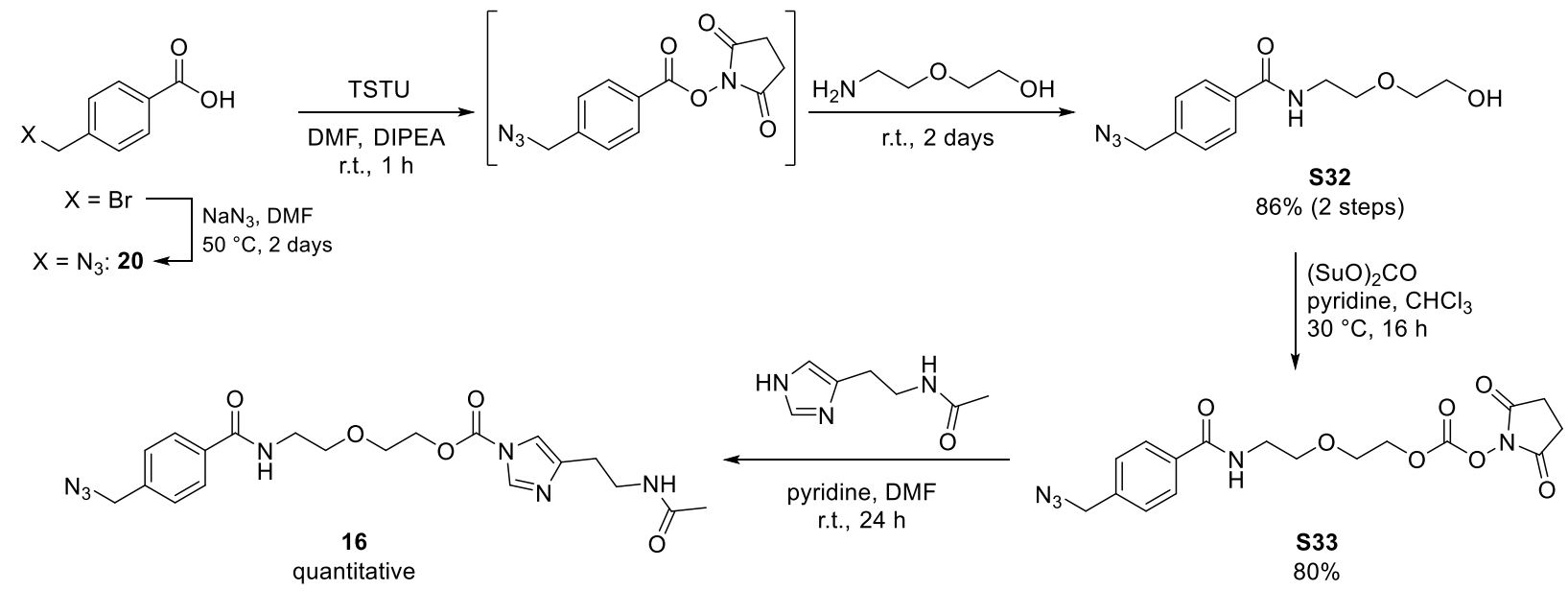

Scheme S10. Synthesis of imidazolyl carbamate 16. 
The pyrrolylquinoxalinedione-based AMPAR ligand S40 was prepared in seven steps according to the general strategy outlined by Lubisch et al. ${ }^{12}$ (Scheme S11). The cleavable acyl imidazole linker with an azido functionality was further installed in four steps according to an approach developed by Wakayama et al. ${ }^{3}$ to yield compound 19 (Scheme S11).
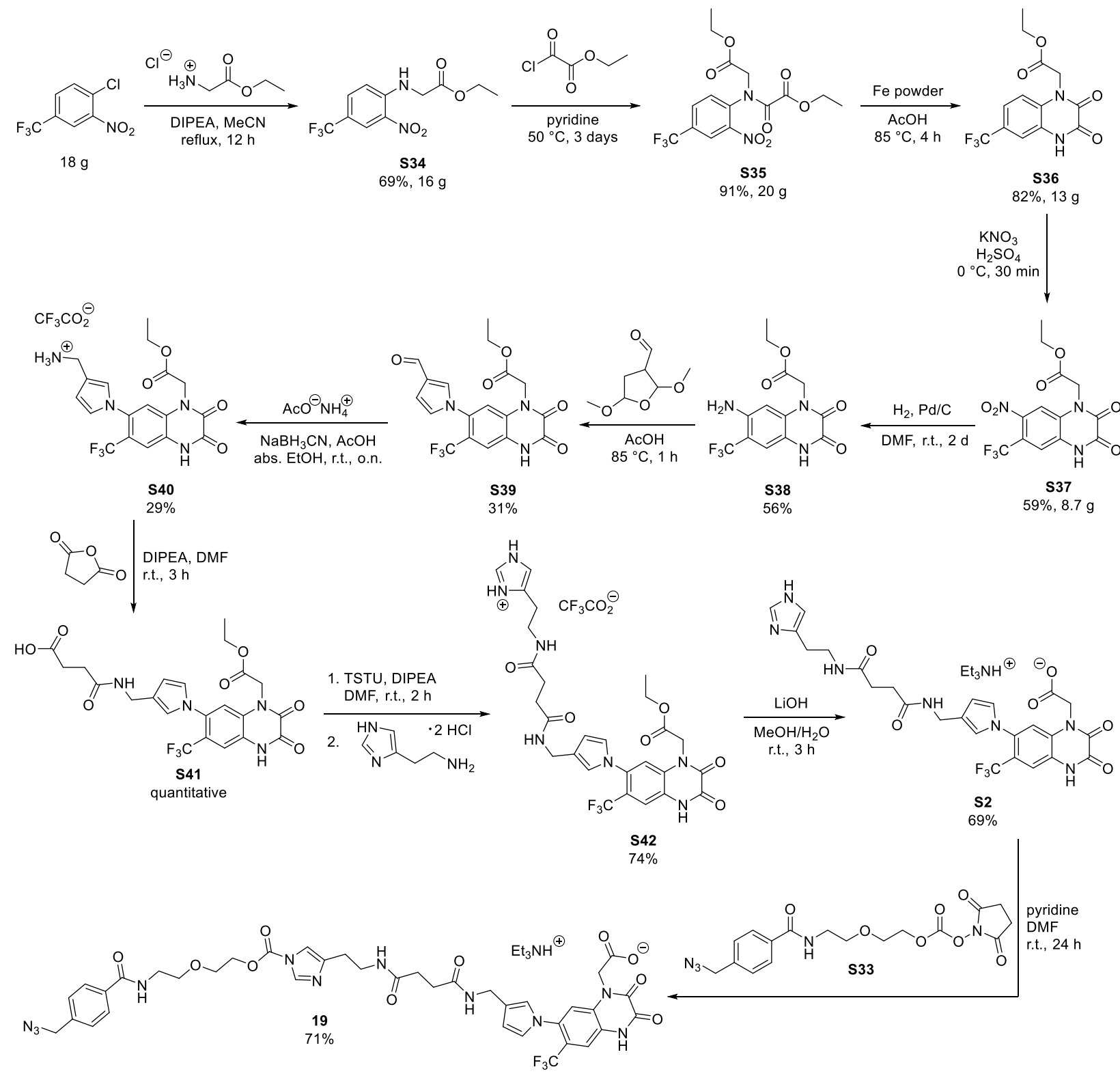

Scheme S11. Synthesis of the pyrrolylquinoxalinedione-based AMPAR ligand 19 containing a cleavable acyl imidazole linker and an azido functionality. 
Azido-functionalized dsRVF5 (dsRVF5-N $\mathrm{N}_{3}, \mathbf{1 8}$ ) was prepared from the Boc protected VSD S26 by acid-mediated deprotection, followed by amidation with activated ester $\mathbf{S 4 5}$, which was prepared in three steps from ethyl 5-bromovalerate (Scheme S12).

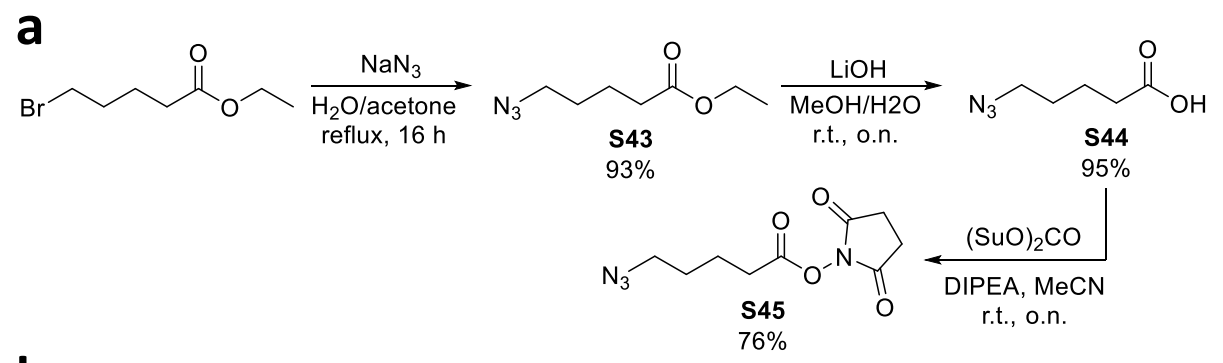

b

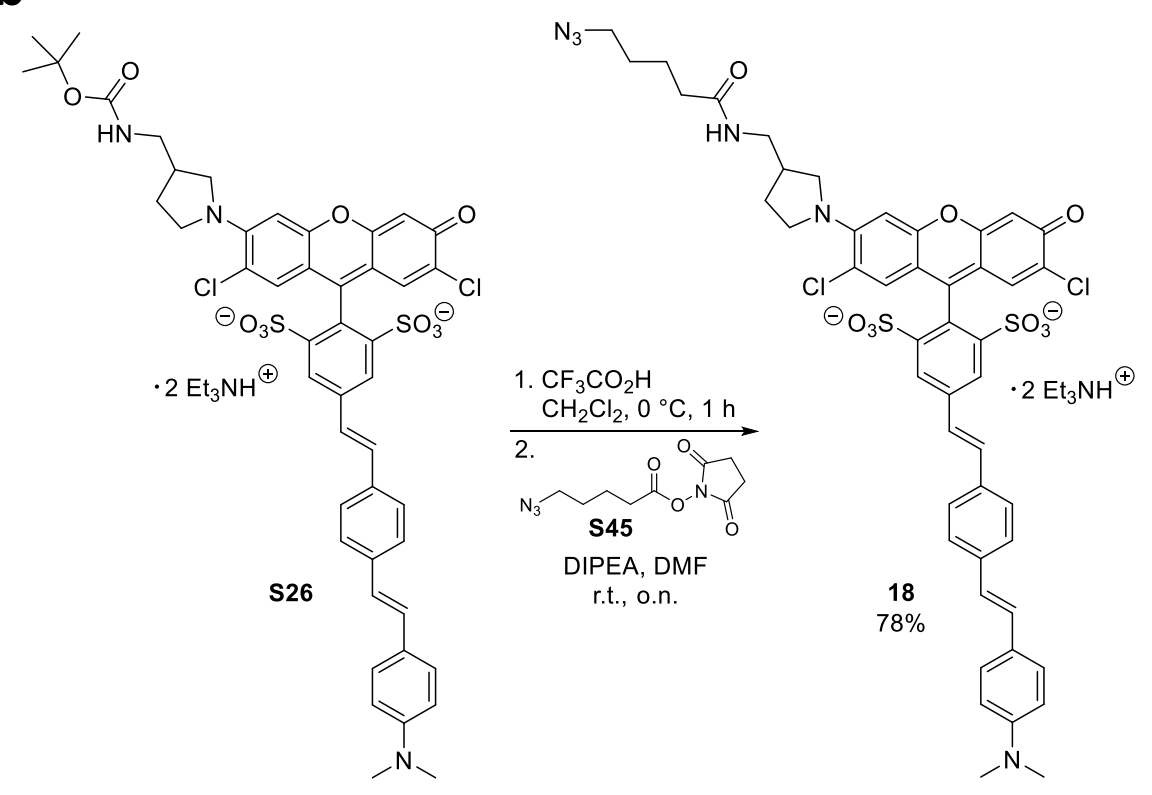

Scheme S12. (a) Synthesis of 5-azidovaleric acid succinimidyl ester (S45). (b) Synthesis of azido-functionalized dsRVF5 (dsRVF5-N 3,18 ). 
Azido-functionalized sulforhodamine B (S46) was prepared from sulforhodamine B sodium salt (Scheme S13).
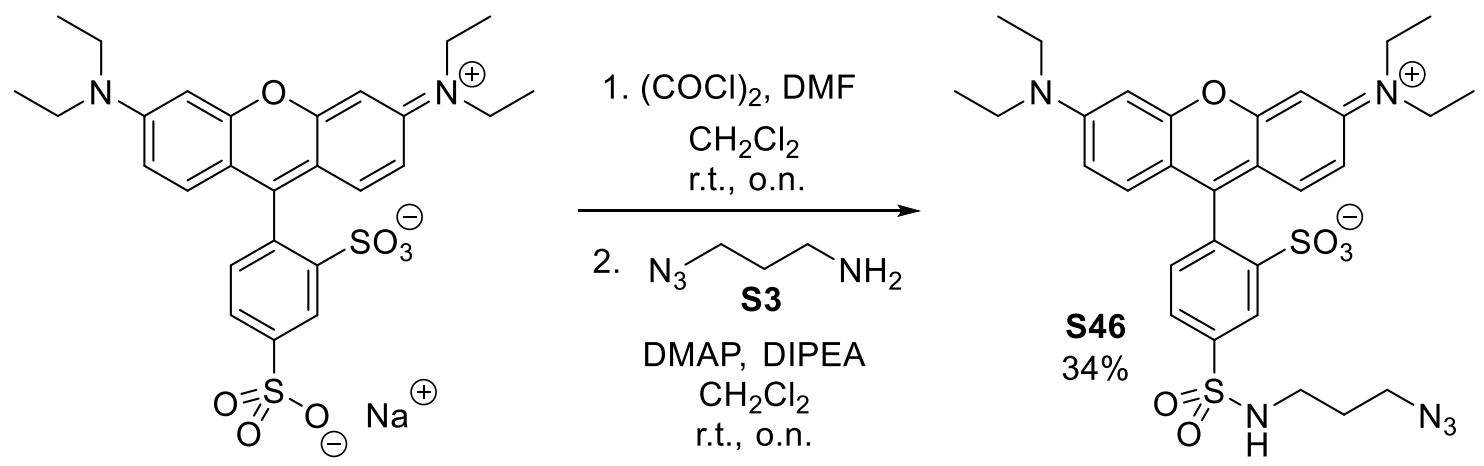

Scheme S13. Synthesis of sulforhodamine B 3-azidopropyl amide (S46). 


\section{Synthetic Protocols}

\section{3-Azidopropyl Amine (S3)}

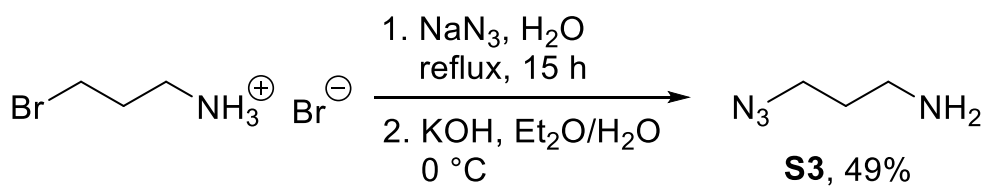

Compound $\mathbf{S 3}$ was prepared according to a modified procedure from the literature. ${ }^{13}$ 3-Bromopropylamine hydrobromide (3.28 g, $15.0 \mathrm{mmol}, 1.0$ eq.) and $\mathrm{NaN}_{3}$ (3.30 g, $50.8 \mathrm{mmol}$, 3.4 eq.) were dissolved in $\mathrm{H}_{2} \mathrm{O}(25 \mathrm{~mL})$. The resulting solution was heated at reflux for $15 \mathrm{~h}$. After cooling down to r.t., the reaction mixture was diluted with $\mathrm{Et}_{2} \mathrm{O}(25 \mathrm{~mL})$ and further cooled to $0{ }^{\circ} \mathrm{C}$. $\mathrm{KOH}(4.0 \mathrm{~g})$ was added slowly at $0{ }^{\circ} \mathrm{C}$. After complete dissolution of $\mathrm{KOH}$, the mixture was diluted with further $\mathrm{Et}_{2} \mathrm{O}(25 \mathrm{~mL})$ and the organic layer was separated. The aqueous layer was extracted with more $\mathrm{Et}_{2} \mathrm{O}(2 \times 30 \mathrm{~mL})$. The combined organic extracts were dried over anhydrous $\mathrm{MgSO}_{4}$, filtered and carefully concentrated by rotary evaporation (Caution: The product is volatile!) to provide 3-azidopropyl amine (S3) as a yellowish liquid. The compound was used in the next step without further purification. Yield: $0.74 \mathrm{~g} \mathrm{(49 \% ).}$

${ }^{1} \mathbf{H}$ NMR $\left(400 \mathrm{MHz}, \mathrm{CDCl}_{3}\right) \delta 3.35(\mathrm{t}, J=6.7 \mathrm{~Hz}, 2 \mathrm{H}), 2.79(\mathrm{t}, J=6.8 \mathrm{~Hz}, 2 \mathrm{H}), 1.71(\mathrm{p}, J=6.7$ $\mathrm{Hz}, 2 \mathrm{H}), 1.41$ (s, 2H).

${ }^{13} \mathbf{C}\left\{{ }^{1} \mathbf{H}\right\}$ NMR $\left(75 \mathrm{MHz}, \mathrm{CDCl}_{3}\right) \delta 49.3,39.4,32.5$.

MS (APCI+): calculated $m / z$ for $\left[\mathrm{C}_{3} \mathrm{H}_{8} \mathrm{~N}_{4}+\mathrm{H}\right]^{+}=101.1$, found $=101.3$.

\section{$N$-(3-Azidopropyl)-O-(4-nitrophenyl) Carbamate (7)}<smiles>[Y8]NCCCNC(=O)Oc1ccc([N+](=O)[O-])cc1</smiles>

A solution of 3-azidopropyl amine (S3, $100 \mathrm{mg}, 1.00 \mathrm{mmol}, 1.0$ eq.) in $\mathrm{CH}_{2} \mathrm{Cl}_{2}$ (4 mL) was added dropwise over $10 \mathrm{~min}$ to a stirred solution of bis(4-nitrophenyl) carbonate (304 mg, $1.00 \mathrm{mmol}$, 
1.0 eq.) in $\mathrm{CH}_{2} \mathrm{Cl}_{2}(3 \mathrm{~mL})$ and the resulting solution was stirred at r.t. for $4 \mathrm{~h}$. A solution of sodium hydroxide (45 mg, $1.0 \mathrm{mmol}, 1.1$ eq.) in water $(5 \mathrm{~mL})$ was then cautiously added to the reaction mixture and shaken. The organic layer was separated, dried over anhydrous $\mathrm{MgSO}_{4}$, filtered and concentrated by rotary evaporation. The crude oil was purified by silica gel column chromatography using $\mathrm{CH}_{2} \mathrm{Cl}_{2}$ as the mobile phase. Carbamate 7 was obtained as a white crystalline solid. Yield: $171 \mathrm{mg}(61 \%)$.

$\boldsymbol{R}_{f}\left(\right.$ silica, $\left.\mathrm{CH}_{2} \mathrm{Cl}_{2}\right): 0.14$

${ }^{1}$ H NMR (400 MHz, DMSO- $\left.d_{6}\right) \delta 8.30$ - $8.22(\mathrm{~m}, 2 \mathrm{H}), 8.09(\mathrm{t}, J=5.7 \mathrm{~Hz}, 1 \mathrm{H}), 7.44-7.36(\mathrm{~m}$, $2 \mathrm{H}), 3.43(\mathrm{t}, J=6.8 \mathrm{~Hz}, 2 \mathrm{H}), 3.17(\mathrm{q}, J=6.6 \mathrm{~Hz}, 2 \mathrm{H}), 1.75(\mathrm{p}, J=6.8 \mathrm{~Hz}, 2 \mathrm{H})$.

${ }^{13} \mathbf{C}\left\{{ }^{1} \mathbf{H}\right\}$ NMR $\left(75 \mathrm{MHz}, \mathrm{CDCl}_{3}\right) \delta 156.0,153.3,145.0,125.3,122.1,49.4,39.3,29.0$.

HRMS (ESI+): calculated $m / z$ for $\left[\mathrm{C}_{10} \mathrm{H}_{11} \mathrm{~N}_{5} \mathrm{O}_{4}+\mathrm{H}\right]^{+}=266.0889$, found $=266.0882$.

FTIR (ATR, diamond, $\mathrm{cm}^{-1}$ ): $2102\left(\mathrm{~N}_{3}\right)$ (Fig. S34 in the Infrared Spectra section).

m.p. $\left(\mathrm{CH}_{2} \mathrm{Cl}_{2}\right): 97-98^{\circ} \mathrm{C}$.

$(1 R, 8 S, 9 s, Z)$-Ethyl bicyclo[6.1.0]non-4-ene-9-carboxylate (endo-S4) and (1R,8S,9r,Z)-ethyl bicyclo[6.1.0]non-4-ene-9-carboxylate (exo-S4)

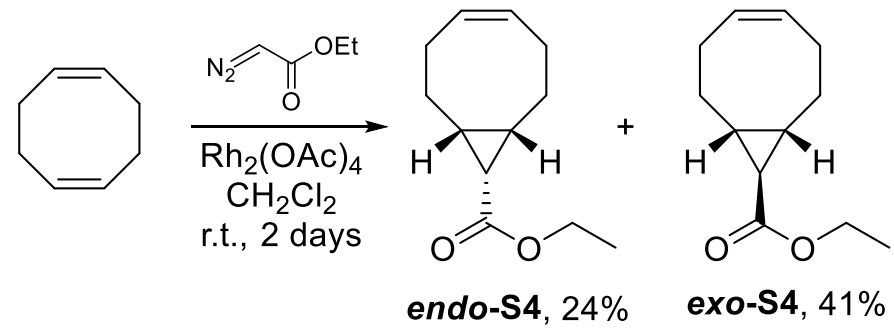

Compounds endo-S4 and exo-S4 were prepared according to a modified procedure from the literature. ${ }^{10} \mathrm{Rh}_{2}(\mathrm{OAc})_{4}(790 \mathrm{mg}, 1.79 \mathrm{mmol}, 4.3 \mathrm{~mol}$. \%) was put under argon atmosphere in an oven-dried $500 \mathrm{~mL}$ round bottomed flask followed by addition of dry $\mathrm{CH}_{2} \mathrm{Cl}_{2}(20 \mathrm{~mL})$ and cycloocta-1,5-diene (41 mL, $0.33 \mathrm{~mol}, 8.1$ eq.). A solution of ethyl diazoacetate (Aldrich, cont. 13 wt. $\% \mathrm{CH}_{2} \mathrm{Cl}_{2}, 5.4 \mathrm{~g}, 41 \mathrm{mmol}, 1.0$ eq.) in dry $\mathrm{CH}_{2} \mathrm{Cl}_{2}(20 \mathrm{~mL})$ was added dropwise slowly over 
the course of $7 \mathrm{~h}$ at constant stirring. The reaction mixture was stirred at r.t. for further 2 days. The resulting solution was concentrated by rotary evaporation $\left(40^{\circ} \mathrm{C}\right)$. Silica gel column chromatography using hexanes-AcOEt (200:1 to 20:1, v/v) provided endo-S4 (higher $R_{f}, 1.96 \mathrm{~g}$, $24 \%$ ) and $e x o-\mathbf{S 4}$ (lower $R_{f}, 3.28 \mathrm{~g}, 41 \%$ ) as colorless liquids.

endo-S4:

${ }^{1} \mathbf{H}$ NMR $\left(500 \mathrm{MHz}, \mathrm{CDCl}_{3}\right) \delta 5.67-5.57(\mathrm{~m}, 2 \mathrm{H}), 4.12(\mathrm{q}, J=7.1 \mathrm{~Hz}, 2 \mathrm{H}), 2.57-2.45(\mathrm{~m}, 2 \mathrm{H})$, $2.26-2.15(\mathrm{~m}, 2 \mathrm{H}), 2.12-2.00(\mathrm{~m}, 2 \mathrm{H}), 1.89-1.77(\mathrm{~m}, 2 \mathrm{H}), 1.71(\mathrm{t}, J=8.8 \mathrm{~Hz}, 1 \mathrm{H}), 1.45-1.34$ $(\mathrm{m}, 2 \mathrm{H}), 1.26(\mathrm{t}, J=7.1 \mathrm{~Hz}, 3 \mathrm{H})$.

MS (APCI+): calculated $m / z$ for $\left[\mathrm{C}_{12} \mathrm{H}_{19} \mathrm{O}_{2}+\mathrm{H}\right]^{+}=195.1$, found $=195.1$.

exo-S4:

${ }^{1} \mathbf{H}$ NMR $\left(500 \mathrm{MHz}, \mathrm{CDCl}_{3}\right) \delta 5.68-5.60(\mathrm{~m}, 2 \mathrm{H}), 4.10(\mathrm{q}, J=7.1 \mathrm{~Hz}, 2 \mathrm{H}), 2.35-2.26(\mathrm{~m}, 2 \mathrm{H})$, $2.24-2.16(\mathrm{~m}, 2 \mathrm{H}), 2.13-2.04(\mathrm{~m}, 2 \mathrm{H}), 1.60-1.53(\mathrm{~m}, 2 \mathrm{H}), 1.52-1.44(\mathrm{~m}, 2 \mathrm{H}), 1.25(\mathrm{t}, J=$ $7.1 \mathrm{~Hz}, 3 \mathrm{H}), 1.18(\mathrm{t}, J=4.6 \mathrm{~Hz}, 1 \mathrm{H})$.

${ }^{13} \mathbf{C}\left\{{ }^{1} \mathbf{H}\right\}$ NMR $\left(126 \mathrm{MHz}, \mathrm{CDCl}_{3}\right) \delta 174.6,130.1,60.4,28.4,28.0,27.9,26.8,14.5$.

MS (APCI+): calculated $m / z$ for $\left[\mathrm{C}_{12} \mathrm{H}_{19} \mathrm{O}_{2}+\mathrm{H}\right]^{+}=195.1$, found $=195.1$.

$(1 R, 8 S, 9 s, Z)$-Bicyclo[6.1.0]non-4-en-9-ylmethanol (S5)

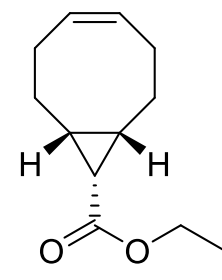

endo-S4

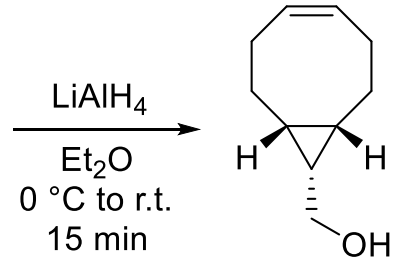

S5, quant.

Compound S5 was prepared according to a modified procedure from the literature. ${ }^{10} \mathrm{LiAlH}_{4}$ $(0.38 \mathrm{~g}, 10 \mathrm{mmol}, 1.0 \mathrm{eq}$.$) was put under argon atmosphere in an oven-dried 500 \mathrm{~mL}$ round bottomed flask followed by addition of $\operatorname{dry} \mathrm{Et}_{2} \mathrm{O}(35 \mathrm{~mL})$. The suspension was cooled to $0{ }^{\circ} \mathrm{C}$, after which a solution of endo-S4 $\left(1.95 \mathrm{~g}, 10 \mathrm{mmol}, 1.0\right.$ eq.) in dry $\mathrm{Et}_{2} \mathrm{O}(35 \mathrm{~mL})$ was added 
dropwise over $30 \mathrm{~min}$. Cooling was removed, and the reaction mixture was stirred at r.t. for $15 \mathrm{~min}$. The resulting suspension was cooled to $0{ }^{\circ} \mathrm{C}$ and $\mathrm{H}_{2} \mathrm{O}(4 \mathrm{~mL})$ was added dropwise. The reaction mixture was stirred at $0{ }^{\circ} \mathrm{C}$ until the color of the present solid changed from grey to white. $\mathrm{Et}_{2} \mathrm{O}$ $(100 \mathrm{~mL})$ was added and the reaction mixture was allowed to warm-up to r.t. After drying with anhydrous $\mathrm{Na}_{2} \mathrm{SO}_{4}$ for $1 \mathrm{~h}$, the solution was filtered and concentrated by rotary evaporation to provide alcohol S5 as a white solid. Yield: $1.52 \mathrm{~g}$ (quantitative).

${ }^{1} \mathrm{H}$ NMR $\left(500 \mathrm{MHz}, \mathrm{CDCl}_{3}\right) \delta 5.68-5.59(\mathrm{~m}, 2 \mathrm{H}), 3.71(\mathrm{dd}, J=7.7,3.6 \mathrm{~Hz}, 2 \mathrm{H}), 2.40-2.32(\mathrm{~m}$, 2H), $2.15-2.05(\mathrm{~m}, 2 \mathrm{H}), 2.03-1.95(\mathrm{~m}, 2 \mathrm{H}), 1.60-1.53(\mathrm{~m}, 2 \mathrm{H}), 1.16$ (br s, 1H), $1.15-1.09$ $(\mathrm{m}, 1 \mathrm{H}), 1.06-0.97(\mathrm{~m}, 2 \mathrm{H})$.

MS (APCI+): calculated $m / z$ for $\left[\mathrm{C}_{10} \mathrm{H}_{16} \mathrm{O}-\mathrm{OH}\right]^{+}=135.1$, found $=135.1$.

\section{$((1 R, 8 S, 9 s)-4,5-D i b r o m o b i c y c l o[6.1 .0]$ nonan-9-yl)methanol (S6)}

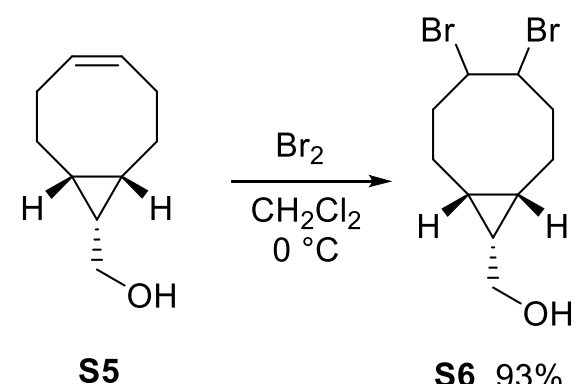

Compound S6 was prepared according to a modified procedure from the literature. ${ }^{10}$ A solution of S5 (1.52 g, $9.98 \mathrm{mmol})$ in $\mathrm{CH}_{2} \mathrm{Cl}_{2}(75 \mathrm{~mL})$ was cooled down to $0{ }^{\circ} \mathrm{C}$ followed by dropwise addition of a $\mathrm{Br}_{2}$ solution in $\mathrm{CH}_{2} \mathrm{Cl}_{2}(0.26 \mathrm{~g} / \mathrm{mL})$ at constant stirring as long as the resulting solution was decolorizing. When a yellowish color persisted, a solution of $\mathrm{Na}_{2} \mathrm{~S}_{2} \mathrm{O}_{3} \cdot 5 \mathrm{H}_{2} \mathrm{O}(2.0 \mathrm{~g})$ in $\mathrm{H}_{2} \mathrm{O}(20 \mathrm{~mL})$ was added to quench excess $\mathrm{Br}_{2}$. The organic layer was separated, and the aqueous phase was extracted with further $2 \times 30 \mathrm{~mL}$ of $\mathrm{CH}_{2} \mathrm{Cl}_{2}$. The combined organic extracts were dried over anhydrous $\mathrm{Na}_{2} \mathrm{SO}_{4}$, filtered and concentrated by rotary evaporation to provide dibromide S6 (mixture of diastereomers) as a white solid which was used in the next step without further purification. Yield: $2.90 \mathrm{~g}(93 \%)$. 
${ }^{1} \mathbf{H}$ NMR $\left(500 \mathrm{MHz}, \mathrm{CDCl}_{3}\right) \delta 4.88-4.78(\mathrm{~m}, 2 \mathrm{H}), 3.81-3.72(\mathrm{~m}, 2 \mathrm{H}), 2.76-2.63(\mathrm{~m}, 2 \mathrm{H}), 2.32$ $-2.23(\mathrm{~m}, 1 \mathrm{H}), 2.20-2.13(\mathrm{~m}, 1 \mathrm{H}), 1.99-1.86(\mathrm{~m}, 2 \mathrm{H}), 1.70-1.56(\mathrm{~m}, 2 \mathrm{H}), 1.26$ (br s, $1 \mathrm{H})$, $1.24-1.15(\mathrm{~m}, 2 \mathrm{H}), 1.15-1.06(\mathrm{~m}, 2 \mathrm{H}), 0.90-0.78(\mathrm{~m}, 1 \mathrm{H})$.

\section{$(1 R, 8 S, 9 p)$-Bicyclo[6.1.0]non-4-yn-9-ylmethanol (S7, BCN-OH)}

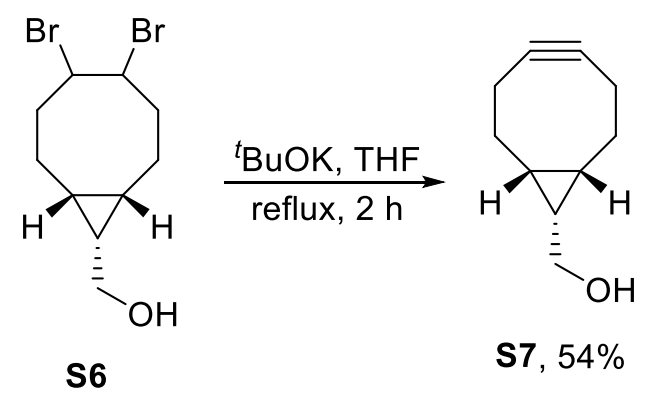

Compound $\mathbf{S 7}$ was prepared according to a modified procedure from the literature. ${ }^{10}$ A solution of S6 $(2.90 \mathrm{~g}, 9.29 \mathrm{mmol}, 1.0$ eq. $)$ in dry THF $(100 \mathrm{~mL})$ was cooled to $0{ }^{\circ} \mathrm{C}$ under dry Ar atmosphere. A solution of ${ }^{t} \mathrm{BuOK}(1 \mathrm{M}$ in THF, $31 \mathrm{~mL}, 31 \mathrm{mmol}, 3.3 \mathrm{eq}$.) was added dropwise at constant stirring. Cooling was removed, and the reaction mixture was heated at reflux $\left(85^{\circ} \mathrm{C}\right)$ for $2 \mathrm{~h}$. After cooling to room temperature, sat. aq. $\mathrm{NH}_{4} \mathrm{Cl}(100 \mathrm{~mL})$ was added with vigorous stirring. The organic layer was separated, and the aqueous layer was extracted with further $3 \times 70 \mathrm{~mL}$ of $\mathrm{CH}_{2} \mathrm{Cl}_{2}$. The combined organic extracts were dried over anhydrous $\mathrm{Na}_{2} \mathrm{SO}_{4}$, filtered and concentrated by rotary evaporation to provide a brownish liquid. Purification by silica gel column chromatography using hexanes/ethyl acetate (9:1 to $2: 1, v / v)$ as a mobile phase provided product $\mathbf{S 7}$ as an off-white solid. Yield: $750 \mathrm{mg}(54 \%)$.

${ }^{1} \mathrm{H}$ NMR $\left(500 \mathrm{MHz}, \mathrm{CDCl}_{3}\right) \delta 3.73(\mathrm{~d}, J=7.9 \mathrm{~Hz}, 2 \mathrm{H}), 2.36-2.18(\mathrm{~m}, 6 \mathrm{H}), 1.67-1.56(\mathrm{~m}, 2 \mathrm{H})$, 1.34 (tt, $J=9.2,7.9 \mathrm{~Hz}, 1 \mathrm{H}), 1.14$ (br s, 1H), $0.99-0.89$ (m, 2H).

${ }^{13} \mathbf{C}\left\{{ }^{1} \mathbf{H}\right\}$ NMR $\left(126 \mathrm{MHz}, \mathrm{CDCl}_{3}\right) \delta 99.0,60.1,29.2,21.65,21.55,20.2$.

MS (APCI+): calculated $m / z$ for $\left[\mathrm{C}_{10} \mathrm{H}_{14} \mathrm{O}-\mathrm{OH}\right]^{+}=133.1$, found $=133.1$; calculated $\mathrm{m} / z$ for $\left[\mathrm{C}_{10} \mathrm{H}_{14} \mathrm{O}-\mathrm{OH}+\mathrm{CH}_{3} \mathrm{CN}\right]^{+}=174.1$, found $=174.2$. 


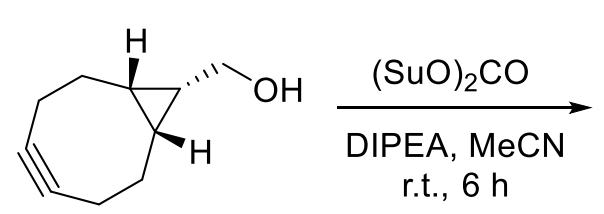

S7

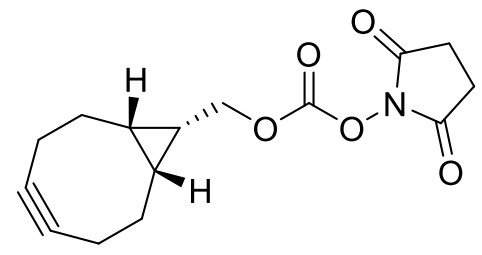

S8, $90 \%$

Compound S8 was prepared according to a modified procedure from the literature. ${ }^{8}$ A solution of BCN-OH (S7, $96 \mathrm{mg}, 0.64 \mathrm{mmol}, 1.0$ eq.) and dry DIPEA (450 $\mu \mathrm{L}, 2.6 \mathrm{mmol}, 4.0$ eq.) in dry $\mathrm{MeCN}(2.0 \mathrm{~mL})$ was cooled to $0{ }^{\circ} \mathrm{C}$ under dry Ar atmosphere. $(\mathrm{SuO})_{2} \mathrm{CO}(242 \mathrm{mg}, 0.94 \mathrm{mmol}$, 1.5 eq.) was added in one portion, cooling was removed, and the resulting mixture was stirred at r.t. under dry Ar atmosphere for 6 h. After concentrating by rotary evaporation $\left(25^{\circ} \mathrm{C}\right)$, the product was isolated by silica gel column chromatography using hexanes/ethyl acetate $(3: 2, v / v)$ as the mobile phase. BCN-OSu (S8) was obtained as a white solid. Yield: $167 \mathrm{mg}$ (90\%).

${ }^{1} \mathbf{H}$ NMR $\left(500 \mathrm{MHz}, \mathrm{CDCl}_{3}\right) \delta 4.45(\mathrm{~d}, J=8.3 \mathrm{~Hz}, 2 \mathrm{H}), 2.84(\mathrm{~s}, 4 \mathrm{H}), 2.37-2.19(\mathrm{~m}, 6 \mathrm{H}), 1.63-$ $1.56(\mathrm{~m}, 2 \mathrm{H}), 1.50(\mathrm{p}, J=8.7 \mathrm{~Hz}, 1 \mathrm{H}), 1.11-1.02(\mathrm{~m}, 2 \mathrm{H})$.

MS (APCI+): calculated $m / z$ for $\left[\mathrm{C}_{17} \mathrm{H}_{15} \mathrm{NO}_{5}-\mathrm{CO}_{2}+\mathrm{H}\right]^{+}=248.1$, found $=248.5$.

\section{$N$-Boc-4-aminobutanol (S9)}<smiles>CC(C)(C)OC(=O)NCCCCO</smiles>

Compound S9 was prepared according to a modified procedure from the literature. ${ }^{14}$ A solution of 4-aminobutanol (3.7 g, $42 \mathrm{mmol}, 1.0$ eq.) in $\mathrm{CH}_{2} \mathrm{Cl}_{2}(50 \mathrm{~mL})$ was cooled to $0{ }^{\circ} \mathrm{C}$. $\mathrm{Boc}_{2} \mathrm{O}(9.1 \mathrm{~g}$, $42 \mathrm{mmol}, 1.0$ eq.) was added dropwise followed by dropwise addition of $\mathrm{Et}_{3} \mathrm{~N}$ (6.9 mL, $50 \mathrm{mmol}$, 1.2 eq.). The resulting solution was stirred at r.t. for $18 \mathrm{~h}$ and subsequently concentrated by rotary evaporation to provide $N$-Boc-4-aminobutanol (S9) as a colorless oil which was used in the next step without purification. Yield: $7.9 \mathrm{~g}$ (quantitative). 
${ }^{1} \mathbf{H}$ NMR $\left(500 \mathrm{MHz}, \mathrm{CDCl}_{3}\right) \delta 4.66$ (br s, 1H), $3.72-3.63(\mathrm{~m}, 2 \mathrm{H}), 3.21-3.09$ (m, 2H), 1.92 (br $\mathrm{s}, 1 \mathrm{H}), 1.64-1.53(\mathrm{~m}, 4 \mathrm{H}), 1.44(\mathrm{~s}, 9 \mathrm{H})$.

MS (APCI+): calculated $m / z$ for $\left[\mathrm{C}_{9} \mathrm{H}_{19} \mathrm{NO}_{3}+\mathrm{H}\right]^{+}=190.1$, found $=190.1$.

\section{$N$-Boc-4-bromobutyl amine (S10)}<smiles>CC(C)(C)OC(=O)NCCCCBr</smiles>

Compound S10 was prepared according to a modified procedure from the literature. ${ }^{15}$ To a stirred solution of $\mathbf{S 9}$ (7.8 g, $41 \mathrm{mmol}, 1.0$ eq.) in THF (300 mL) was in portions added $\mathrm{PPh}_{3}(20.5 \mathrm{~g}$, $78.2 \mathrm{mmol}, 1.9$ eq.), followed by $\mathrm{CBr}_{4}$ (26 g, $78 \mathrm{mmol}, 1.9$ eq.). The reaction mixture was stirred at r.t. for $3 \mathrm{~h}$. Subsequently, the mixture was filtered through a short pad of celite and concentrated by rotary evaporation $\left(30^{\circ} \mathrm{C}\right)$. The crude product was purified by silica gel column chromatography using hexanes/AcOEt $(9: 1$ to $4: 1, v / v)$ as the mobile phase, providing $N$-Boc-4-bromobutyl amine (S10) as a yellowish oil. Yield: $10.2 \mathrm{~g}(98 \%)$.

${ }^{1} \mathrm{H}$ NMR $\left(500 \mathrm{MHz}, \mathrm{CDCl}_{3}\right) \delta 4.54(\mathrm{br} \mathrm{s}, 1 \mathrm{H}), 3.43(\mathrm{t}, J=6.7 \mathrm{~Hz}, 2 \mathrm{H}), 3.16(\mathrm{q}, J=6.7 \mathrm{~Hz}, 2 \mathrm{H})$, $1.94-1.85(\mathrm{~m}, 2 \mathrm{H}), 1.65(\mathrm{dd}, J=15.1,7.5 \mathrm{~Hz}, 3 \mathrm{H}), 1.44(\mathrm{~s}, 9 \mathrm{H})$.

${ }^{13} \mathbf{C}\left\{{ }^{1} \mathbf{H}\right\}$ NMR $\left(126 \mathrm{MHz}, \mathrm{CDCl}_{3}\right) \delta 154.8,79.0,46.1,45.8,28.7,25.9,25.1$.

MS (APCI + ): calculated $m / z$ for $\left[\mathrm{C}_{9} \mathrm{H}_{18} \mathrm{BrNO}_{2}+\mathrm{H}\right]^{+}=252.1$, found $=252.0$.

\section{$N$-(N'-Boc-aminobutyl)-4-methylpyridinium bromide (S11)}<smiles>CC(C)(C)OC(=O)NCCCCBr</smiles>

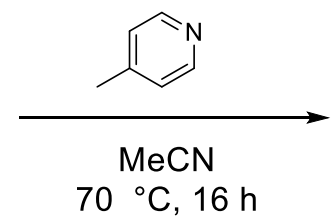<smiles>Cc1cc[n+](CCCCNC(=O)OC(C)(C)C)cc1</smiles> 
A solution of $\mathbf{S 1 0}$ (1.40 g, $5.55 \mathrm{mmol}, 1.3$ eq.) and 4-picoline (0.42 mL, $4.3 \mathrm{mmol}, 1.0$ eq.) in $\operatorname{MeCN}(5 \mathrm{~mL})$ was heated at $70{ }^{\circ} \mathrm{C}$ for $16 \mathrm{~h}$. After cooling to r.t., the reaction mixture was added dropwise into stirred $\mathrm{Et}_{2} \mathrm{O}(50 \mathrm{~mL})$ resulting in precipitation of a brown solid. The precipitate was collected by vacuum filtration and washed with $\mathrm{Et}_{2} \mathrm{O}(2 \times 50 \mathrm{~mL})$. The crude product was purified by chromatography on basic alumina using $\mathrm{CH}_{2} \mathrm{Cl}_{2} / \mathrm{MeOH}(50: 1$ to $9: 1, v / v)$ as the mobile phase, providing S11 as a reddish-brown oil. Yield: $118 \mathrm{mg}$ (7.9\%).

$\boldsymbol{R}_{\boldsymbol{f}}$ (alumina, $\mathrm{CH}_{2} \mathrm{Cl}_{2} / \mathrm{MeOH}$ 9:1, v/v): 0.42 .

${ }^{1} \mathbf{H}$ NMR (400 MHz, $\left.\mathrm{CDCl}_{3}\right) \delta 9.41(\mathrm{~d}, J=6.4 \mathrm{~Hz}, 2 \mathrm{H}), 7.79(\mathrm{~d}, J=6.2 \mathrm{~Hz}, 2 \mathrm{H}), 5.15(\mathrm{br} \mathrm{t}, J=$ $6.3 \mathrm{~Hz}, 1 \mathrm{H}), 5.07-4.98(\mathrm{~m}, 2 \mathrm{H}), 3.22(\mathrm{q}, J=6.4 \mathrm{~Hz}, 2 \mathrm{H}), 2.09(\mathrm{p}, J=7.1 \mathrm{~Hz}, 2 \mathrm{H}), 1.68(\mathrm{p}, J=$ $6.7 \mathrm{~Hz}, 2 \mathrm{H}), 1.43(\mathrm{~s}, 9 \mathrm{H})$.

${ }^{13} \mathbf{C}\left\{{ }^{1} \mathbf{H}\right\} \mathbf{N M R}\left(126 \mathrm{MHz}, \mathrm{CDCl}_{3}\right) \delta 158.8,156.6,144.4,128.7,79.2,60.2,38.7,29.0,28.5,26.6$, 22.3.

HRMS (ESI+): calculated $m / z$ for $\left[\mathrm{C}_{15} \mathrm{H}_{25} \mathrm{~N}_{2} \mathrm{O}_{2}\right]^{+}=265.1916$, found $=265.1912$.

6-Bromo- $N, N$-diethylnaphthalen-2-amine (S12) and 6-bromo- $N$-ethylnaphthalen-2-amine (S12b)

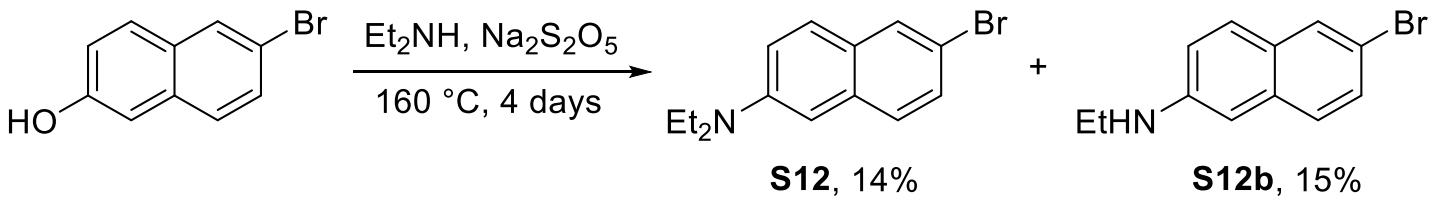

The following procedure was adopted from Kim, Ahn et. al. ${ }^{16}$ 6-Bromo-naphth-2-ol (10.0 g, $44.8 \mathrm{mmol}, 1.0$ eq.) and sodium metabisulfite (17.9 g, $93.9 \mathrm{mmol}, 2.1$ eq.) were weighted into a heavy-walled high-pressure vessel and suspended in water $(100 \mathrm{~mL})$. Then, diethylamine $(23 \mathrm{~mL}$, $224 \mathrm{mmol}, 5.0$ eq.) was added in one batch and the vessel was sealed. The reaction mixture was stirred for 4 days at $200^{\circ} \mathrm{C}$, and then cooled down to r.t. The crude mixture was extracted with $\mathrm{CH}_{2} \mathrm{Cl}_{2}(3 \times 50 \mathrm{~mL})$. The combined organic layers were washed with brine $(50 \mathrm{~mL})$, dried over anhydrous $\mathrm{Na}_{2} \mathrm{SO}_{4}$, filtered and volatiles were removed under reduced pressure. The obtained 
waxy residuum was separated by silica gel column chromatography using ethyl acetate/hexanes $(1: 19, v / v)$ to yield pure products.

6-Bromo- $N, N$-diethylnaphthalen-2-amine (S12). Yield: $1.67 \mathrm{~g}$ (14\%). Yellowish crystalline solid. This compound has also been characterized elsewhere. ${ }^{17}$

$\boldsymbol{R}_{\boldsymbol{f}}$ (silica; ethyl acetate/hexanes 1:19, v/v): 0.2.

${ }^{1} \mathbf{H}$ NMR (400 MHz, $\left.\mathrm{CDCl}_{3}\right): \delta(\mathrm{ppm}) 1.23(\mathrm{t}, 6 \mathrm{H}, J=7.0 \mathrm{~Hz}), 3.46(\mathrm{q}, 4 \mathrm{H}, J=7.0 \mathrm{~Hz}), 6.82(\mathrm{~d}$, $1 \mathrm{H}, J=2.4 \mathrm{~Hz}), 7.11\left(\mathrm{dd}, 1 \mathrm{H}, J_{1}=9.1 \mathrm{~Hz}, J_{2}=2.6 \mathrm{~Hz}\right), 7.39\left(\mathrm{dd}, 1 \mathrm{H}, J_{1}=8.7 \mathrm{~Hz}, J_{2}=2.0 \mathrm{~Hz}\right)$, $7.48(\mathrm{~d}, 1 \mathrm{H}, J=8.8 \mathrm{~Hz}), 7.59(\mathrm{~d}, 1 \mathrm{H}, J=9.2 \mathrm{~Hz}), 7.80(\mathrm{~d}, 1 \mathrm{H}, J=1.8 \mathrm{~Hz})$.

${ }^{13} \mathbf{C}\left\{{ }^{1} \mathbf{H}\right\}$ NMR $\left(100 \mathrm{MHz}, \mathrm{CDCl}_{3}\right): \delta(\mathrm{ppm})$ 14.9, 38.6, 104.2, 115.21, 119.0, 127.8, 128.2, 128.7, 129.6, 129.8, 134.0, 146.6.

MS (ESI+): calculated $m / z$ for $\left[\mathrm{C}_{14} \mathrm{H}_{16} \mathrm{BrN}+\mathrm{H}\right]^{+}=278.1$, found $=278.3$

IR (ATR, diamond, $\mathrm{cm}^{-1}$ ): 2968, 2928, 2886, 1618, 1582, 1498, 1446, 1398, 1350, 1268, 1224, $1176,1150,1124,1056,1010,924,872,838,808,792,650,468$.

m.p. (AcOEt/hexanes): $59.5-61.0^{\circ} \mathrm{C}$.

6-Bromo-N-ethylnaphthalen-2-amine (S12b). Yield: $1.64 \mathrm{~g}$ (15\%). White crystalline solid. This compound has also been characterized elsewhere. ${ }^{17}$

$\boldsymbol{R}_{\boldsymbol{f}}$ (silica; ethyl acetate/hexanes 1:19, v/v): 0.1 .

${ }^{1} \mathbf{H}$ NMR (400 MHz, $\left.\mathrm{CDCl}_{3}\right): \delta(\mathrm{ppm}) 1.33(\mathrm{t}, 3 \mathrm{H}, J=7.1 \mathrm{~Hz}), 3.26(\mathrm{q}, 2 \mathrm{H}, J=7.1 \mathrm{~Hz}), 3.79$ (bs, $1 \mathrm{H},>\mathrm{NH}), 6.75(\mathrm{~d}, 1 \mathrm{H}, J=2.2 \mathrm{~Hz}), 6.88\left(\mathrm{dd}, 1 \mathrm{H}, J_{1}=8.8 \mathrm{~Hz}, J_{2}=2.3 \mathrm{~Hz}\right), 7.41\left(\mathrm{dd}, 1 \mathrm{H}, J_{1}=8.8\right.$ $\left.\mathrm{Hz}, J_{2}=1.9 \mathrm{~Hz}\right), 7.49(\mathrm{~d}, 1 \mathrm{H}, J=8.8 \mathrm{~Hz}), 7.53(\mathrm{~d}, 1 \mathrm{H}, J=8.8 \mathrm{~Hz}), 7.81(\mathrm{~d}, 1 \mathrm{H}, J=1.7 \mathrm{~Hz})$.

${ }^{13} \mathbf{C}\left\{{ }^{1} \mathbf{H}\right\} \mathbf{N M R}\left(100 \mathrm{MHz}, \mathrm{CDCl}_{3}\right): \delta(\mathrm{ppm}) 14.9,38.6,104.2,115.1,119.0,127.8,128.2,128.7$, 129.6, 129.8, 134.0, 146.6. 


\section{6-(Diethylamino)-2-naphthaldehyde (S13)}

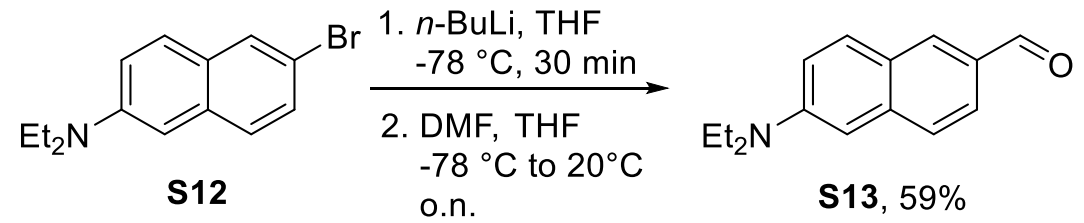

$n$-Butyllithium (1.6 mL, $2.5 \mathrm{~mol} \mathrm{dm}^{-3}$ in hexanes, $4.05 \mathrm{mmol}, 1.2 \mathrm{eq}$.) was dropwise added to a solution of 6-bromo- $N, N$-diethylnaphthalen-2-amine (S12, $0.94 \mathrm{~g}, 3.38 \mathrm{mmol}, 1.0$ eq.) in dry THF $(20 \mathrm{~mL})$ at $-78^{\circ} \mathrm{C}$ under dry Ar atmosphere and was further stirred for 30 minutes at $-78^{\circ} \mathrm{C}$. Then, dry DMF (0.32 mL, $4.05 \mathrm{mmol}, 1.2$ eq.) was added dropwise and the reaction mixture was stirred overnight, allowed to warm up to r.t. and subsequently diluted with sat. aq. $\mathrm{NH}_{4} \mathrm{Cl}(30 \mathrm{~mL})$. The aqueous layer was extracted with $\mathrm{CH}_{2} \mathrm{Cl}_{2}(3 \times 30 \mathrm{~mL})$. The combined organic layers were washed with brine $(30 \mathrm{~mL})$, dried over anhydrous $\mathrm{Na}_{2} \mathrm{SO}_{4}$, filtered and volatiles were removed under reduced pressure. The obtained residuum was purified by silica gel column chromatography (AcOEt/hexanes, $1: 9$ to $1: 4, v / v$ ) to afford pure product. White crystalline solid. Yield: $0.45 \mathrm{~g}$ $(59 \%)$.

$\boldsymbol{R}_{\boldsymbol{f}}($ silica; AcOEt/hexanes 1:4,v/v): 0.5.

${ }^{1} \mathbf{H}$ NMR (400 MHz, $\left.\mathrm{CDCl}_{3}\right): \delta(\mathrm{ppm}) 1.29(\mathrm{t}, 6 \mathrm{H}, J=7.1 \mathrm{~Hz}), 3.54(\mathrm{q}, 4 \mathrm{H}, J=7.1 \mathrm{~Hz}), 6.87$ (d, $1 \mathrm{H}, J=2.6 \mathrm{~Hz}), 7.15\left(\mathrm{dd}, 1 \mathrm{H}, J_{1}=9.1 \mathrm{~Hz}, J_{2}=2.6 \mathrm{~Hz}\right), 7.62(\mathrm{~d}, 1 \mathrm{H}, J=8.6 \mathrm{~Hz}), 7.81\left(\mathrm{dd}, 1 \mathrm{H}, J_{1}\right.$ $\left.=8.8 \mathrm{~Hz}, J_{2}=1.7 \mathrm{~Hz}\right), 8.13(\mathrm{~s}, 1 \mathrm{H}), 10.00(\mathrm{~s}, 1 \mathrm{H})$.

${ }^{13} \mathbf{C}\left\{{ }^{1} \mathbf{H}\right\}$ NMR $\left(100 \mathrm{MHz}, \mathrm{CDCl}_{3}\right): \delta(\mathrm{ppm}) 12.9,44.8,104.7,116.1,123.7,124.8,126.7,130.5$, $131.2,135.0,139.2,148.5,198.0$.

HRMS $(\mathrm{ESI}+)$ calculated $\mathrm{m} / z$ for $\left[\mathrm{C}_{15} \mathrm{H}_{18} \mathrm{NO}+\mathrm{H}\right]^{+}=228.1388$; found $=228.1383$.

IR (ATR, diamond, $\mathrm{cm}^{-1}$ ): 2972, 2931, 2898, 2869, 2813, 1680, 1614, 1507, 1492, 1478, 1428, 1400, 1377, 1354, 1276, 1206, 1168, 114, 1076, 1013, 848, 806, 689, 475.

m.p. (AcOEt/hexanes): $101.5-102.5^{\circ} \mathrm{C}$. 


\section{(E)-N,N-Diethyl-6-(2-(pyridin-4-yl)vinyl)naphthalen-2-amine (S14).}

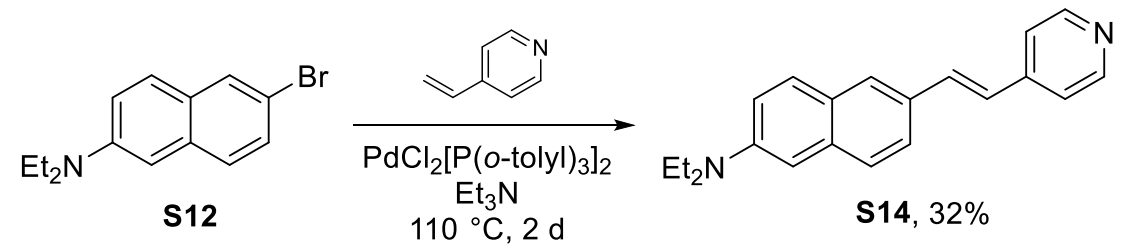

This procedure was adopted from Loew et al. ${ }^{18}$ 4-Vinylpyridine $(0.19 \mathrm{~g}, 1.80 \mathrm{mmol}, 1.25 \mathrm{eq}$.$) was$ added in one batch to a suspension of 6-bromo- $N, N$-diethylnaphthalen-2-amine (S12; $0.40 \mathrm{~g}$, 1.44 mmol, 1.0 eq. $)$ and $\mathrm{PdCl}_{2}\left[\mathrm{P}(o \text {-tolyl })_{3}\right]_{2}(0.28 \mathrm{~g}, 0.36 \mathrm{mmol}, 25 \mathrm{~mol} . \%)$ in $\mathrm{Et}_{3} \mathrm{~N}(50 \mathrm{~mL})$ in a pre-dried high-pressure reaction vessel and stirred for two days at $110^{\circ} \mathrm{C}$. The reaction mixture was then allowed to cool down to r.t. and volatiles were removed under reduced pressure. The obtained residuum was dissolved in $\mathrm{CH}_{2} \mathrm{Cl}_{2}(50 \mathrm{~mL})$ and washed with $\mathrm{H}_{2} \mathrm{O}(50 \mathrm{~mL})$. The aqueous phase was further extracted with $\mathrm{CH}_{2} \mathrm{Cl}_{2}(2 \times 50 \mathrm{~mL})$. The combined organic extracts were dried over anhydrous $\mathrm{Na}_{2} \mathrm{SO}_{4}$, filtered and volatiles were removed under reduced pressure. The obtained residuum was purified by silica gel column chromatography $\left(\mathrm{CH}_{2} \mathrm{Cl}_{2} /\right.$ hexanes $1: 1, v / v \rightarrow 5$ vol. \% $\mathrm{Et}_{3} \mathrm{~N}$ in $\mathrm{CH}_{2} \mathrm{Cl}_{2} /$ hexanes $\left.1: 1, v / v\right)$. Yellow crystalline solid. Yield: $0.14 \mathrm{~g}(32 \%)$.

$\boldsymbol{R}_{\mathbf{f}}$ (silica; 5 vol.\% $\mathrm{Et}_{3} \mathrm{~N}$ in $\mathrm{CH}_{2} \mathrm{Cl}_{2} /$ hexanes $\left.1: 1, v / v\right): 0.25$.

${ }^{1} \mathbf{H}$ NMR (400 MHz, C6 $\left.\mathrm{D}_{6}\right): \delta(\mathrm{ppm}) 0.95(\mathrm{t}, 6 \mathrm{H}, J=7.1 \mathrm{~Hz}), 3.07$ (q, 4H, J = 7.1 Hz), 6.81 (d, $1 \mathrm{H}, J=16.3 \mathrm{~Hz}), 6.89(\mathrm{~d}, 1 \mathrm{H}, \mathrm{J}=2.4 \mathrm{~Hz}), 6.94\left(\mathrm{dd}, 1 \mathrm{H}, J_{1}=9.2 \mathrm{~Hz}, J_{2}=2.6 \mathrm{~Hz}\right), 6.98(\mathrm{~d}, 1 \mathrm{H}, J$ $=1.75 \mathrm{~Hz}), 6.99(\mathrm{~d}, 1 \mathrm{H}, J=1.5 \mathrm{~Hz}), 7.22(\mathrm{~d}, 1 \mathrm{H}, J=16.3 \mathrm{~Hz}), 7.54\left(\mathrm{dd}, 1 \mathrm{H}, J_{1}=8.6 \mathrm{~Hz}, J_{2}=1.6\right.$ $\mathrm{Hz}), 7.59-7.66(\mathrm{~m}, 3 \mathrm{H}), 8.62(\mathrm{~d}, 1 \mathrm{H}, J=1.5 \mathrm{~Hz}), 8.63(\mathrm{~d}, 1 \mathrm{H}, \mathrm{J}=1.6 \mathrm{~Hz})$.

MS (APCI+): calculated $m / z$ for $\left[\mathrm{C}_{21} \mathrm{H}_{22} \mathrm{~N}_{2}+\mathrm{H}\right]^{+}=303.2$, found $=303.3$.

m.p. $\left(\mathrm{CH}_{2} \mathrm{Cl}_{2} /\right.$ hexanes/Et $\left.3 \mathrm{~N}\right)$ : $>200^{\circ} \mathrm{C}$ (decomp.). 


\section{(E)-1-(5-(tert-Butoxy)-5-oxopentyl)-4-(2-(6-(diethylamino)naphthalen-2-yl)vinyl)pyridin-1-}

ium bromide (S15).

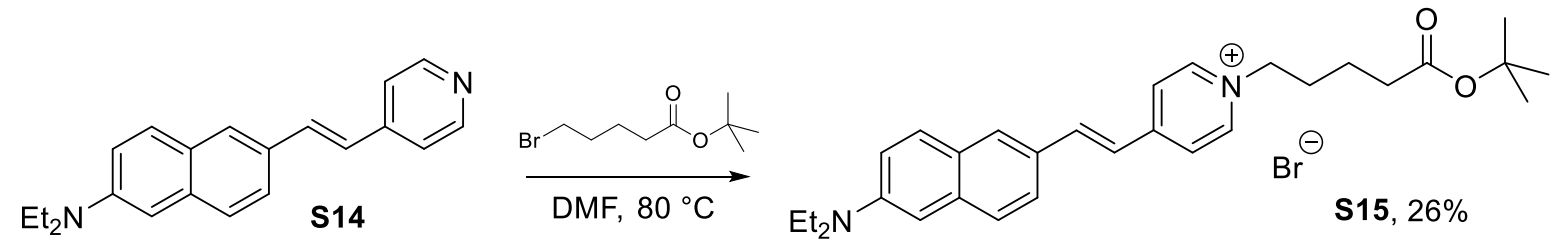

A flask with solid S14 (139 mg, $0.46 \mathrm{mmol}, 1.0$ eq.) was dried under high vacuum and back-filled with dry Ar. Dry DMF (5 mL) was added followed by tert-butyl 5-bromopentanoate (218 mg, $0.92 \mathrm{mmol}, 2.0 \mathrm{eq}$.) and the reaction mixture was further stirred overnight at $80^{\circ} \mathrm{C}$. The reaction mixture was then allowed to cool down to r.t. and volatiles were removed under reduced pressure. The obtained residuum was purified by column chromatography on basic alumina $\left(\mathrm{MeOH} / \mathrm{CH}_{2} \mathrm{Cl}_{2}\right.$ $1: 49 \rightarrow 1: 19, v / v)$ to yield pure product. Dark violet crystalline solid. Yield: $65 \mathrm{mg}(26 \%)$.

$\boldsymbol{R}_{\mathbf{f}}$ (alumina; $\mathrm{MeOH} / \mathrm{CH}_{2} \mathrm{Cl}_{2}$ 1:49, v/v): 0.05 .

${ }^{1} \mathbf{H}$ NMR (400 MHz, $\left.\mathrm{CDCl}_{3}\right): \delta(\mathrm{ppm}) 1.22(\mathrm{t}, 6 \mathrm{H}, J=7.0 \mathrm{~Hz}), 1.39$ (s, 9H), $1.62(\mathrm{p}, 2 \mathrm{H}, J=7.4$ $\mathrm{Hz}), 1.96-2.06(\mathrm{~m}, 2 \mathrm{H}), 2.27$ (t, 2H, $J=7.2 \mathrm{~Hz}), 3.45(\mathrm{q}, 4 \mathrm{H}, J=7.0 \mathrm{~Hz}), 4.71(\mathrm{t}, 2 \mathrm{H}, J=7.2 \mathrm{~Hz})$, $6.77(\mathrm{~d}, 1 \mathrm{H}, J=2.0 \mathrm{~Hz}), 7.02-7.08(\mathrm{~m}, 2 \mathrm{H}), 7.52(\mathrm{~d}, 1 \mathrm{H}, J=8.8 \mathrm{~Hz}), 7.57(\mathrm{~d}, 1 \mathrm{H}, J=8.8 \mathrm{~Hz})$, $7.67(\mathrm{~d}, 1 \mathrm{H}, J=9.1 \mathrm{~Hz}), 7.75(\mathrm{~d}, 1 \mathrm{H}, J=16.0 \mathrm{~Hz}), 7.79$ (s, 1H), 7.96 (d, 2H, $J=6.3 \mathrm{~Hz}), 9.04$ (d, $1 \mathrm{H}, J=6.3 \mathrm{~Hz})$.

${ }^{13} \mathbf{C}\left\{{ }^{1} \mathbf{H}\right\}$ NMR $\left(100 \mathrm{MHz}, \mathrm{CDCl}_{3}\right): \delta(\mathrm{ppm}) 12.9,21.5,28.3,31.0,34.6,44.7,60.1,80.7,105.0$, 116.1, 119.3, 123.6, 124.0, 125.6, 127.0, 127.7, 130.5, 131.6, 137.2, 143.3, 143.9, 147.6, 154.0, 172.4 .

MS (ESI + ) calculated $m / z$ for $\left[\mathrm{C}_{30} \mathrm{H}_{39} \mathrm{~N}_{2} \mathrm{O}_{2}\right]^{+}=459.3$; found $=459.4$.

HRMS (ESI+) calculated $\mathrm{m} / z$ for $\left[\mathrm{C}_{30} \mathrm{H}_{39} \mathrm{~N}_{2} \mathrm{O}_{2}\right]^{+}=459.3006$; found $=459.3011$

IR (ATR, diamond, $\mathrm{cm}^{-1}$ ): 3366 (br), 2973, 2927, 1724, 1642, 1593, 1490, 1466, 1360, 1262, 1206, 1149, 970, 854, 809, 666, 579, 526, 474.

m.p. $\left(\mathrm{MeOH} / \mathrm{CH}_{2} \mathrm{Cl}_{2}\right): 196-199{ }^{\circ} \mathrm{C}$. 
(E)-4-(2-(6-(Diethylamino)naphthalen-2-yl)vinyl)-1-(4-carboxybutyl)pyridin-1-ium bromide (S16).

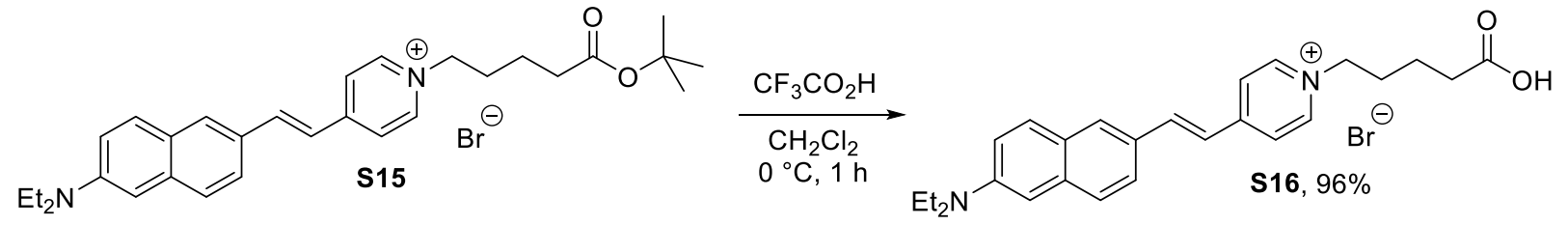

Trifluoroacetic acid (5 mL) was dropwise added to a stirred solution of $\mathbf{S 1 5}$ (63 $\mathrm{mg}, 0.117 \mathrm{mmol}$, 1.0 eq. ) in $\mathrm{CH}_{2} \mathrm{Cl}_{2}(25 \mathrm{~mL})$ at $0^{\circ} \mathrm{C}$ and stirred for $1 \mathrm{~h}$ at $0{ }^{\circ} \mathrm{C}$. Volatiles were then carefully removed under reduced pressure and the obtained residuum was co-evaporated with toluene $(1.0 \mathrm{~mL})$. The obtained residuum was further dried under high vacuum to yield pure product. Red waxy solid. Yield: $54 \mathrm{mg}(96 \%)$.

${ }^{1} \mathbf{H}$ NMR (400 MHz, CD 3 OD): $\delta(\mathrm{ppm}) 1.18$ (t, 6H, J = 7.0 Hz), 1.68 (p, 2H, J = 7.5 Hz), 2.06 (p, $2 \mathrm{H}, \mathrm{J}=7.5 \mathrm{~Hz}), 2.40(\mathrm{t}, 2 \mathrm{H}, \mathrm{J}=7.1 \mathrm{~Hz}), 3.74(\mathrm{q}, 4 \mathrm{H}, \mathrm{J}=7.0 \mathrm{~Hz}), 4.55(\mathrm{t}, 2 \mathrm{H}, \mathrm{J}=7.3 \mathrm{~Hz}), 7.58$ (d, $1 \mathrm{H}, \mathrm{J}=16.3 \mathrm{~Hz}), 7.65(\mathrm{~d}, 1 \mathrm{H}, \mathrm{J}=8.7 \mathrm{~Hz}), 7.94-8.05(\mathrm{~m}, 3 \mathrm{H}), 8.09(\mathrm{~d}, 1 \mathrm{H}, \mathrm{J}=16.3 \mathrm{~Hz}), 8.15(\mathrm{~d}$, $1 \mathrm{H}, \mathrm{J}=8.8 \mathrm{~Hz}), 8.20-8.26(\mathrm{~m}, 3 \mathrm{H}), 8.81(\mathrm{~d}, 2 \mathrm{H}, \mathrm{J}=6.2 \mathrm{~Hz})$.

${ }^{13} \mathbf{C}\left\{{ }^{1} \mathbf{H}\right\}$ NMR (100 MHz, CD $\left.3 \mathrm{OD}\right): \delta(\mathrm{ppm}) 11.1,22.4,31.6,33.9,53.2,61.6,120.0,124.9,125.5$, $126.5,129.87,129.92,131.1,132.9,133.6,135.2$, 135.66, 135.72, 142.4, 145.3, 155.2, 176.6 .

HRMS (ESI+) calculated $m / z$ for $\left[\mathrm{C}_{26} \mathrm{H}_{31} \mathrm{~N}_{2} \mathrm{O}_{2}\right]^{+}=403.2380$; found $=403.2385$.

IR (ATR, diamond, $\mathrm{cm}^{-1}$ ): 3412, 3052, 2968, 2931, 1667, 1618, 1588, 1470, 1412, 1355, 1168, 1126, 966, 878, 829, 798, 719, 518, 478. 


\section{(E)-4-(2-(6-(Diethylamino)naphthalen-2-yl)vinyl)-1-(5-((2,5-dioxopyrrolidin-1-yl)oxy)-5-}

oxopentyl)pyridin-1-ium bromide (S17).

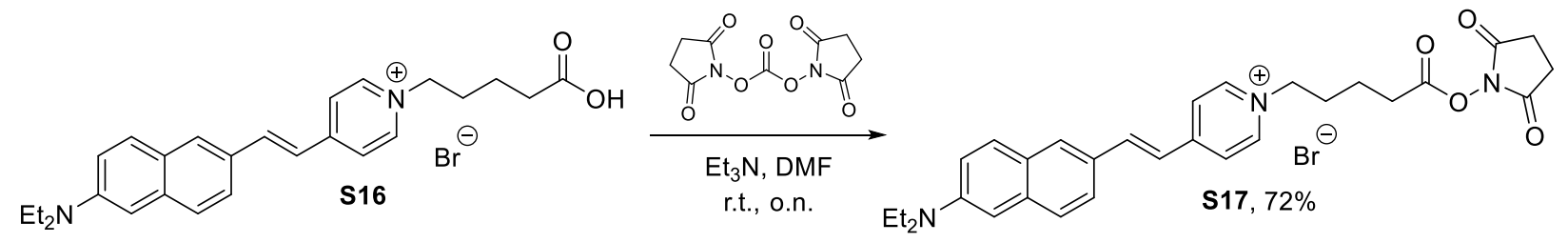

This procedure was adopted from Mascareñas et al. ${ }^{19}$ A flask with solid $\mathbf{S 1 6}$ (76 mg, $0.157 \mathrm{mmol}$, 1.0 eq.) was dried under high vacuum and back-filled with dry Ar. Dry DMF (5 mL) and $\mathrm{Et}_{3} \mathrm{~N}$ (90 $\mu \mathrm{L}, 0.63 \mathrm{mmol}, 4.0$ eq.) were added and reaction mixture was stirred $5 \mathrm{~min}$ at $20^{\circ} \mathrm{C}$. Disuccinimidyl carbonate [( $\left.\mathrm{SuO})_{2} \mathrm{CO}, 48 \mathrm{mg}, 0.189 \mathrm{mmol}, 1.2 \mathrm{eq}.\right]$ was added in one batch and the reaction mixture was further stirred overnight at $20^{\circ} \mathrm{C}$. The reaction mixture was loaded on a short silica pad $(\sim 2 \mathrm{~cm})$ and washed with AcOEt. The silica (red colored) was collected, carefully dried under high vacuum and extracted with dimethylformamide $(3 \times 10 \mathrm{~mL})$. All extracts were combined and filtered. Volatiles were removed under reduced pressure and the obtained residuum was further dried under high vacuum to yield a ca. 1:1 mixture of pure product and triethylammonium bromide. This mixture was used in the next step without further purification. Red waxy solid. Yield: $64 \mathrm{mg}$ (72\%).

$\boldsymbol{R}_{\mathbf{f}}$ (silica; AcOEt): 0.05 .

${ }^{1} \mathbf{H}$ NMR (400 MHz, acetone- $\left.d_{6}\right): \delta(\mathrm{ppm}) 1.22\left(\mathrm{t}, 6 \mathrm{H}, J=7.0 \mathrm{~Hz} ;\left(\mathrm{CH}_{3} \mathrm{CH}_{2}\right)_{3} \mathrm{NH}^{+} \mathrm{Br}^{-}\right), 1.32(\mathrm{t}$, $6 \mathrm{H}, J=7.3 \mathrm{~Hz}), 1.80-1.89$ (m, 2H), 2.16-2.25 (m, 2H), 2.76 (t, 2H, $J=7.2 \mathrm{~Hz}), 2.88$ (s, 4H), 3.19 (q, $4 \mathrm{H}, J=7.2 \mathrm{~Hz}), 3.54$ (q, 4H, $\left.J=7.0 \mathrm{~Hz} ;\left(\mathrm{CH}_{3} \mathrm{CH}_{2}\right)_{3} \mathrm{NH}^{+} \mathrm{Br}^{-}\right), 4.75(\mathrm{t}, 2 \mathrm{H}, J=7.2 \mathrm{~Hz}), 6.97(\mathrm{~d}$, $1 \mathrm{H}, J=2.1 \mathrm{~Hz}), 7.14-7.26(\mathrm{~m}, 2 \mathrm{H}), 7.45(\mathrm{~d}, 1 \mathrm{H}, J=16.2 \mathrm{~Hz}) 7.65(\mathrm{~d}, 1 \mathrm{H}, J=8.8 \mathrm{~Hz}), 7.75(\mathrm{dd}$, $\left.2 \mathrm{H}, J_{1}=9.1 \mathrm{~Hz}, J_{2}=2.7 \mathrm{~Hz}\right), 7.98(\mathrm{~s}, 1 \mathrm{H}), 8.12(\mathrm{~d}, 1 \mathrm{H}, J=16.2 \mathrm{~Hz}), 8.25(\mathrm{~d}, 2 \mathrm{H}, J=6.0 \mathrm{~Hz})$, $9.02(\mathrm{~d}, 2 \mathrm{H}, J=6.0 \mathrm{~Hz})$.

${ }^{13} \mathbf{C}\left\{{ }^{1} \mathbf{H}\right\}$ NMR (100 MHz, $\left.\mathrm{CDCl}_{3}\right): \delta(\mathrm{ppm})$ 8.8, 13.0, 22.0, 26.0, 26.3, 30.7, 30.9, 45.1, 46.5, 60.4, 105.7, 116.6, 117.0, 119.5, 121.0, 124.4, 124.7, 126.4, 127.7, 129.2, 131.0, 131.8, 138.0, 143.6, $144.9,148.4,155.2,161.6,169.4,170.6$.

HRMS (ESCI+) calculated $m / z$ for $\left[\mathrm{C}_{30} \mathrm{H}_{34} \mathrm{~N}_{3} \mathrm{O}_{4}\right]^{+}=500.2544$; found $=500.2548$. 
(E)-1-(4-((tert-Butoxycarbonyl)amino)butyl)-4-(2-(6-(diethylamino)naphthalen-2yl)vinyl)pyridin-1-ium bromide (S18; di-2-ANEPB-NHBoc)

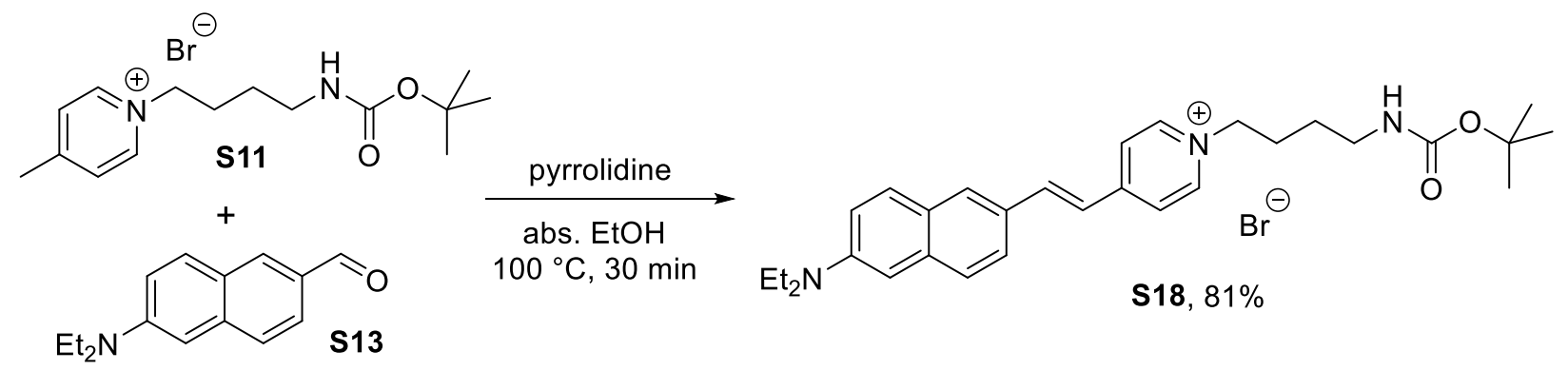

A solution of $\mathbf{S 1 1}$ (75 mg, $0.22 \mathrm{mmol}, 1.0$ eq.), $\mathbf{S 1 3}$ (59 mg, $0.26 \mathrm{mmol}, 1.2 \mathrm{eq}$.$) and pyrrolidine$ $(250 \mu \mathrm{L})$ in abs. EtOH $(25 \mathrm{~mL})$ was sealed in a pressure vessel and heated at $100{ }^{\circ} \mathrm{C}$ for $30 \mathrm{~min}$ during which the solution turned dark red. After cooling to r.t., the reaction mixture was concentrated by rotary evaporation. Purification by chromatography on basic alumina using $\mathrm{CH}_{2} \mathrm{Cl}_{2} / \mathrm{MeOH}(50: 1$ to $12: 1, v / v)$ as the mobile phase provided di-2-ANEPB-NHBoc (S18) as a dark red solid. Yield: $98 \mathrm{mg},(81 \%)$.

$\boldsymbol{R}_{\mathbf{f}}$ (alumina; $\mathrm{CH}_{2} \mathrm{Cl}_{2} / \mathrm{MeOH}$ 19:1, v/v): 0.26 .

${ }^{1} \mathrm{H}$ NMR $\left(500 \mathrm{MHz}, \mathrm{CD}_{3} \mathrm{OD}\right) \delta 8.62(\mathrm{~d}, J=6.4 \mathrm{~Hz}, 2 \mathrm{H}), 8.05(\mathrm{~d}, J=6.4 \mathrm{~Hz}, 2 \mathrm{H}), 7.98(\mathrm{~d}, J=$ $16.0 \mathrm{~Hz}, 1 \mathrm{H}), 7.90(\mathrm{~s}, 1 \mathrm{H}), 7.76-7.70(\mathrm{~m}, 2 \mathrm{H}), 7.61(\mathrm{~d}, J=8.7 \mathrm{~Hz}, 1 \mathrm{H}), 7.30(\mathrm{~d}, J=16.1 \mathrm{~Hz}$, 1H), 7.17 (dd, $J=9.2,2.6 \mathrm{~Hz}, 1 \mathrm{H}), 6.90(\mathrm{~d}, J=2.5 \mathrm{~Hz}, 1 \mathrm{H}), 4.43$ (t, $J=7.6 \mathrm{~Hz}, 2 \mathrm{H}), 3.53$ (q, $J=$ $7.1 \mathrm{~Hz}, 4 \mathrm{H}), 3.10(\mathrm{t}, J=6.8 \mathrm{~Hz}, 2 \mathrm{H}), 1.96(\mathrm{p}, J=7.7 \mathrm{~Hz}, 2 \mathrm{H}), 1.53(\mathrm{p}, J=6.9 \mathrm{~Hz}, 2 \mathrm{H}), 1.43$ (s, $9 \mathrm{H}), 1.24(\mathrm{t}, J=7.1 \mathrm{~Hz}, 6 \mathrm{H})$.

${ }^{13} \mathbf{C}\left\{{ }^{1} \mathbf{H}\right\}$ NMR (126 MHz, CD $\left.{ }_{3} \mathrm{OD}\right) \delta 158.6,155.9,148.8,144.6,144.3,138.4,132.3,131.3,129.4$, 127.9, 127.0, 124.7, 124.4, 120.7, 117.2, 106.0, 80.0, 61.0, 45.5, 40.2, 29.5, 28.8, 27.7, 13.1.

HRMS (ESI+): calculated $m / z$ for $\left[\mathrm{C}_{30} \mathrm{H}_{40} \mathrm{~N}_{3} \mathrm{O}_{2}\right]^{+}=474.3121$, found $=474.3119$.

FTIR (ATR, diamond, $\left.\mathrm{cm}^{-1}\right)$ : $1690(\mathrm{C}=\mathrm{O})$ (Fig. S35 in the Infrared Spectra section). 
1-(4-(((((1R,8S,9S)-Bicyclo[6.1.0]non-4-yn-9-yl)methoxy)carbonyl)amino)butyl)-4-((E)-2-(6(diethylamino)naphthalen-2-yl)vinyl)pyridin-1-ium bromide (9; di-2-ANEPB-BCN)<smiles>CCNc1ccc2cc(/C=C/c3cc[n+](CCCCNC(=O)OC(C)(C)C)cc3)ccc2c1</smiles>

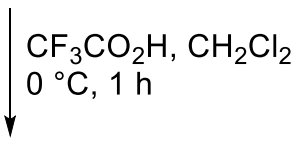<smiles>CCNc1ccc2cc(/C=C/c3cc[n+](CCCC[NH-])cc3)ccc2c1</smiles><smiles>CCNc1ccc2cc(/C=C/c3cc[n+](CCCCNC(=O)OCC4[C@H]5CCC#CCC[C@H]45)cc3)ccc2c1</smiles>

A solution of $\mathbf{S 1 8}$ (40 mg, $72 \mu \mathrm{mol}, 1.0$ eq.) in $\mathrm{CH}_{2} \mathrm{Cl}_{2}(1.0 \mathrm{~mL})$ was cooled to $0{ }^{\circ} \mathrm{C}$, followed by dropwise addition of $\mathrm{CF}_{3} \mathrm{CO}_{2} \mathrm{H}(2.0 \mathrm{~mL})$ during which the originally red solution turned yellow. The reaction mixture was stirred at $0{ }^{\circ} \mathrm{C}$ for $1 \mathrm{~h}$. The volatiles were removed by rotary evaporation $\left(30{ }^{\circ} \mathrm{C}\right)$ and the crude mixture was co-evaporated with toluene $(2 \times 1.5 \mathrm{~mL})$ to remove residual $\mathrm{CF}_{3} \mathrm{CO}_{2} \mathrm{H}$ (indicated by the color of the mixture changing from yellow to red). Without purification, the ammonium salt intermediate was dissolved in dry DIPEA $(125 \mu \mathrm{L}, 718 \mu \mathrm{mol}$, 10 eq.) and dry DMF (250 $\mu \mathrm{L})$. BCN-OSu (S8, $23 \mathrm{mg}, 79 \mu \mathrm{mol}, 1.1$ eq.) was added and the reaction mixture was stirred at r.t. for $67 \mathrm{~h}$. The volatiles were removed by rotary evaporation $\left(40{ }^{\circ} \mathrm{C}\right)$, after which the crude product was purified by chromatography on basic alumina using $\mathrm{MeOH}$ (0 to 8 vol. \%) in $\mathrm{CH}_{2} \mathrm{Cl}_{2}$ as the mobile phase. di-2-ANEPB-BCN (9) was obtained as a dark red solid. Yield: $21 \mathrm{mg}(46 \%)$.

$\boldsymbol{R} \mathbf{f}$ (alumina, $\mathrm{CH}_{2} \mathrm{Cl}_{2} / \mathrm{MeOH} 19: 1, v / v$ ): 0.28. 
${ }^{1} \mathbf{H}$ NMR $\left(500 \mathrm{MHz}, \mathrm{CD}_{3} \mathrm{OD}\right) \delta 8.61(\mathrm{~d}, J=6.4 \mathrm{~Hz}, 2 \mathrm{H}), 8.03(\mathrm{~d}, J=6.4 \mathrm{~Hz}, 2 \mathrm{H}), 7.96(\mathrm{~d}, J=$ $16.1 \mathrm{~Hz}, 1 \mathrm{H}), 7.91-7.87(\mathrm{~m}, 1 \mathrm{H}), 7.75-7.68(\mathrm{~m}, 2 \mathrm{H}), 7.61(\mathrm{~d}, J=8.7 \mathrm{~Hz}, 1 \mathrm{H}), 7.29(\mathrm{~d}, J=16.1$ $\mathrm{Hz}, 1 \mathrm{H}), 7.16(\mathrm{dd}, J=9.2,2.6 \mathrm{~Hz}, 1 \mathrm{H}), 6.89(\mathrm{~d}, J=2.5 \mathrm{~Hz}, 1 \mathrm{H}), 4.42$ (t, $J=7.5 \mathrm{~Hz}, 2 \mathrm{H}), 4.12(\mathrm{~d}$, $J=8.1 \mathrm{~Hz}, 2 \mathrm{H}), 3.52(\mathrm{q}, J=7.1 \mathrm{~Hz}, 4 \mathrm{H}), 3.16(\mathrm{t}, J=6.8 \mathrm{~Hz}, 2 \mathrm{H}), 2.28-2.08(\mathrm{~m}, 6 \mathrm{H}), 1.96$ (p, $J$ $=7.8 \mathrm{~Hz}, 2 \mathrm{H}), 1.62-1.51(\mathrm{~m}, 4 \mathrm{H}), 1.35(\mathrm{p}, J=8.7 \mathrm{~Hz}, 1 \mathrm{H}), 0.91(\mathrm{ddd}, J=10.7,6.0,2.3 \mathrm{~Hz}, 2 \mathrm{H})$.

${ }^{13} \mathbf{C}\left\{{ }^{1} \mathbf{H}\right\}$ NMR (126 MHz, CD $\left.3 \mathrm{OD}\right) \delta 159.4,155.9,148.8,144.6,144.3,138.4,132.3,131.3,129.4$, 127.9, 127.0, 124.8, 124.5, 124.4, 120.7, 117.2 , 106.0, 99.5, 63.7, 60.9, 45.5, 40.6, 30.1, 29.4, 27.6, 21.9, 21.4, 18.9, 13.1.

HRMS (ESI+): calculated $m / z$ for $\left[\mathrm{C}_{36} \mathrm{H}_{44} \mathrm{~N}_{3} \mathrm{O}_{2}\right]^{+}=550.3434$, found $=550.3440$.

\section{$N, N$-Dimethyl-4-vinylaniline (S19)}

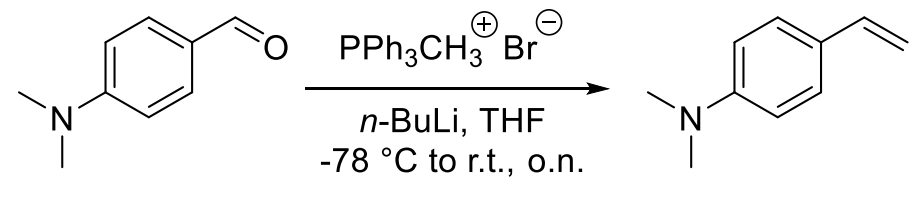

$\mathbf{S 1 9}, 95 \%$

Compound S19 was prepared according to a modified procedure from the literature. ${ }^{11}$ Triphenylmethylphosphonium bromide ( $4.37 \mathrm{~g}, 12.2 \mathrm{mmol}, 1.8$ eq.) was suspended in dry THF $(21 \mathrm{~mL})$ under $\mathrm{Ar}$ atmosphere and cooled to $-78^{\circ} \mathrm{C}$. A $2.5 \mathrm{M}$ solution of $n$-BuLi in hexanes (4.8 mL, $12 \mathrm{mmol}, 1.8$ eq.) was added dropwise. The mixture was stirred for $5 \mathrm{~min}$ at $-78^{\circ} \mathrm{C}$, then for $30 \mathrm{~min}$ at r.t. resulting in formation of a yellow solution of the corresponding phosphonium ylide. 4-(Dimethylamino)benzaldehyde (1.00 g, $6.70 \mathrm{mmol}, 1.0 \mathrm{eq}$.) was added in one portion. The reaction mixture was stirred under Ar at r.t. overnight $(16 \mathrm{~h})$ and subsequently poured into hexanes $(100 \mathrm{~mL})$ resulting in precipitation of a white solid. The precipitate was filtered off on a short pad of celite and washed with hexanes $(2 \times 15 \mathrm{~mL})$. The combined filtrates were concentrated by rotary evaporation $\left(30^{\circ} \mathrm{C}\right)$. The crude yellow oil was passed through a pad of silica gel $(\varnothing 24 \mathrm{~mm}$, height $20 \mathrm{~mm}$ ) eluting with AcOEt providing $N, N$-dimethyl-4-vinylaniline (S19) as a yellowish liquid. Yield: $937 \mathrm{mg},(95 \%)$. 
${ }^{1} \mathbf{H}$ NMR $\left(400 \mathrm{MHz}, \mathrm{CDCl}_{3}\right) \delta 7.36-7.28(\mathrm{~m}, 2 \mathrm{H}), 6.74-6.66(\mathrm{~m}, 2 \mathrm{H}), 6.65(\mathrm{dd}, J=17.6$, $10.9 \mathrm{~Hz}, 1 \mathrm{H}), 5.55(\mathrm{dd}, J=17.6,1.1 \mathrm{~Hz}, 1 \mathrm{H}), 5.03(\mathrm{dd}, J=10.9,1.1 \mathrm{~Hz}, 1 \mathrm{H}), 2.97$ (s, 6H).

${ }^{13} \mathbf{C}\left\{{ }^{1} \mathbf{H}\right\}$ NMR $\left(101 \mathrm{MHz}, \mathrm{CDCl}_{3}\right) \delta 150.4,136.7,127.3,126.4,112.5,109.5,40.7$.

MS (APCI+): calculated $m / z$ for $\left[\mathrm{C}_{10} \mathrm{H}_{13} \mathrm{~N}+\mathrm{H}\right]^{+}=148.1$, found $=148.3$.

\section{(E)-4-(4-(Dimethylamino)styryl)benzaldehyde (S20)}

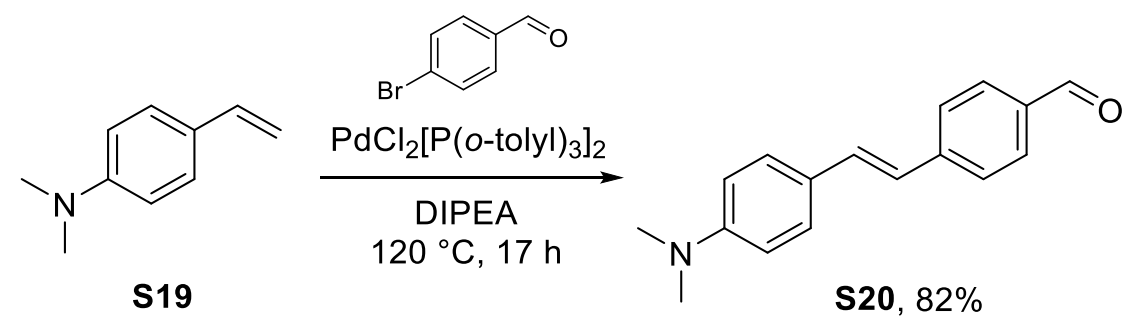

Compound $\mathbf{S 2 0}$ was prepared according to a modified procedure from the literature. ${ }^{11}$ A solution of S19 (897 mg, $6.09 \mathrm{mmol}, 1.2$ eq.), 4-bromobenzaldehyde (939 mg, $5.08 \mathrm{mmol}, 1.0$ eq.) and $\mathrm{PdCl}_{2}\left[\mathrm{P}(o \text {-tolyl })_{3}\right]_{2}(100 \mathrm{mg}, 0.127 \mathrm{mmol}, 2.5 \mathrm{~mol}$ \% $)$ in dry DIPEA (3.0 mL) was stirred at $120{ }^{\circ} \mathrm{C}$ under dry Ar atmosphere for $17 \mathrm{~h}$. After cooling to r.t., the resulting orange to brownish slurry was dissolved in $\mathrm{CH}_{2} \mathrm{Cl}_{2}(30 \mathrm{~mL})$, washed with sat. aq. $\mathrm{NH}_{4} \mathrm{Cl}(2 \times 10 \mathrm{~mL})$ and brine $(2 \times 10 \mathrm{~mL})$. The organic layer was dried over anhydrous $\mathrm{Na}_{2} \mathrm{SO}_{4}$, filtered and concentrated by rotary evaporation $\left(30{ }^{\circ} \mathrm{C}\right)$ until an orange solid started emerging. The suspension was diluted with hexanes $(100 \mathrm{~mL})$ resulting in further precipitation of an orange solid which was collected by filtration, washed with hexanes $(2 \times 10 \mathrm{~mL})$ and dried under high vacuum to yield aldehyde $\mathbf{S 2 0}$. Yield: $1.05 \mathrm{~g}(82 \%)$.

${ }^{1} \mathbf{H}$ NMR $\left(500 \mathrm{MHz}, \mathrm{CDCl}_{3}\right) \delta 9.96(\mathrm{~s}, 1 \mathrm{H}), 7.83(\mathrm{~d}, J=8.3 \mathrm{~Hz}, 2 \mathrm{H}), 7.60(\mathrm{~d}, J=8.3 \mathrm{~Hz}, 2 \mathrm{H})$, $7.44(\mathrm{~d}, J=8.8 \mathrm{~Hz}, 2 \mathrm{H}), 7.21(\mathrm{~d}, J=16.2 \mathrm{~Hz}, 1 \mathrm{H}), 6.93(\mathrm{~d}, J=16.2 \mathrm{~Hz}, 1 \mathrm{H}), 6.72(\mathrm{~d}, J=8.8 \mathrm{~Hz}$, 2H), $3.01(\mathrm{~s}, 6 \mathrm{H})$.

${ }^{13} \mathbf{C}\left\{{ }^{1} \mathbf{H}\right\}$ NMR $\left(126 \mathrm{MHz}, \mathrm{CDCl}_{3}\right) \delta 191.7,150.8,144.7,134.7,132.7,130.4,128.3,126.4,124.9$, $122.8,112.4,40.5$.

MS (APCI+): calculated $m / z$ for $\left[\mathrm{C}_{17} \mathrm{H}_{17} \mathrm{NO}+\mathrm{H}\right]^{+}=252.1$, found $=252.3$. 
m.p. (hexanes $/ \mathrm{CH}_{2} \mathrm{Cl}_{2}$ ): $>210{ }^{\circ} \mathrm{C}$ (decomp.).

\section{(E)-N,N-Dimethyl-4-(4-vinylstyryl)aniline (S21)}<smiles>C=Cc1ccc(/C=C/c2ccc(N(C)C)cc2)cc1</smiles>

Compound S21 was prepared according to a modified procedure from the literature. ${ }^{11}$ Triphenylmethylphosphonium bromide $(2.55 \mathrm{~g}, 7.14 \mathrm{mmol}, 1.8 \mathrm{eq}$.) was suspended in dry THF $(15 \mathrm{~mL})$ under dry $\mathrm{Ar}$ atmosphere and cooled to $-78^{\circ} \mathrm{C}$. A $2.5 \mathrm{M}$ solution of $n$-BuLi in hexanes ( $2.8 \mathrm{~mL}, 7.0 \mathrm{mmol}, 1.8 \mathrm{eq}$.) was added dropwise. The reaction mixture was stirred for $2 \mathrm{~h}$ at $0{ }^{\circ} \mathrm{C}$ resulting in formation of the corresponding phosphonium ylide. A suspension of $\mathbf{S 2 0}(1.0 \mathrm{~g}$, $4.0 \mathrm{mmol}, 1.0$ eq. $)$ in dry THF $(5 \mathrm{~mL})$ was added dropwise at $0{ }^{\circ} \mathrm{C}$. The reaction mixture was stirred at r.t. overnight. The resulting suspension was dissolved in $\mathrm{CH}_{2} \mathrm{Cl}_{2}(30 \mathrm{~mL})$ and passed through a pad of basic alumina (ø $40 \mathrm{~mm}$, height $20 \mathrm{~mm}$ ) eluting with $\mathrm{CH}_{2} \mathrm{Cl}_{2}(75 \mathrm{~mL})$. After concentrating by rotary evaporation $\left(30^{\circ} \mathrm{C}\right)$, the crude yellow solid was triturated with ice-cold $95 \%$ aq. EtOH $(2 \times 20 \mathrm{~mL})$ providing styrene $\mathbf{S 2 1}$ as a yellow crystalline solid. Yield: $0.83 \mathrm{~g}$ $(84 \%)$.

${ }^{1} \mathbf{H}$ NMR $\left(500 \mathrm{MHz}, \mathrm{CDCl}_{3}\right) \delta 7.33-7.27(\mathrm{~m}, 2 \mathrm{H}), 6.70-6.56(\mathrm{~m}, 3 \mathrm{H}), 5.51(\mathrm{dd}, J=17.5,1.2$ $\mathrm{Hz}, 1 \mathrm{H}), 4.99(\mathrm{dd}, J=10.9,1.2 \mathrm{~Hz}, 1 \mathrm{H}), 3.37$ (q, $J=7.1 \mathrm{~Hz}, 4 \mathrm{H}), 1.17$ (t, $J=7.1 \mathrm{~Hz}, 6 \mathrm{H})$.

${ }^{13} \mathbf{C}\left\{{ }^{1} \mathbf{H}\right\}$ NMR $\left(126 \mathrm{MHz}, \mathrm{CDCl}_{3}\right) \delta 150.1,137.8,136.6,135.9,128.8,127.6,126.5,126.1,125.7$, $123.9,113.1,112.4,40.5$.

MS (APCI+): calculated $m / z$ for $\left[\mathrm{C}_{18} \mathrm{H}_{19} \mathrm{~N}+\mathrm{H}\right]^{+}=250.2$, found $=250.4$.

m.p. $\left(\mathrm{THF} / \mathrm{CH}_{2} \mathrm{Cl}_{2}\right): 189-191^{\circ} \mathrm{C}$. 


\section{4-Bromo-2,6-disulfobenzaldehyde disodium salt (S22)}

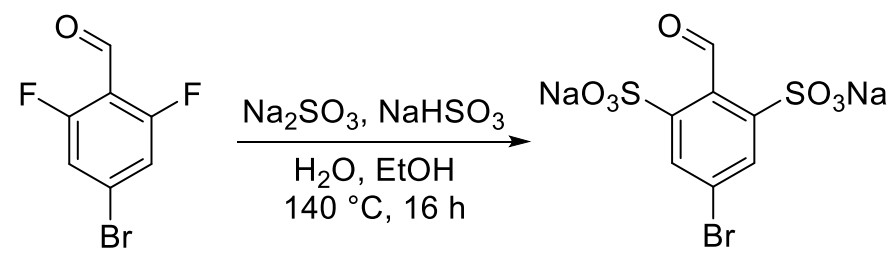

S22, $93 \%$

(80\% purity)

Compound S22 was prepared according to a modified procedure from the literature. ${ }^{7}$ 4-Bromo2,6-difluorobenzaldehyde (1.0 g, $4.5 \mathrm{mmol}, 1.0$ eq.), $\mathrm{Na}_{2} \mathrm{SO}_{3}$ (1.14 g, $9.04 \mathrm{mmol}, 2.0$ eq.) and $\mathrm{NaHSO}_{3}$ (94 mg, $0.90 \mathrm{mmol}, 0.2$ eq.) in $\mathrm{H}_{2} \mathrm{O} / \mathrm{EtOH}(1: 1, v / v ; 10 \mathrm{~mL})$ were sealed in a pressure vessel and heated at $140{ }^{\circ} \mathrm{C}$ for $16 \mathrm{~h}$. After cooling to r.t., the reaction mixture was poured into $\mathrm{MeOH}(15 \mathrm{~mL})$ to precipitate out the inorganic salts. The suspension was filtered, and the filtrate was concentrated by rotary evaporation. The resulting white solid was dissolved in $\mathrm{MeOH}(5 \mathrm{~mL})$ and reprecipitated by addition of $\mathrm{Et}_{2} \mathrm{O}(20 \mathrm{~mL})$. Product $\mathbf{S 2 2}$ was collected by filtration and dried under high vacuum. Yield: $1.64 \mathrm{~g}(93 \%)$, purity: 80\% ( $\left.{ }^{1} \mathrm{H} \mathrm{NMR}\right)$.

${ }^{1} \mathbf{H}$ NMR (400 MHz, DMSO-d6) $\delta 10.26(\mathrm{~s}, 1 \mathrm{H}), 7.75$ (s, 2H).

MS (ESI-): calculated $m / z$ for $\left[\mathrm{C}_{7} \mathrm{H}_{3} \mathrm{BrO}_{7} \mathrm{~S}_{2}+\mathrm{H}\right]^{-}=342.9$, found $=342.9$.

m.p. $(\mathrm{MeOH}):>260{ }^{\circ} \mathrm{C}$.

\section{4'-Bromo-2,7-dichloro-2',6'-disulfofluorescein (S23)}<smiles>O=Cc1c(S(=O)(=O)O[Na])cc(Br)cc1S(=O)(=O)O[Na]</smiles>

S23, $31 \%$

Compound $\mathbf{S 2 3}$ was prepared according to a modified procedure from the literature. ${ }^{7}$ A solution of aldehyde S22 (1.01 g, $2.60 \mathrm{mmol}, 1.0$ eq.) and 4-chlororesorcinol (838 mg, $5.80 \mathrm{mmol}, 2.2$ eq.) 
in methanesulfonic acid $(12 \mathrm{~mL})$ was stirred at $120^{\circ} \mathrm{C}$ in an open flask for $18 \mathrm{~h}$. After cooling to r.t., the reaction mixture was diluted with $\mathrm{CH}_{2} \mathrm{Cl}_{2}(12 \mathrm{~mL})$ and subsequently added dropwise into stirred $\mathrm{Et}_{2} \mathrm{O}(200 \mathrm{~mL})$ resulting in precipitation of a brown solid. The precipitate was collected by filtration, washed with $\mathrm{Et}_{2} \mathrm{O}(2 \times 20 \mathrm{~mL})$ and dried under high vacuum. The crude product at this stage could be used in subsequent steps but with highly reduced yields. To obtain pure $\mathbf{S 2 3}$, the crude solid was co-evaporated with conc. aq. $\mathrm{HCl}(10 \mathrm{~mL})$ in $\mathrm{MeOH}(50 \mathrm{~mL})$, followed by $\mathrm{RP}$ $\mathrm{C}_{18}$-silica column chromatography using $0.035 \%$ aq. $\mathrm{HCl}$ as the mobile phase. Pure $\mathbf{S 2 3}$ was obtained as an orange solid. Yield: $475 \mathrm{mg}(31 \%)$.

${ }^{1}$ H NMR (500 MHz, DMSO-d $d_{6} \delta 8.14(\mathrm{~s}, 2 \mathrm{H}), 6.98(\mathrm{~s}, 2 \mathrm{H}), 6.91$ (br s, 3H+H $\left.\mathrm{H}_{2} \mathrm{O}\right), 6.85$ (s, 2H).

${ }^{13} \mathbf{C}\left\{{ }^{1} \mathbf{H}\right\}$ NMR $\left(126 \mathrm{MHz}, \mathrm{DMSO}-d_{6}\right) \delta 166.4,154.9,154.3,149.5,131.4,130.7,124.1,123.4$, 122.3, 117.7, 102.4 .

MS (ESI-): calculated $\mathrm{m} / \mathrm{z}$ for $\left[\mathrm{C}_{19} \mathrm{H}_{9} \mathrm{BrCl}_{2} \mathrm{O}_{9} \mathrm{~S}_{2}-\mathrm{H}\right]^{-}=592.8$, found $=593.6$; calculated $\mathrm{m} / \mathrm{z}$ for $\left[\mathrm{C}_{19} \mathrm{H}_{9} \mathrm{BrCl}_{2} \mathrm{O}_{9} \mathrm{~S}_{2}-2 \mathrm{H}\right]^{2-}=295.9$, found $=296.2$.

m.p. $(\mathrm{MeOH}):>260^{\circ} \mathrm{C}$.

\section{5-Bromo-2-(2,7-dichloro-3-oxo-6-(pyrrolidin-1-yl)-3H-xanthen-9-yl)benzene-1,3-disulfonate} (S24)

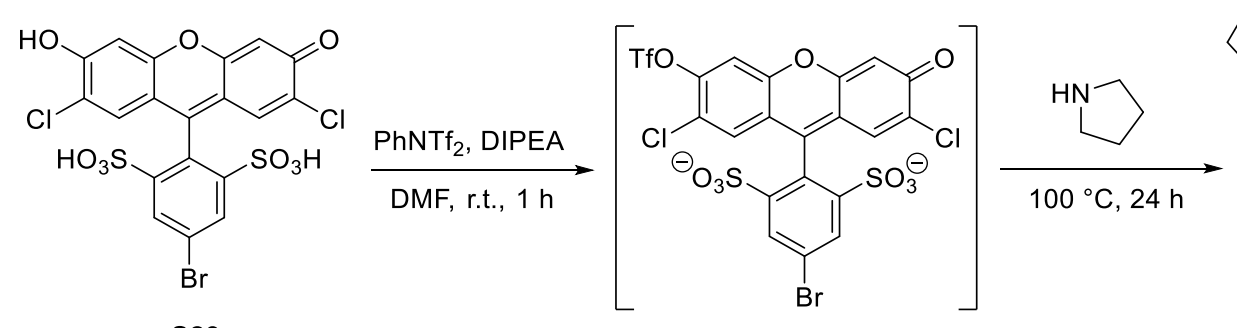

S23

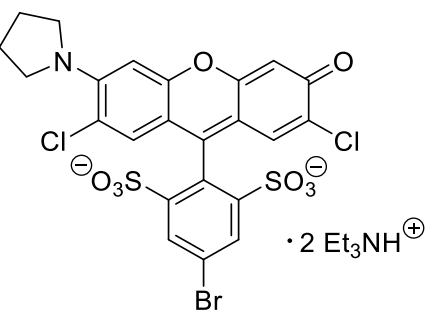

S24, $14 \%$

S23 (51 mg, $86 \mu \mathrm{mol}, 1.0$ eq.) and $N$-phenyl-bis(trifluoromethanesulfonimide) (PhNTf $2,46 \mathrm{mg}$, $0.13 \mathrm{mmol}, 1.5$ eq. $)$ were dissolved in dry DIPEA $(150 \mu \mathrm{L}, 0.861 \mathrm{mmol}, 10$ eq. $)$ and dry DMF $(500 \mu \mathrm{L})$ in an oven-dried screw-cap vial with a stir bar under Ar atmosphere. The reaction mixture was stirred at r.t. for 60 min during which the color of the solution turned from pink to dark orange indicating formation of the intermediate triflate ester. Pyrrolidine $(1.0 \mathrm{~mL}, 12.2 \mathrm{mmol}, \sim 100$ eq. $)$ 
was added, resulting in almost immediate change of color to pink. The resulting solution was heated in the sealed vial at $100{ }^{\circ} \mathrm{C}$ for $24 \mathrm{~h}$. Volatiles were removed by rotary evaporation $\left(40{ }^{\circ} \mathrm{C}\right)$, after which the crude oil was purified by $\mathrm{RP} \mathrm{C}_{18}$-silica column chromatography using $0.1 \%$ aq. $\mathrm{Et}_{3} \mathrm{~N}$ as the mobile phase. Rhodol $\mathbf{S 2 4}$ was obtained as a pink solid in sufficient purity to be used in the next step. Yield: $10 \mathrm{mg}(14 \%)$.

$\boldsymbol{R}_{\mathbf{f}}\left(\mathrm{C}_{18}\right.$-silica, $\left.\mathrm{H}_{2} \mathrm{O} / \mathrm{MeCN} 2: 1, v / v\right): 0.69$.

${ }^{1} \mathbf{H}$ NMR $\left(500 \mathrm{MHz}, \mathrm{CD}_{3} \mathrm{OD}\right) \delta 8.46(\mathrm{~s}, 2 \mathrm{H}), 7.14(\mathrm{~s}, 1 \mathrm{H}), 7.00(\mathrm{~s}, 1 \mathrm{H}), 6.85(\mathrm{~s}, 1 \mathrm{H}), 6.51(\mathrm{~s}, 1 \mathrm{H})$, $3.77-3.68(\mathrm{~m}, 4 \mathrm{H}), 3.17(\mathrm{q}, J=7.3 \mathrm{~Hz}, 12 \mathrm{H}), 2.05-1.98(\mathrm{~m}, 4 \mathrm{H}), 1.28(\mathrm{t}, J=7.3 \mathrm{~Hz}, 18 \mathrm{H})$.

HRMS (ESI-): calculated $m / z$ for $\left[\mathrm{C}_{23} \mathrm{H}_{14} \mathrm{BrCl}_{2} \mathrm{NO}_{8} \mathrm{~S}_{2}+\mathrm{H}\right]^{+}=645.8800$, found $=645.8806$.

\section{5-Bromo-2-(6-(3-(((tert-butoxycarbonyl)amino)methyl)pyrrolidin-1-yl)-2,7-dichloro-3-oxo- 3H-xanthen-9-yl)benzene-1,3-disulfonate (S25)}
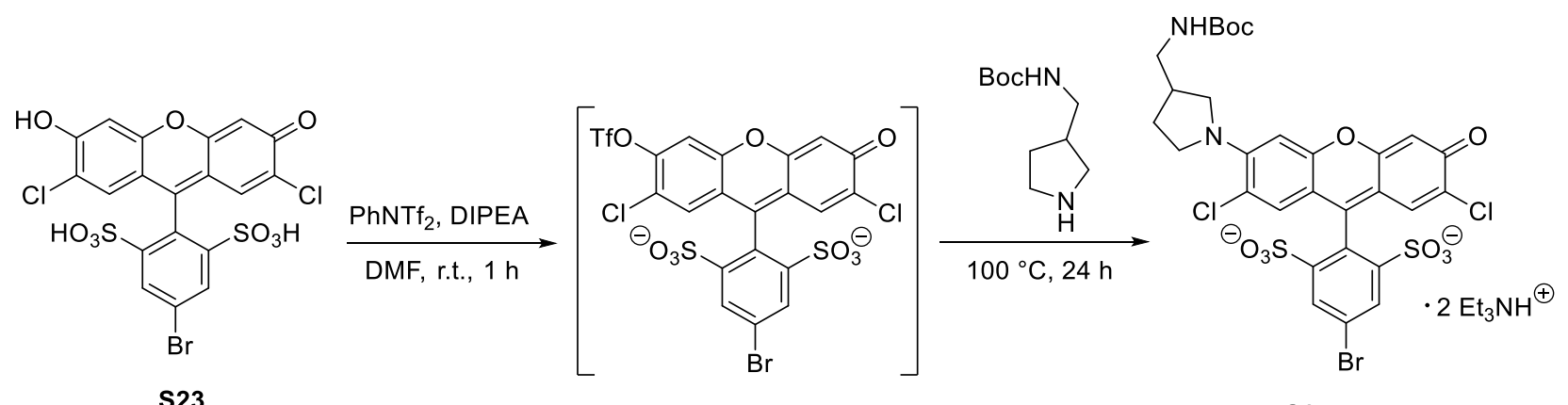

$\mathbf{S 2 5}, 39 \%$

The purity of commercial 3-(N-Boc-aminomethyl)pyrrolidine (Small Molecules, Inc.) was unsatisfactory as revealed by ${ }^{1} \mathrm{H}$ NMR (not shown). Therefore, the commercial chemical was purified by column chromatography on basic alumina using $\mathrm{MeOH}$ in $\mathrm{CH}_{2} \mathrm{Cl}_{2}$ (0 to 15 vol. \%) as the mobile phase prior to use.

S23 (209 mg, $0.351 \mathrm{mmol}, 1.0$ eq.) and $\mathrm{PhNTf}_{2}$ (154 mg, $0.431 \mathrm{mmol}, 1.2$ eq.) were dissolved in dry DIPEA (305 $\mu \mathrm{L}, 1.75 \mathrm{mmol}, 5.0$ eq.) and dry DMF (1.5 mL) in an oven-dried screw-cap vial with a stir bar under Ar atmosphere. The reaction mixture was stirred at r.t. for 60 min during which the color of the solution turned from pink to dark orange indicating formation of the intermediate triflate ester. The solution was transferred into a $25 \mathrm{~mL}$ round-bottom flask with dried 
3-( $N$-Boc-aminomethyl)pyrrolidine $(370 \mathrm{mg}, 1.85 \mathrm{mmol}, 5.3 \mathrm{eq}$.) in dry DMF $(0.5 \mathrm{~mL})$ under dry Ar atmosphere. The resulting mixture was heated at $100{ }^{\circ} \mathrm{C}$ for $24 \mathrm{~h}$. After cooling to r.t., the crude mixture was diluted with $\mathrm{Et}_{3} \mathrm{~N}(10 \mathrm{~mL})$ and partitioned between $\mathrm{H}_{2} \mathrm{O}(70 \mathrm{~mL})$ and $\mathrm{Et}_{2} \mathrm{O}(50 \mathrm{~mL})$. Some precipitates were formed which were insoluble in either of the two phases; their dissolution was aided by adding a small amount of $\mathrm{MeCN}$ and $\mathrm{CH}_{2} \mathrm{Cl}_{2}$. The organic layer was separated, and the aqueous phase was washed with further $\mathrm{Et}_{2} \mathrm{O}(2 \times 50 \mathrm{~mL})$. The combined organic washes were then extracted with $\mathrm{H}_{2} \mathrm{O}(2 \times 40 \mathrm{~mL})$. All aqueous phases were combined, and volatiles were removed by rotary evaporation $\left(40{ }^{\circ} \mathrm{C}\right)$ with azeotropic co-evaporation with plenty of $\mathrm{MeCN}$. The resulting pink solid was dissolved in $\mathrm{DMF} / \mathrm{H}_{2} \mathrm{O}$ 1:5 and pre-purified by passing through a short column with $\mathrm{RP} \mathrm{C}_{18}$-silica eluting with aq. $\mathrm{MeCN}$ (5 to 30 vol. \% $\mathrm{MeCN}$, containing $0.1 \% \mathrm{Et}_{3} \mathrm{~N}$ ). All fractions containing the desired product were collected, concentrated and dissolved in $\mathrm{MeOH}$ ( $20 \mathrm{~mL}$; some colorless solid remained undissolved). The suspension was filtered through a $0.45 \mu \mathrm{m}$ hydrophilic filter. After concentrating the filtrate by rotary evaporation, the pink solid was subject to purification by $\mathrm{RP} \mathrm{C}_{18}$-silica column chromatography using aq. $\mathrm{MeCN}$ (0 to 3 vol. \%, containing $0.75 \% \mathrm{Et}_{3} \mathrm{~N}$ ) as the mobile phase. Rhodol $\mathbf{S 2 5}$ was obtained as a pink solid. Yield: $134 \mathrm{mg}(39 \%)$.

$\boldsymbol{R}_{\mathbf{f}}\left(\mathrm{C}_{18}\right.$-silica, $\left.\mathrm{H}_{2} \mathrm{O} / \mathrm{MeCN} 2: 1, v / v\right): 0.54$.

${ }^{1}$ H NMR (500 MHz, DMSO- $\left.d_{6}\right) \delta 8.84(\mathrm{br} \mathrm{s}, 2 \mathrm{H}), 8.14(\mathrm{~s}, 2 \mathrm{H}), 7.02(\mathrm{t}, J=5.8 \mathrm{~Hz}, 1 \mathrm{H}), 6.83(\mathrm{~s}$, $1 \mathrm{H}), 6.75(\mathrm{~s}, 1 \mathrm{H}), 6.68(\mathrm{~s}, 1 \mathrm{H}), 6.22(\mathrm{~s}, 1 \mathrm{H}), 3.70-3.56(\mathrm{~m}, 3 \mathrm{H}), 3.40(\mathrm{dd}, J=10.6,7.0 \mathrm{~Hz}, 1 \mathrm{H})$, $3.07(\mathrm{q}, J=7.3 \mathrm{~Hz}, 12 \mathrm{H}), 3.03-2.97(\mathrm{~m}, 2 \mathrm{H}), 2.42-2.33(\mathrm{~m}, 1 \mathrm{H}), 2.04-1.96(\mathrm{~m}, 1 \mathrm{H}), 1.72-$ $1.63(\mathrm{~m}, 1 \mathrm{H}), 1.39(\mathrm{~s}, 9 \mathrm{H}), 1.15(\mathrm{t}, J=7.3 \mathrm{~Hz}, 18 \mathrm{H})$.

$\left.{ }^{13} \mathbf{C}_{\{}{ }^{1} \mathbf{H}\right\}$ NMR (126 MHz, DMSO- $\left.d_{6}\right) \delta 175.9,157.8,155.8,151.7,149.7,149.6,149.5,148.9$, 132.2, 131.0, 130.7, 124.8, 121.7, 116.9, 115.4, 114.9, 102.9, 100.7, 77.6, 54.6, 50.6, 45.8, 42.1, $38.8,28.6,28.2,8.7$.

HRMS (ESI-): calculated $m / z$ for $\left[\mathrm{C}_{29} \mathrm{H}_{25} \mathrm{BrCl}_{2} \mathrm{~N}_{2} \mathrm{O}_{10} \mathrm{~S}_{2}+\mathrm{H}\right]^{-}=774.9589$, found $=774.9615$. 


\section{2-(2,7-Dichloro-3-oxo-6-(pyrrolidin-1-yl)-3H-xanthen-9-yl)-5-((E)-4-((E)-4-}

(dimethylamino)styryl)styryl)benzene-1,3-disulfonate (dsRVF5, 2)

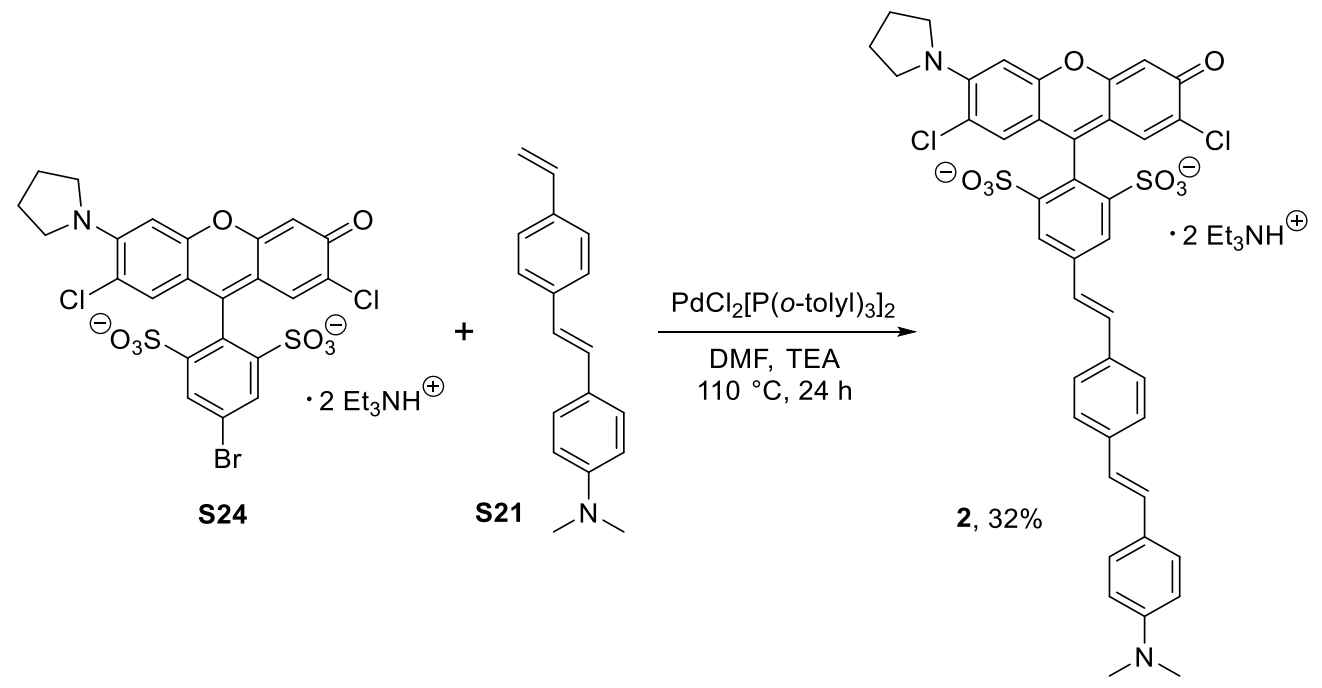

dsRhodol S24 (9.0 mg, $11 \mu \mathrm{mol}, 1.0$ eq.), $\pi$-wire $\mathbf{S 2 1}\left(3.8 \mathrm{mg}, 15 \mu \mathrm{mol}, 1.4\right.$ eq.) and $\mathrm{PdCl}_{2}[\mathrm{P}(o-$ tolyl $\left.)_{3}\right]_{2}(2.8 \mathrm{mg}, 3.6 \mu \mathrm{mol}, 34 \mathrm{~mol} . \%)$ were dissolved in dry Et $3 \mathrm{~N}(70 \mu \mathrm{L})$ and dry DMF $(70 \mu \mathrm{L})$ in an oven-dried screw-cap vial with a stir bar under Ar atmosphere. The reaction mixture was stirred at $110{ }^{\circ} \mathrm{C}$ for $24 \mathrm{~h}$. After cooling to r.t., the crude mixture was diluted with water $(2 \mathrm{~mL})$ and purified by $\mathrm{RP} \mathrm{C}_{18}$-silica column chromatography using aq. $\mathrm{MeCN}$ (5 to $25 \mathrm{vol}$. \%, containing $0.1 \% \mathrm{Et}_{3} \mathrm{~N}$ ) as the mobile phase. Pure dsRVF5 (2) was obtained as a red solid. Yield: $3.3 \mathrm{mg}$ $(32 \%)$.

$\boldsymbol{R}_{\mathbf{f}}\left(\mathrm{C}_{18}\right.$-silica; $\left.\mathrm{H}_{2} \mathrm{O} / \mathrm{MeCN} 2: 1, v / v\right): 0.34$.

${ }^{1} \mathbf{H}$ NMR $\left(500 \mathrm{MHz}, \mathrm{CD}_{3} \mathrm{OD}\right) \delta 8.52(\mathrm{~s}, 2 \mathrm{H}), 7.64(\mathrm{~d}, J=8.4 \mathrm{~Hz}, 2 \mathrm{H}), 7.55(\mathrm{~d}, J=8.3 \mathrm{~Hz}, 2 \mathrm{H})$, $7.47(\mathrm{~d}, J=16.2 \mathrm{~Hz}, 1 \mathrm{H}), 7.44(\mathrm{~d}, J=8.6 \mathrm{~Hz}, 2 \mathrm{H}), 7.39$ (d, $J=16.3 \mathrm{~Hz}, 1 \mathrm{H}), 7.24(\mathrm{~s}, 1 \mathrm{H}), 7.15$ (d, $J=16.3 \mathrm{~Hz}, 1 \mathrm{H}), 7.10(\mathrm{~s}, 1 \mathrm{H}), 6.98(\mathrm{~d}, J=16.3 \mathrm{~Hz}, 1 \mathrm{H}), 6.86(\mathrm{~s}, 1 \mathrm{H}), 6.78(\mathrm{~d}, J=8.9 \mathrm{~Hz}, 2 \mathrm{H})$, $6.53(\mathrm{~s}, 1 \mathrm{H}), 3.77-3.68(\mathrm{~m}, 4 \mathrm{H}), 3.00(\mathrm{q}, J=7.3 \mathrm{~Hz}, 12 \mathrm{H}), 2.98(\mathrm{~s}, 6 \mathrm{H}), 2.04-1.99(\mathrm{~m}, 4 \mathrm{H})$, $1.27(\mathrm{t}, J=7.3 \mathrm{~Hz}, 18 \mathrm{H})$.

HRMS (ESI-): calculated $m / z$ for $\left[\mathrm{C}_{41} \mathrm{H}_{32} \mathrm{Cl}_{2} \mathrm{~N}_{2} \mathrm{O}_{8} \mathrm{~S}_{2}+\mathrm{H}\right]^{-}=815.1055$, found $=815.1027$. 


\section{2-(6-(3-(((tert-Butoxycarbonyl)amino)methyl)pyrrolidin-1-yl)-2,7-dichloro-3-oxo-3H-}

xanthen-9-yl)-5-((E)-4-((E)-4-(dimethylamino)styryl)styryl)benzene-1,3-disulfonate (dsRVF5-NHBoc, S26)

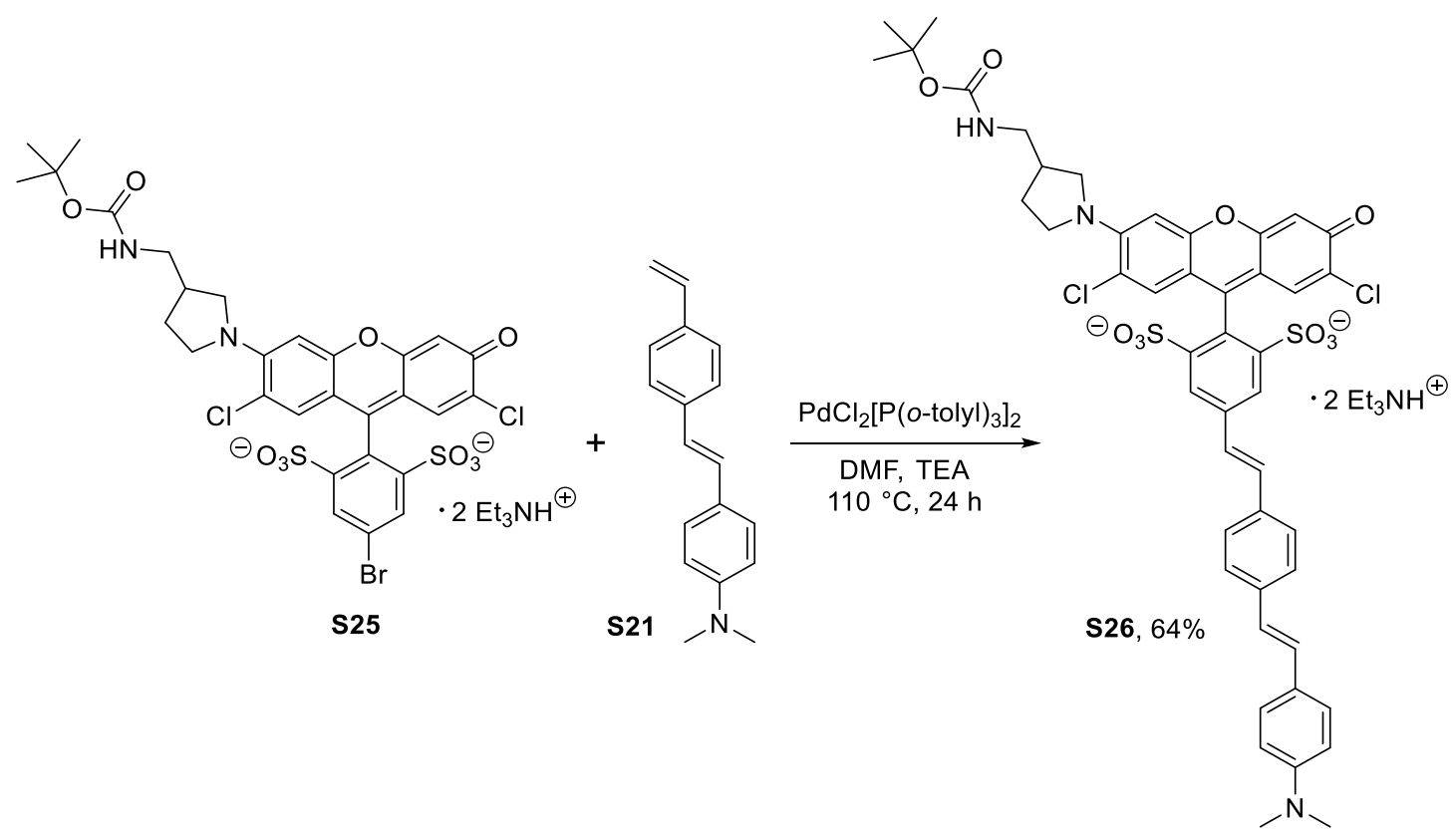

dsRhodol S25 (7.9 mg, $8.1 \mu \mathrm{mol}, 1.0$ eq.), $\pi$-wire $\mathbf{S 2 1}$ (3.5 mg, $14 \mu \mathrm{mol}, 1.7$ eq. $)$ and $\mathrm{PdCl}_{2}[(\mathrm{P}(o$ tolyl $\left.)_{3}\right]_{2}\left(2.1 \mathrm{mg}, 2.7 \mu \mathrm{mol}, 33 \mathrm{~mol}\right.$. \%) were dissolved in dry $\mathrm{Et}_{3} \mathrm{~N}(60 \mu \mathrm{L})$ and dry DMF $(60 \mu \mathrm{L})$ in an oven-dried screw-cap vial with a stir bar under dry Ar atmosphere. The reaction mixture was stirred at $110{ }^{\circ} \mathrm{C}$ for $24 \mathrm{~h}$. After cooling to r.t., the crude mixture was diluted with water ( $\left.1.5 \mathrm{~mL}\right)$ and purified by $\mathrm{RP} \mathrm{C}_{18}$-silica column chromatography using aq. MeCN (10 to 20 vol. \% MeCN, containing $\left.0.1 \% \mathrm{Et}_{3} \mathrm{~N}\right)$ as the mobile phase. Pure dsRVF5-NHBoc (S26) was obtained as a red solid. Yield: $5.9 \mathrm{mg}(64 \%)$.

$\boldsymbol{R} \mathbf{f}\left(\mathrm{C}_{18}\right.$-silica; $\left.\mathrm{H}_{2} \mathrm{O} / \mathrm{MeCN} 2: 1, v / v\right): 0.29$.

${ }^{1}$ H NMR (500 MHz, CD 3 OD) $\delta 8.52(\mathrm{~s}, 2 \mathrm{H}), 7.64(\mathrm{~d}, J=8.4 \mathrm{~Hz}, 2 \mathrm{H}), 7.55(\mathrm{~d}, J=8.4 \mathrm{~Hz}, 2 \mathrm{H})$, $7.49-7.37$ (m, 4H), 7.24 (s, 1H), 7.15 (d, $J=16.3 \mathrm{~Hz}, 1 \mathrm{H}), 7.09$ (s, 1H), 6.97 (d, J = 16.2 Hz, $1 \mathrm{H}), 6.86(\mathrm{~s}, 1 \mathrm{H}), 6.77(\mathrm{~d}, J=8.9 \mathrm{~Hz}, 2 \mathrm{H}), 6.53(\mathrm{~s}, 1 \mathrm{H}), 3.82-3.71(\mathrm{~m}, 3 \mathrm{H}), 3.52(\mathrm{dd}, J=10.7$, $7.4 \mathrm{~Hz}, 1 \mathrm{H}), 3.23-3.17(\mathrm{~m}, 2 \mathrm{H}), 3.14(\mathrm{q}, J=7.3 \mathrm{~Hz}, 12 \mathrm{H}), 2.98$ (s, 6H), 2.47 (dt, $J=14.5,7.1$ $\mathrm{Hz}, 1 \mathrm{H}), 2.11(\mathrm{dq}, J=11.9,6.1 \mathrm{~Hz}, 1 \mathrm{H}), 1.76(\mathrm{dq}, J=16.0,8.1 \mathrm{~Hz}, 1 \mathrm{H}), 1.45(\mathrm{~s}, 9 \mathrm{H}), 1.26(\mathrm{t}, J=$ $7.3 \mathrm{~Hz}, 18 \mathrm{H})$. 
${ }^{13} \mathbf{C}\left\{{ }^{1} \mathbf{H}\right\}$ NMR (126 MHz, CD 3 OD) $\delta 179.0,160.4,158.6,154.9,154.1,152.3,151.9,147.4,147.3$, 141.0, 140.0, 136.4, 134.2, 133.0, 132.6, 131.1, 130.4, 128.7, 128.5, 128.4, 127.4, 126.7, 126.4, 124.8, 119.2, 118.8, 117.0, 113.8, 104.5, 101.7, 80.1, 56.3, 52.3, 47.9, 46.6, 43.5, 43.4, 40.8, 35.4, $30.0,28.8,25.0,11.6,9.2$.

HRMS (ESI-): calculated $m / z$ for $\left[\mathrm{C}_{47} \mathrm{H}_{43} \mathrm{Cl}_{2} \mathrm{~N}_{3} \mathrm{O}_{10} \mathrm{~S}_{2}+\mathrm{H}\right]^{-}=944.1845$, found $=944.1829$.

\section{2-(6-(3-)((((1R,8p,9s)-Bicyclo[6.1.0]non-4-yn-9-}

ylmethoxy)carbonyl)amino)methyl)pyrrolidin-1-yl)-2,7-dichloro-3-oxo-3H-xanthen-9-yl)-5-

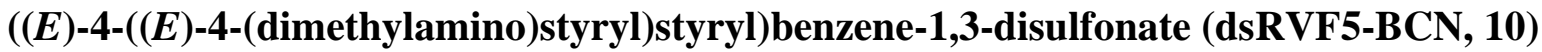

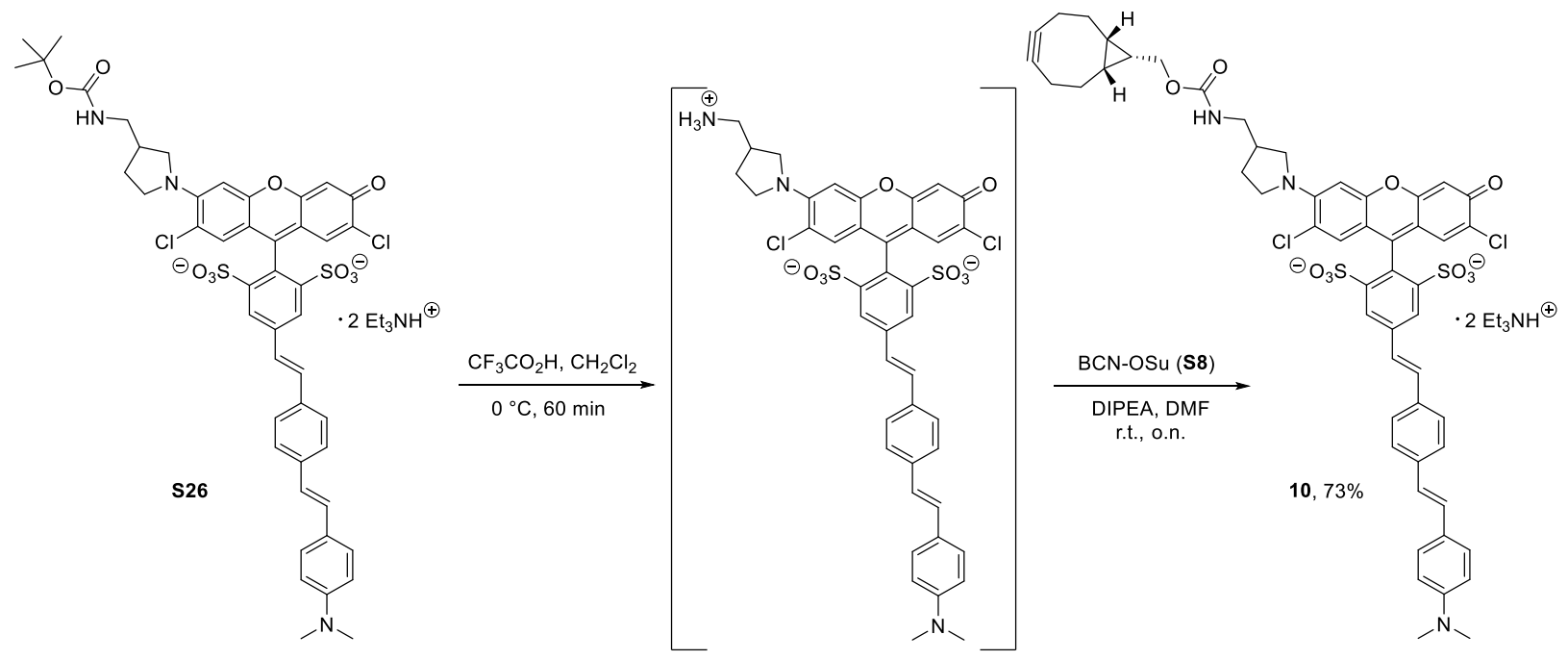

A suspension of dsRVF5-NHBoc ( $\mathbf{S 2 6}, 5.5 \mathrm{mg}, 4.8 \mu \mathrm{mol}, 1.0$ eq.) in $\mathrm{CH}_{2} \mathrm{Cl}_{2}(0.5 \mathrm{~mL})$ was cooled to $0{ }^{\circ} \mathrm{C}$ followed by dropwise addition of $\mathrm{CF}_{3} \mathrm{CO}_{2} \mathrm{H}(1 \mathrm{~mL})$. The resulting solution was stirred at $0{ }^{\circ} \mathrm{C}$ for $60 \mathrm{~min}$. Subsequently, volatiles were removed by rotary evaporation $\left(30^{\circ} \mathrm{C}\right)$ and the solid material was co-evaporated with toluene $(2 \times 1.5 \mathrm{~mL})$ to remove residual acid, providing the intermediate ammonium salt. After short drying under high vacuum $(1 \mathrm{~h})$, the dark purple solid was dissolved in dry DMF $(500 \mu \mathrm{L})$ and dry DIPEA $(8.3 \mu \mathrm{L}, 48 \mu \mathrm{mol}, 10$ eq. $)$ under argon atmosphere, followed by addition of BCN-OSu (S8, $4.0 \mathrm{mg}, 14 \mu \mathrm{mol}, 2.9$ eq.). The resulting solution was stirred at r.t. for $19 \mathrm{~h}$. After diluting with water $(3 \mathrm{~mL})$, the crude product was purified by $\mathrm{RP} \mathrm{C}_{18}$-silica column chromatography using aq. $\mathrm{MeCN}$ (10 to 30 vol. \% MeCN, containing 
$\left.0.1 \% \mathrm{Et}_{3} \mathrm{~N}\right)$ as the mobile phase. Pure dsRVF5-BCN (10) was obtained as a red solid. Yield: $4.3 \mathrm{mg}$ $(73 \%)$.

$\boldsymbol{R} \mathbf{f}\left(\mathrm{C}_{18}\right.$-silica; $\left.\mathrm{H}_{2} \mathrm{O} / \mathrm{MeCN} 1: 1, v / v\right)$ : 0.66.

${ }^{1}$ H NMR (500 MHz, CD 3 OD) $\delta 8.54(\mathrm{~s}, 2 \mathrm{H}), 7.66(\mathrm{~d}, J=8.5 \mathrm{~Hz}, 2 \mathrm{H}), 7.57(\mathrm{~d}, J=8.3 \mathrm{~Hz}, 2 \mathrm{H})$, $7.49(\mathrm{~d}, J=16.2 \mathrm{~Hz}, 1 \mathrm{H}), 7.46(\mathrm{~d}, J=8.6 \mathrm{~Hz}, 2 \mathrm{H}), 7.41(\mathrm{~d}, J=16.3 \mathrm{~Hz}, 1 \mathrm{H}), 7.26(\mathrm{~s}, 1 \mathrm{H}), 7.17$ $(\mathrm{d}, J=16.2 \mathrm{~Hz}, 1 \mathrm{H}), 7.12(\mathrm{~s}, 1 \mathrm{H}), 7.00(\mathrm{~d}, J=16.3 \mathrm{~Hz}, 1 \mathrm{H}), 6.88(\mathrm{~s}, 1 \mathrm{H}), 6.80(\mathrm{~d}, J=8.9 \mathrm{~Hz}, 2 \mathrm{H})$, $6.55(\mathrm{~s}, 1 \mathrm{H}), 4.18(\mathrm{~d}, J=8.2 \mathrm{~Hz}, 2 \mathrm{H}), 3.85-3.74(\mathrm{~m}, 3 \mathrm{H}), 3.66(\mathrm{~s}, 6 \mathrm{H}), 3.54(\mathrm{dd}, J=10.7,7.3 \mathrm{~Hz}$, $1 \mathrm{H}), 3.18(\mathrm{q}, J=7.3 \mathrm{~Hz}, 12 \mathrm{H}), 2.52$ (p, $J=6.9 \mathrm{~Hz}, 1 \mathrm{H}), 2.33-2.10(\mathrm{~m}, 7 \mathrm{H}), 1.85-1.73(\mathrm{~m}, 1 \mathrm{H})$, $1.64(\mathrm{~d}, J=12.2 \mathrm{~Hz}, 2 \mathrm{H}), 1.46-1.39(\mathrm{~m}, 1 \mathrm{H}), 1.30(\mathrm{t}, J=7.3 \mathrm{~Hz}, 18 \mathrm{H}), 1.03-0.92(\mathrm{~m}, 2 \mathrm{H})$.

HRMS (ESI-): calculated $m / z$ for $\left[\mathrm{C}_{53} \mathrm{H}_{47} \mathrm{Cl}_{2} \mathrm{~N}_{3} \mathrm{O}_{10} \mathrm{~S}_{2}+\mathrm{H}\right]^{-}=1020.2158$, found $=1020.2162$.

rac-N-Methyl-2-carbomethoxy-8-azabicyclo[3.2.1] octanone (rac-S27).

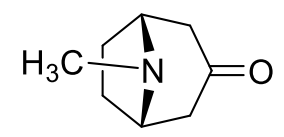

Tropinone

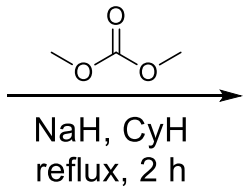

reflux, $2 \mathrm{~h}$

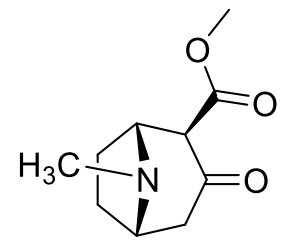

$\mathbf{S 2 7}+$ enantiomer $68 \%$

This procedure was adopted from Lewin et al. ${ }^{20}$ Dimethyl carbonate (24.4 mL, $0.29 \mathrm{~mol}, 2.2$ eq.) was added dropwise to a slurry of $\mathrm{NaH}$ (10.6 g, 0.264 mol, 2.0 eq., unwashed $60 \%$ dispersion in oil) in cyclohexane $(30 \mathrm{~mL})$ and heated to reflux. A cyclohexane $(75 \mathrm{~mL})$ solution of dried tropinone $^{21}$ (18.36 g, $0.132 \mathrm{mmol}, 1.0$ eq.) was added dropwise into the reaction mixture and refluxed for further $2 \mathrm{~h}$ (evolution of gas finished within this time). $\mathrm{MeOH}(0.5 \mathrm{~mL})$ was added to the reaction mixture and stirred for further $30 \mathrm{~min}$ while allowing to cool down to r.t. Water $(200 \mathrm{~mL})$ was then carefully added to the reaction mixture and the organic layer was separated and extracted with more water $(2 \times 50 \mathrm{~mL})$. The combined aqueous layers were saturated with $\mathrm{NH}_{4} \mathrm{Cl}$ ( $~ 90$ g was necessary) and extracted with $\mathrm{CH}_{2} \mathrm{Cl}_{2}(8 \times 20 \mathrm{~mL})$. The combined organic layers were washed with brine $(50 \mathrm{~mL})$, dried over anhydrous $\mathrm{Na}_{2} \mathrm{SO}_{4}$, filtered and volatiles were removed 
under reduced pressure. The material was judged to be sufficiently pure by ${ }^{1} \mathrm{H}$ NMR. An analytical sample was obtained by silica gel column chromatography purification $\left(\mathrm{MeOH} / \mathrm{CH}_{2} \mathrm{Cl}_{2}\right.$ 3:97 to 1:9, $v / v ; R_{f}=0.15$ in $\left.\mathrm{MeOH} / \mathrm{CH}_{2} \mathrm{Cl}_{2} 1: 19, v / v\right)$. Yield: $17.7 \mathrm{~g}(68 \%)$. Yellowish powder.

The spectral characteristics were the same as those found for (2R)- $N$-methyl-2-carbomethoxy-8azabicyclo[3.2.1] octanone (S27).

(2R)-N-Methyl-2-carbomethoxy-8-azabicyclo[3.2.1]octanone (S27).

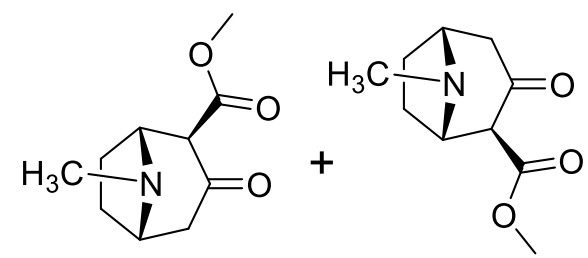

racemic $\mathbf{S 2 7}$

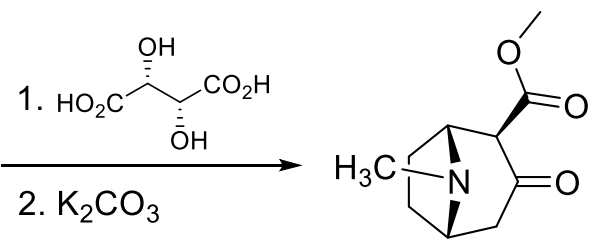

S27, $\sim 9 \%$ yield $>90 \%$ ee

This procedure was adopted from Kuznetsov. ${ }^{22}$ A solution of (+)-tartaric acid (7.32 g, $48.8 \mathrm{mmol}$, 1.0 eq.) in water (19 mL) was added to a stirred solution of rac- $N$-methyl-2-carbomethoxy-8azabicyclo[3.2.1] octanone (rac-S27, $9.62 \mathrm{~g}, 48.8 \mathrm{mmol}, 1.0$ eq.) in $\mathrm{CH}_{2} \mathrm{Cl}_{2}(60 \mathrm{~mL})$ and stirred for $15 \mathrm{~min}$ at r.t. The aqueous layer (including grown crystals) was transferred to a round bottom flask and diluted with methanol $(59 \mathrm{~mL})$. The slurry was heated to reflux until all the crystals dissolved and was subsequently allowed to cool down to r.t. with slow stirring. Crystals emerged in $\sim 1 \mathrm{~h}$ and the mixture was stirred for another 30 minutes and crystals were collected by vacuum filtration on a Büchner funnel. The crystalline solid was further washed with cold methanol $(2 \times 5 \mathrm{~mL})$ and acetone $(2 \times 10 \mathrm{~mL})$ and dried under high vacuum to yield white crystals predominantly composed of the (+)-tartarate salt of $\mathbf{S 2 7}$ (7.49 g, 44\% yield). The obtained crystals were suspended in water $(100 \mathrm{~mL})$ and slowly added to a stirred sat. aq. $\mathrm{K}_{2} \mathrm{CO}_{3}(50 \mathrm{~mL})$. When the evolution of carbon dioxide stopped ( $\sim 2 \mathrm{~min}), \mathrm{pH}$ was checked to be in the basic region $(\sim 9)$ and the solution was extracted with $\mathrm{CH}_{2} \mathrm{Cl}_{2}(6 \times 20 \mathrm{~mL})$. The combined organic phases were washed with brine $(50 \mathrm{~mL})$, dried over anhydrous magnesium sulfate, filtered and the solvent was removed under reduced pressure. The solid was further dried under high vacuum and judged to be chemically pure by ${ }^{1} \mathrm{H}$ NMR. Yield: $3.05 \mathrm{~g}(32 \%)$ after one crystallization. White powder. 
Note: Three crystallization cycles were necessary to obtain samples with $>90 \%$ ee of $\mathbf{S 2 7}$. The overall yield after three crystallization cycles was $9 \%$. The enantiomeric composition of the material was checked after each crystallization cycle.

$\boldsymbol{R}$ (silica; $\left.\mathrm{MeOH} / \mathrm{CH}_{2} \mathrm{Cl}_{2} 1: 19, v / v\right): 0.15$.

${ }^{1} \mathbf{H}$ NMR (400 MHz, CDCl $): \delta(\mathrm{ppm}) 1.49-1.58(\mathrm{~m}, 1 \mathrm{H}), 1.75-1.83(\mathrm{~m}, 1 \mathrm{H}), 1.89(\mathrm{~d}, 1 \mathrm{H}, J=$ $18.5 \mathrm{~Hz}$ ), 2.05-2.27 (m, 3H), 2.35 (s, 3H), $2.76\left(\mathrm{ddd}, 1 \mathrm{H}, J_{1}=18.6 \mathrm{~Hz}, J_{2}=4.8 \mathrm{~Hz}, J_{3}=1.4 \mathrm{~Hz}\right)$, $3.31-3.36(\mathrm{~m}, 1 \mathrm{H}), 3.77(\mathrm{~s}, 3 \mathrm{H}), 3.78(\mathrm{~d}, 1 \mathrm{H}, J=5.3 \mathrm{~Hz})$.

FTIR $\left(\right.$ ATR, $\left.\mathrm{cm}^{-1}\right)=3089,2996,2950,2727,1652,1517,1428,1405,1348,1246,1222,1174$, $1118,1042,965,926,909,832,816,784,764$.

MS (ESI+): calculated $m / z$ for $\left[\mathrm{C}_{10} \mathrm{H}_{15} \mathrm{NO}_{3}+\mathrm{H}\right]^{+}=198.1$, found $=198.3$.

m.p. $\left(\mathrm{CH}_{2} \mathrm{Cl}_{2}\right): 111.5-112.5^{\circ} \mathrm{C}$ (ref. ${ }^{23} 111{ }^{\circ} \mathrm{C}$; ref. $\left..^{24} 104-105^{\circ} \mathrm{C}\right)$.

Note: ${ }^{13} \mathrm{C}\left\{{ }^{1} \mathrm{H}\right\}$ NMR gave complicated spectra, showing the enol tautomer ${ }^{25}\left(>20 \%\right.$ in $\mathrm{CDCl}_{3}$ and $\sim 50 \%$ in DMSO- $d_{6}$ ).

The optical purity of samples of (2R)-N-methyl-2-carbomethoxy-8-azabicyclo[3.2.1]octanone (S27) was determined by two independent methods:

1) Determination of optical activity in methanolic solution $(c=1.0 \mathrm{~g} / 100 \mathrm{~mL})$ and comparison to published data. However, the published values range from $[\alpha]^{21} \mathrm{D}=+18.3^{\circ}(c=1 \mathrm{~g} / 100 \mathrm{~mL}$, $\mathrm{MeOH})^{26}$ and $[\alpha]^{21} \mathrm{D}=+18.6^{\circ}(c=1 \mathrm{~g} / 100 \mathrm{~mL}, \mathrm{MeOH})^{24}$ to $+25.4^{\circ}(c=1 \mathrm{~g} / 100 \mathrm{~mL}, \mathrm{MeOH}),{ }^{27}$ thus optical activity was used solely as a qualitative, not quantitative, indicator of optical purity.

2) A sample of $\mathbf{S} 27$ (20 mg, $0.101 \mathrm{mmol}, 1.0$ eq.) was dried under high vacuum, dissolved in dry THF ( $5 \mathrm{~mL}$ ) under dry Ar atmosphere. $n$-Butyllithium ( $2.5 \mathrm{M}$ in hexanes; $45 \mu \mathrm{L}, 0.115 \mathrm{mmol}$, 1.1 eq.) was dropwise added to the solution of $\mathbf{S} 27$ at $-78^{\circ} \mathrm{C}$ and stirred for further $15 \mathrm{~min}$. (1S)-(-)-Camphanic chloride ( $24 \mathrm{mg}, 0.115 \mathrm{mmol}, 1.1 \mathrm{eq}$.) was then added in one portion and the reaction mixture was allowed to warm-up to r.t. Water $(5 \mathrm{~mL})$ was added to the reaction mixture and the organic layer was separated, dried over anhydrous $\mathrm{Na}_{2} \mathrm{SO}_{4}$, filtered and volatiles were 
removed under reduced pressure. The obtained residuum was dissolved in $\mathrm{CDCl}_{3}$ and integrals of diagnostic camphanate methyl groups: $\delta(\mathrm{ppm}) 1.04$ (s, 3H), for $\left(1 S, 1^{\prime} S\right)-\mathbf{S 4 7}$ and 1.05 (s, 3H) for $(1 R, 1 ' S)-\mathbf{S 4 7}$; were used to calculate enantiomeric excess (ee). This procedure was adopted from Meltzer et al. ${ }^{28}$

Methyl (1R,5S)-8-methyl-3-(((4R)-4,7,7-trimethyl-3-oxo-2-oxabicyclo[2.2.1]heptane-1carbonyl)oxy)-8-azabicyclo[3.2.1]oct-2-ene-2-carboxylate (S47).

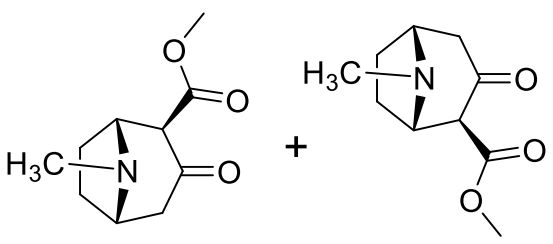

S27
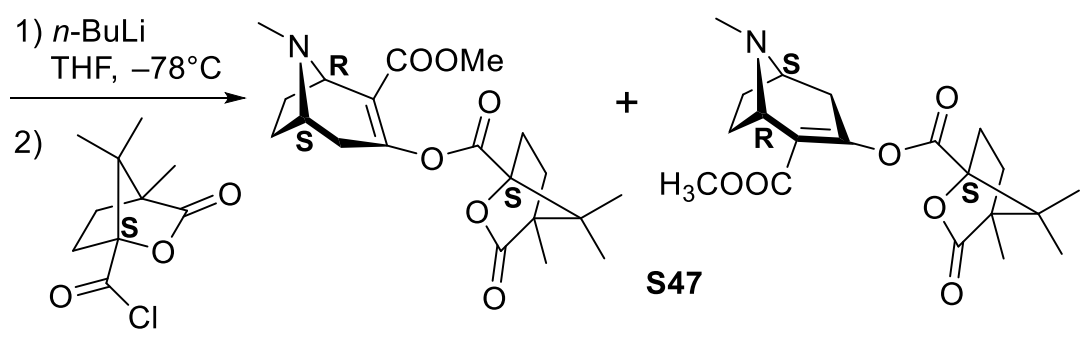

Yield: $35 \mathrm{mg}$ (90\%). Off-white powder.

${ }^{1} \mathbf{H}$ NMR (400 MHz, $\mathrm{CDCl}_{3}$ ): $\delta(\mathrm{ppm}) 1.06$ (s, 3H), 1.13 (s, 3H), 1.14 (s, 3H), 1.62-1.69 (m, 1H), 1.69-1.77 (m, 1H), $1.82(\mathrm{~d}, 1 \mathrm{H}, J=18.4 \mathrm{~Hz}), 1.91-2.01(\mathrm{~m}, 2 \mathrm{H}), 2.11-2.21(\mathrm{~m}, 3 \mathrm{H}), 2.45(\mathrm{~s}, 3 \mathrm{H})$, 2.46-2.55 (m, 1H), $2.74\left(\mathrm{dd}, 1 \mathrm{H}, J_{1}=18.3 \mathrm{~Hz}, J_{2}=4.6 \mathrm{~Hz}\right), 3.38(\mathrm{~m}, 1 \mathrm{H}), 3.71(\mathrm{~s}, 3 \mathrm{H}), 3.92(\mathrm{~d}$, $1 \mathrm{H}, J=5.4 \mathrm{~Hz})$.

${ }^{13} \mathbf{C}\left\{{ }^{1} \mathbf{H}\right\}$ NMR $\left(100 \mathrm{MHz}, \mathrm{CDCl}_{3}\right): \delta(\mathrm{ppm})$ 9.9, 16.85, 16.96, 29.1, 30.5, 31.3, 33.4, 35.08, 35.16, 51.9, 54.5, 55.1, 57.3, 59.5, 91.0, 121.6, 152.1, 164.5, 165.1, 178.0.

FTIR (ATR, diamond, $\mathrm{cm}^{-1}$ ) = 2956, 2886, 1790, 1760, 1718, 1656, 1448, 1428, 1304, 1256, 1224, 1146, 1104, 1054, 1038, 956, 932, 828, 512.

m.p. (THF): $>270{ }^{\circ} \mathrm{C}$.

Methyl (1R,5S)-8-methyl-3-(((trifluoromethyl)sulfonyl)oxy)-8-azabicyclo[3.2.1]oct-2-ene-2carboxylate $(\mathbf{S 2 8})$. 


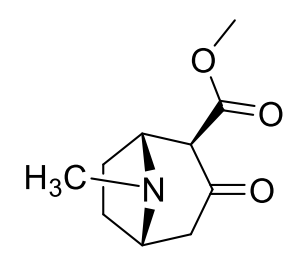

S27

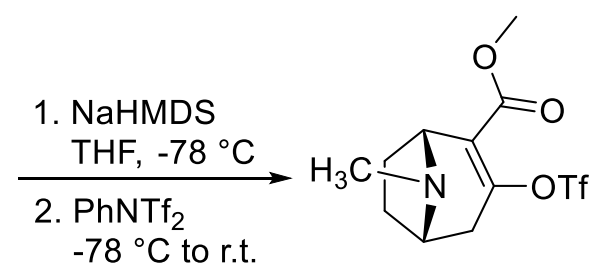

S28, $90 \%$

This procedure was adopted from Meltzer et al. ${ }^{29}$ Sodium bis(trimethylsilyl)amide (1.0 M in dry THF; $67.5 \mathrm{~mL}, 67.5 \mathrm{mmol}, 1.15$ eq.) was dropwise added to a stirred solution of $\mathbf{S 2 7}$ (11.47 g, $58.2 \mathrm{mmol}, 1.0$ eq. $)$ in dry $\mathrm{THF}(160 \mathrm{~mL})$ at $-78^{\circ} \mathrm{C}$ under dry Ar atmosphere and further stirred for 30 minutes. Solid $N$-phenyl-trifluoromethanesulfonimide (22.9 g, $64.0 \mathrm{mmol}, 1.15 \mathrm{eq}$.) was then added in one portion and further stirred overnight, while allowing to warm-up to r.t. Volatiles were then removed under reduced pressure and the obtained residuum was dissolved in AcOEt $(200 \mathrm{~mL})$, washed with water $(200 \mathrm{~mL})$, brine $(100 \mathrm{~mL})$, dried over anhydrous $\mathrm{Na}_{2} \mathrm{SO}_{4}$, filtered and volatiles were removed under vacuum to yield a brown oil. The crude product was further dissolved in $\mathrm{Et}_{2} \mathrm{O}(200 \mathrm{~mL})$, washed with water $(2 \times 100 \mathrm{~mL})$, brine $(100 \mathrm{~mL})$ and filtered through a short pad of silica $(\sim 3 \mathrm{~cm})$. The product was further eluted with $\mathrm{Et}_{3} \mathrm{~N}_{/} \mathrm{Et}_{2} \mathrm{O}(1: 19, v / v)$ until all the product was recovered (TLC). Fractions containing the product were combined and volatiles were removed under vacuum to yield a yellowish oil which was judged pure ( $>95 \%$ by ${ }^{1} \mathrm{H}$ NMR). Yield: $17.34 \mathrm{~g}(90 \%)$. Yellowish oil.

$\boldsymbol{R}_{\mathbf{f}}($ silica; AcOEt/hexanes 1:1, v/v): 0.1.

${ }^{1} \mathbf{H}$ NMR (400 MHz, $\left.\mathrm{CDCl}_{3}\right): \delta(\mathrm{ppm})$ 1.52-1.62 (m, 1H), 1.90-2.00 (m, 2H), 2.10-2.19 (m, 2H), $2.37(\mathrm{~s}, 3 \mathrm{H}), 2.82\left(\mathrm{dd}, 1 \mathrm{H}, J_{1}=18.8 \mathrm{~Hz}, J_{2}=4.9 \mathrm{~Hz}\right), 3.36-3.42(\mathrm{~m}, 1 \mathrm{H}), 3.78(\mathrm{~s}, 3 \mathrm{H}), 3.90(\mathrm{~d}$, $1 \mathrm{H}, J=5.2 \mathrm{~Hz})$.

${ }^{13} \mathbf{C}\left\{{ }^{1} \mathbf{H}\right\}$ NMR $\left(100 \mathrm{MHz}, \mathrm{CDCl}_{3}\right): \delta(\mathrm{ppm}) 30.1,33.3,34.8,35.1,52.3,57.7,60.3,118.4$ (q, $\left.{ }^{1} J_{\mathrm{C}-\mathrm{F}}=320 \mathrm{~Hz}\right), 125.4,149.3,164.0$.

${ }^{19}$ F NMR $\left(376 \mathrm{MHz}, \mathrm{CDCl}_{3}\right): \delta(\mathrm{ppm})-74.9$ (s).

FTIR $\left(A T R\right.$, diamond $\mathrm{cm}^{-1}$ ) = 2952, 2804, 1720, 1420, 1266, 1202, 1140, 1078, 1032, 878, 850, $828,620,584,502$.

MS (ESI+): calculated $m / z$ for $\left[\mathrm{C}_{11} \mathrm{H}_{14} \mathrm{~F}_{3} \mathrm{NO}_{5} \mathrm{~S}+\mathrm{H}\right]^{+}=330.1$, found $=330.1$. 
Methyl (1R,5S)-3-(3,4-dichlorophenyl)-8-methyl-8-azabicyclo[3.2.1]oct-2-ene-2-carboxylate (S29).

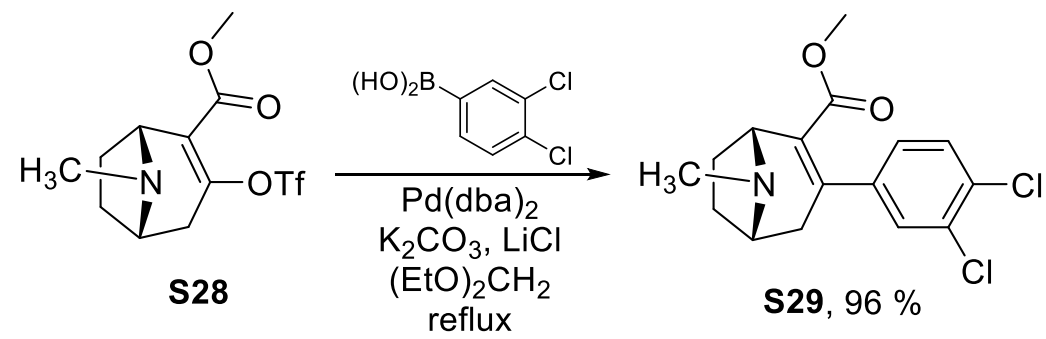

This procedure was adopted from Meltzer et al. ${ }^{30}$ Aqueous $\mathrm{Na}_{2} \mathrm{CO}_{3}(2.0 \mathrm{M} ; 48.5 \mathrm{~mL}, 97 \mathrm{mmol}$, 2.0 eq.) was added in one portion to a suspension of $\mathbf{S 2 8}$ (15.96 g, $48.5 \mathrm{mmol}, 1.0$ eq.), 3,4-dichlorophenylboronic acid (12.49 g, $65.4 \mathrm{mmol})$ and $\mathrm{LiCl}(4.52 \mathrm{~g}, 107 \mathrm{mmol}, 2.2 \mathrm{eq}$.$) in$ diethoxymethane $(130 \mathrm{~mL})$ and was bubbled with dry Ar for 30 minutes. Then, bis(dibenzylideneacetone)palladium( $(0)\left(\operatorname{Pd}(\mathrm{dba})_{2}, 1.77 \mathrm{~g}, 1.9 \mathrm{mmol}, 4 \mathrm{~mol} . \%\right)$ was added in one portion and the reaction mixture was refluxed for 2 hours (the reaction progress was monitored by $\mathrm{TLC}\left(\mathrm{Et}_{3} \mathrm{~N} / \mathrm{Et}_{2} \mathrm{O} 1: 19, v / v\right)$. The reaction mixture was then cooled down to r.t. and filtered through a short pad of celite $(\sim 2 \mathrm{~cm})$. The celite pad was further washed with $\mathrm{Et}_{2} \mathrm{O}(200 \mathrm{~mL})$. The combined filtrates were basified with conc. aq. $\mathrm{NH}_{3}$ (until $\mathrm{pH} \sim 9$ ) and washed with brine (100 mL). The organic phase was dried over anhydrous $\mathrm{Na}_{2} \mathrm{SO}_{4}$, filtered and volatiles were removed under vacuum. The obtained orange oil was purified by silica gel column chromatography $\left(\mathrm{Et}_{3} \mathrm{~N} / \mathrm{Et}_{2} \mathrm{O}\right.$ 1:99 to $1: 19, v / v)$ to provide pure product. Yield: $15.82 \mathrm{~g}(96 \%)$. Yellowish viscous oil.

$\boldsymbol{R}_{\mathbf{f}}\left(\right.$ silica; $\left.\mathrm{Et}_{3} \mathrm{~N} / \mathrm{Et}_{2} \mathrm{O} 2.5: 97.5, v / v\right): 0.25$.

${ }^{1} \mathbf{H}$ NMR (400 MHz, $\left.\mathrm{CDCl}_{3}\right): \delta(\mathrm{ppm}) 1.51-1.59(\mathrm{~m}, 1 \mathrm{H}), 1.85-1.93(\mathrm{~m}, 2 \mathrm{H}), 2.05-2.19(\mathrm{~m}, 2 \mathrm{H})$, $2.35(\mathrm{~s}, 3 \mathrm{H}), 2.65\left(\mathrm{dd}, 1 \mathrm{H}, J_{1}=18.8 \mathrm{~Hz}, J_{2}=4.4 \mathrm{~Hz}\right), 3.24-3.29(\mathrm{~m}, 1 \mathrm{H}), 3.44(\mathrm{~s}, 3 \mathrm{H}), 3.77(\mathrm{~d}$, $1 \mathrm{H}, J=5.4 \mathrm{~Hz}), 6.90\left(\mathrm{dd}, 1 \mathrm{H}, J_{1}=8.2 \mathrm{~Hz}, J_{2}=2.0 \mathrm{~Hz}\right), 7.15(\mathrm{~d}, J=2.0 \mathrm{~Hz}), 7.29(\mathrm{~d}, 1 \mathrm{H}, J=8.2$ $\mathrm{Hz})$.

${ }^{13} \mathbf{C}\left\{{ }^{1} \mathbf{H}\right\}$ NMR (100 MHz, $\left.\mathrm{CDCl}_{3}\right): \delta(\mathrm{ppm}) 30.0,34.1,36.1,37.6,51.4,57.3,60.2,126.4,128.7$, 129.9, 131.2, 131.8, 132.0, 141.2, 141.5, 167.4. 
FTIR $\left(\right.$ ATR, diamond, $\mathrm{cm}^{-1}$ ) $=2946,2876,2796,1706,1468,1434,1352,1258,1222,1152$, 1112, 1044, 1030, 948, 810, 678, 596, 496, 442.

MS (APCI+): calculated $m / z$ for $\left[\mathrm{C}_{16} \mathrm{H}_{17} \mathrm{Cl}_{2} \mathrm{NO}_{2}+\mathrm{H}\right]^{+}=326.1$, found $=326.2$.

Methyl

$(1 R, 2 S, 3 S, 5 S)-3-(3,4-d i c h l o r o p h e n y l)-8-m e t h y l-8$-azabicyclo[3.2.1]octane-2carboxylate (3).

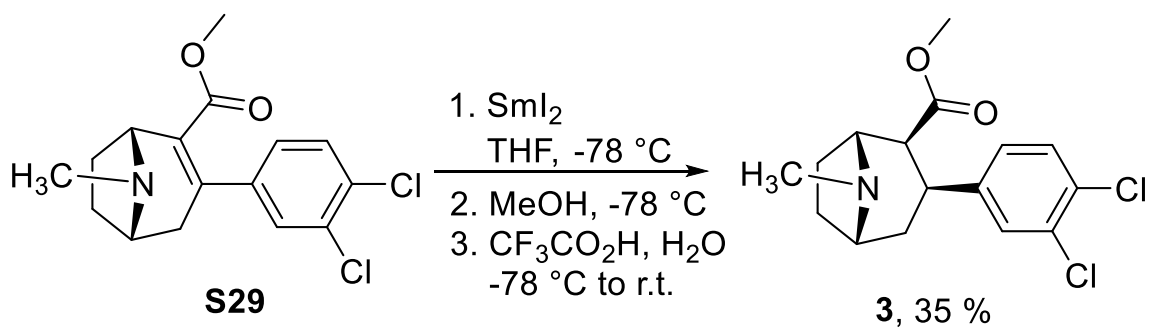

This procedure was adopted from Meltzer et al. ${ }^{30}$ A solution of $\mathbf{S 2 9}(7.23 \mathrm{~g}, 22.2 \mathrm{mmol}, 1.0$ eq. $)$ in dry THF $(57 \mathrm{~mL})$ was dropwise added to a solution of $\mathrm{SmI}_{2}(0.1 \mathrm{M}$ in dry THF; $842 \mathrm{~mL}$, $84.2 \mathrm{mmol}, 3.8$ eq.) at $-78^{\circ} \mathrm{C}$ and under dry $\mathrm{Ar}$ atmosphere and stirred for $45 \mathrm{~min}$. Then, dry $\mathrm{MeOH}(57 \mathrm{~mL})$ was added and the reaction mixture was further stirred for $3 \mathrm{~h}$ at $-78^{\circ} \mathrm{C}$. Then, $\mathrm{CF}_{3} \mathrm{CO}_{2} \mathrm{H}(28.5 \mathrm{~mL})$ was cautiously added dropwise, followed by $\mathrm{H}_{2} \mathrm{O}(581 \mathrm{~mL})$ and the reaction mixture was allowed to warm up to r.t. and subsequently basified with conc. aq. $\mathrm{NH}_{3}$ until $\mathrm{pH} \sim 10$ and filtered through a short silica pad $(\sim 3 \mathrm{~cm})$. The product was further eluted with $\mathrm{Et}_{2} \mathrm{O}(300 \mathrm{~mL})$ and sat. aq. $\mathrm{NaHSO}_{3}(150 \mathrm{~mL})$. The aqueous solution was extracted with $\mathrm{Et}_{2} \mathrm{O}(2 \times 200 \mathrm{~mL})$. The combined organic layers were washed with brine $(200 \mathrm{~mL})$, dried over anhydrous $\mathrm{Na}_{2} \mathrm{SO}_{4}$, filtered and volatiles were evaporated under reduced pressure. The obtained yellow oil was purified by silica gel column chromatography $\left(\mathrm{Et}_{3} \mathrm{~N} / \mathrm{Et}_{2} \mathrm{O} 1: 399\right.$ to $\left.1: 99, v / v\right)$ to give pure product. Yield: $2.51 \mathrm{~g}(35 \%)$. Yellowish viscous oil.

$\boldsymbol{R}_{\mathbf{f}}\left(\right.$ silica; $\left.\mathrm{Et}_{3} \mathrm{~N} / \mathrm{Et}_{2} \mathrm{O} 2.5: 97.5, v / v\right): 0.3$.

${ }^{1} \mathbf{H}$ NMR (400 MHz, $\left.\mathrm{CDCl}_{3}\right): \delta(\mathrm{ppm}) 1.55-1.75(\mathrm{~m}, 3 \mathrm{H}), 2.05-2.21(\mathrm{~m}, 2 \mathrm{H}), 2.22(\mathrm{~s}, 3 \mathrm{H}), 2.52$ $\left(\mathrm{dt}, 1 \mathrm{H}, J_{1}=12.5 \mathrm{~Hz}, J_{2}=2.7 \mathrm{~Hz}\right), 2.85-2.89(\mathrm{~m}, 1 \mathrm{H}), 2.94\left(\mathrm{td}, 1 \mathrm{H}, J_{1}=12.5 \mathrm{~Hz}, J_{2}=5.2 \mathrm{~Hz}\right)$, $3.33-3.38(\mathrm{~m}, 1 \mathrm{H}), 3.52(\mathrm{~s}, 3 \mathrm{H}), 3.58\left(\mathrm{dd}, 1 \mathrm{H}, J_{1}=6.8 \mathrm{~Hz}, J_{2}=2.7 \mathrm{~Hz}\right), 7.09\left(\mathrm{dd}, 1 \mathrm{H}, J_{1}=8.4 \mathrm{~Hz}\right.$, $\left.J_{2}=2.1 \mathrm{~Hz}\right), 7.29-7.33(\mathrm{~m}, 2 \mathrm{H})$. 
${ }^{13} \mathbf{C}\left\{{ }^{1} \mathbf{H}\right\}$ NMR $\left(100 \mathrm{MHz}, \mathrm{CDCl}_{3}\right): \delta(\mathrm{ppm}) 25.3,26.0,33.5,34.1,42.0,51.4,52.7,62.3,65.4$, 126.9, 129.6, 129.8, 130.0, 132.0, 143.8, 171.9.

FTIR $\left(\right.$ ATR, diamond, $\mathrm{cm}^{-1}$ ) $=2948,2878,2847,2798,1742,1472,1434,1352,1234,1174$, 1132, 1122, 1029, 1009, 818, 753, 672, 604.

MS $\left(\mathrm{APCI}^{+}\right)$: calculated $m / z$ for $\left[\mathrm{C}_{16} \mathrm{H}_{19} \mathrm{Cl}_{2} \mathrm{NO}_{2}+\mathrm{H}\right]^{+}=328.1$, found $=328.2$.

Note: methyl (1R,2S,3R,5S)-3-(3,4-dichlorophenyl)-8-methyl-8-azabicyclo[3.2.1]octane-2-

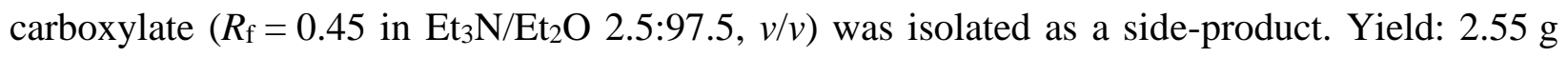
(35\%). Off-white solid.

${ }^{1} \mathbf{H}$ NMR (400 MHz, $\left.\mathrm{CDCl}_{3}\right): \delta(\mathrm{ppm}) 1.26-1.37(\mathrm{~m}, 1 \mathrm{H}), 1.44-1.52(\mathrm{~m}, 1 \mathrm{H}), 1.55-1.63(\mathrm{~m}, 1 \mathrm{H})$, 2.08-2.18 (m, 1H), 2.20-2.30 (m, 1H), 2.25 (s, 3H), 2.40-2.50 (m, 2H), 3.24-3.40 (m, 3H), 3.61 (s, 3H), $7.05\left(\mathrm{dd}, 1 \mathrm{H}, J_{1}=8.3 \mathrm{~Hz}, J_{2}=2.0 \mathrm{~Hz}\right), 7.28(\mathrm{~d}, 1 \mathrm{H}, J=2.0 \mathrm{~Hz}), 7.31(\mathrm{~d}, 1 \mathrm{H}, J=8.3 \mathrm{~Hz})$.

${ }^{13} \mathbf{C}\left\{{ }^{1} \mathbf{H}\right\}$ NMR (100 MHz, $\left.\mathrm{CDCl}_{3}\right): \delta(\mathrm{ppm})$ 29.0, 29.1, 35.8, 41.1, 52.1, 56.2, 59.6, 63.3, 127.5, $129.8,130.3,130.4,132.4,144.9,174.8$.

FTIR $\left(\right.$ ATR, $\left.\mathrm{cm}^{-1}\right)=2953,2876,2847,2796,1725,1469,1434,1306,1193,1165,1131,1027$, $836,744,672,599,475,440$.

MS $\left(\mathrm{APCI}^{+}\right)$: calculated $m / z$ for $\left[\mathrm{C}_{16} \mathrm{H}_{19} \mathrm{Cl}_{2} \mathrm{NO}_{2}+\mathrm{H}\right]^{+}=328.1$, found $=328.2$.

m.p. $\left(\mathrm{Et}_{3} \mathrm{~N} / \mathrm{Et}_{2} \mathrm{O}\right): 89-90{ }^{\circ} \mathrm{C}$. 
Methyl (1R,2S,3S,5S)-3-(3,4-dichlorophenyl)-8-azabicyclo[3.2.1]octane-2-carboxylate (S30).
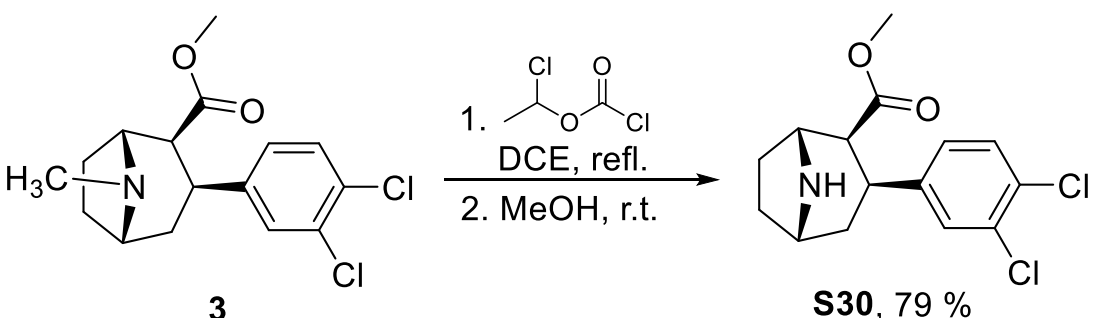

This procedure was adopted from Cha et al. ${ }^{31}$ Sodium carbonate $(2.44 \mathrm{~g}, 23.0 \mathrm{mmol}, 4.0 \mathrm{eq}$.$) and$ 1-chloroethyl chloroformate $(2.51 \mathrm{~mL}, 23.0 \mathrm{mmol}, 4.0$ eq.) were consecutively added to a solution of 3 (1.89 g, $5.76 \mathrm{mmol}, 1.0$ eq.) in dry 1,2-dichloroethane (25 mL) under dry Ar atmosphere, and the reaction mixture was refluxed for $18 \mathrm{~h}$. The reaction mixture was then cooled down to r.t. and volatiles were removed under reduced pressure. The obtained brownish oil $(3.09 \mathrm{~g})$ was dissolved in methanol $(20 \mathrm{~mL})$ and further stirred overnight at r.t. The solvent was removed under reduced pressure and the obtained oily residuum was purified by silica gel column chromatography $\left(\mathrm{Et}_{3} \mathrm{~N} / \mathrm{Et}_{2} \mathrm{O} 1: 9, v / v\right)$ to give pure product. Yield: $1.26 \mathrm{~g}$ (79\% at $88 \%$ conversion). White crystalline solid. A small amount of starting material $3(0.22 \mathrm{~g}, 12 \%)$ was regenerated.

$\boldsymbol{R}_{\mathbf{f}}\left(\right.$ silica; $\left.\mathrm{Et}_{3} \mathrm{~N} / \mathrm{Et}_{2} \mathrm{O} 1: 9, v / v\right): 0.15$.

${ }^{1} \mathbf{H}$ NMR (400 MHz, $\left.\mathrm{CDCl}_{3}\right): \delta(\mathrm{ppm})$ 1.57-1.78 (m, 3H), 1.95-2.05 (m, 1H), 2.06-2.13 (m, 1H), $2.35\left(\mathrm{dt}, 1 \mathrm{H}, J_{1}=12.9 \mathrm{~Hz}, J_{2}=2.9 \mathrm{~Hz}\right), 2.48($ br s, $1 \mathrm{H},>\mathrm{NH}), 3.17\left(\mathrm{td}, 1 \mathrm{H}, J_{1}=12.9 \mathrm{~Hz}, J_{2}=5.4\right.$ $\mathrm{Hz}), 3.44(\mathrm{~s}, 3 \mathrm{H}), 3.70-3.75(\mathrm{~m}, 2 \mathrm{H}), 7.04\left(\mathrm{dd}, 1 \mathrm{H}, J_{1}=8.4 \mathrm{~Hz}, J_{2}=2.1 \mathrm{~Hz}\right), 7.28(\mathrm{~d}, 1 \mathrm{H}, J=2.1$ $\mathrm{Hz}), 7.33(\mathrm{~d}, 1 \mathrm{H}, J=8.4 \mathrm{~Hz})$.

${ }^{13} \mathbf{C}\left\{{ }^{1} \mathbf{H}\right\}$ NMR $\left(100 \mathrm{MHz}, \mathrm{CDCl}_{3}\right): \delta$ (ppm) 27.9, 29.3, 33.8, 35.2, 51.0, 51.5, 53.8, 56.6, 127.0, $129.7,130.3,130.5,132.4,143.0,173.5$.

FTIR (ATR, diamond, $\mathrm{cm}^{-1}$ ) = 3320, 2948, 2874, 1718, 1470, 1443, 1406, 1376, 1232, 1168, $1078,1028,962,872,818,760,684,602$.

MS $\left(\mathrm{APCI}^{+}\right)$: calculated $m / z$ for $\left[\mathrm{C}_{15} \mathrm{H}_{17} \mathrm{Cl}_{2} \mathrm{NO}_{2}+\mathrm{H}\right]^{+}=314.1$, found $=314.2$.

m.p. $\left(\mathrm{Et}_{3} \mathrm{~N} / \mathrm{Et}_{2} \mathrm{O}\right): 94-95.5{ }^{\circ} \mathrm{C}$. 
Methyl (1R,2S,3S,5S)-8-(4-((tert-butoxycarbonyl)amino)butyl)-3-(3,4-dichlorophenyl)-8azabicyclo[3.2.1]octane-2-carboxylate (5).
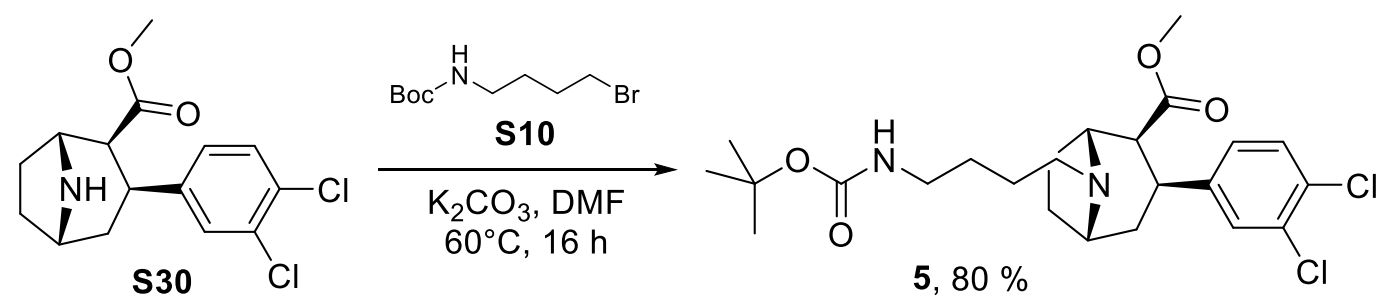

This procedure was adopted from Cha et al. ${ }^{31}$ A solution of $\mathbf{S 3 0}$ (1.17 g, $3.72 \mathrm{mmol}, 1.0$ eq.), 4-(Boc-amino)butyl bromide (S10, $1.13 \mathrm{~g}, 4.47$ mmol, 1.2 eq.) and $\mathrm{K}_{2} \mathrm{CO}_{3}$ (1.03 g, 7.45 mmol, 2.0 eq.) in DMF (20 mL) was stirred for $16 \mathrm{~h}$ at $60^{\circ} \mathrm{C}$. The reaction mixture was then cooled down to r.t. and water $(50 \mathrm{~mL})$ was added and subsequently extracted with ethyl acetate $(3 \times 50 \mathrm{~mL})$. The combined organic extracts were dried over anhydrous $\mathrm{Na}_{2} \mathrm{SO}_{4}$, filtered and solvents were removed under reduced pressure. The obtained yellow oil was dissolved in $\mathrm{Et}_{2} \mathrm{O}(50 \mathrm{~mL})$ and washed with sat. aq. $\mathrm{LiCl}(3 \times 20 \mathrm{~mL}), \mathrm{H}_{2} \mathrm{O}(3 \times 20 \mathrm{~mL})$ and brine $(20 \mathrm{~mL})$. Volatiles were removed under reduced pressure and the obtained viscous oil was purified by silica gel column chromatography $\left(\mathrm{Et}_{3} \mathrm{~N} / \mathrm{Et}_{2} \mathrm{O} 1: 399, v / v\right)$ to give pure product. Yield: $1.42 \mathrm{~g}(80 \%)$. Yellowish crystalline solid.

$\boldsymbol{R}_{\mathbf{f}}\left(\right.$ silica; $\mathrm{Et}_{3} \mathrm{~N} / \mathrm{Et}_{2} \mathrm{O}$ 2.5:97.5, v/v): 0.7.

${ }^{1} \mathbf{H}$ NMR (400 MHz, $\left.\mathrm{CDCl}_{3}\right): \delta$ (ppm) 1.36-1.42 (m, 2H), 1.44 (s, 9H), 1.47-1.54 (m, 2H), 1.57$1.73(\mathrm{~m}, 2 \mathrm{H}), 1.94-2.15(\mathrm{~m}, 2 \mathrm{H}), 2.21-2.30(\mathrm{~m}, 2 \mathrm{H}), 2.53\left(\mathrm{dt}, 1 \mathrm{H}, J_{1}=12.5 \mathrm{~Hz}, J_{2}=2.1 \mathrm{~Hz}\right)$, 2.85-2.90 (m, 1H), $2.95\left(\mathrm{td}, 1 \mathrm{H}, J_{1}=12.6 \mathrm{~Hz}, J_{2}=4.8 \mathrm{~Hz}\right), 3.05-3.13(\mathrm{~m}, 2 \mathrm{H}), 3.35-3.40(\mathrm{~m}, 1 \mathrm{H})$, 3.50 (s, 3H), 3.65-3.70 (m, 1H), 4.85 (br s, 1H, -NH-CO-), 7.09 (dd, 1H, $J_{1}=8.4 \mathrm{~Hz}, J_{2}=1.6$ $\mathrm{Hz})$.

${ }^{13} \mathbf{C}\left\{{ }^{1} \mathbf{H}\right\}$ NMR $\left(100 \mathrm{MHz}, \mathrm{CDCl}_{3}\right): \delta(\mathrm{ppm})$ 26.08, 26.16, 26.33, 27.4, 28.7, 34.0, 34.2, 40.5, 51.3, $52.85,52.94,61.5,62.8,79.0,127.0,129.68,129.78,129.91,132.0,144.0,156.2$, 172.0 .

FTIR (ATR, diamond, $\mathrm{cm}^{-1}$ ) = 3362, 1743, 1694, 1512, 1472, 1391, 1364, 1349, 1249, 1169, $1135,1029,867,783,673,604,438$.

HRMS (APCI+): calculated $m / z$ for $\left[\mathrm{C}_{24} \mathrm{H}_{34} \mathrm{Cl}_{2} \mathrm{~N}_{2} \mathrm{O}_{4}+\mathrm{H}\right]^{+}=485.1974$, found $=485.1977$. 
m.p. $\left(\mathrm{Et}_{3} \mathrm{~N} / \mathrm{Et}_{2} \mathrm{O}\right): 86-88^{\circ} \mathrm{C}$.

$(1 R, 2 S, 3 S, 5 S)$-Methyl

8-(4-((((1R,8S,9s)-bicyclo[6.1.0]non-4-yn-9-

ylmethoxy)carbonyl)amino)butyl)-3-(3,4-dichlorophenyl)-8-azabicyclo[3.2.1]octane-2carboxylate (dichloropane-BCN, 11)
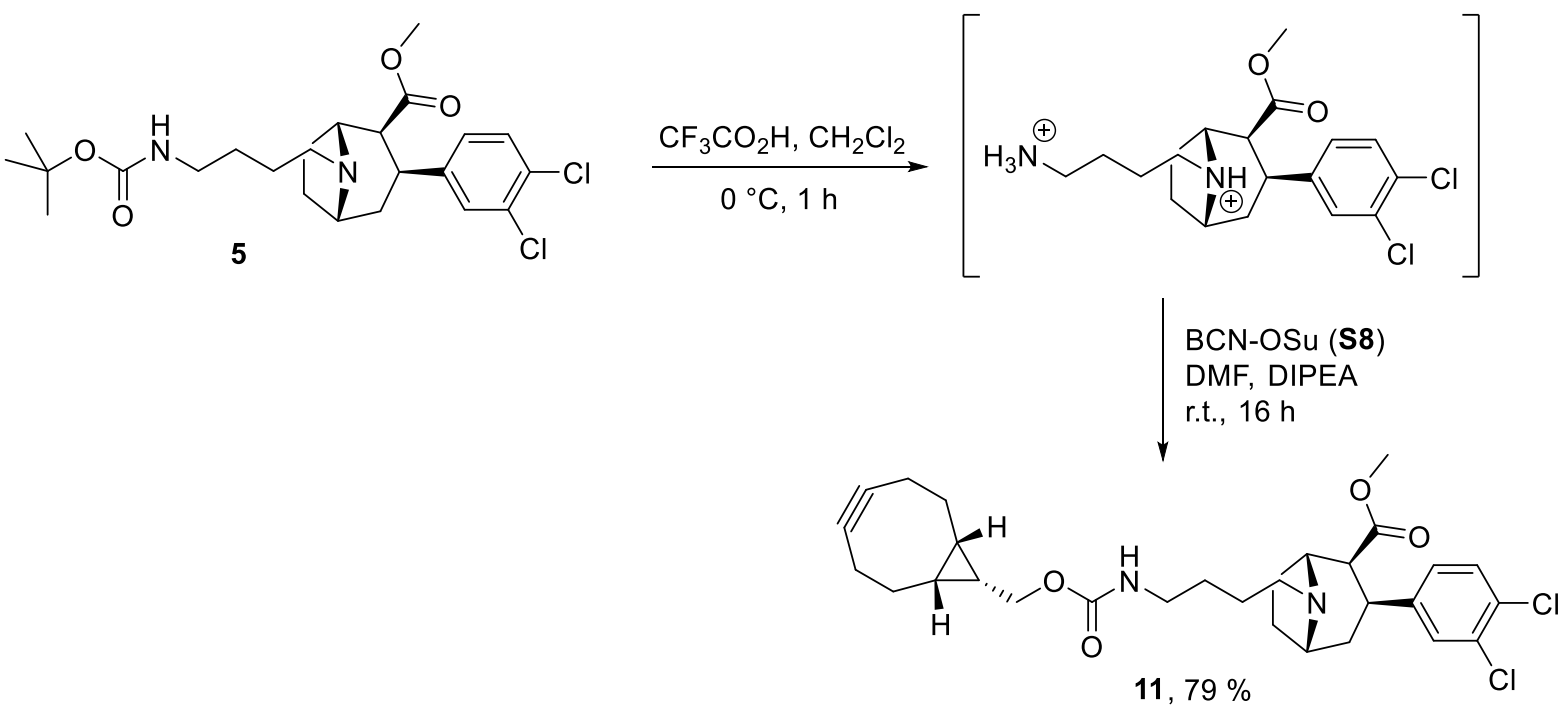

A solution of dichloropane-NHBoc $\left(5,13.6 \mathrm{mg}, 28.0 \mu \mathrm{mol}, 1.0\right.$ eq.) in $\mathrm{CH}_{2} \mathrm{Cl}_{2}(0.5 \mathrm{~mL})$ was cooled to $0{ }^{\circ} \mathrm{C}$ followed by dropwise addition of $\mathrm{CF}_{3} \mathrm{CO}_{2} \mathrm{H}(1 \mathrm{~mL})$. The resulting solution was stirred at $0{ }^{\circ} \mathrm{C}$ for $60 \mathrm{~min}$. Subsequently, volatiles were removed by rotary evaporation $\left(40{ }^{\circ} \mathrm{C}\right)$ and the solid material was co-evaporated with toluene $(2 \times 1.5 \mathrm{~mL})$ to remove residual acid, providing the intermediate ammonium salt. The colorless amorphous solid was dissolved in dry DMF $(1.0 \mathrm{~mL})$ and DIPEA ( $20 \mu \mathrm{L}, 0.11 \mathrm{mmol}, 4.0$ eq.) under dry Ar atmosphere, followed by addition of a solution of BCN-OSu (S8, $10 \mathrm{mg}, 34 \mu \mathrm{mol}, 1.2 \mathrm{eq}$.) in dry DMF $(0.4 \mathrm{~mL})$. The reaction mixture was stirred at r.t. under dry Ar for $16 \mathrm{~h}$. Subsequently, the solution was diluted with $\mathrm{Et}_{2} \mathrm{O}$ (20 mL), washed with $\mathrm{H}_{2} \mathrm{O}(2 \times 10 \mathrm{~mL})$, sat. aq. $\mathrm{LiCl}(10 \mathrm{~mL})$ and brine $(10 \mathrm{~mL})$ and dried over anhydrous $\mathrm{Na}_{2} \mathrm{SO}_{4}$. After filtration and removal of volatiles by rotary evaporation, the crude product was purified by silica gel column chromatography using $\mathrm{CH}_{2} \mathrm{Cl}_{2} / \mathrm{MeOH}(15: 1, v / v)$ as the mobile phase. Pure dichloropane-BCN (11) was obtained as a thick colorless oil. Yield: $12.5 \mathrm{mg}$ $(79 \%)$.

$\boldsymbol{R} \mathbf{f}\left(\right.$ silica; $\mathrm{CH}_{2} \mathrm{Cl}_{2} / \mathrm{MeOH}$ 9:1, v/v): 0.18 . 
${ }^{1} \mathbf{H}$ NMR (400 MHz, $\left.\mathrm{CDCl}_{3}\right) \delta 7.35-7.28(\mathrm{~m}, 2 \mathrm{H}), 7.08(\mathrm{dd}, J=8.4,2.1 \mathrm{~Hz}, 1 \mathrm{H}), 5.10(\mathrm{br} \mathrm{s}, 1 \mathrm{H})$, $4.13(\mathrm{~d}, J=8.0 \mathrm{~Hz}, 2 \mathrm{H}), 3.73-3.62(\mathrm{~m}, 1 \mathrm{H}), 3.49(\mathrm{~s}, 3 \mathrm{H}), 3.42-3.33(\mathrm{~m}, 1 \mathrm{H}), 3.22-3.09(\mathrm{~m}$, 2H), $3.00-2.91(\mathrm{~m}, 1 \mathrm{H}), 2.91-2.85(\mathrm{~m}, 1 \mathrm{H}), 2.54(\mathrm{t}, J=12.4 \mathrm{~Hz}, 1 \mathrm{H}), 2.35-2.15(\mathrm{~m}, 8 \mathrm{H}), 2.15$ $-1.93(\mathrm{~m}, 1 \mathrm{H}), 1.75-1.48(\mathrm{~m}, 7 \mathrm{H}), 1.46-1.30(\mathrm{~m}, 3 \mathrm{H}), 1.00-0.89(\mathrm{~m}, 2 \mathrm{H})$.

${ }^{13} \mathbf{C}\left\{{ }^{1} \mathbf{H}\right\}$ NMR $\left(101 \mathrm{MHz}, \mathrm{CDCl}_{3}\right) \delta 171.9,156.9,143.9,132.0,129.9,129.8,129.6,126.9,99.0$, 99.0, 77.4, 62.8, 62.6, 61.5, 52.8 (2 inequivalent C), 51.3, 40.9, 34.1, 33.9, 29.2, 27.3, 26.0, 21.6, 20.2, 18.0.

FTIR (ATR, diamond, $\mathrm{cm}^{-1}$ ): 1707 (C=O) (Fig. S36 in the Infrared Spectra section).

HRMS (ESI+): calculated $m / z$ for $\left[\mathrm{C}_{30} \mathrm{H}_{38} \mathrm{Cl}_{2} \mathrm{~N}_{2} \mathrm{O}_{4}+\mathrm{H}\right]^{+}=561.2287$, found $=561.2289$.

\section{5-(N-(6-((4-((1R,2S,3S,5S)-3-(3,4-dichlorophenyl)-2-(methoxycarbonyl)-8-}

azabicyclo[3.2.1]octan-8-yl)butyl)amino)-6-oxohexyl)sulfamoyl)-2-(6-(diethylamino)-3(diethyliminio)-3H-xanthen-9-yl)benzenesulfonate (6)

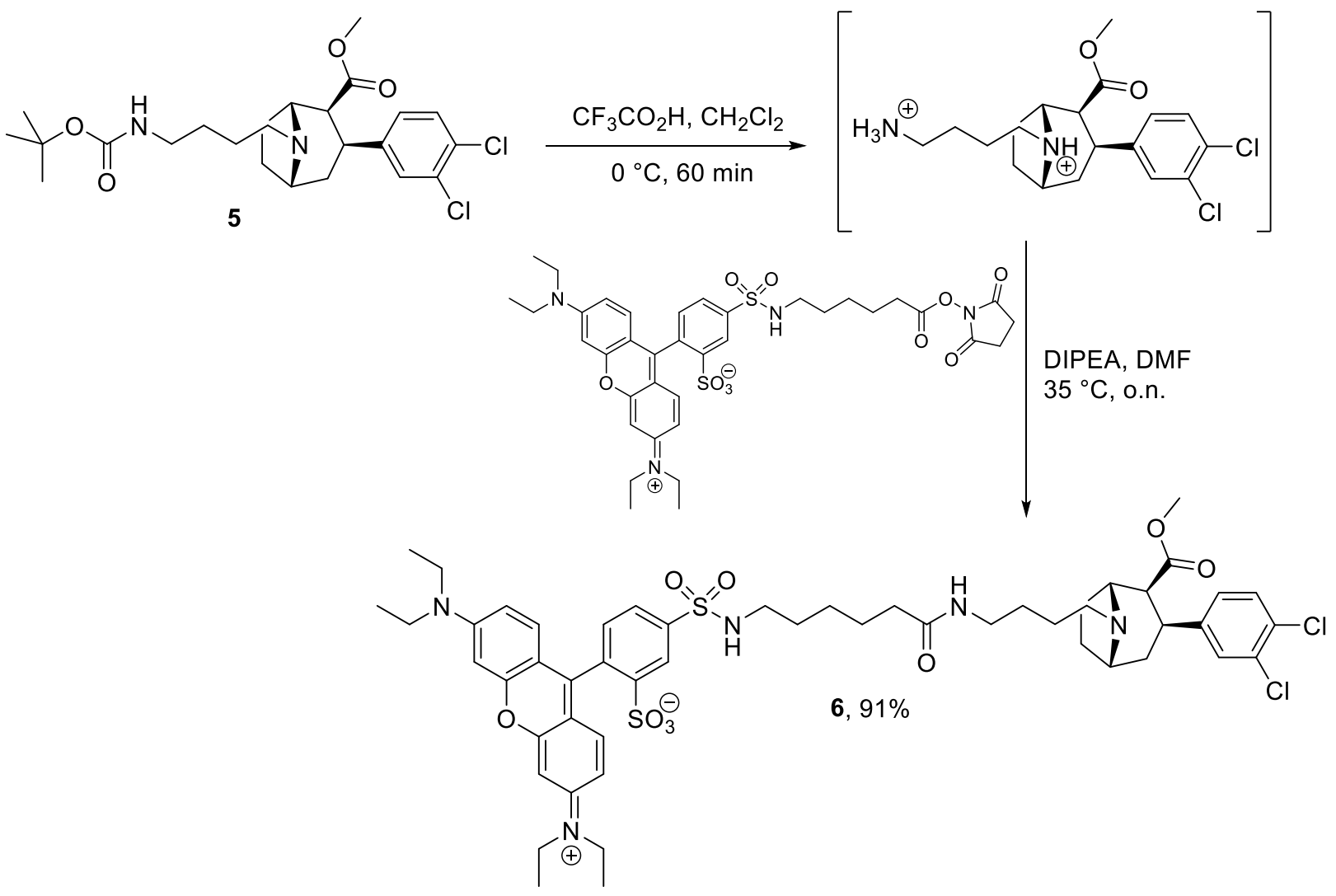


A solution of dichloropane-NHBoc $\left(5,5.0 \mathrm{mg}, 10.0 \mu \mathrm{mol}, 1.3 \mathrm{eq}\right.$.) in $\mathrm{CH}_{2} \mathrm{Cl}_{2}(0.5 \mathrm{~mL})$ was cooled to $0{ }^{\circ} \mathrm{C}$ followed by dropwise addition of $\mathrm{CF}_{3} \mathrm{CO}_{2} \mathrm{H}(1 \mathrm{~mL})$. The resulting solution was stirred at $0{ }^{\circ} \mathrm{C}$ for $60 \mathrm{~min}$. Subsequently, volatiles were removed by rotary evaporation (r.t.) and the solid material was co-evaporated with toluene $(2 \times 1.5 \mathrm{~mL})$ to remove residual acid, providing the intermediate ammonium salt. The colorless amorphous solid was dissolved in dry DMF (700 $\mu \mathrm{L})$ and DIPEA $(57 \mu \mathrm{L}, 0.33 \mathrm{mmol}, 42 \mathrm{eq}$.$) , followed by addition of rhodamine red-X$ $N$-hydroxysuccinimidyl ester $(6.0 \mathrm{mg}, 7.8 \mu \mathrm{mol}, 1.0$ eq. $)$. The reaction mixture was stirred at $35^{\circ} \mathrm{C}$ overnight (19 h). After removal of volatiles by rotary evaporation, the crude product was purified by column chromatography on basic alumina using $\mathrm{CH}_{2} \mathrm{Cl}_{2} / \mathrm{MeOH}(30: 1, v / v)$ as the mobile phase. Pure 6 was obtained as a dark purple solid. Yield: $7.4 \mathrm{mg}(91 \%)$.

$\boldsymbol{R}$ f (alumina; $\left.\mathrm{CH}_{2} \mathrm{Cl}_{2} / \mathrm{MeOH} 19: 1, v / v\right): 0.35$.

${ }^{1}$ H NMR (500 MHz, CD $\left.{ }_{3} \mathrm{OD}\right) \delta 8.65(\mathrm{~d}, J=1.9 \mathrm{~Hz}, 1 \mathrm{H}), 8.09$ (dd, $\left.J=8.0,1.9 \mathrm{~Hz}, 1 \mathrm{H}\right), 7.48$ (d, $J=7.9 \mathrm{~Hz}, 1 \mathrm{H}), 7.40(\mathrm{~d}, J=2.2 \mathrm{~Hz}, 1 \mathrm{H}), 7.34(\mathrm{~d}, J=8.4 \mathrm{~Hz}, 1 \mathrm{H}), 7.16(\mathrm{dd}, J=8.5,2.1 \mathrm{~Hz}, 1 \mathrm{H})$, $7.10(\mathrm{dd}, J=9.5,2.0 \mathrm{~Hz}, 2 \mathrm{H}), 6.99(\mathrm{dt}, J=9.6,2.0 \mathrm{~Hz}, 2 \mathrm{H}), 6.93(\mathrm{~d}, J=2.5 \mathrm{~Hz}, 2 \mathrm{H}), 3.75-3.65$ (m, 1H), $3.67(\mathrm{q}, J=7.2 \mathrm{~Hz}, 8 \mathrm{H}), 3.46(\mathrm{~s}, 3 \mathrm{H}), 3.42-3.36(\mathrm{~m}, 1 \mathrm{H}), 3.19-2.97(\mathrm{~m}, 6 \mathrm{H}), 2.44(\mathrm{t}$, $J=12.3 \mathrm{~Hz}, 1 \mathrm{H}), 2.38-2.23(\mathrm{~m}, 2 \mathrm{H}), 2.18(\mathrm{t}, J=7.5 \mathrm{~Hz}, 2 \mathrm{H}), 2.15-2.08(\mathrm{~m}, 1 \mathrm{H}), 2.08-1.98$ $(\mathrm{m}, 1 \mathrm{H}), 1.87-1.76(\mathrm{~m}, 1 \mathrm{H}), 1.71-1.64(\mathrm{~m}, 2 \mathrm{H}), 1.60(\mathrm{p}, J=7.6 \mathrm{~Hz}, 2 \mathrm{H}), 1.56-1.46(\mathrm{~m}, 4 \mathrm{H})$, $1.41-1.34(\mathrm{~m}, 4 \mathrm{H}), 1.30(\mathrm{t}, J=7.2 \mathrm{~Hz}, 12 \mathrm{H})$.

${ }^{13} \mathbf{C}\left\{{ }^{1} \mathbf{H}\right\}$ NMR $\left(126 \mathrm{MHz}, \mathrm{CD}_{3} \mathrm{OD}\right) \delta 175.9,173.8,159.4,157.9,157.2,147.3,145.8,144.3,135.3$, 133.6, 132.6, 132.4, 130.8, 130.6, 130.3, 129.2, 128.4, 127.7, 115.3, 115.1, 97.0, 64.0, 62.8, 53.9, 51.7, 46.8, 44.0, 40.1, 37.0, 34.9, 34.7, 30.7, 30.4, 27.9, 27.3, 26.9, 26.7, 26.6, 26.4, 12.9 .

HRMS (ESI+): calculated $m / z$ for $\left[\mathrm{C}_{52} \mathrm{H}_{65} \mathrm{~N}_{5} \mathrm{O}_{9} \mathrm{~S}_{2} \mathrm{Cl}_{2}+\mathrm{H}\right]^{+}=1038.3679$, found $=1038.3683$. 
dichloropane-PEG(3400)-di-2-ANEP (S1).

管

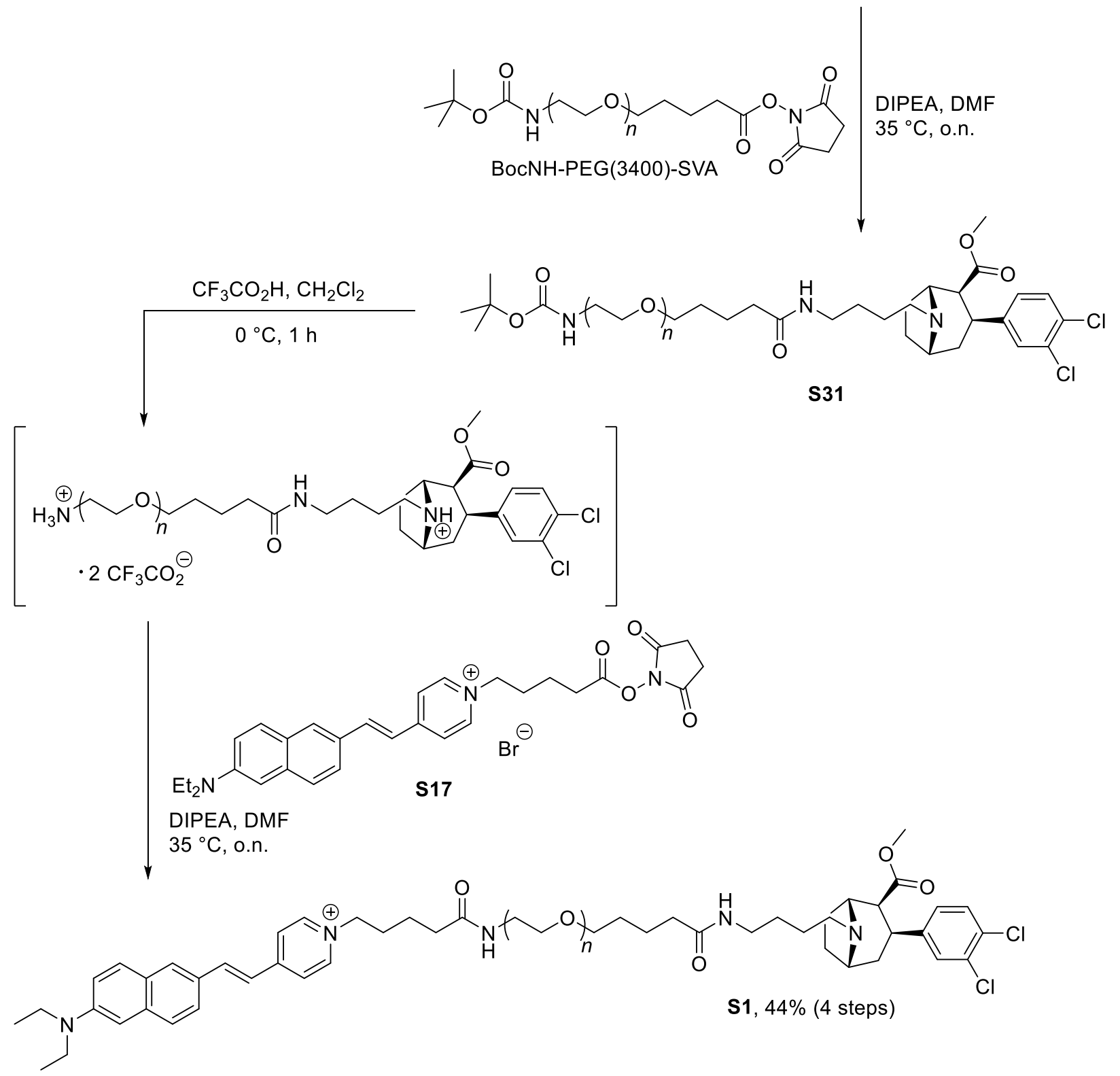

Trifluoroacetic acid (1.0 mL) was slowly dropwise added (over $\sim 5 \mathrm{~min}$ ) to a stirred solution of 5 (3.2 mg, $6.50 \mu \mathrm{mol}, 1.25$ eq.) in dry dichloromethane $(0.5 \mathrm{~mL})$ at $0{ }^{\circ} \mathrm{C}$ under dry Ar atmosphere and the reaction mixture was further stirred for $1 \mathrm{~h}$ at $0{ }^{\circ} \mathrm{C}$. Volatiles were removed under reduced 
pressure and the obtained residuum was co-evaporated with toluene $(2 \times 2 \mathrm{~mL})$. The obtained slightly orange residuum was dried under high vacuum overnight. The dried ammonium trifluoroacetate salt intermediate was dissolved in dry DMF (0.5 mL), DIPEA (2 drops) was added and the reaction mixture was stirred for 5 min at r.t. under dry Ar atmosphere. BocNH-PEG(3400)SVA (17.7 mg, $5.2 \mu \mathrm{mol}, 1.0$ eq.) was added in one portion and the reaction mixture was further stirred at $35^{\circ} \mathrm{C}$ overnight under dry $\mathrm{Ar}$ atmosphere. Volatiles were removed under reduced pressure and the obtained residuum was dissolved in water $(2.5 \mathrm{~mL})$. This solution was transferred into a dialysis cassette (Slide-A-Lyzer, $2000 \mathrm{MWCO}$, ThermoFisher) and purified by dialysis in water $(400 \mathrm{~mL})$. The dialysate was exchanged every $2 \mathrm{~h}$ during the first five cycles and every $12 \mathrm{~h}$ for the next three days. The solution from the dialysis cassette was then transferred into a $25 \mathrm{~mL}$ flask, volatiles were removed under reduced pressure and the obtained residuum was further dried under high vacuum overnight to yield S31 as a white solid. Yield: $20 \mathrm{mg}$. Purity of the obtained material was satisfactory $\left({ }^{1} \mathrm{H} \mathrm{NMR}, \mathrm{CDCl}_{3}\right)$ to be used in the next step without further purification.

Note: We did not notice any decomposition of $\mathbf{S 3 1}$ upon long-term storage (3 months) at $-20{ }^{\circ} \mathrm{C}$.

Trifluoroacetic acid (1.0 mL) was slowly dropwise added (over $\sim 5 \mathrm{~min}$ ) to a stirred solution of S31 (3.2 mg, $6.50 \mu \mathrm{mol}, 1.25$ eq.) in dry $\mathrm{CH}_{2} \mathrm{Cl}_{2}(0.5 \mathrm{~mL})$ at $0{ }^{\circ} \mathrm{C}$ under dry Ar atmosphere and the reaction mixture was stirred for $1 \mathrm{~h}$ at $0{ }^{\circ} \mathrm{C}$. Volatiles were removed under reduced pressure and the obtained residuum was co-evaporated with toluene $(2 \times 1 \mathrm{~mL})$. The obtained residuum was further dried under high vacuum overnight. The dried material was dissolved in dry DMF $(0.5 \mathrm{~mL})$ and DIPEA ( 2 drops) was added and the reaction mixture was stirred for $5 \mathrm{~min}$ at $20^{\circ} \mathrm{C}$ under dry Ar atmosphere. di-2-ANEPB- $\mathrm{CO}_{2} \mathrm{Su}(\mathbf{S 1 7}, 5.0 \mathrm{mg}, 6.50 \mu \mathrm{mol}, 1.25$ eq.) was added in one portion and the reaction mixture was further stirred at $35^{\circ} \mathrm{C}$ overnight under dry $\mathrm{Ar}$ atmosphere. Volatiles were removed under reduced pressure and the obtained residuum was dissolved in water $(2.5 \mathrm{~mL})$ and transferred into a dialysis cassette (Slide-A-Lyzer, $2000 \mathrm{MWCO}$, ThermoFisher) and purified by dialysis in water $(400 \mathrm{~mL})$. The dialysate was exchanged every $2 \mathrm{~h}$ during the first five cycles and every $12 \mathrm{~h}$ for the next three days (the dialysate solution was turning red for the first 1.5 days, with gradually decreasing color density). The solution from the dialysis cassette was then transferred into a $25 \mathrm{~mL}$-flask, volatiles were removed under reduced pressure and the obtained residuum was further dried under high vacuum overnight to yield $15 \mathrm{mg}$ of $\mathbf{S 1}$. 
Purity of the obtained material was satisfactory $\left({ }^{1} \mathrm{H} \mathrm{NMR}, \mathrm{CDCl}_{3}\right)$ to be used without further purification.

${ }^{1} \mathbf{H}$ NMR (400 MHz, $\left.\mathrm{CDCl}_{3}\right) \delta$ (only diagnostic signals in the aromatic region are shown and aliphatic region is omitted for clarity) $6.84(\mathrm{~d}, 1 \mathrm{H}, J=1.7 \mathrm{~Hz}), 7.09\left(\mathrm{dt}, 2 \mathrm{H}, J_{1}=8.5 \mathrm{~Hz}, J_{2}=1.4\right.$ $\mathrm{Hz}), 7.32(\mathrm{~d}, 1 \mathrm{H}, J=1.1 \mathrm{~Hz}), 7.37$ (d, 1H, $J=8.3 \mathrm{~Hz}), 7.58-7.68(\mathrm{~m}, 2 \mathrm{H}), 7.70-7.81(\mathrm{~m}, 2 \mathrm{H})$, $7.84(\mathrm{~s}, 1 \mathrm{H}), 7.90$ (br, 1H), 8.93 (br s, 1H).

\section{$(1 R, 8 S, 9 s)$-Bicyclo[6.1.0]non-4-yn-9-ylmethyl 4-nitrophenyl carbonate (14, BCN-OpNP)}

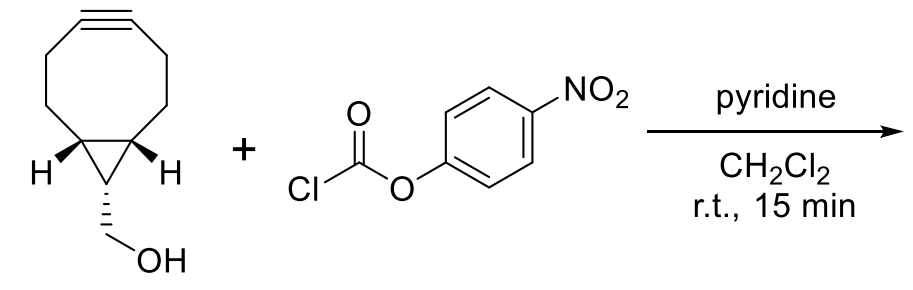

$\mathrm{BCN}-\mathrm{OH}(\mathbf{S} 7)$

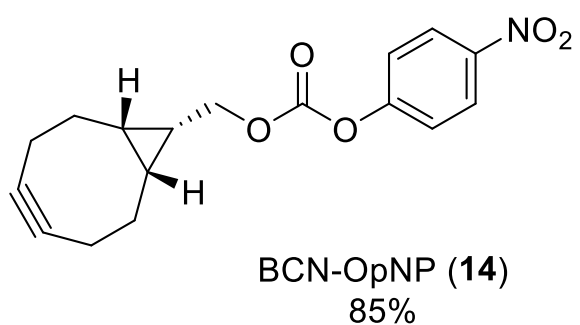

$85 \%$

This procedure was adopted from Dommerholt et al. ${ }^{10} \mathrm{BCN}-\mathrm{OH}(\mathbf{S 7}, 56 \mathrm{mg}, 0.37 \mathrm{mmol}, 1.0$ eq.) was dissolved in $\mathrm{CH}_{2} \mathrm{Cl}_{2}(8 \mathrm{~mL})$, followed by addition of dry pyridine (75 $\mu \mathrm{L}, 0.93 \mathrm{mmol}, 2.5$ eq.) and 4-nitrophenyl chloroformate (96 mg, $0.48 \mathrm{mmol}, 1.3 \mathrm{eq}$.). The resulting solution was stirred at r.t. for $15 \mathrm{~min}$, after which it was quenched with sat. aq. $\mathrm{NH}_{4} \mathrm{Cl}(10 \mathrm{~mL})$. The organic layer was separated, and the aqueous phase was extracted with further $\mathrm{CH}_{2} \mathrm{Cl}_{2}(3 \times 5 \mathrm{~mL})$. The combined org. extracts were dried over anhydrous $\mathrm{Na}_{2} \mathrm{SO}_{4}$, filtered, and volatiles were removed by rotary evaporation $\left(30^{\circ} \mathrm{C}\right)$. The crude product was purified by silica gel column chromatography using pentane/AcOEt $(5: 1, v / v)$ as the mobile phase. Pure BCN-OpNP (14) was obtained as a white crystalline solid. Yield: $100 \mathrm{mg}(85 \%)$.

$\boldsymbol{R}_{\mathbf{f}}($ silica; hexanes/AcOEt 2:1, v/v): 0.53.

${ }^{1} \mathbf{H}$ NMR $\left(500 \mathrm{MHz}, \mathrm{CDCl}_{3}\right) \delta 8.28(\mathrm{~d}, J=9.2 \mathrm{~Hz}, 2 \mathrm{H}), 7.39(\mathrm{~d}, J=9.2 \mathrm{~Hz}, 2 \mathrm{H}), 4.41(\mathrm{~d}, J=8.2$ $\mathrm{Hz}, 2 \mathrm{H}), 2.44-2.10(\mathrm{~m}, 6 \mathrm{H}), 1.67-1.56(\mathrm{~m}, 2 \mathrm{H}), 1.52$ (p, J=8.6 Hz, 1H), $1.12-1.01$ (m, 2H).

MS (APCI+): calculated $m / z$ for $\left[\mathrm{C}_{10} \mathrm{H}_{15} \mathrm{O}\right]^{+}=151.1$, found $=151.0\left(\mathrm{BCN}-\mathrm{OH}_{2}{ }^{+}\right)$.

MS (APCI-): calculated $m / z$ for $\left[\mathrm{C}_{6} \mathrm{H}_{4} \mathrm{NO}_{3}\right]^{-}=138.0$, found $=137.9\left(\mathrm{O}_{2} \mathrm{~N}_{-} \mathrm{C}_{6} \mathrm{H}_{4}-\mathrm{O}^{-}\right)$. 
4-(Azidomethyl)benzoic acid (20)<smiles>O=C(O)c1ccc(CBr)cc1</smiles>

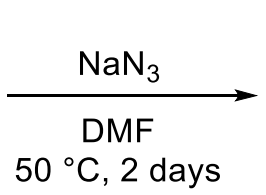<smiles>N#Cc1ccc(C(=O)O)cc1</smiles>

4-(Bromomethyl)benzoic acid (997 mg, $4.64 \mathrm{mmol}, 1.0$ eq.) and $\mathrm{NaN}_{3}$ (945 mg, $14.5 \mathrm{mmol}$, 3.1 eq.) were dissolved in dry DMF $(10 \mathrm{~mL})$, and the reaction mixture was heated under $\mathrm{Ar}$ atmosphere at $50{ }^{\circ} \mathrm{C}$ for 2 days. The resulting suspension was diluted with $1 \mathrm{M} \mathrm{aq} . \mathrm{HCl}(100 \mathrm{~mL})$ and extracted with $\mathrm{Et}_{2} \mathrm{O}(3 \times 50 \mathrm{~mL})$. The combined organic extracts were washed with brine $(50 \mathrm{~mL})$, dried over anhydrous $\mathrm{MgSO}_{4}$ and filtered. Removing volatiles by rotary evaporation $\left(30^{\circ} \mathrm{C}\right)$ provided 4 -(azidomethyl)benzoic acid $(\mathbf{2 0})$ as a white crystalline solid. Yield: $780 \mathrm{mg}$ $(95 \%)$.

${ }^{1}$ H NMR (500 MHz, DMSO-d6) $\delta 13.01$ (br s, 1H), $7.96(\mathrm{~d}, J=8.3 \mathrm{~Hz}, 2 \mathrm{H}), 7.49$ (d, $J=8.3 \mathrm{~Hz}$, $2 \mathrm{H}), 4.56(\mathrm{~s}, 2 \mathrm{H})$.

${ }^{13} \mathbf{C}\left\{{ }^{1} \mathbf{H}\right\}$ NMR $\left(126 \mathrm{MHz}, \mathrm{DMSO}-d_{6}\right) \delta 167.0,140.6,130.4,129.7,128.3,53.1$.

\section{4-(Azidomethyl)-N-(2-(2-hydroxyethoxy)ethyl)benzamide (S32)}
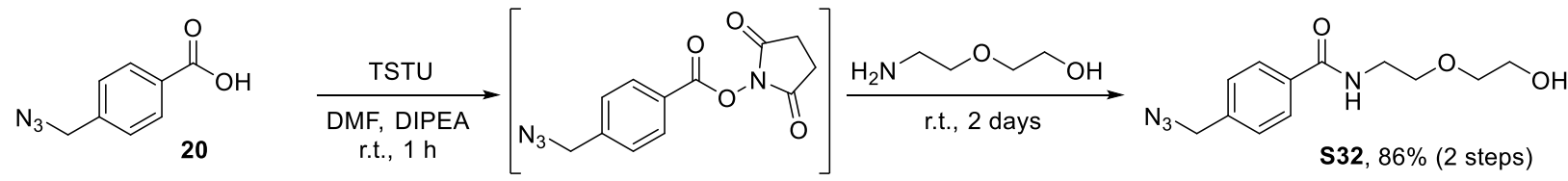

4-(Azidomethyl)benzoic acid (20, $150 \mathrm{mg}, 847 \mu \mathrm{mol}, 1.0$ eq.) and $N, N, N^{\prime}, N^{\prime}$-tetramethyl- $O-(N-$ succinimidyl)uronium tetrafluoroborate (TSTU, $283 \mathrm{mg}, 940 \mu \mathrm{mol}, 1.1 \mathrm{eq}$.) were dissolved in dry DMF (3.0 mL) and dry DIPEA (177 $\mu \mathrm{L}, 1.02 \mathrm{mmol}, 1.2$ eq.) under dry Ar atmosphere. The resulting solution was stirred at r.t. for 60 min resulting in formation of the corresponding NHS ester. 2-(2-Aminoethoxy)ethanol (126 $\mu \mathrm{L}, 1.27 \mathrm{mmol}, 1.5 \mathrm{eq}$.) was added to the reaction mixture and the resulting solution was stirred for further 2 days. After diluting with $0.5 \mathrm{M} \mathrm{aq} . \mathrm{HCl}(50 \mathrm{~mL})$, the product was extracted with $\mathrm{CH}_{2} \mathrm{Cl}_{2}(3 \times 30 \mathrm{~mL})$. The combined organic extracts were washed 
with brine, dried over anhydrous $\mathrm{MgSO}_{4}$ and filtered. After removing the volatiles by rotary evaporation $\left(30^{\circ} \mathrm{C}\right)$, the crude product was purified by silica gel column chromatography (short column: $40 \mathrm{~mm} ø, 2.5 \mathrm{~cm}$ silica layer) using AcOEt as the mobile phase. 4-(Azidomethyl)- $\mathrm{N}$-(2(2-hydroxyethoxy)ethyl)benzamide (S32) was obtained as a thick colorless oil. Yield: $193 \mathrm{mg}$ (86\%). Note: The purified product contained $10 \mathrm{~mol} . \% \mathrm{~N}$-hydroxysuccinimide as an impurity and was used in the next step without further purification.

$\boldsymbol{R}_{\mathbf{f}}$ (silica; AcOEt): 0.12 .

${ }^{1} \mathbf{H}$ NMR $\left(500 \mathrm{MHz}, \mathrm{CDCl}_{3}\right) \delta 7.81(\mathrm{~d}, J=8.3 \mathrm{~Hz}, 2 \mathrm{H}), 7.39(\mathrm{~d}, J=8.3 \mathrm{~Hz}, 2 \mathrm{H}), 6.65(\mathrm{br} \mathrm{s}, 1 \mathrm{H})$, $4.40(\mathrm{~s}, 2 \mathrm{H}), 3.81-3.75(\mathrm{~m}, 2 \mathrm{H}), 3.72-3.66(\mathrm{~m}, 4 \mathrm{H}), 3.66-3.60(\mathrm{~m}, 2 \mathrm{H}), 1.83(\mathrm{br} \mathrm{s}, \mathrm{ROH}+$ $\mathrm{H}_{2} \mathrm{O}$ signal with fast exchange).

${ }^{13} \mathbf{C}\left\{{ }^{1} \mathbf{H}\right\}$ NMR $\left(126 \mathrm{MHz}, \mathrm{CDCl}_{3}\right) \delta 167.2,139.0,134.5,128.4,127.7,72.4,70.0,62.0,54.4,40.0$.

MS $\left(\mathrm{APCI}^{+}\right)$: calculated $m / z$ for $\left[\mathrm{C}_{12} \mathrm{H}_{16} \mathrm{~N}_{4} \mathrm{O}_{3}+\mathrm{H}\right]^{+}=265.1$, found $=264.8$.

\section{2-(2-(4-(Azidomethyl)benzamido)ethoxy)ethyl (2,5-dioxopyrrolidin-1-yl) carbonate (S33)}

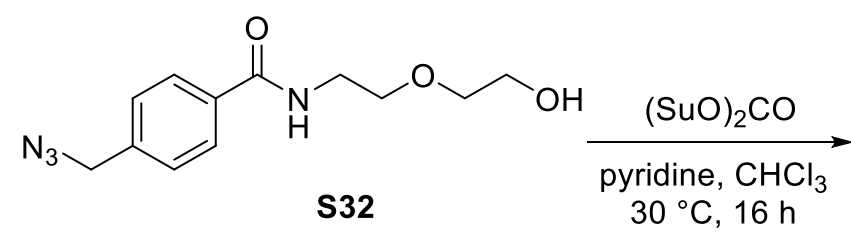

S32

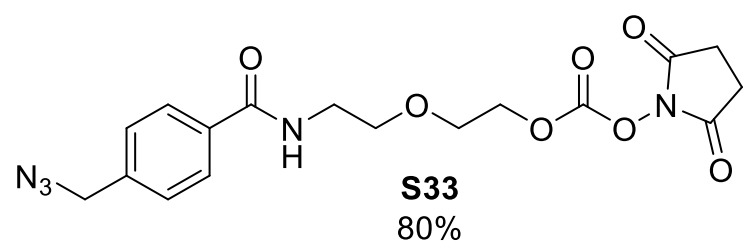

Alcohol S32 (190 mg, $549 \mu \mathrm{mol}, 1.0$ eq.) and $(\mathrm{SuO})_{2} \mathrm{CO}(169 \mathrm{mg}, 660 \mu \mathrm{mol}, 1.2$ eq. $)$ were suspended in dry $\mathrm{CHCl}_{3}(2.8 \mathrm{~mL})$ and dry pyridine ( $88 \mu \mathrm{L}, 1.1 \mathrm{mmol}, 2.0$ eq.) under dry $\mathrm{Ar}$ atmosphere. The resulting mixture was stirred at $30^{\circ} \mathrm{C}$ for $16 \mathrm{~h}$ during which all the solid fully dissolved. After diluting with $\mathrm{CH}_{2} \mathrm{Cl}_{2}(50 \mathrm{~mL})$, the solution was washed with $0.5 \mathrm{M}$ aq. $\mathrm{HCl}$ $(3 \times 30 \mathrm{~mL})$ and brine $(30 \mathrm{~mL})$, dried over anhydrous $\mathrm{MgSO}_{4}$ and filtered. After removing the volatiles by rotary evaporation $\left(30^{\circ} \mathrm{C}\right)$, the crude product was purified by silica gel column chromatography (short column: $40 \mathrm{~mm} ø, 3.5 \mathrm{~cm}$ silica layer) using AcOEt as the mobile phase. Succinimidyl carbonate $\mathbf{S 3 3}$ was obtained as a thick colorless oil. Yield: $178 \mathrm{mg}(80 \%)$.

$\boldsymbol{R}_{\mathbf{f}}$ (silica; AcOEt): 0.41 . 
${ }^{1} \mathbf{H}$ NMR $\left(500 \mathrm{MHz}, \mathrm{CDCl}_{3}\right) \delta 7.84(\mathrm{~d}, J=7.9 \mathrm{~Hz}, 2 \mathrm{H}), 7.37(\mathrm{~d}, J=7.9 \mathrm{~Hz}, 2 \mathrm{H}), 6.87$ (br s, 1H), $4.56-4.46(\mathrm{~m}, 2 \mathrm{H}), 4.38(\mathrm{~s}, 2 \mathrm{H}), 3.81-3.73(\mathrm{~m}, 2 \mathrm{H}), 3.68(\mathrm{~s}, 4 \mathrm{H}), 2.81(\mathrm{~s}, 4 \mathrm{H})$.

${ }^{13} \mathbf{C}\left\{{ }^{1} \mathbf{H}\right\}$ NMR $\left(126 \mathrm{MHz}, \mathrm{CDCl}_{3}\right) \delta 168.8,167.2,151.9,138.7,134.7,128.3,127.9,70.3,69.9$, $68.4,54.4,39.9,25.6$.

2-(2-(4-(Azidomethyl)benzamido)ethoxy)ethyl

4-(2-acetamidoethyl)-1H-imidazole-1carboxylate (16)

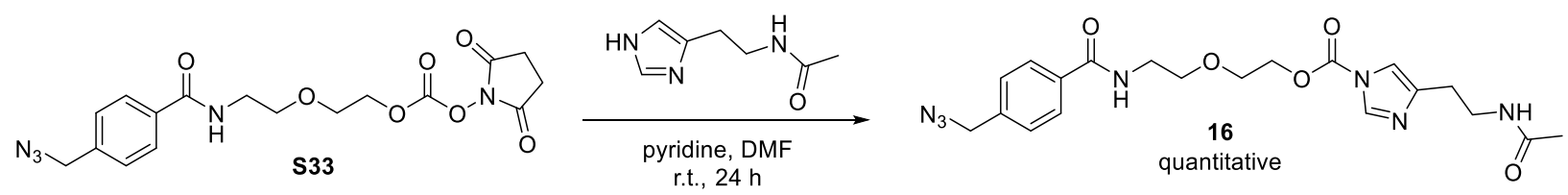

Succinimidyl carbonate S33 (22.4 mg, $55.3 \mu \mathrm{mol}, 1.0$ eq.) and $N \omega$-acetylhistamine (12.9 mg, $84.2 \mu \mathrm{mol}, 1.5$ eq.) were dissolved in dry DMF $(250 \mu \mathrm{L})$ and dry pyridine $(44.5 \mu \mathrm{L}, 552 \mu \mathrm{mol}$, 10 eq.) under dry Ar atmosphere. The resulting solution was stirred at r.t. for $24 \mathrm{~h}$. After removing volatiles under high vacuum, the crude oil was purified by $\mathrm{RP} \mathrm{C}_{18}$-silica column chromatography using aq. $\mathrm{MeCN}$ (17 to $25 \mathrm{vol} \% \mathrm{MeCN}$ ) as the mobile phase. Imidazolyl carbamate 16 was obtained as a thick colorless oil. Yield: $25 \mathrm{mg}$ (quantitative).

$\boldsymbol{R}_{\mathbf{f}}\left(\mathrm{C}_{18}\right.$-silica, $\left.\mathrm{H}_{2} \mathrm{O} / \mathrm{MeCN} 1: 1, v / v\right): 0.56$.

${ }^{1}$ H NMR (500 MHz, DMSO-d $\left.d_{6}\right) \delta 8.55$ (br t, $\left.J=5.6 \mathrm{~Hz}, 1 \mathrm{H}\right), 8.15$ (d, $\left.J=1.3 \mathrm{~Hz}, 1 \mathrm{H}\right), 7.89$ (br t, $J=5.7 \mathrm{~Hz}, 1 \mathrm{H}), 7.83(\mathrm{~d}, J=8.3 \mathrm{~Hz}, 2 \mathrm{H}), 7.43(\mathrm{~d}, J=8.3 \mathrm{~Hz}, 2 \mathrm{H}), 7.32(\mathrm{~d}, J=1.3 \mathrm{~Hz}, 1 \mathrm{H}), 4.52$ $(\mathrm{s}, 2 \mathrm{H}), 4.50-4.44(\mathrm{~m}, 2 \mathrm{H}), 3.80-3.74(\mathrm{~m}, 2 \mathrm{H}), 3.61(\mathrm{t}, J=5.9 \mathrm{~Hz}, 2 \mathrm{H}), 3.43(\mathrm{q}, J=5.8 \mathrm{~Hz}$, $2 \mathrm{H}), 3.30-3.24(\mathrm{~m}, 2 \mathrm{H}), 2.62-2.55(\mathrm{~m}, 2 \mathrm{H}), 1.78(\mathrm{~s}, 3 \mathrm{H})$.

${ }^{13} \mathbf{C}\left\{{ }^{1} \mathbf{H}\right\}$ NMR $\left(126 \mathrm{MHz}, \mathrm{DMSO}-d_{6}\right) \delta 169.1,165.9,148.3,141.3,138.7,136.8,134.1,128.1$, 127.6, 113.4, 68.8, 67.6, 67.0, 53.1, 39.1, 37.9, 27.9, 22.6. 


\section{Ethyl (2-nitro-4-(trifluoromethyl)phenyl)glycinate (S34)}<smiles>CCOC(=O)CNc1ccc(C(F)(F)F)cc1[N+](=O)[O-]</smiles>

A solution of 1-chloro-2-nitro-4-trifluoromethylbenzene (18.41 g, $81.62 \mathrm{mmol}, 1.0$ eq.) and ethyl glycinate hydrochloride (13.72 g, 98.29 mmol, 1.2 eq.) in DIPEA (34 mL, 195 mmol, 2.4 eq.) and MeCN (120 mL) was heated at reflux for $12 \mathrm{~h}$ with TLC monitoring (silica, hexane/AcOEt 4:1 $v / v$; starting material spot at $R_{f}=0.42$ ). After cooling to room temperature, volatiles were removed by rotary evaporation. The resulting solid was recrystallized from 95\% aq. EtOH (20 mL) providing pure compound S34 as a yellow crystalline solid. Yield: $16.4 \mathrm{~g}$ (69\%).

${ }^{1}$ H NMR (500 MHz, DMSO- $\left.d_{6}\right) \delta 8.70(\mathrm{t}, J=5.9 \mathrm{~Hz}, 1 \mathrm{H}), 8.34(\mathrm{~d}, J=2.3 \mathrm{~Hz}, 1 \mathrm{H}), 7.81(\mathrm{dd}, J=$ 9.1, $2.3 \mathrm{~Hz}, 1 \mathrm{H}), 7.13(\mathrm{~d}, J=9.1 \mathrm{~Hz}, 1 \mathrm{H}), 4.35(\mathrm{~d}, J=5.9 \mathrm{~Hz}, 2 \mathrm{H}), 4.18(\mathrm{q}, J=7.1 \mathrm{~Hz}, 2 \mathrm{H}), 1.23$ (t, $J=7.1 \mathrm{~Hz}, 3 \mathrm{H})$.

${ }^{13} \mathbf{C}\left\{{ }^{1} \mathbf{H}\right\}$ NMR $\left(126 \mathrm{MHz}, \mathrm{DMSO}-d_{6}\right) \delta 169.4,146.4,131.9$ (q, $\left.J=2.4 \mathrm{~Hz}\right), 130.6,123.9$ (q, $J=$ $4.0 \mathrm{~Hz}), 123.7(\mathrm{q}, J=270.7 \mathrm{~Hz}), 116.5,115.7(\mathrm{q}, J=33.9 \mathrm{~Hz}), 61.1,44.2,14.0$.

Ethyl 2-((2-ethoxy-2-oxoethyl)(2-nitro-4-(trifluoromethyl)phenyl)amino)-2-oxoacetate (S35)<smiles>CCOC(=O)CN(c1ccc(C(F)(F)F)cc1[N+](=O)[O-])c1ccc(C(F)(F)F)cc1[N+](=O)[O-]</smiles>

S34 (16.3 g, $55.8 \mathrm{mmol}, 1.0$ eq.) was dissolved (5 min stirring) in dry pyridine (65 mL). Ethyl oxalyl chloride (9.4 mL, $84 \mathrm{mmol}, 1.5$ eq.) was added dropwise over $10 \mathrm{~min}$. The reaction mixture was stirred at r.t. with TLC monitoring (silica, $\mathrm{CH}_{2} \mathrm{Cl}_{2} / \mathrm{MeOH} 50: 1, v / v$ ). Due to slow progression 
of the reaction, the temperature was elevated to $50{ }^{\circ} \mathrm{C}$ and more ethyl oxalyl chloride was added (9.4 mL, $84 \mathrm{mmol}, 1.5$ eq. after $24 \mathrm{~h}$ of heating; and another $18.8 \mathrm{~mL}, 168 \mathrm{mmol}, 3.0$ eq. after another $30 \mathrm{~h}$ of heating). After a total of 3 days of heating at $50{ }^{\circ} \mathrm{C}$, TLC (silica; $\mathrm{CH}_{2} \mathrm{Cl}_{2} / \mathrm{MeOH}$ $50: 1 \mathrm{v} / \mathrm{v})$ showed completion of the reaction. The reaction mixture was poured on ice $(400 \mathrm{~g})$, followed by slow addition of $4 \mathrm{M}$ aq. $\mathrm{HCl}(100 \mathrm{~mL})$. After the ice has melted, the emulsion was extracted with AcOEt $(2 \times 200 \mathrm{~mL}+100 \mathrm{~mL})$. The combined org. extracts were washed with $1 \mathrm{M}$ aq. $\mathrm{HCl}(100 \mathrm{~mL})$ and brine $(100 \mathrm{~mL})$, dried over anhydrous $\mathrm{MgSO}_{4}$ and filtered. After removing the volatiles by rotary evaporation $\left(30^{\circ} \mathrm{C}\right)$, the crude product was purified by silica gel column chromatography using $\mathrm{CH}_{2} \mathrm{Cl}_{2}$ in hexanes (30 to 100 vol. $\% \mathrm{CH}_{2} \mathrm{Cl}_{2}$ ) as the mobile phase. Compound S35 was obtained as a yellowish oil. Yield: 20.0 g (91\%). Variable temperature (VT) ${ }^{1} \mathrm{H}$ NMR $\left(500 \mathrm{MHz}, \mathrm{DMSO}-d_{6}, 27^{\circ} \mathrm{C}\right.$ and $120^{\circ} \mathrm{C}$ ) revealed the existence of rotamers (Fig. S114 and S115 in the NMR Spectra section).

$\boldsymbol{R} \mathbf{f}\left(\right.$ silica, $\left.\mathrm{CH}_{2} \mathrm{Cl}_{2}\right): 0.42$.

${ }^{1} \mathbf{H}$ NMR ${ }^{1} \mathrm{H}$ NMR $\left(500 \mathrm{MHz}, \mathrm{DMSO}-d_{6}, \mathbf{2 7}^{\circ} \mathbf{C}\right) \delta$ major $8.57(\mathrm{~d}, J=2.2 \mathrm{~Hz}, 1 \mathrm{H}), 8.33-8.29$ $(\mathrm{m}, 1 \mathrm{H}), 7.94(\mathrm{dd}, J=8.3,0.9 \mathrm{~Hz}, 1 \mathrm{H}), 4.61(\mathrm{~d}, J=17.5 \mathrm{~Hz}, 1 \mathrm{H}), 4.54(\mathrm{~d}, J=17.5 \mathrm{~Hz}, 1 \mathrm{H}), 4.17$ $-4.11(\mathrm{~m}, 2 \mathrm{H}), 4.01(\mathrm{q}, J=7.1 \mathrm{~Hz}, 2 \mathrm{H}), 1.20(\mathrm{t}, J=7.1 \mathrm{~Hz}, 3 \mathrm{H}), 0.94(\mathrm{t}, J=7.1 \mathrm{~Hz}, 3 \mathrm{H}) ; \delta$ minor $8.50(\mathrm{~d}, J=2.2 \mathrm{~Hz}, 1 \mathrm{H}), 8.30-8.26(\mathrm{~m}, 1 \mathrm{H}), 7.90(\mathrm{~d}, J=8.3 \mathrm{~Hz}, 1 \mathrm{H}), 4.79(\mathrm{br} \mathrm{s}, 2 \mathrm{H}), 4.32(\mathrm{q}, J$ $=7.1 \mathrm{~Hz}, 2 \mathrm{H}), 4.22-4.15(\mathrm{~m}, 2 \mathrm{H}), 1.28(\mathrm{t}, J=7.1 \mathrm{~Hz}, 3 \mathrm{H}), 1.22(\mathrm{t}, J=7.1 \mathrm{~Hz}, 3 \mathrm{H})$.

${ }^{1} \mathrm{H}$ NMR ${ }^{1} \mathrm{H}$ NMR $\left(500 \mathrm{MHz}, \mathrm{DMSO}-d_{6}, \mathbf{1 2 0}^{\circ} \mathrm{C}\right) \delta 8.46(\mathrm{~d}, J=2.1 \mathrm{~Hz}, 1 \mathrm{H}), 8.22(\mathrm{dd}, J=8.3$, $2.1 \mathrm{~Hz}, 1 \mathrm{H}), 7.94(\mathrm{~d}, J=8.3 \mathrm{~Hz}, 1 \mathrm{H}), 4.57$ (br s, 2H), 4.20 (q, $J=7.1 \mathrm{~Hz}, 2 \mathrm{H}), 4.06$ (br s, 2H), $1.25(\mathrm{t}, J=7.1 \mathrm{~Hz}, 3 \mathrm{H}), 1.02$ (br s, 3H).

${ }^{13} \mathbf{C}\left\{{ }^{1} \mathbf{H}\right\}$ NMR (126 MHz, DMSO-d $\left.d_{6}, 27{ }^{\circ} \mathbf{C}\right) \delta 168.6$ (minor), 167.8 (major), 160.4 (minor), 160.2 (minor), 159.8 (major), 159.2 (major), 146.2 (major), 146.0 (minor), 137.3 (major), 137.0 (minor), 133.0 (major), 131.7 (major, q, $J=3.6 \mathrm{~Hz}$ ), 131.0 (minor), 130.3 (major, q, $J=33.7 \mathrm{~Hz}$ ), 123.2 (major, q, $J=3.7 \mathrm{~Hz}$ ), 122.7 (minor, q, $J=3.3 \mathrm{~Hz}$ ), 122.6 (major, q, $J=273.2 \mathrm{~Hz}$ ), 62.8 (minor), 62.4 (major), 61.6 (minor), 61.3 (major), 52.0 (minor), 50.7 (major), 13.9 (major + minor), 13.6 (minor), 13.2 (major).

MS $\left(\mathrm{APCI}^{+}\right)$: calculated $m / z$ for $\left[\mathrm{C}_{15} \mathrm{H}_{15} \mathrm{~F}_{3} \mathrm{~N}_{2} \mathrm{O}_{7}+\mathrm{H}\right]^{+}=393.1$, found $=392.8$. 
Ethyl 2-(2,3-dioxo-6-(trifluoromethyl)-3,4-dihydroquinoxalin-1(2H)-yl)acetate (S36)<smiles>CCOC(=O)CN(C(=O)C(=O)OCC)c1ccc(C(F)(F)F)cc1[N+](=O)[O-]</smiles>

A stirred solution of $\mathbf{S 3 5}$ (19.9 g, $50.7 \mathrm{mmol}, 1.0$ eq.) in glacial acetic acid (150 mL) was heated to $85^{\circ} \mathrm{C}$. Iron powder $(2.83 \mathrm{~g}, 50.7 \mathrm{mmol}, 1.0 \mathrm{eq}$. $)$ was added in small portions during which the color of the solution turned from orange to dark brown. The reaction mixture was heated at $85^{\circ} \mathrm{C}$ with TLC monitoring (silica, $\left.\mathrm{CH}_{2} \mathrm{Cl}_{2} / \mathrm{MeOH} 50: 1, v / v\right)$. After $2 \mathrm{~h}$, more iron powder $(2.83 \mathrm{~g}$, $50.7 \mathrm{mmol}$, 1.0 eq.) was added in portions. The reaction was completed (TLC; silica; $\mathrm{CH}_{2} \mathrm{Cl}_{2} / \mathrm{MeOH} 50: 1 \mathrm{v} / v$ ) after another $2 \mathrm{~h}$ of heating at $85{ }^{\circ} \mathrm{C}$. The mixture was poured into an ice/water mixture $(500 \mathrm{~g})$ resulting in a tan precipitate. After most of the ice has melted, the precipitate was collected by filtration, washed with ice-cold water $(2 \times 200 \mathrm{~mL})$ and dried in vacuo. Compound S36 was obtained as an off-white solid. Yield: $13.1 \mathrm{~g}(82 \%)$.

${ }^{1}$ H NMR $\left(400 \mathrm{MHz}\right.$, DMSO- $\left.d_{6}\right) \delta 12.37$ (br s, 1H), $7.69-7.34(\mathrm{~m}, 3 \mathrm{H}), 5.02(\mathrm{~s}, 2 \mathrm{H}), 4.18(\mathrm{q}, J=$ $7.1 \mathrm{~Hz}, 2 \mathrm{H}), 1.22(\mathrm{t}, J=7.1 \mathrm{~Hz}, 3 \mathrm{H})$.

${ }^{13} \mathbf{C}\left\{{ }^{1} \mathbf{H}\right\}$ NMR (101 MHz, DMSO- $\left.d_{6}\right) \delta 167.3,155.2,153.0,129.5,126.0,124.1(\mathrm{q}, J=32.7 \mathrm{~Hz})$, $123.9(\mathrm{q}, J=271.5 \mathrm{~Hz}), 119.9(\mathrm{q}, J=3.6 \mathrm{~Hz}), 115.9,112.5(\mathrm{q}, J=3.9 \mathrm{~Hz}), 61.4,44.4$, 14.0. 
Ethyl 2-(7-nitro-2,3-dioxo-6-(trifluoromethyl)-3,4-dihydroquinoxalin-1(2H)-yl)acetate (S37)<smiles>CCOC(=O)Cn1c(=O)c(=O)[nH]c2cc(C(F)(F)F)ccc21</smiles>

S36<smiles>CCOC(=O)Cn1c(=O)c(=O)[nH]c2cc(C(F)(F)F)c([N+](=O)[O-])cc21</smiles>

S37

$59 \%$

S36 (12.9 g, 40.8 mmol, 1.0 eq.) was stirred with $96 \%$ aq. $\mathrm{H}_{2} \mathrm{SO}_{4}$ until dissolution of most of the solid material (clumps formed which did not dissolve well; partially broken down using a spatula). After cooling to $0{ }^{\circ} \mathrm{C}, \mathrm{KNO}_{3}(4.12 \mathrm{~g}$, $40.8 \mathrm{mmol}, 1.0$ eq.) was added in small portions. The reaction mixture was stirred at $0{ }^{\circ} \mathrm{C}$ for $30 \mathrm{~min}$, after which it was poured into a water/ice mixture (400 g) resulting in a white precipitate to emerge. The suspension was extracted with AcOEt $(250 \mathrm{~mL}+$ $2 \times 100 \mathrm{~mL})$. The combined org. extracts were washed with $\mathrm{H}_{2} \mathrm{O}(200 \mathrm{~mL})$ and brine $(150 \mathrm{~mL})$, dried over anhydrous $\mathrm{MgSO}_{4}$ and filtered. After removing the volatiles by rotary evaporation $\left(30{ }^{\circ} \mathrm{C}\right)$, the crude solid was recrystallized from 95\% aq. EtOH $(175 \mathrm{~mL})$. Compound $\mathbf{S 3 7}$ was obtained as a white crystalline solid. Yield: $8.73 \mathrm{~g}(59 \%)$. Note: The purified product contained 14 mol. \% S36 as an impurity ( ${ }^{1} \mathrm{H}$ NMR) and was used in the next step without further purification.

$\boldsymbol{R} \mathbf{f}\left(\right.$ silica, $\left.\mathrm{CH}_{2} \mathrm{Cl}_{2} / \mathrm{MeOH} 9: 1, v / v\right): 0.49$.

${ }^{1}$ H NMR (400 MHz, DMSO-d $d_{6} \delta 12.68$ (br s, 1H), 8.23 (s, 1H), 7.67 (s, 1H), 5.04 (s, 2H), 4.19 (q, $J=7.1 \mathrm{~Hz}, 2 \mathrm{H}), 1.23(\mathrm{t}, J=7.1 \mathrm{~Hz}, 3 \mathrm{H})$.

${ }^{13} \mathbf{C}\left\{{ }^{1} \mathbf{H}\right\}$ NMR $\left(126 \mathrm{MHz}, \mathrm{DMSO}-d_{6}\right) \delta 167.0,154.9,152.9,142.0,129.8,129.2,121.9(\mathrm{q}, J=$ $271.6 \mathrm{~Hz}), 116.7$ (q, $J=34.3 \mathrm{~Hz}), 114.7$ (q, $J=5.6 \mathrm{~Hz}), 113.1,61.5,61.5,44.9,14.0$. 
Ethyl 2-(7-amino-2,3-dioxo-6-(trifluoromethyl)-3,4-dihydroquinoxalin-1(2H)-yl)acetate (S38)<smiles>CCOC(=O)Cn1c(=O)c(=O)[nH]c2cc(C(F)(F)F)c(N)cc21</smiles>

S37 (0.99 g, $2.7 \mathrm{mmol}, 1.0$ eq.) and 10\% Pd/C (45 mg) in DMF (7 mL) was stirred under $\mathrm{H}_{2}$ atmosphere (balloon) for 2 days with TLC monitoring (silica, $\mathrm{CH}_{2} \mathrm{Cl}_{2} / \mathrm{MeOH} 50: 1, v / v$ ). The resulting solution was filtered through celite and the volatiles were removed by rotary evaporation $\left(30{ }^{\circ} \mathrm{C}\right)$ followed by co-evaporation with $\mathrm{MeCN}(10 \mathrm{~mL})$. The resulting yellow solid was suspended in $95 \%$ aq. EtOH $(20 \mathrm{~mL})$ and heated at reflux for $10 \mathrm{~min}$, after which it was collected by vacuum filtration while still hot. After washing with $95 \%$ aq. EtOH $(2 \times 10 \mathrm{~mL})$ and drying in vасио, S38 was obtained as a yellow solid. Yield: $508 \mathrm{mg}$ (56\%). Note: The product contained impurities and was used in the next step without further purification.

${ }^{1}$ H NMR $\left(500 \mathrm{MHz}, \mathrm{DMSO}-d_{6}\right) \delta 11.96(\mathrm{~s}, 1 \mathrm{H}), 7.21(\mathrm{~s}, 1 \mathrm{H}), 6.65(\mathrm{~s}, 1 \mathrm{H}), 5.50(\mathrm{~s}, 2 \mathrm{H}), 4.82(\mathrm{~s}$, $2 \mathrm{H}), 4.19$ (q, $J=7.2 \mathrm{~Hz}, 2 \mathrm{H}), 1.23(\mathrm{t}, J=7.2 \mathrm{~Hz}, 3 \mathrm{H})$.

MS $\left(\mathrm{APCI}^{+}\right)$: calculated $m / z$ for $\left[\mathrm{C}_{13} \mathrm{H}_{12} \mathrm{~F}_{3} \mathrm{~N}_{3} \mathrm{O}_{4}+\mathrm{H}\right]^{+}=332.1$, found $=331.7$. 
Ethyl 2-(7-(3-formyl-1H-pyrrol-1-yl)-2,3-dioxo-6-(trifluoromethyl)-3,4-dihydroquinoxalin$1(2 H)$-yl)acetate (S39)<smiles>CCOC(=O)Cn1c(=O)c(=O)[nH]c2cc(C(F)(F)F)c(N)cc21</smiles>

S38

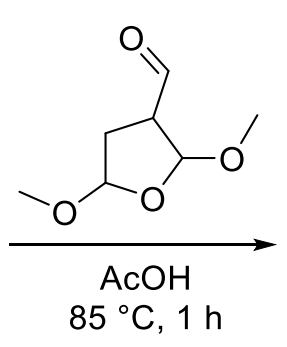

$85^{\circ} \mathrm{C}, 1 \mathrm{~h}$<smiles>CCOC(=O)Cn1c(=O)c(=O)[nH]c2cc(C(F)(F)F)c(-n3ccc(C=O)c3)cc21</smiles>

S39

$31 \%$

A solution of $\mathbf{S 3 8}$ ( $0.50 \mathrm{~g}, 1.5 \mathrm{mmol}, 1.0 \mathrm{eq}$.) and 2,5-dimethoxytetrahydrofuran-3-carbaldehyde (Acros, $90 \%$ pure technical; $0.28 \mathrm{~g}, 1.6 \mathrm{mmol}, 1.04$ eq.) in $\mathrm{AcOH}(6 \mathrm{~mL})$ was heated at $85^{\circ} \mathrm{C}$ for $60 \mathrm{~min}$. Volatiles were subsequently removed by rotary evaporation $\left(40^{\circ} \mathrm{C}\right)$ and the crude product was purified by silica gel column chromatography using acetone in $\mathrm{CH}_{2} \mathrm{Cl}_{2}$ (10 to 35 vol. \% acetone) as the mobile phase. S39 was obtained as a yellow solid. Yield: $190 \mathrm{mg}$ (31\%). Note: Further purification could be achieved by $\mathrm{RP} \mathrm{C}_{18}$-silica column chromatography using aq. $\mathrm{MeCN}$ (24 to 30 vol. \% $\mathrm{MeCN}+0.1 \% \mathrm{CF}_{3} \mathrm{CO}_{2} \mathrm{H}$ ) as the mobile phase.

$\boldsymbol{R}_{\mathbf{f}}$ (silica, $\mathrm{CH}_{2} \mathrm{Cl}_{2} /$ acetone $\left.3: 1, v / v\right): 0.21$.

${ }^{1}$ H NMR $\left(500 \mathrm{MHz}\right.$, DMSO- $\left.d_{6}\right) \delta 12.55(\mathrm{~s}, 1 \mathrm{H}), 9.78(\mathrm{~s}, 1 \mathrm{H}), 7.85-7.82(\mathrm{~m}, 1 \mathrm{H}), 7.80(\mathrm{~s}, 1 \mathrm{H})$, $7.67-7.63(\mathrm{~m}, 1 \mathrm{H}), 7.09-7.05(\mathrm{~m}, 1 \mathrm{H}), 6.67-6.63(\mathrm{~m}, 1 \mathrm{H}), 5.00(\mathrm{~s}, 2 \mathrm{H}), 4.15(\mathrm{q}, J=7.1 \mathrm{~Hz}$, $2 \mathrm{H}), 1.19(\mathrm{t}, J=7.1 \mathrm{~Hz}, 3 \mathrm{H})$. 


\section{(1-(4-(2-Ethoxy-2-oxoethyl)-2,3-dioxo-7-(trifluoromethyl)-1,2,3,4-tetrahydroquinoxalin-6-}

yl)-1 $H$-pyrrol-3-yl)methanaminium trifluoroacetate (S40)
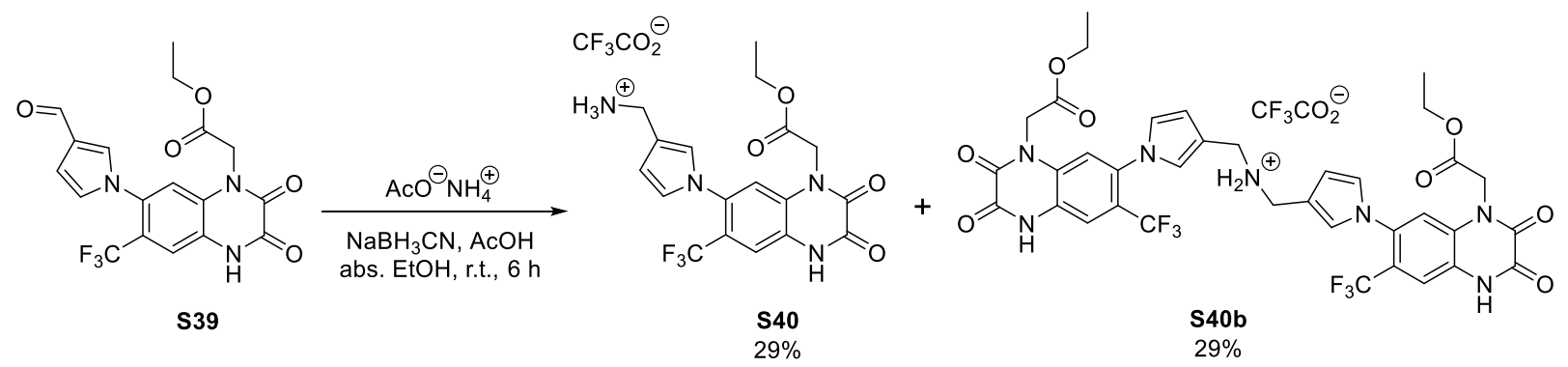

S39 (51 mg, $0.12 \mathrm{mmol}, 1.0$ eq.) and ammonium acetate ( $255 \mathrm{mg}, 3.31 \mathrm{mmol}, 27 \mathrm{eq}$.$) were stirred$ with abs. EtOH $(2.5 \mathrm{~mL})$ and glacial $\mathrm{AcOH}(0.25 \mathrm{~mL})$ at r.t. under dry Ar atmosphere until complete dissolution (30 min). $\mathrm{NaBH}_{3} \mathrm{CN}$ (33 mg, $0.53 \mathrm{mmol}, 4.2 \mathrm{eq}$.) was added, and the resulting solution was stirred at r.t. for further $6 \mathrm{~h}$. The volatiles were removed by rotary evaporation $\left(30^{\circ} \mathrm{C}\right)$, and the resulting brown oil was purified by $\mathrm{RP} \mathrm{C}_{18}$-silica column chromatography using aq. $\mathrm{MeCN}$ (18 to 34 vol. $\% \mathrm{MeCN}$, containing $0.1 \% \mathrm{CF}_{3} \mathrm{CO}_{2} \mathrm{H}$ ) as the mobile phase. Compound $\mathbf{S 4 0}$ was obtained as colorless amorphous solid. Yield: $18.7 \mathrm{mg}$ (29\%). Note: The dimerized secondary amine $\mathbf{S 4 0 b}$ was obtained as an unwanted side product (yellowish amorphous solid) in $29 \%$ yield.

S40:

$\boldsymbol{R}_{\mathbf{f}}\left(\mathrm{C}_{18}\right.$-silica, $\left.\mathrm{H}_{2} \mathrm{O} / \mathrm{MeCN} 1: 1, v / v+0.1 \% \mathrm{CF}_{3} \mathrm{CO}_{2} \mathrm{H}\right): 0.69$.

${ }^{1}$ H NMR (400 MHz, DMSO-d $)_{6} \delta 12.54(\mathrm{~s}, 1 \mathrm{H}), 8.10(\mathrm{br} \mathrm{s}, 3 \mathrm{H}), 7.66(\mathrm{~s}, 1 \mathrm{H}), 7.48(\mathrm{~s}, 1 \mathrm{H}), 7.02$ (s, 1H), 6.93 (s, 1H), 6.37 (s, 1H), $5.01(\mathrm{~s}, 2 \mathrm{H}), 4.15(\mathrm{q}, J=7.1 \mathrm{~Hz}, 2 \mathrm{H}), 3.93(\mathrm{q}, J=5.7 \mathrm{~Hz}, 2 \mathrm{H})$, $1.19(\mathrm{t}, J=7.1 \mathrm{~Hz}, 3 \mathrm{H})$.

${ }^{13} \mathbf{C}\left\{{ }^{1} \mathbf{H}\right\}$ NMR $\left(101 \mathrm{MHz}\right.$, DMSO- $\left.d_{6}\right) \delta 167.3,155.2,152.8,133.3(\mathrm{q}, J=1.5 \mathrm{~Hz}), 129.9,125.4$, $124.5,123.2,122.8(\mathrm{q}, J=272.8 \mathrm{~Hz}), 119.9(\mathrm{q}, J=31.1 \mathrm{~Hz}), 116.9,116.3,114.1(\mathrm{q}, J=5.2 \mathrm{~Hz})$, 109.7, 61.4, 44.7, 35.5, 14.0 .

${ }^{19} \mathbf{F}\left\{{ }^{1} \mathrm{H}\right\}$ NMR $\left(376 \mathrm{MHz}, \mathrm{DMSO}-d_{6}\right) \delta-57.5\left(\mathrm{Ar}^{-} \mathrm{CF}_{3}\right),-73.9\left(\mathrm{CF}_{3} \mathrm{CO}_{2}{ }^{-}\right)$.

HRMS (ESI+): calculated $m / z$ for $\left[\mathrm{C}_{18} \mathrm{H}_{18} \mathrm{~N}_{4} \mathrm{O}_{4} \mathrm{~F}_{3}-\mathrm{H}+\mathrm{Na}\right]^{+}=433.1100$, found $=433.1098$; calculated $m / z$ for $\left[\mathrm{C}_{18} \mathrm{H}_{18} \mathrm{~N}_{4} \mathrm{O}_{4} \mathrm{~F}_{3}-\mathrm{NH}_{3}\right]^{+}=394.1015$, found $=394.1007$ 
S40b:

$\boldsymbol{R}_{\mathbf{f}}\left(\mathrm{C}_{18}\right.$-silica, $\left.\mathrm{H}_{2} \mathrm{O} / \mathrm{MeCN} 1: 1, v / v+0.1 \% \mathrm{CF}_{3} \mathrm{CO}_{2} \mathrm{H}\right): 0.40$.

${ }^{1} \mathrm{H}$ NMR $\left(500 \mathrm{MHz}, \mathrm{DMSO}-d_{6}\right) \delta 12.54(\mathrm{~s}, 2 \mathrm{H}), 9.03(\mathrm{br} \mathrm{s}, 2 \mathrm{H}), 7.64(\mathrm{~s}, 2 \mathrm{H}), 7.56(\mathrm{~s}, 2 \mathrm{H}), 7.05$ (s, 2H), 6.98 (s, 2H), $6.38(\mathrm{~s}, 2 \mathrm{H}), 5.01(\mathrm{~s}, 4 \mathrm{H}), 4.13(\mathrm{q}, J=7.1 \mathrm{~Hz}, 4 \mathrm{H}), 3.99$ (t, $J=5.3 \mathrm{~Hz}, 4 \mathrm{H})$, $1.18(\mathrm{t}, J=7.1 \mathrm{~Hz}, 6 \mathrm{H})$.

MS (ESI+): calculated $m / z$ for $\left[\mathrm{C}_{36} \mathrm{H}_{32} \mathrm{~N}_{7} \mathrm{O}_{8} \mathrm{~F}_{6}\right]^{+}=804.2$, found $=804.1$.

\section{4-(((1-(4-(2-Ethoxy-2-oxoethyl)-2,3-dioxo-7-(trifluoromethyl)-1,2,3,4-tetrahydroquinoxalin-} 6-yl)-1H-pyrrol-3-yl)methyl)amino)-4-oxobutanoic acid (S41)

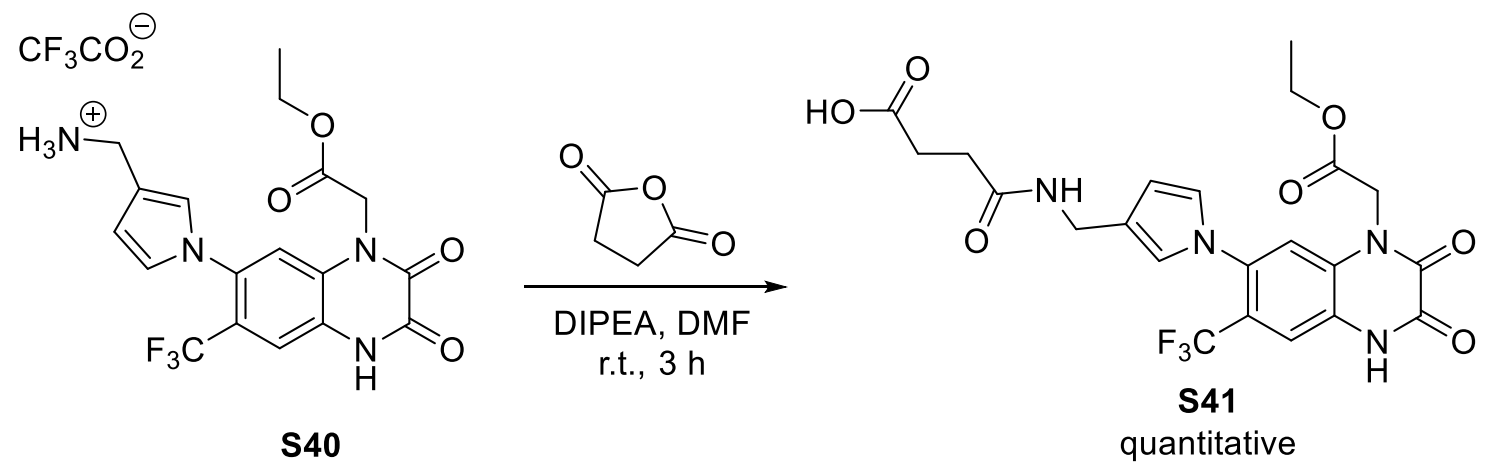

Compound $\mathbf{S 4 1}$ was prepared according to a modified procedure from the literature. ${ }^{3}$ A solution of $\mathbf{S 4 0}$ (18.5 mg, $35.3 \mu \mathrm{mol}, 1.0$ eq.) and succinic anhydride ( $8.9 \mathrm{mg}, 89 \mu \mathrm{mol}, 2.5$ eq.) in dry $\operatorname{DMF}(550 \mu \mathrm{L})$ and dry DIPEA $(30.7 \mu \mathrm{L}, 176 \mu \mathrm{mol}, 5.0$ eq.) was stirred at r.t. under Ar atmosphere for $3 \mathrm{~h}$. After removing volatiles under high vacuum, the residue was dissolved in $18 \%$ aq. MeCN ( $3 \mathrm{~mL}$ ) and acidified with $\mathrm{CF}_{3} \mathrm{CO}_{2} \mathrm{H}$ (10 drops). The product was purified by $\mathrm{RP} \mathrm{C}_{18}$-silica column chromatography using aq. $\mathrm{MeCN}$ (18 to 30 vol. $\% \mathrm{MeCN}$, containing $0.1 \% \mathrm{CF}_{3} \mathrm{CO}_{2} \mathrm{H}$ ) as the mobile phase. Compound $\mathbf{S 4 1}$ was obtained as a slightly yellowish amorphous solid. Yield: $18 \mathrm{mg}$ (quantitative).

${ }^{1}$ H NMR $\left(500 \mathrm{MHz}\right.$, DMSO- $\left.d_{6}\right) \delta 12.49(\mathrm{~s}, 1 \mathrm{H}), 8.14(\mathrm{t}, J=5.8 \mathrm{~Hz}, 1 \mathrm{H}), 7.62(\mathrm{~s}, 1 \mathrm{H}), 7.51(\mathrm{~s}$, $1 \mathrm{H}), 6.80(\mathrm{~d}, J=2.7 \mathrm{~Hz}, 1 \mathrm{H}), 6.79(\mathrm{~s}, 1 \mathrm{H}), 6.17(\mathrm{~s}, 1 \mathrm{H}), 5.02(\mathrm{~s}, 2 \mathrm{H}), 4.24-4.07(\mathrm{~m}, 4 \mathrm{H}), 2.44(\mathrm{t}$, $J=6.9 \mathrm{~Hz}, 2 \mathrm{H}), 2.35(\mathrm{t}, J=6.9 \mathrm{~Hz}, 2 \mathrm{H}), 1.19(\mathrm{t}, J=7.1 \mathrm{~Hz}, 3 \mathrm{H})$. 
${ }^{13} \mathbf{C}\left\{{ }^{1} \mathbf{H}\right\}$ NMR (126 MHz, DMSO- $\left.d_{6}\right) \delta 173.9,170.7,167.4,155.3,152.8,133.8,129.9,125.1$, 123.7, 122.9 (q, $J=273.2 \mathrm{~Hz}), 122.5,121.2,119.9(\mathrm{q}, J=31.2 \mathrm{~Hz}), 116.4,114.0(\mathrm{q}, J=5.3 \mathrm{~Hz})$, $109.2,61.4,44.7,35.3,30.0,29.2,14.0$.

\section{4-(2-(4-)((1-(4-(2-Ethoxy-2-oxoethyl)-2,3-dioxo-7-(trifluoromethyl)-1,2,3,4-} tetrahydroquinoxalin-6-yl)-1 $H$-pyrrol-3-yl)methyl)amino)-4-oxobutanamido)ethyl)-1 $H$ imidazol-3-ium trifluoroacetate (S42)

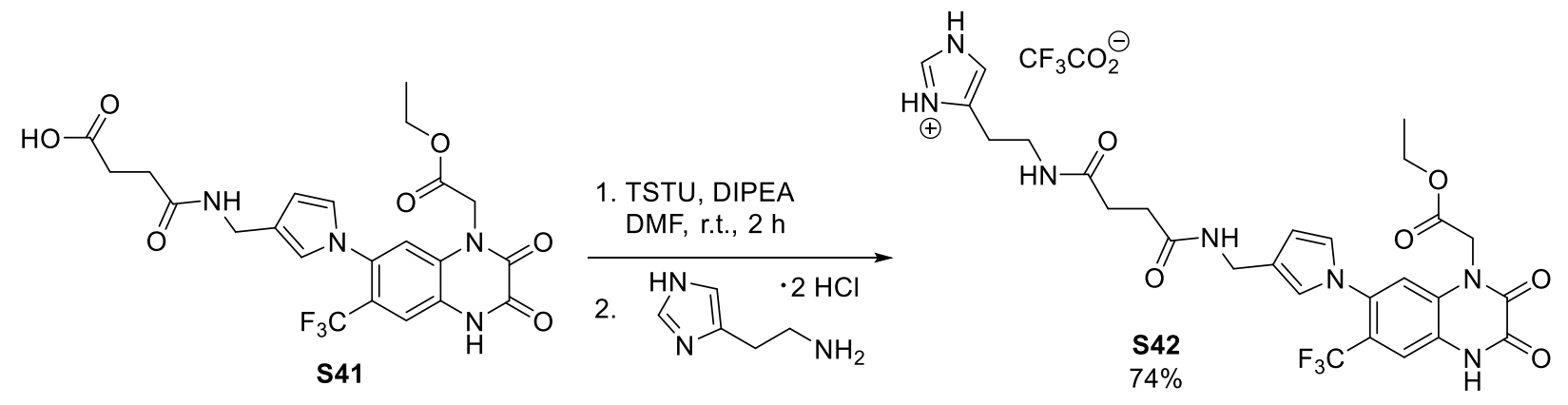

A solution of $\mathbf{S 4 1}$ (18 mg, $35.3 \mu \mathrm{mol}, 1.0$ eq.) and TSTU (11.6 mg, $38.5 \mu \mathrm{mol}, 1.1$ eq.) in dry DMF $(250 \mu \mathrm{L})$ and dry DIPEA $(9.2 \mu \mathrm{L}, 53 \mu \mathrm{mol}, 1.5$ eq. $)$ was stirred at r.t. under Ar atmosphere for $2 \mathrm{~h}$ resulting in the formation of the intermediate NHS ester. Dry DIPEA ( $30.7 \mu \mathrm{L}, 176 \mu \mathrm{mol}, 5.0$ eq.) and histamine dihydrochloride $(9.9 \mathrm{mg}, 54 \mu \mathrm{mol}, 1.5 \mathrm{eq}$.) were added, and the resulting solution was stirred at r.t. for $22 \mathrm{~h}$. After removing volatiles under high vacuum, the residue was dissolved in $15 \%$ aq. $\mathrm{MeCN}(3 \mathrm{~mL})$ and acidified with $\mathrm{CF}_{3} \mathrm{CO}_{2} \mathrm{H}$ (10 drops). The product was purified by $\mathrm{RP} \mathrm{C}_{18}$-silica column chromatography using aq. $\mathrm{MeCN}$ (15 to 25 vol. \% MeCN, containing $0.1 \%$ $\left.\mathrm{CF}_{3} \mathrm{CO}_{2} \mathrm{H}\right)$ as the mobile phase. Compound $\mathbf{S 4 2}$ was obtained as a thick colorless oil. Yield: $18.7 \mathrm{mg}(74 \%)$.

$\boldsymbol{R}_{\mathbf{f}}\left(\mathrm{C}_{18}\right.$-silica, $\left.\mathrm{H}_{2} \mathrm{O} / \mathrm{MeCN} 2: 1, v / v+0.1 \% \mathrm{CF}_{3} \mathrm{CO}_{2} \mathrm{H}\right): 0.32$.

${ }^{1}$ H NMR $\left(500 \mathrm{MHz}, \mathrm{DMSO}-d_{6}\right) \delta 14.33$ (br s, 2H), $12.51(\mathrm{~s}, 1 \mathrm{H}), 8.99(\mathrm{~s}, 1 \mathrm{H}), 8.15(\mathrm{t}, J=5.7 \mathrm{~Hz}$, $1 \mathrm{H}), 8.00(\mathrm{t}, J=5.8 \mathrm{~Hz}, 1 \mathrm{H}), 7.62(\mathrm{~s}, 1 \mathrm{H}), 7.50(\mathrm{~s}, 1 \mathrm{H}), 7.43(\mathrm{~s}, 1 \mathrm{H}), 6.81(\mathrm{t}, J=2.6 \mathrm{~Hz}, 1 \mathrm{H}), 6.79$ (s, 1H), 6.16 (t, $J=2.2 \mathrm{~Hz}, 1 \mathrm{H}), 5.01(\mathrm{~s}, 2 \mathrm{H}), 4.21-4.06(\mathrm{~m}, 4 \mathrm{H}), 3.30(\mathrm{q}, J=6.5 \mathrm{~Hz}, 2 \mathrm{H}), 2.75$ (t, $J=6.8 \mathrm{~Hz}, 2 \mathrm{H}), 2.40-2.23(\mathrm{~m}, 4 \mathrm{H}), 1.19(\mathrm{t}, J=7.1 \mathrm{~Hz}, 3 \mathrm{H})$. 
${ }^{13} \mathbf{C}\left\{{ }^{1} \mathbf{H}\right\}$ NMR $\left(126 \mathrm{MHz}\right.$, DMSO- $\left.d_{6}\right) \delta 172.1,171.5,167.8,158.5\left(\mathrm{q}, J=34.4 \mathrm{~Hz}, \mathrm{CF}_{3} \underline{C O}_{2}{ }^{-}\right)$, 155.7, 153.3, 134.3, 134.2, 131.5, 130.3, 125.6, 124.2, 123.3 (q, $J=272.7 \mathrm{~Hz}), 122.9,121.6,120.3$ $(\mathrm{q}, J=31.1 \mathrm{~Hz}), 116.8,116.6,114.5(\mathrm{q}, J=5.5 \mathrm{~Hz}), 109.7,61.8,45.2,37.9,35.7,31.2,31.0,24.8$, 14.4 .

2-(7-(3-((4-((2-(1H-Imidazol-4-yl)ethyl)amino)-4-oxobutanamido)methyl)-1 $H$-pyrrol-1-yl)2,3-dioxo-6-(trifluoromethyl)-3,4-dihydroquinoxalin-1(2H)-yl)acetic acid triethylammonium salt (S2)
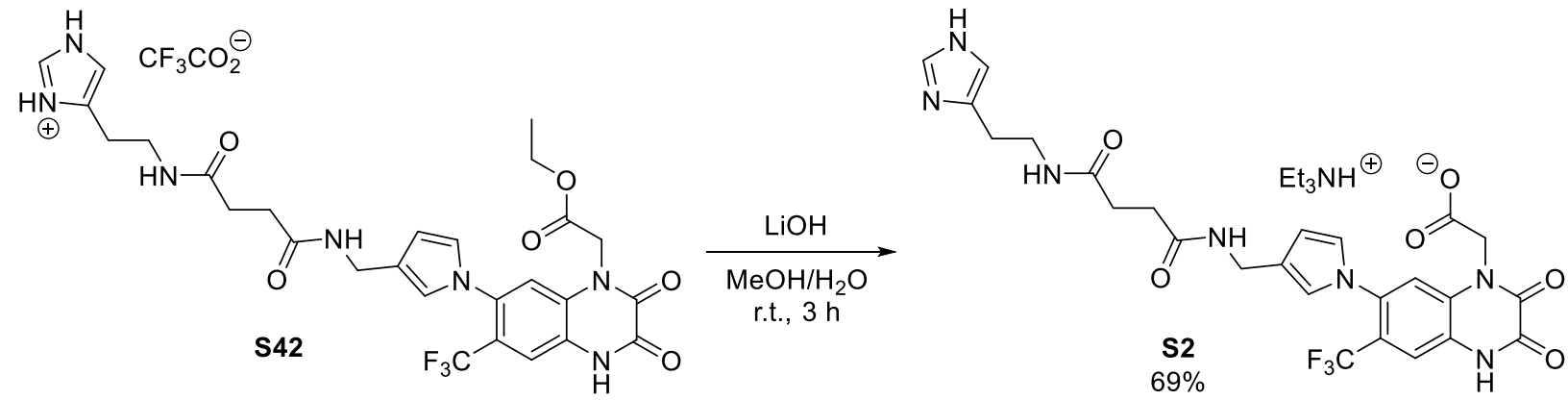

To a solution of $\mathbf{S 4 2}(18.5 \mathrm{mg}, 25.8 \mu \mathrm{mol}, 1.0$ eq.) in $\mathrm{MeOH}(600 \mu \mathrm{L})$ was added $0.5 \mathrm{M}$ aq. $\mathrm{LiOH}$ ( $258 \mu \mathrm{L}, 129 \mu \mathrm{mol} \mathrm{LiOH}, 5.0$ eq.), which resulted in precipitation of a white solid. The reaction mixture was stirred at r.t. for $3 \mathrm{~h}$, after which it was quenched with aq. triethylamine hydrochloride (177 $\mathrm{mg} \mathrm{Et}_{3} \mathrm{NH}^{+} \mathrm{Cl}^{-}, 1.30 \mathrm{mmol}, 50$ eq. in $6 \mathrm{~mL} \mathrm{H}_{2} \mathrm{O}$ ). The product was purified by $\mathrm{RP} \mathrm{C}_{18}$-silica column chromatography using aq. $\mathrm{MeCN}$ (10 to 14 vol. $\% \mathrm{MeCN}$, containing $0.1 \% \mathrm{Et}_{3} \mathrm{~N}$ ) as the mobile phase. $15.4 \mathrm{mg}$ of a white solid was obtained which exhibited low solubility in most solvents (including DMF and DMSO), likely the Li-salt. Therefore, the compound was suspended in $10 \%$ aq. $\mathrm{MeCN}(3 \mathrm{~mL})$ and acidified with $\mathrm{CF}_{3} \mathrm{CO}_{2} \mathrm{H}$ (10 drops). The Li-salts were removed by $\mathrm{RP} \mathrm{C}_{18}$-silica column chromatography using aq. $\mathrm{MeCN}$ (10 to 18 vol. \% MeCN, containing $0.1 \%$ $\left.\mathrm{CF}_{3} \mathrm{CO}_{2} \mathrm{H}\right)$ as the mobile phase. $18 \mathrm{mg}$ of the $\mathrm{CF}_{3} \mathrm{CO}_{2}^{-}$salt was obtained as a light tan oil. The desired $\mathrm{Et}_{3} \mathrm{NH}^{+}$salt was obtained by another $\mathrm{RP} \mathrm{C}_{18}$-silica column chromatography using aq. $\mathrm{MeCN}$ ( 8 to 14 vol. \% MeCN, containing $0.1 \% \mathrm{Et}_{3} \mathrm{~N}$ ) as the mobile phase. Compound $\mathbf{S 2}$ was obtained as a white solid. Yield: $12.0 \mathrm{mg}(69 \%)$. Note: ${ }^{1} \mathrm{H}$ NMR $\left(500 \mathrm{MHz}, \mathrm{DMSO}-d_{6}\right)$ of the final product revealed lower integral intensities of the $\mathrm{Et}_{3} \mathrm{NH}^{+}$counter-cations than expected $(\sim 2 / 3)$. We 
hypothesize that the zwitterionic form of the product or other residual counter-cations were present.

\section{$\mathrm{CF}_{3} \mathrm{CO}_{2}^{-}$salt:}

$\boldsymbol{R}_{\mathbf{f}}\left(\mathrm{C}_{18}\right.$-silica, $\left.\mathrm{H}_{2} \mathrm{O} / \mathrm{MeCN} 2: 1, v / v\right): 0.49$.

${ }^{1}$ H NMR (500 MHz, DMSO- $\left.d_{6}\right) \delta 14.28(\mathrm{br} \mathrm{s}, 2 \mathrm{H}), 12.50(\mathrm{~s}, 1 \mathrm{H}), 8.99(\mathrm{~d}, J=1.4 \mathrm{~Hz}, 1 \mathrm{H}), 8.16$ $(\mathrm{t}, J=5.7 \mathrm{~Hz}, 1 \mathrm{H}), 8.00(\mathrm{t}, J=5.8 \mathrm{~Hz}, 1 \mathrm{H}), 7.61(\mathrm{~s}, 1 \mathrm{H}), 7.43(\mathrm{~s}, 1 \mathrm{H}), 7.42(\mathrm{~s}, 1 \mathrm{H}), 6.83-6.81$ (m, 1H), $6.80(\mathrm{~s}, 1 \mathrm{H}), 6.21-6.12(\mathrm{~m}, 1 \mathrm{H}), 4.95(\mathrm{~s}, 2 \mathrm{H}), 4.14(\mathrm{~d}, J=5.5 \mathrm{~Hz}, 2 \mathrm{H}), 3.30(\mathrm{q}, J=6.5$ $\mathrm{Hz}, 2 \mathrm{H}), 2.75(\mathrm{t}, J=6.8 \mathrm{~Hz}, 2 \mathrm{H}), 2.39-2.23(\mathrm{~m}, 4 \mathrm{H})$.

${ }^{13} \mathbf{C}\left\{{ }^{1} \mathbf{H}\right\}$ NMR (126 MHz, DMSO- $\left.d_{6}\right) \delta 171.7,171.1,168.7,158.2(\mathrm{q}, J=35.6 \mathrm{~Hz}), 155.3,152.9$, 133.8, 131.0, 129.9, 125.1, 123.8, 122.9 (q, $J=273.0 \mathrm{~Hz}), 122.4,121.2,119.7$ (q, $J=30.4 \mathrm{~Hz})$, $116.3,116.2,114.3(\mathrm{q}, J=285.4 \mathrm{~Hz}), 114.0(\mathrm{q}, J=5.1 \mathrm{~Hz}), 109.3,55.1,44.7,37.5,35.3,30.8$, 30.6, 24.4.

\section{$\mathrm{Et}_{3} \mathrm{NH}^{+}$salt:}

$\boldsymbol{R}_{\mathbf{f}}\left(\mathrm{C}_{18}\right.$-silica, $\left.\mathrm{H}_{2} \mathrm{O} / \mathrm{MeCN} 2: 1, v / v+0.1 \% \mathrm{CF}_{3} \mathrm{CO}_{2} \mathrm{H}\right): 0.49$.

${ }^{1} \mathrm{H}$ NMR $\left(500 \mathrm{MHz}, \mathrm{DMSO}-d_{6}\right) \delta 8.18(\mathrm{t}, J=5.7 \mathrm{~Hz}, 1 \mathrm{H}), 8.08(\mathrm{br} \mathrm{t}, J=5.7 \mathrm{~Hz}, 1 \mathrm{H}), 7.64(\mathrm{br} \mathrm{s}$, 1H), $7.58(\mathrm{~s}, 1 \mathrm{H}), 7.09(\mathrm{~s}, 1 \mathrm{H}), 6.87-6.77(\mathrm{~m}, 2 \mathrm{H}), 6.75(\mathrm{~s}, 1 \mathrm{H}), 6.14(\mathrm{~s}, 1 \mathrm{H}), 4.57$ (br s, 2H), $4.12(\mathrm{~d}, J=5.3 \mathrm{~Hz}, 2 \mathrm{H}), 3.22(\mathrm{q}, J=6.6 \mathrm{~Hz}, 2 \mathrm{H}), 2.93(\mathrm{q}, J=7.2 \mathrm{~Hz}, 4 \mathrm{H}[6 \mathrm{H}$ expected]), $2.68-$ $2.55(\mathrm{~m}, 2 \mathrm{H}), 2.30(\mathrm{~s}, 4 \mathrm{H}), 1.09(\mathrm{t}, J=7.2 \mathrm{~Hz}, 6 \mathrm{H}[9 \mathrm{H}$ expected]). 
2-(7-(3-((4-((2-(1-((2-(2-(4-(Azidomethyl)benzamido)ethoxy)ethoxy)carbonyl)-1H-imidazol4-yl)ethyl)amino)-4-oxobutanamido)methyl)-1H-pyrrol-1-yl)-2,3-dioxo-6-(trifluoromethyl)3,4-dihydroquinoxalin-1(2H)-yl)acetic acid triethylammonium salt (19)

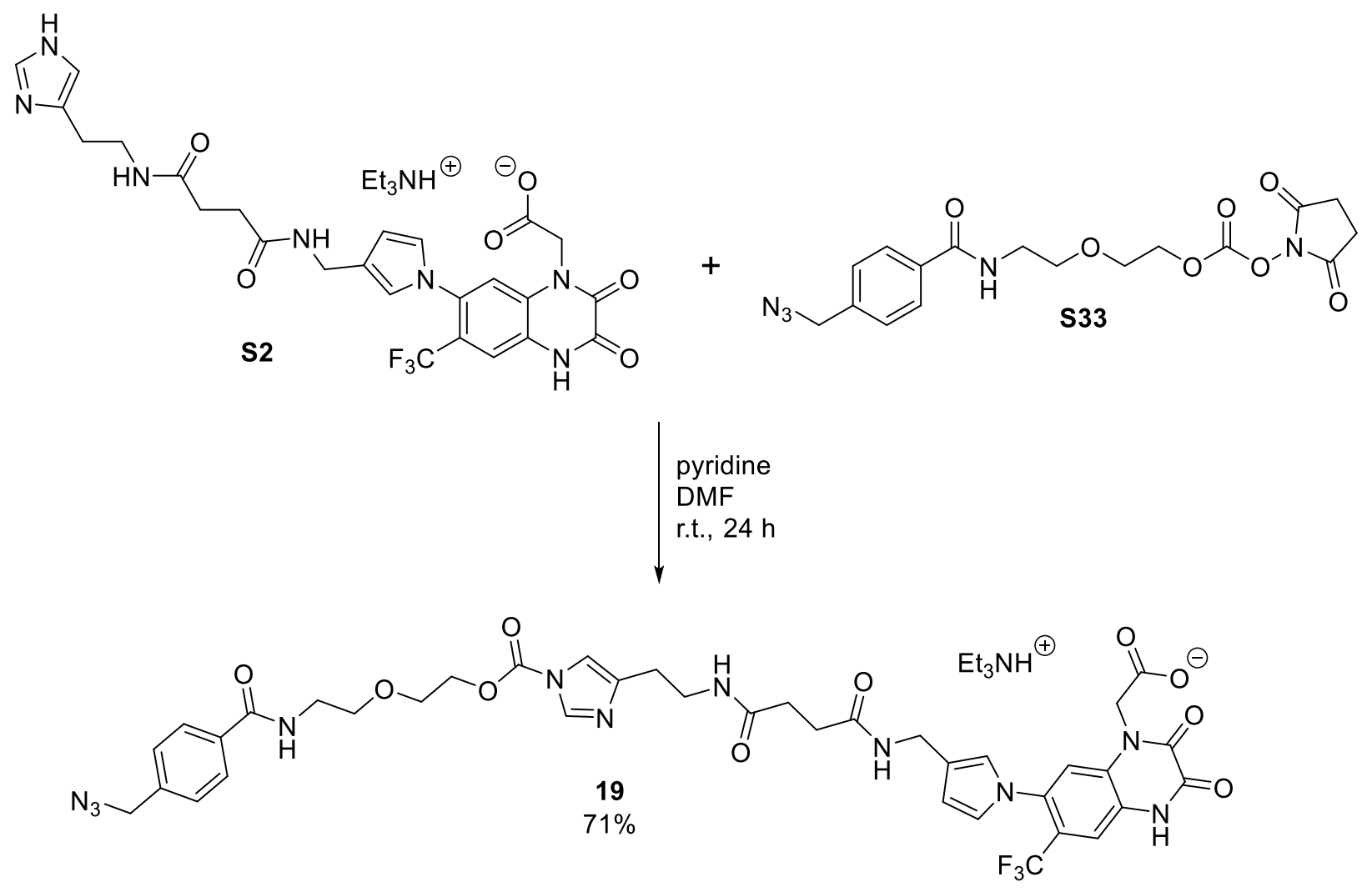

A solution of $\mathbf{S 3 3}(5.0 \mathrm{mg}, 12 \mu \mathrm{mol}, 2.2$ eq.) in dry DMF (150 $\mu \mathrm{L})$ was added to a mixture of $\mathbf{S 2}$ (3.7 mg, $5.6 \mu \mathrm{mol}, 1.0$ eq.) and dry pyridine ( $4.5 \mu \mathrm{L}, 56 \mu \mathrm{mol}, 10$ eq.) under dry Ar atmosphere. The resulting solution was stirred at r.t. under dry Ar for $48 \mathrm{~h}$. After quenching with aq. $\mathrm{Et}_{3} \mathrm{NH}^{+} \mathrm{Cl}^{-}$ ( $c=10 \mathrm{mg} / \mathrm{mL}, 3 \mathrm{~mL}$ ), the crude mixture was purified by $\mathrm{RP} \mathrm{C}_{18}$-silica column chromatography using aq. $\mathrm{MeCN}$ (5 to 17 vol. \% MeCN, no other additive) as the mobile phase. Compound 19 was obtained as a colorless amorphous solid. Yield: $3.8 \mathrm{mg}(71 \%)$.

$\boldsymbol{R} \mathbf{f}\left(\mathrm{C}_{18}\right.$-silica, $\left.\mathrm{H}_{2} \mathrm{O} / \mathrm{MeCN} 2: 1, v / v\right): 0.42$.

${ }^{1} \mathrm{H}$ NMR $\left(500 \mathrm{MHz}, \mathrm{DMSO}-d_{6}\right) \delta 8.61(\mathrm{t}, J=5.6 \mathrm{~Hz}, 1 \mathrm{H}), 8.16(\mathrm{t}, J=5.6 \mathrm{~Hz}, 1 \mathrm{H}), 8.13(\mathrm{~s}, 1 \mathrm{H})$, $8.08(\mathrm{t}, J=5.7 \mathrm{~Hz}, 1 \mathrm{H}), 7.83(\mathrm{~d}, J=8.3 \mathrm{~Hz}, 2 \mathrm{H}), 7.56(\mathrm{~s}, 1 \mathrm{H}), 7.41(\mathrm{~d}, J=8.3 \mathrm{~Hz}, 2 \mathrm{H}), 7.31(\mathrm{~s}$, 1H), $7.06(\mathrm{~s}, 1 \mathrm{H}), 6.80(\mathrm{~d}, J=2.7 \mathrm{~Hz}, 1 \mathrm{H}), 6.77(\mathrm{~s}, 1 \mathrm{H}), 6.14(\mathrm{dd}, J=2.9,1.7 \mathrm{~Hz}, 1 \mathrm{H}), 4.51$ (s, $2 \mathrm{H}), 4.49-4.44(\mathrm{~m}, 2 \mathrm{H}), 4.12(\mathrm{~d}, J=5.5 \mathrm{~Hz}, 2 \mathrm{H}), 3.78-3.74(\mathrm{~m}, 2 \mathrm{H}), 3.60(\mathrm{t}, J=5.9 \mathrm{~Hz}, 2 \mathrm{H})$, 
$3.42(\mathrm{q}, J=5.9 \mathrm{~Hz}, 2 \mathrm{H}), 3.25(\mathrm{q}, J=7.0 \mathrm{~Hz}, 2 \mathrm{H}), 2.94-2.80(\mathrm{~m}, 6 \mathrm{H}), 2.57(\mathrm{t}, J=7.3 \mathrm{~Hz}, 2 \mathrm{H})$, $2.31(\mathrm{~s}, 4 \mathrm{H}), 1.08(\mathrm{t}, J=7.2 \mathrm{~Hz}, 9 \mathrm{H})$.

HRMS (ESI+): calculated $m / z$ for $\left[\mathrm{C}_{38} \mathrm{H}_{37} \mathrm{~N}_{11} \mathrm{O}_{10} \mathrm{~F}_{3}+2 \mathrm{H}\right]^{+}=866.2833$, found $=866.2841$.

\section{Ethyl 5-azidovalerate (S43)}

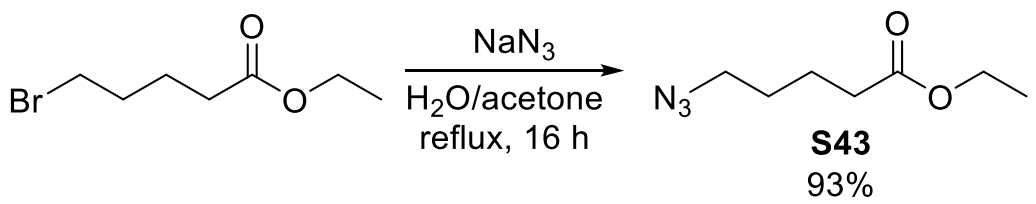

A solution of ethyl 5-bromovalerate $\left(5.07 \mathrm{~g}, 24.2 \mathrm{mmol}, 1.0\right.$ eq.) and $\mathrm{NaN}_{3}$ (4.70 g, $72.3 \mathrm{mmol}$, 3.0 eq.) in $\mathrm{H}_{2} \mathrm{O}(15 \mathrm{~mL})$ and acetone $(25 \mathrm{~mL})$ was heated at reflux for $16 \mathrm{~h}$. The resulting mixture was concentrated by rotary evaporation to a $\sim 20 \mathrm{~mL}$ volume, extracted with $\mathrm{CH}_{2} \mathrm{Cl}_{2}(3 \times 20 \mathrm{~mL})$, dried over anhydrous $\mathrm{MgSO}_{4}$, and filtered. Removing volatiles by rotary evaporation provided compound $\mathbf{S 4 3}$ as a colorless liquid. Yield: $3.87 \mathrm{~g}(93 \%)$.

${ }^{1} \mathbf{H}$ NMR $\left(400 \mathrm{MHz}, \mathrm{CDCl}_{3}\right) \delta 4.14(\mathrm{q}, J=7.2 \mathrm{~Hz}, 2 \mathrm{H}), 3.30(\mathrm{t}, J=6.6 \mathrm{~Hz}, 2 \mathrm{H}), 2.34(\mathrm{t}, J=7.2$ $\mathrm{Hz}, 2 \mathrm{H}), 1.78-1.55(\mathrm{~m}, 4 \mathrm{H}), 1.26(\mathrm{t}, J=7.1 \mathrm{~Hz}, 3 \mathrm{H})$.

${ }^{13} \mathbf{C}\left\{{ }^{1} \mathbf{H}\right\}$ NMR $\left(75 \mathrm{MHz}, \mathrm{CDCl}_{3}\right) \delta 173.3,60.5,51.2,33.8,28.4,22.3,14.4$.

MS (APCI+): calculated $m / z$ for $\left[\mathrm{C}_{7} \mathrm{H}_{13} \mathrm{~N}_{3} \mathrm{O}_{2}+\mathrm{H}\right]^{+}=172.1$, found $=172.2$.

\section{5-Azidovaleric acid (S44)}

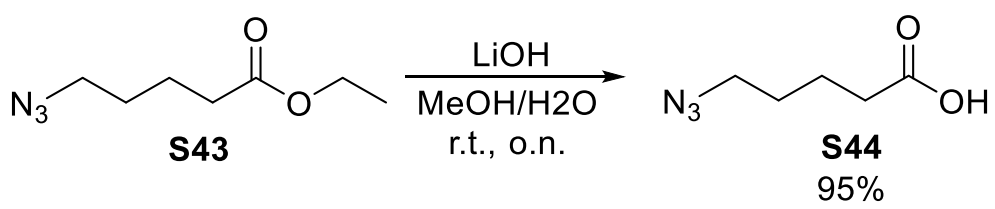

S43 (3.85 g, $22.5 \mathrm{mmol}, 1.0$ eq.) was dissolved in $\mathrm{MeOH}(40 \mathrm{~mL})$ and ice (35 g) was added. $\mathrm{LiOH} \cdot \mathrm{H}_{2} \mathrm{O}(2.83 \mathrm{~g}, 67.5 \mathrm{mmol}, 3.0$ eq.) was added to the stirred mixture in portions. The resulting solution was further stirred at r.t. for $19 \mathrm{~h}$. The solution was concentrated by rotary evaporation 
$\left(30{ }^{\circ} \mathrm{C}\right)$ to a $\sim 40 \mathrm{~mL}$ volume, diluted with $\mathrm{H}_{2} \mathrm{O}(10 \mathrm{~mL})$ and washed with $\mathrm{CH}_{2} \mathrm{Cl}_{2}(30 \mathrm{~mL})$. The aqueous phase was acidified with $36 \%$ aq. $\mathrm{HCl}$ to $\mathrm{pH}=1(\sim 6 \mathrm{~mL})$ and subsequently extracted with $\mathrm{CH}_{2} \mathrm{Cl}_{2}(3 \times 30 \mathrm{~mL})$. The combined org. extracts were washed with brine $(30 \mathrm{~mL})$, dried over

anhydrous $\mathrm{MgSO}_{4}$, and filtered. Removing volatiles by rotary evaporation $\left(30^{\circ} \mathrm{C}\right)$ provided compound $\mathbf{S 4 4}$ as a colorless liquid. Yield: $3.07 \mathrm{~g}(95 \%)$.

${ }^{1} \mathbf{H}$ NMR $\left(400 \mathrm{MHz}, \mathrm{CDCl}_{3}\right) \delta 3.31(\mathrm{t}, J=6.5 \mathrm{~Hz}, 2 \mathrm{H}), 2.41(\mathrm{t}, J=7.1 \mathrm{~Hz}, 2 \mathrm{H}), 1.84-1.40(\mathrm{~m}$, $4 \mathrm{H})$.

${ }^{13} \mathbf{C}\left\{{ }^{1} \mathbf{H}\right\}$ NMR $\left(101 \mathrm{MHz}, \mathrm{CDCl}_{3}\right) \delta 179.4,51.0,33.4,28.2,21.8$.

MS (APCI-): calculated $m / z$ for $\left[\mathrm{C}_{5} \mathrm{H}_{9} \mathrm{~N}_{3} \mathrm{O}_{2}-\mathrm{H}\right]^{-}=142.1$, found $=142.2$.

\section{5-Azidovaleric acid $N$-hydroxysuccinimidyl ester (S45)}

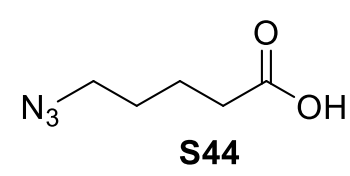

S44

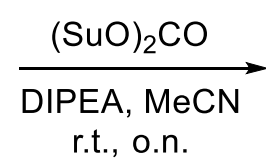

r.t., o.n.

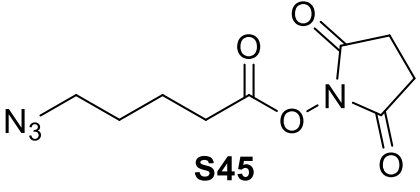

$76 \%$

$(\mathrm{SuO})_{2} \mathrm{CO}$ (545 mg, $2.13 \mathrm{mmol}, 1.5$ eq.) was added to a solution of $\mathbf{S 4 4}$ (209 $\mathrm{mg}, 1.46 \mathrm{mmol}$, 1.0 eq.) and DIPEA (490 $\mu \mathrm{L}, 2.80 \mathrm{mmol}, 1.9$ eq.) in $\mathrm{MeCN}(2 \mathrm{~mL})$ under $\mathrm{Ar}$ atmosphere. The resulting reaction mixture was stirred under Ar at r.t. for $16 \mathrm{~h}$. After diluting with $\mathrm{Et}_{2} \mathrm{O}(12 \mathrm{~mL})$, the solution was washed with $1 \mathrm{M}$ aq. $\mathrm{HCl}(2 \times 1.5 \mathrm{~mL})$, sat. aq. $\mathrm{NaHCO}_{3}(2 \times 1.5 \mathrm{~mL})$ and brine (1.5 mL), dried over anhydrous $\mathrm{MgSO}_{4}$ and filtered. Removing volatiles by rotary evaporation $\left(35^{\circ} \mathrm{C}\right)$ provided compound $\mathbf{S 4 5}$ as a colorless oil. Yield: $268 \mathrm{mg}(76 \%)$.

${ }^{1} \mathbf{H}$ NMR $\left(400 \mathrm{MHz}, \mathrm{CDCl}_{3}\right) \delta 3.34(\mathrm{t}, J=6.6 \mathrm{~Hz}, 2 \mathrm{H}), 2.84(\mathrm{~s}, 4 \mathrm{H}), 2.66(\mathrm{t}, J=7.2 \mathrm{~Hz}, 2 \mathrm{H}), 1.91$ $-1.80(\mathrm{~m}, 2 \mathrm{H}), 1.77-1.67(\mathrm{~m}, 2 \mathrm{H})$.

${ }^{13} \mathbf{C}\left\{{ }^{1} \mathbf{H}\right\}$ NMR $\left(101 \mathrm{MHz}, \mathrm{CDCl}_{3}\right) \delta 169.2,168.3,51.0,30.6,28.0,25.7,22.0$.

MS (APCI+): calculated $m / z$ for $\left[\mathrm{C}_{9} \mathrm{H}_{12} \mathrm{~N}_{4} \mathrm{O}_{4}+\mathrm{H}\right]^{+}=241.1$, found $=241.2$. 
dsRVF5-N3 (18)

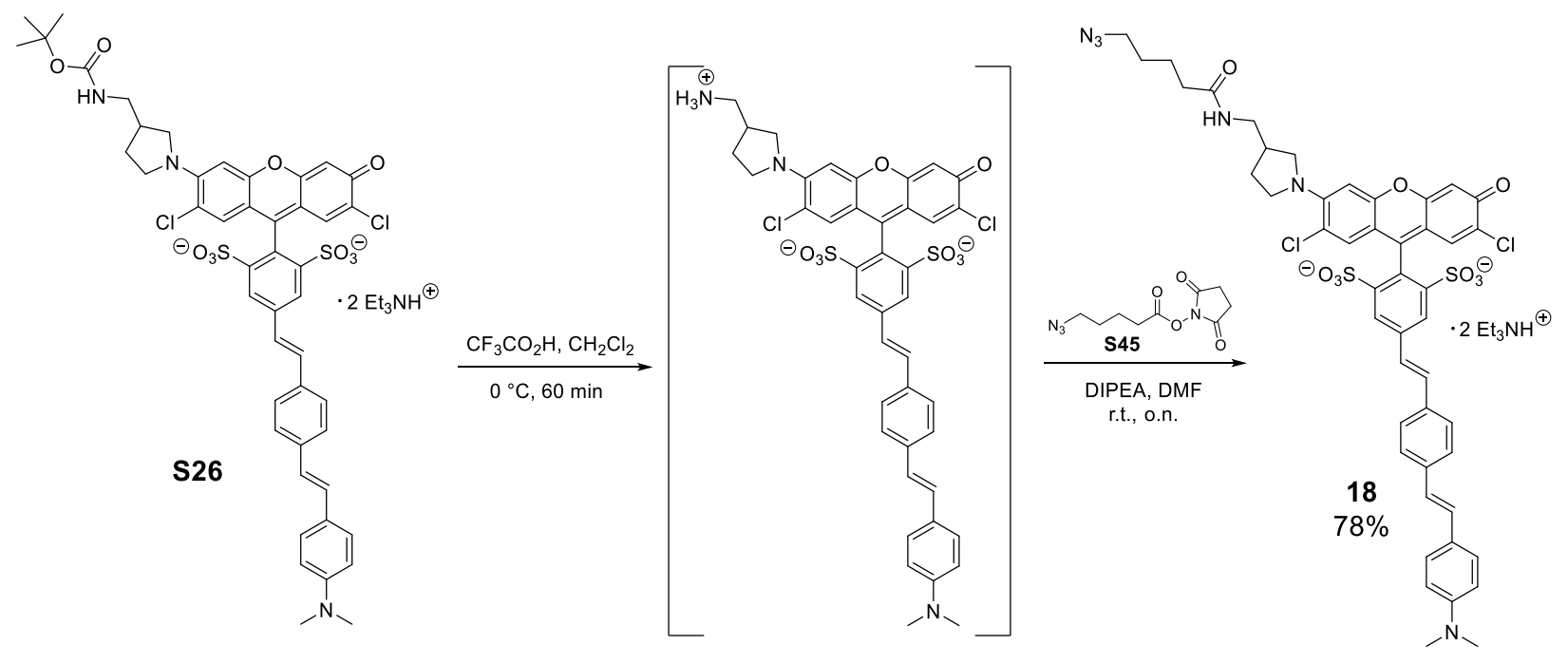

A suspension of dsRVF5-NHBoc (S26, $11 \mathrm{mg}, 9.6 \mu \mathrm{mol}, 1.0$ eq.) in $\mathrm{CH}_{2} \mathrm{Cl}_{2}(0.5 \mathrm{~mL})$ was cooled to $0{ }^{\circ} \mathrm{C}$ followed by dropwise addition of $\mathrm{CF}_{3} \mathrm{CO}_{2} \mathrm{H}(1 \mathrm{~mL})$. The resulting solution was stirred at $0{ }^{\circ} \mathrm{C}$ for $60 \mathrm{~min}$. Subsequently, volatiles were removed by rotary evaporation $\left(30^{\circ} \mathrm{C}\right)$ and the solid material was co-evaporated with toluene $(2 \times 1.5 \mathrm{~mL})$ to remove residual acid, providing the intermediate ammonium salt. After short drying under high vacuum $(1 \mathrm{~h})$, the dark purple solid was dissolved in dry DMF $(220 \mu \mathrm{L})$ and dry DIPEA (17 $\mu \mathrm{L}, 98 \mu \mathrm{mol}$, 10 eq.) under argon atmosphere, followed by addition of $\mathbf{S 4 5}$ ( $6.8 \mathrm{mg}, 28 \mu \mathrm{mol}, 3.0$ eq.). The resulting solution was stirred at r.t. under dry Ar for $18 \mathrm{~h}$. After diluting with water $(3 \mathrm{~mL})$, the crude product was purified by $\mathrm{RP} \mathrm{C}_{18}$-silica column chromatography using aq. $\mathrm{MeCN}$ (20 to 26 vol. \% MeCN, containing $\left.0.1 \% \mathrm{Et}_{3} \mathrm{~N}\right)$ as the mobile phase. Pure dsRVF5- $\mathrm{N}_{3}(\mathbf{1 8})$ was obtained as a red solid. Yield: $8.8 \mathrm{mg}$ $(78 \%)$.

$\boldsymbol{R}_{\mathbf{f}}\left(\mathrm{C} 18\right.$-silica; $\left.\mathrm{H}_{2} \mathrm{O} / \mathrm{MeCN} 2: 1, v / v\right): 0.29$.

${ }^{1}$ H NMR (500 MHz, DMSO- $\left.d_{6}\right) \delta 8.24(\mathrm{~s}, 2 \mathrm{H}), 8.02(\mathrm{t}, J=5.8 \mathrm{~Hz}, 1 \mathrm{H}), 7.72(\mathrm{~d}, J=8.2 \mathrm{~Hz}, 2 \mathrm{H})$, $7.57(\mathrm{~d}, J=8.1 \mathrm{~Hz}, 2 \mathrm{H}), 7.52-7.43(\mathrm{~m}, 3 \mathrm{H}), 7.32(\mathrm{~d}, J=16.3 \mathrm{~Hz}, 1 \mathrm{H}), 7.21(\mathrm{~d}, J=16.3 \mathrm{~Hz}, 1 \mathrm{H})$, $7.01(\mathrm{~d}, J=16.3 \mathrm{~Hz}, 1 \mathrm{H}), 6.92(\mathrm{~s}, 1 \mathrm{H}), 6.77$ (s, 2H), $6.74(\mathrm{~d}, J=9.0 \mathrm{~Hz}, 2 \mathrm{H}), 6.23$ (s, 1H), $3.69-$ $3.60(\mathrm{~m}, 3 \mathrm{H}), 3.42-3.36(\mathrm{~m}, 1 \mathrm{H}), 3.33(\mathrm{t}, J=6.5 \mathrm{~Hz}, 2 \mathrm{H}), 3.23-3.17(\mathrm{~m}, 1 \mathrm{H}), 3.11-3.07(\mathrm{~m}$, 1H), 3.03 (q, $J=7.4 \mathrm{~Hz}, 12 \mathrm{H}), 2.95$ (s, 6H), $2.46-2.34(\mathrm{~m}, 1 \mathrm{H}), 2.12(\mathrm{t}, J=7.0 \mathrm{~Hz}, 2 \mathrm{H}), 2.06-$ $1.97(\mathrm{~m}, 1 \mathrm{H}), 1.73-1.63(\mathrm{~m}, 1 \mathrm{H}), 1.61-1.47(\mathrm{~m}, 4 \mathrm{H}), 1.14(\mathrm{t}, J=7.3 \mathrm{~Hz}, 18 \mathrm{H})$. 
${ }^{13} \mathbf{C}\left\{{ }^{1} \mathbf{H}\right\}$ NMR $\left(126 \mathrm{MHz}, \mathrm{DMSO}-d_{6}\right) \delta 175.9,171.9,157.9,151.6,151.5,150.0,148.8,148.1$, 148.0, 137.7, 137.4, 135.1, 132.5, 131.4, 129.7, 129.3, 129.0, 127.6, 127.3, 126.6, 126.2, 125.8, $125.0,124.3$, 123.2, 117.1, 115.5, 115.4, 112.2, 102.8, 100.7, 54.7, 50.5, 50.4, 45.8, 40.6, 40.1, $40.0,38.4,34.7,28.7,27.8,22.5,8.8$.

HRMS (ESI-): calculated $m / z$ for $\left[\mathrm{C}_{47} \mathrm{H}_{42} \mathrm{Cl}_{2} \mathrm{~N}_{6} \mathrm{O}_{9} \mathrm{~S}_{2}+\mathrm{H}\right]^{-}=969.1910$, found $=969.1912$.

Sulforhodamine B 3-azidopropyl amide (S46)
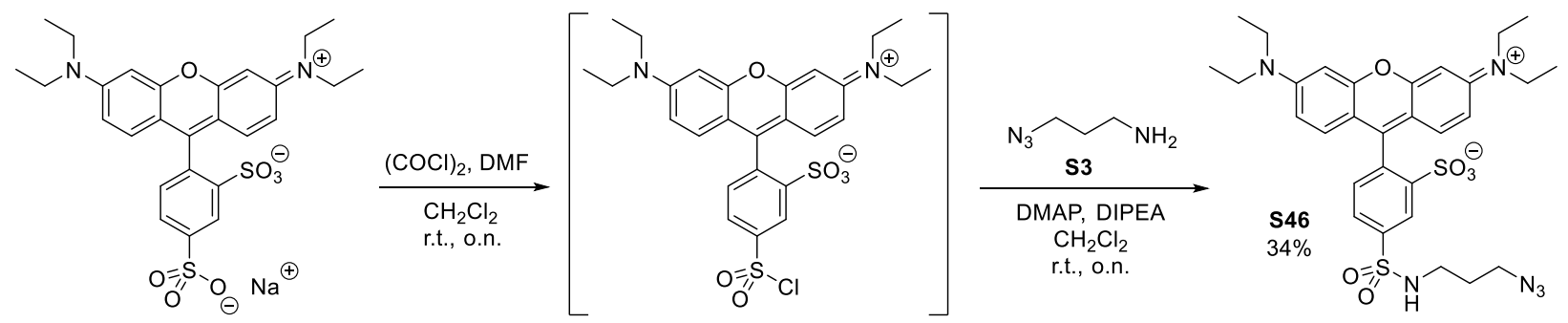

Sulforhodamine B sodium salt (786 mg, $1.35 \mathrm{mmol}, 1.0$ eq.) was dissolved in $\mathrm{CH}_{2} \mathrm{Cl}_{2}(10 \mathrm{~mL})$ under dry Ar atmosphere. Subsequently, oxalyl chloride (470 $\mu \mathrm{L}, 5.48 \mathrm{mmol}, 4.0$ eq.) and DMF (18 $\mu \mathrm{L}, 0.23 \mathrm{mmol}, 17 \mathrm{~mol}$. \%) were added. The resulting solution was stirred under Ar at r.t. for $22 \mathrm{~h}$. The volatiles were removed by rotary evaporation $\left(30^{\circ} \mathrm{C}\right)$, followed by co-evaporation with toluene $(20 \mathrm{~mL})$. The crude sulfonyl chloride was dissolved in $\mathrm{CH}_{2} \mathrm{Cl}_{2}(10 \mathrm{~mL})$. A solution of $\mathbf{S 3}$ (166 mg, $1.65 \mathrm{mmol}, 1.2$ eq.) in $\mathrm{CH}_{2} \mathrm{Cl}_{2}(5 \mathrm{~mL})$ was added, followed by addition of DMAP (10 mg, $82 \mu \mathrm{mol}, 6 \mathrm{~mol} . \%)$ and DIPEA (282 $\mu \mathrm{L}, 1.62 \mathrm{mmol}, 1.2$ eq.). The resulting solution was stirred at r.t. for $22 \mathrm{~h}$. Volatiles were removed by rotary evaporation $\left(30^{\circ} \mathrm{C}\right)$ and the crude product was purified by silica gel column chromatography using $\mathrm{MeOH}$ in $\mathrm{CH}_{2} \mathrm{Cl}_{2}$ (0 to 10 vol. \% $\mathrm{MeOH}$ ) as the mobile phase. The product was further purified by partially dissolving in $\mathrm{CH}_{2} \mathrm{Cl}_{2}(20 \mathrm{~mL})$ and reprecipitation in $\mathrm{Et}_{2} \mathrm{O}(200 \mathrm{~mL})$. Pure sulforhodamine B 3-azidopropyl amide (S46) was obtained as a dark purple solid. Yield: $295 \mathrm{mg}(34 \%)$.

$\boldsymbol{R} \mathbf{f}\left(\right.$ silica; $\left.\mathrm{CH}_{2} \mathrm{Cl}_{2} / \mathrm{MeOH} 9: 1, v / v\right): 0.34$.

${ }^{1}$ H NMR (500 MHz, DMSO- $\left.d_{6}\right) \delta 8.42(\mathrm{~d}, J=1.9 \mathrm{~Hz}, 1 \mathrm{H}), 8.00(\mathrm{t}, J=5.8 \mathrm{~Hz}, 1 \mathrm{H}), 7.94(\mathrm{dd}, J=$ 7.9, $2.0 \mathrm{~Hz}, 1 \mathrm{H}), 7.48(\mathrm{~d}, J=7.9 \mathrm{~Hz}, 1 \mathrm{H}), 7.04(\mathrm{dd}, J=9.6,2.4 \mathrm{~Hz}, 2 \mathrm{H}), 6.98(\mathrm{~d}, J=9.5 \mathrm{~Hz}, 2 \mathrm{H})$, 
$6.94(\mathrm{~d}, J=2.4 \mathrm{~Hz}, 2 \mathrm{H}), 3.64(\mathrm{~m}, 8 \mathrm{H}), 3.41(\mathrm{t}, J=6.7 \mathrm{~Hz}, 2 \mathrm{H}), 2.93(\mathrm{q}, J=6.6 \mathrm{~Hz}, 2 \mathrm{H}), 1.70(\mathrm{p}$, $J=6.8 \mathrm{~Hz}, 2 \mathrm{H}), 1.21(\mathrm{t}, J=7.0 \mathrm{~Hz}, 12 \mathrm{H})$.

MS (ESI+): calculated $m / z$ for $\left[\mathrm{C}_{30} \mathrm{H}_{36} \mathrm{~N}_{6} \mathrm{O}_{6} \mathrm{~S}_{2}+\mathrm{H}\right]^{+}=641.2$, found $=641.2$.

FTIR (ATR, diamond, $\mathrm{cm}^{-1}$ ): $2096\left(\mathrm{~N}_{3}\right)$ (Fig. S37 in the Infrared Spectra section). 


\section{Dextran Chemistry}

\section{Functionalization of dextran with azidopropyl carbamate linkers}

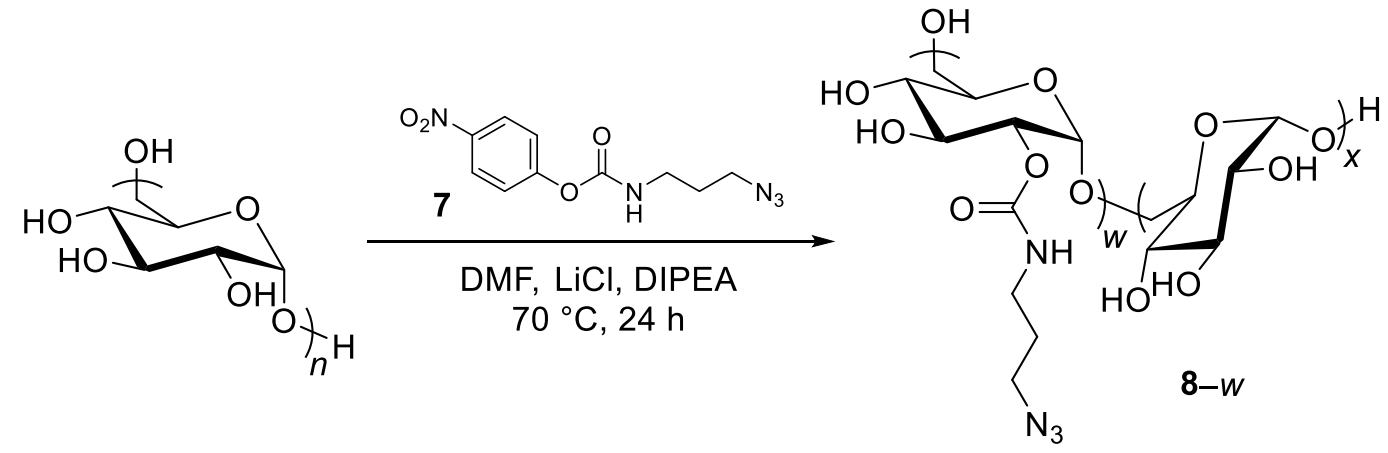

Commercial dextran (avg. $M_{w}=6000$, Chem-Impex International) was dried under high vacuum for 4 days (until constant mass, $\sim 6 \%$ weight loss) prior to use.

Commercial LiCl (anhydrous, Alfa Aesar) was dried under high vacuum at $150{ }^{\circ} \mathrm{C}$ overnight prior to use.

General procedure for synthesis of azidopropyl-carbamate dextran(6000) (8-w): Dried dextran(6000) (1.0 eq.) and dried $\mathrm{LiCl}(1.0 \mathrm{mg} / \mathrm{mg}$ dextran) were dissolved in dry DMF (20 $\mu \mathrm{L} / \mathrm{mg}$ dextran) at $70{ }^{\circ} \mathrm{C}$ under dry Ar atmosphere (dissolution was completed within $10 \mathrm{~min}$ ). Dry DIPEA (1.0 $\mu \mathrm{L} / \mathrm{mg}$ dextran) was added, followed by addition of $N$-(3-azidopropyl)-O-(4-nitrophenyl) carbamate (7, 1.1 eq. with respect to expected coverage) resulting in immediate change of color from colorless to yellow. The resulting solution was stirred at $70{ }^{\circ} \mathrm{C}$ for 24 h. After cooling to r.t., volatiles were removed by rotary evaporation $\left(40{ }^{\circ} \mathrm{C}\right)$. The resulting thick yellow-brown oil was dissolved in $\mathrm{H}_{2} \mathrm{O}\left(2.0 \mathrm{~mL}\right.$ ) and dialyzed against $\mathrm{H}_{2} \mathrm{O}$ (Slide-A-Lyzer G2, Thermo Fisher, MWCO $2000 \mathrm{Da}$ ) for 3 days with $2 \times$ per day exchange of dialysate for fresh $\mathrm{H}_{2} \mathrm{O}$. Removing the aqueous solvent by rotary evaporation $\left(40^{\circ} \mathrm{C}\right)$ provided azidopropyl carbamate dextran $(8-w)$ as a white solid. Number $w$ in 8-w designates the average number of azidopropyl groups per 6000 Da dextran polymer molecule as determined by ${ }^{1} \mathrm{H}$ NMR (vide infra). The $\mathrm{N}_{3}$ coverage yield and the total yield were highly reproducible for a range of dextran-7 ratios (84-87\% and $87-90 \%$ respectively). IR (ATR, diamond) showed a band at $2103 \mathrm{~cm}^{-1}$ corresponding to the $\mathrm{N}_{3}$ asymmetric stretch. 
IR characterization: IR (ATR, diamond) spectra of all 8-w samples showed a band at $2103 \mathrm{~cm}^{-1}$ corresponding to the $\mathrm{N}_{3}$ asymmetric stretch. Representative spectra of $8-2.15$ and 8-4.42 are shown in Fig. S38 and S39 respectively in the Infrared Spectra section.

NMR determination of coverage with azidopropyl groups: All samples of 8-w were coevaporated with $\mathrm{D}_{2} \mathrm{O}(1.5 \mathrm{~mL})$ prior to NMR analysis to reduce the intensity of the $-\mathrm{OH}$ signal via $\mathrm{H} / \mathrm{D}$ exchange. ${ }^{1} \mathrm{H}$ NMR $\left(500 \mathrm{MHz} ; \mathrm{D}_{2} \mathrm{O} / \mathrm{CD}_{3} \mathrm{CN} 5: 1, v / v\right)$ provided clearly separated signals of the dextran backbone and all azidopropyl $\mathrm{CH}_{2}$ groups (the zoomed-in spectrum of $8-2.15$ is shown in Fig. S28; full spectra of 8-w with five different $w$ are shown in Fig. S142 to S146 in the NMR spectra section). The total integration of dextran $\mathrm{C}-\mathrm{H}$ signals was set to 258.26 (the theoretical number of glucose units in a $6000 \mathrm{Da}$ dextran is 36.89 , each of which has seven non-exchangeable $\mathrm{C}-\mathrm{H}$ protons). The coverage with azidopropyl linker groups was determined from integration of the $\mathrm{CH}_{2}$ signal at $\delta 1.81 \mathrm{ppm}$ (most separated from the dextran signals; Fig. S28) and interpreted as an average number of these linker groups per ideal 6000 Da dextran polymer molecule ( $\mathrm{N}_{3} /$ polymer). 


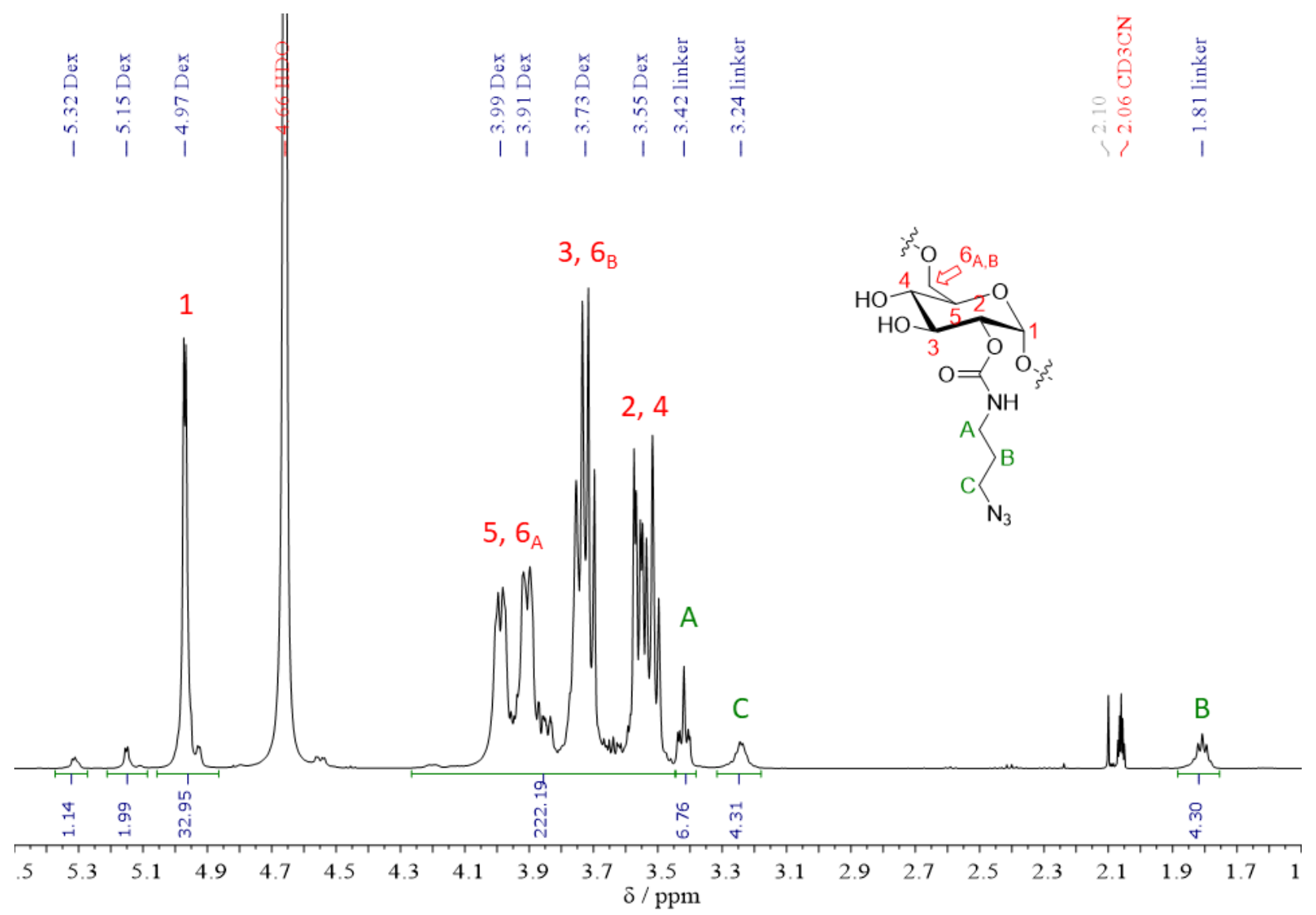

Figure S28. ${ }^{1} \mathrm{H}$ NMR $\left(500 \mathrm{MHz} ; \mathrm{D}_{2} \mathrm{O} / \mathrm{CD}_{3} \mathrm{CN} 5: 1, v / v\right)$ of 8-2.15. The total integration of all dextran $\mathrm{C}-\mathrm{H}$ signals (red numbers, assignment based on ref. ${ }^{32}$ ) was set to 258.26 ( $7 \mathrm{C}-\mathrm{H}$ protons $\times 36.89$ glucose units per $6000 \mathrm{Da}$ dextran). The integral of signal (green B) was used to determine the average number of azidopropyl linker groups per ideal 6000 Da dextran - 2.15 (4.30/2).

N3 coverage yield: Defined as the ratio between linker coverage as determined by ${ }^{1} \mathrm{H}$ NMR (vide supra) and maximum theoretical coverage $(n(7) / n($ dextran $))$. Example: To prepare 8-2.15, $41.1 \mu \mathrm{mol}$ of 7 was allowed to react with $16.8 \mu \mathrm{mol}$ of dextran(6000), while the experimental coverage is $2.15 \mathrm{~N}_{3} /$ pol. The $\mathrm{N}_{3}$ coverage yield is thus $2.15 \times 16.8 \mu \mathrm{mol} / 41.1 \mu \mathrm{mol}=0.88(88 \%)$.

Total yield: $100 \%$ total yield was defined as $100 \%$ material recovery with a maximum theoretical linker coverage. Attachment of one azidopropoyl carbamate group increases the $M_{w}$ by $126.12 \mathrm{~g} \mathrm{~mol}^{-1}$. Example: For 8-2.15, the maximum theoretical linker coverage was $41.1 \mu \mathrm{mol} 7$ / $16.8 \mu \mathrm{mol}$ of dextran $(6000)=2.44$. The theoretical average $M_{w}$ of the product is thus $6000 \mathrm{~g} \mathrm{~mol}^{-1}+2.44 \times 126.12 \mathrm{~g} \mathrm{~mol}^{-1}=6308 \mathrm{~g} \mathrm{~mol}^{-1} .101 \mathrm{mg}$ of used dextran(6000) implies a 
theoretical total yield of $(6308 / 6000) \times 101 \mathrm{mg}=106 \mathrm{mg}$. An isolated yield of $92 \mathrm{mg}$ thus corresponds to $87 \%$.

Regioselectivity of functionalization: We did not study the regioselectivity of this reaction. Dupayage et al. ${ }^{33}$ reported that the nucleophilicity of dextran-OH groups follows the following trend: $\mathrm{C}^{2}>\mathrm{C}^{4}>\mathrm{C}^{3}$ (see Fig. S28 for numbering). Therefore, we assume that attachment of azidopropyl carbamate linkers presented herein follows this trend. For simplicity and clarity, only the azidopropyl carbamate attached to $C^{2}$ is shown in all figures throughout this article. However, it is likely that a minor portion of linkers is also attached to $\mathrm{C}^{4}, \mathrm{C}^{3}$ and the terminal $\mathrm{C}^{6}$.

\section{Specific examples of $8-w$ synthesis:}

8-2.15: $101 \mathrm{mg}$ of dextran(6000) (16.8 $\mu \mathrm{mol}, 1.00$ eq.), $10.9 \mathrm{mg}$ of 7 (41.1 $\mu \mathrm{mol}, 2.44$ eq.), $103 \mathrm{mg}$ of dry $\mathrm{LiCl}, 100 \mu \mathrm{L}$ of dry DIPEA and $2.0 \mathrm{~mL}$ of dry DMF were used. $92 \mathrm{mg}$ of product was isolated. Coverage: $2.15 \mathrm{~N}_{3} /$ polymer, $\mathrm{N}_{3}$ coverage yield: $88 \%$, total yield: $87 \%$.

8-3.25: $100 \mathrm{mg}$ of dextran(6000) (16.7 $\mathrm{mol}, 1.00$ eq.), $16.3 \mathrm{mg}$ of 7 (61.5 $\mu \mathrm{mol}, 3.69$ eq.), $106 \mathrm{mg}$ of dry LiCl, $100 \mu \mathrm{L}$ of dry DIPEA and $2.0 \mathrm{~mL}$ of dry DMF were used. $92 \mathrm{mg}$ of product was isolated. Coverage: $3.25 \mathrm{~N}_{3}$ / polymer, $\mathrm{N}_{3}$ coverage yield: $88 \%$, total yield: $85 \%$.

8-4.42: $110 \mathrm{mg}$ of dextran(6000) (18.3 $\mu \mathrm{mol}, 1.00$ eq.), $23.8 \mathrm{mg}$ of 7 (89.7 $\mu \mathrm{mol}, 4.90$ eq.), $131 \mathrm{mg}$ of dry LiCl, $100 \mu \mathrm{L}$ of dry DIPEA and $2.0 \mathrm{~mL}$ of dry DMF were used. $102 \mathrm{mg}$ of product was isolated. Coverage: $4.42 \mathrm{~N}_{3}$ / polymer, $\mathrm{N}_{3}$ coverage yield: $90 \%$, total yield: $84 \%$.

8-5.37: $103 \mathrm{mg}$ of dextran(6000) (17.2 $\mu \mathrm{mol}, 1.00$ eq.), $27.3 \mathrm{mg}$ of 7 (103 $\mu \mathrm{mol}, 5.99$ eq.), $125 \mathrm{mg}$ of dry $\mathrm{LiCl}, 100 \mu \mathrm{L}$ of dry DIPEA and $2.0 \mathrm{~mL}$ of dry DMF were used. $100 \mathrm{mg}$ of product was isolated. Coverage: $5.37 \mathrm{~N}_{3}$ / polymer, $\mathrm{N}_{3}$ coverage yield: $90 \%$, total yield: $86 \%$.

8-5.94: $108 \mathrm{mg}$ of dextran(6000) (18.0 $\mu \mathrm{mol}, 1.00$ eq.), $32.7 \mathrm{mg}$ of 7 (123 $\mu \mathrm{mol}, 6.85$ eq.), $108 \mathrm{mg}$ of dry $\mathrm{LiCl}, 100 \mu \mathrm{L}$ of dry DIPEA and $2.0 \mathrm{~mL}$ of dry DMF were used. $107 \mathrm{mg}$ of product was isolated. Coverage: $5.94 \mathrm{~N}_{3}$ / polymer, $\mathrm{N}_{3}$ coverage yield: $87 \%$, total yield: $87 \%$. 


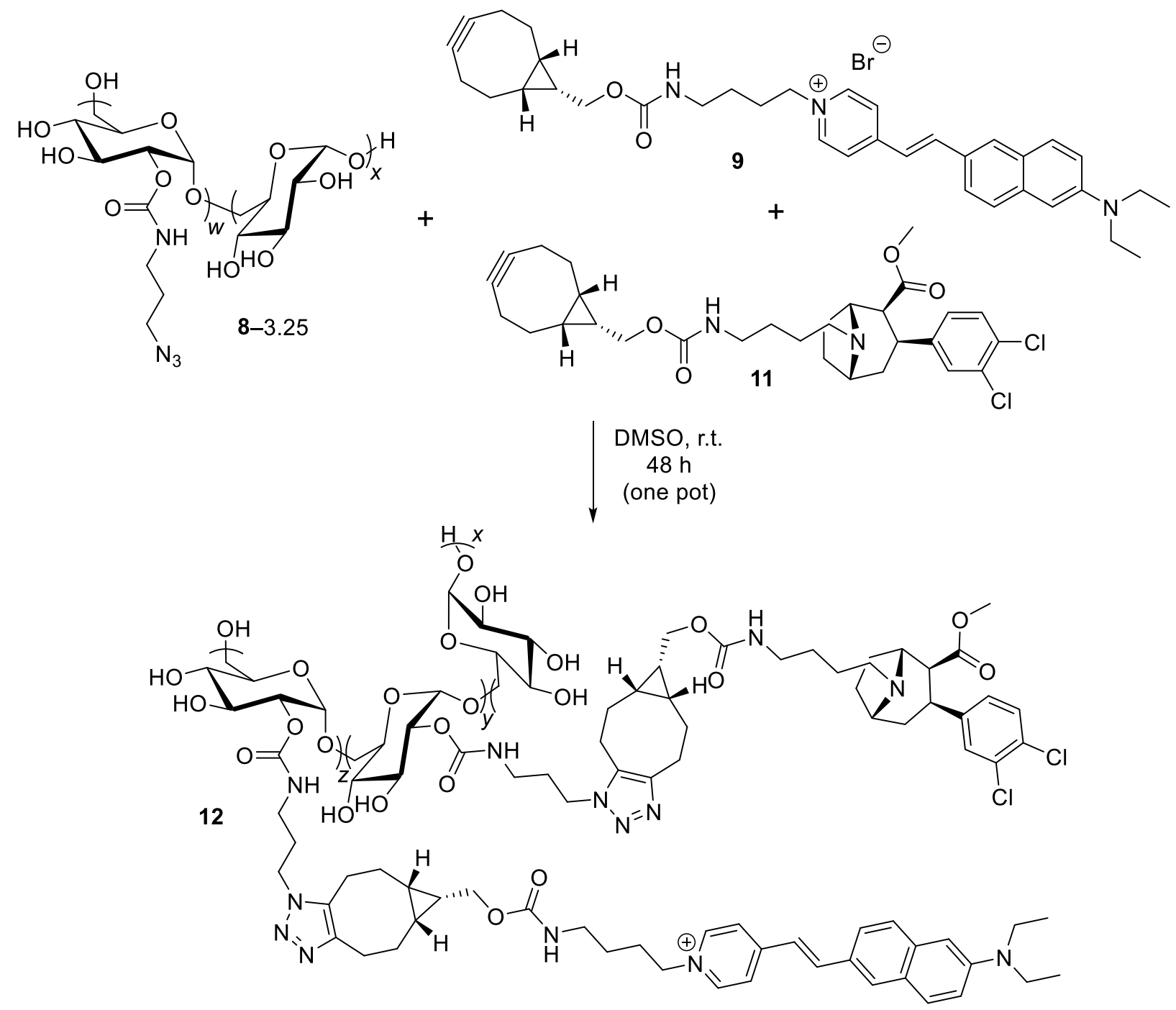

Synthesis of di-2-ANEP-VoLDeMo (12). To 8-3.25 (6.3 mg, $3.2 \mu \mathrm{mol}$ of $\mathrm{N}_{3}, 1.0$ eq. $\left.\mathrm{N}_{3}\right)$ in a $1 \mathrm{~mL} \mathrm{~V}$-shaped vial with a stir bar was added di-2-ANEPB-BCN $(9,73.9 \mu \mathrm{L}$ of a $10 \mathrm{mg} / \mathrm{mL}$ solution in DMSO, $0.74 \mathrm{mg}, 1.2 \mu \mathrm{mol}, 1.1 \times 1 / 3$ eq. per $\mathrm{N}_{3}$ group) and dichloropane-BCN (11, $170.8 \mu \mathrm{L}$ of a $7.7 \mathrm{mg} / \mathrm{mL}$ solution in DMSO, $1.3 \mathrm{mg}, 2.3 \mu \mathrm{mol}, 1.1 \times 2 / 3$ eq. per $\mathrm{N}_{3}$ group). The resulting solution was stirred at r.t. for $48 \mathrm{~h}$. After diluting with $\mathrm{H}_{2} \mathrm{O}(2.0 \mathrm{~mL})$, the crude solution was dialyzed against $\mathrm{H}_{2} \mathrm{O}$ (Slide-A-Lyzer G2, Thermo Fisher, MWCO $3500 \mathrm{Da}$ ) for 3 days with $2 \times$ per day exchange of dialysate for fresh $\mathrm{H}_{2} \mathrm{O}$. Post-dialysis, the membrane remained red indicating some extent of non-specific binding of ANEP to the dialysis membrane. Removing the aqueous solvent by rotary evaporation $\left(40{ }^{\circ} \mathrm{C}\right)$ provided di-2-ANEP-VoLDeMo $(\mathbf{1 2})$ as a red solid. 
Yield: $6.3 \mathrm{mg}$ (77\%). No signal at $2103 \mathrm{~cm}^{-1}$ was observed in the IR (ATR, diamond) spectrum of 12 which would correspond to the $\mathrm{N}_{3}$ asymmetric stretch of the parent 8-3.25. di-2-ANEP-VoLDeMo (12) was obtained with a coverage of 0.74 ANEP/polymer and 1.38 ligand/polymer as determined by ${ }^{1} \mathrm{H}$ NMR (Fig. S5).

IR characterization: No signal at $2103 \mathrm{~cm}^{-1}$ was observed in the IR (ATR, diamond) spectrum of di-2-ANEP-VoLDeMo (12) corresponding to the $\mathrm{N}_{3}$ asymmetric stretch in the parent 8-3.25, indicating full functionalization of linker groups in the starting material (Fig. S40).

NMR characterization: The coverage of di-2-ANEP-VoLDeMo (12) with di-2-ANEP VSD and dichloropane ligand was determined by ${ }^{1} \mathrm{H}$ NMR (500 MHz; DMSO- $d_{6} / \mathrm{D}_{2} \mathrm{O}$ 5:1, v/v) as follows: The integration of the major dextran acetal $\mathrm{C}-\mathrm{H}$ peak was set to the same value as observed for the corresponding parent azidopropyl carbamate dextran 8-3.25 (vide supra). Such integration corresponds to an ideal dextran(6000) polymer molecule with 36.89 glucose units. di-2-ANEP and dichloropane peaks in the aromatic region ( 6.5 to $9.0 \mathrm{ppm})$ were used to determine the average number of these tags per polymer molecule (Fig. S5; see Fig. S147 in the NMR Spectra section for the full spectrum). The ${ }^{1} \mathrm{H}$ NMR signals at $\delta 6.88,7.64,7.73,7.79,8.09$ and $8.69 \mathrm{ppm}$ correspond exclusively to ANEP. Therefore, a normalized (to $1 \mathrm{H}$ ) average integral of these signals was used to determine the average ANEP coverage (0.74 ANEP/pol.). The multiplets at 7.17 and $7.40 \mathrm{ppm}$ are overlapping signals of ANEP (1H in both signals) and the dichloropane ligand $(1 \mathrm{H}$ and $2 \mathrm{H}$ respectively). The average ANEP integral of 0.74 was thus subtracted from each of the multiplet's integrals. Then, a normalized average integral of these signals provided the average dichloropane ligand coverage (1.38 ligand/pol.). The expected 1:2 ANEP-ligand ratio thus translated into the final product. The overall coverage $(0.74+1.38=2.12)$ in the product is lower than the original coverage of the parent azidopropyl carbamate dextran (3.25 $\mathrm{N}_{3} /$ pol.) likely due to non-specific binding of higher coverage species to the dialysis membrane (vide supra).

Yield: $100 \%$ yield was defined as all dextran material recovered with 3.25 linkers/polymer functionalized in a 1:2 ratio with di-2-ANEP (i.e. 1.08 ANEP/polymer) and dichloropane (2.17 ligand/polymer).

Concentration and average molecular weight: Concentrations of di-2-ANEP-VoLDeMo (12) solutions used for biological characterization were calculated both with respect to the VSD, as well 
as the dextran polymer. To determine the latter, the average molecular weight of the product was estimated from the coverage of dextran with di-2-ANEP and dichloropane as determined by ${ }^{1} \mathrm{H}$ NMR. The dextran average molecular weight (6000 Da) was added to the molecular weight of the linkers $(2.12 \times 261.12 \mathrm{Da})$, di-2-ANEPB-BCN $(0.74 \times 630.67 \mathrm{Da})$ and dichloropane-BCN $(1.38 \times 561.54 \mathrm{Da}) \quad$ resulting in an average molecular weight of $7509 \mathrm{Da}$ for di-2-ANEP-VoLDeMo (12). Independent determination of the average molecular weight was not possible, because a suitable gel permeation chromatography (GPC) standard does not exist for such a dextran polymer functionalized with lipophilic groups; and poor ionization and fragmentation was observed by matrix-assisted laser desorption ionization mass spectrometry (MALDI-MS). 


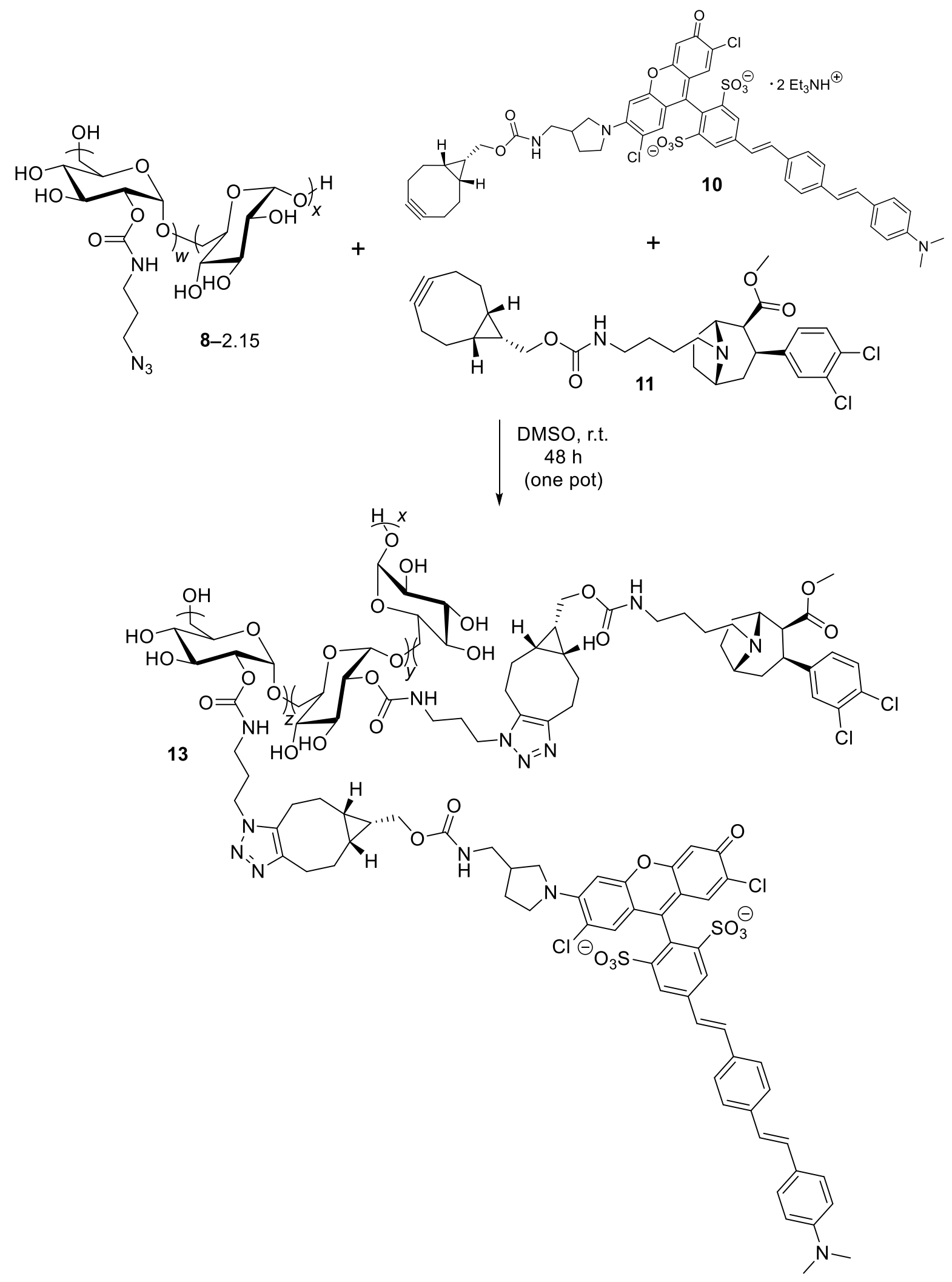


Synthesis of dsRVF5-VoLDeMo (13). The synthesis was repeated twice with a similar outcome in terms of yield and dextran coverage with the VSD and ligand. Targeting and voltage sensing properties of the two samples were also similar. A representative example: To 8-2.15 (5.1 mg, $1.7 \mu \mathrm{mol}$ of $\mathrm{N}_{3}, 1.0$ eq. $\left.\mathrm{N}_{3}\right)$ in a $1 \mathrm{~mL} \mathrm{~V}$-shaped vial with a stir bar was added dsRVF5-BCN (10, $76.7 \mu \mathrm{L}$ of a $10 \mathrm{mg} / \mathrm{mL}$ sol. in DMSO, $0.77 \mathrm{mg}, 0.63 \mu \mathrm{mol}, 1.1 \times 1 / 3$ eq. per $\mathrm{N}_{3}$ group) and dichloropane-BCN $(\mathbf{1 1}, 94.6 \mu \mathrm{L}$ of a $7.7 \mathrm{mg} / \mathrm{mL}$ sol. in DMSO, $0.73 \mathrm{mg}, 1.3 \mu \mathrm{mol}, 1.1 \times 2 / 3$ eq. per $\mathrm{N}_{3}$ group). The resulting solution was stirred at r.t. for $48 \mathrm{~h}$. After diluting with $\mathrm{H}_{2} \mathrm{O}(2.0 \mathrm{~mL})$, the crude solution was dialyzed against $\mathrm{H}_{2} \mathrm{O}$ (Slide-A-Lyzer G2, Thermo Fisher, MWCO 3500 Da) for 3 days with $2 \times$ per day exchange of dialysate for fresh $\mathrm{H}_{2} \mathrm{O}$. The membrane was colorless postdialysis, indicating negligible non-specific binding of dsRVF5 to the dialysis membrane (unlike ANEP, vide supra $)$. Removing the aqueous solvent by rotary evaporation $\left(40^{\circ} \mathrm{C}\right)$ provided dsRVF5-VoLDeMo (13) as a red solid. Yield: $5.9 \mathrm{mg}(91 \%)$. No signal at $2103 \mathrm{~cm}^{-1}$ was observed in the IR (ATR, diamond) spectrum of $\mathbf{1 3}$ which would correspond to the $\mathrm{N}_{3}$ asymmetric stretch of the parent 8-2.15. dsRVF5-VoLDeMo (13) was obtained with a coverage of $0.63 \mathrm{ANEP} /$ polymer and 1.39 ligand/polymer as determined by ${ }^{1} \mathrm{H}$ NMR (Fig. S6).

IR characterization: No signal at $2103 \mathrm{~cm}^{-1}$ was observed in the IR (ATR, diamond) spectrum of dsRVF5-VoLDeMo (13) corresponding to the $\mathrm{N}_{3}$ asymmetric stretch in the parent $8-2.15$, indicating full functionalization of linker groups in the starting material (Fig. S41).

NMR characterization: The coverage of dsRVF5-VoLDeMo (13) with dsRVF5 VSD and dichloropane ligand was determined by ${ }^{1} \mathrm{H}$ NMR $\left(500 \mathrm{MHz}\right.$, DMSO- $\left.d_{6} / \mathrm{D}_{2} \mathrm{O} 5: 1 v / v\right)$ similarly as for di-2-ANEP-VoLDeMo (12): The integration of the major dextran acetal $\mathrm{C}-\mathrm{H}$ peak was set to the same value as observed for the corresponding parent azidopropyl carbamate dextran 8-2.15 (vide supra). dsRVF5 and dichloropane peaks in the aromatic region ( 6.0 to $8.5 \mathrm{ppm})$ were used to determine the average number of these tags per polymer molecule (Fig. S6 and S7; see Fig. $S 148$ and $S 149$ in the NMR Spectra section for the full spectra). The ${ }^{1} \mathrm{H}$ NMR signals at $\delta 6.31$, $6.74,6.95$ and $8.23 \mathrm{ppm}$ correspond exclusively to dsRVF5. Therefore, a normalized (to $1 \mathrm{H}$ ) average integral of these signals was used to determine the average dsRVF5 coverage ( $0.63 \mathrm{dsRVF5/pol}$. and $0.74 \mathrm{dsRVF5} /$ pol. respectively). In the latter case, the signal at $8.23 \mathrm{ppm}$ was not used for the calculation due to its overlap with a residual -OH/-NH signal (confirmed by ${ }^{1} \mathrm{H}$ NMR in pure DMSO- $d_{6}$ where the signals were separated, Fig. S150). The multiplets at 7.16 
ppm and 7.35-7.65 ppm are overlapping signals of dsRVF5 (1H and $8 \mathrm{H}$ respectively) and the dichloropane ligand $(1 \mathrm{H}$ and $2 \mathrm{H}$ respectively). Therefore, the corresponding multiple of the dsRVF5 coverage value ( 0.63 or 0.74 respectively) was subtracted from each of the multiplet's integrals. Then, a normalized average integral of these signals provided the average dichloropane ligand coverage (1.39 ligand/polymer and 1.45 ligand/polymer respectively). The expected 1:2 dsRVF5-dichloropane ratio thus translated into the final product. The overall coverage values $(0.63+1.39=2.02$; and $0.74+1.45=2.19$ respectively $)$ for the products are close to the original coverage of the parent azidopropyl carbamate dextran (2.15 $\mathrm{N}_{3} /$ polymer). A sharp contrast in the ${ }^{1} \mathrm{H}$ NMR spectra of dsRVF5-VoLDeMo in $\mathrm{D}_{2} \mathrm{O}$ vs. DMSO- $d_{6}$ was observed (Fig. S8; compare to Fig. $3 a, b$ in the main text for di-2-ANEP-VoLDeMo).

Yield: The per cent yield of dsRVF5-VoLDeMo (13) was defined analogously to the one of di-2-ANEP-VoLDeMo (12; vide supra).

Concentration and average molecular weight: Concentrations of dsRVF5-VoLDeMo (13) solutions used for biological characterization were calculated both with respect to the VSD, as well as the dextran polymer. To determine the latter, the average molecular weight of dsRVF5-VoLDeMo (13) was calculated analogously to the one of di-2-ANEP-VoLDeMo (12). The dextran average molecular weight $(6000 \mathrm{Da})$ was added to the molecular weight of the linkers ( 2.02 or $2.19 \times 261.12 \mathrm{Da})$, dsRVF5-BCN (0.63 or $0.74 \times 1225.39 \mathrm{Da})$ and dichloropane-BCN $(1.39$ or $1.45 \times 561.54 \mathrm{Da})$ resulting in an average molecular weight of $8080 \mathrm{Da}$ and $8293 \mathrm{Da}$ respectively for the two samples of dsRVF5-VoLDeMo (13). Independent determination of the average molecular weight was not possible, because a suitable GPC standard does not exist for such a dextran polymer functionalized with lipophilic groups; and poor ionization and fragmentation was observed by MALDI-MS. 


\section{Functionalization of dextran with BCN carbonate linkers}

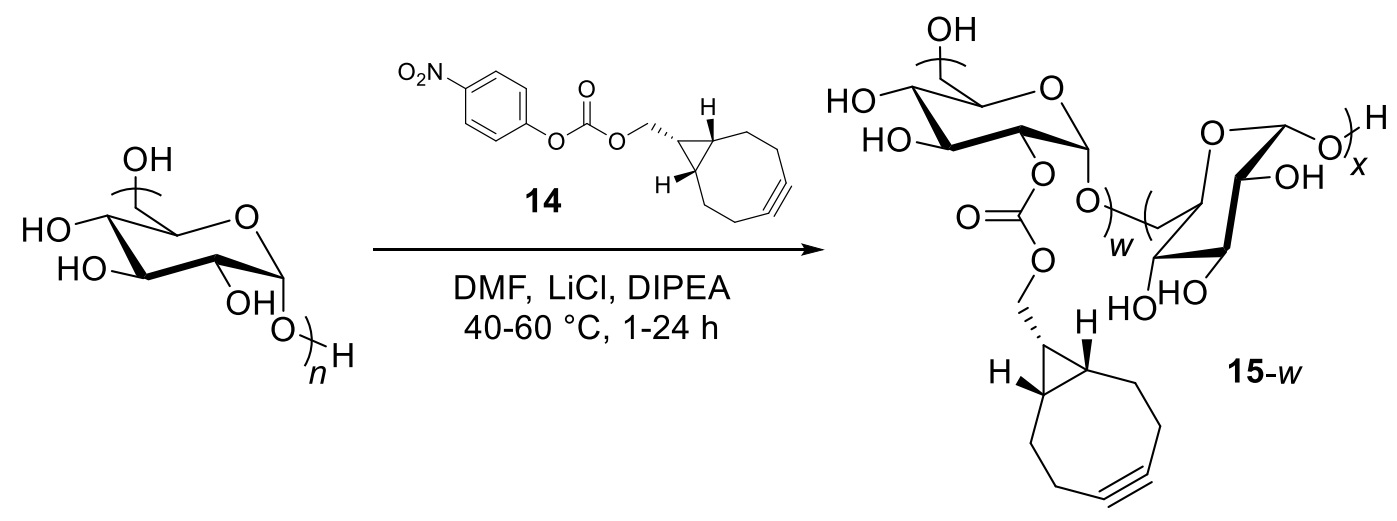

Commercial dextran (avg. $M_{w}=6000$, Chem-Impex International) was dried under high vacuum for 4 days (until constant mass, $\sim 6 \%$ weight loss) prior to use.

Commercial $\mathrm{LiCl}$ (anhydrous, Alfa Aesar) was dried under high vacuum at $150{ }^{\circ} \mathrm{C}$ overnight prior to use.

\section{General procedure for synthesis of BCN-carbonate dextran(6000) (15-w). Dried} dextran(6000) (1.0 eq.) and dried $\mathrm{LiCl}(1.0 \mathrm{mg} / \mathrm{mg}$ dextran) were dissolved in dry DMF (20 $\mu \mathrm{L} / \mathrm{mg}$ dextran) at $80-90{ }^{\circ} \mathrm{C}$ under Ar atmosphere (dissolution was completed within $10 \mathrm{~min}$ ). Dry DIPEA (1.0 $\mu \mathrm{L} / \mathrm{mg}$ dextran) was added, followed by addition of (1R,8S,9s)-bicyclo[6.1.0]non-4-yn-9yl)methyl (4-nitrophenyl) carbamate (BCN-OpNP, 14, amount specified in each example) resulting in immediate change of color from colorless to yellow. The resulting solution was stirred at $40-60^{\circ} \mathrm{C}$ for $1-24 \mathrm{~h}$. After cooling to r.t., volatiles were removed under high vacuum. The resulting thick yellow to brown oil was dissolved in $\mathrm{H}_{2} \mathrm{O}(2.0 \mathrm{~mL})$ and dialyzed against $\mathrm{H}_{2} \mathrm{O}$ (Slide-A-Lyzer G2, Thermo Fisher, MWCO $2000 \mathrm{Da}$ ) for 3 days with $2 \times$ per day exchange of dialysate for fresh $\mathrm{H}_{2} \mathrm{O}$. After microfiltration $(0.45 \mu \mathrm{m}$ nylon filter) and removal of the aqueous solvent by rotary evaporation $\left(30^{\circ} \mathrm{C}\right), \mathrm{BCN}$-carbonate dextran $(\mathbf{1 5}-w)$ was obtained as a white solid. Number $w$ in 15-w designates the average number of BCN groups per 6000 Da dextran polymer molecule available for click chemistry. While the number of $\mathrm{BCN}$ groups attached to dextran could be determined by ${ }^{1} \mathrm{H}$ NMR spectroscopy, only $72-84 \%$ of these $\mathrm{BCN}$ groups were able to further react with organic azides by SP-AAC click chemistry (as determined by a test reaction with excess organic azide, vide infra). A higher percentage of available $\mathrm{BCN}$ groups was 
generally observed for shorter reaction times or lower reaction temperatures, pointing to some degree of instability of the BCN moiety under the reaction conditions.

NMR characterization: All samples of $\mathbf{1 5}-w$ were co-evaporated with $\mathrm{D}_{2} \mathrm{O}(1.5 \mathrm{~mL})$ prior to NMR analysis to reduce the intensity of the $-\mathrm{OH}$ signal via $\mathrm{H} / \mathrm{D}$ exchange. ${ }^{1} \mathrm{H}$ NMR $(500 \mathrm{MHz}$; $\left.\mathrm{D}_{2} \mathrm{O} / \mathrm{CD}_{3} \mathrm{CN} 5: 1, v / v\right)$ provided clearly separated signals of the dextran backbone and all BCN groups (the zoomed-in spectrum of $\mathbf{1 5 - 3 . 5 4}$ is shown in Fig. S29; full spectra of 15-w with four different $w$ are shown in Fig. S151 to S154 in the NMR spectra section). The integration of dextran acetal $\mathrm{C}-\mathrm{H}$ signal at $4.97 \mathrm{ppm}$ was set to 33.49 (the same integration value as found in the corresponding unsubstituted dextran(6000)). The total coverage with BCN carbamate groups was obtained as the average integration value of all $\mathrm{BCN}$ groups normalized to $1 \mathrm{H}$.

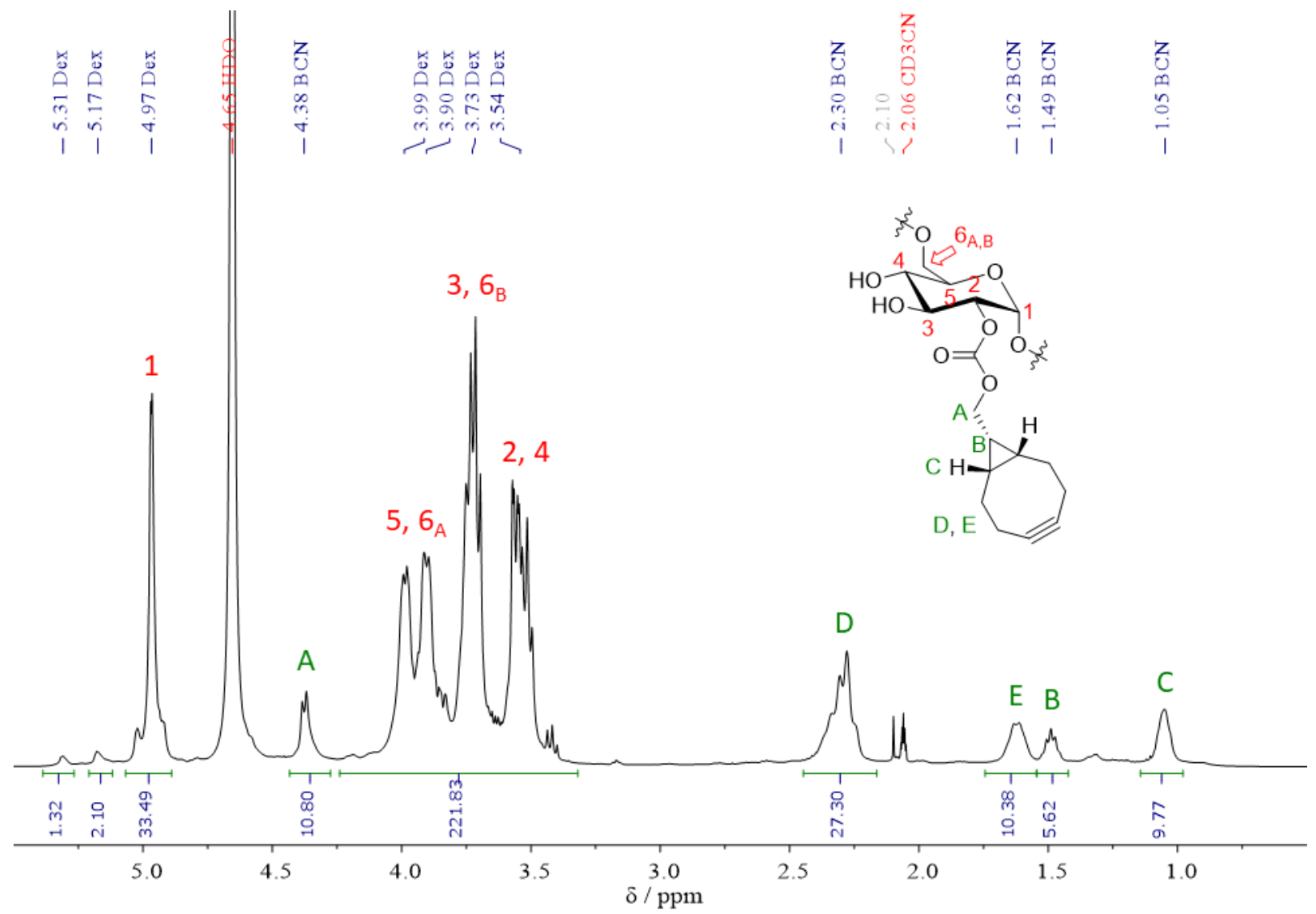

Figure S29. ${ }^{1} \mathrm{H}$ NMR $\left(500 \mathrm{MHz} ; \mathrm{D}_{2} \mathrm{O} / \mathrm{CD}_{3} \mathrm{CN} 5: 1, v / v\right)$ of $15-3.54$. Dextran $\mathrm{C}-\mathrm{H}$ signals $=$ red numbers, assignment based on ref. ${ }^{32}$ Integration of the acetal $\mathrm{C}^{1}-\mathrm{H}$ (4.97 ppm) was set to 33.49 (same integration value as in the parent non-functionalized $6 \mathrm{kDa}$ dextran). $\mathrm{BCN} \mathrm{C}-\mathrm{H}$ signals $=$ green letters. The total coverage with $\mathrm{BCN}$ 
carbamate groups (4.91) was obtained as the average integration value of all BCN groups normalized to $1 \mathrm{H}$. The number of available BCN groups for further click chemistry $(w=3.54)$ was determined by a subsequent test reaction with organic azide (vide infra).

Determination of the number of available BCN groups $(w)$. In initial experiments, we were not able to achieve full conversion of BCN groups in 15- $w$ in SP-AAC click reactions with organic azides even with excess azide and prolonged reaction times. We therefore characterized every sample of $\mathbf{1 5}-w$ by the following test reaction using excess azide:

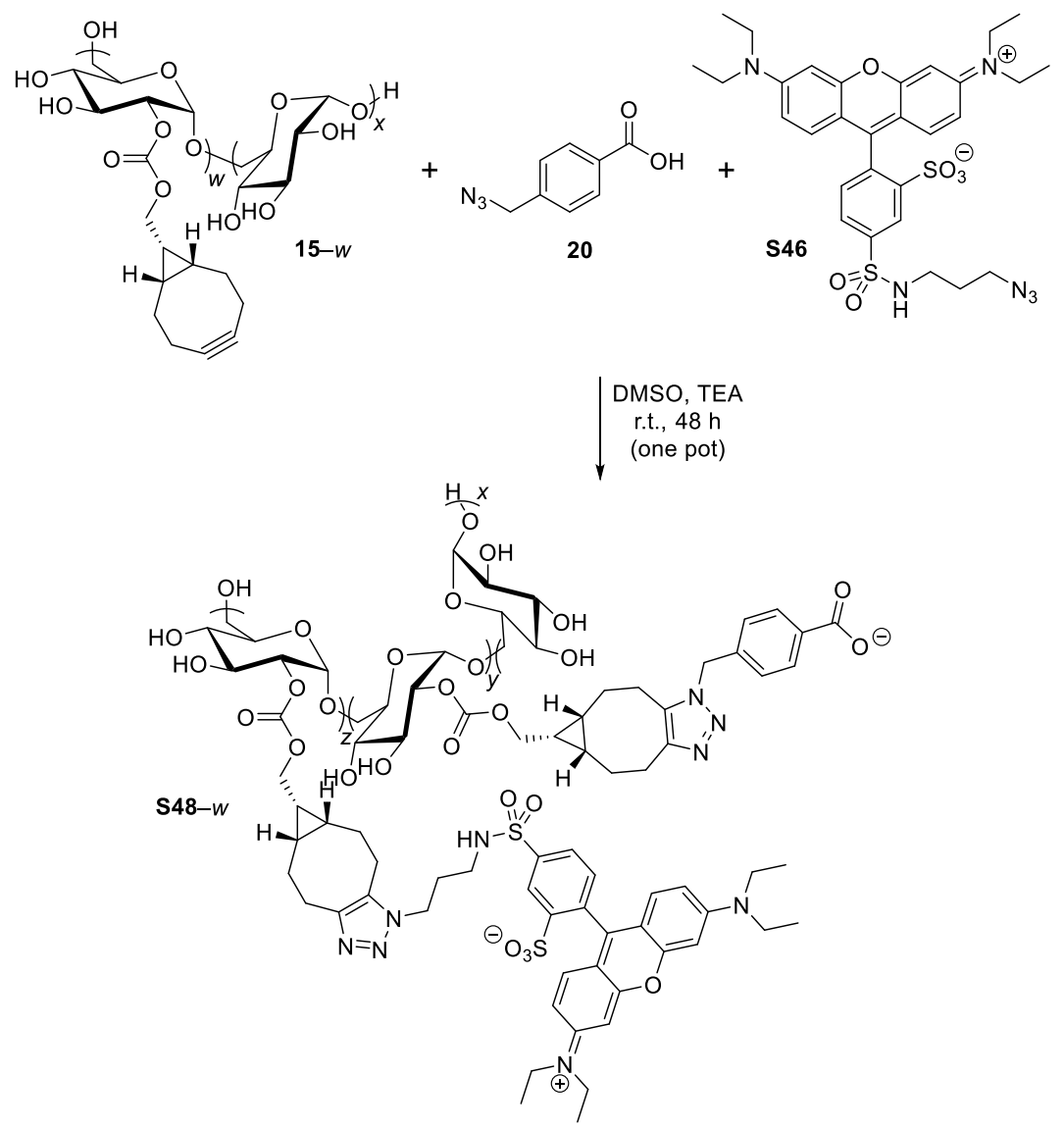

To $15-w(5.0 \mathrm{mg})$ in a $1 \mathrm{~mL} \mathrm{~V}$-shaped vial with a stir bar was added $20(5.0 \mathrm{mg} / \mathrm{mL}$ solution in DMSO, 1.35 eq. per BCN group) and $\mathbf{S 4 6}$ (10 mg/mL solution in DMSO, 0.15 eq. per BCN group) and $\mathrm{Et}_{3} \mathrm{~N}$ (4.0 eq. with respect to 20). The resulting solution was stirred at r.t. for $48 \mathrm{~h}$. After diluting with $\mathrm{H}_{2} \mathrm{O}(1.0 \mathrm{~mL})$, the crude solution was passed through a Sephadex column (G-25, Medium, GE Healthcare; $4 \mathrm{~mL}$ of Sephadex in a $14 \mathrm{~mm} \varnothing$ column) using aq. $\mathrm{Et}_{3} \mathrm{NH}^{+} \mathrm{Cl}^{-}$ $(20 \mathrm{mg} / 100 \mathrm{~mL})$ as the eluent. The first colored fraction was collected, and volatiles were removed 
by rotary evaporation $\left(30^{\circ} \mathrm{C}\right)$. The resulting solid was washed with $\mathrm{MeOH}(3 \times 1 \mathrm{~mL}$; sonication and centrifugation), co-evaporated with $\mathrm{D}_{2} \mathrm{O}(2 \mathrm{~mL})$ and analyzed by ${ }^{1} \mathrm{H}$ NMR (500 MHz; DMSO$d_{6} / \mathrm{D}_{2} \mathrm{O} 5: 1, v / v$ ). The zoomed-in spectrum of $\mathbf{S 4 8}-3.54$ (corresponding to analysis of 15-3.54) is shown in Fig. S30; full spectra of $\mathbf{S 4 8}-w$ with four different $w$ are shown in Fig. S155 to S158 in the NMR spectra section. The number $(w)$ of BCN groups in 15-w available for SP-AAC click chemistry was determined from the weighted average of the integration values of the nonoverlapping signals at 7.14 and 6.94 ppm (Fig. S30).

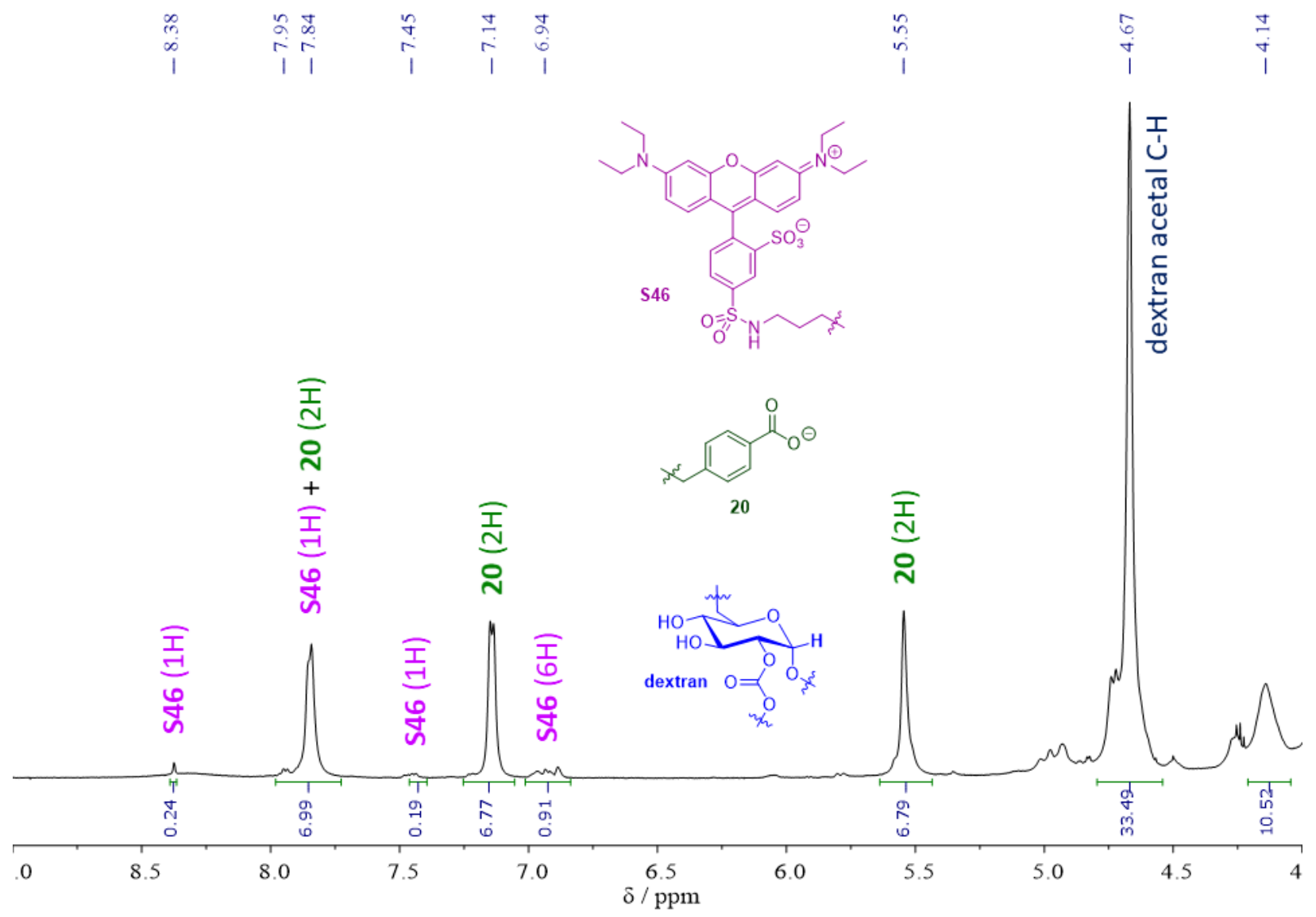

Figure S30. ${ }^{1} \mathrm{H}$ NMR $\left(500 \mathrm{MHz}\right.$; DMSO- $\left.d_{6} / \mathrm{D}_{2} \mathrm{O} 5: 1, v / v\right)$ of $\mathbf{S 4 8}-3.54$. Integration of the acetal $\mathrm{C}-\mathrm{H}$ (4.67 ppm) was set to 33.49 as in the corresponding starting material (15-3.54). The non-overlapping signals at $7.14 \mathrm{ppm}$ and $6.94 \mathrm{ppm}$ were used to calculate the number of available BCN groups for SP-AAC click chemistry $(w=6.77 / 2$ $+0.91 / 6=3.54)$.

BCN coverage yield: Defined as the ratio between linker coverage as determined by ${ }^{1} \mathrm{H}$ NMR (vide supra) and maximum theoretical coverage $(n(\mathbf{1 4}) / n$ (dextran)). Example: To prepare 15- 
3.54, $24.4 \mu \mathrm{mol}$ of $\mathbf{1 4}$ was allowed to react with $3.43 \mu \mathrm{mol}$ of dextran(6000), while the experimental coverage (as determined by NMR) is $4.91 \mathrm{BCN} /$ pol. The $\mathrm{BCN}$ coverage yield is thus $4.91 \times 3.43 \mu \mathrm{mol} / 24.4 \mu \mathrm{mol}=0.69(69 \%)$.

Total yield: $100 \%$ total yield was defined as $100 \%$ material recovery with a maximum theoretical linker coverage. Attachment of one BCN carbonate group increases the $M_{w}$ by $176.22 \mathrm{~g} \mathrm{~mol}^{-1}$. Example: For 15-3.54, the maximum theoretical linker coverage was $24.4 \mu \mathrm{mol} 14$ / $3.43 \mu \mathrm{mol}$ of dextran $(6000)=7.1$. The theoretical average $M_{w}$ of the product is thus $6000 \mathrm{~g} \mathrm{~mol}^{-1}+$ $7.1 \times 176.22 \mathrm{~g} \mathrm{~mol}^{-1}=7251 \mathrm{~g} \mathrm{~mol}^{-1} .20 .6 \mathrm{mg}$ of used dextran(6000) implies a theoretical total yield of $(7251 / 6000) \times 20.6 \mathrm{mg}=24.9 \mathrm{mg}$. An isolated yield of $20.4 \mathrm{mg}$ thus corresponds to $82 \%$.

Regioselectivity of functionalization: We did not study the regioselectivity of this reaction. Dupayage et al. ${ }^{33}$ reported that the nucleophilicity of dextran - $\mathrm{OH}$ groups follows the following trend: $\mathrm{C}^{2}>\mathrm{C}^{4}>\mathrm{C}^{3}$ (see Fig. S29 for numbering). Therefore, we assume that attachment of BCN carbamate linkers presented herein follows this trend. For simplicity and clarity, only the BCNcarbonate attached to $\mathrm{C}^{2}$ is shown in all figures throughout this article. However, it is likely that a minor portion of linkers is also attached to $\mathrm{C}^{4}, \mathrm{C}^{3}$ and the terminal $\mathrm{C}^{6}$.

\section{Specific examples of $15-w$ synthesis:}

15-2.58: $104 \mathrm{mg}$ of dextran(6000) (17.3 $\mu \mathrm{mol}, 1.0$ eq.), $26.2 \mathrm{mg}$ of 14 (83.1 $\mu \mathrm{mol}, 4.8$ eq.), $104 \mathrm{mg}$ of dry $\mathrm{LiCl}, 100 \mu \mathrm{L}$ of dry DIPEA and $2.0 \mathrm{~mL}$ of dry DMF were heated at $60{ }^{\circ} \mathrm{C}$ for $60 \mathrm{~min} .90 \mathrm{mg}$ of product was isolated. Total BCN coverage (NMR): $3.08 \mathrm{BCN} /$ polymer, BCN coverage yield: $64 \%$, total yield: $76 \%$, available BCN coverage (w): $2.58 \mathrm{BCN} /$ polymer, per cent available BCN: $84 \%$.

15-2.95: $52.0 \mathrm{mg}$ of dextran(6000) (8.67 $\mu \mathrm{mol}, 1.0$ eq.), $12.6 \mathrm{mg}$ of $\mathbf{1 4}$ (40.0 $\mu \mathrm{mol}, 4.6 \mathrm{eq}$.), $49 \mathrm{mg}$ of dry $\mathrm{LiCl}, 50 \mu \mathrm{L}$ of dry DIPEA and $1.0 \mathrm{~mL}$ of dry DMF were heated at $40{ }^{\circ} \mathrm{C}$ for $24 \mathrm{~h} .45 .7 \mathrm{mg}$ of product was isolated. Total BCN coverage (NMR): $3.54 \mathrm{BCN} /$ polymer, BCN coverage yield: $77 \%$, total yield: $77 \%$, available $\mathrm{BCN}$ coverage $(w): 2.95 \mathrm{BCN} /$ polymer, per cent available BCN: $83 \%$. 
15-3.28: $20.6 \mathrm{mg}$ of dextran(6000) (3.43 $\mu \mathrm{mol}, 1.0$ eq.), $7.7 \mathrm{mg}$ of 14 (24.4 $\mu \mathrm{mol}, 7.1$ eq.), $20 \mathrm{mg}$ of dry $\mathrm{LiCl}, 20 \mu \mathrm{L}$ of dry DIPEA and $400 \mu \mathrm{L}$ of dry DMF were heated at $60{ }^{\circ} \mathrm{C}$ for $60 \mathrm{~min} .18 .1 \mathrm{mg}$ of product was isolated. Total BCN coverage (NMR): $4.01 \mathrm{BCN} /$ polymer, BCN coverage yield: $56 \%$, total yield: $73 \%$, available BCN coverage $(w): 3.28 \mathrm{BCN} /$ polymer, per cent available BCN: $82 \%$.

15-3.54: $20.6 \mathrm{mg}$ of dextran(6000) (3.43 $\mu \mathrm{mol}, 1.0$ eq.), $7.7 \mathrm{mg}$ of 14 (24.4 $\mu \mathrm{mol}, 7.1$ eq.), $20 \mathrm{mg}$ of dry LiCl, $20 \mu \mathrm{L}$ of dry DIPEA and $400 \mu \mathrm{L}$ of dry DMF were heated at $60{ }^{\circ} \mathrm{C}$ for $24 \mathrm{~h} .20 .4 \mathrm{mg}$ of product was isolated. Total BCN coverage (NMR): $4.91 \mathrm{BCN} /$ polymer, BCN coverage yield: $69 \%$, total yield: $82 \%$, available $\mathrm{BCN}$ coverage $(w)$ : $3.54 \mathrm{BCN} /$ polymer, per cent available $\mathrm{BCN}$ : $72 \%$. 


\section{Compatibility of acyl imidazole linkers with dextran}

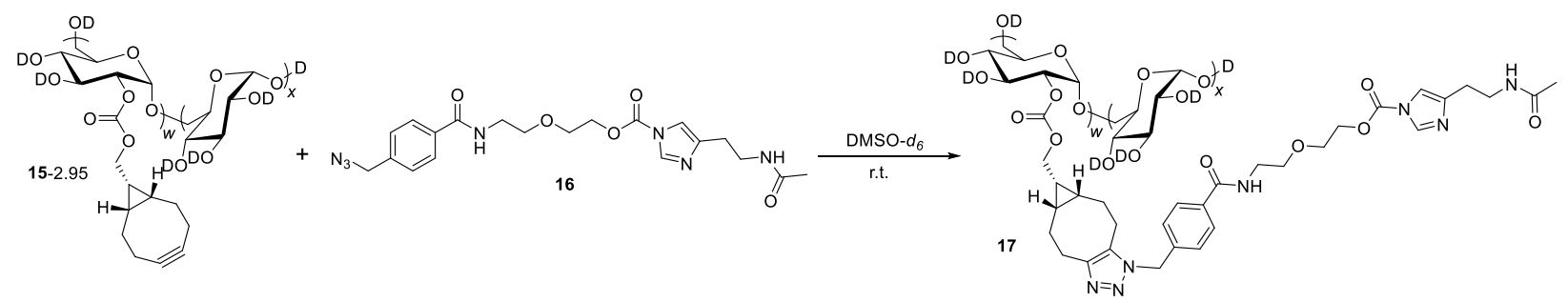

To 15-2.95 (6.2 mg, $2.8 \mu \mathrm{mol} \mathrm{BCN}, 1.0$ eq.) was added a solution of $16(220.4 \mu \mathrm{L}, c=5.0 \mathrm{mg} / \mathrm{mL}$ in DMSO- $d_{6}, 2.5 \mu \mathrm{mol}, 0.9$ eq.). The reaction mixture was sonicated and stirred until complete dissolution of 15-2.95 ( 20-25 min). The solution was transferred into a DMSO- $d_{6}$-matched Shigemi NMR cuvette and the reaction progress was monitored by ${ }^{1} \mathrm{H}$ NMR (Fig. $7 c$ in the main text) for 31 days. Subsequently, the DMSO- $d_{6}$ solvent was removed under high vacuum overnight. The product was redissolved in $\mathrm{D}_{2} \mathrm{O}(220 \mu \mathrm{L})$, transferred into a $\mathrm{D}_{2} \mathrm{O}$-matched Shigemi NMR cuvette and the stability of the product was monitored for four days (Fig. S20). Gradual decrease in intensity of the signals of $\mathbf{1 7}$ and increase in intensity of AcHA signals was observed on the timescale of hours to days (Fig. S20). We hypothesize that the model acyl imidazole product 17 partially decomposed during DMSO- $d_{6}$ solvent removal under high vacuum (i.e. at a higher concentration in DMSO- $d_{6}$ or in the solid state), as $14 \%$ decomposition was observed already in the first ${ }^{1} \mathrm{H}$ NMR spectrum in $\mathrm{D}_{2} \mathrm{O}$ (after $10 \mathrm{~min}$ in aqueous solution) which is not in line with the further observed decomposition kinetics in $\mathrm{D}_{2} \mathrm{O}$. 


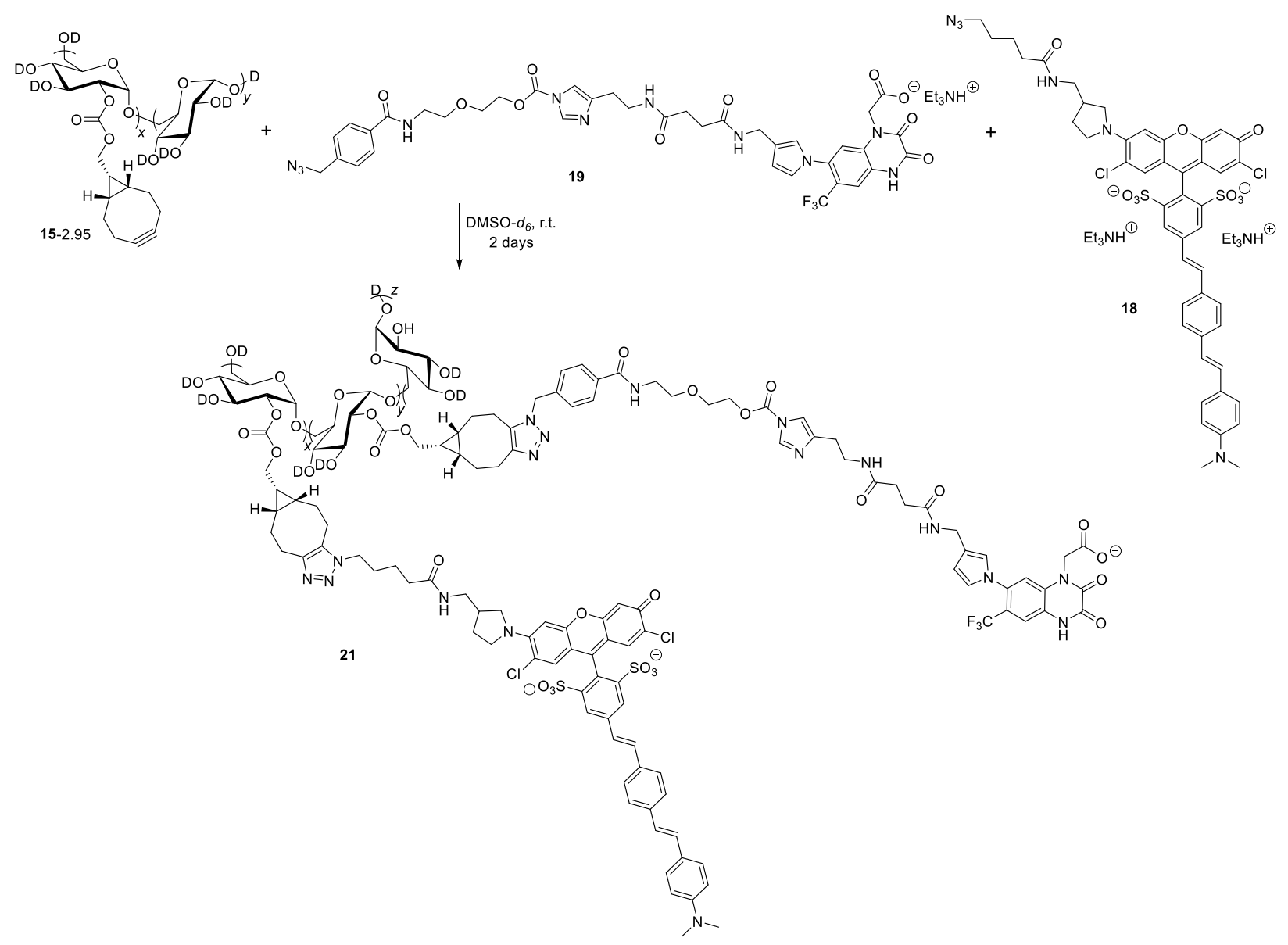

To 15-2.95 (4.9 mg, $2.2 \mu \mathrm{mol}$ of $\mathrm{BCN}, 1.0$ eq.) in a $1 \mathrm{~mL}$ V-shaped vial were added $19(217.5 \mu \mathrm{L}$ of a $6.9 \mathrm{mg} / \mathrm{mL}$ solution in DMSO-d $d_{6}, 1.6 \mu \mathrm{mol}, 0.71$ eq.) and $18(40.5 \mu \mathrm{L}$ of a $15 \mathrm{mg} / \mathrm{mL}$ solution in DMSO- $d_{6}, 0.52 \mu \mathrm{mol}, 0.24$ eq.). The overall concentration of organic azides was $8 \mathrm{mM}(6 \mathrm{mM}$ 19 and $2 \mathrm{~mm} \mathrm{18}$ ). The reaction mixture was stirred at r.t. until complete dissolution of 15-2.95 ( 20 min). Subsequently, the solution was transferred into a DMSO-d6-matched Shigemi NMR cuvette and the reaction progress was monitored by ${ }^{1} \mathrm{H}$ NMR (Fig. S22). The reaction was completed within the $50 \mathrm{~h}$ reaction time and no sign of $\mathbf{S 2}$ (the expected product of acyl imidazole decomposition) formation was observed. The resulting solution was stored as a stock at $-80{ }^{\circ} \mathrm{C}$ as obtained ( $c=2 \mathrm{mM}$ with respect to the VSD). 


\section{Control probe 22}

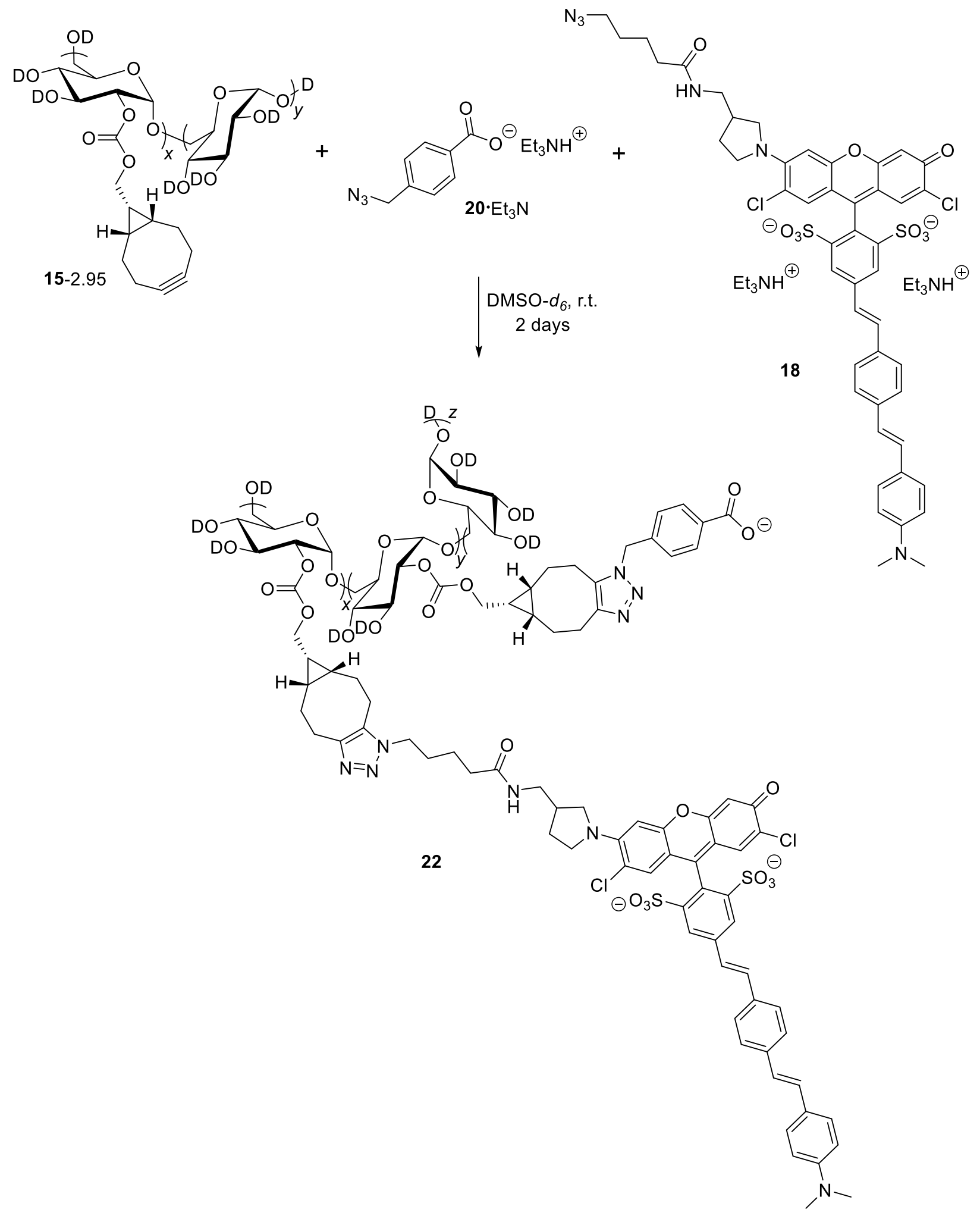


To 15-2.95 (5.1 mg, $2.3 \mu \mathrm{mol}$ of BCN, 1.0 eq.) in a $1 \mathrm{~mL}$ V-shaped vial were added DMSO- $d_{6}$ $(169.4 \mu \mathrm{L}), 20 \cdot \mathrm{Et}_{3} \mathrm{~N}\left(57.3 \mu \mathrm{L}\right.$ of a $5.0 \mathrm{mg} / \mathrm{mL}$ solution in DMSO- $d_{6}, 1.6 \mu \mathrm{mol}, 0.71$ eq.) and $\mathbf{1 8}$ (42.2 $\mu \mathrm{L}$ of a $15 \mathrm{mg} / \mathrm{mL}$ solution in DMSO- $d_{6}, 0.54 \mu \mathrm{mol}, 0.24 \mathrm{eq}$.). The overall concentration of organic azides was $8 \mathrm{mM}(6 \mathrm{mM} 20$ and $2 \mathrm{mM} \mathrm{18})$. The reaction mixture was stirred at r.t. until complete dissolution of 15-2.95 ( $20 \mathrm{~min})$. Subsequently, the solution was transferred into a DMSO- $d_{6}$-matched Shigemi NMR cuvette and the reaction progress was monitored by ${ }^{1} \mathrm{H}$ NMR (Fig. S23). The reaction was completed within the $48 \mathrm{~h}$ reaction time. The resulting solution was stored as a stock at $-80{ }^{\circ} \mathrm{C}$ as obtained ( $c=2 \mathrm{mM}$ with respect to the VSD). 


\section{Biological Characterizations}

\section{Preparation and Storage of Stock Solutions}

Stock solutions of VoLDeMo probes (12 and 13), di-2-ANEPEQ (1, AnaSpec, Fremont, CA), and dsRVF5 (2) were prepared at $200 \mu \mathrm{M}$ in DMSO and stored at $-80{ }^{\circ} \mathrm{C}$. Stock solutions of nomifensine (Sigma-Aldrich, St. Louis, MO), APP+, and FFN102 were prepared at $10 \mathrm{~mm}$ in DMSO and stored at $-80{ }^{\circ} \mathrm{C}$. Stock solutions of CAM2(Fl) (Fukanoshi, Tokyo, Japan) were prepared at $1 \mathrm{mM}$ in DMSO and stored at $-80{ }^{\circ} \mathrm{C}$. Solutions of dsRVF5-VoLDeCAM (21) and control probe 22 were obtained at $2 \mathrm{mM}$ in DMSO- $d_{6}$ (with respect to VSD) from their synthesis and stored as stock solutions at $-80^{\circ} \mathrm{C}$. Stock solutions of NBQX disodium salt (Tocris Bioscience, Bristol, UK) were prepared at $10 \mathrm{mM}$ in distilled water and stored at $-80{ }^{\circ} \mathrm{C}$. Stock solutions of tetrodotoxin (TTX; Tocris Bioscience, Bristol, UK) were prepared at $1 \mathrm{mM}$ in distilled water and stored at $-20{ }^{\circ} \mathrm{C}$.

\section{In HEK Cell Culture}

Growth of HEK cell culture. A human embryonic kidney 293 (HEK) EM4 cell line stably transfected with human dopamine transporter (hDAT) and an empty-vector transfected EM4 cell line ${ }^{34}$ were provided by Dr. Jonathan Javitch and Dr. Mark Sonders of the Department of Psychiatry at New York State Psychiatric Institute/Columbia University Medical Center. They were cultured in Dulbecco's modified Eagle medium (DMEM) with GlutaMAX (Gibco-Thermo Fisher Scientific, Waltham, MA) supplemented with 10\% FBS and 1\% Penicillin-Streptomycin.

Determination of $\boldsymbol{I C}_{50}$ of compound 5 to hDAT. hDAT-HEK cells (Passage \# 15 to 20) were plated at a density of $1.5 \times 10^{5}$ cells/well onto a white, clear-bottom 96-well plate pre-coated with Poly-D-lysine solution $(0.1 \mathrm{mg} / \mathrm{mL}$, Sigma-Aldrich) and allowed to grow for $24 \mathrm{~h}$ to provide around $100 \%$ confluency. Prior to experiment, experimental media [phenol red-free DMEM (Gibco) supplemented with 1\% FBS and 1\% Penicillin-Streptomycin] was first prepared containing eight different concentrations of $5(0.1 \mathrm{nM}, 1 \mathrm{nM}, 5 \mathrm{nM}, 10 \mathrm{nM}, 25 \mathrm{nM}, 50 \mathrm{nM}, 100 \mathrm{nM}$, and $1000 \mathrm{nM}$ ) plus either nomifensine (a potent inhibitor of DAT; ${ }^{1} 2 \mu \mathrm{M}, 0.05 \% \mathrm{w} / \mathrm{v}$ ) or DMSO (vehicle, $0.02 \% \mathrm{v} / \mathrm{v}$ ). Each of the sixteen experimental conditions (eight concentrations of $\mathbf{5}$, nomifensine/DMSO) were carried out in six replicates in each plate utilizing all 96 wells. During 
the experiment, the growth media was replaced with experimental media with sixteen different conditions and pre-incubated for $30 \mathrm{~min}$ at $37^{\circ} \mathrm{C}$. APP+ (a known fluorescent hDAT substrate; ${ }^{34}$ $2 \mu \mathrm{M})$ was then added to all wells and cells were further incubated for $10 \mathrm{~min}$ at room temperature. After incubation, cells were washed $3 \times$ with Dulbecco's phosphate-buffered saline (DPBS; HyClone, GE Healthcare Life Sciences, Pittsburgh, PA) and maintained in DPBS during the remainder of APP+ fluorescence uptake measurement, which was performed with a H1MF plate reader (BioTek, Winooski, VT) using $3 \times 3$ area scan and bottom-read mode. The excitation and emission wavelengths were set at $420 \mathrm{~nm}$ and $500 \mathrm{~nm}$, respectively. For data analysis, the mean fluorescence of six inhibited wells (containing nomifensine) was subtracted from that of six noninhibited wells (with vehicle) for each probe concentration. The same experiment was performed on three different days for $n=3$ experiments and all data were normalized to the maximum fluorescence intensity of each experiment. The $I C_{50}$ of dichloropane derivative 5 to hDAT was determined by non-linear regression using a 1:1 binding model (Fig. S1) measuring the dosedependent inhibition of APP+ uptake (GraphPad Prism version 6, GraphPad Software Inc., San Diego, CA).

Determination of $I C_{50}$ of compounds 6, 12 and 13 to hDAT. hDAT-HEK cells (Passage \# 15 to 20) were plated at a density of $1.5 \times 10^{5}$ cells/well onto a white, clear-bottom 96-well plate precoated with poly-D-lysine solution $(0.1 \mathrm{mg} / \mathrm{mL}$, Sigma-Aldrich) and allowed to grow for $24 \mathrm{~h}$ to provide around $100 \%$ confluency. Prior to the experiment, experimental media [phenol red-free DMEM (Gibco) supplemented with 1\% FBS and 1\% Penicillin-Streptomycin] was first prepared containing different concentrations of $6(0.1 \mathrm{nM}, 1 \mathrm{nM}, 10 \mathrm{nM}, 20 \mathrm{nM}, 50 \mathrm{nM}, 100 \mathrm{nM}, 250 \mathrm{nM}$ and $1000 \mathrm{nM}), 12$ or 13 (1 nM, $3.3 \mathrm{nM}, 10 \mathrm{nM}, 20 \mathrm{nM}, 50 \mathrm{nM}, 100 \mathrm{nM}, 250 \mathrm{nM}, 500 \mathrm{~nm}$ and $1000 \mathrm{nM})$; or DMSO (vehicle; same volume as added stock solutions of 6, 12 or 13). During the experiment, the cells were washed with DPBS $(120 \mu \mathrm{L})$ and pre-incubated with experimental media with the tested compounds or vehicle $(63 \mu \mathrm{L})$ for $45 \mathrm{~min}$ at $37^{\circ} \mathrm{C}$. APP+ $(1.1 \mu \mathrm{M})$ was then added to all wells and cells were further incubated for $25 \mathrm{~min}$ at $37^{\circ} \mathrm{C}$. After incubation, cells were washed with DPBS $(120 \mu \mathrm{L})$ and maintained in DPBS $(120 \mu \mathrm{L})$ during the remainder of APP+ fluorescence uptake measurement, which was performed with a H1MF plate reader (BioTek, Winooski, VT) using $3 \times 3$ area scan and bottom-read mode. The excitation and emission wavelengths were set at $420 \mathrm{~nm}$ and $500 \mathrm{~nm}$, respectively. For data analysis, the mean 
fluorescence of wells containing tested probes was subtracted from that of the respective wells containing DMSO (vehicle) for each probe concentration. All data were normalized to the maximum fluorescence intensity of a well containing nomifensine as an internal standard at $1 \mu \mathrm{M}$ concentration. The $I C_{50}$ of probes $\mathbf{6 , 1 2}$ and 13 to hDAT was determined by non-linear regression using a 1:1 binding model measuring the dose-dependent inhibition of APP+ uptake ([inhibitor] vs. response - Variable slope function; GraphPad Prism version 8, GraphPad Software Inc., San Diego, CA).

Measurement of excitation and fluorescence spectra of fluorescent probes. hDAT-HEK cells (Passage \# 15-20) were plated at a density of $1.5 \times 10^{5}$ cells/well onto a white, clear-bottom 96well plate pre-coated with poly-D-lysine solution $(0.1 \mathrm{mg} / \mathrm{mL}$, Sigma-Aldrich $)$ and allowed to grow for $24 \mathrm{~h}$ to provide around 100\% confluency. During the experiment, the growth media was replaced with experimental media and pre-incubated for $30 \mathrm{~min}$ at $37^{\circ} \mathrm{C}$. Dye solutions were then added at defined concentration and cells were further incubated for $15 \mathrm{~min}$ at room temperature. After incubation, cells were washed $3 \times$ with DPBS and maintained in DPBS during the remainder of the measurement, which was performed with a $\mathrm{H} 1 \mathrm{MF}$ plate reader (BioTek) at $25^{\circ} \mathrm{C}$ using bottom-read mode. Fluorescence emission spectra were measured using an excitation source with $\lambda_{\text {exc }}=550 \mathrm{~nm}$ for $6, \lambda_{\text {exc }}=480 \mathrm{~nm}$ for di-2-ANEPEQ (1) and di-2-ANEP-VoLDeMo (12), and $\lambda_{\text {exc }}=530 \mathrm{~nm}$ for dsRVF5 (2), dsRVF5-VoLDeMo (13), dsRVF5-VoLDeCAM (21) and control probe 22. Excitation spectra were measured using the emission at $\lambda_{\mathrm{em}}=605 \mathrm{~nm}$ for $6, \lambda_{\mathrm{em}}=600 \mathrm{~nm}$ for di-2-ANEPEQ (1) and di-2-ANEP-VoLDeMo (12), and $\lambda_{\mathrm{em}}=590 \mathrm{~nm}$ for dsRVF5 (2), dsRVF5-VoLDeMo (13), dsRVF5-VoLDeCAM (21) and control probe 22. All spectra were measured with a $1 \mathrm{~nm}$-resolution and normalized.

Preparation of HEK cells for imaging and electrophysiological experiments. Cells (passage \# 15-20) were plated at a density of $4 \times 10^{5}$ cells/coverslip or $1.5 \times 10^{5}$ cells/coverslip onto acidwashed $15 \mathrm{~mm}$ coverslips pre-coated with poly-D-lysine solution $(0.1 \mathrm{mg} / \mathrm{mL}$, Sigma-Aldrich, St. Louis, MO) and allowed to grow for $24 \mathrm{~h}$ to provide around $70 \%$ or $30 \%$ confluency for imaging or electrophysiological experiments, respectively. Prior to imaging, the growth media were replaced with experimental media containing either nomifensine $(2 \mu \mathrm{M}, 0.05 \% w / v)$ or DMSO (vehicle, $0.02 \% \mathrm{v} / \mathrm{v}$ ) as control. After $30 \mathrm{~min}$ of pre-incubation at $37^{\circ} \mathrm{C}$, stock solution of the dye was added to the cells and incubated at room temperature (for 5 min for di-2-ANEP-VoLDeMo 
and for 15 min for dsRVF5-VoLDeMo). After incubation, cells were washed $3 \times$ with DPBS (HyClone) and maintained in HEPES-buffered Tyrode's solution (in mM: $118.6 \mathrm{NaCl}, 3 \mathrm{KCl}$, 2.7 HEPES sodium salt, 3.3 HEPES, 1.2 $\mathrm{MgCl}_{2}, 2 \mathrm{CaCl}_{2}, 10$ D-Glucose; $\mathrm{pH}=7.30 ; 250 \mathrm{mOsm}$ ) during the course of the imaging and electrophysiological experiments.

Imaging HEK cells with one-photon epifluorescence. Imaging was carried out with a BX51WI epifluorescence microscope (Olympus, Tokyo, Japan) equipped with a mercury lamp (Olympus). Images were obtained with a LUMFL water-immersion objective $(60 \times / 1.10 \mathrm{NA}$; Olympus $)$ and an ORCA-Flash4.0 sCMOS camera (Hamamatsu Photonics, Hamamatsu City, Japan). Filters used for di-2-ANEP-VoLDeMo include a $480 \mathrm{~nm}$ excitation filter (40 nm bandpass, Chroma Technology Corp., Bellows Falls, VT), a $570 \mathrm{~nm}$ dichroic (Olympus), and a $590 \mathrm{~nm}$ longpass emission filter (Olympus). Filters used for dsRVF5-VoLDeMo include a $540 \mathrm{~nm}$ excitation filter (20 nm bandpass), a $570 \mathrm{~nm}$ dichroic, and a $590 \mathrm{~nm}$ longpass emission filter (all from Olympus). Images were collected using the HCImage software (Hamamatsu Photonics). Figures showing specific labeling (Fig. 4a and $d$ ) were resolved in $2048 \times 2048$ pixel $^{2}$ in a $225 \times 225 \mu \mathrm{m}^{2}$ field of view. For voltage imaging, regions of interest (ROIs) of $400 \times 100 \mathrm{pixel}^{2}$ were used to achieve fast sampling rates of $200 \mathrm{~Hz}$ for simulated voltage steps and $1 \mathrm{kHz}$ for simulated spiking events.

Whole-cell voltage clamping of HEK cells. Patching pipettes were pulled using a DMZUniversal Puller (Zeitz Instruments, Martinsried, Germany) with borosilicate glass (Sutter Instrument Company, Novato, CA; O.D. $=1.5 \mathrm{~mm}$, I.D. $=0.86 \mathrm{~mm}$ ) to the resistance of 5-7 $\mathrm{M} \Omega$. Internal solution (in mM: $115 \mathrm{~K}$-gluconate, $20 \mathrm{KCl}, 10 \mathrm{HEPES}, 2 \mathrm{MgCl}_{2}, 4 \mathrm{MgATP}, 0.3 \mathrm{NaGTP}$, 10 EGTA; $\mathrm{pH}=7.25 ; 280 \mathrm{mOsm})$ was filtered with Ultrafree Centrifugal Filters $(0.22 \mu \mathrm{m}$; MilliporeSigma, Burlington, MA) before every experiment. Electrical signals were obtained with a Multiclamp 700B amplifier (Molecular Devices, San Jose, CA) and digitized with a BNC-2090 (National Instruments, Austin, TX) with a sampling rate of $10 \mathrm{kHz}$. Electrical signals were recorded with PackIO, a PC-based open-source software developed by Adam Packer of the Yuste lab (www.packio.org). ${ }^{35}$ Pipette resistance was corrected by adjusting the bridge balance and fast capacitance was compensated in cell-attached mode. Recordings were only collected from cells when the access resistance of the whole-cell voltage clamp was below $30 \mathrm{M} \Omega$. 
Photostability in HEK cells. hDAT-HEK cells were incubated with experimental media [phenol red-free DMEM (Gibco) supplemented with 1\% FBS and 1\% Penicillin-Streptomycin] containing di-2-ANEPEQ (1; $500 \mathrm{nM})$, dsRVF5 (2; $500 \mathrm{nM})$, di-2-ANEP-VoLDeMo (12; $100 \mathrm{nM}$ dextran) or dsRVF5-VoLDeMo (13; $100 \mathrm{nM}$ dextran) at $37{ }^{\circ} \mathrm{C}$ for 15 min. Imaging was carried out with a Leica DMI4000 epifluorescence microscope (Leica Microsystems, Wetzlar, Germany) equipped with a xenon lamp (Sutter Lambda LS; Sutter Instrument, Novato, CA). Images were obtained with a Leica objective (40×/0.45 HI Plan; Leica Microsystems, Wetzlar, Germany) and a PCO.Panda 4.2 camera (PCO, Kelheim, Germany). Filters used for di-2-ANEP VSDs 1 and 12 include a $450 \mathrm{~nm}$ excitation filter (50 nm bandpass), a $480 \mathrm{~nm}$ dichroic, and a $580 \mathrm{~nm}$ emission filter (40 nm bandpass; all Chroma Technology Corp., Bellows Falls, VT). Filters used for dsRVF5 VSDs include a $540 \mathrm{~nm}$ excitation filter ( $25 \mathrm{~nm}$ bandpass), a $570 \mathrm{~nm}$ dichroic, and a $605 \mathrm{~nm}$ emission filter ( $55 \mathrm{~nm}$ bandpass; all Chroma Technology Corp., Bellows Falls, VT). Images were taken in $2048 \times 2048$ pixel $^{2}$ in a $225 \times 225 \mu \mathrm{m}^{2}$ field of view every 5 seconds for 15 min with constant illumination. For each image, the average fluorescence intensity from the entire field of view (background subtracted) was normalized to the fluorescence intensity at $t=0$. Averages from $n=4$ independent experiments were plotted vs. time to provide the photostability curves in Fig. S13.

\section{In Acute Murine Brain Slice}

Preparation of acute murine brain slices. All animal protocols were approved by the Institutional Animal Care and Use Committee of New York State Psychiatric Institute/Columbia University Medical Center. All protocols followed the Guide for the Care and Use of Laboratory Animals by National Institute of Health (NIH). All animals used were C57BL/6, wild-type mice from The Jackson Laboratory (Bar Harbor, ME), included both males and females, and sacrificed between 8 to 10 weeks. Coronal slices ( $300 \mu \mathrm{m}$ thick) were cut by a Leica VT1200 vibratome in ice-cold slicing solution (in mM: 194 sucrose, $30 \mathrm{NaCl}, 4.5 \mathrm{KCl}, 26 \mathrm{NaHCO}_{3}, 1 \mathrm{MgCl}_{2} \cdot 6 \mathrm{H}_{2} \mathrm{O}$, $\left.1.2 \mathrm{NaH}_{2} \mathrm{PO}_{4}, 10 \mathrm{D}-\mathrm{Glucose}\right)$, and transferred to oxygenated $\left(95 \% \mathrm{O}_{2}+5 \% \mathrm{CO}_{2}\right), 37{ }^{\circ} \mathrm{C}$ artificial cerebrospinal fluid (ACSF, in mM: $124 \mathrm{NaCl}, 4.5 \mathrm{KCl}, 26 \mathrm{NaHCO}_{3}, 1 \mathrm{MgCl}_{2} \cdot 6 \mathrm{H}_{2} \mathrm{O}, 2 \mathrm{CaCl}_{2}$, 
1.2 $\mathrm{NaH}_{2} \mathrm{PO}_{4}, 10 \mathrm{D}-\mathrm{Glucose} ; \mathrm{pH}=7.30 ; 295 \mathrm{mOsm}$ ) in a slice chamber for $15 \mathrm{~min}$. Slices were then incubated in oxygenated ACSF at room temperature for 30 min before imaging.

Electrical stimulation and two-photon imaging of acute murine brain slices. Slices were transferred to a QE-1 imaging chamber and held in place by a SHD-26H/2 slice anchor (both by Warner Instruments LLC, Hamden, CT). Slices were maintained in a bath of $\sim 2 \mathrm{~mL}$, oxygenated ACSF during probe incubation. Slices were perfused with oxygenated ACSF at $2 \mathrm{~mL} / \mathrm{min}$ during the course of imaging, with tetrodotoxin (TTX; $1 \mu \mathrm{M}$ ) added to the perfusate only for TTX-treated conditions. For inhibited conditions, slices were first pre-incubated in a bath of $\sim 2 \mathrm{~mL}$, oxygenated ACSF with nomifensine $(2 \mu \mathrm{M}, 0.05 \% \mathrm{w} / \mathrm{v})$ for $15 \mathrm{~min}$. The probe was then added to the bath so that slices were co-incubated with nomifensine during probe incubation (at $100 \mathrm{nM}$ dextran concentration for $30 \mathrm{~min}$, same for all probes shown in Fig. 5). Slices were imaged with a Prairie Ultima Multiphoton Microscopy System (Bruker Corp., Billerica, MA), a Ti:sapphire Chameleon Ultra II laser (Coherent Inc., Santa Clara, CA), a Zeiss water-immersion objective (63×/0.9 NA; Zeiss, Oberkochen, Germany), and the Prairie View software (version 5.4). di-2-ANEP-VoLDeMo (12) was excited at $920 \mathrm{~nm}$ and emission collected at $570-610 \mathrm{~nm}$. dsRVF5-VoLDeMo (13) and dsRVF5-VoLDeCAM (21) was excited at $820 \mathrm{~nm}$ and emission collected at $570-610 \mathrm{~nm}$. High-resolution images were collected with the galvo scanning mode in the format of 16-bit, $1024 \times 1024$ pixel $^{2}, 10 \mu$ s/pixel dwell time, and $4 \times$ optical zoom $(55 \times 55$ $\mu \mathrm{m}^{2}$ field of view; Fig. 5b, c, e, and f; Fig. S14). To achieve a faster sampling rate (30 frames/second) during the electrical stimulation experiment, images were collected with the spiral scanning mode in the format of 16-bit, $10.4 \mu$ s/pixel dwell time, and $1 \times$ optical zoom with circular ROIs of 16 pixel in diameter ( $84 \mu \mathrm{m}$ in diameter field of view; Fig. $6 b$ and $9 b$ ). Each t-series was imaged for $5 \mathrm{~s}$ total, where a single electrically stimulated train was delivered from seconds 2 to $2.5(50 \mathrm{~Hz}, 25$ pulses, each pulse $100 \mu \mathrm{s} \times 180-200 \mathrm{~mA}, 0.5 \mathrm{~s}$ total $)$ with a bipolar stainless steel electrode, each pole of the electrode controlled by an ISO-Flex stimulus isolator triggered by the "Voltage Output" application of the Prairie View software. Here, one of the two isolators were set to be positive and the other negative, and each individual pulse was comprised of a positive pulse immediately followed by a negative pulse (i.e. $100 \mu$ s delay for the negative pulse). For each condition, 50 different ROIs/t-series were taken $25-35 \mu \mathrm{m}$ below slice surface with 10 ROIs per slice, 5 total slices across $2-3$ days. 
Electrophysiology. Whole-cell patch clamp recordings were performed on layers V-VI prefrontal cortex through an upright Olympus BX50WI (Olympus, Tokyo, Japan) differential interference contrast microscope with a $40 \times$ water immersion objective and an IR-sensitive video camera. For the recording, slices were submerged in a flowing ACSF $(2 \mathrm{~mL} / \mathrm{min})$ and all experiments were conducted at $35-36{ }^{\circ} \mathrm{C}$. Patch pipettes (3-5 M ) were made with P-97 pipette puller (Sutter Instruments, Novato, CA). Whole-cell patch clamp recording was performed with a multiclamp 700B amplifier (Molecular Devices, Forster City, CA) and digitized at $10 \mathrm{kHz}$ with a ITC-18 (HEKA Instruments, Bellmore, NY). Data were acquired using WinWCP software (developed by Dr. John Dempster, University of Strathclyde, UK) for subsequent analysis. For eEPSC and sEPSC recording, cells were held at $-70 \mathrm{mV}$ in the voltage clamp mode and recordings were performed in the presence of $25 \mu \mathrm{M}$ picrotoxin. Bipolar tungsten stimulation electrode placed $\sim 100 \mu \mathrm{m}$ distance from the patch pipette and stimuli $(0.1 \mathrm{~ms})$ were delivered every $20 \mathrm{~s}$ by Master-9 (A.M.P.I, Jerusalem, Israel) and stimulation intensity (0-0.03 mA) was adjusted by Isoflex (A.M.P.I). Pipette solution contained (in mM): $120 \mathrm{CsMeSO}_{3}, 5 \mathrm{NaCl}, 10 \mathrm{HEPES}, 1.1 \mathrm{EGTA}, 2$ ATP-Mg ${ }^{2+}, 0.3$ GTP-Na2, 2 ATP-Na2, 5 QX314, pH=7.3; $280 \pm 5$ mOsm. For optical voltage recording with dsRVF5-VoLDeCAM (21), cells were held at $-60 \mathrm{mV}$ and pipettes were filled with (in mM): $115 \mathrm{~K}$-gluconate, $10 \mathrm{HEPES}, 2 \mathrm{MgCl}_{2}, 20 \mathrm{KCl}, 2 \mathrm{ATP}-\mathrm{Mg}^{2+}, 1$ ATP-Na, $0.3 \mathrm{GTP}-\mathrm{Na}_{2}$, $\mathrm{pH}=7.3 ; 280 \pm 5$ mOsm. eEPSCs were analyzed with Clampfit 10.2 software (Molecular Devices) and sEPSCs were analyzed using Mini Analysis program (Synaptosoft, Decatur, GA). sEPSC detection threshold was generally adjusted to two-fold the RMS noise level (typically 8 pA). Collected data arranged and illustrated in graphs with GraphPad Prism 8 software (San Diego, CA). eEPSC and sEPSC data was expressed as mean \pm SEM and median $\pm 95 \%$ confidence interval respectively. Two-way ANOVA was used to compare groups of eEPSCs and Kolmogorov-Smirnov test was used to compare groups of sEPSC amplitude and frequency. The difference was considered statistically significant when $p<0.05$.

\section{In Drosophila Brain}

Targeting of VoLDeMo probes in whole Drosophila brains. The brain of the adult fruit fly (Drosophila melanogaster) is small $(\sim 200 \mu \mathrm{m}$ in depth). Combined with the fly's genetic 
tractability, this organism serves as a useful model for studying intact circuitry in the whole brain via multiphoton microscopy. ${ }^{36-38}$ Therefore, we tested targeting of VoLDeMo probes in whole fly brains. We used adult brains from Drosophila melanogaster expressing $\mathrm{ArcLight}^{38}$ under the TH promotor (marker of catecholaminergic neurons) and focused on the suboesophageal ganglion (SOG) region, due to its accessibility for multiphoton imaging and high density of dopamine axonal varicosities.

Incubating a TH-driven ArcLight fly brain with di-2-ANEP-VoLDeMo (12, $100 \mathrm{~nm}$ dextran/74 nM VSD, $30 \mathrm{~min})$ resulted in sparse punctate staining with partial overlap with ArcLight $(n=4$ brains, Fig. S18a), which was abolished by pre- and co-incubation with excess competing ligand, dichloropane derivative $5(2 \mu \mathrm{M}, 30 \mathrm{~min}$ pre-incubation, $n=3$ brains, Fig. S18b), leaving only a diffuse labeling pattern consistent with background fluorescence. Incubation of the brains with untethered di-2-ANEPEQ $(\mathbf{1}, 100 \mathrm{nM}, 30 \mathrm{~min})$ resulted in similar non-punctate, non-specific labeling ( $n=3$ brains, Fig. $S 18 c$ ), thus highlighting the labeling selectivity of the VoLDeMo probe, and indicating that the labeling is ligand-dependent. While there is partial co-localization of VoLDeMo with TH-driven ArcLight, which suggests co-localization of the two fluorescent signals to dopamine nerve varicosities, we also observed VoLDeMo puncta that were not co-labeled by Arclight. Given that the VoLDeMo probe labels other monoaminergic transporters including the serotonin transporter, this additional labeling may be due to association with these related monoamine transporters in Drosophila. ${ }^{39,40}$ Incubating a $\mathrm{TH}$-driven ArcLight-expressing Drosophila brain with dsRVF5-VoLDeMo (13, $100 \mathrm{~nm}$ dextran/63 nM VSD, $30 \mathrm{~min})$ resulted in a similar punctate staining pattern as for the di-2-ANEP targeted probe ( $n=3$ brains, Fig. S18d). The sparse punctate staining of fly brains with VoLDeMo probes and significant movement artifacts during stimulation, did not allow us to obtain functional recordings in this system.

Drosophila strains. All Drosophila melanogaster strains were grown and maintained on standard cornmeal-molasses media at $25{ }^{\circ} \mathrm{C}$ under a $12: 12 \mathrm{~h}$ light-dark schedule. Strains include UASArcLight (a gift of Dr. M. Nitabach, Yale University, New Haven, CT), ${ }^{38}$ and the expression driver line TH-GAL4 (a gift of Dr. S. Birman, Université Aix-Marseille II-III, Marseille, France) ${ }^{41}$ for expression in presynaptic neurons.

Compound treatments. All drugs were diluted in adult hemolymph-like saline (AHL; in mM: 108 $\mathrm{NaCl}, 5 \mathrm{KCl}, 2 \mathrm{CaCl}_{2}, 8.2 \mathrm{MgCl}_{2}, 1 \mathrm{NaH}_{2} \mathrm{PO}_{4}, 10$ sucrose, 5 trehalose, 5 HEPES, 4 NaHCO ; $\mathrm{pH}$ 
7.50, $265 \mathrm{mOsm}$ ) as described previously. ${ }^{42}$ For VSD experiments, brains were loaded to steady state with the respective VSDs [100 nM of di-2-ANEPEQ (1), di-2-ANEP-VoLDeMo (12), or dsRVF5-VoLDeMo (13)] which were applied to the brain by bath superfusion into a flow chamber at $50 \mathrm{~mL} /$ hour at room temperature $\left(25^{\circ} \mathrm{C}\right)$. This was followed by $30 \mathrm{~min}$ of washing in AHL to remove potential non-specific background staining. To further validate the specificity of di-2-ANEP-VoLDeMo (12) staining, brains were first pre-treated with the targeting ligand of the construct, dichloropane derivative 5 ( $2 \mu \mathrm{M}, 30 \mathrm{~min}$ pre-incubation), followed by co-incubation with di-2-ANEP-VoLDeMo (12, $100 \mathrm{nM}$ dextran/74 nM VSD, $30 \mathrm{~min})$. Brains were subsequently washed for $30 \mathrm{~min}$ in AHL prior to imaging.

Imaging. An isolated, ex vivo whole adult fly brain preparation was obtained by rapid removal and microdissection of the brain from decapitated flies as described previously. ${ }^{37,42}$ The brain was placed in a recording chamber (JG-23, Warner Instruments, Hamden, CT) for rapid drug application and exchange. Brain preparations were imaged under continuous flow on a Bergamo II resonant-scanning two-photon microscope (Thorlabs, Inc., Newton, NJ) using a 20× (1.0 NA) water immersion objective lens (Olympus) and the tunable output of an Insight X3 laser (SpectraPhysics) mode-locked at $920 \mathrm{~nm}$. Typically, $<50 \mathrm{~mW}$ mean power was delivered through the objective. Fluorescent emission was collected using a $607 / 70 \mathrm{~nm}$ full-width half-maximum (FWHM) bandpass emission filter for VSDs and a 525/50 nm FWHM bandpass filter for ArcLight. Data acquisition was performed with ThorImage 3.1 software (Thorlabs, Inc.). Images were 16bit, $1024 \times 1024$ pixels ${ }^{2}, 100 \mathrm{~nm}$ per xy pixel with $4 \times$ frame averaging. Z-stacks of optical sections were acquired through a $20 \mu \mathrm{m}$ depth in $1 \mu \mathrm{m}$ steps to capture the entire dopaminergic innervation of the SOG region. Z-projections of the respective image stacks were generated using the Fiji/ImageJ image-processing package (National Institutes of Health, Bethesda, MD). VoLDeMo probes produced punctate staining (puncta $\sim 1 \mu \mathrm{m}$ size), some of which co-localized with puncta in the TH promoter-driven ArcLight channel. Examples of such co-localizing puncta are highlighted in Fig. S18a and $d$ by white arrowheads. 


\section{Data Analysis}

Quantification of VoLDeMo labeling in HEK cells. For each experimental condition (hDAT, hDAT + Nomi, or Null), ROIs strictly surrounding individual cell bodies were selected using the "Freehand selections" tool from the brightfield (BF) image without prior knowledge of the respective epifluorescence (FL) image. Only then were these ROIs applied to the corresponding FL image to measure the mean fluorescence intensity within each ROI (ImageJ, NIH). Each data point $(n)$ represented the average of 10 ROIs (five ROIs per image, two images per experiment). The same number of ROIs were also selected in cell-free areas following the same procedure and this average used for background subtraction. In very rare circumstances, ROIs selected from the BF image included exceedingly uniform bright circular spots in the corresponding FL image, and these ROIs were discarded. The resulting fluorescence intensity for each probe was normalized to the mean of the hDAT-transfected, non-inhibited condition (hDAT). The quantified results were displayed using GraphPad Prism 6 software (Fig. S2b and S9).

Analysis of whole-cell voltage clamp in HEK cells and in acute murine brain slices. ROIs of background strictly following the outer contour of the clamped cell body were drawn using the "Freehand selections" tool in ImageJ. The background for each time frame was calculated using the Nonuniform Background Removal plugin (Cory Quammen, UNC) and subtracted with the Image Calculator (both in ImageJ). The resulting image stack was analyzed together with the electrical recording file using a custom MATLAB routine with MATLAB 9.0 software. Specifically, ROIs strictly surrounding individual cell bodies were manually drawn and the mean $\Delta F / F$ calculated over all pixels within each ROI over all time frames at known stimulation profiles. Correction for photobleaching was done using one-phase exponential decay function (GraphPad Prism 8). For HEK cell data, imaging frames from all stimulated epochs were overlaid to give Fig. $4 b, e$ and Fig. $S 12 b, d$. The mean $\Delta F / F$ over all frames within each stimulated voltage step was calculated and plotted against the corresponding applied voltage to give Fig. $4 c$ and $f$. For acute slice data, imaging frames of $1-1.5,2-2.5,3-3.5,4-4.5$, and $5-5.5$ seconds of all cells for $+100 \mathrm{mV}$ holdings and $+0 \mathrm{mV}$ holdings were averaged to give Fig. 10b. For Fig. 10e and 10f, each cell was treated separately where the imaging frames of $1-1.5,2-2.5,3-3.5,4-4.5$, and $5-$ 5.5 seconds and imaging frames of $1.5-2,2.5-3,3.5-4,4.5-5$, and 5.5 - 6 seconds were each averaged and shown as a pair of connected datapoints. The averages of all datapoints from imaging 
frames of $1-1.5,2-2.5,3-3.5,4-4.5$, and $5-5.5$ seconds and imaging frames of $1.5-2,2.5$ $-3,3.5-4,4.5-5$, and $5.5-6$ seconds were plotted and its statistical significance analyzed with two-tailed paired t-test (Fig. 10c, d).

Colocalization of di-2-ANEP-VoLDeMo labeling with FFN102 labeling. The Multiple Thresholds plugin for ImageJ (Damon Poburko, Simon Fraser University, Burnaby, BC, Canada) was used to identify biologically-relevant puncta from FFN102 labeled images based on the following criteria: > 0.6 SD above background in fluorescence intensity, $0.5-4 \mu \mathrm{m}^{2}$ in size, $\geq 0.6$ circularity in shape, and well-defined boundaries. The auto-picked puncta (ROIs) were then applied as a mask to the corresponding di-2-ANEP-VoLDeMo (12) labeled images. Each punctum was defined as positively colocalized if more than $50 \%$ of the pixels within the punctum were more than two SDs brighter than the background fluorescence. The quantified results were displayed using GraphPad Prism 6 software (Fig. S15b). Error bars are \pm SD for $n=45$ images from 9 slices from 3 animals.

Analysis of electrical stimulation experiments in acute murine brain slices. The total fluorescence of each timeframe of each t-series was plotted as the raw data trace. The normalized raw traces for each condition are shown collectively in Fig. S19a, b and Fig. S27a, b. Each raw trace was corrected for photobleaching using the two-phase exponential decay function in GraphPad Prism 8. The change in fluorescence $(\Delta F / F)$ at each time frame for each bleachingcorrected trace was then calculated. The average of all $\Delta F / F$ traces for each brain region was shown in Fig. S19c and Fig. S27c. For each brain region, the averaged $\Delta F / F$ trace of the TTX-added condition was subtracted from the $\Delta F / F$ trace of the same condition but with no TTX added and the results shown in Fig. S19d and Fig. S27d. Then a 2-points moving average (of each frame and its previous frame) was applied to each $\Delta F / F$ trace, and for each brain region, the average $\Delta F / F$ trace of the TTX-added condition was subtracted from the $\Delta F / F$ trace of the same condition but with no TTX added and the results shown in Fig. $6 c$ and Fig. 9c. Finally, for each brain region, the average of all data points from the subtracted $\Delta F / F$ traces from the pre-stimulation period and those from the stimulation period were shown in Fig. $6 d$ and Fig. $9 d$ with \pm SEM, and the statistical significance analyzed with a two-tailed unpaired t-test using GraphPad Prism 8. 


\section{Excitation and Emission Spectra}

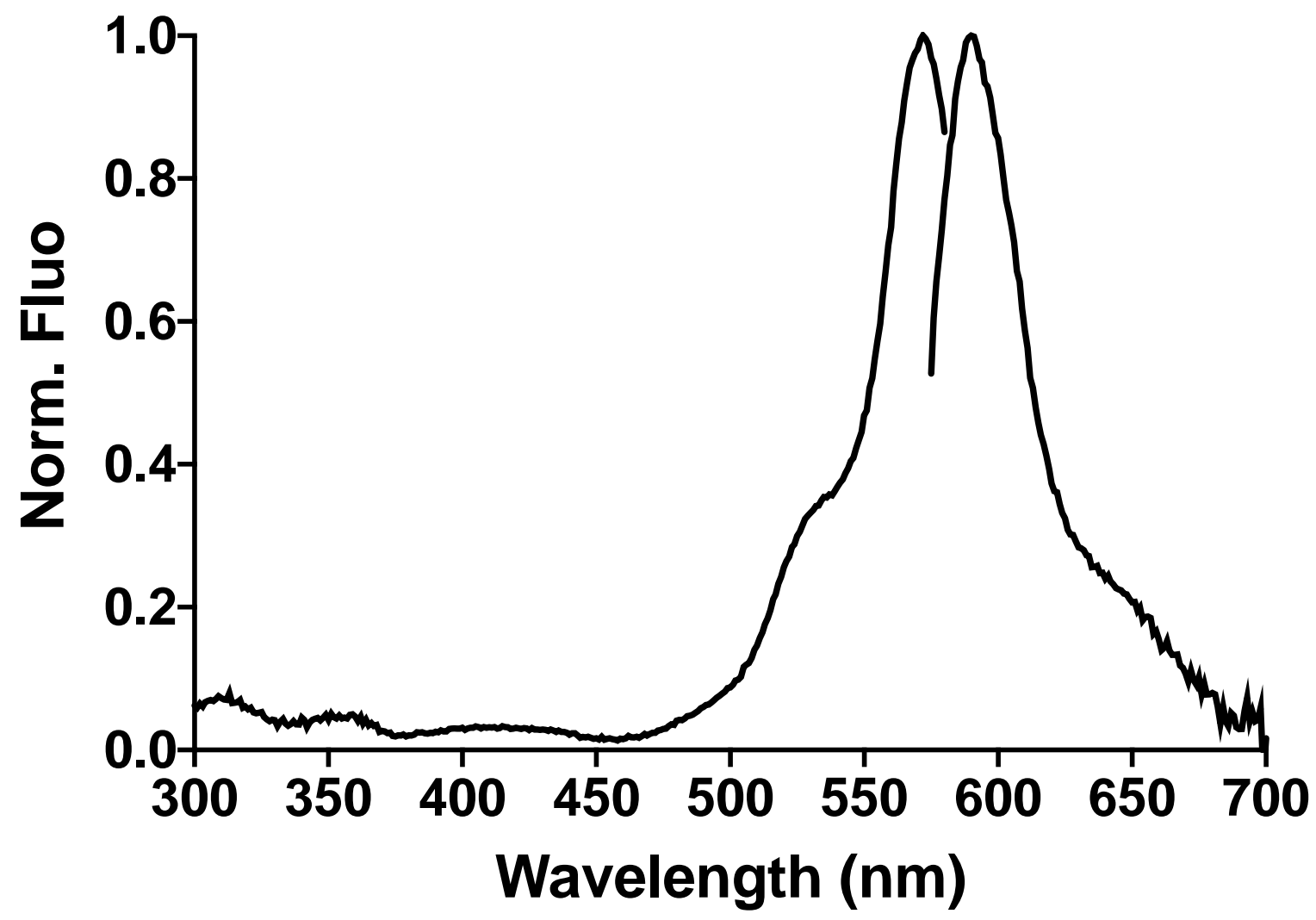

Figure S31. Normalized excitation and fluorescence emission spectra of rhodamine-labeled dichloropane (6) in hDAT-HEK cells. Measured in hDAT-HEK cells maintained in DPBS. Rhodamine-labeled dichloropane $(6,2 \mu \mathrm{M})$, excitation spectrum at $605 \mathrm{~nm}$ emission, emission spectrum with $550 \mathrm{~nm}$ excitation. 

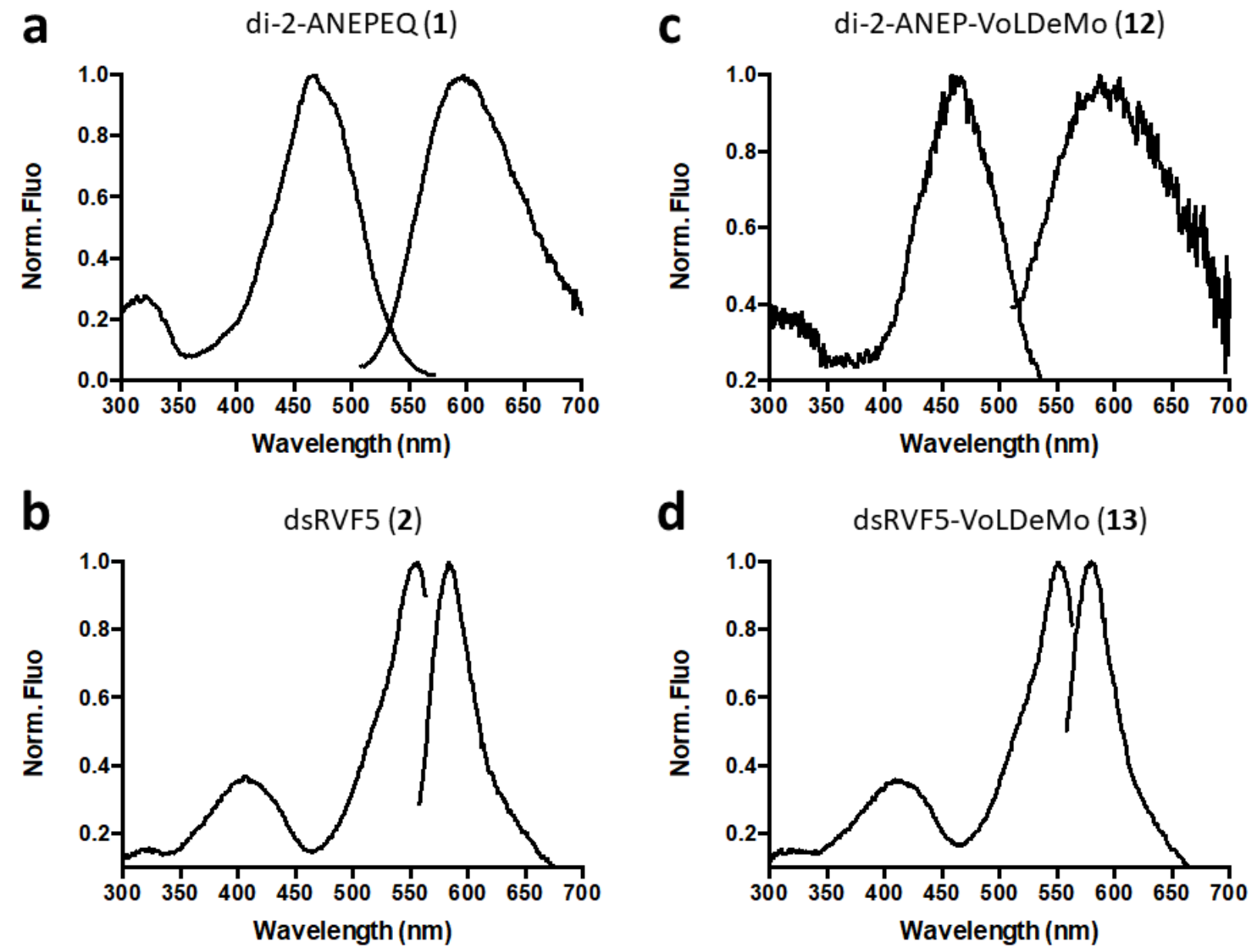

Figure S32. Normalized excitation and fluorescence emission spectra of VoLDeMo probes and untethered VSDs in hDAT-HEK cells. All spectra were measured in hDAT-HEK cells maintained in DPBS. (a) di-2-ANEPEQ $(\mathbf{1}, 100 \mu \mathrm{M})$, excitation spectrum at $600 \mathrm{~nm}$ emission, emission spectrum with $480 \mathrm{~nm}$ excitation. (b) dsRVF5 $(2,100 \mu \mathrm{M})$, excitation spectrum at $590 \mathrm{~nm}$ emission, emission spectrum with $530 \mathrm{~nm}$ excitation. (c) di-2-ANEP-VoLDeMo (12, $100 \mu \mathrm{M})$, excitation spectrum at $600 \mathrm{~nm}$ emission, emission spectrum with $480 \mathrm{~nm}$ excitation. (d) dsRVF5-VoLDeMo $(13,100 \mu \mathrm{M})$, excitation spectrum at $590 \mathrm{~nm}$ emission, emission spectrum with $530 \mathrm{~nm}$ excitation. 

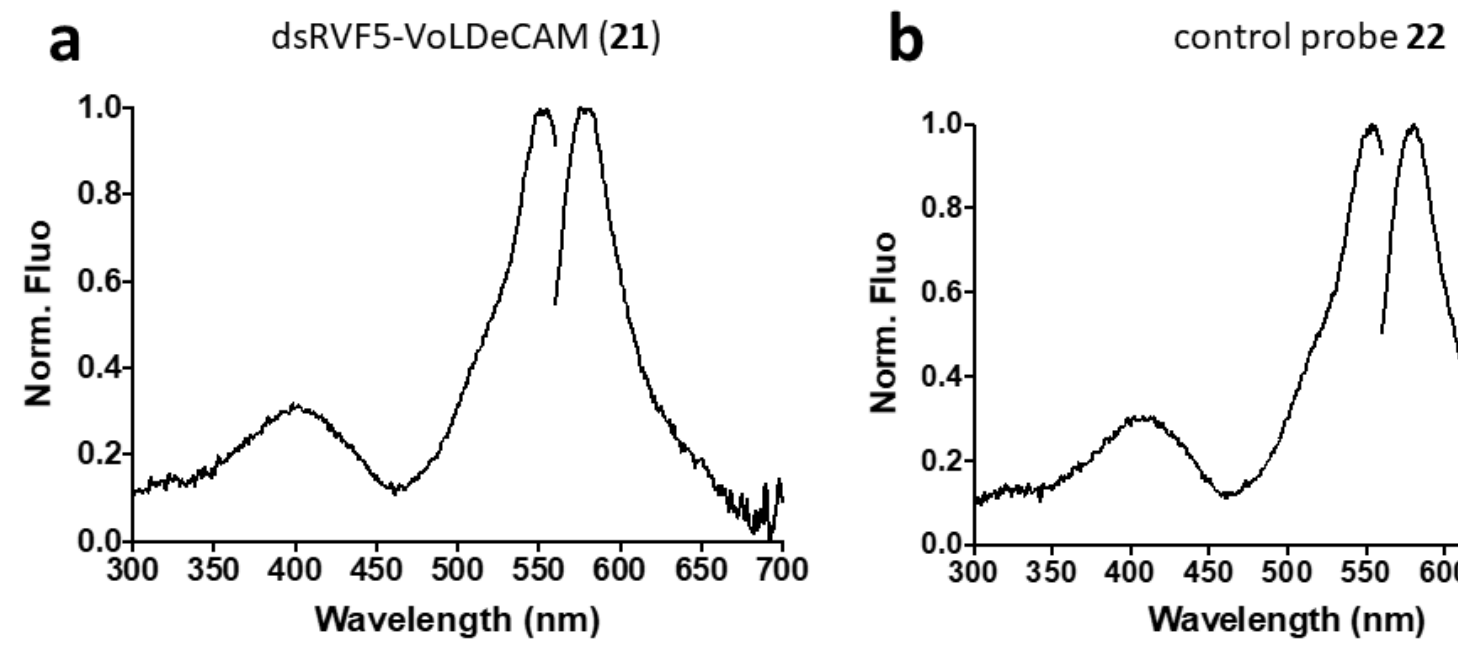

Figure S33. Normalized excitation and fluorescence emission spectra of VoLDeCAM probes in HEK cells. All spectra were measured in HEK cells maintained in DPBS. (a) dsRVF5-VoLDeCAM (21, $2 \mu \mathrm{M})$, excitation spectrum at $590 \mathrm{~nm}$ emission, emission spectrum with $530 \mathrm{~nm}$ excitation. (b) control probe 22 $(2 \mu \mathrm{M})$, excitation spectrum at $590 \mathrm{~nm}$ emission, emission spectrum with $530 \mathrm{~nm}$ excitation. 


\section{Infrared Spectra}

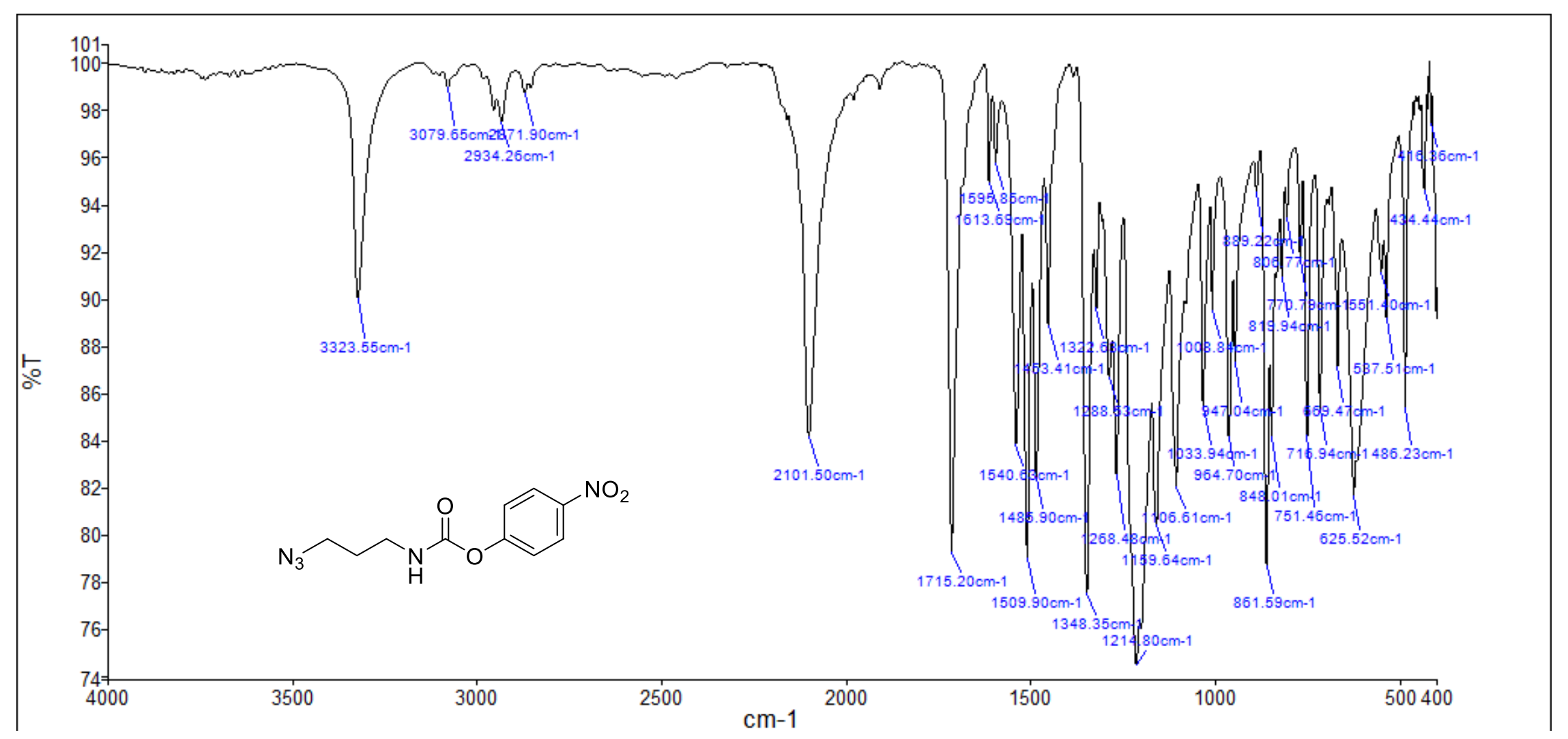

Figure S34. FTIR (ATR, diamond) spectrum of carbamate 7. 


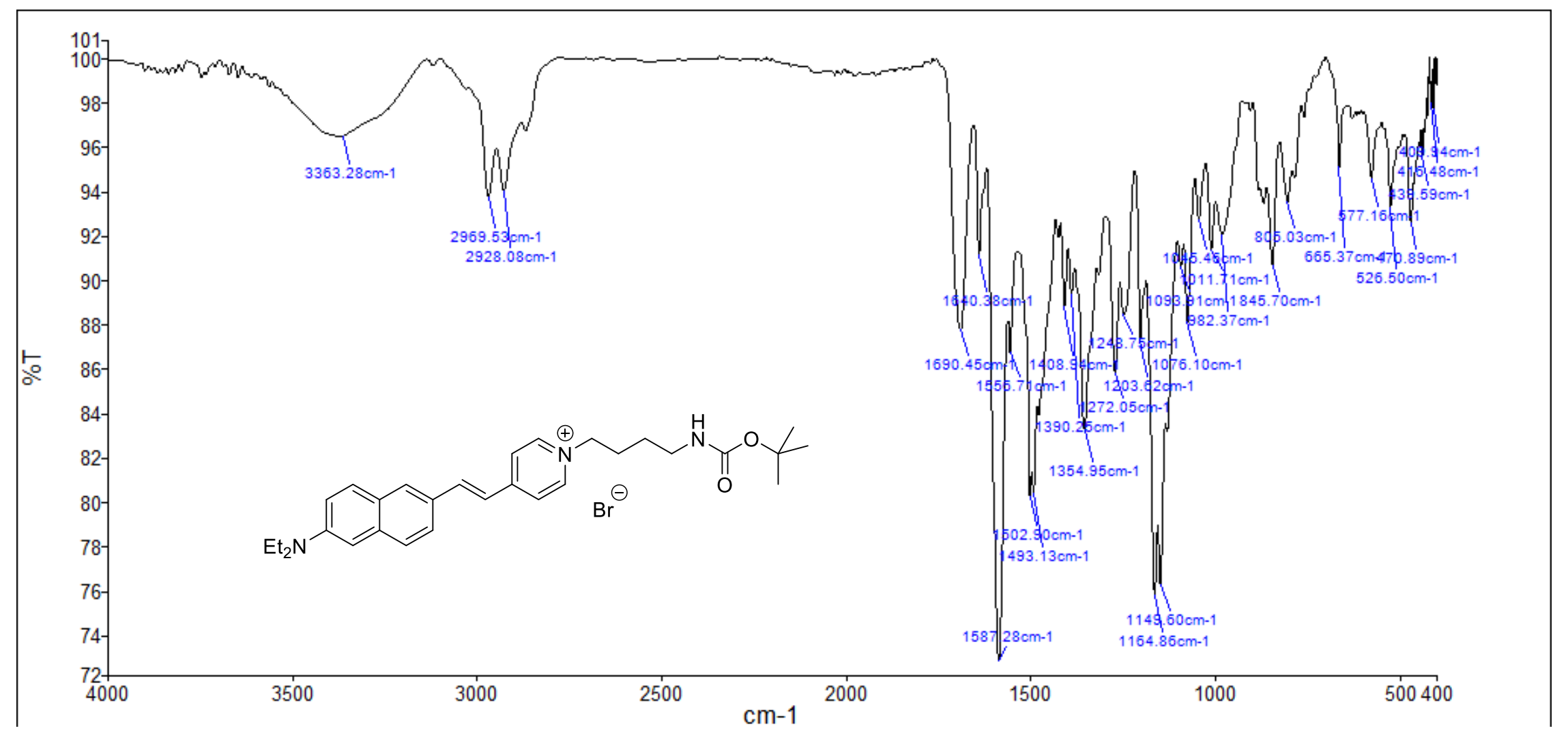

Figure S35. FTIR (ATR, diamond) spectrum of di-2-ANEPB-NHBoc (S18). 


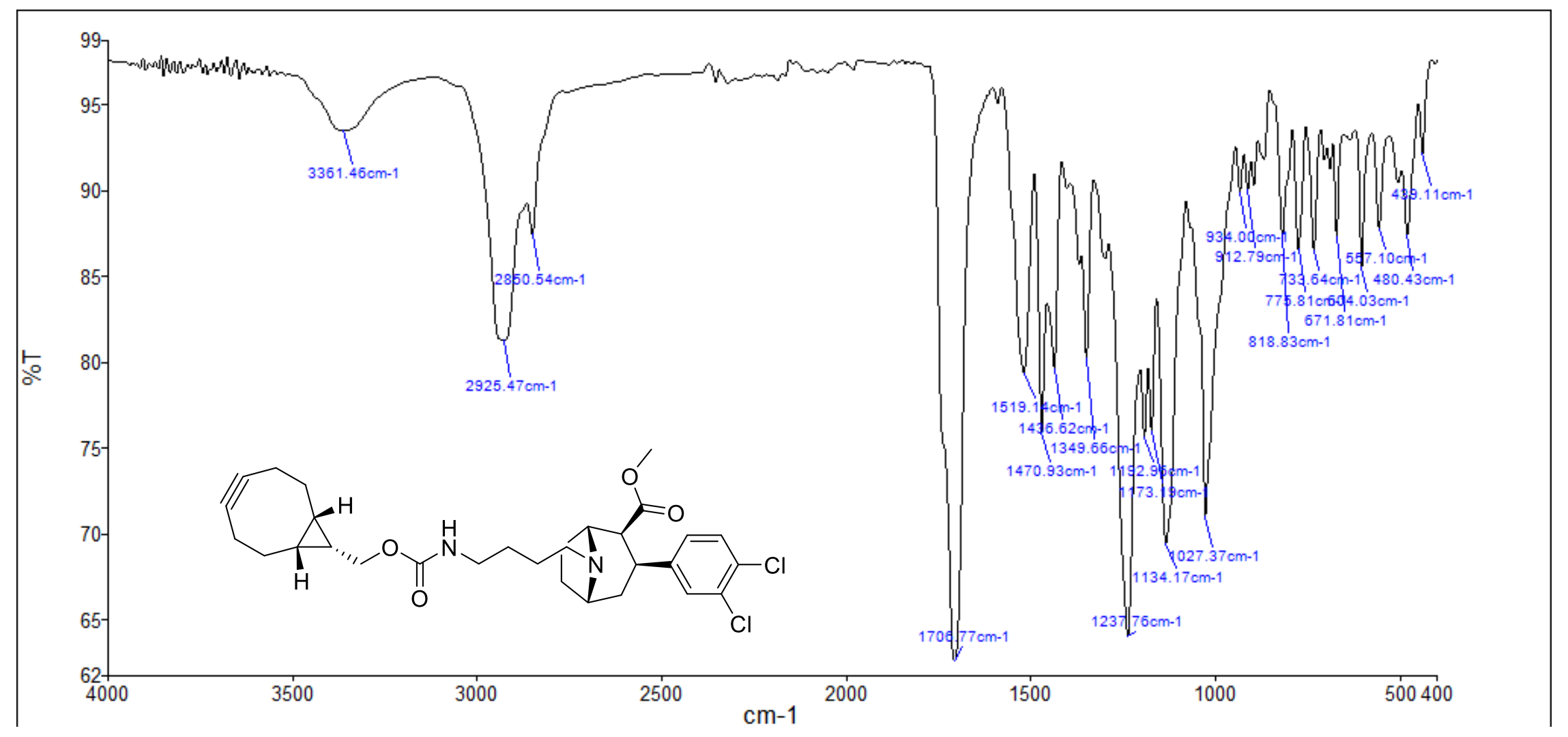

Figure S36. FTIR (ATR, diamond) spectrum of dichloropane-BCN (11). 


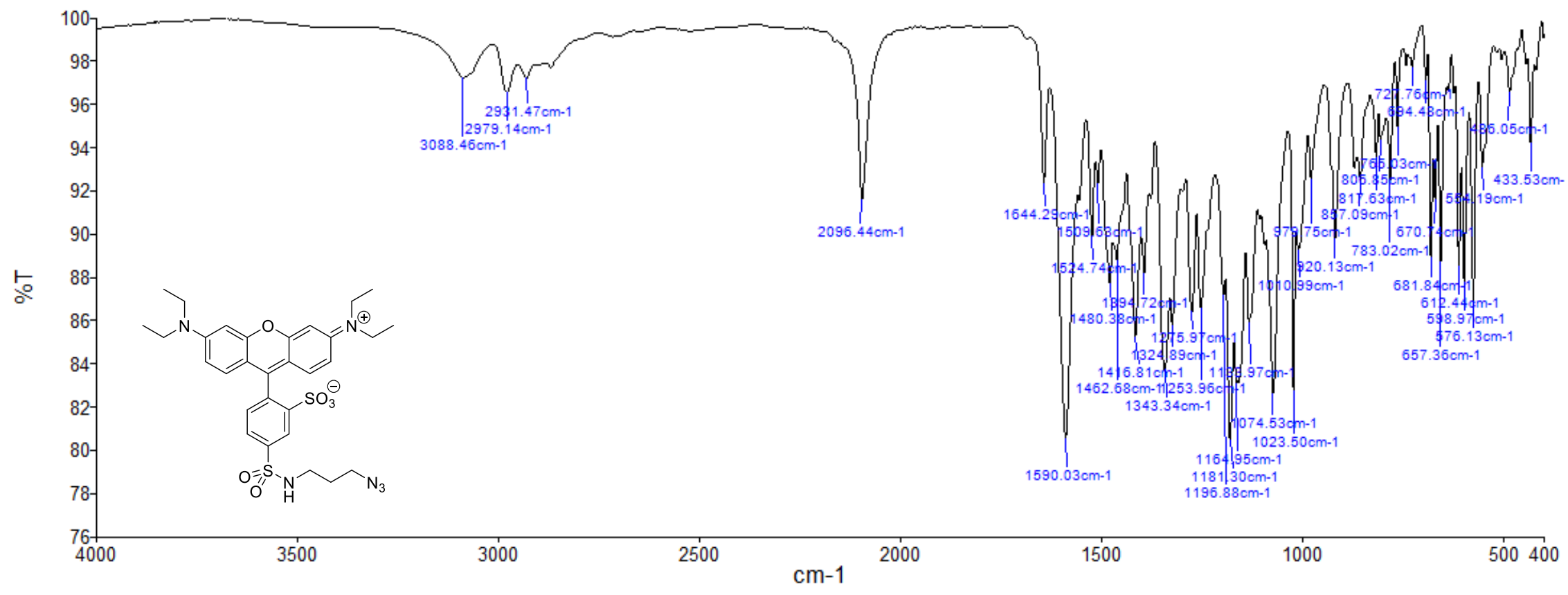

Figure S37. FTIR (ATR, diamond) spectrum of compound S46. 


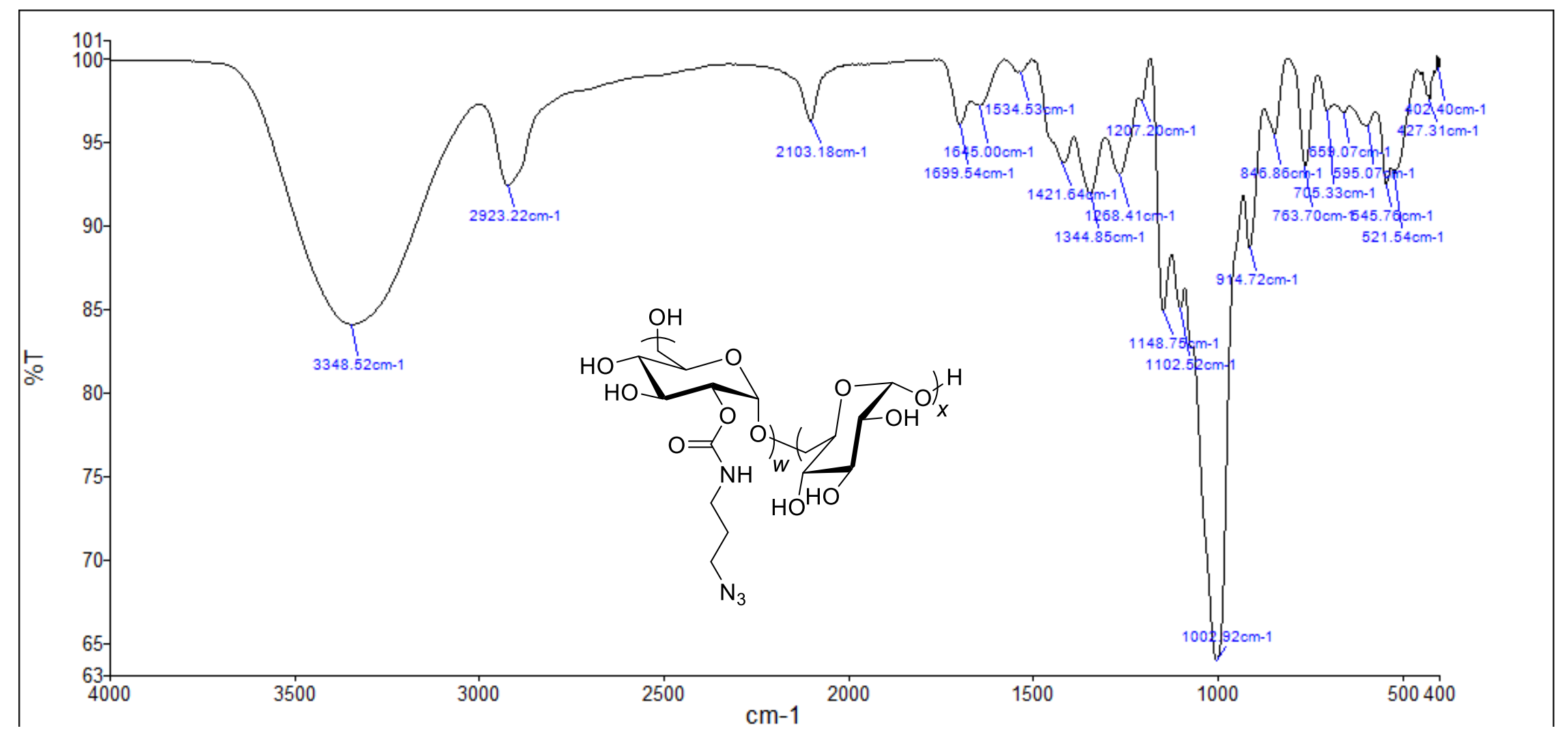

Figure S38. FTIR (ATR, diamond) spectrum of azidopropyl-carbamate dextran(6000) (8-2.15) containing 2.15 N $\mathrm{N}_{3} /$ polymer. 


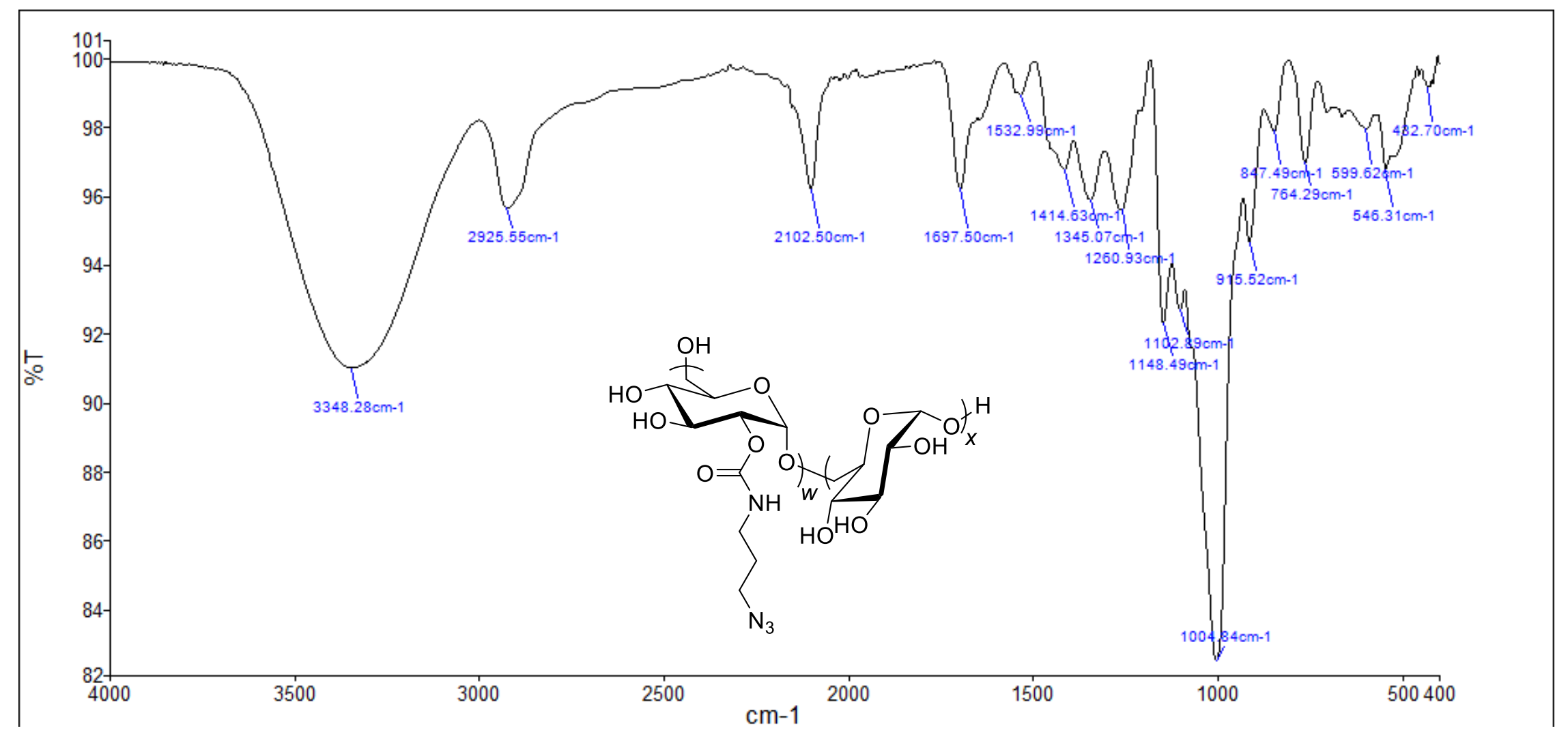

Figure S39. FTIR (ATR, diamond) spectrum of azidopropyl-carbamate dextran(6000) (8-4.42) containing 4.42 N N$_{3} /$ polymer. 


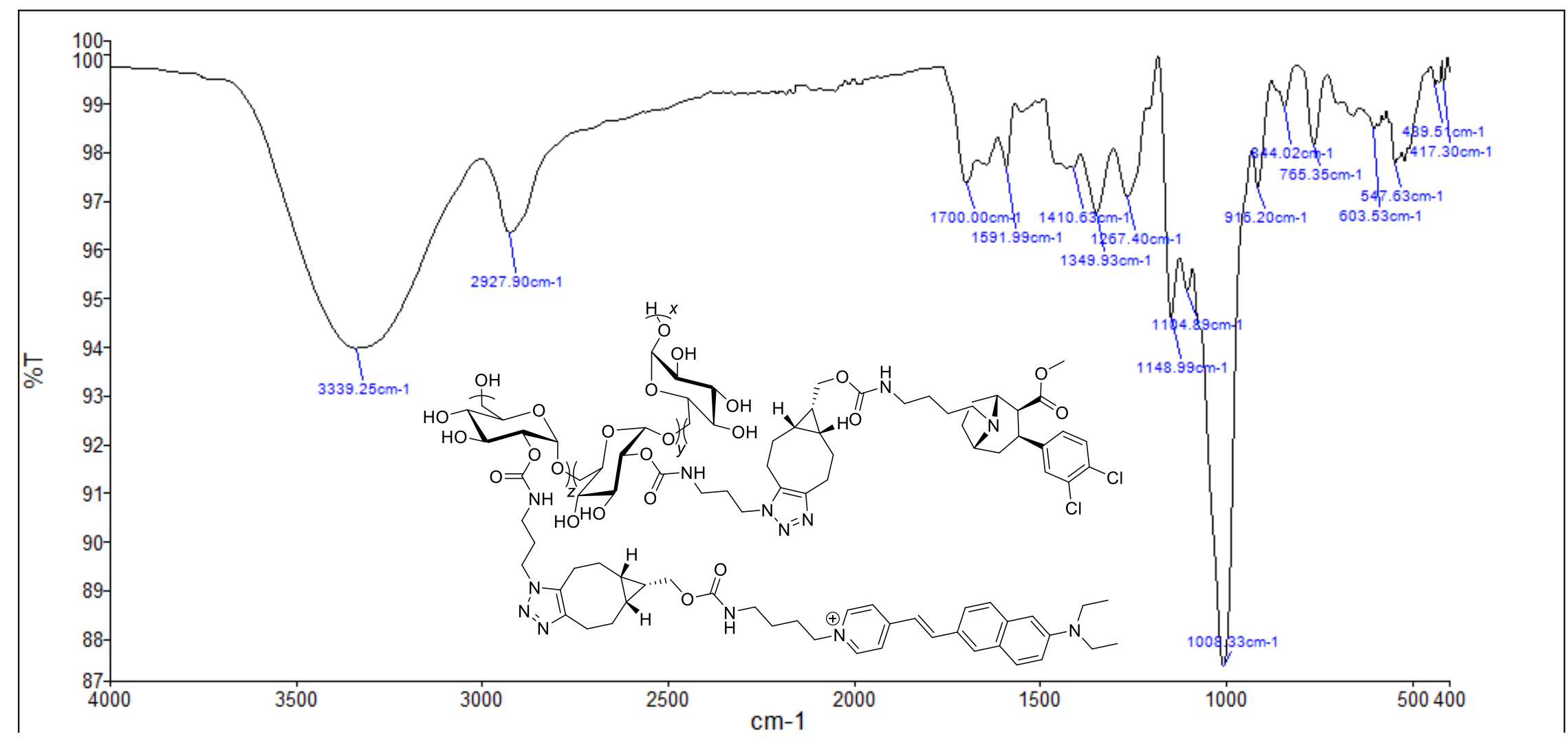

Figure S40. FTIR (ATR, diamond) spectrum of di-2-ANEP-VoLDeMo (12). 


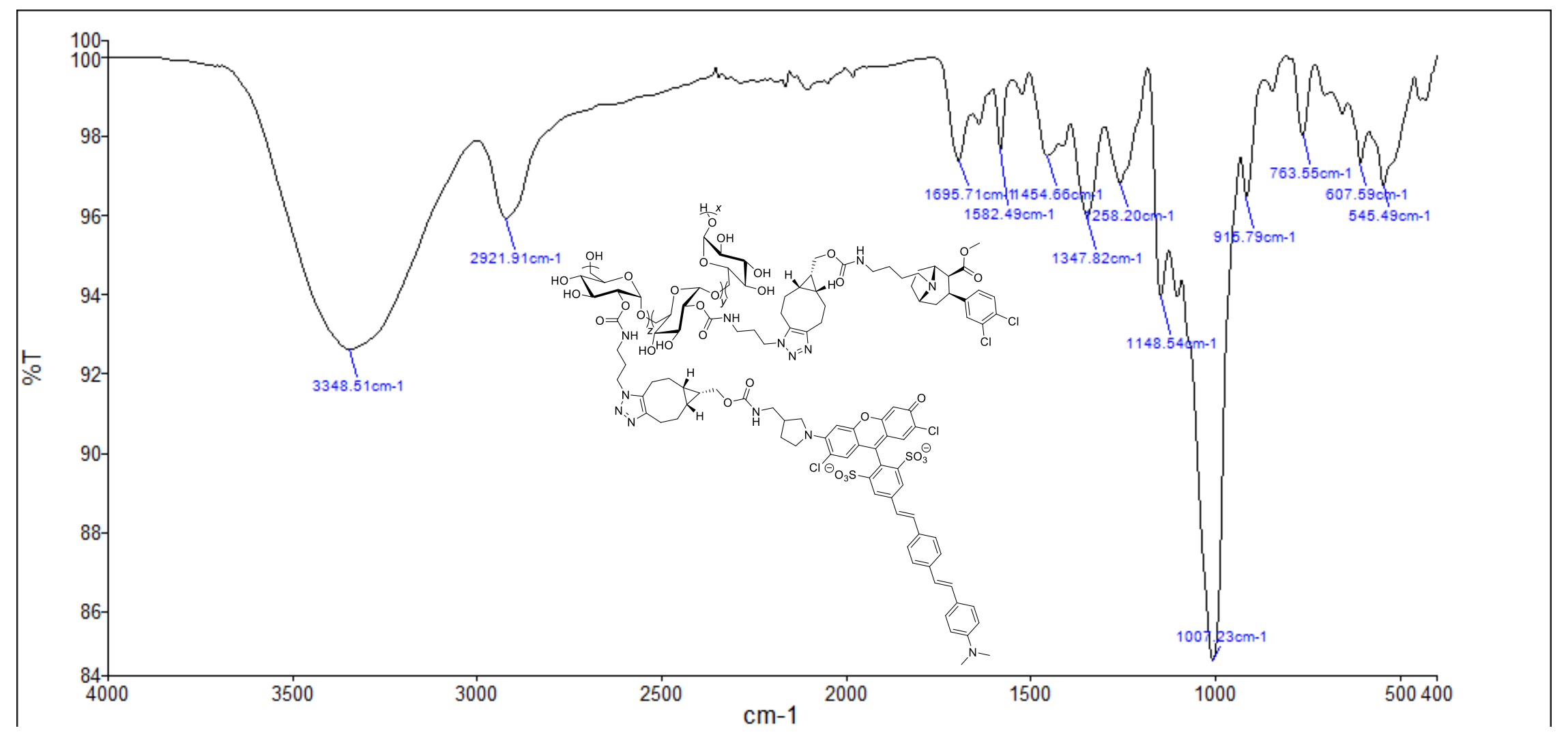

Figure S41. FTIR (ATR, diamond) spectrum of dsRVF5-VoLDeMo (13). 


\section{NMR Spectra}

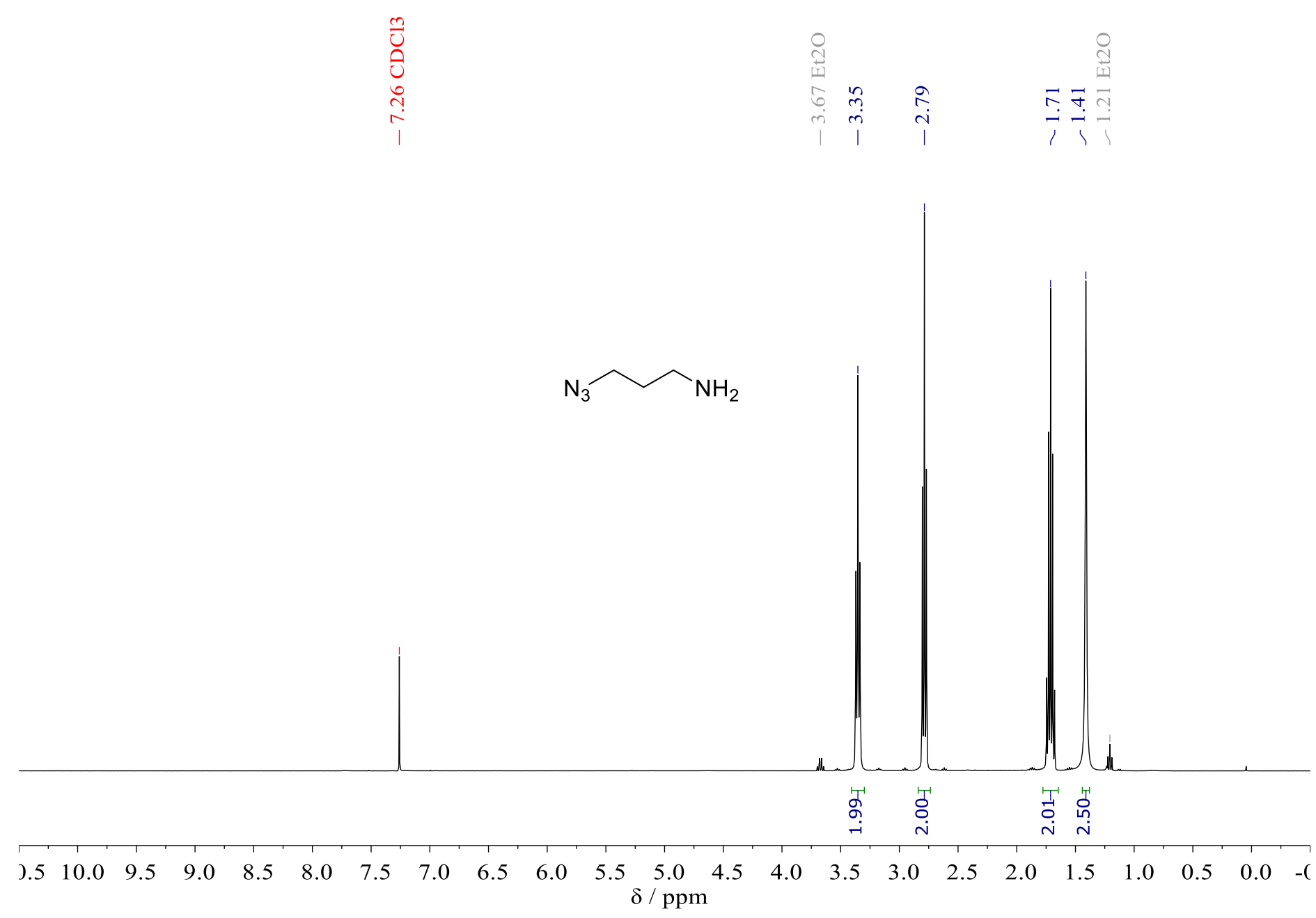

Figure S42. ${ }^{1} \mathrm{H}$ NMR (400 MHz, $\left.\mathrm{CDCl}_{3}\right)$ of 3-azidopropyl amine (S3). 

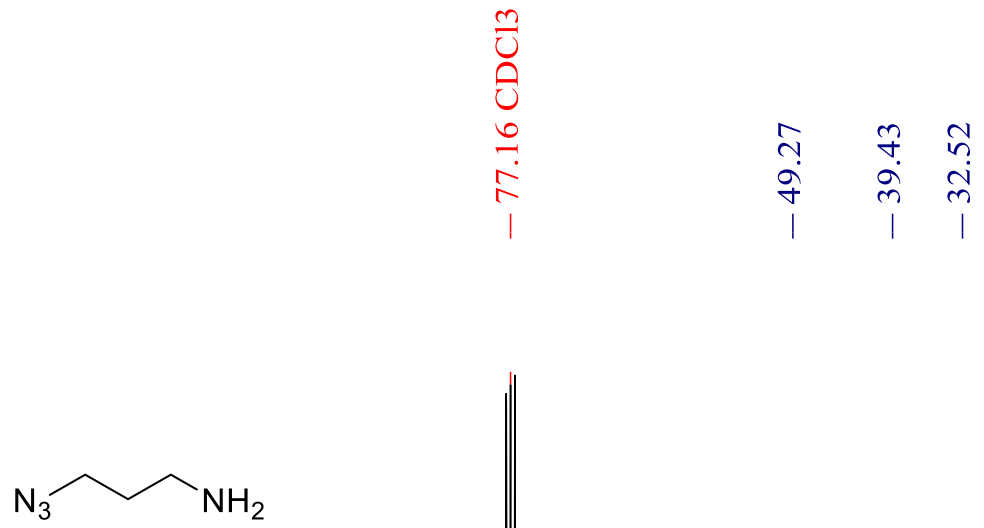

Ww.

$\begin{array}{llllllllllllllllllllllll}200 & 190 & 180 & 170 & 160 & 150 & 140 & 130 & 120 & \begin{array}{c}110 \\ \delta / \mathrm{ppm}\end{array} & \begin{array}{c}100 \\ \delta 0\end{array} & 80 & 70 & 60 & 50 & 40 & 30 & 20 & 10 & 0\end{array}$

Figure S43. ${ }^{13} \mathrm{C}\left\{{ }^{1} \mathrm{H}\right\}$ NMR (75 $\left.\mathrm{MHz}, \mathrm{CDCl}_{3}\right)$ of 3-azidopropyl amine (S3). 


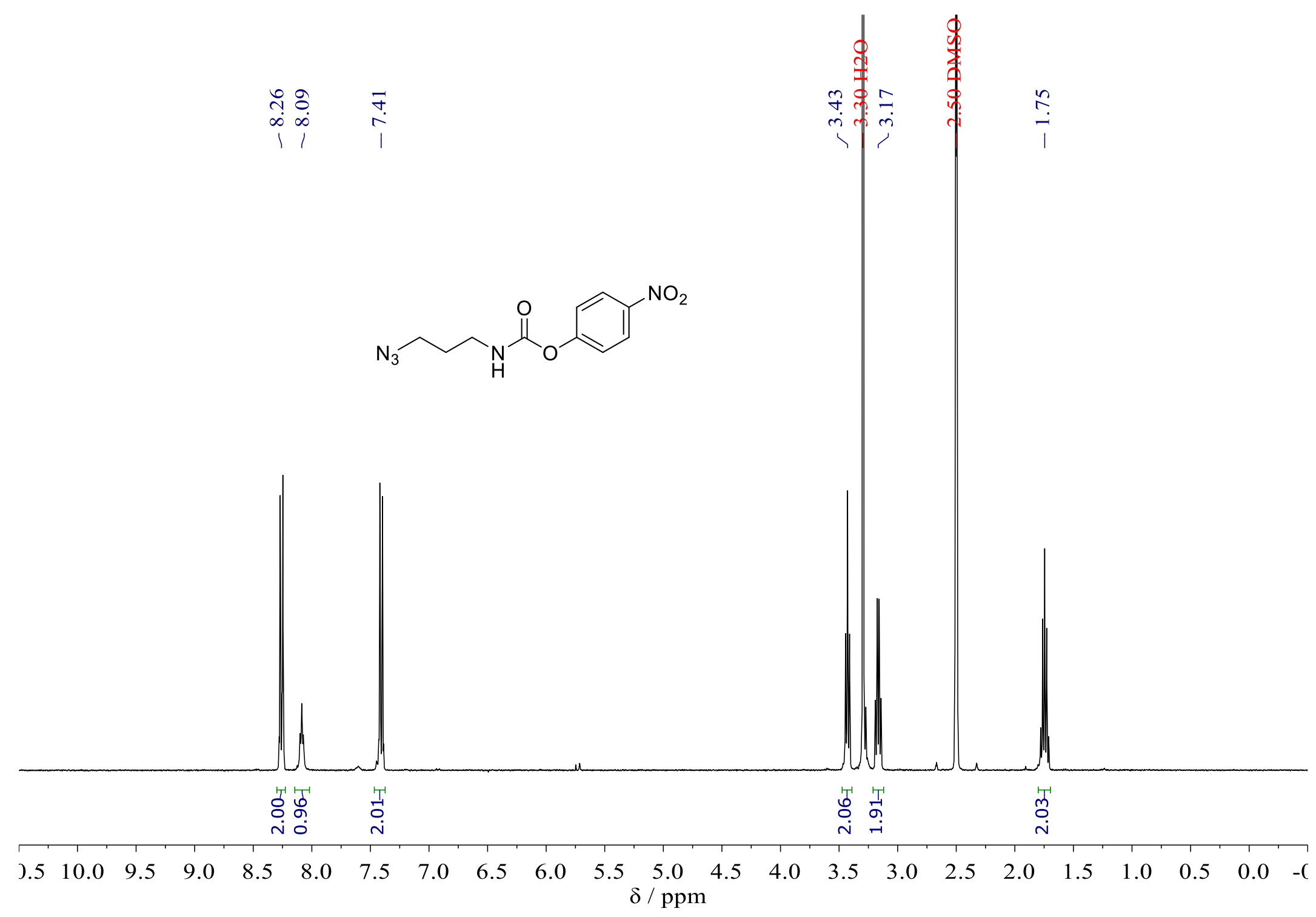

Figure S44. ${ }^{1} \mathrm{H}$ NMR (400 MHz, DMSO- $\left.d_{6}\right)$ of $N$-(3-azidopropyl)- $O$-(4-nitrophenyl) carbamate (7). 


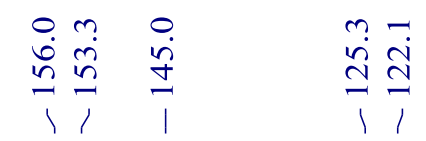
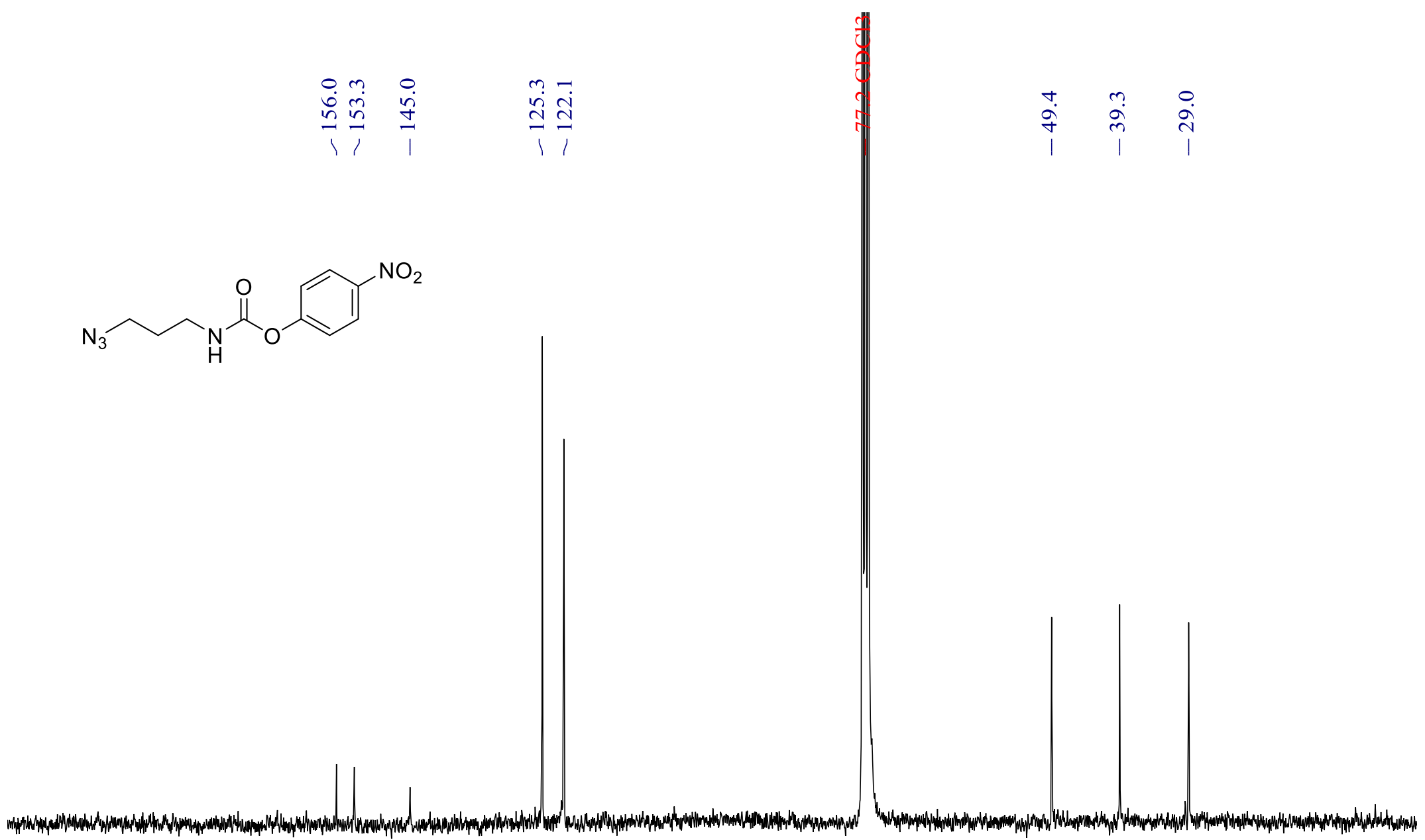

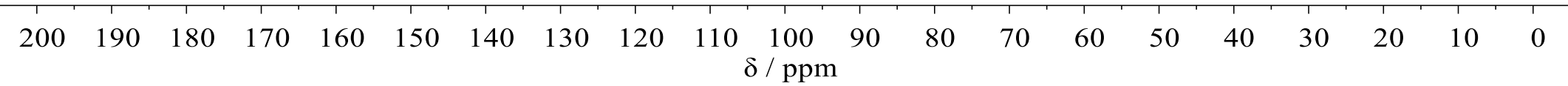

Figure S45. ${ }^{13} \mathrm{C}\left\{{ }^{1} \mathrm{H}\right\}$ NMR (75 MHz, $\mathrm{CDCl}_{3}$ ) of $N$-(3-azidopropyl)-O-(4-nitrophenyl) carbamate (7). 


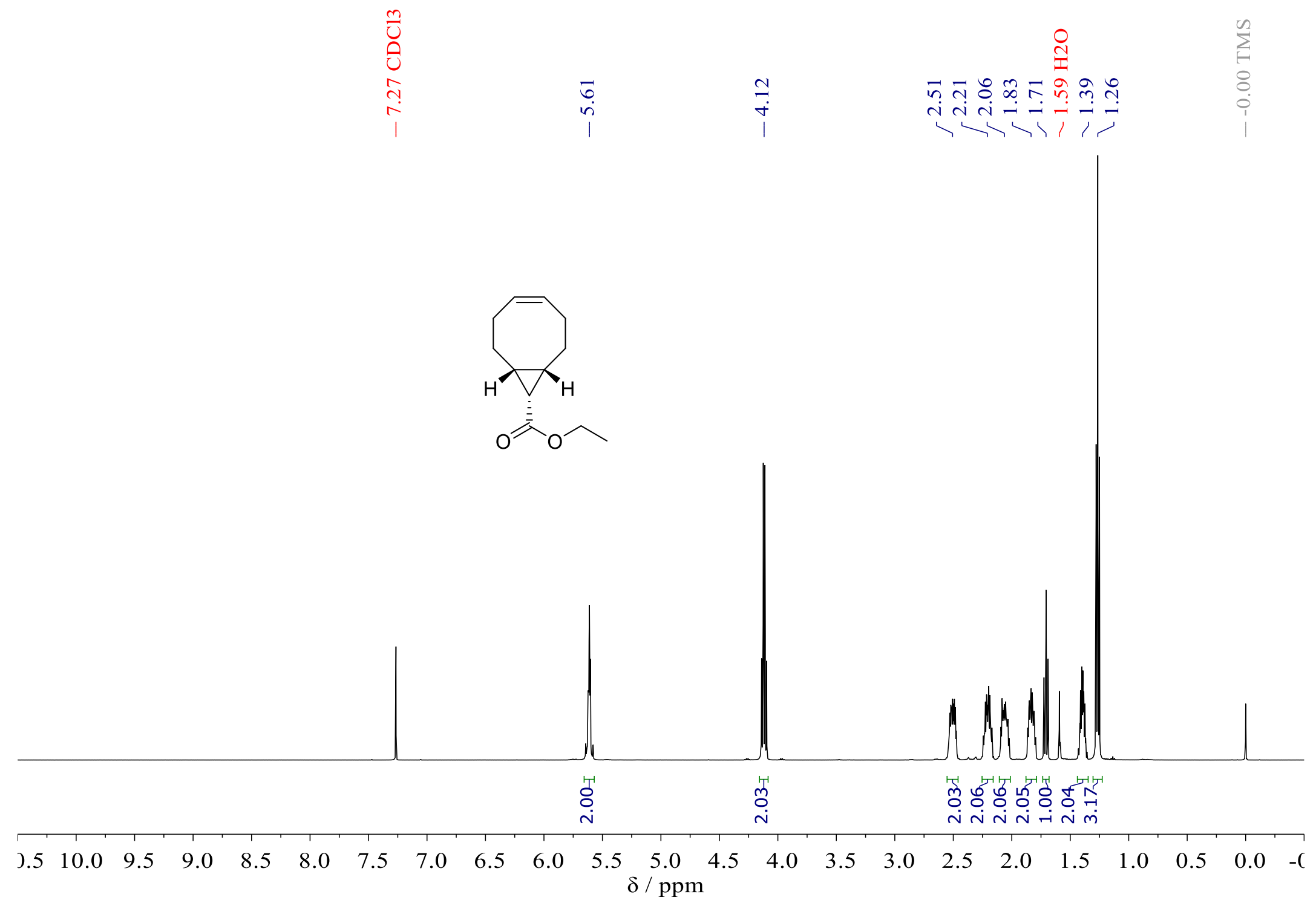

Figure S46. ${ }^{1} \mathrm{H}$ NMR $\left(500 \mathrm{MHz}, \mathrm{CDCl}_{3}\right)$ of compound endo-S4. 

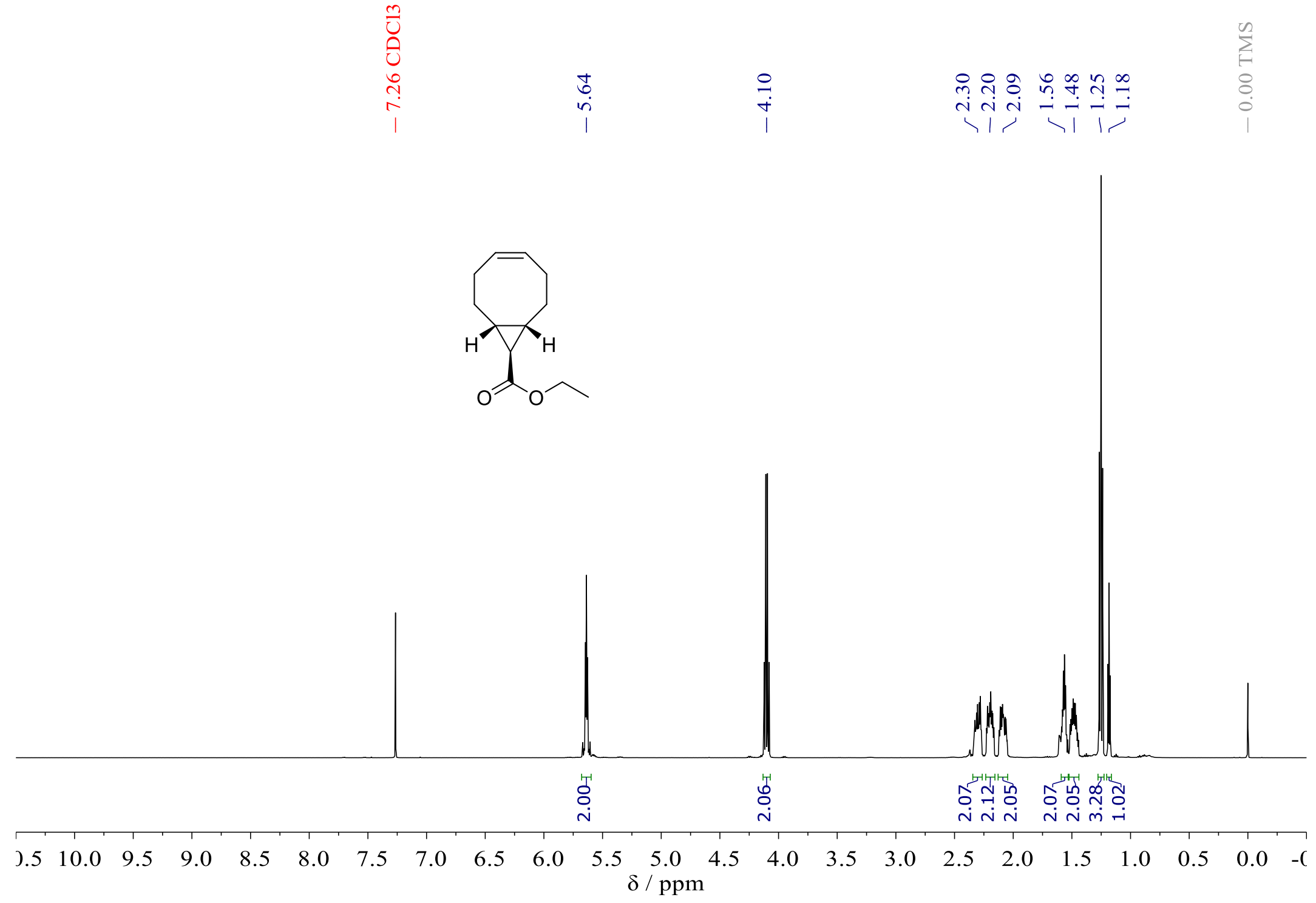

Figure S47. ${ }^{1} \mathrm{H}$ NMR $\left(500 \mathrm{MHz}, \mathrm{CDCl}_{3}\right)$ of compound exo-S4. 


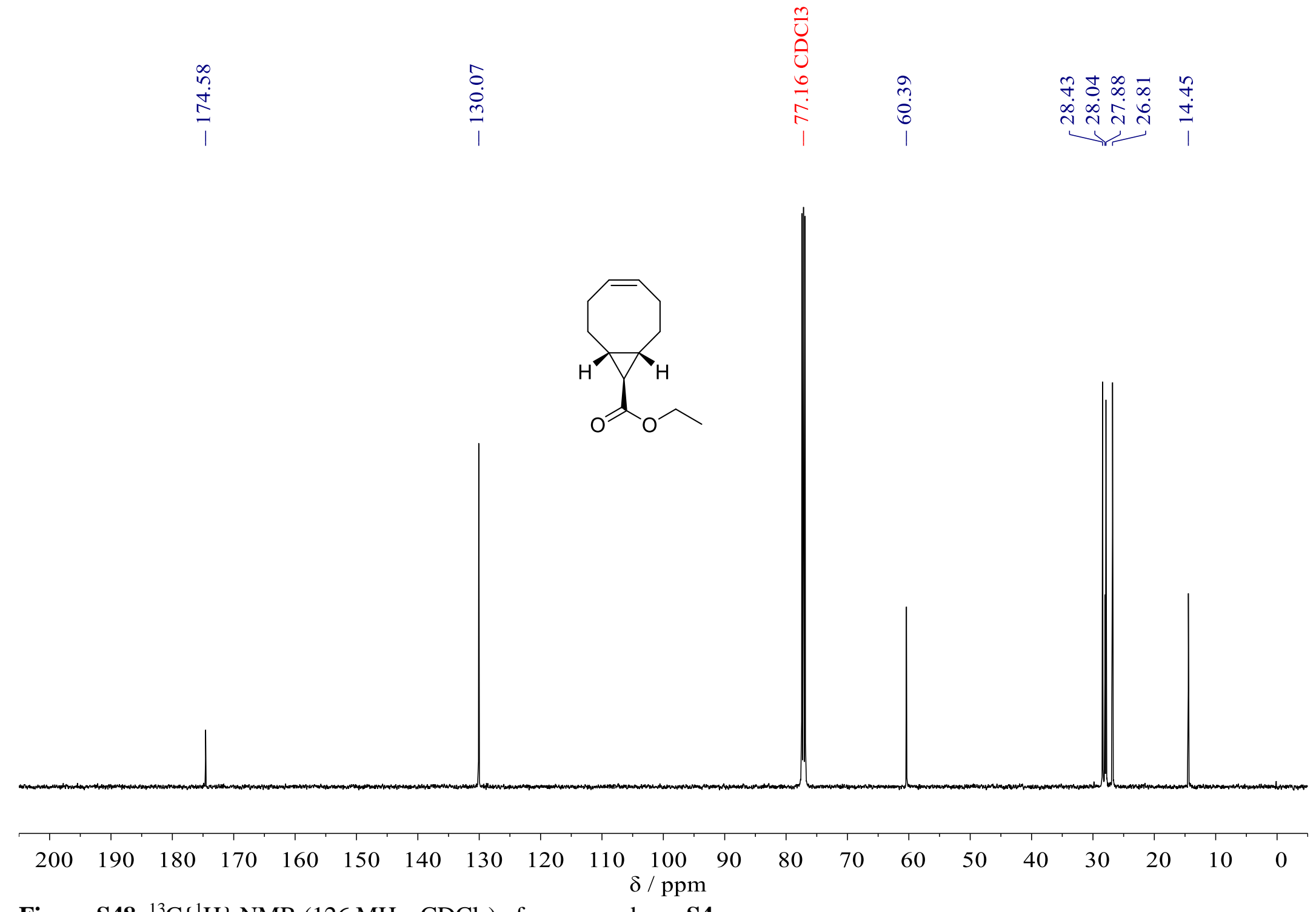

Figure S48. ${ }^{13} \mathrm{C}\left\{{ }^{1} \mathrm{H}\right\} \mathrm{NMR}\left(126 \mathrm{MHz}, \mathrm{CDCl}_{3}\right)$ of compound exo-S4. 


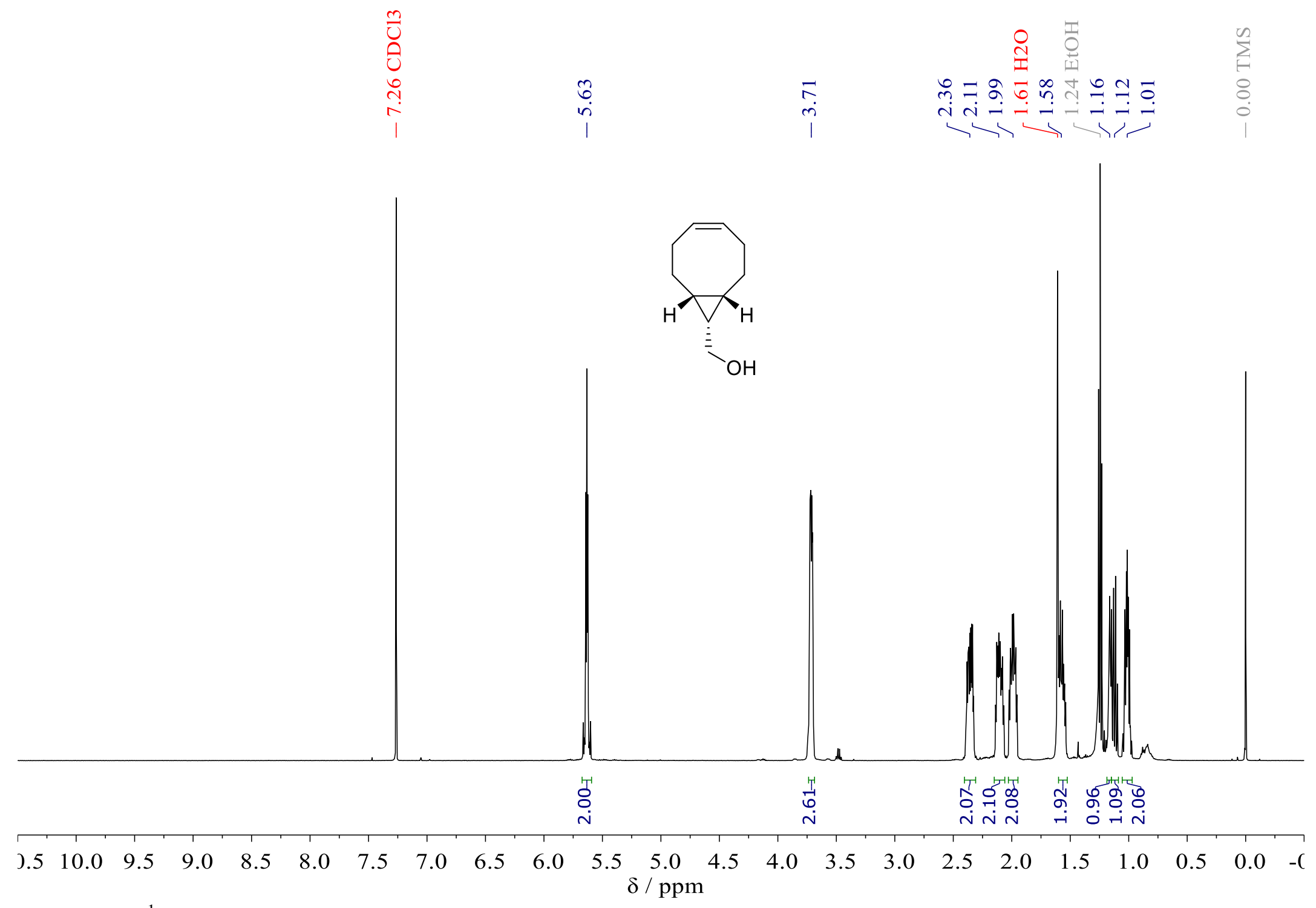

Figure S49. ${ }^{1} \mathrm{H}$ NMR $\left(500 \mathrm{MHz}, \mathrm{CDCl}_{3}\right)$ of compound S5. 


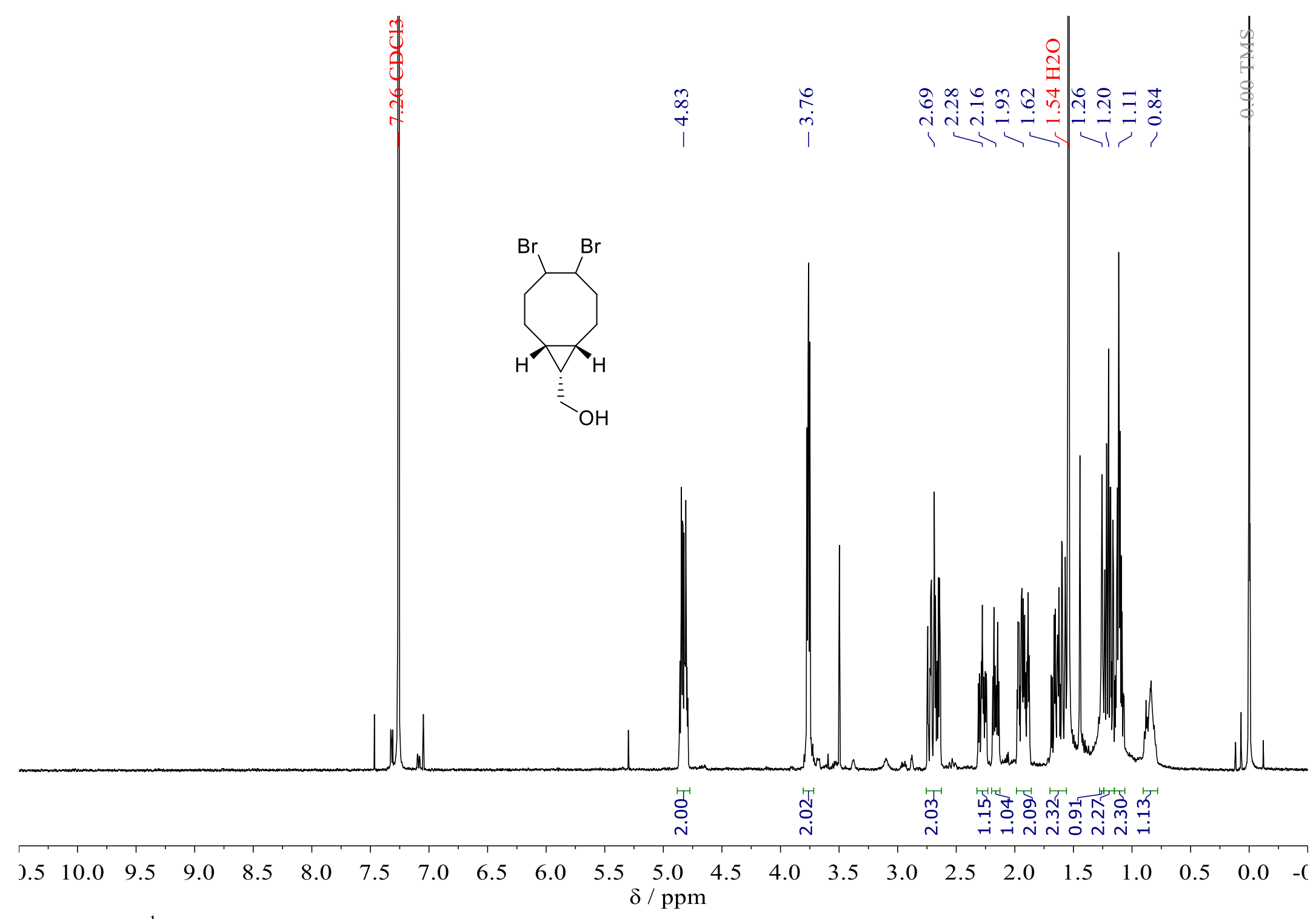

Figure S50. ${ }^{1} \mathrm{H}$ NMR $\left(500 \mathrm{MHz}, \mathrm{CDCl}_{3}\right)$ of compound S6. 


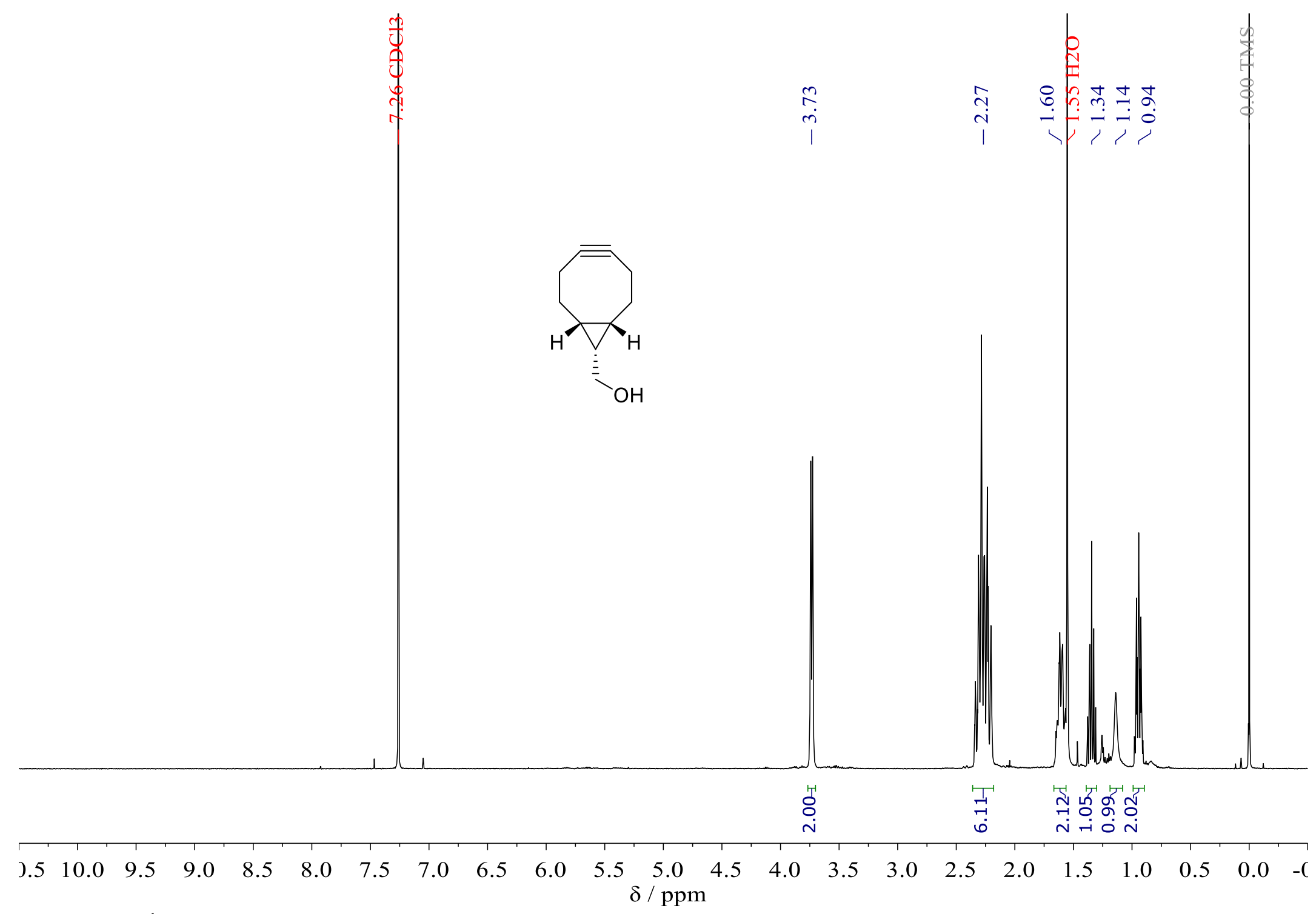

Figure S51. ${ }^{1} \mathrm{H} \mathrm{NMR}\left(500 \mathrm{MHz}, \mathrm{CDCl}_{3}\right)$ of $\mathrm{BCN}-\mathrm{OH}(\mathbf{S 7})$. 


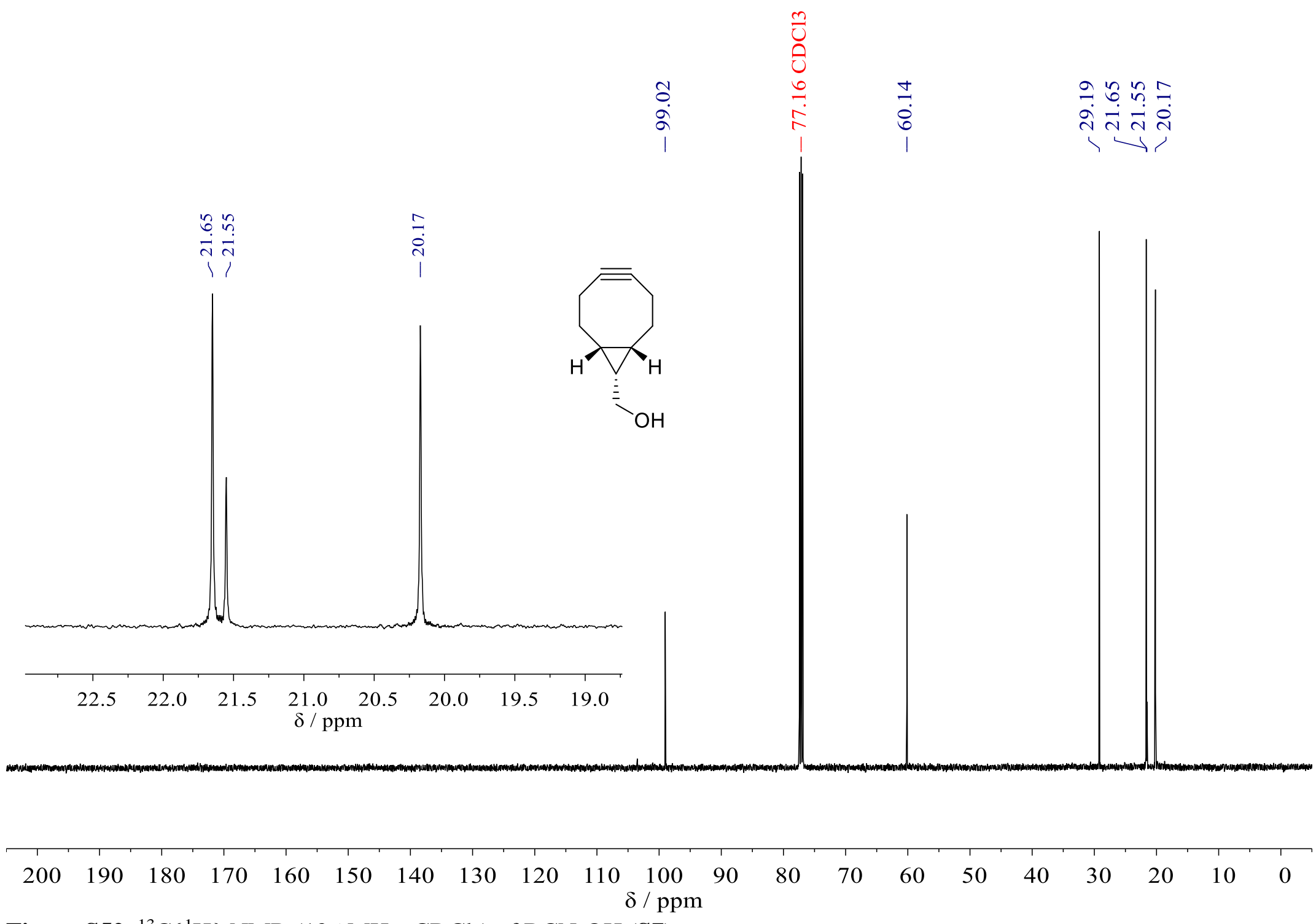

Figure S52. ${ }^{13} \mathrm{C}\left\{{ }^{1} \mathrm{H}\right\} \mathrm{NMR}\left(126 \mathrm{MHz}, \mathrm{CDCl}_{3}\right)$ of $\mathrm{BCN}-\mathrm{OH}(\mathbf{S 7})$. 


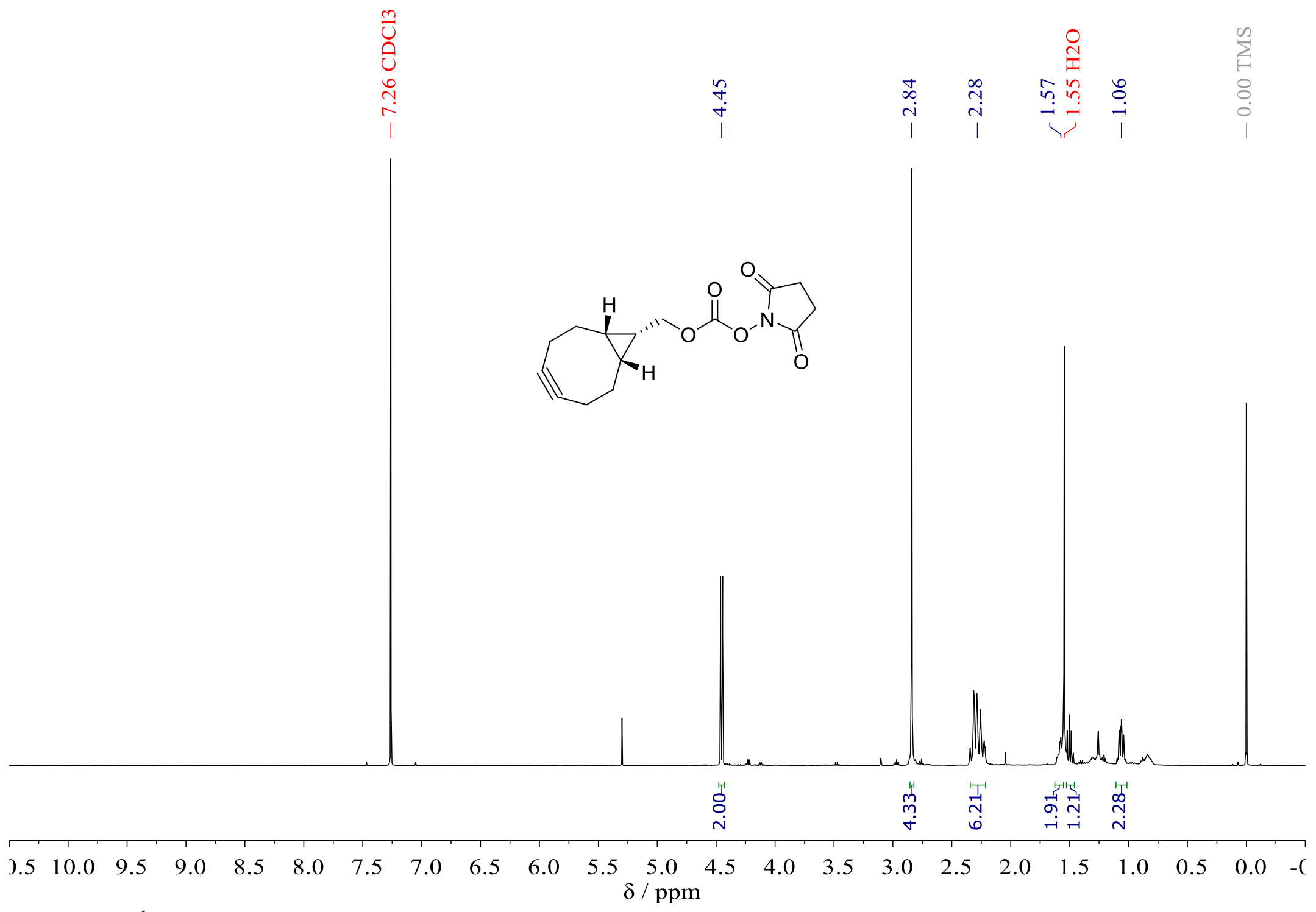

Figure S53. ${ }^{1} \mathrm{H} \mathrm{NMR}\left(500 \mathrm{MHz}, \mathrm{CDCl}_{3}\right)$ of $\mathrm{BCN}-\mathrm{OSu}(\mathbf{S 8})$.

S161 


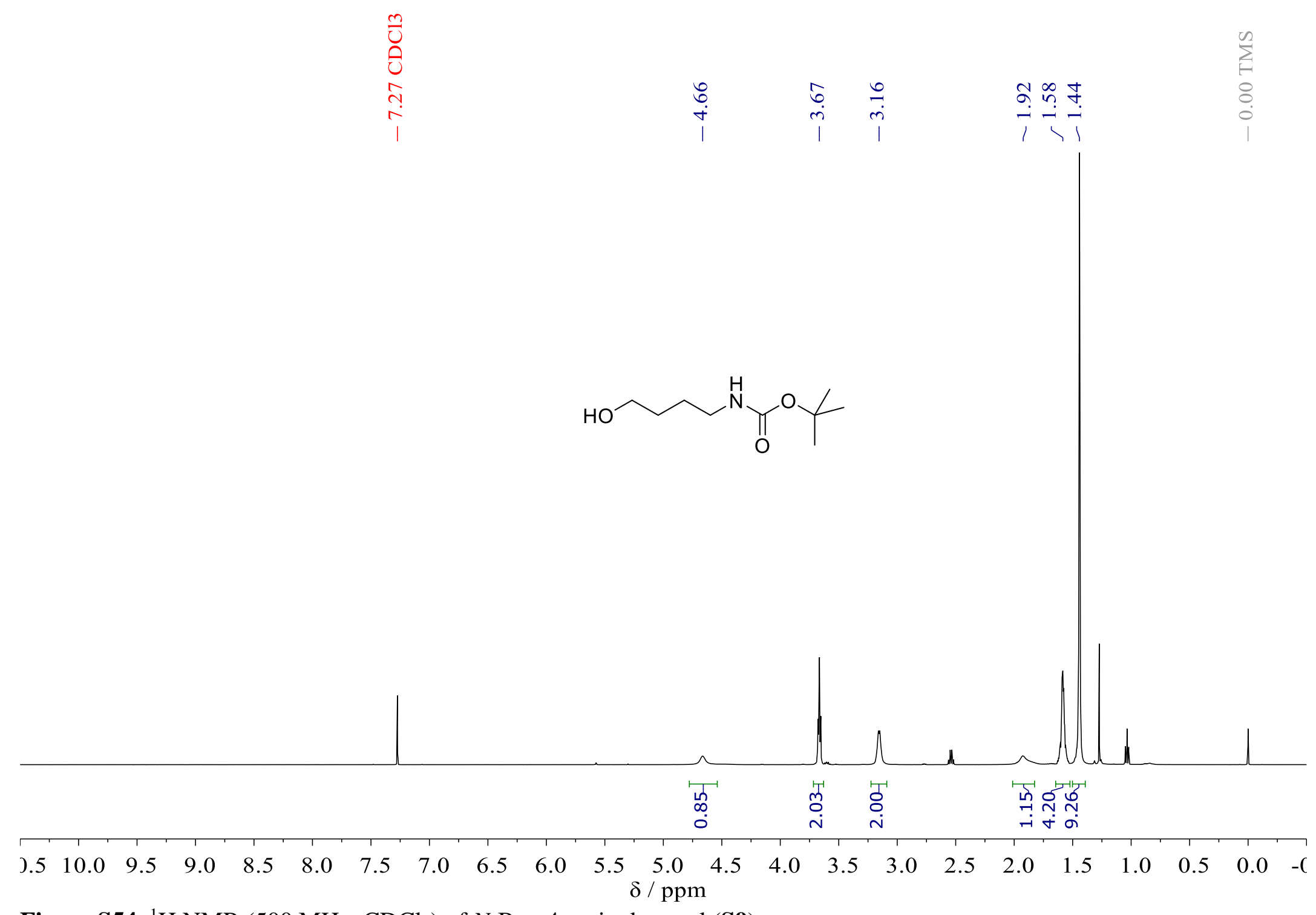

Figure S54. ${ }^{1} \mathrm{H}$ NMR $\left(500 \mathrm{MHz}, \mathrm{CDCl}_{3}\right)$ of $\mathrm{N}$-Boc-4-aminobutanol (S9). 


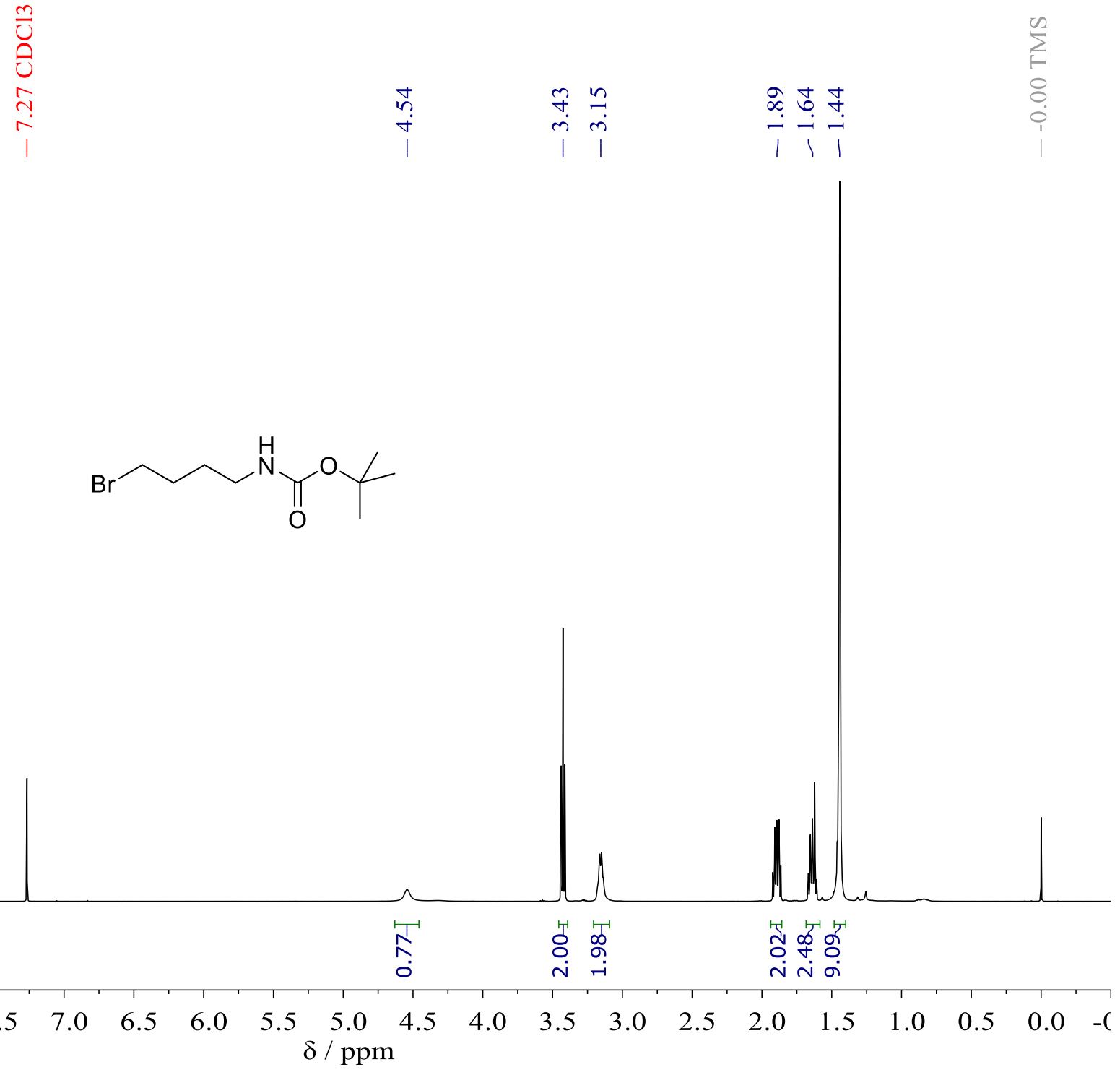

$\begin{array}{lllllllllllllllllllllll}0.5 & 10.0 & 9.5 & 9.0 & 8.5 & 8.0 & 7.5 & 7.0 & 6.5 & 6.0 & 5.5 & \begin{array}{c}5.0 \\ \delta / \mathrm{ppm}\end{array} & 4.5 & 4.0 & 3.5 & 3.0 & 2.5 & 2.0 & 1.5 & 1.0 & 0.5 & 0.0 & -(\end{array}$

Figure S55. ${ }^{1} \mathrm{H}$ NMR $\left(500 \mathrm{MHz}, \mathrm{CDCl}_{3}\right)$ of $N$-Boc-4-bromobutyl amine (S10). 


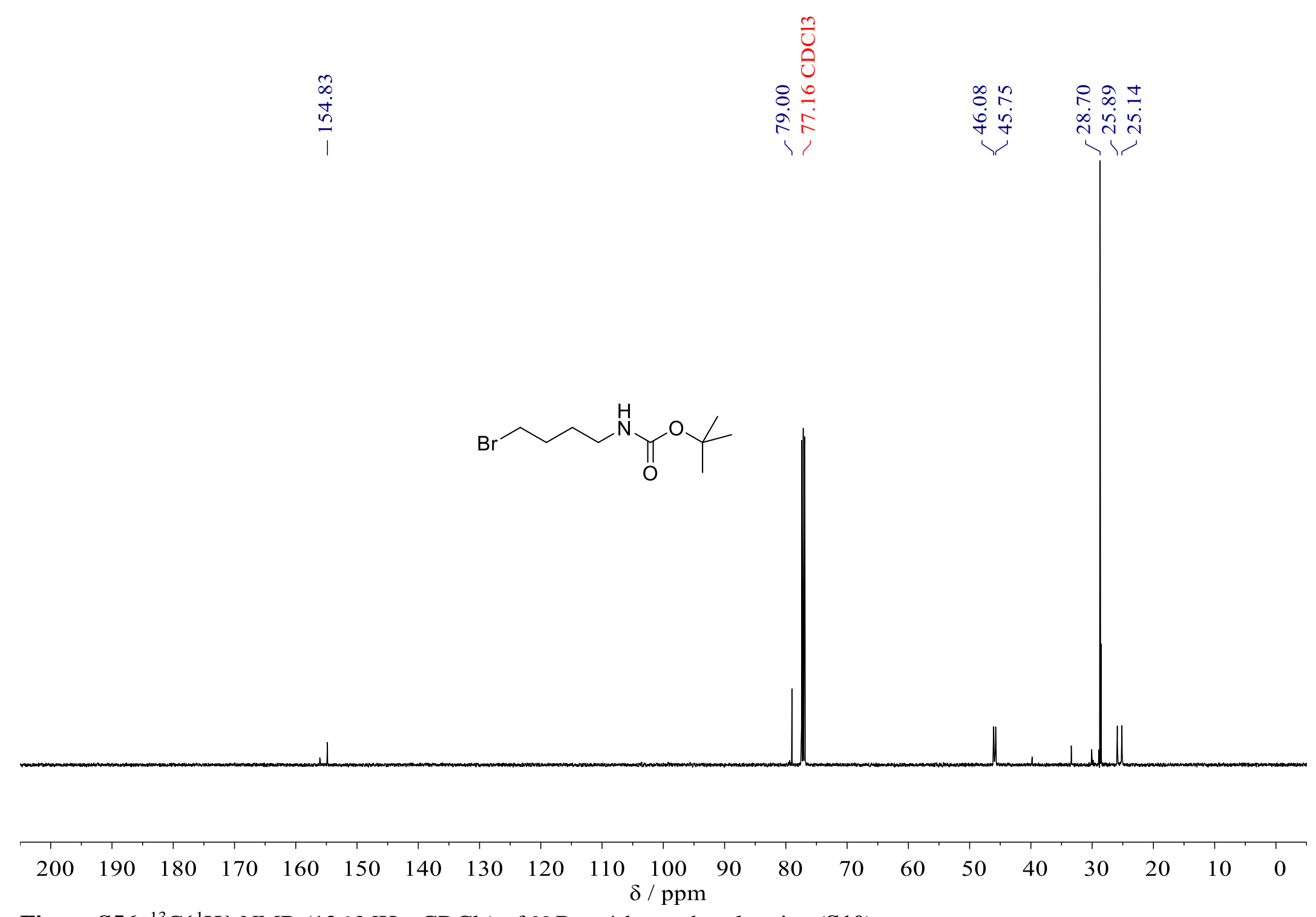

Figure S56. ${ }^{13} \mathrm{C}\left\{{ }^{1} \mathrm{H}\right\}$ NMR $\left(126 \mathrm{MHz}, \mathrm{CDCl}_{3}\right)$ of $\mathrm{N}$-Boc-4-bromobutyl amine (S10). 


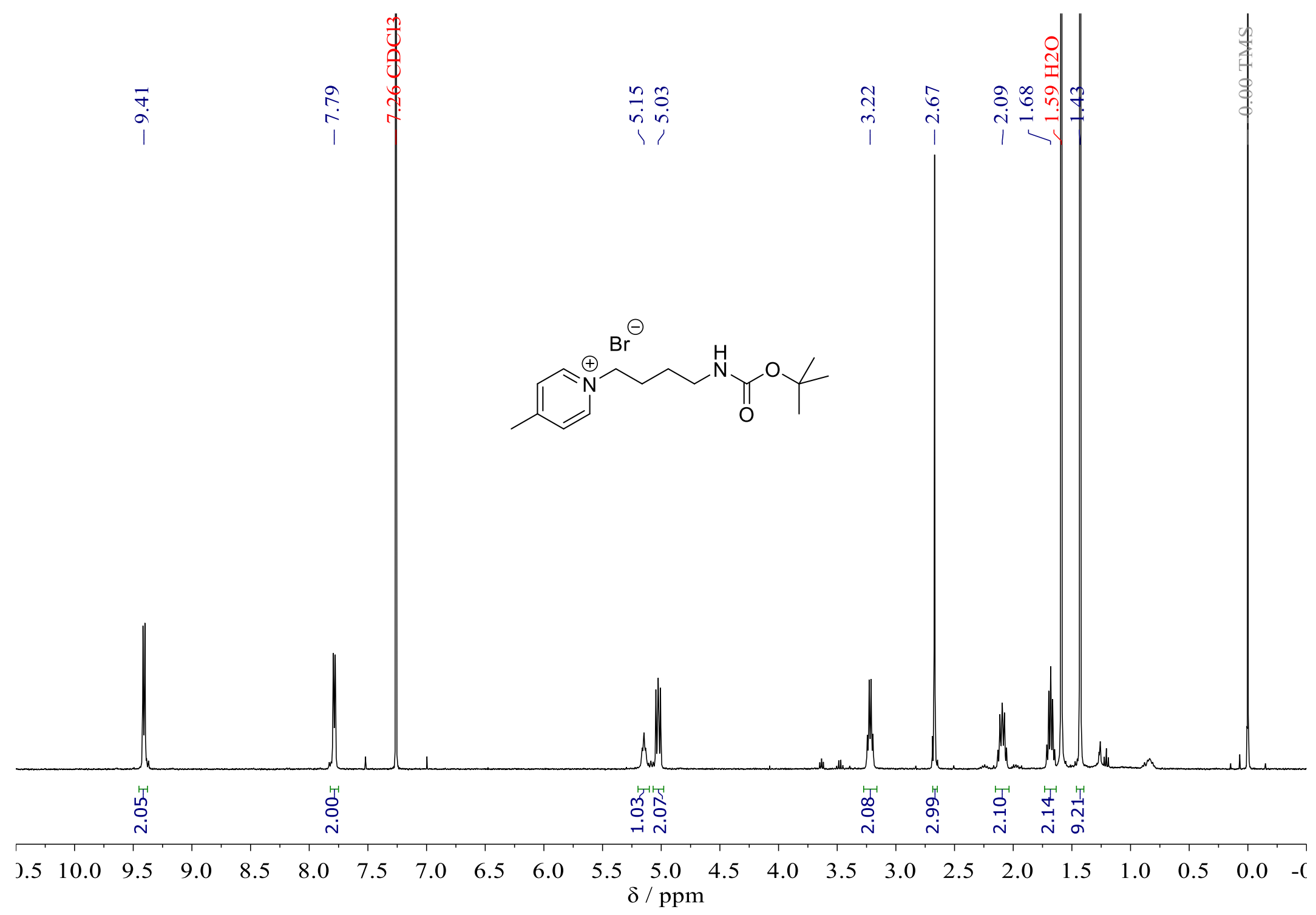

Figure S57. ${ }^{1} \mathrm{H}$ NMR $\left(400 \mathrm{MHz}, \mathrm{CDCl}_{3}\right)$ of $N$-(N'-Boc-aminobutyl)-4-methylpyridinium bromide $(\mathbf{S 1 1})$. 


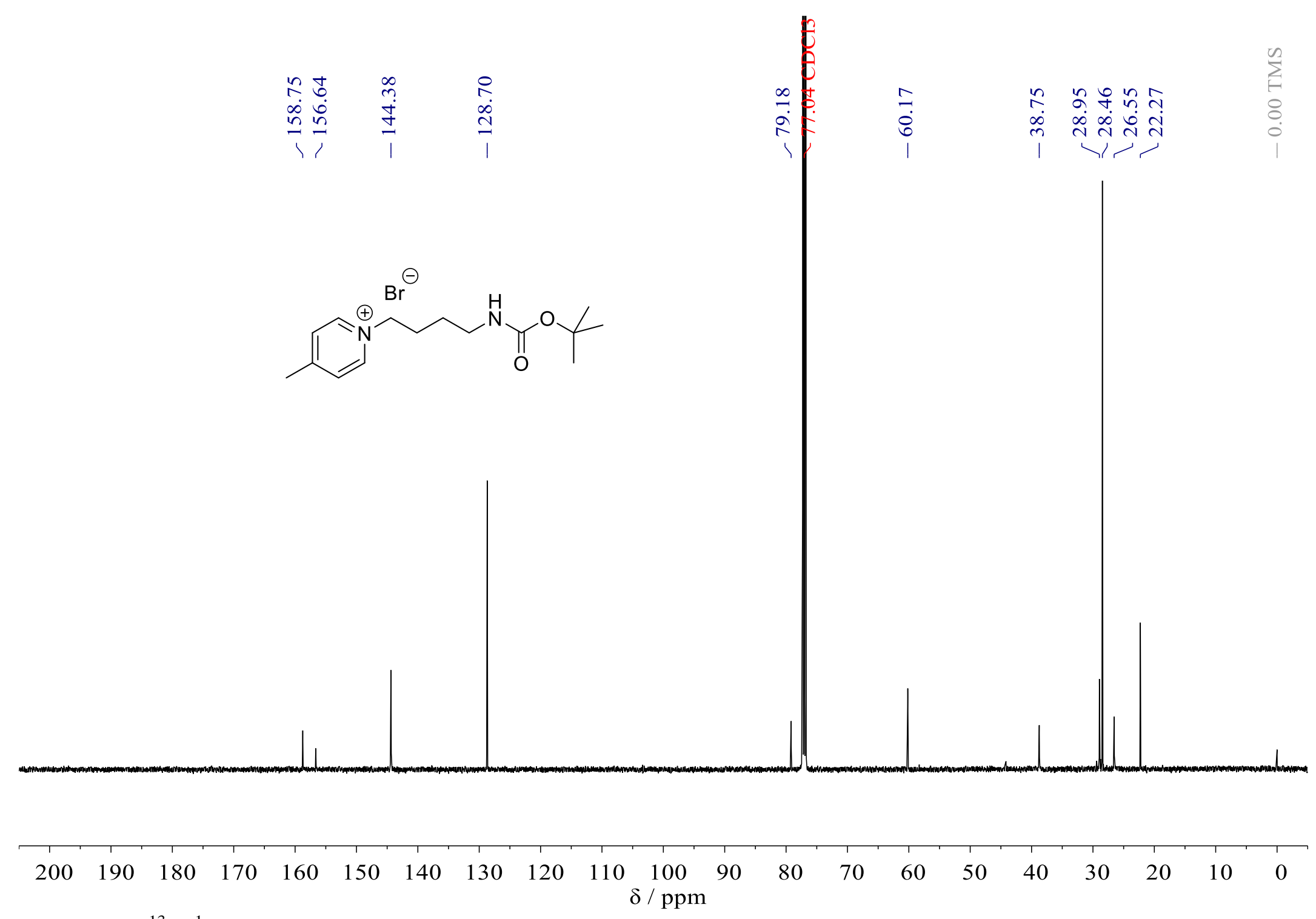

Figure S58. ${ }^{13} \mathrm{C}\left\{{ }^{1} \mathrm{H}\right\}$ NMR $\left(126 \mathrm{MHz}, \mathrm{CDCl}_{3}\right)$ of $N$-(N'-Boc-aminobutyl)-4-methylpyridinium bromide (S11). 


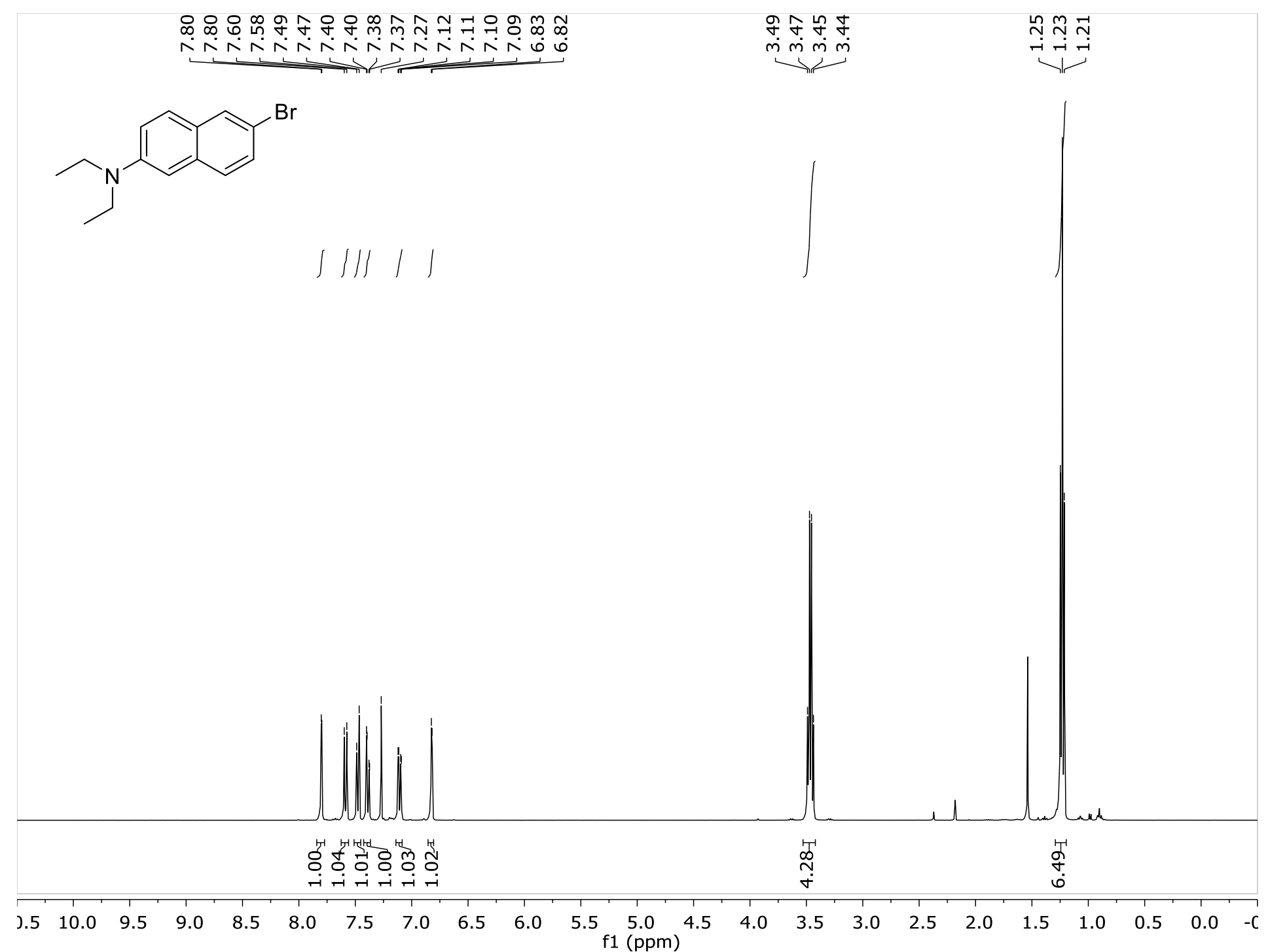

Figure S59. ${ }^{1} \mathrm{H}$ NMR $\left(\mathrm{CDCl}_{3}, 400 \mathrm{MHz}\right)$ of 6-bromo- $N, N$-diethylnaphthalen-2-amine (S12). 


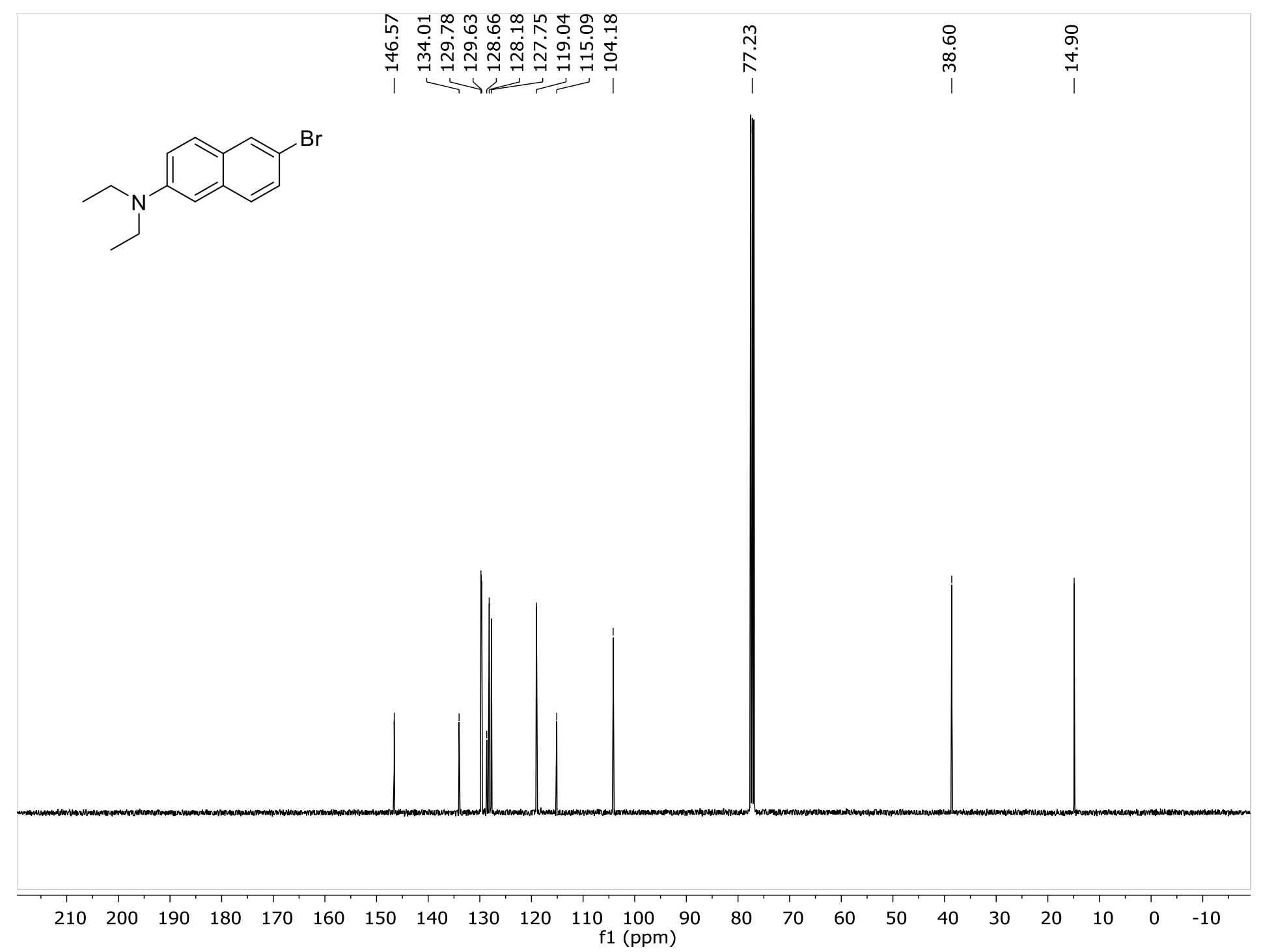

Figure S60. ${ }^{13} \mathrm{C}\left\{{ }^{1} \mathrm{H}\right\}$ NMR $\left(\mathrm{CDCl}_{3}, 100 \mathrm{MHz}\right)$ of 6-bromo- $N, N$-diethylnaphthalen-2-amine (S12). 


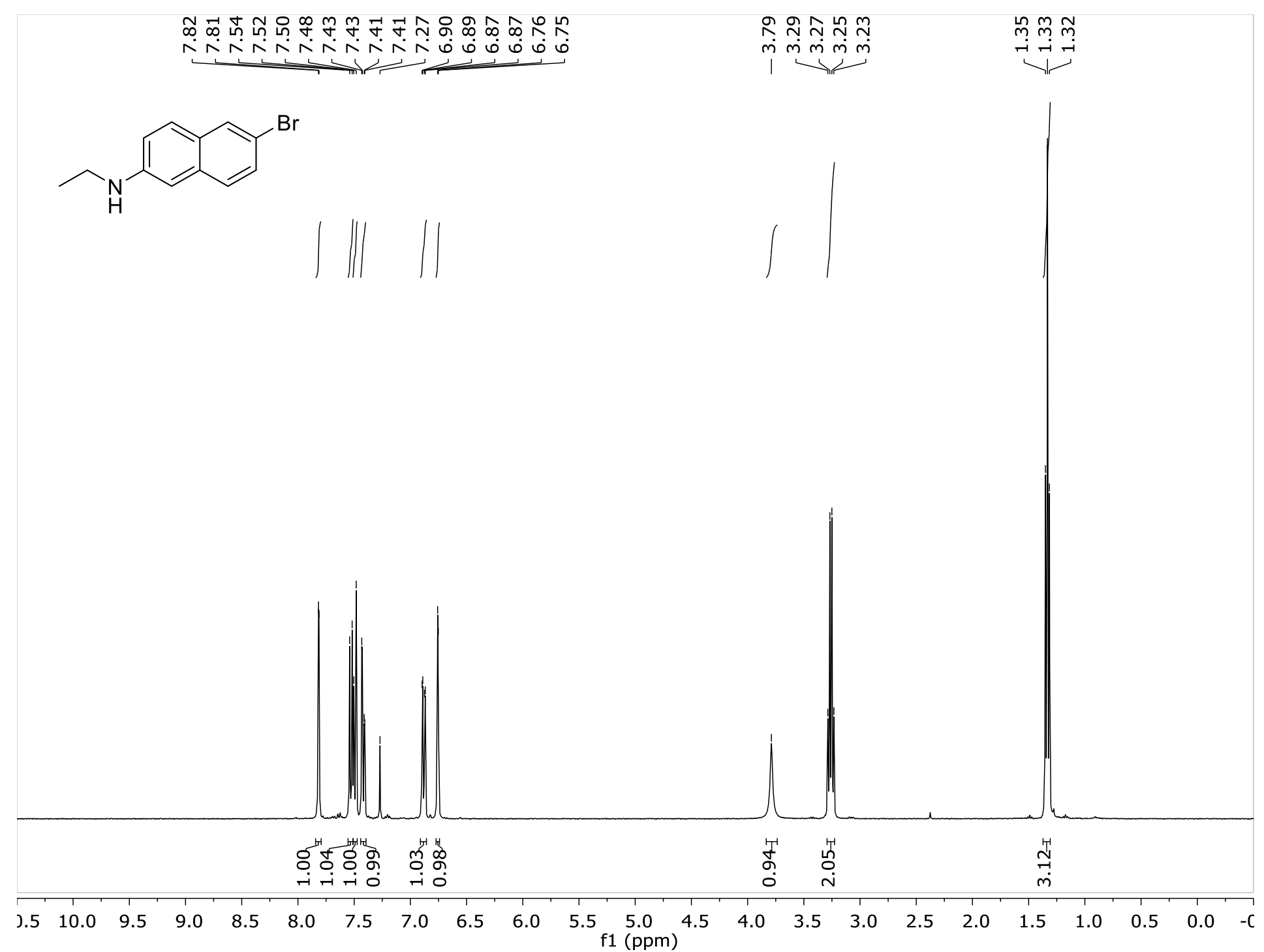

Figure S61. ${ }^{1} \mathrm{H}$ NMR $\left(\mathrm{CDCl}_{3}, 400 \mathrm{MHz}\right)$ of 6-bromo- $N$-ethylnaphthalen-2-amine (S12b). 


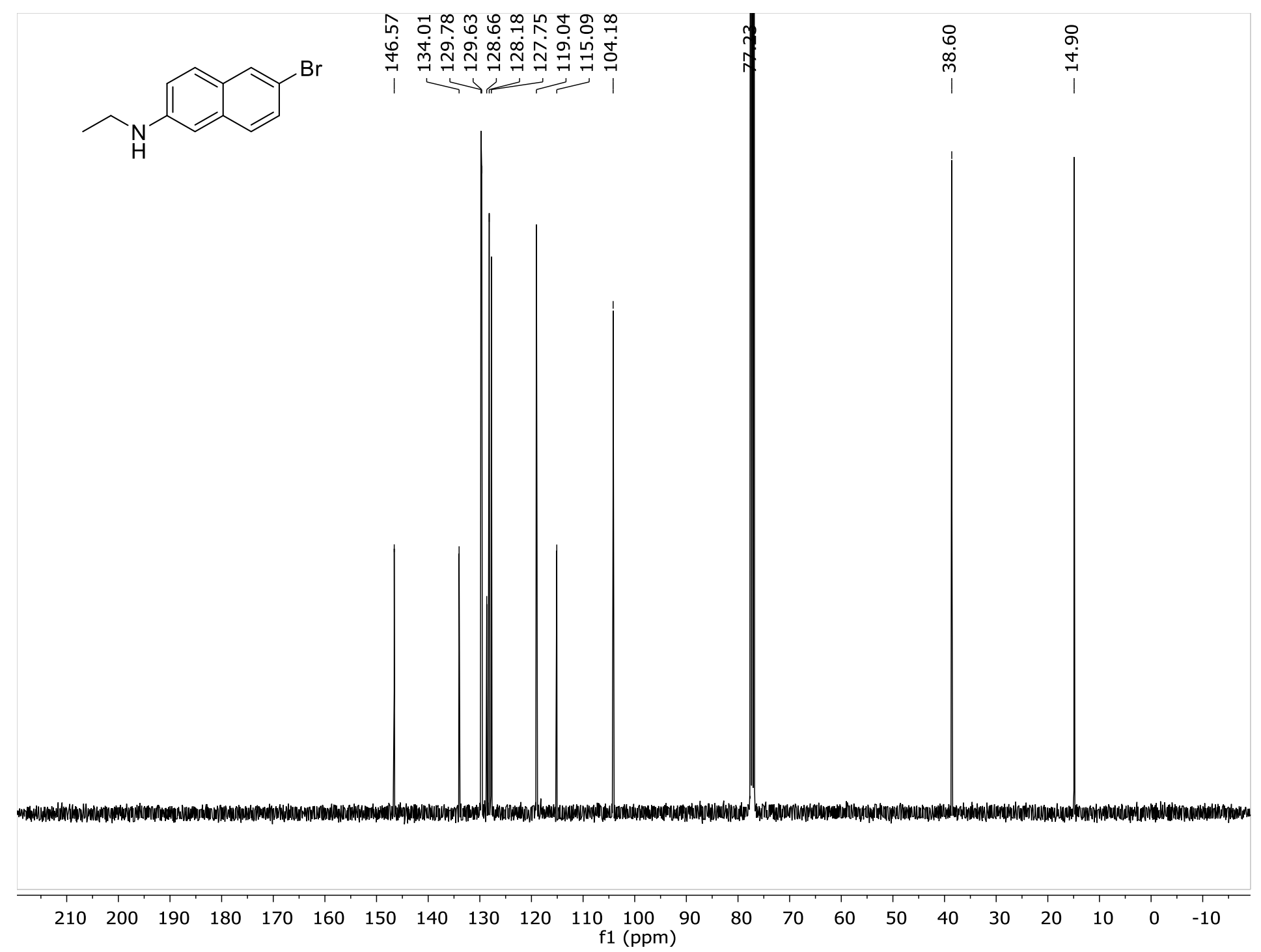

Figure S62. ${ }^{13} \mathrm{C}\left\{{ }^{1} \mathrm{H}\right\} \mathrm{NMR}\left(\mathrm{CDCl}_{3}, 100 \mathrm{MHz}\right)$ of 6-bromo- $N$-ethylnaphthalen-2-amine $(\mathbf{S 1 2 b})$. 


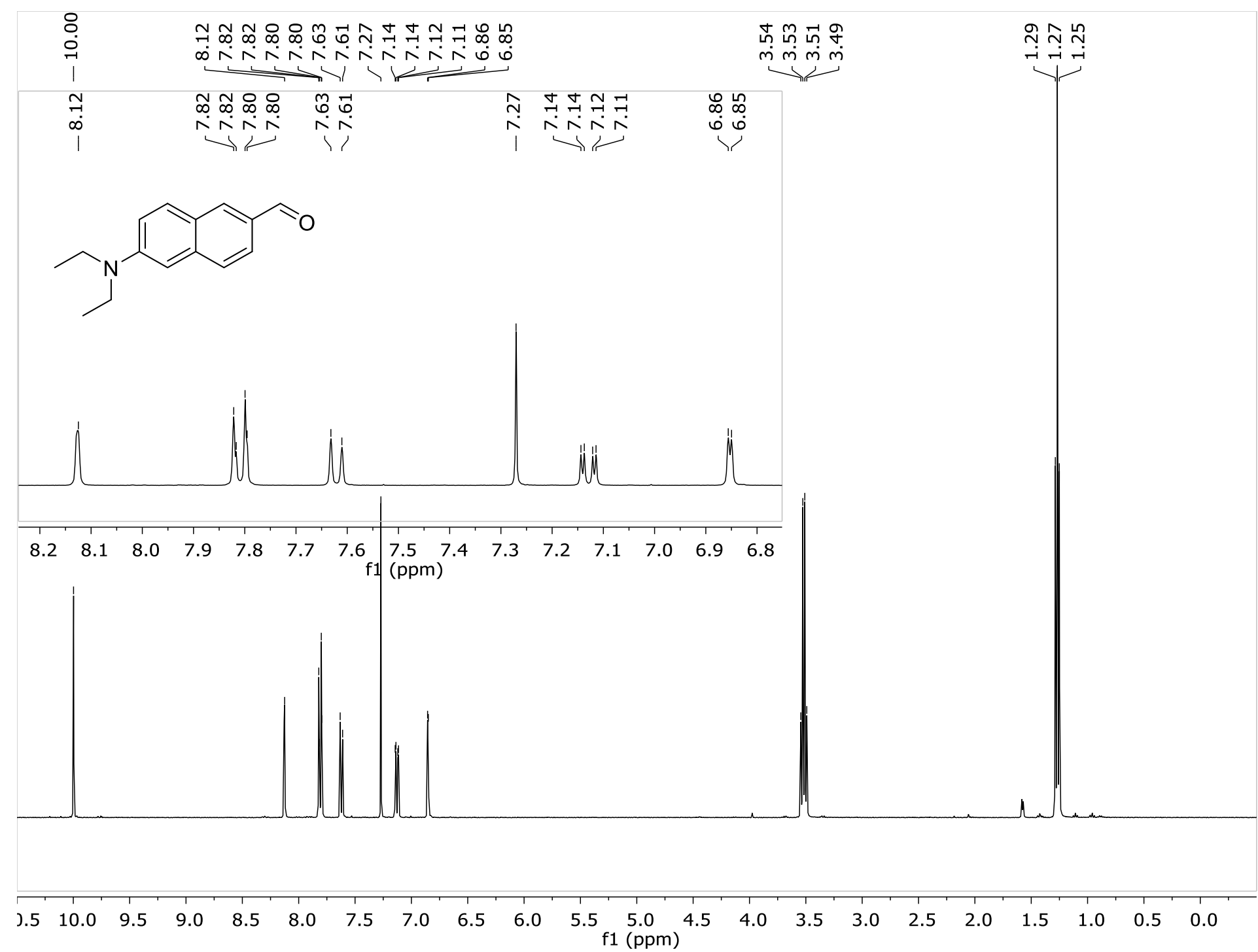

Figure S63. ${ }^{1} \mathrm{H}$ NMR $\left(\mathrm{CDCl}_{3}, 400 \mathrm{MHz}\right)$ of 6-(diethylamino)-2-naphthaldehyde (S13). 


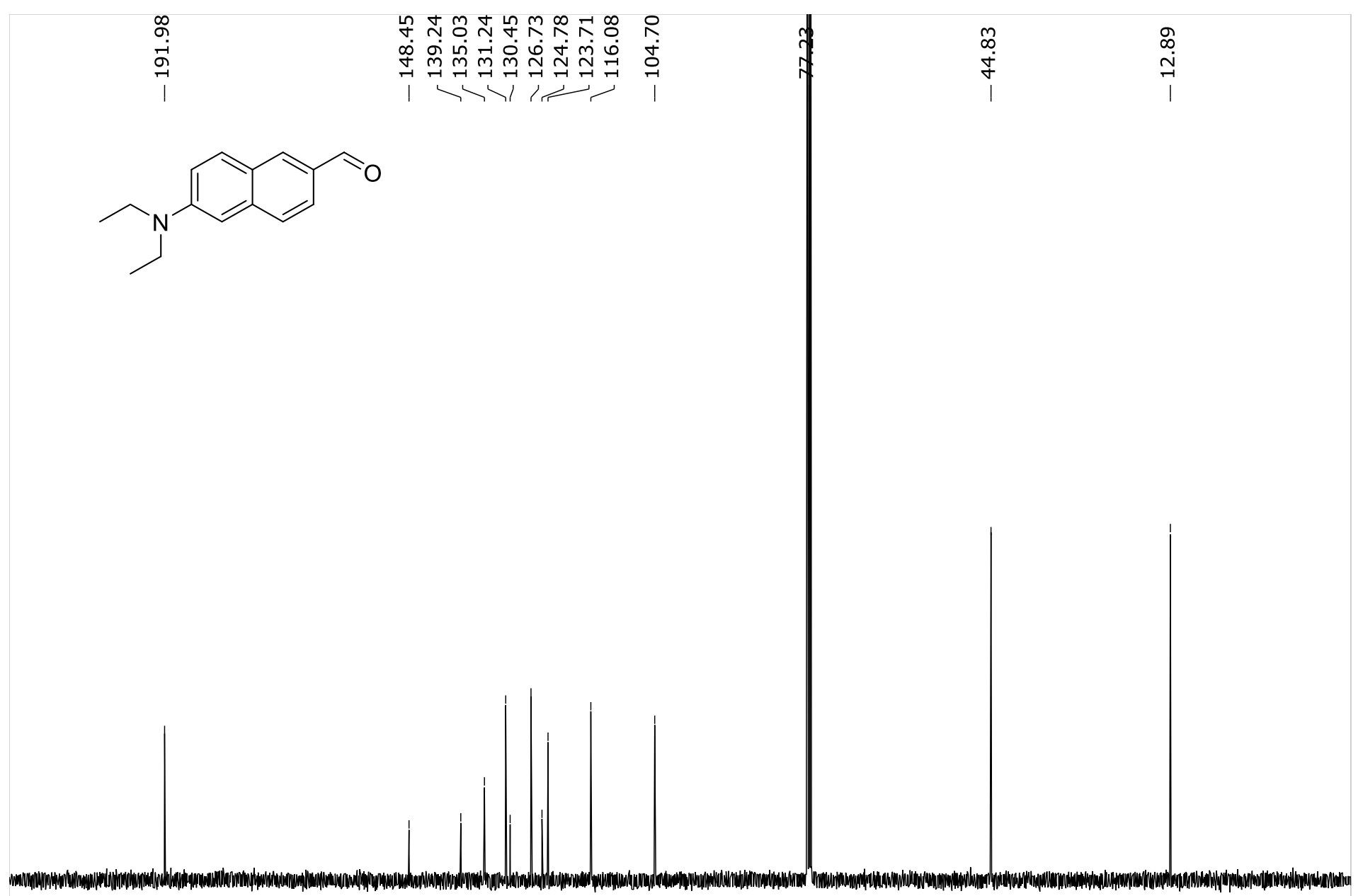

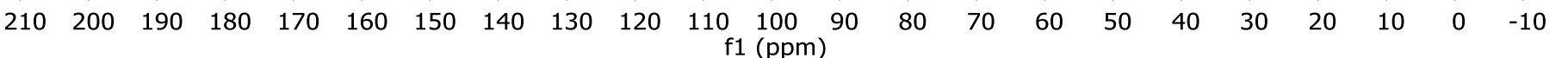

Figure S64. ${ }^{13} \mathrm{C}\left\{{ }^{1} \mathrm{H}\right\}$ NMR $\left(\mathrm{CDCl}_{3}, 100 \mathrm{MHz}\right)$ of 6-(diethylamino)-2-naphthaldehyde (S13). 


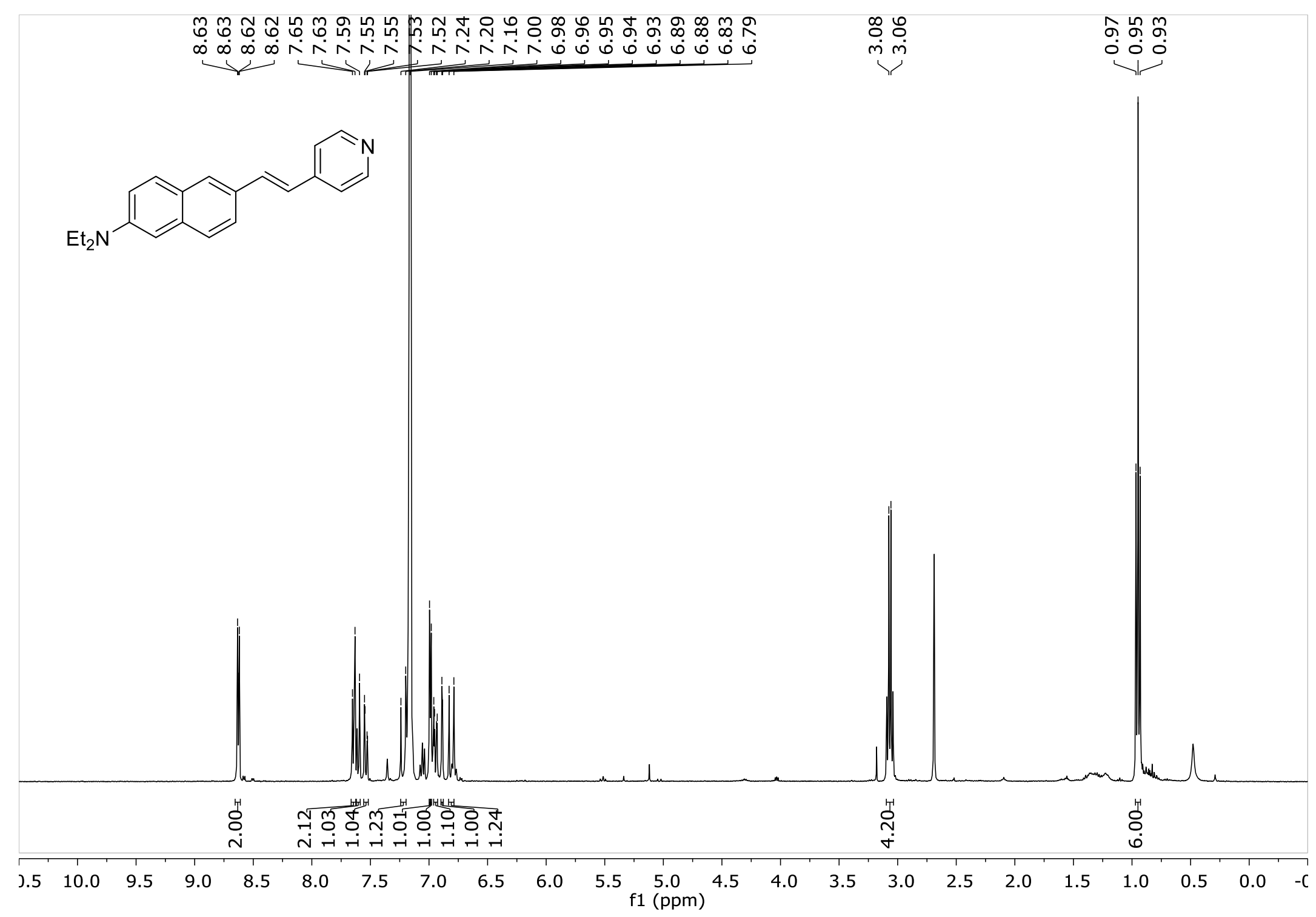

Figure S65. ${ }^{1} \mathrm{H}$ NMR $\left(\mathrm{CDCl}_{3}, 400 \mathrm{MHz}\right)$ of compound $\mathbf{S 1 4}$. 


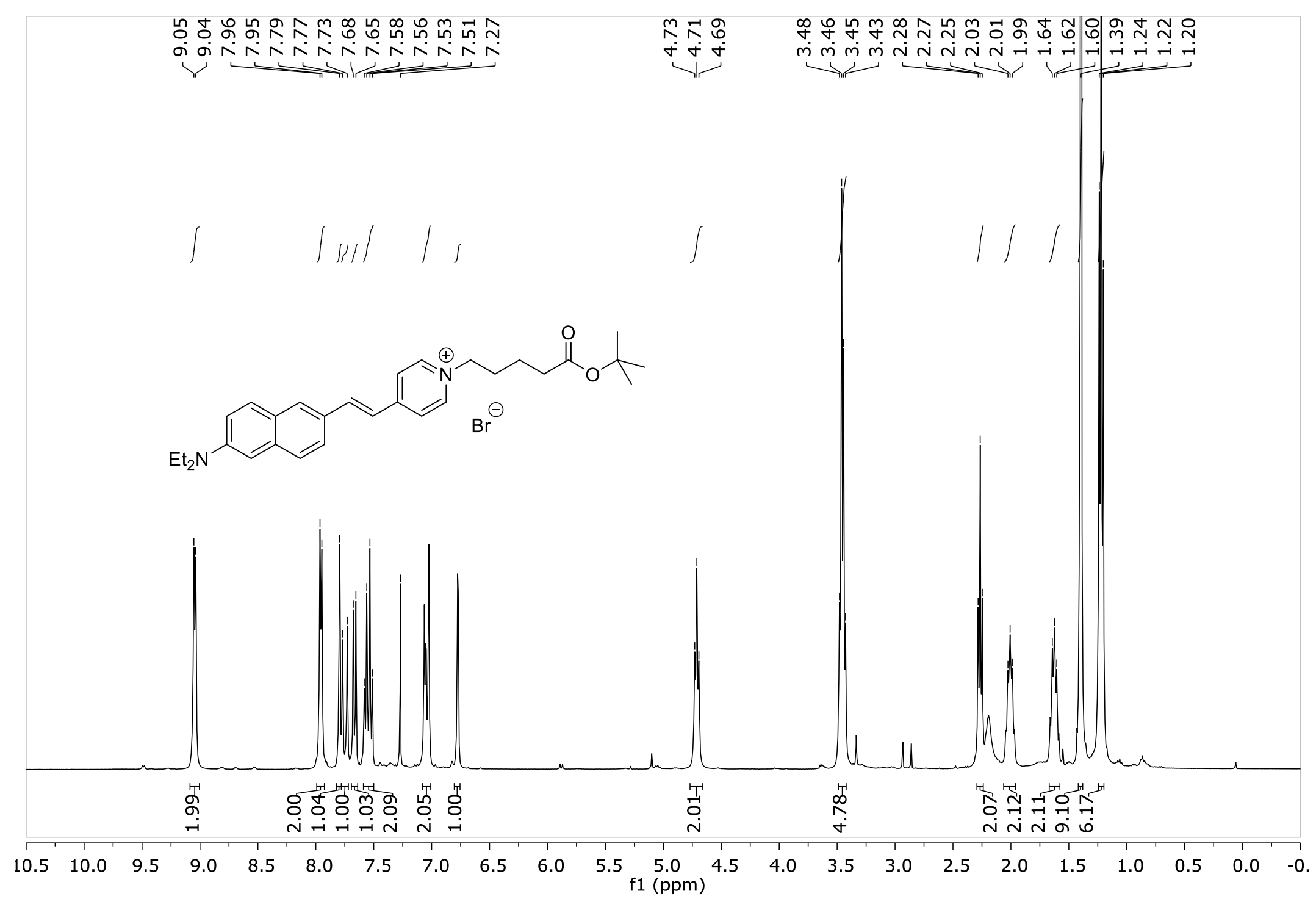

Figure S66. ${ }^{1} \mathrm{H} \mathrm{NMR}\left(\mathrm{CDCl}_{3}, 400 \mathrm{MHz}\right)$ di-2-ANEPB-CO ${ }^{t} \mathrm{Bu}(\mathbf{S 1 5})$. 


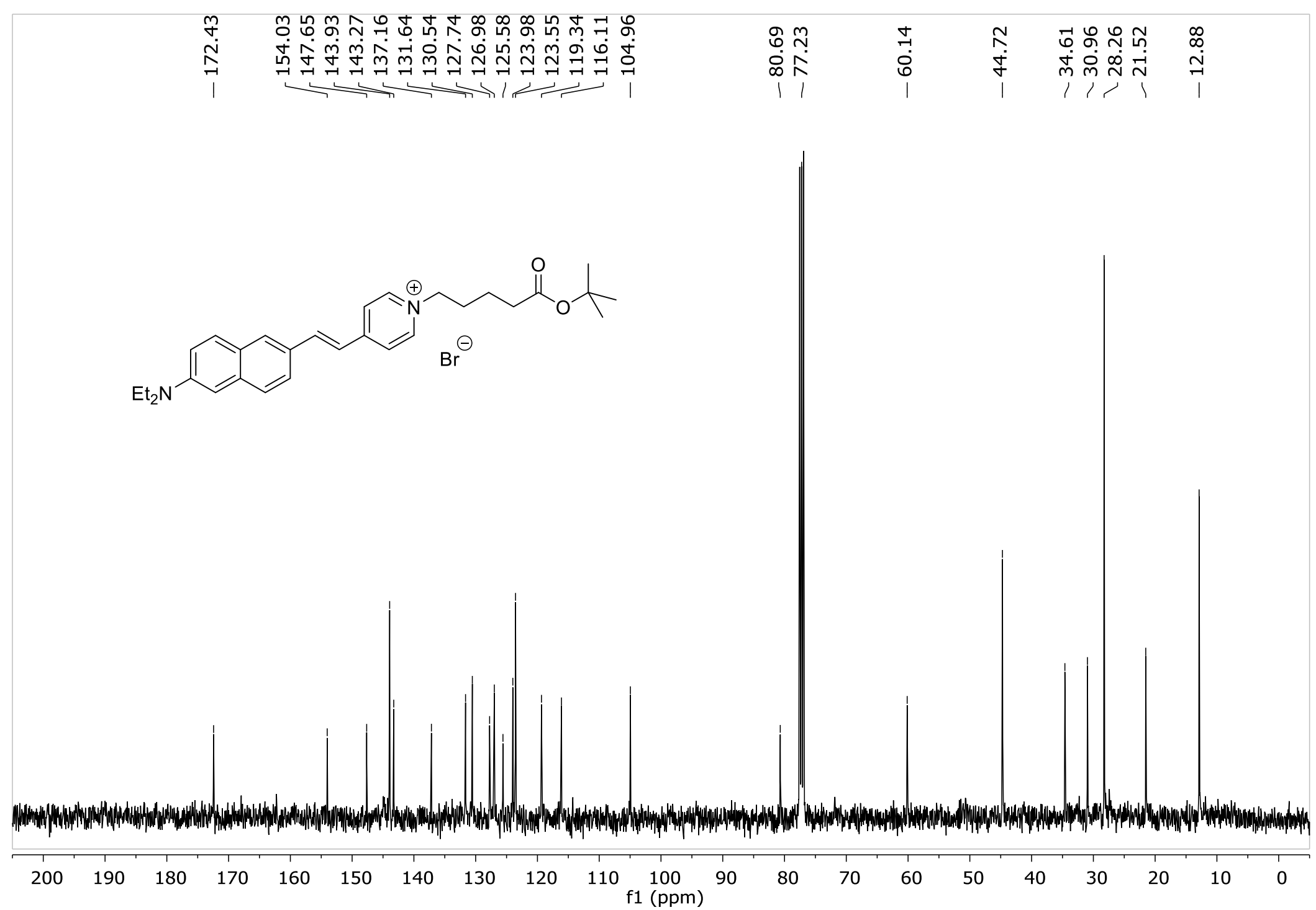

Figure S67. ${ }^{13} \mathrm{C}\left\{{ }^{1} \mathrm{H}\right\}$ NMR $\left(\mathrm{CDCl}_{3}, 100 \mathrm{MHz}\right)$ of di-2-ANEPB- $\mathrm{CO}_{2}{ }^{t} \mathrm{Bu}(\mathbf{S 1 5})$. 


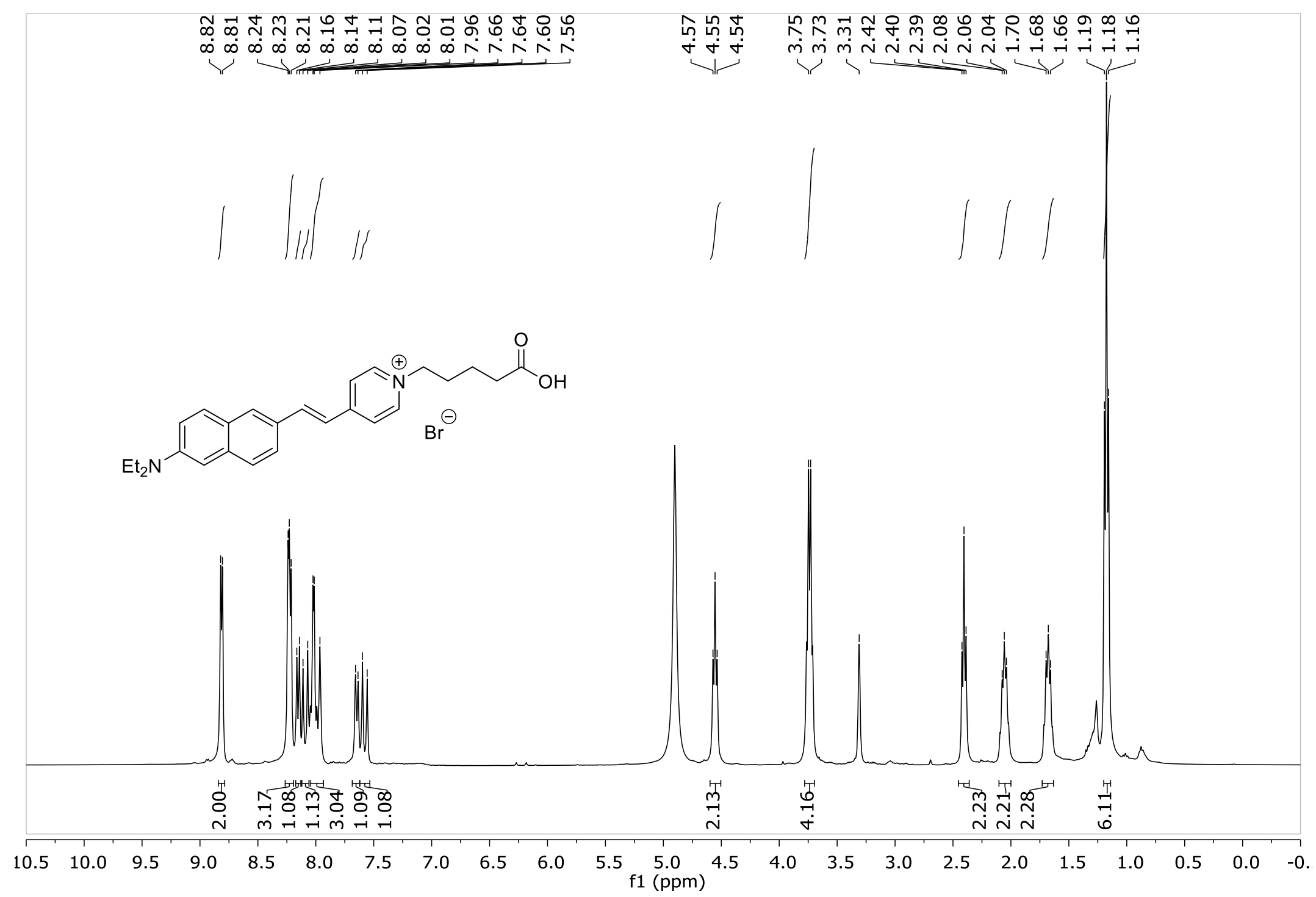

Figure S68. ${ }^{1} \mathrm{H}$ NMR $\left(\mathrm{CD}_{3} \mathrm{OD}, 400 \mathrm{MHz}\right)$ of di-2-ANEPB-CO ${ }_{2} \mathrm{H}(\mathbf{S 1 6})$. 


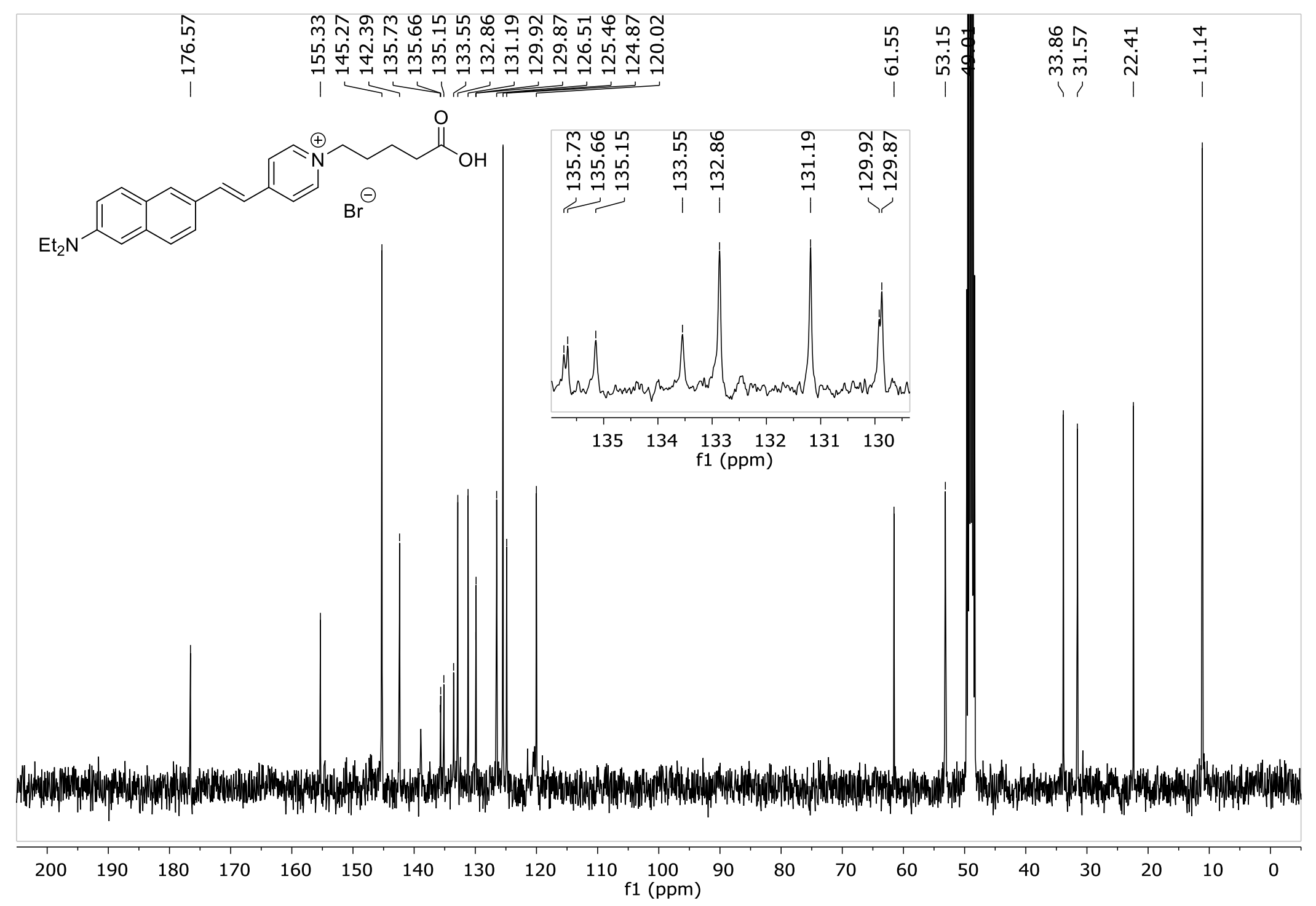

Figure S69. ${ }^{13} \mathrm{C}\left\{{ }^{1} \mathrm{H}\right\}$ NMR $\left(\mathrm{CD}_{3} \mathrm{OD}, 100 \mathrm{MHz}\right)$ of di-2-ANEPB-CO ${ }_{2} \mathrm{H}($ S16). 


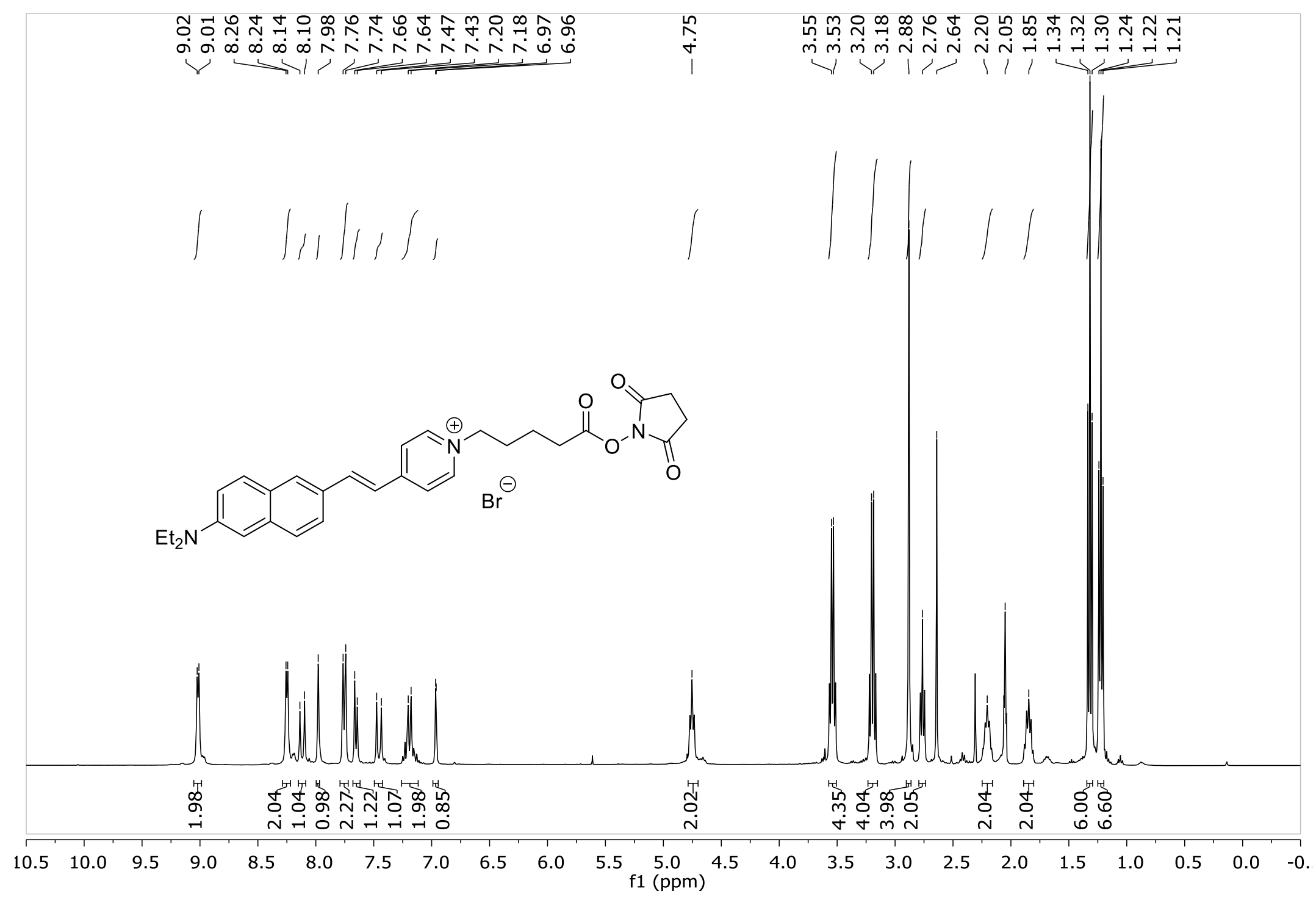

Figure S70. ${ }^{1} \mathrm{H}$ NMR (acetone- $d_{6}, 400 \mathrm{MHz}$ ) of di-2-ANEPB- $\mathrm{CO}_{2} \mathrm{Su}(\mathbf{S 1 7})$. 


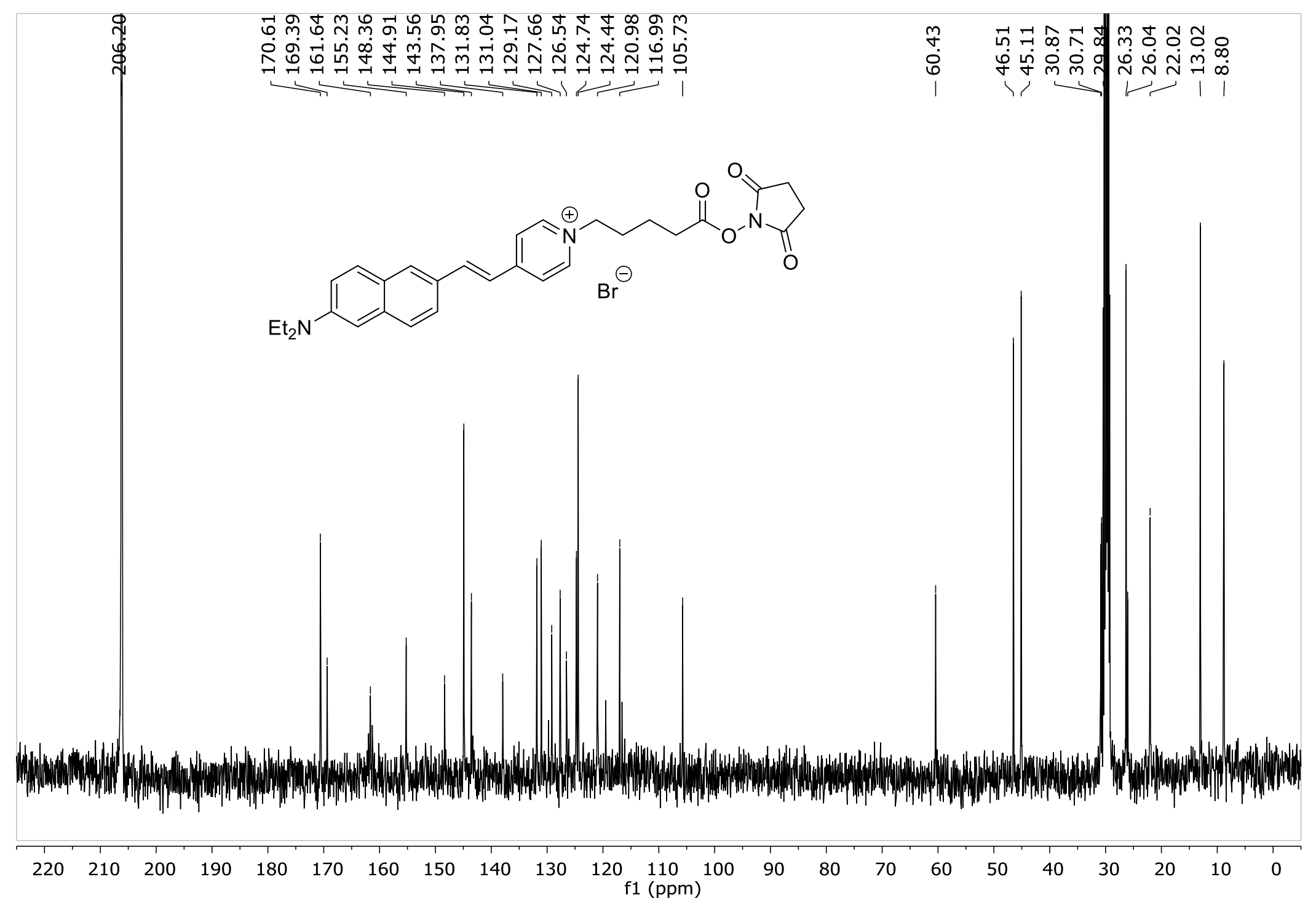

Figure S71. ${ }^{13} \mathrm{C}\left\{{ }^{1} \mathrm{H}\right\}$ NMR (acetone- $d_{6}, 100 \mathrm{MHz}$ ) of di-2-ANEPB-CO $\mathrm{Cu}_{2} \mathrm{Su}$ (S17). 


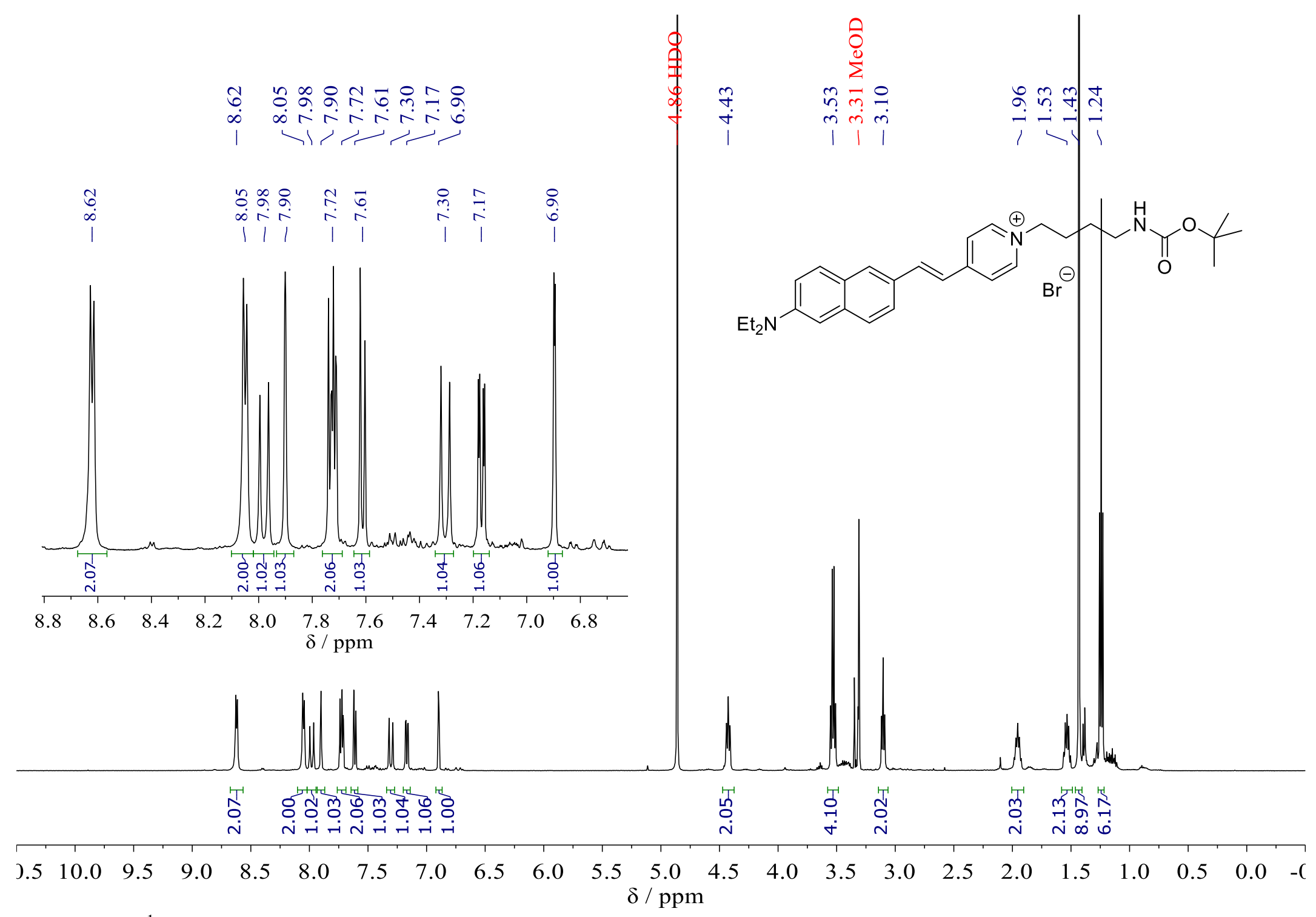

Figure S72. ${ }^{1} \mathrm{H}$ NMR (500 MHz, $\left.\mathrm{CD}_{3} \mathrm{OD}\right)$ of di-2-ANEPB-NHBoc (S18). 


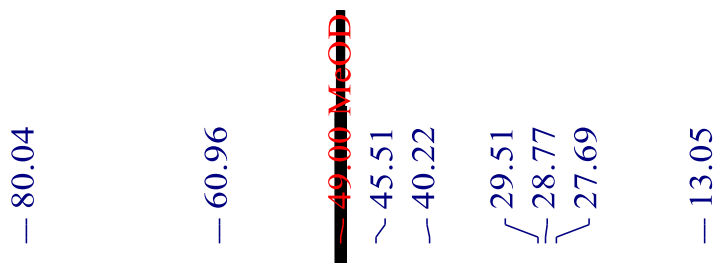
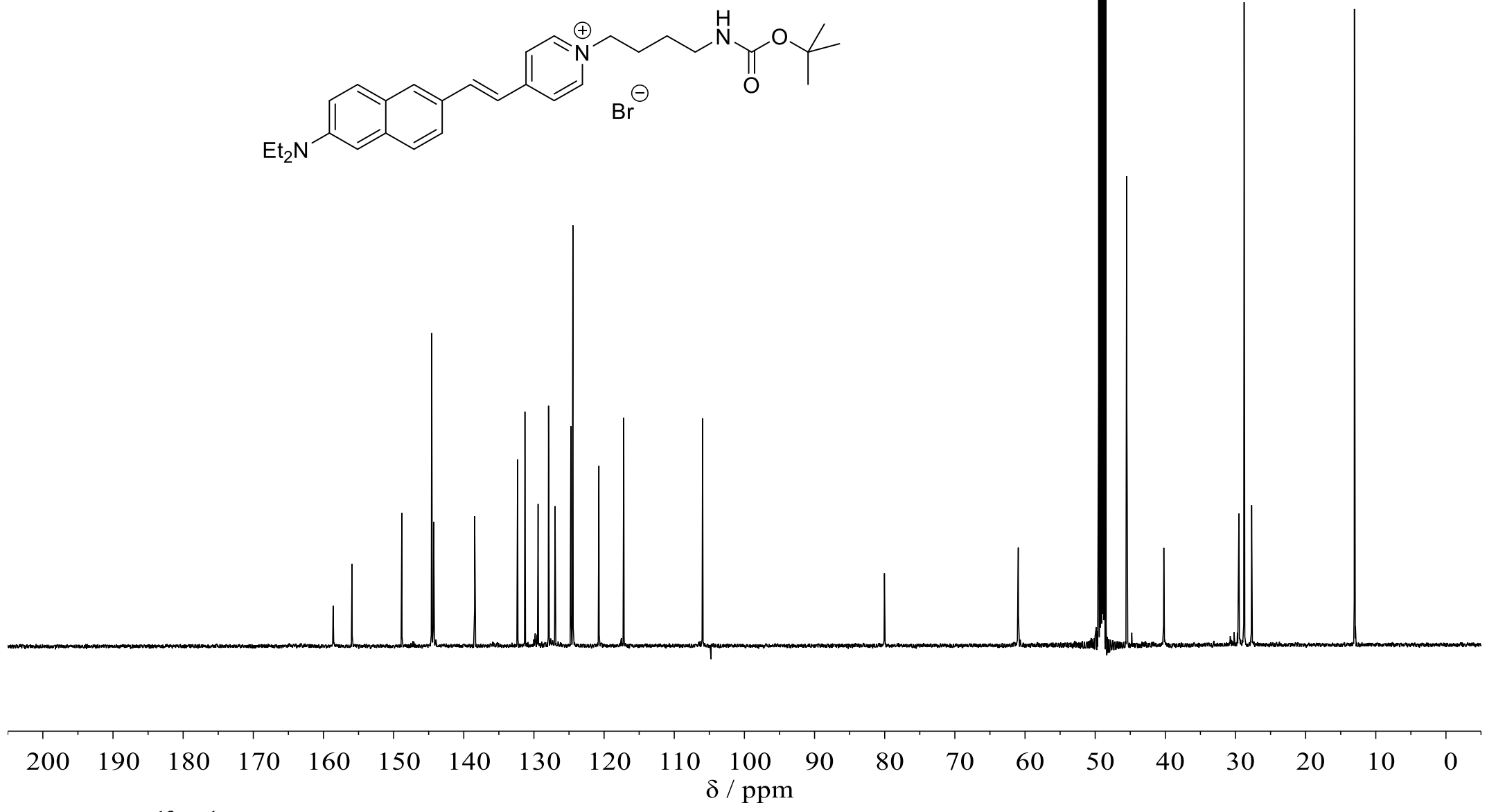

Figure S73. ${ }^{13} \mathrm{C}\left\{{ }^{1} \mathrm{H}\right\}$ NMR $\left(126 \mathrm{MHz}, \mathrm{CD}_{3} \mathrm{OD}\right)$ of di-2-ANEPB-NHBoc (S18). 


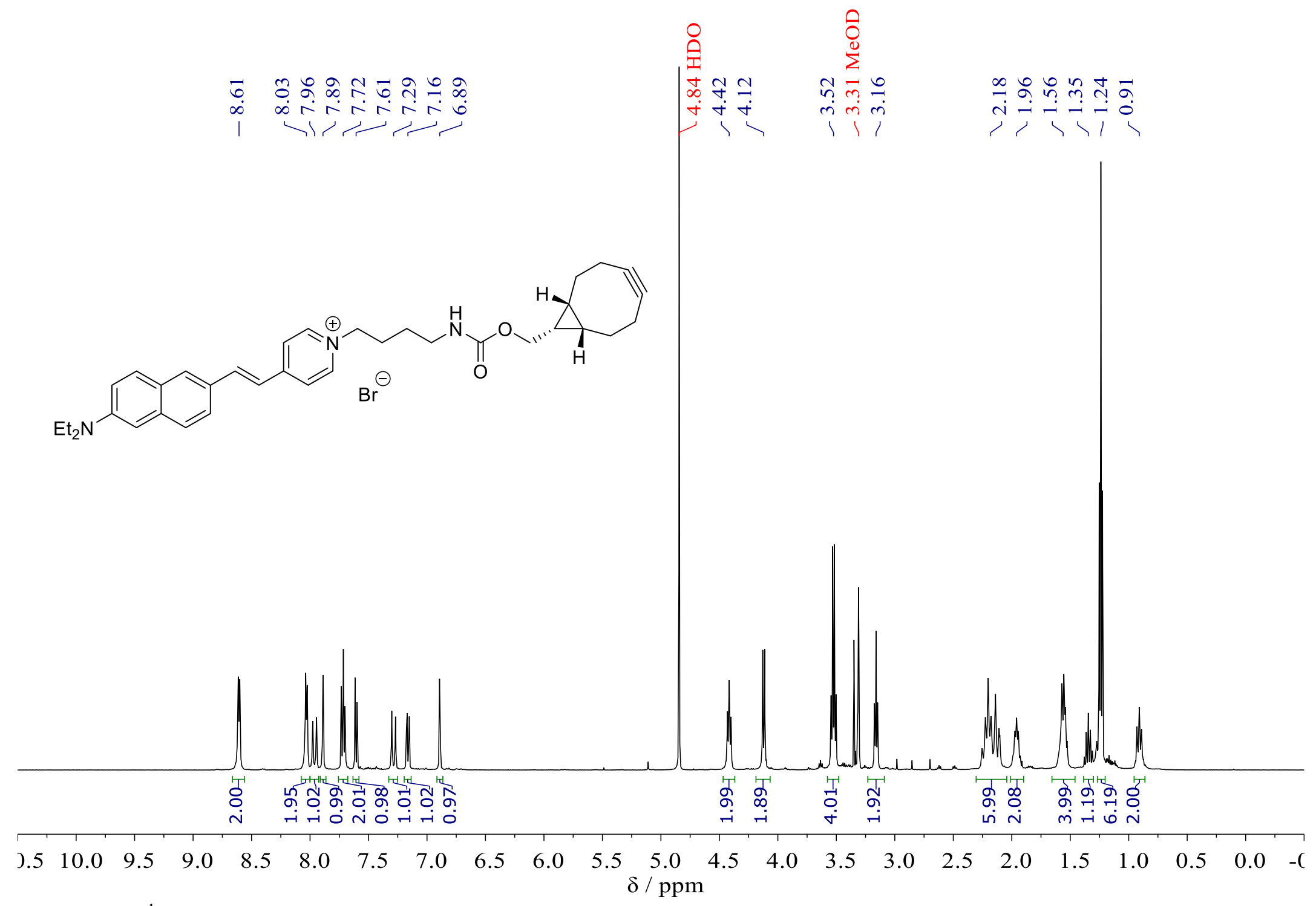

Figure S74. ${ }^{1} \mathrm{H}$ NMR $\left(500 \mathrm{MHz}, \mathrm{CD}_{3} \mathrm{OD}\right)$ of di-2-ANEPB-BCN (9). 


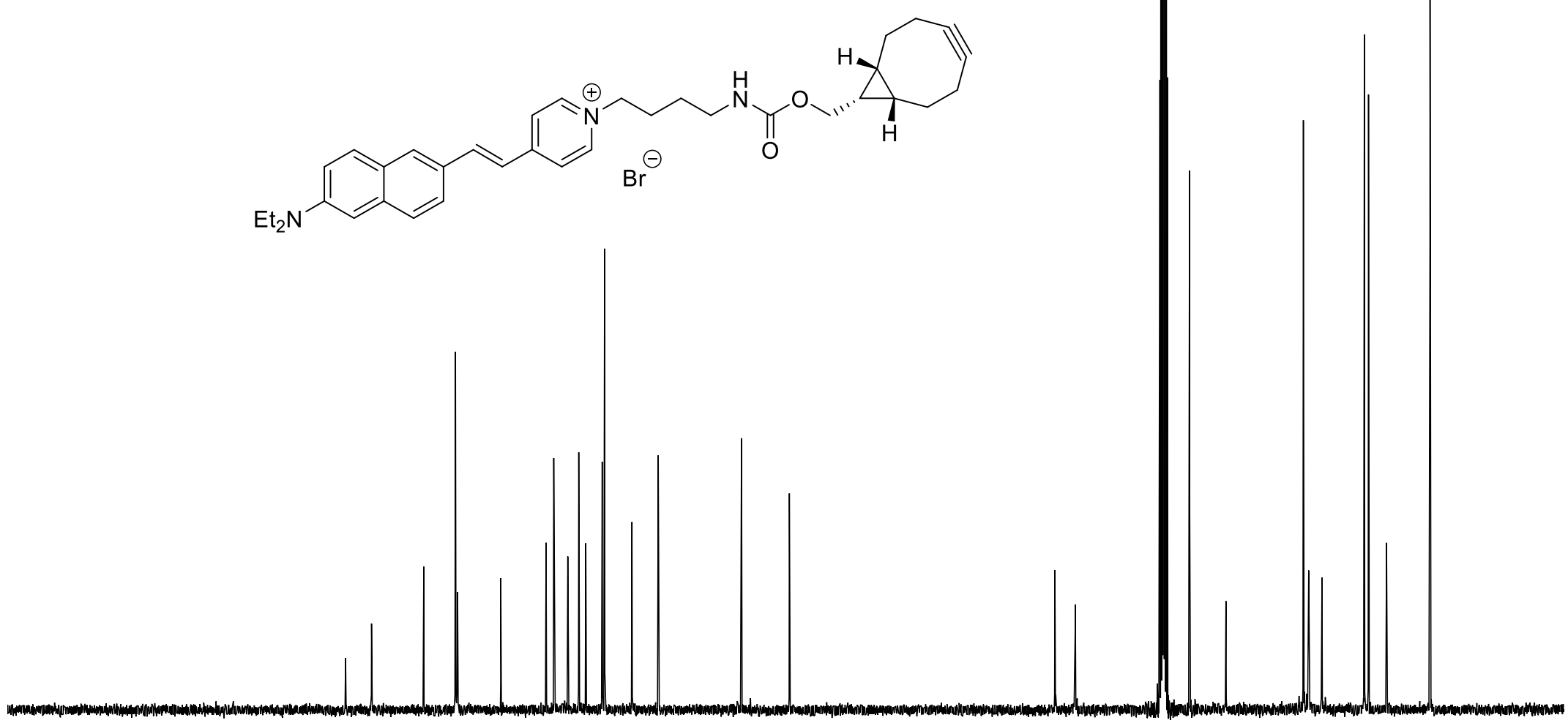

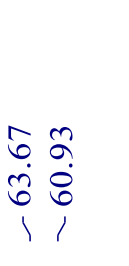

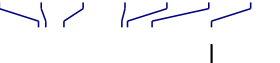

$\begin{array}{llllllll}200 & 190 & 180 & 170 & 160 & 150 & 140 & 130\end{array}$

120110

$$
\delta / \mathrm{ppm}
$$

Figure S75. ${ }^{13} \mathrm{C}\left\{{ }^{1} \mathrm{H}\right\}$ NMR $\left(126 \mathrm{MHz}, \mathrm{CD}_{3} \mathrm{OD}\right)$ of di-2-ANEPB-BCN (9). 


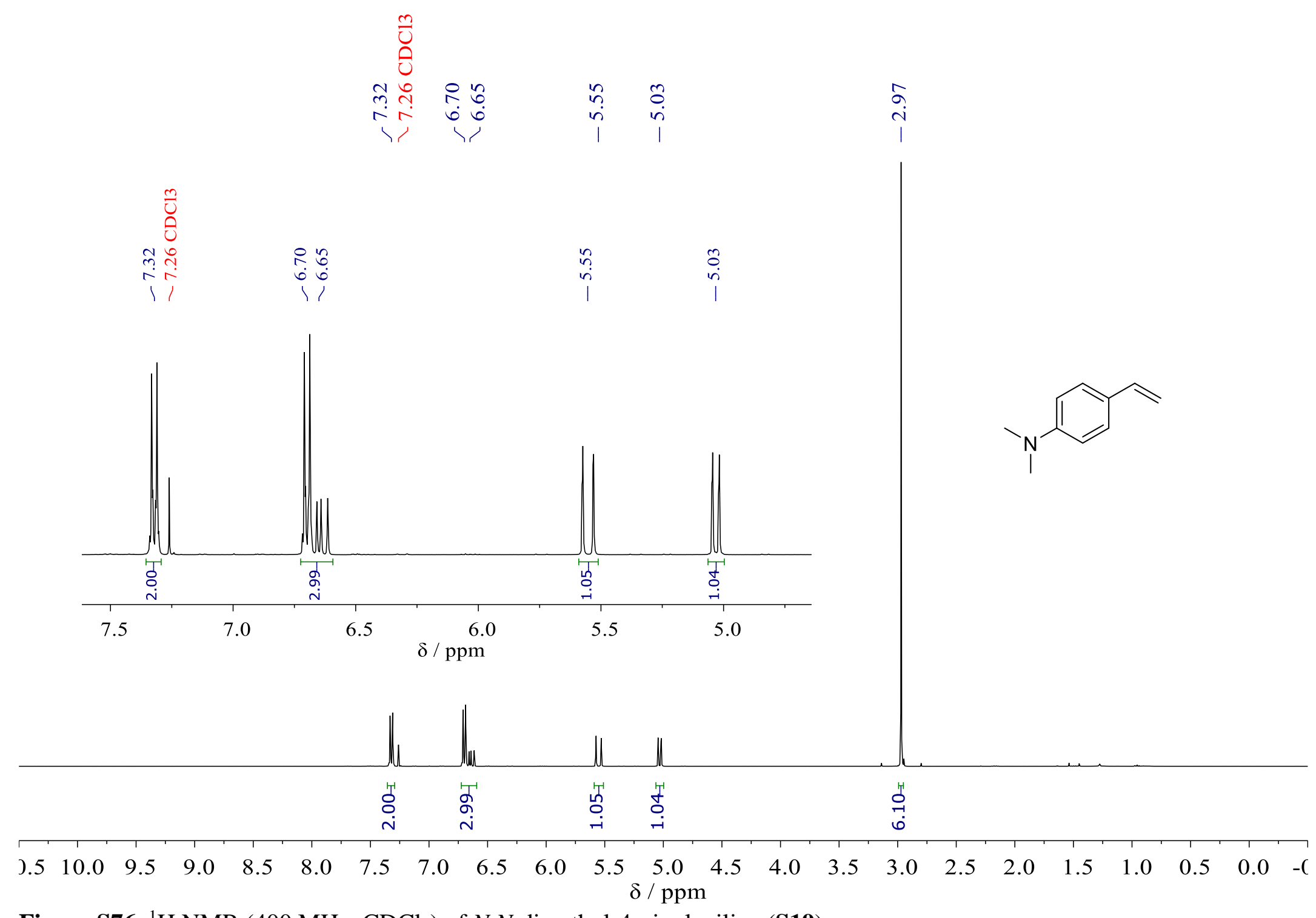

Figure S76. ${ }^{1} \mathrm{H} \mathrm{NMR}\left(400 \mathrm{MHz}, \mathrm{CDCl}_{3}\right)$ of $N, N$-dimethyl-4-vinylaniline (S19). 


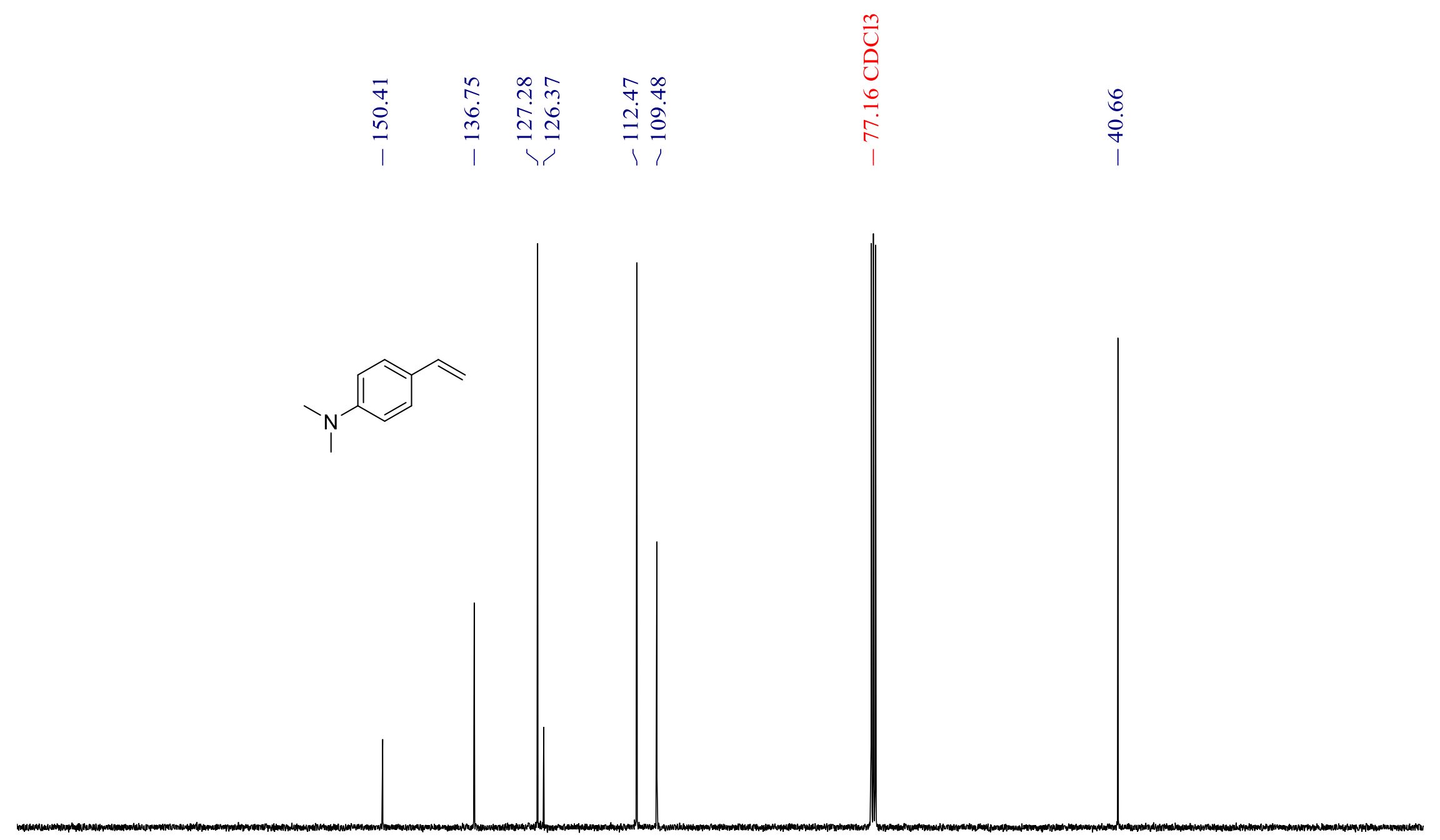

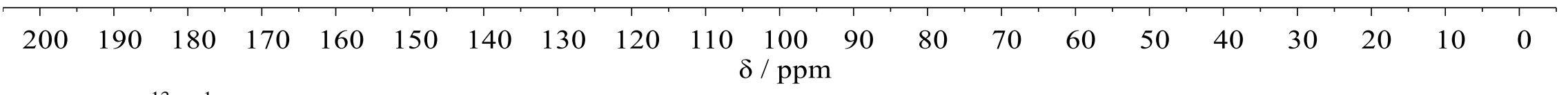

Figure S77. ${ }^{13} \mathrm{C}\left\{{ }^{1} \mathrm{H}\right\}$ NMR (101 MHz, $\left.\mathrm{CDCl}_{3}\right)$ of $N, N$-dimethyl-4-vinylaniline (S19). 


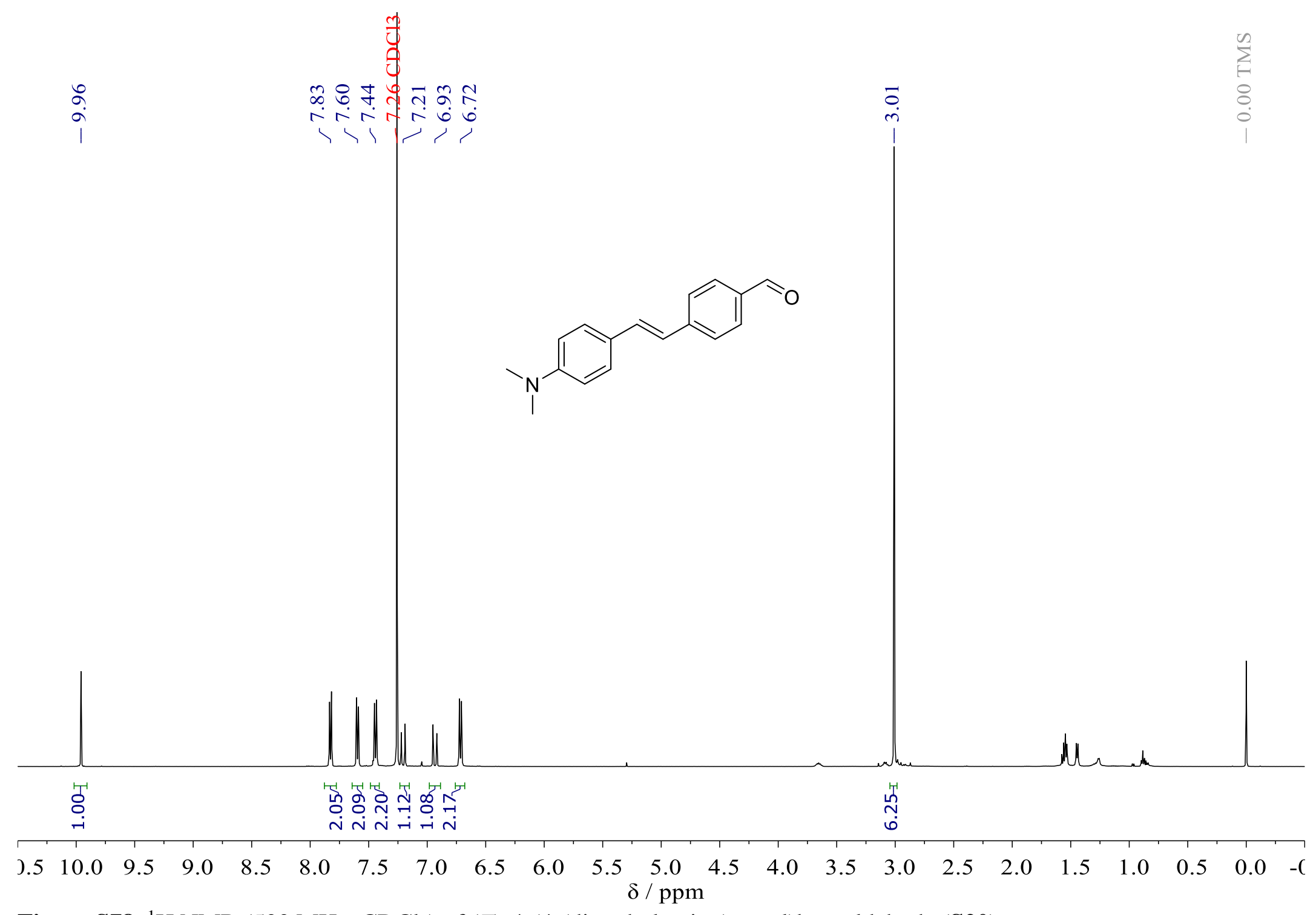

Figure S78. ${ }^{1} \mathrm{H}$ NMR $\left(500 \mathrm{MHz}, \mathrm{CDCl}_{3}\right)$ of (E)-4-(4-(dimethylamino)styryl)benzaldehyde (S20). 


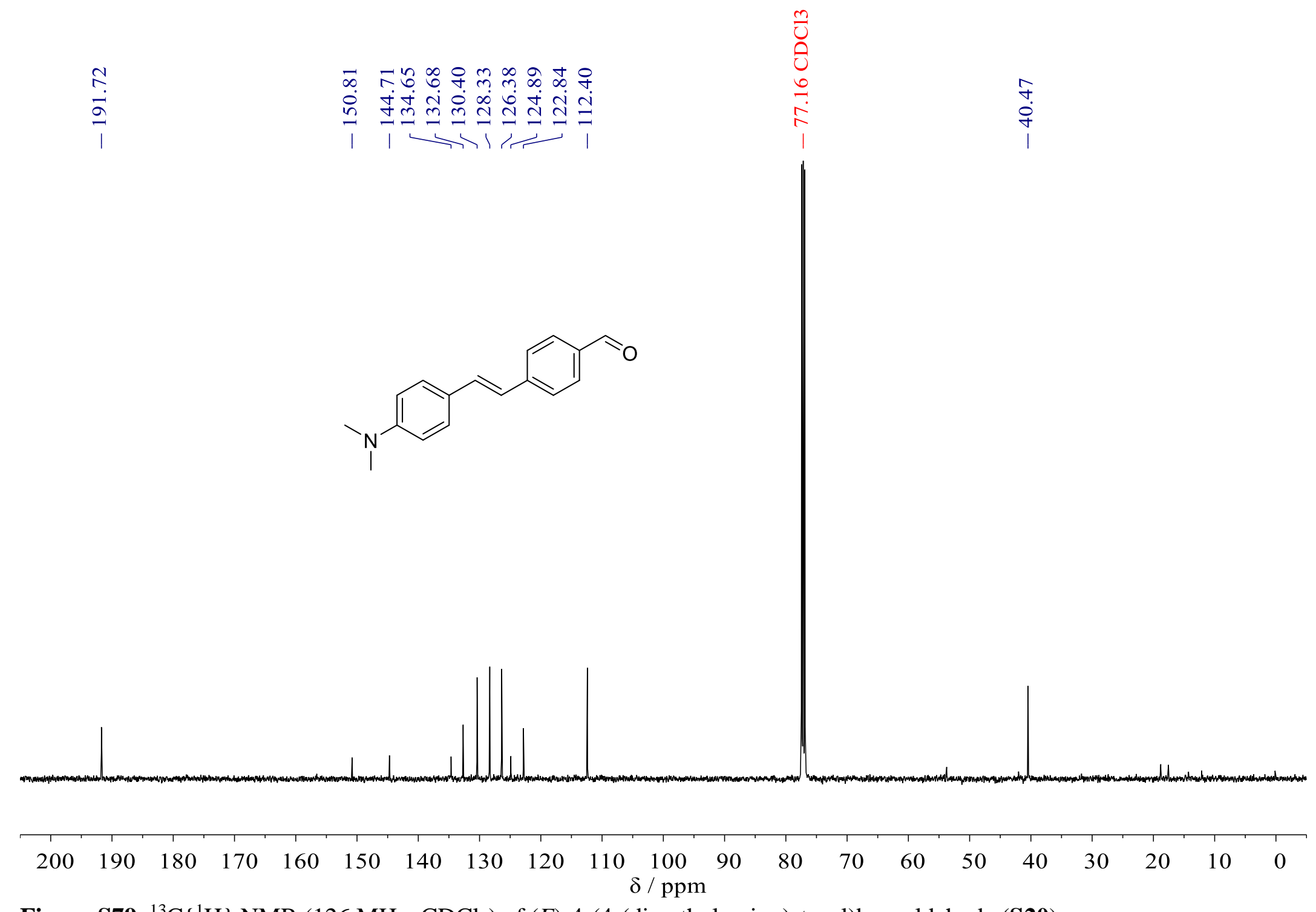

Figure S79. ${ }^{13} \mathrm{C}\left\{{ }^{1} \mathrm{H}\right\}$ NMR (126 MHz, $\left.\mathrm{CDCl}_{3}\right)$ of $(E)-4$-(4-(dimethylamino)styryl)benzaldehyde (S20). 


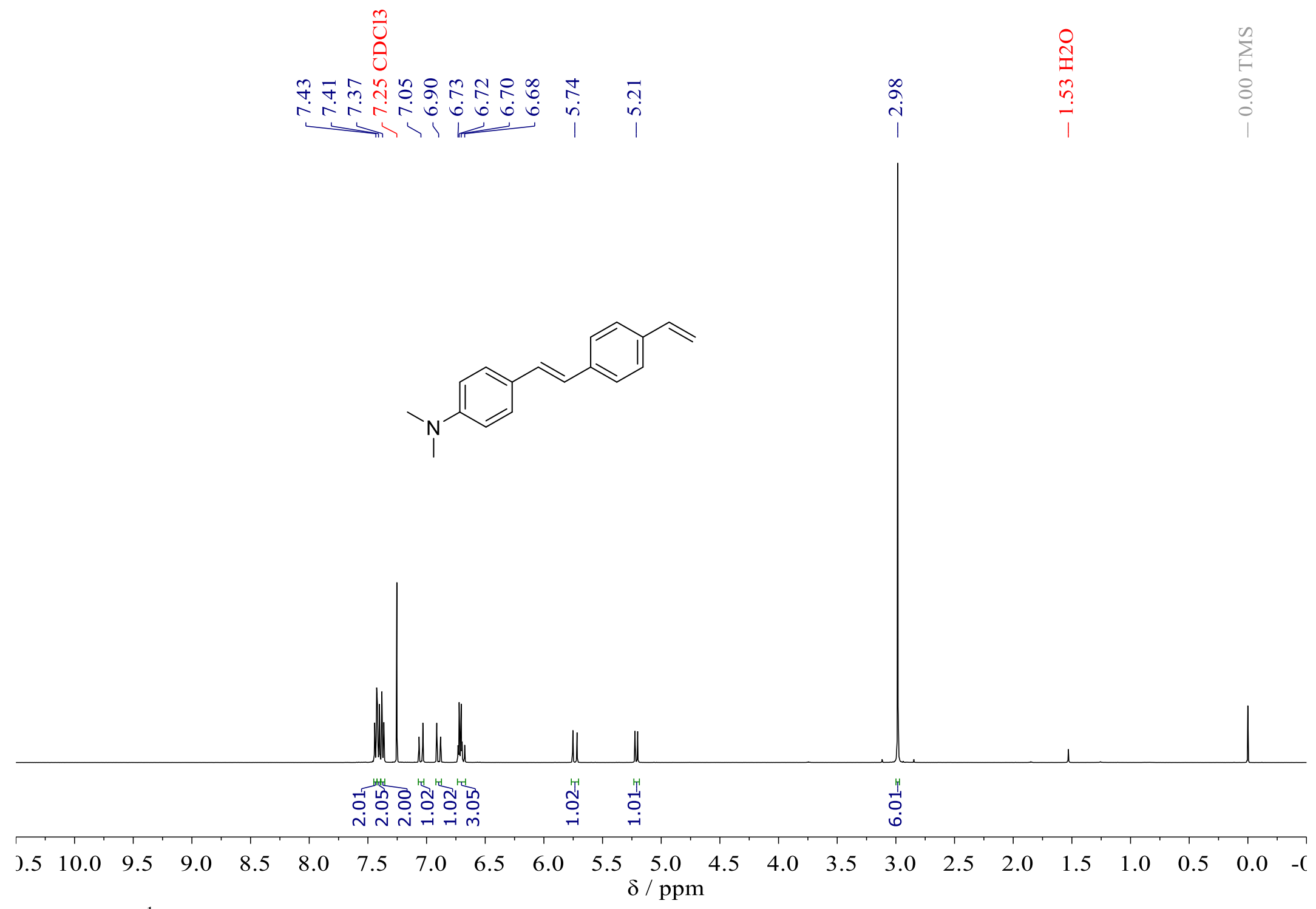

Figure S80. ${ }^{1} \mathrm{H}$ NMR $\left(500 \mathrm{MHz}, \mathrm{CDCl}_{3}\right)$ of $(E)-N, N$-dimethyl-4-(4-vinylstyryl)aniline (S21). 


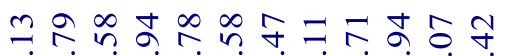

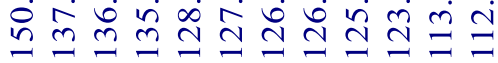

$\frac{0}{0}$

$\sum_{1}^{\infty}$
8
0
0
0
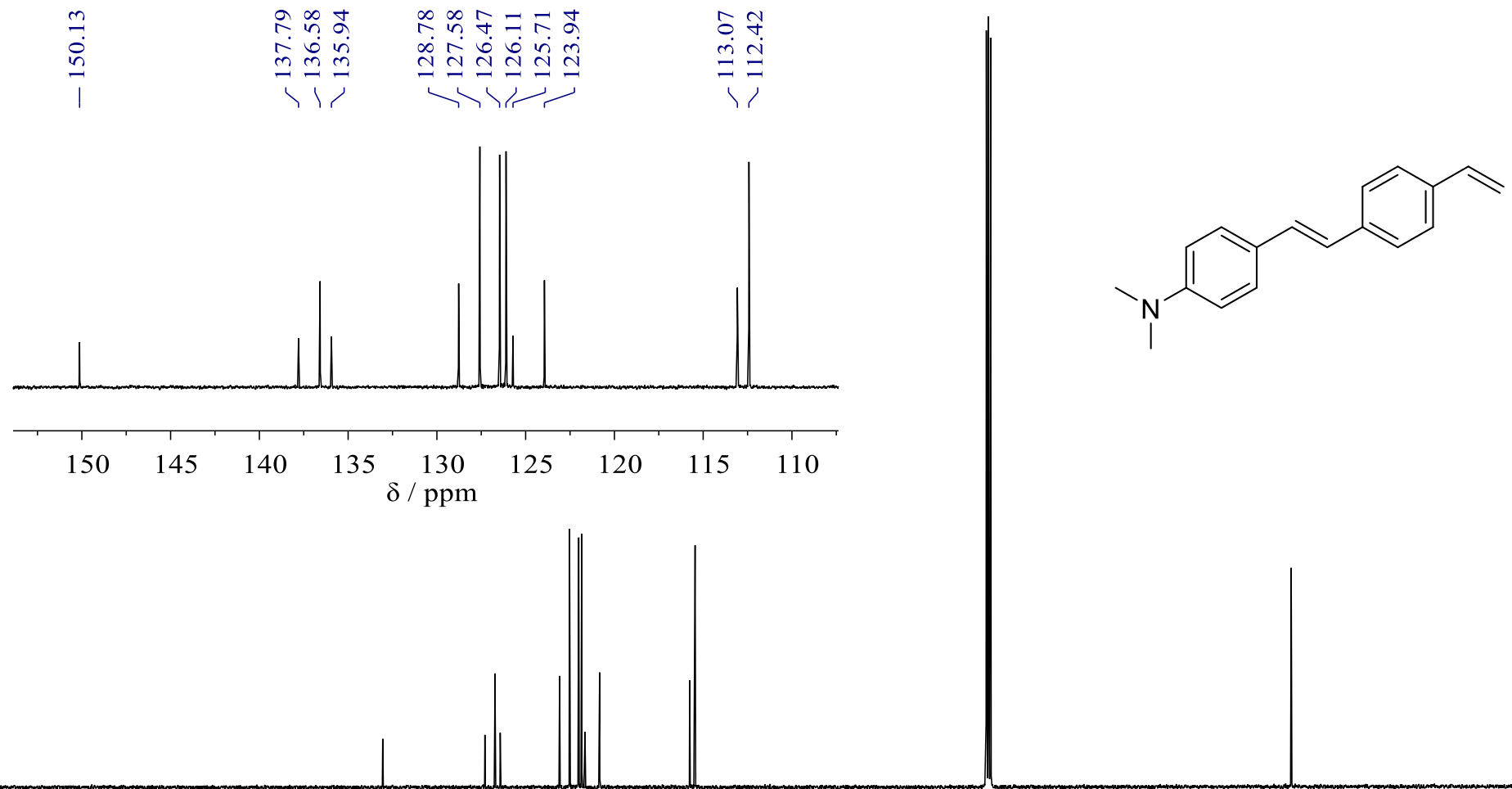

200

$\begin{array}{lllllll}190 & 180 & 170 & 160 & 150 & 140 & 130\end{array}$

120

110100

$\delta / \mathrm{ppm}$

Figure S81. ${ }^{13} \mathrm{C}\left\{{ }^{1} \mathrm{H}\right\}$ NMR (126 MHz, $\left.\mathrm{CDCl}_{3}\right)$ of $(E)-N, N$-dimethyl-4-(4-vinylstyryl)aniline (S21). 


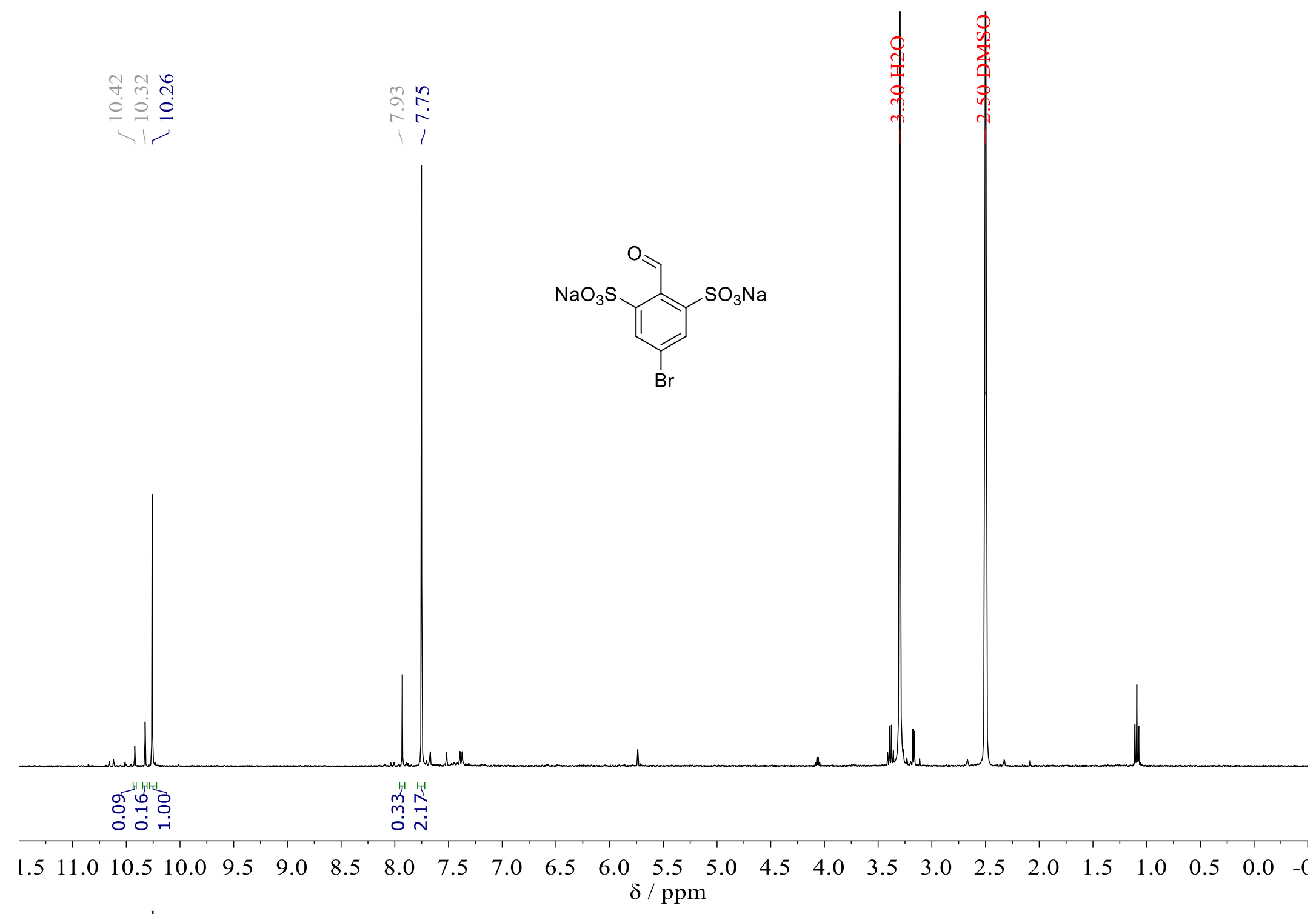

Figure S82. ${ }^{1} \mathrm{H}$ NMR (400 MHz, DMSO- $d_{6}$ ) of 4-bromo-2,6-disulfobenzaldehyde disodium salt (S22). 


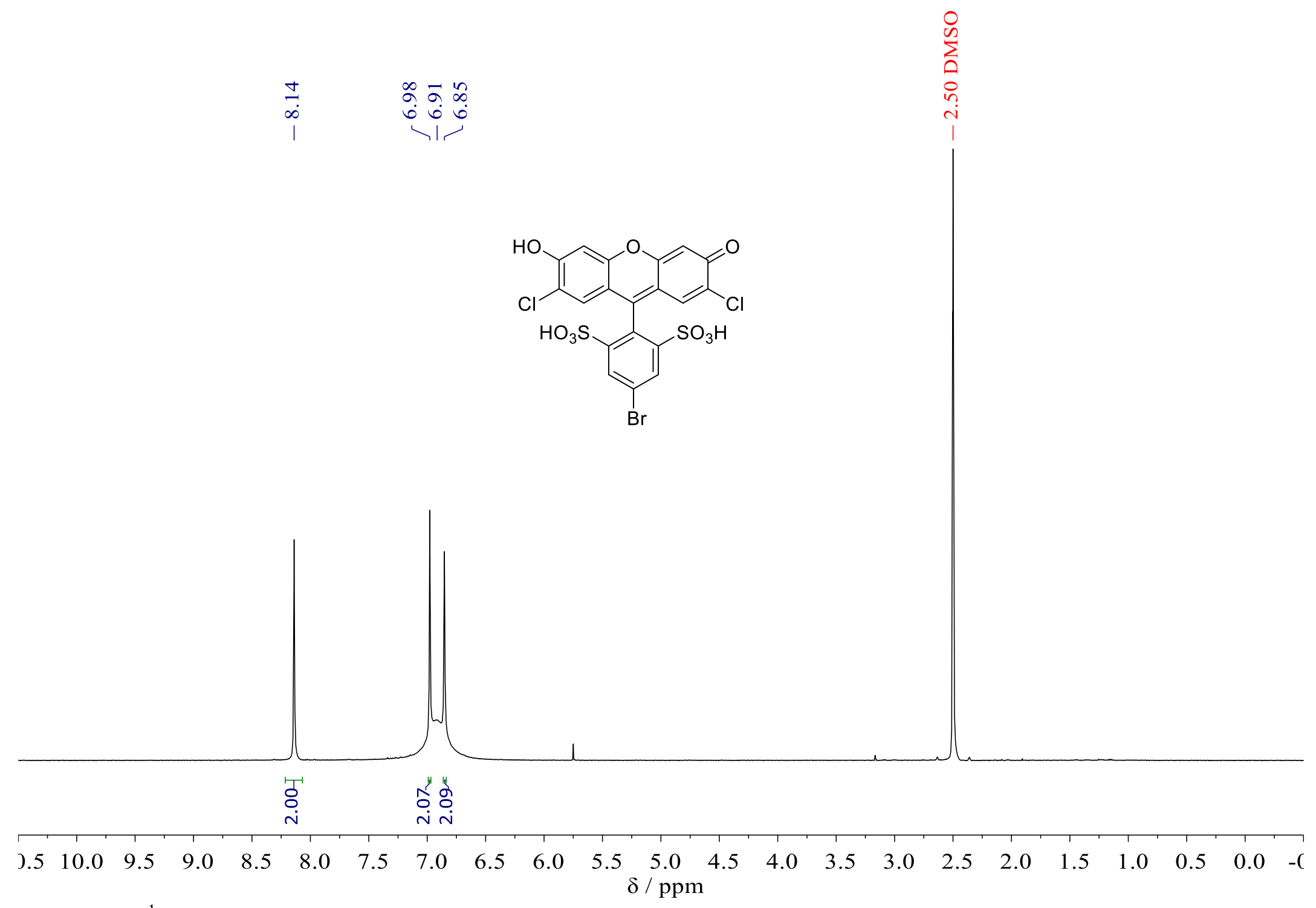

Figure S83. ${ }^{1} \mathrm{H}$ NMR (500 MHz, DMSO-d6) of 4'-bromo-2,7-dichloro-2',6'-disulfofluorescein (S23). 


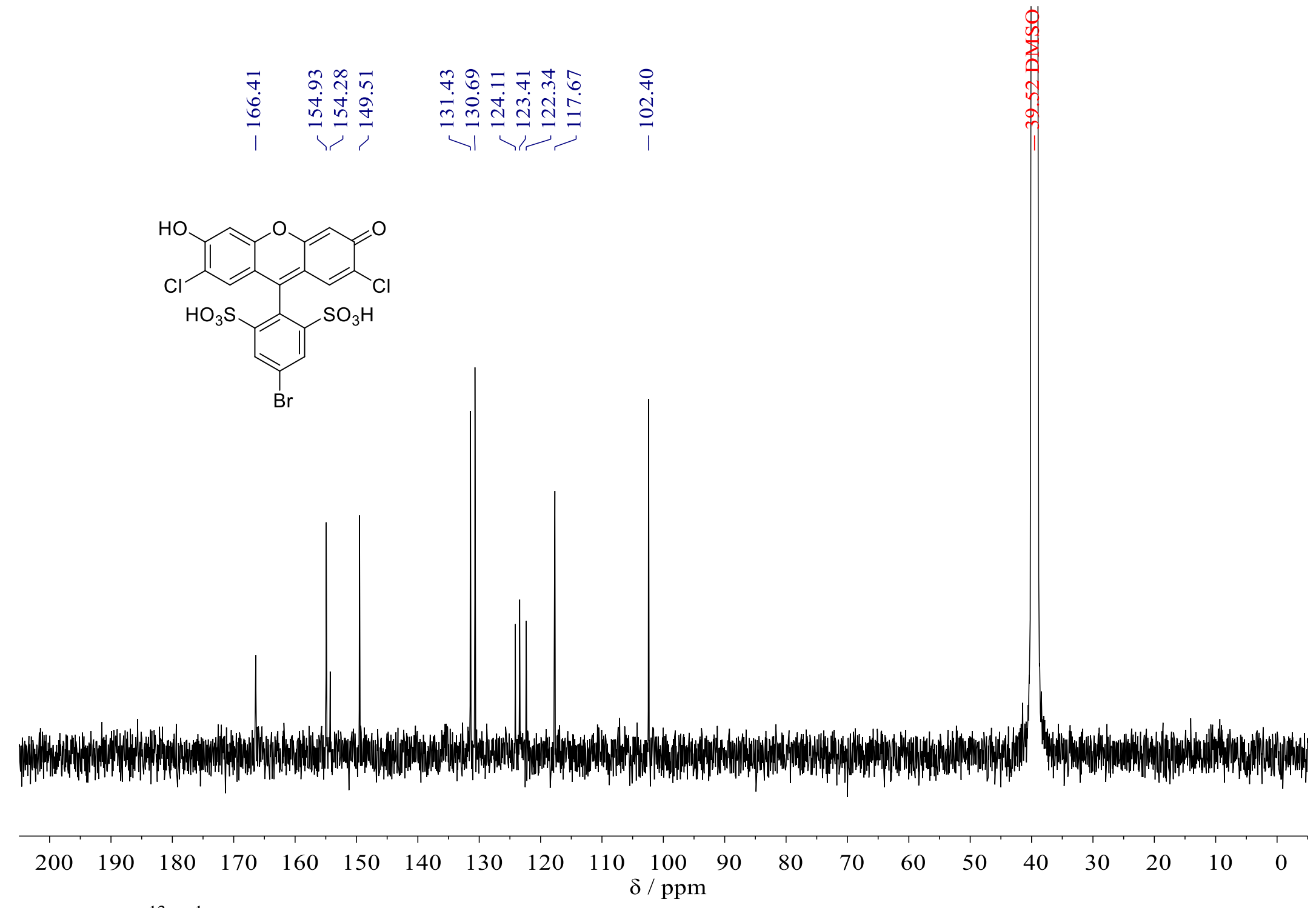

Figure S84. ${ }^{13} \mathrm{C}\left\{{ }^{1} \mathrm{H}\right\}$ NMR (126 MHz, DMSO-d 6 ) of 4'-bromo-2,7-dichloro-2',6'-disulfofluorescein (S23). 


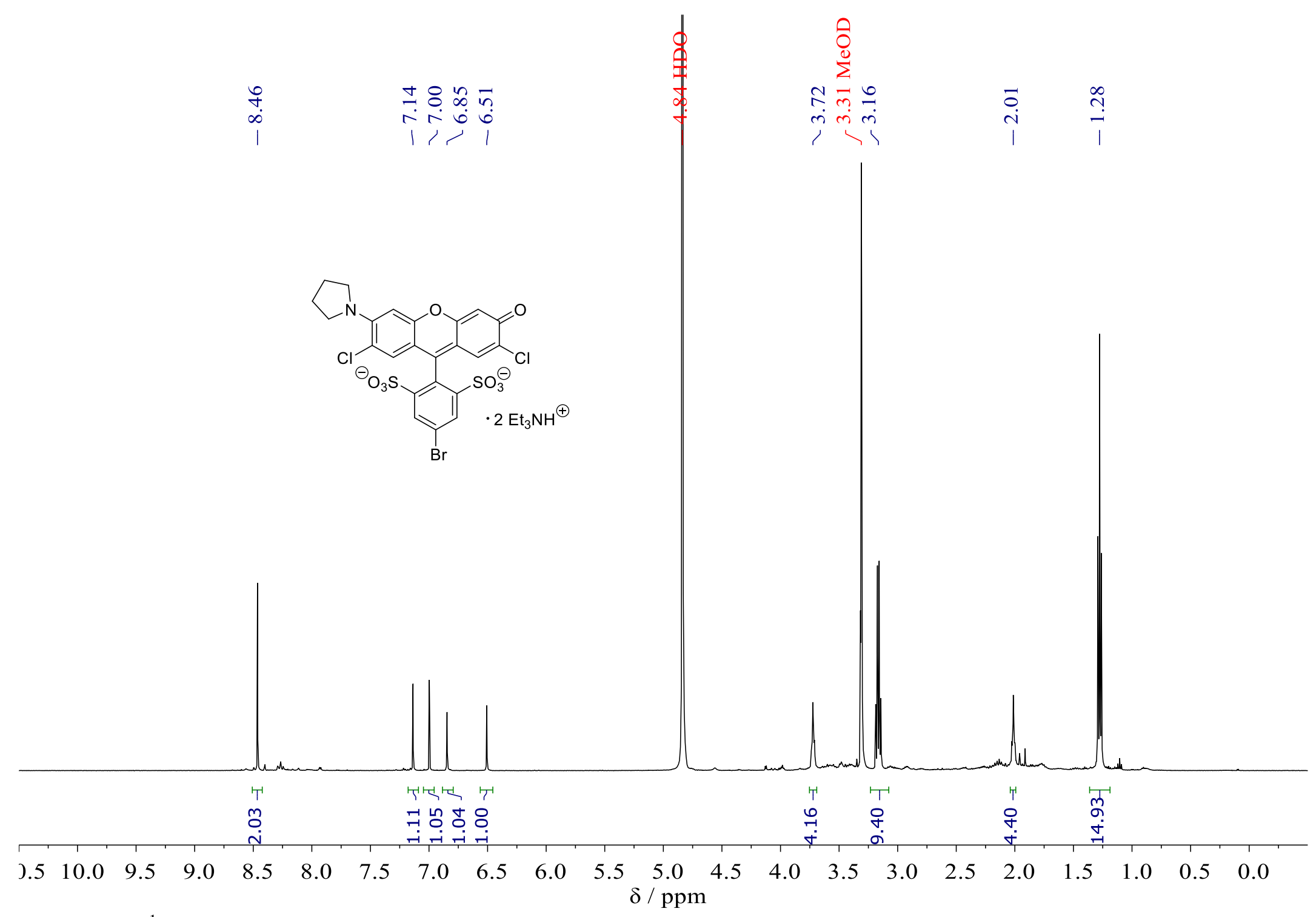

Figure S85. ${ }^{1} \mathrm{H}$ NMR $\left(500 \mathrm{MHz}, \mathrm{CD}_{3} \mathrm{OD}\right)$ of compound $\mathbf{S 2 4}$. 


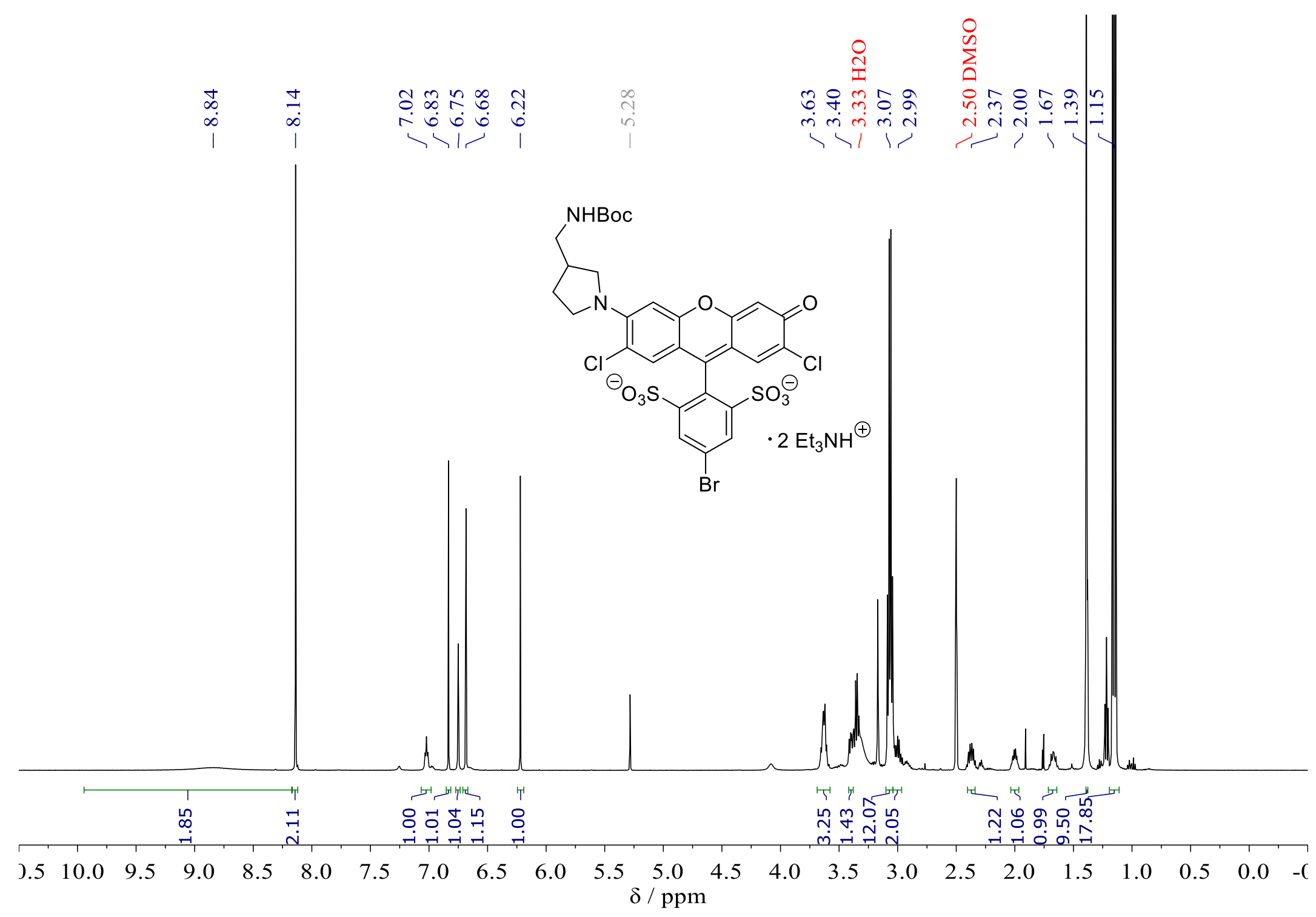

Figure S86. ${ }^{1} \mathrm{H}$ NMR $\left(500 \mathrm{MHz}, \mathrm{CD}_{3} \mathrm{OD}\right)$ of compound $\mathbf{S 2 5}$. 


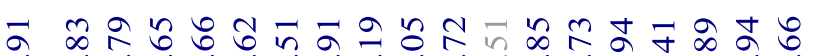

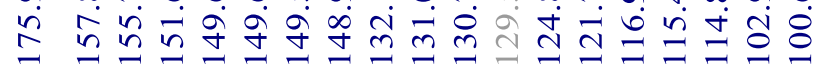

l

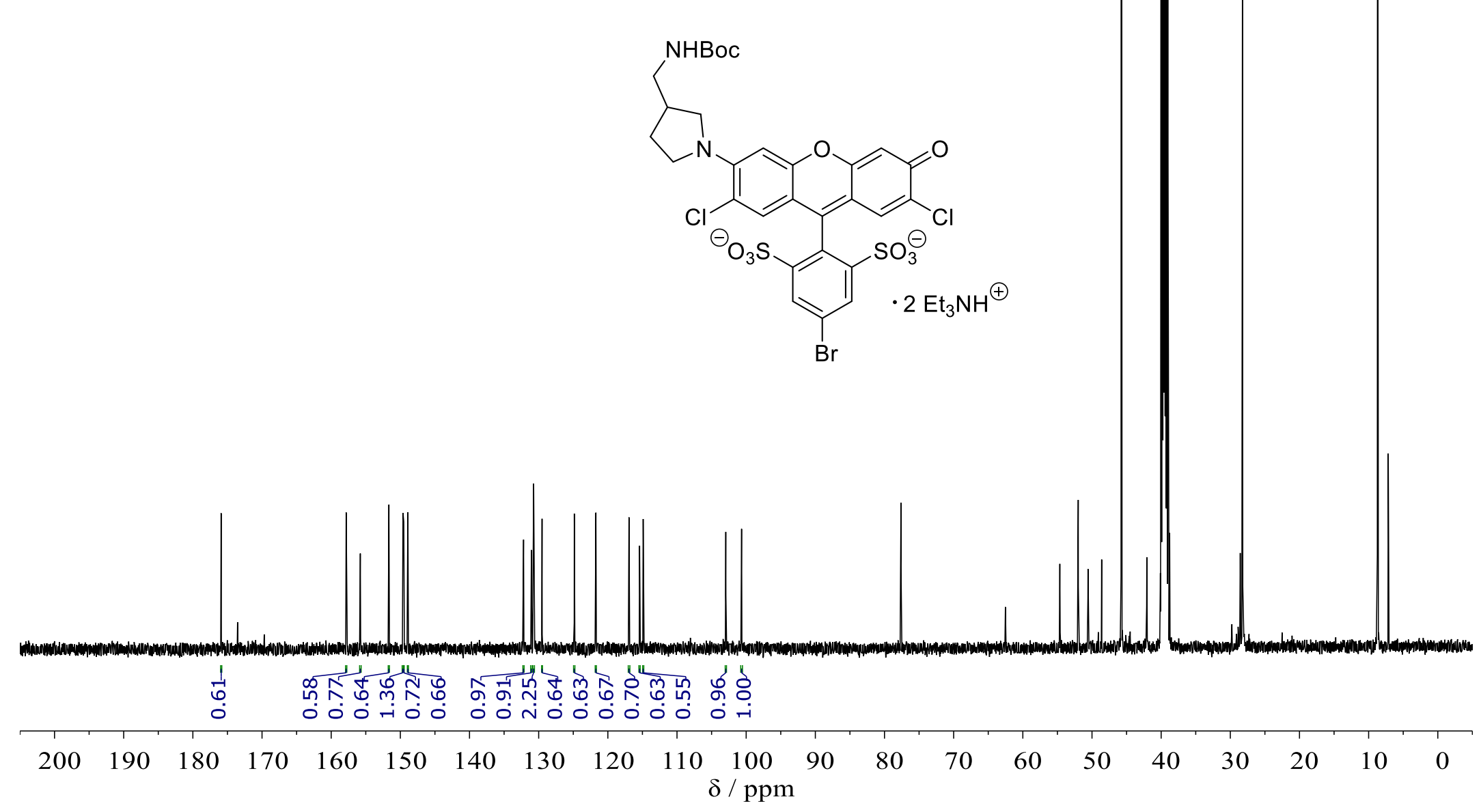

Figure S87. ${ }^{13} \mathrm{C}\left\{{ }^{1} \mathrm{H}\right\}$ NMR (126 MHz, DMSO- $\left.d_{6}\right)$ of compound $\mathbf{S 2 5}$. 


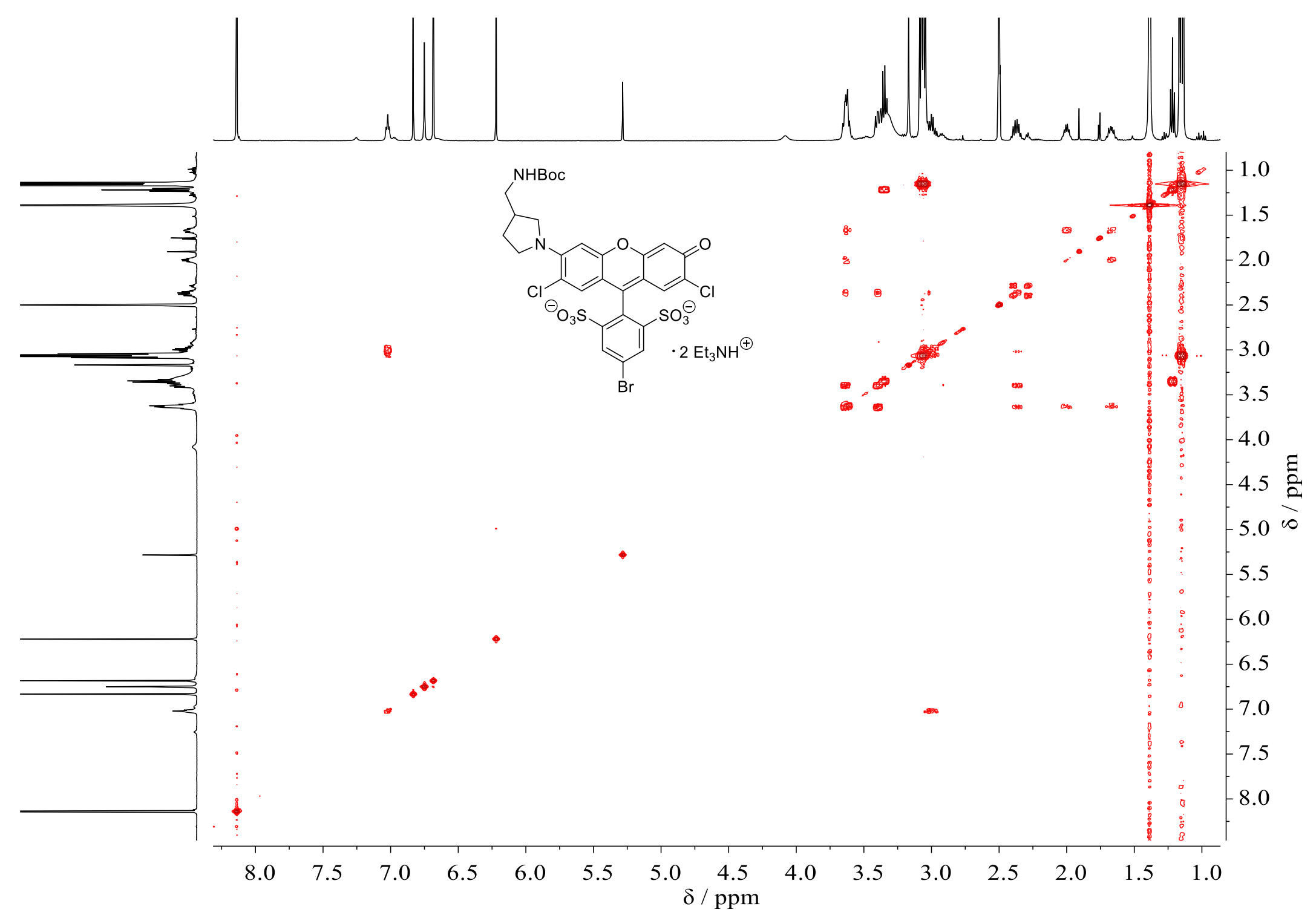

Figure S88. ${ }^{1} \mathrm{H}-{ }^{1} \mathrm{H}$ COSY NMR (500 MHz, $\left.\mathrm{CD}_{3} \mathrm{OD}\right)$ of compound $\mathbf{S 2 5}$. 


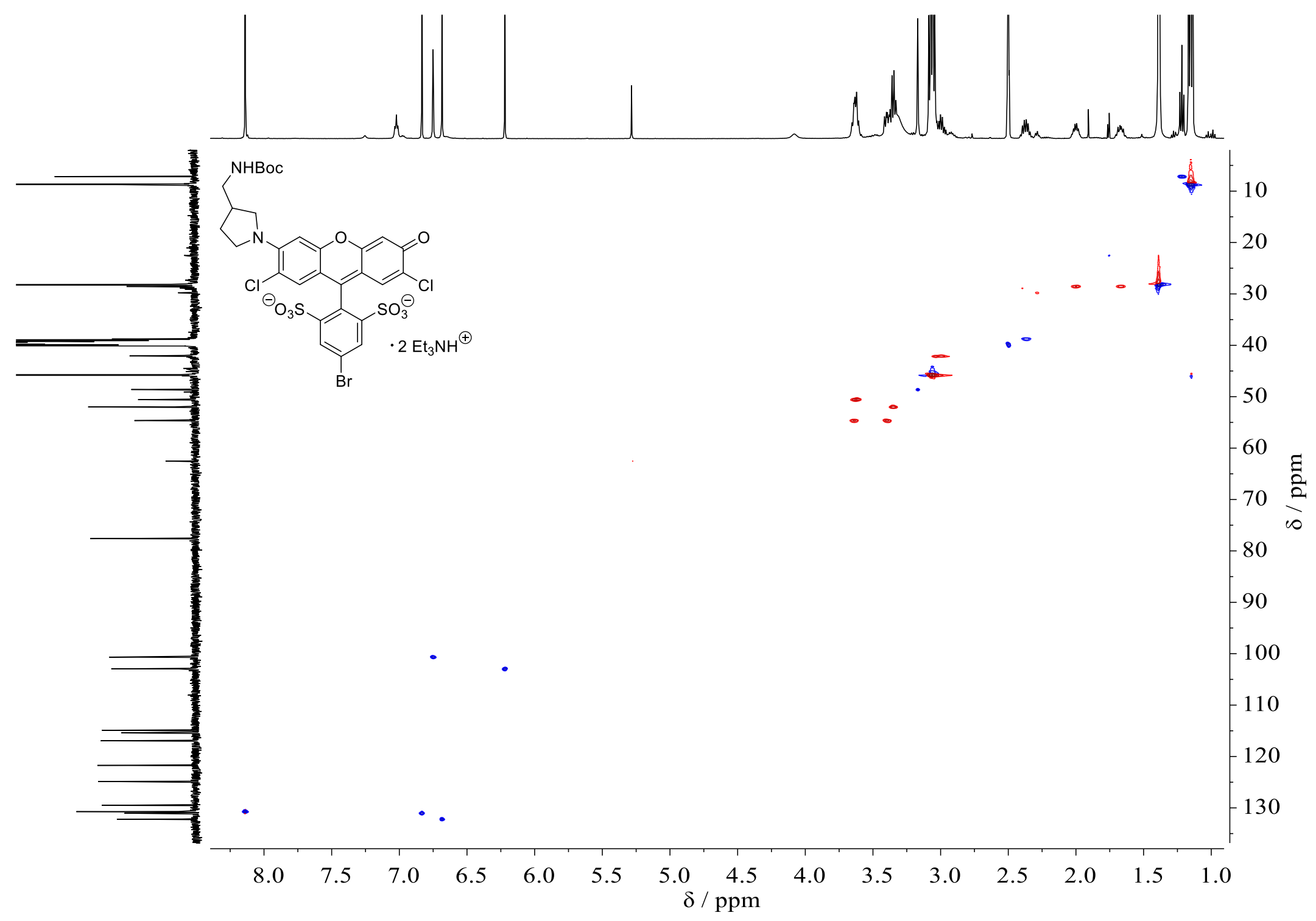

Figure S89. ${ }^{1} \mathrm{H}-{ }^{13} \mathrm{C}$ HSQC NMR $\left(500 \mathrm{MHz}, \mathrm{CD}_{3} \mathrm{OD}\right)$ of compound $\mathbf{S 2 5}$. 


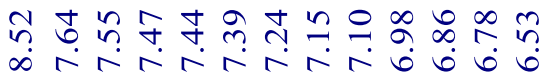

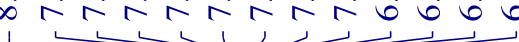

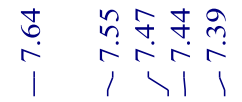

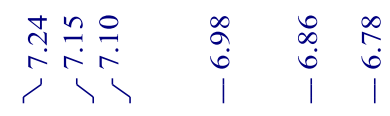
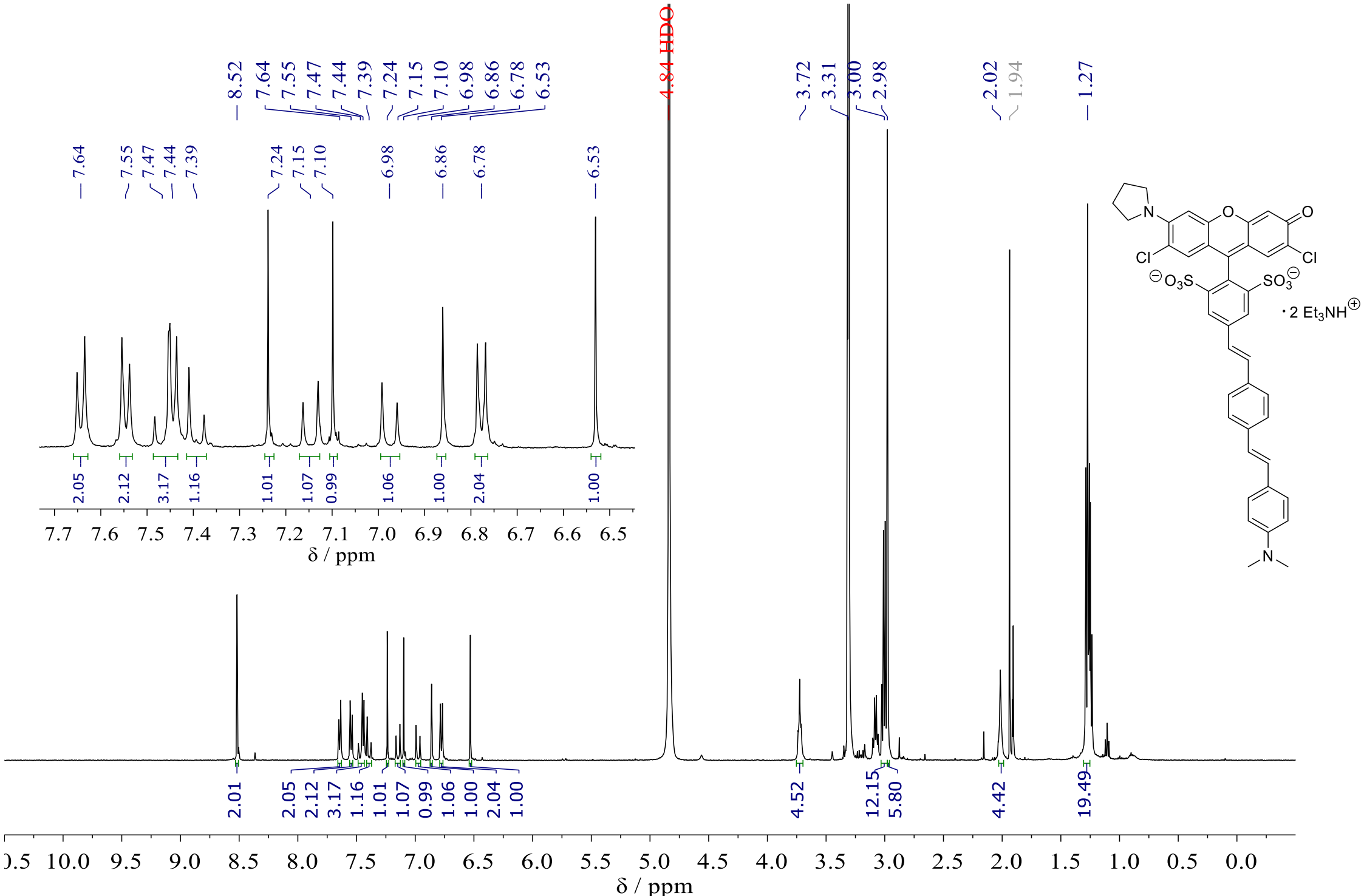

Figure S90. ${ }^{1} \mathrm{H}$ NMR (500 MHz, $\left.\mathrm{CD}_{3} \mathrm{OD}\right)$ of dsRVF5 (2). 


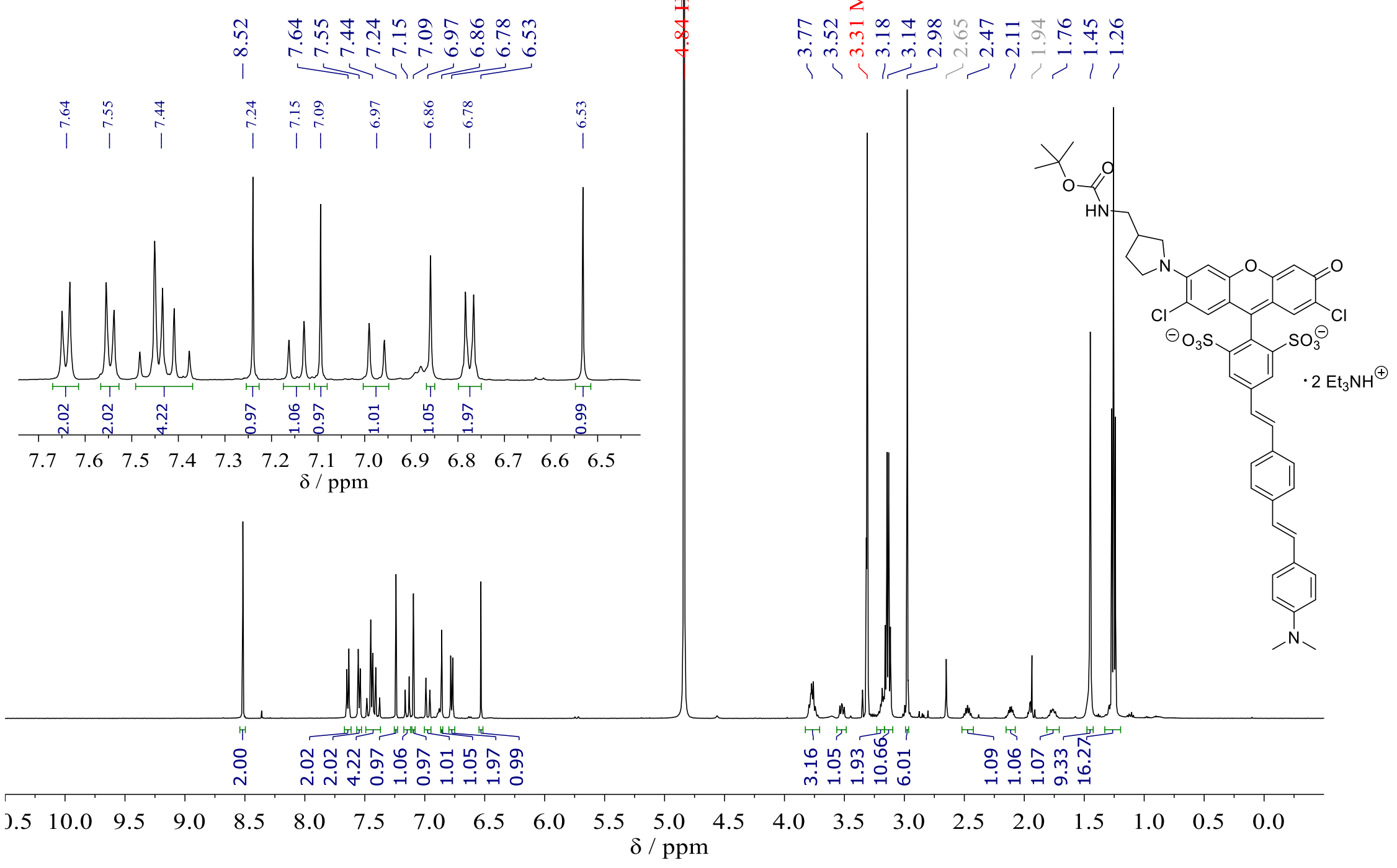

Figure S91. ${ }^{1} \mathrm{H}$ NMR $\left(500 \mathrm{MHz}, \mathrm{CD}_{3} \mathrm{OD}\right)$ of dsRVF5-NHBoc (S26). 


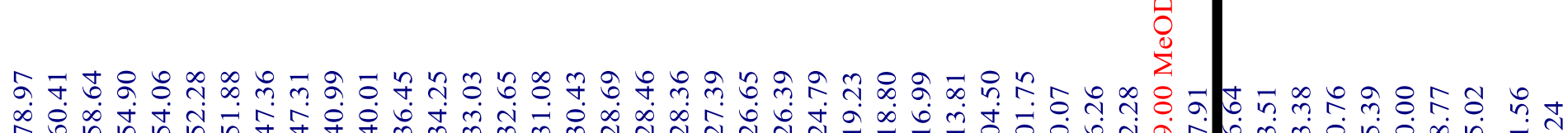

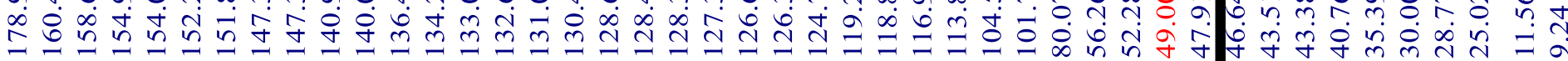

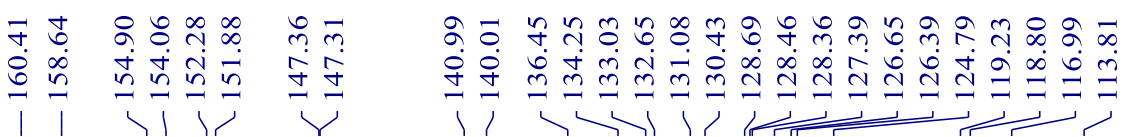
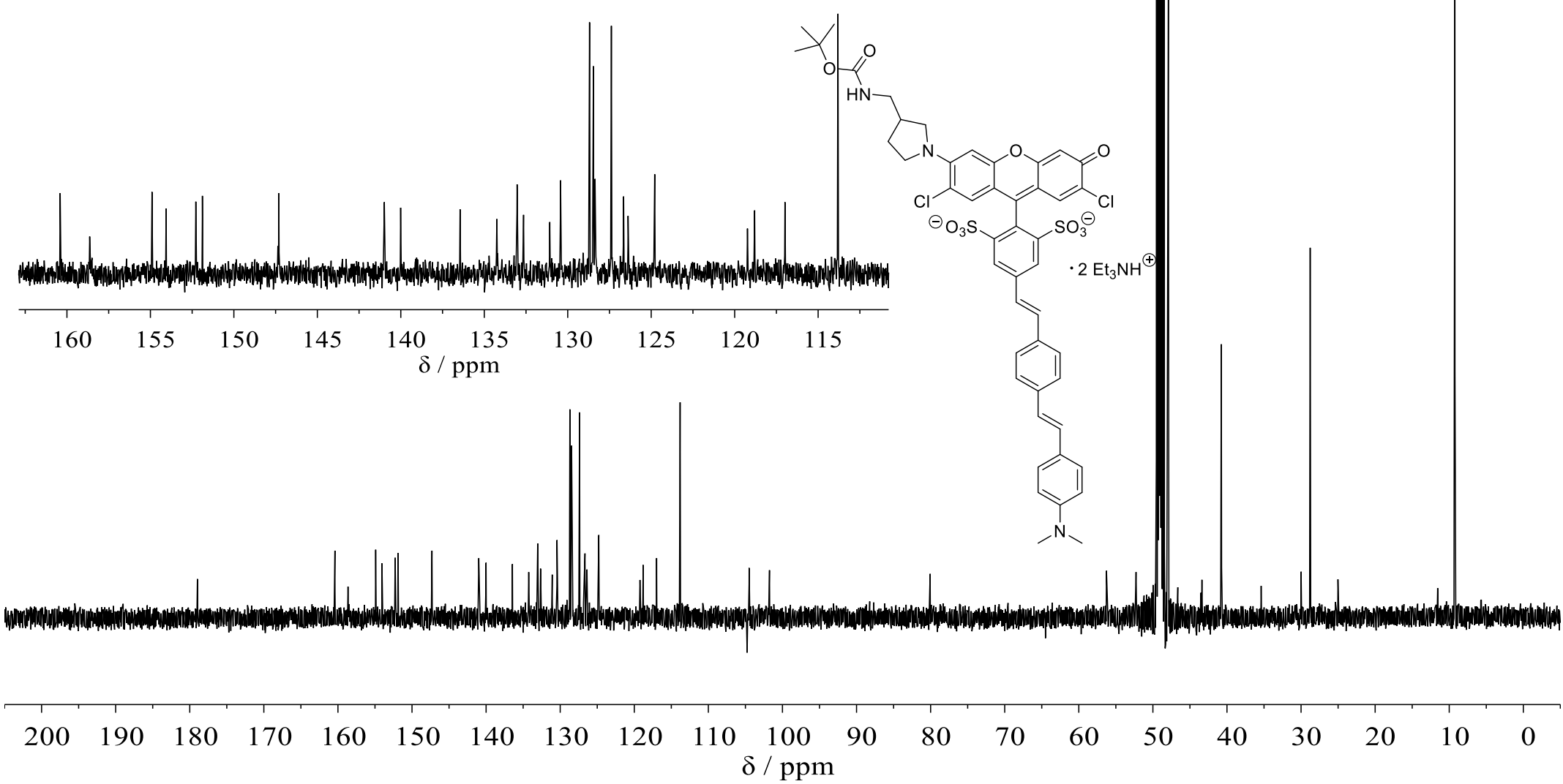

Figure S92. ${ }^{13} \mathrm{C}\left\{{ }^{1} \mathrm{H}\right\}$ NMR (126 MHz, DMSO-d 6 ) of dsRVF5-NHBoc (S26). 


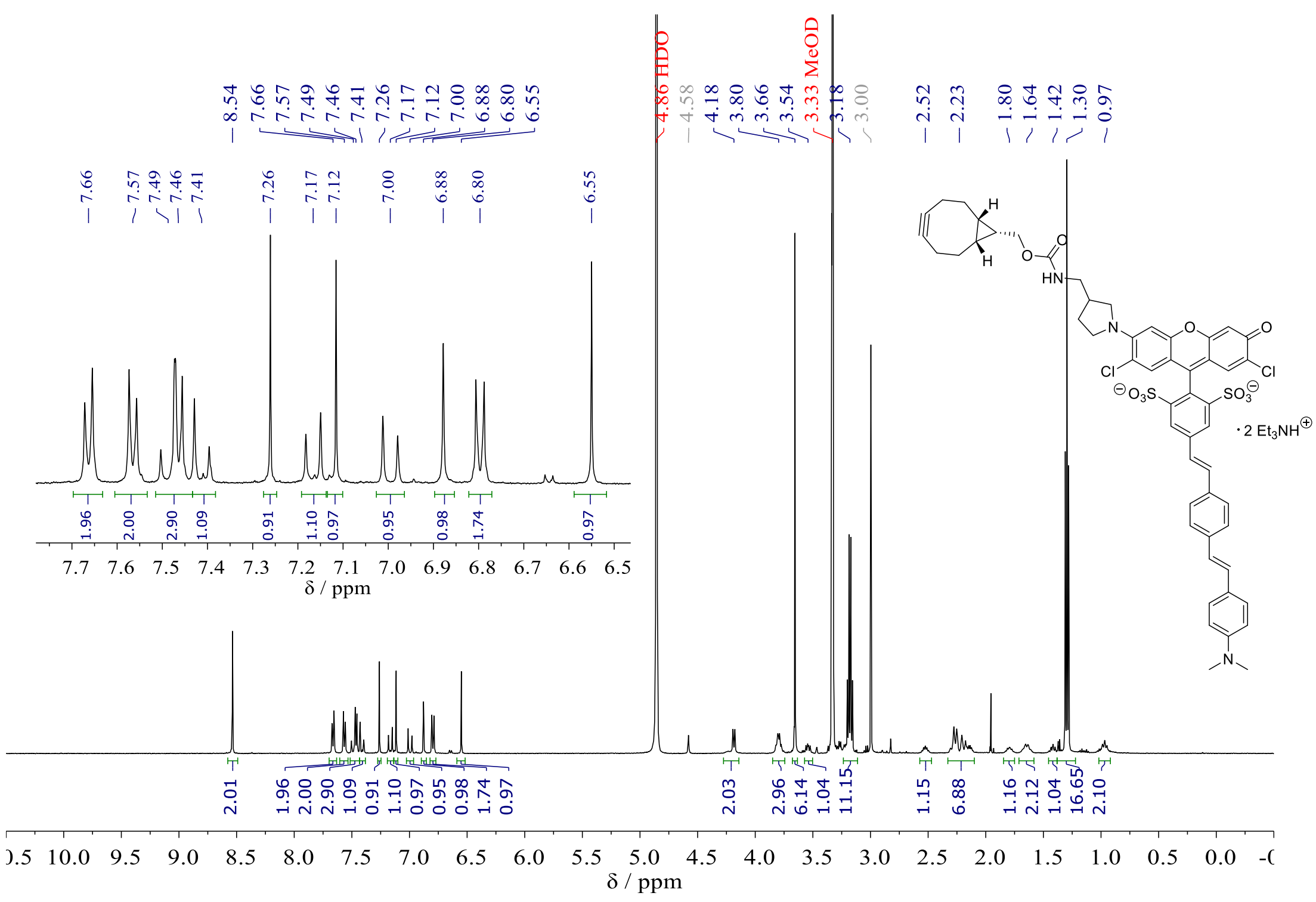

Figure S93. ${ }^{1} \mathrm{H}$ NMR $\left(500 \mathrm{MHz}, \mathrm{CD}_{3} \mathrm{OD}\right)$ of dsRVF5-BCN (10). 


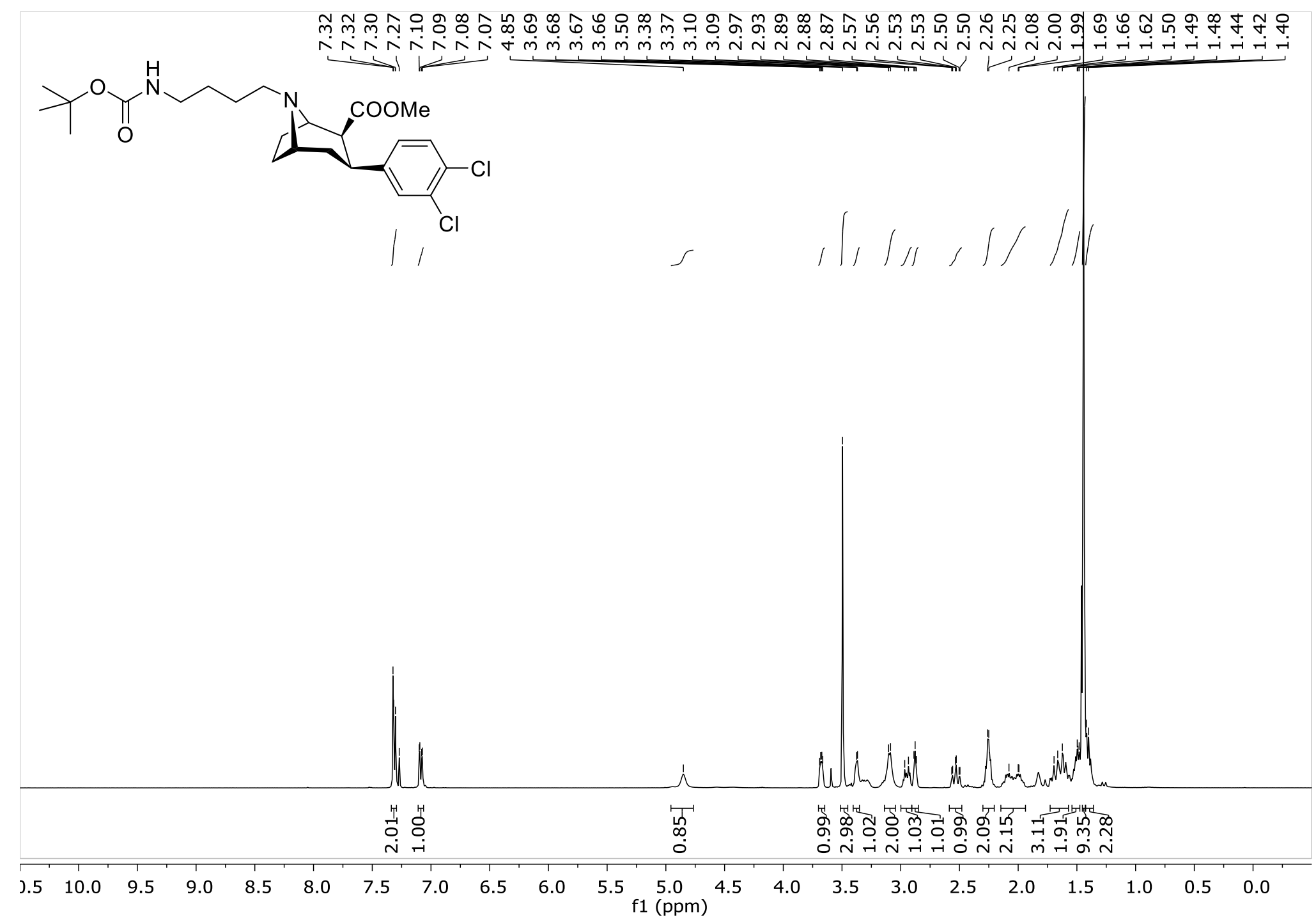

Figure S94. ${ }^{1} \mathrm{H}$ NMR $\left(\mathrm{CDCl}_{3}, 400 \mathrm{MHz}\right)$ of dichloropane-NHBoc (5). 


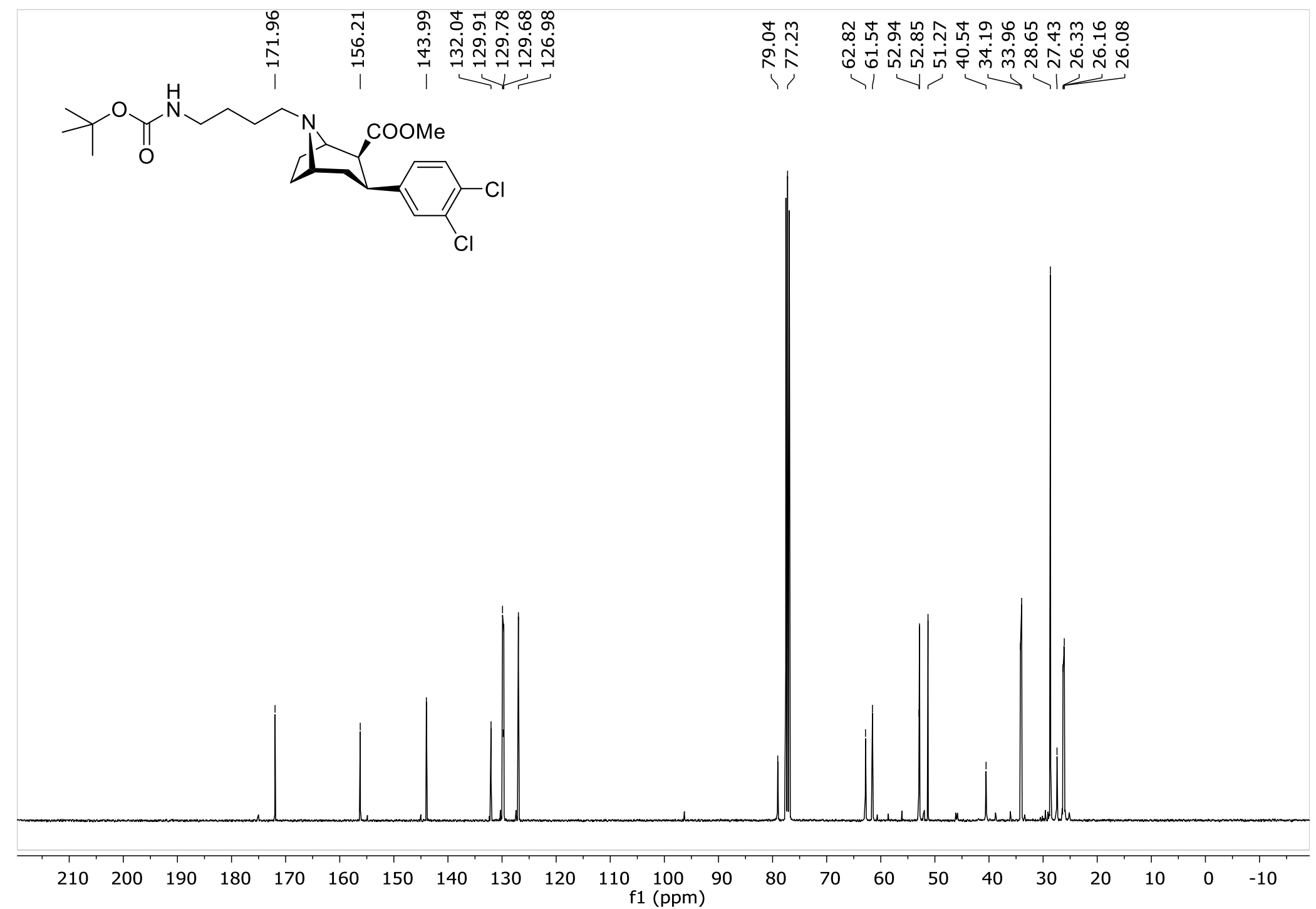

Figure S95. ${ }^{13} \mathrm{C}\left\{{ }^{1} \mathrm{H}\right\} \mathrm{NMR}\left(\mathrm{CDCl}_{3}, 100 \mathrm{MHz}\right)$ of dichloropane-NHBoc (5). 


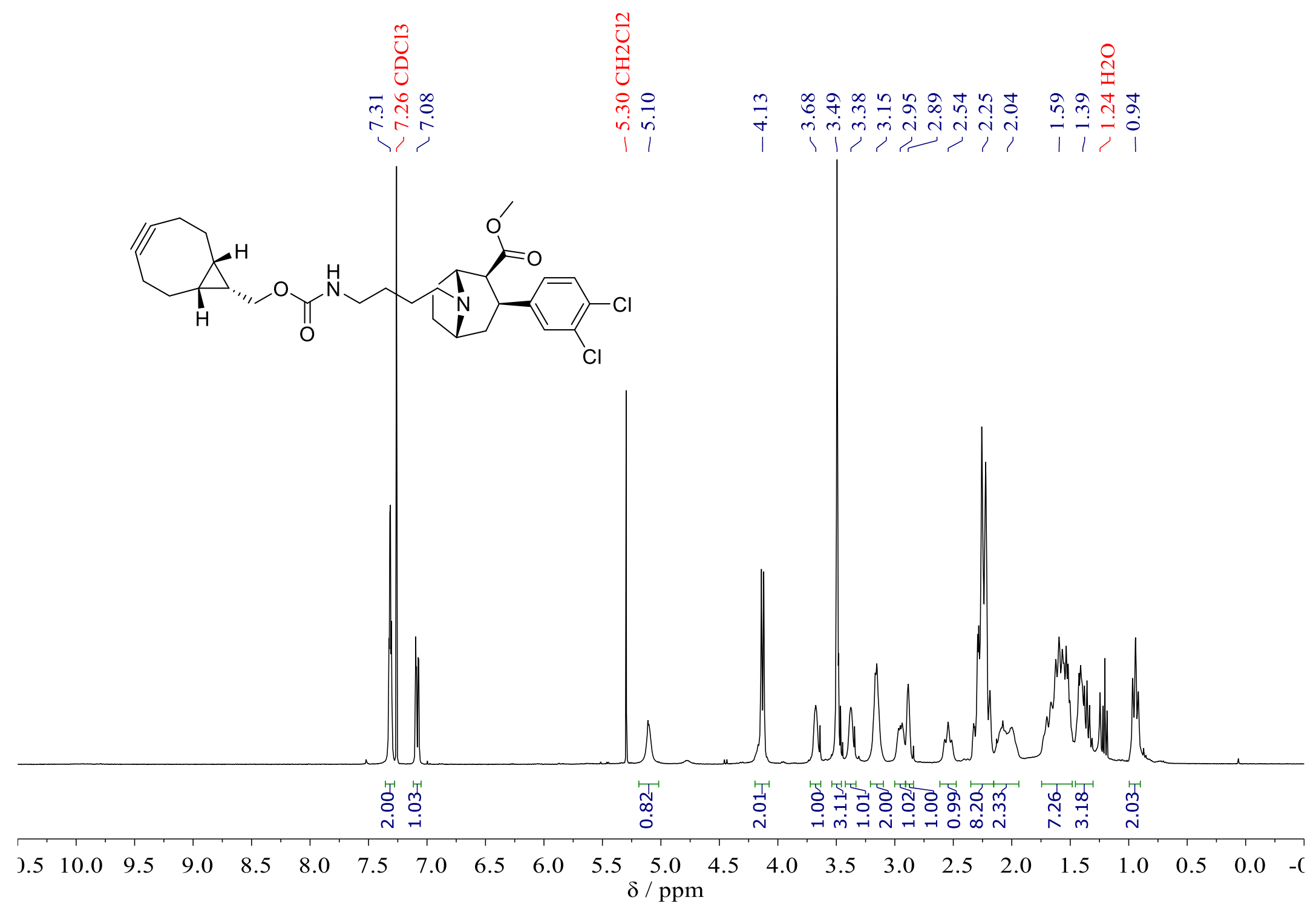

Figure S96. ${ }^{1} \mathrm{H}$ NMR $\left(400 \mathrm{MHz}, \mathrm{CDCl}_{3}\right)$ of dichloropane-BCN (11). 

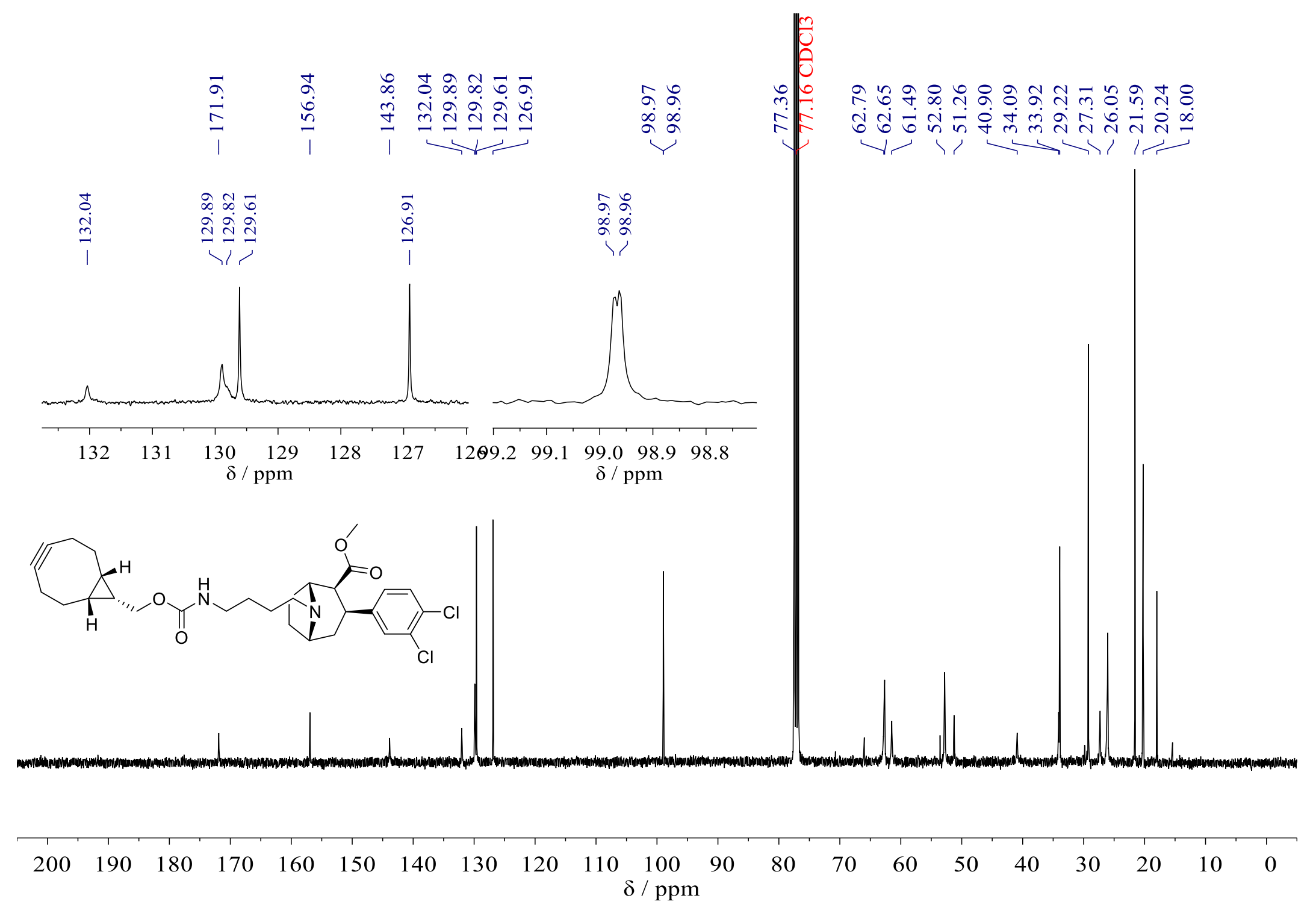

Figure S97. ${ }^{13} \mathrm{C}\left\{{ }^{1} \mathrm{H}\right\}$ NMR $\left(101 \mathrm{MHz}, \mathrm{CDCl}_{3}\right)$ of dichloropane-BCN (11). 


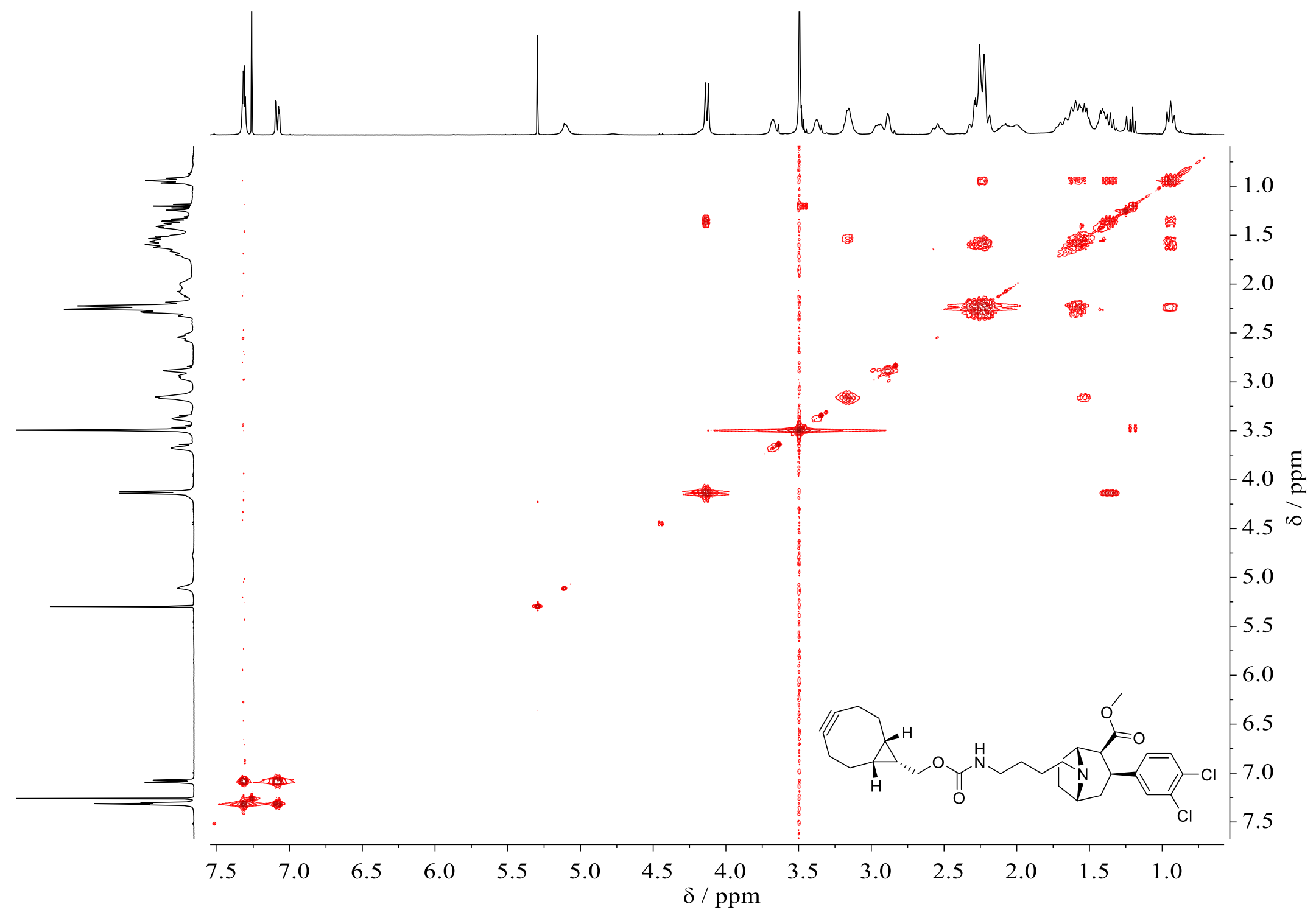

Figure S98. ${ }^{1} \mathrm{H}-{ }^{1} \mathrm{H}$ COSY NMR (400 MHz, $\left.\mathrm{CDCl}_{3}\right)$ of dichloropane-BCN (11). 


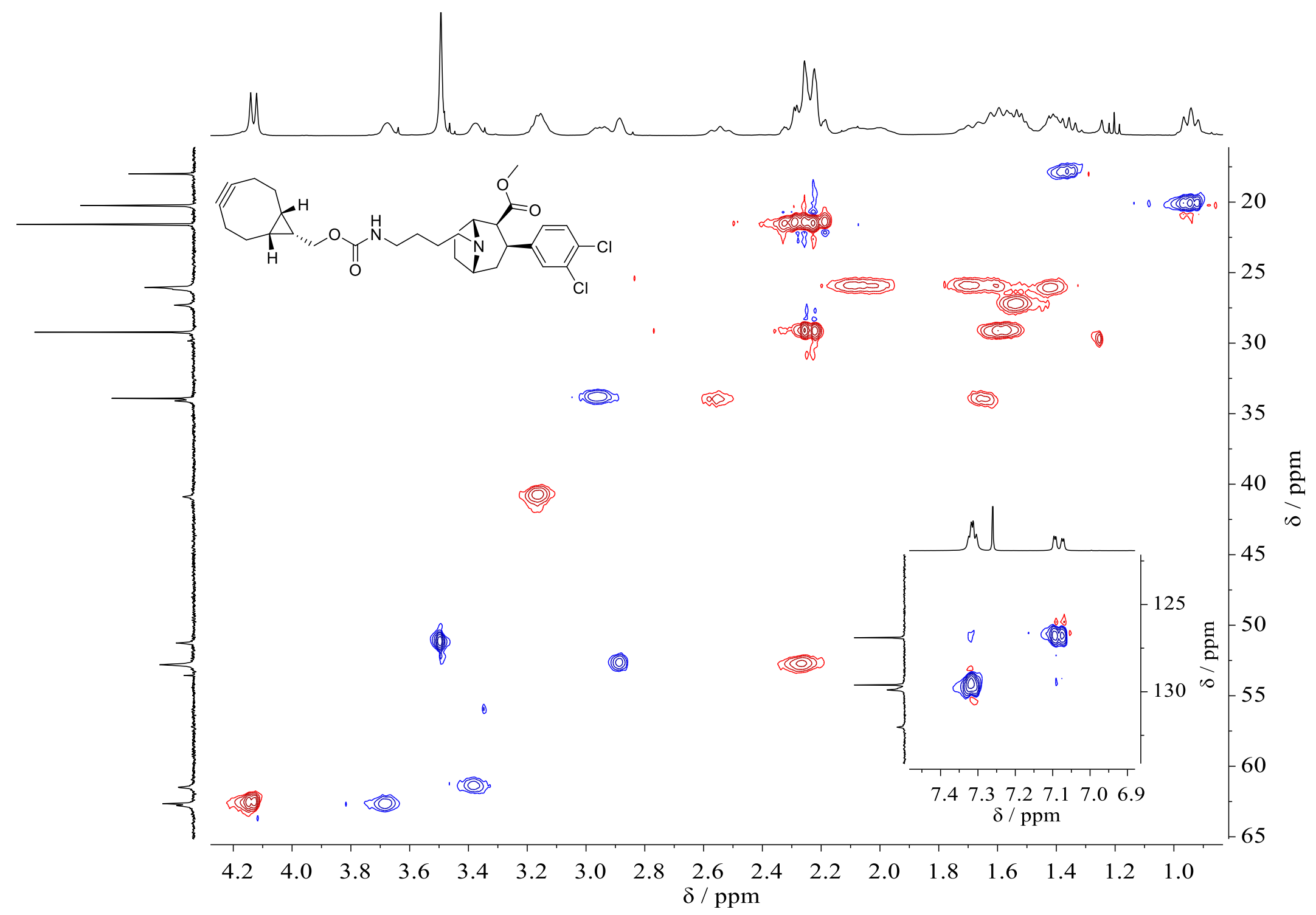

Figure S99. ${ }^{1} \mathrm{H}-{ }^{13} \mathrm{C}$ HSQC NMR $\left(400 \mathrm{MHz}, \mathrm{CDCl}_{3}\right)$ of dichloropane-BCN (11). 


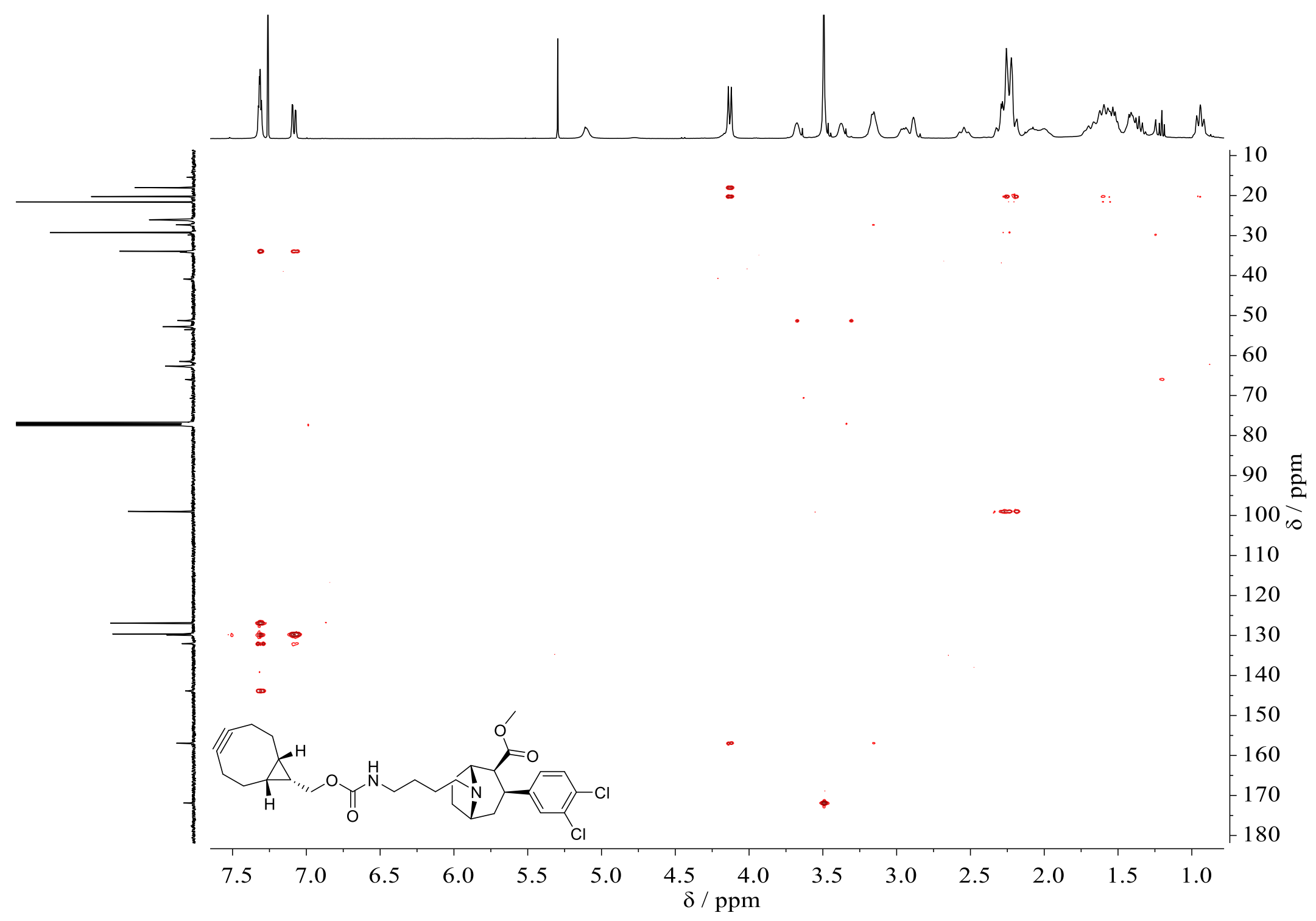

Figure S100. ${ }^{1} \mathrm{H}-{ }^{13} \mathrm{C}$ HMBC NMR $\left(400 \mathrm{MHz}, \mathrm{CDCl}_{3}\right)$ of dichloropane-BCN (11). 


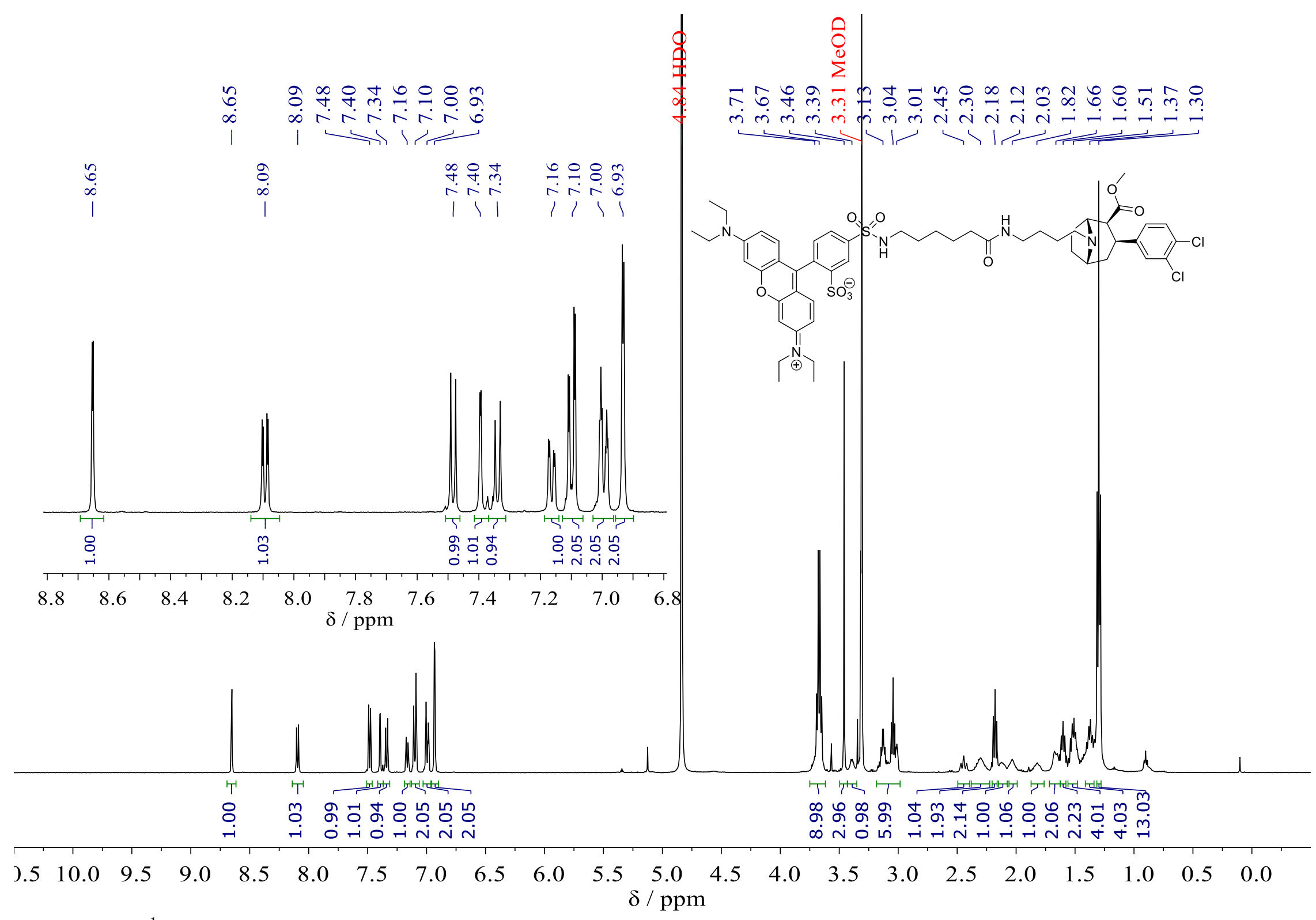

Figure S101. ${ }^{1} \mathrm{H}$ NMR $\left(500 \mathrm{MHz}, \mathrm{CD}_{3} \mathrm{OD}\right)$ of probe 6. 


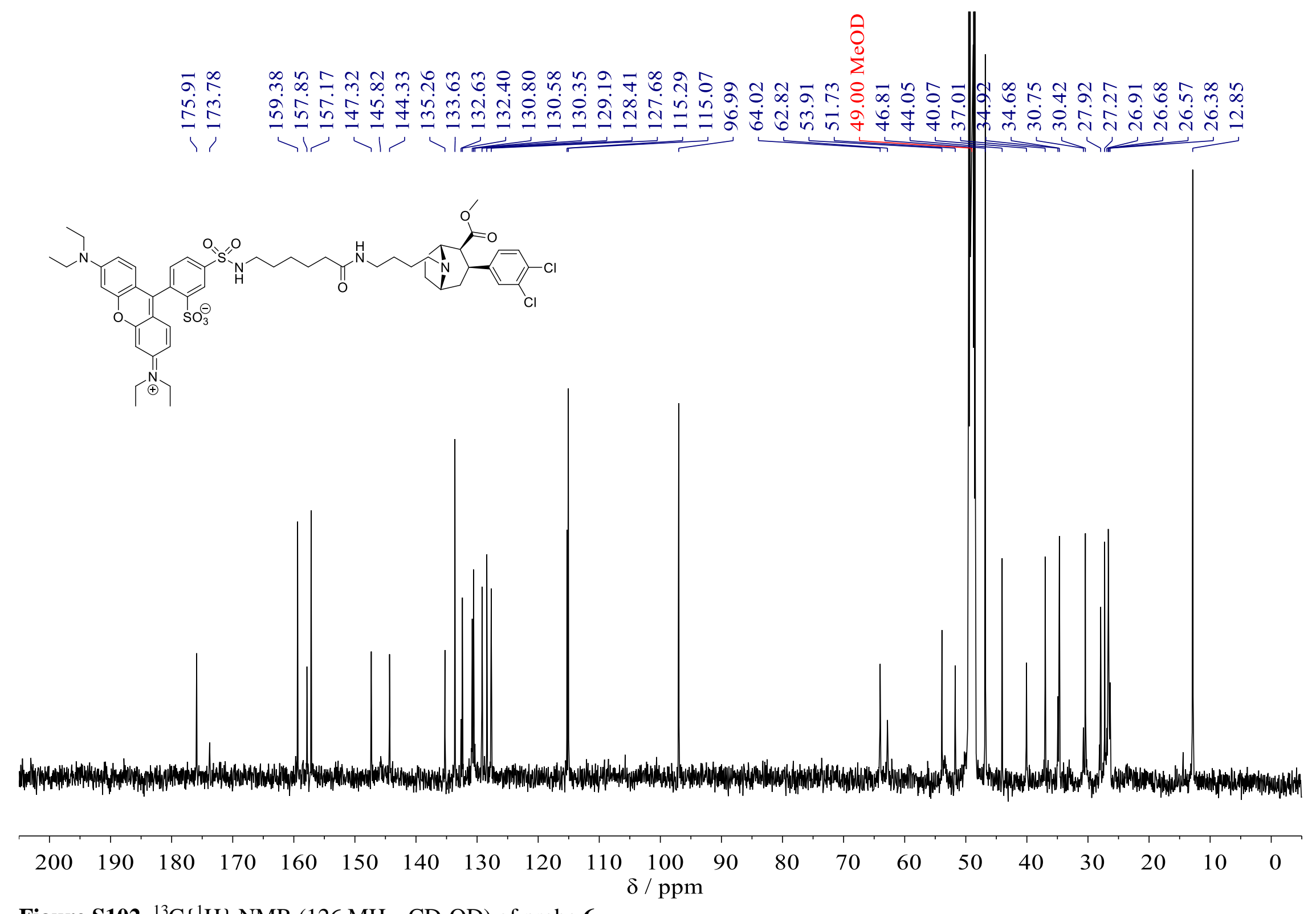

Figure S102. ${ }^{13} \mathrm{C}\left\{{ }^{1} \mathrm{H}\right\}$ NMR (126 MHz, $\left.\mathrm{CD}_{3} \mathrm{OD}\right)$ of probe 6 . 


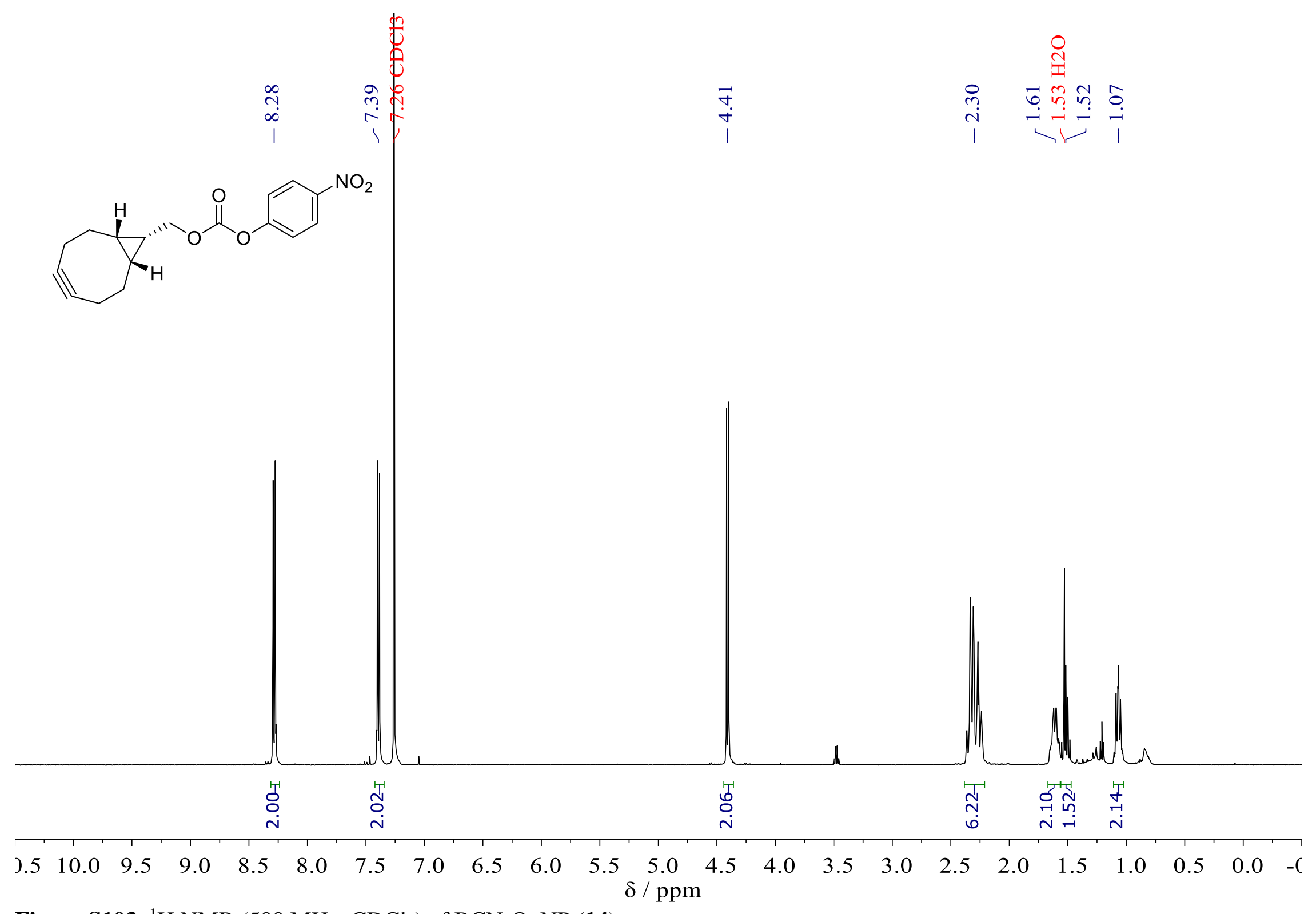

Figure S103. ${ }^{1} \mathrm{H} \mathrm{NMR}\left(500 \mathrm{MHz}, \mathrm{CDCl}_{3}\right)$ of $\mathrm{BCN}-\mathrm{OpNP}(\mathbf{1 4})$. 


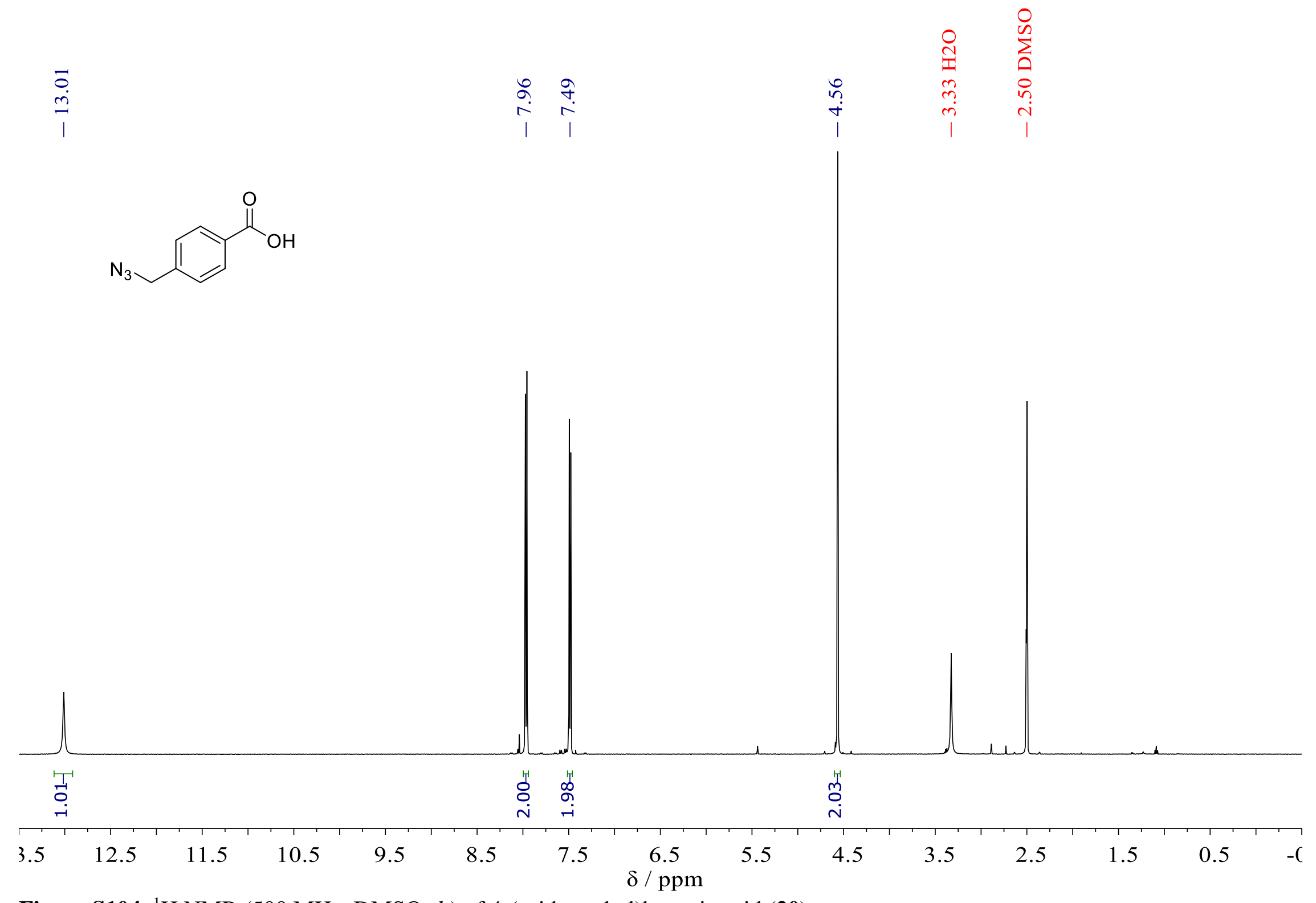

Figure S104. ${ }^{1} \mathrm{H}$ NMR (500 MHz, DMSO- $\left.d_{6}\right)$ of 4-(azidomethyl)benzoic acid (20). 


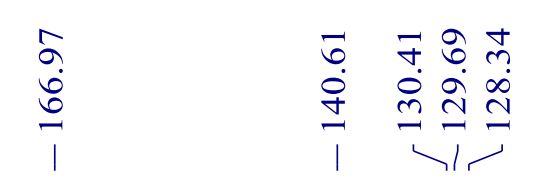
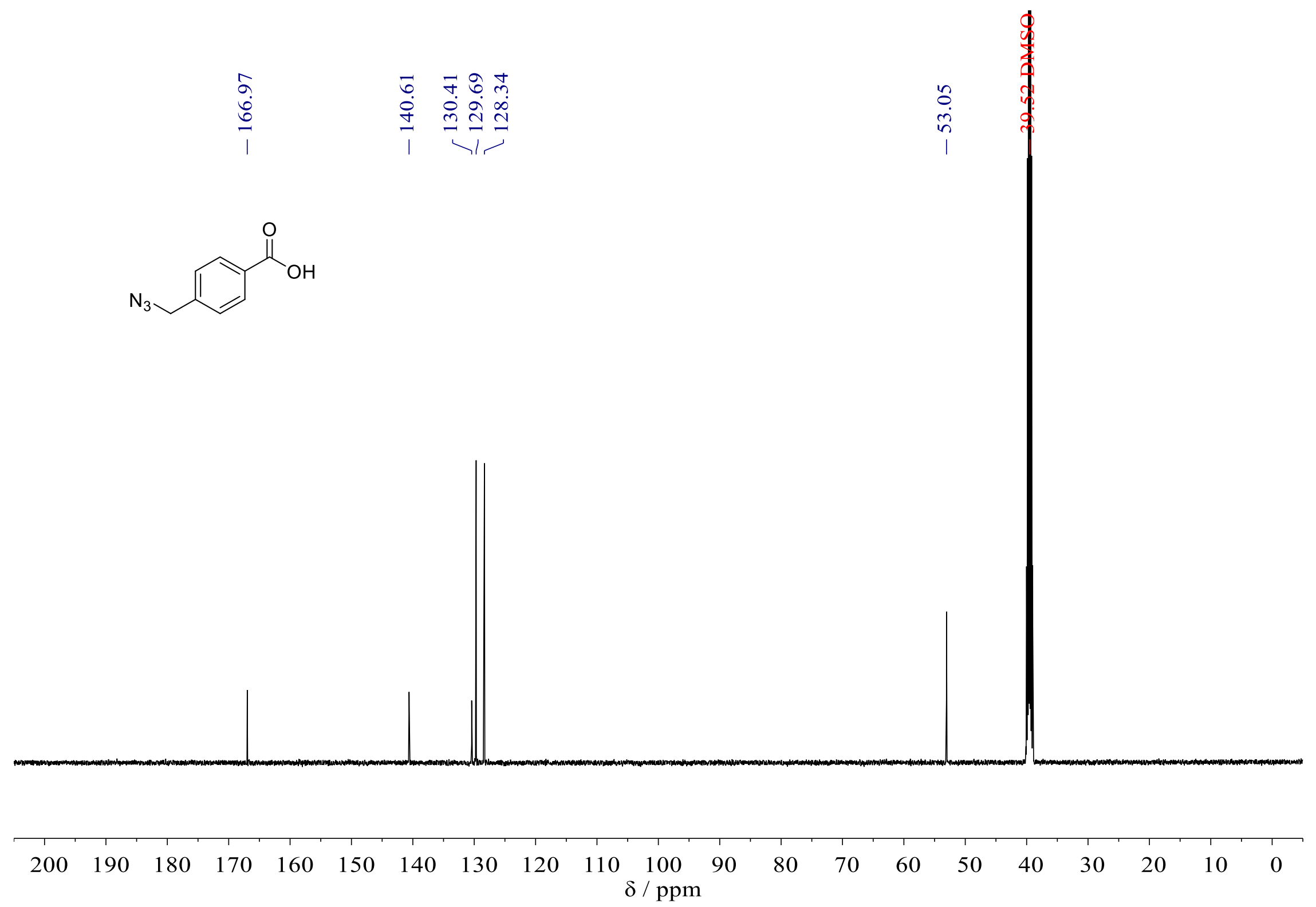

Figure S105. ${ }^{13} \mathrm{C}\left\{{ }^{1} \mathrm{H}\right\}$ NMR (126 MHz, DMSO- $\left.d_{6}\right)$ of 4-(azidomethyl)benzoic acid (20). 


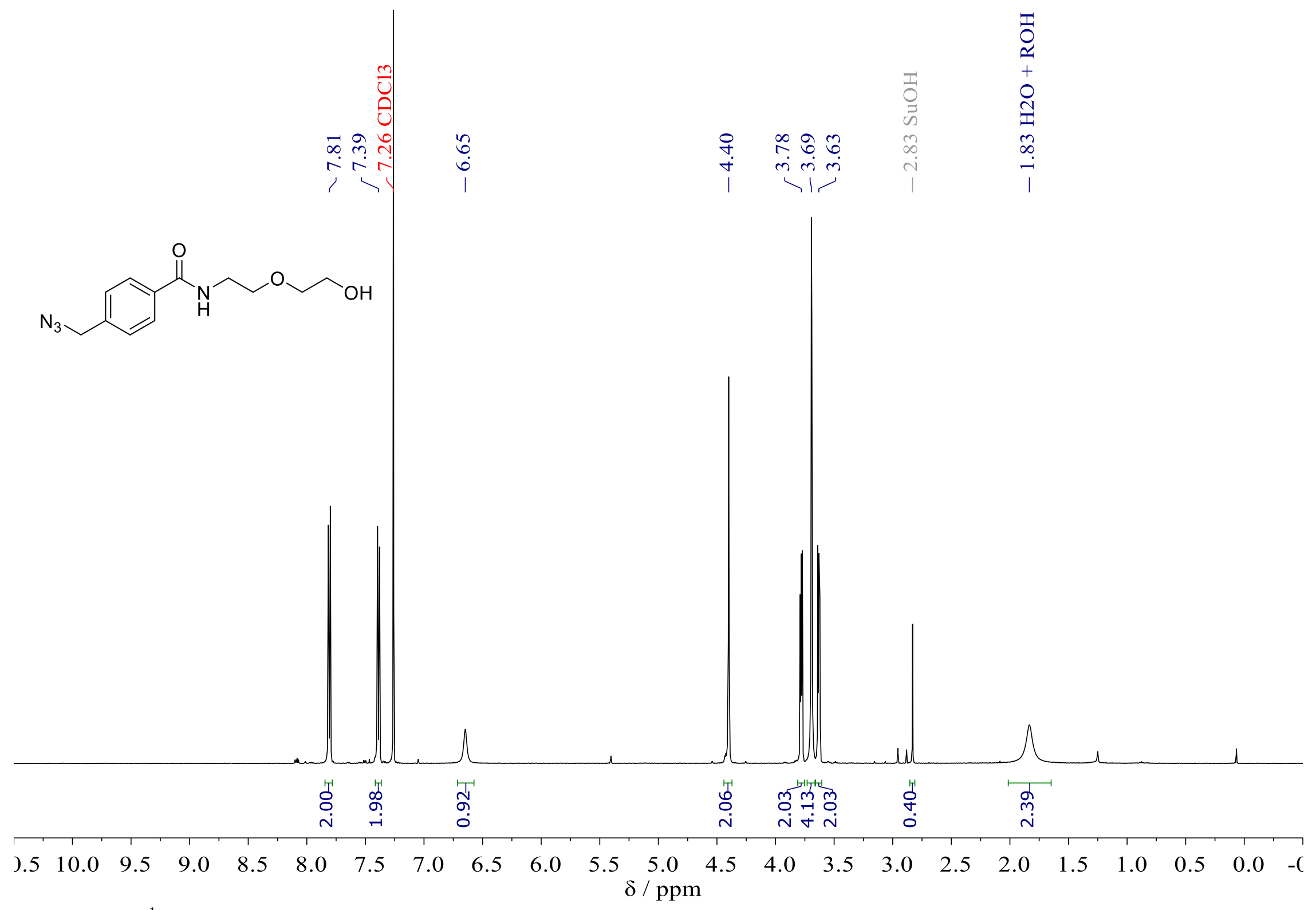

Figure S106. ${ }^{1} \mathrm{H}$ NMR $\left(500 \mathrm{MHz}, \mathrm{CDCl}_{3}\right)$ of compound $\mathbf{S 3 2}$. 


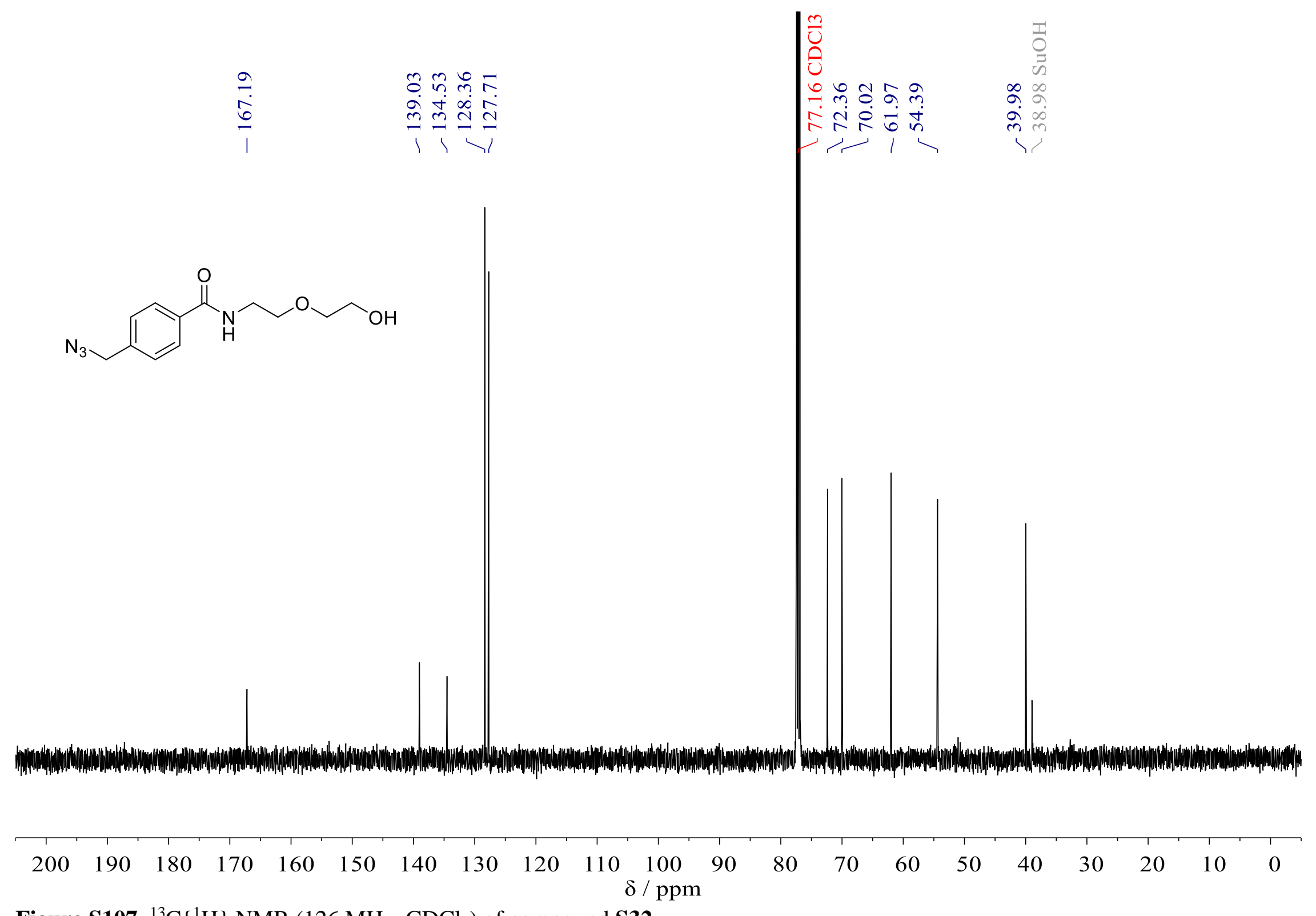

Figure S107. ${ }^{13} \mathrm{C}\left\{{ }^{1} \mathrm{H}\right\}$ NMR (126 MHz, $\mathrm{CDCl}_{3}$ ) of compound $\mathbf{S 3 2}$. 


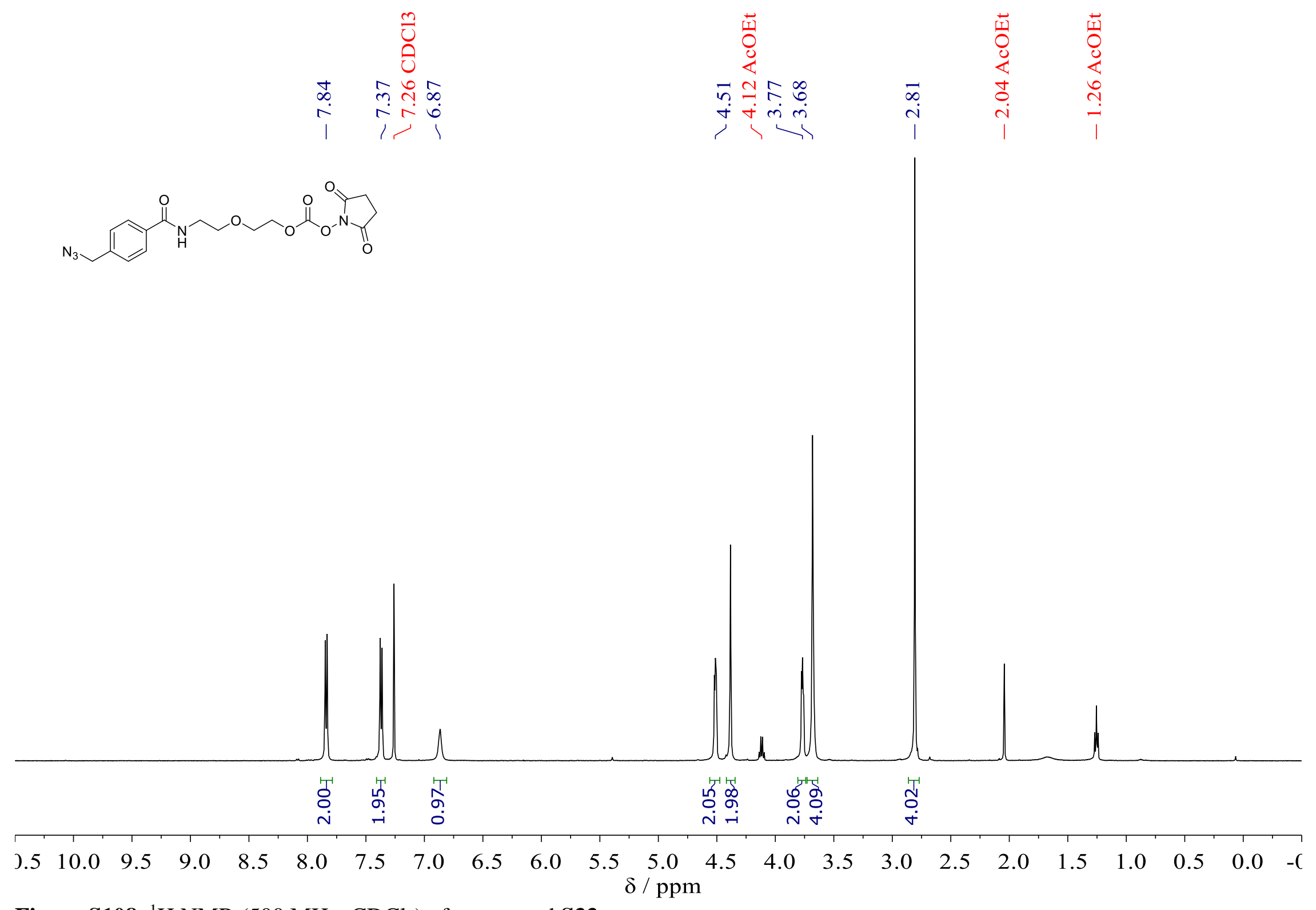

Figure S108. ${ }^{1} \mathrm{H}$ NMR $\left(500 \mathrm{MHz}, \mathrm{CDCl}_{3}\right)$ of compound $\mathbf{S 3 3}$. 


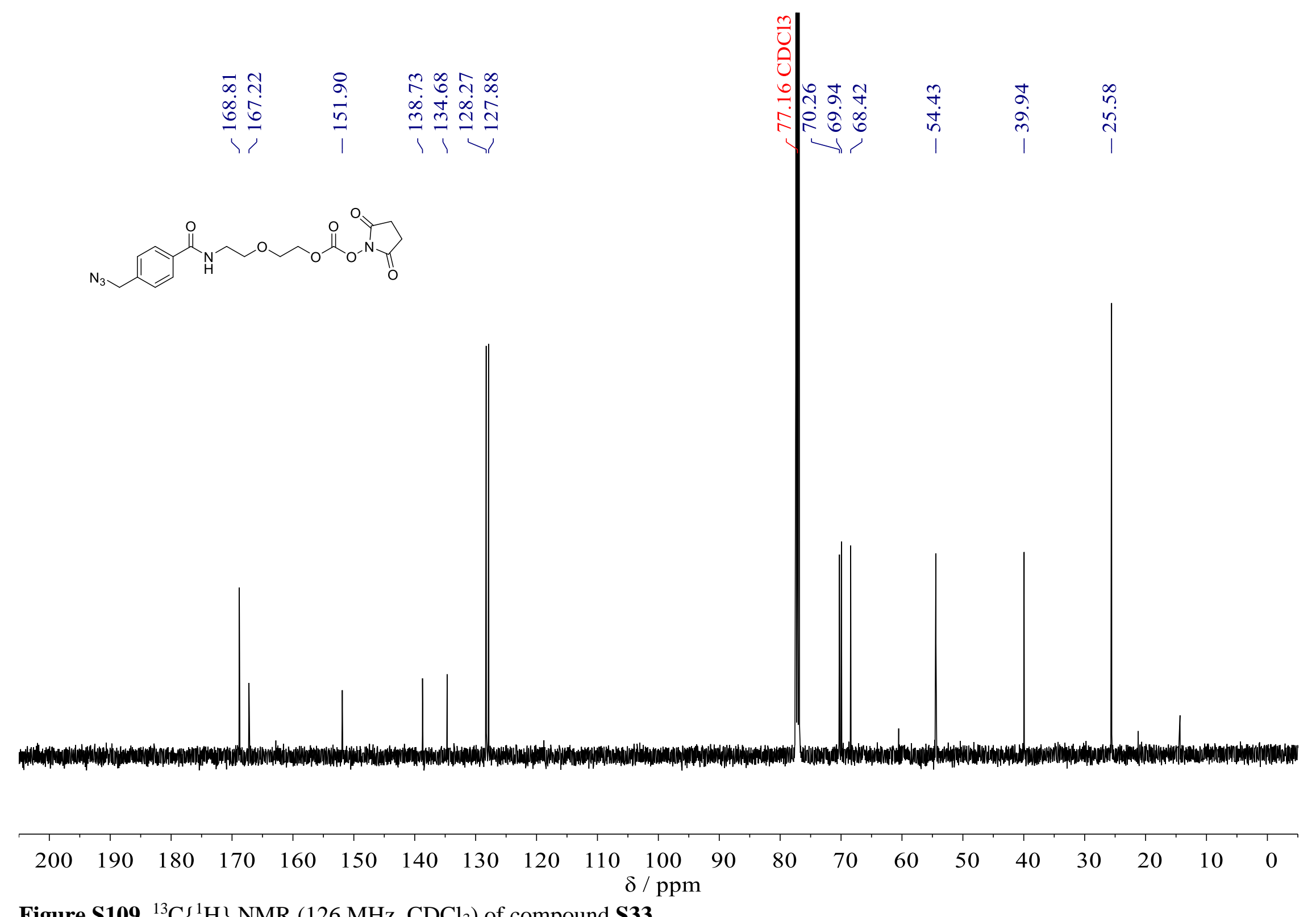

Figure S109. ${ }^{13} \mathrm{C}\left\{{ }^{1} \mathrm{H}\right\}$ NMR (126 MHz, $\left.\mathrm{CDCl}_{3}\right)$ of compound $\mathbf{S 3 3}$. 


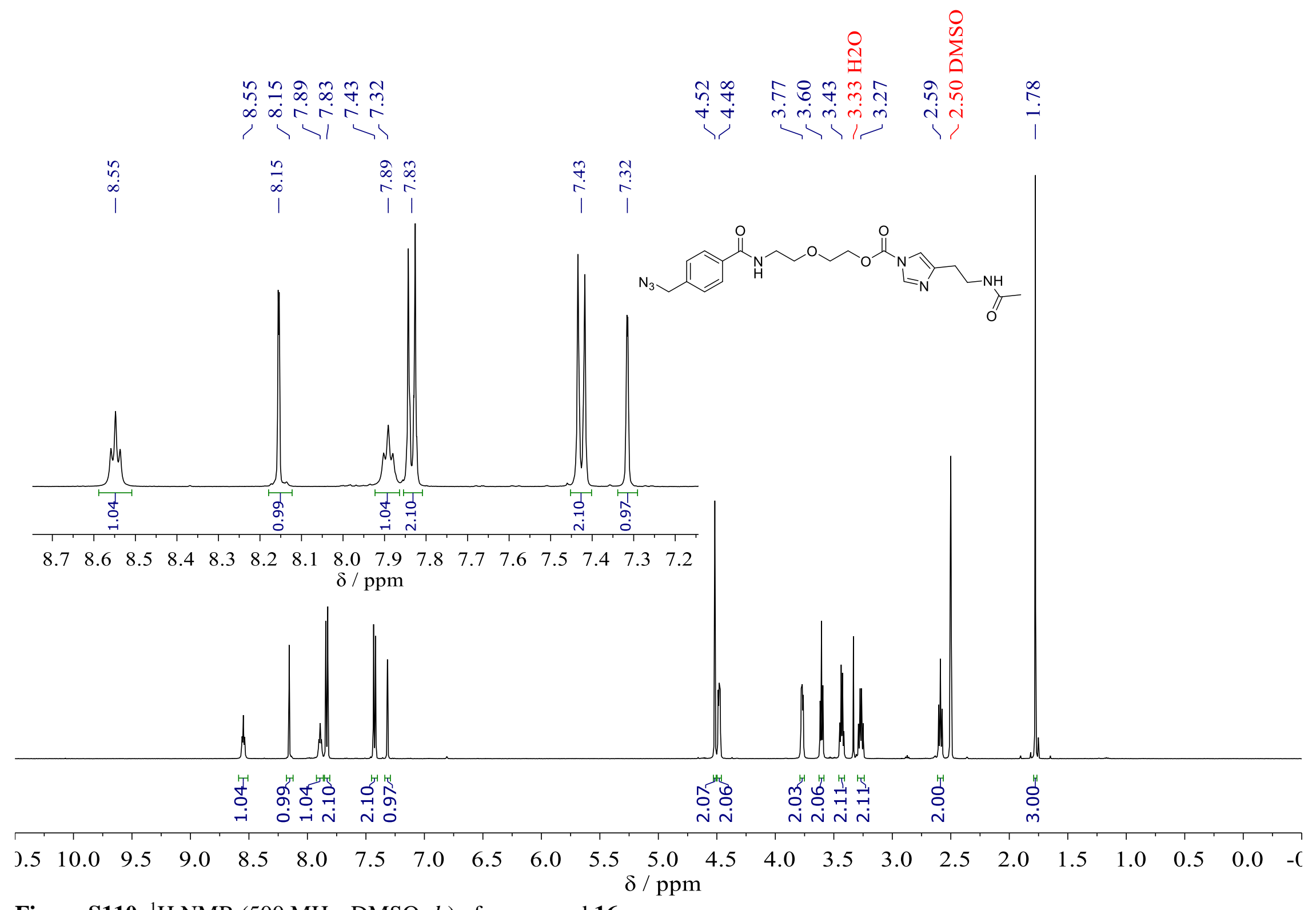

Figure S110. ${ }^{1} \mathrm{H}$ NMR (500 MHz, DMSO-d $)$ of compound 16. 


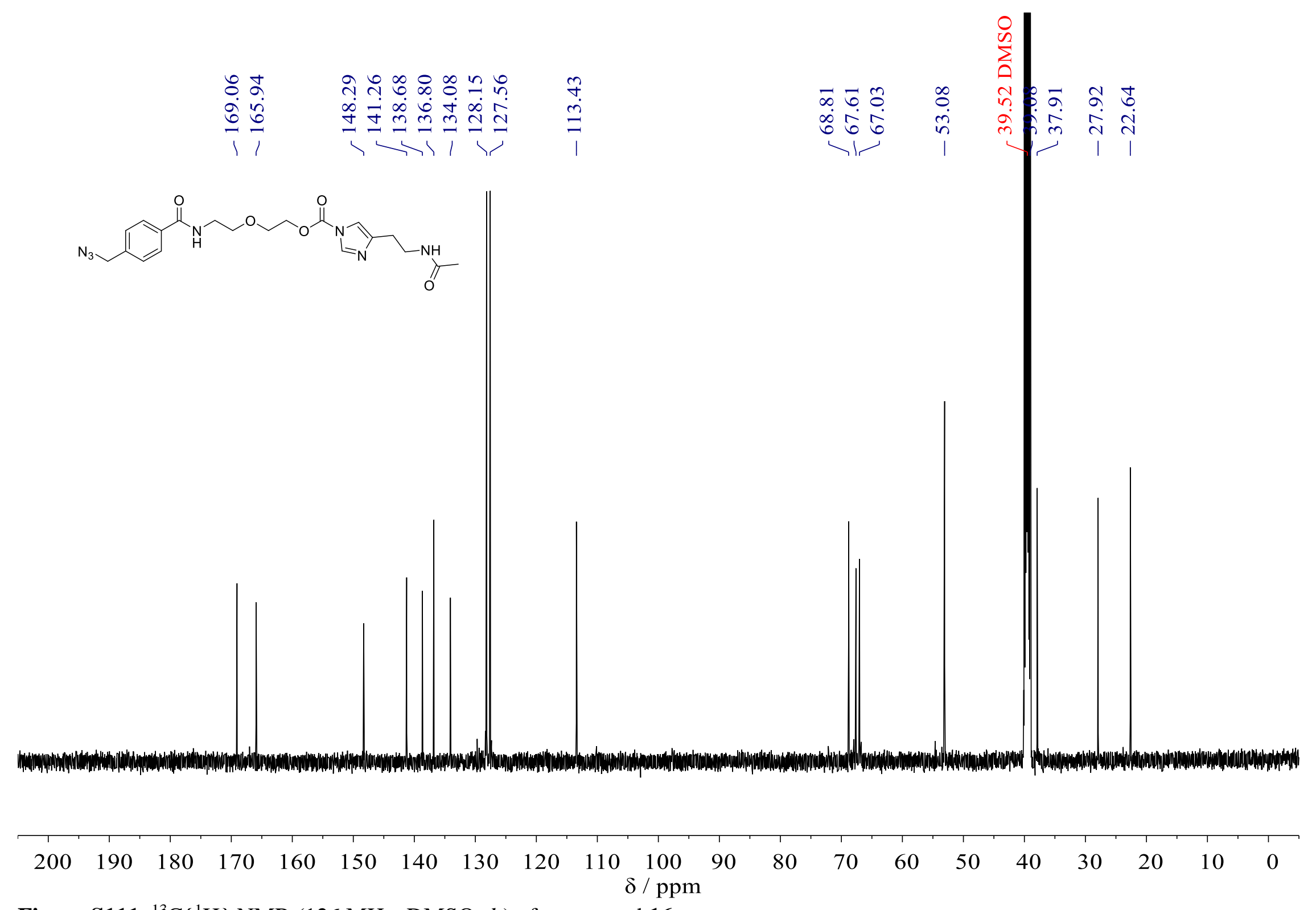

Figure S111. ${ }^{13} \mathrm{C}\left\{{ }^{1} \mathrm{H}\right\}$ NMR (126 MHz, DMSO-d $\left.d_{6}\right)$ of compound 16. 


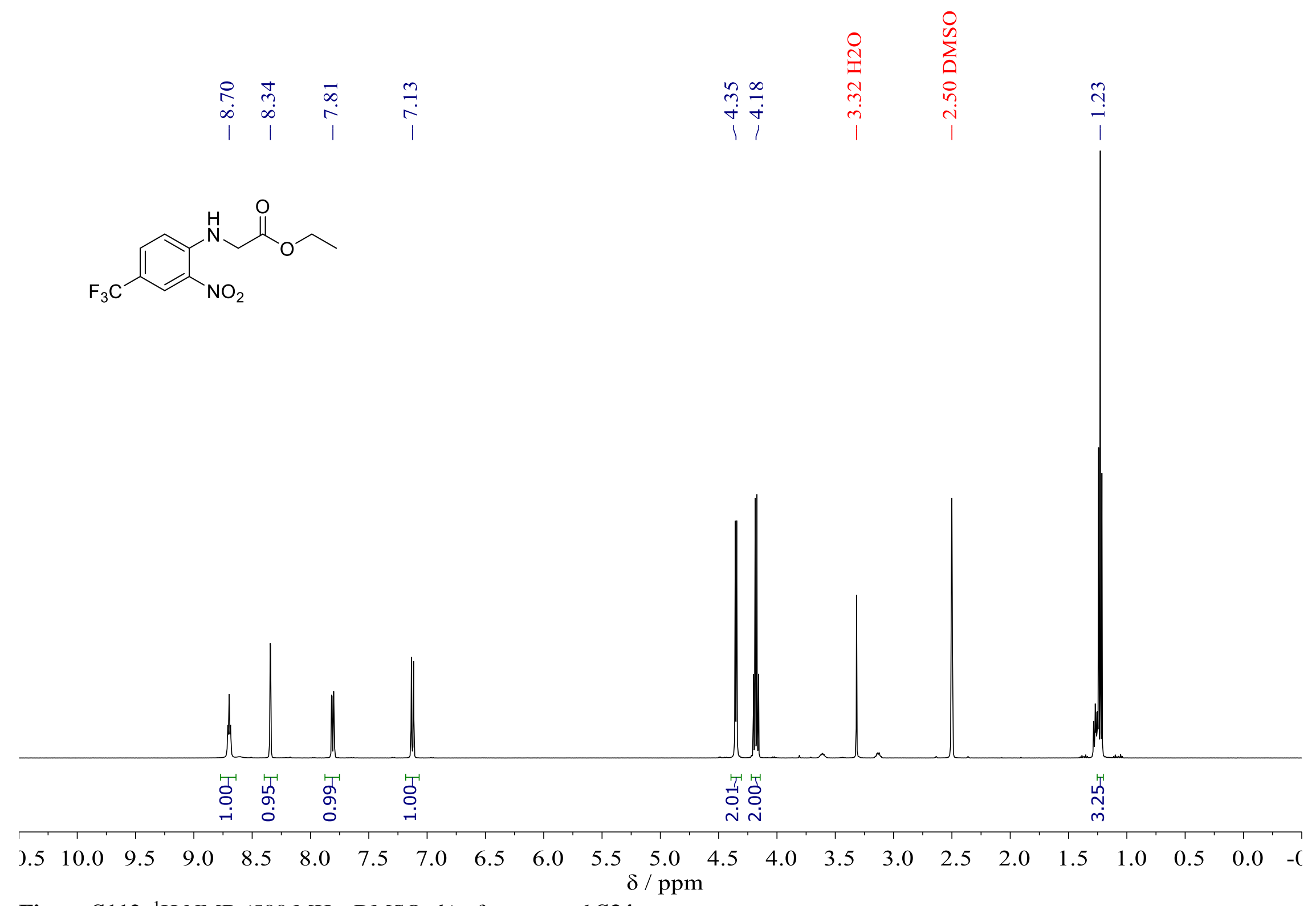

Figure S112. ${ }^{1} \mathrm{H}$ NMR (500 MHz, DMSO- $\left.d_{6}\right)$ of compound S34. 


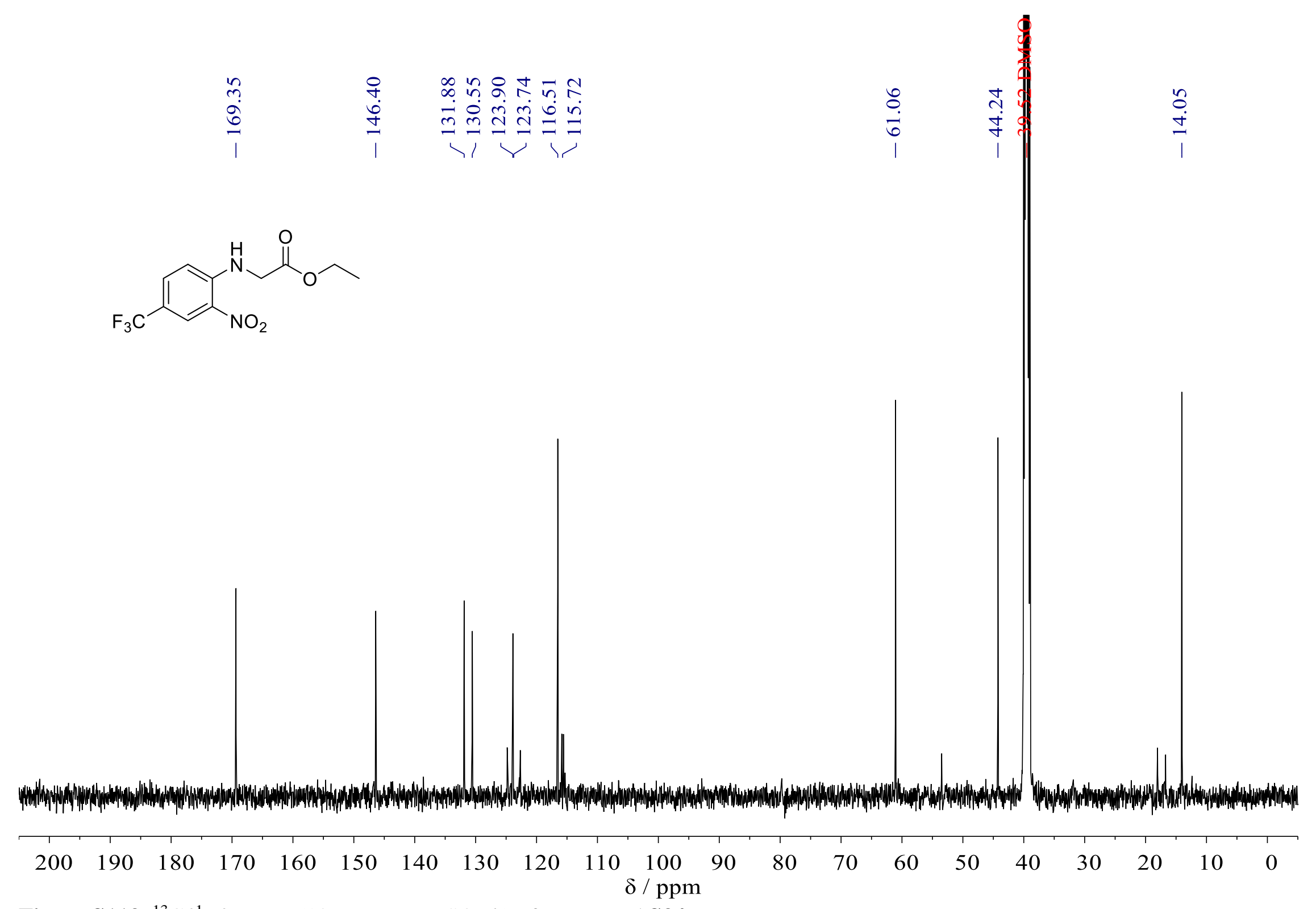

Figure S113. ${ }^{13} \mathrm{C}\left\{{ }^{1} \mathrm{H}\right\}$ NMR (126 MHz, DMSO- $\left.d_{6}\right)$ of compound $\mathbf{S 3 4}$. 


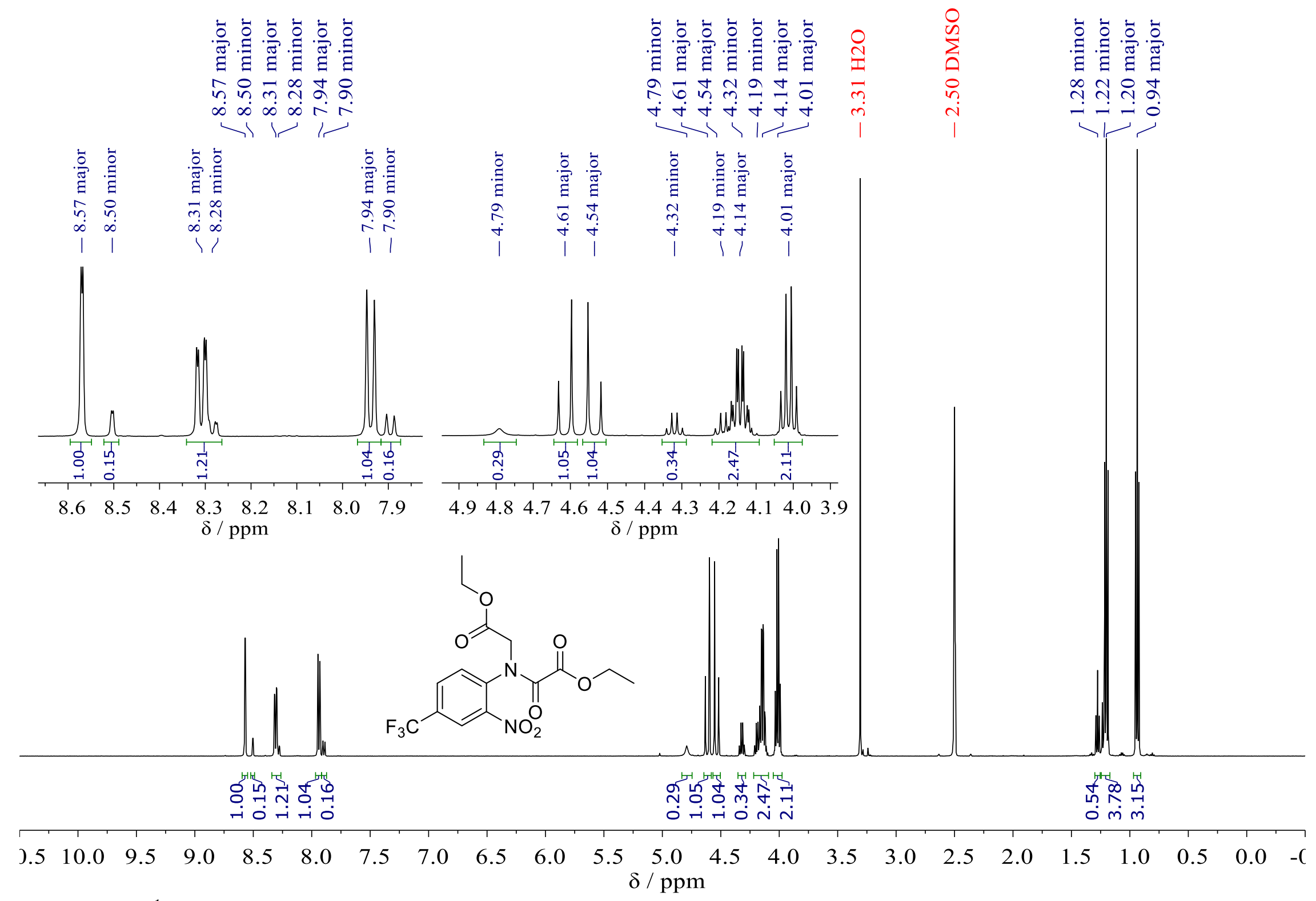

Figure S114. ${ }^{1} \mathrm{H}$ NMR $\left(500 \mathrm{MHz}, \mathrm{DMSO}-d_{6}, 27^{\circ} \mathrm{C}\right)$ of compound $\mathbf{S 3 5}$. 


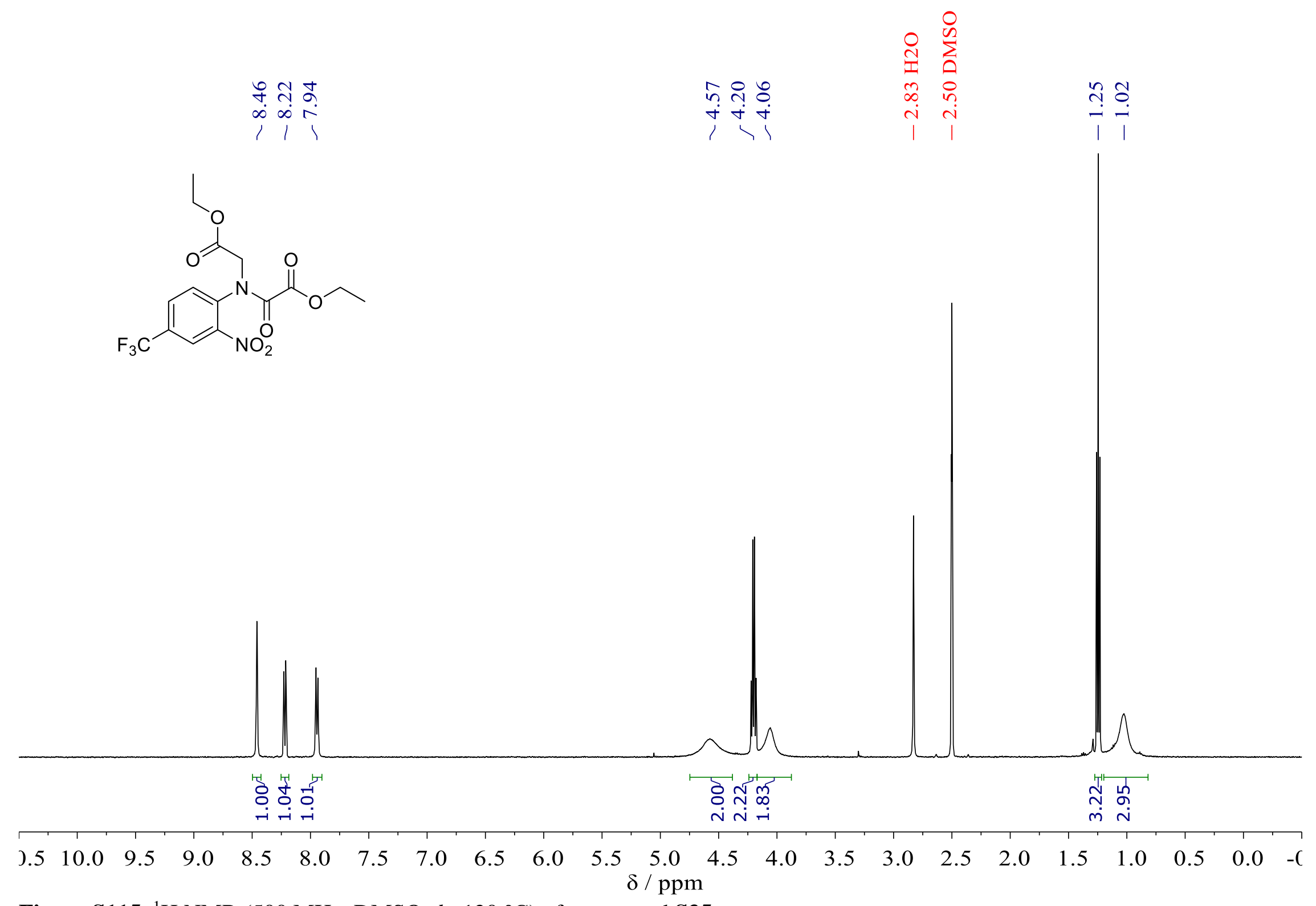

Figure S115. ${ }^{1} \mathrm{H}$ NMR $\left(500 \mathrm{MHz}, \mathrm{DMSO}-d_{6}, 120{ }^{\circ} \mathrm{C}\right)$ of compound $\mathbf{S 3 5}$. 

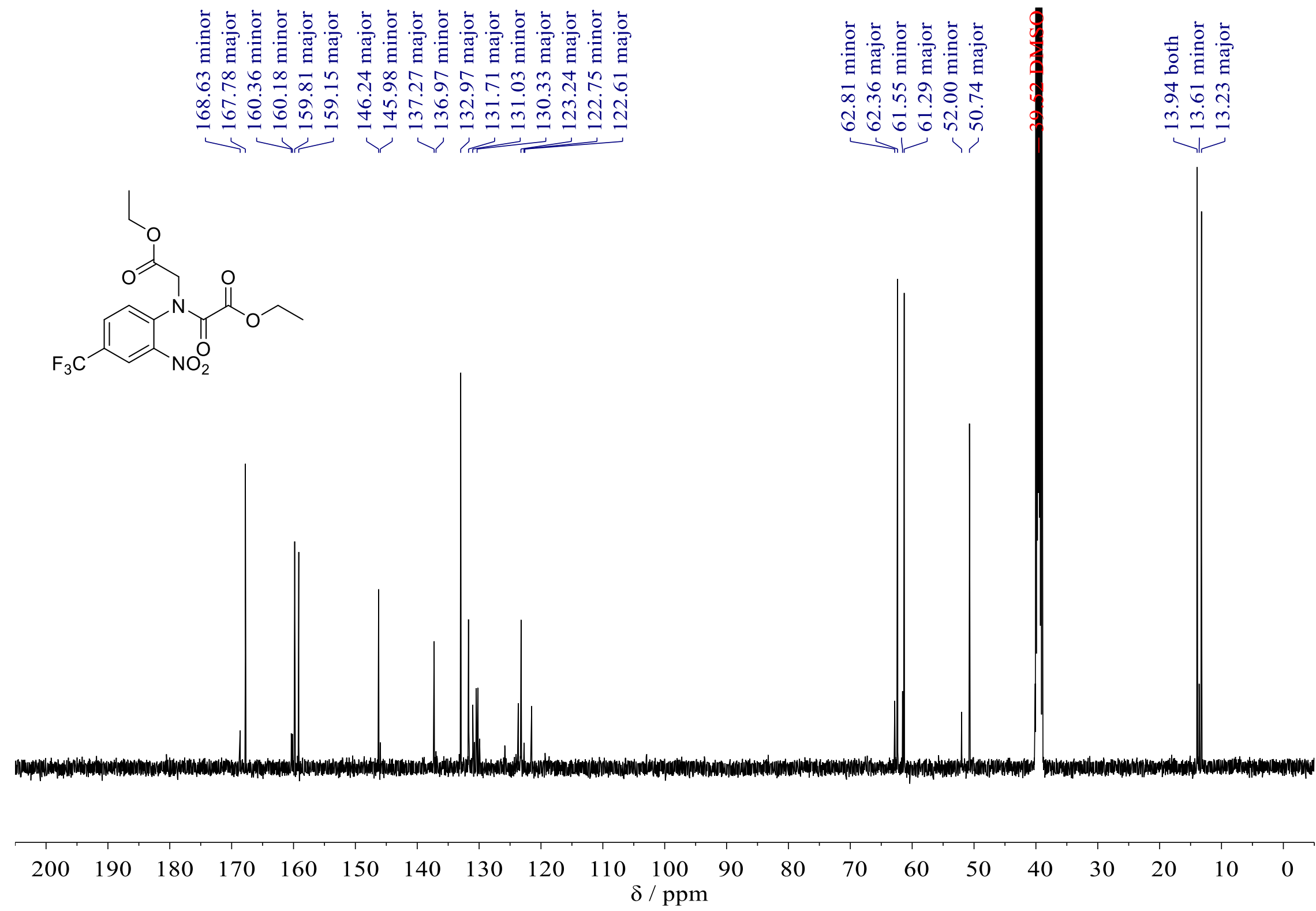

Figure S116. ${ }^{13} \mathrm{C}\left\{{ }^{1} \mathrm{H}\right\}$ NMR $\left(126 \mathrm{MHz}, \mathrm{DMSO}-d_{6}, 27^{\circ} \mathrm{C}\right)$ of compound $\mathbf{S 3 5}$. 


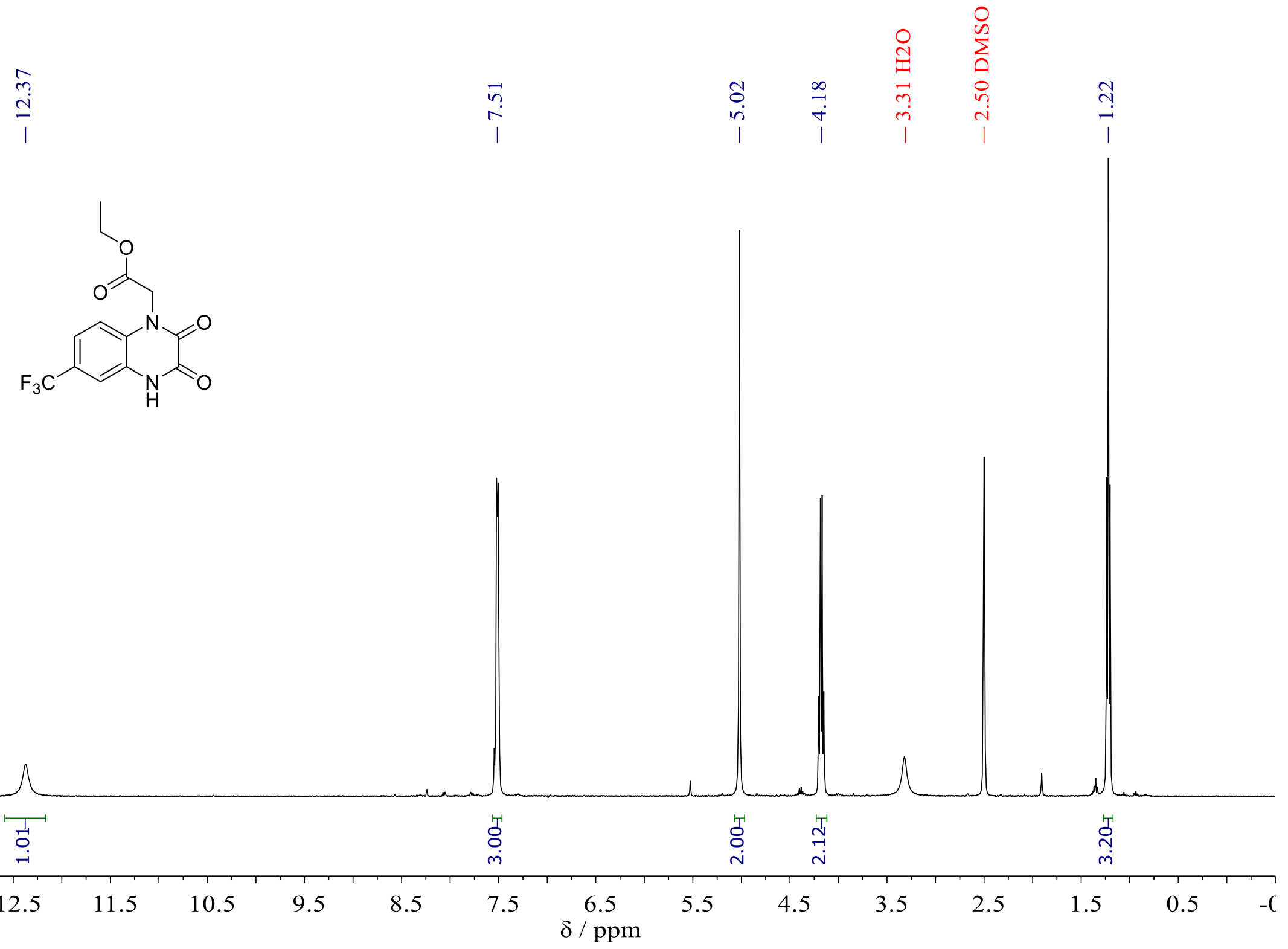

Figure S117. ${ }^{1} \mathrm{H}$ NMR (400 MHz, DMSO- $\left.d_{6}\right)$ of compound S36. 


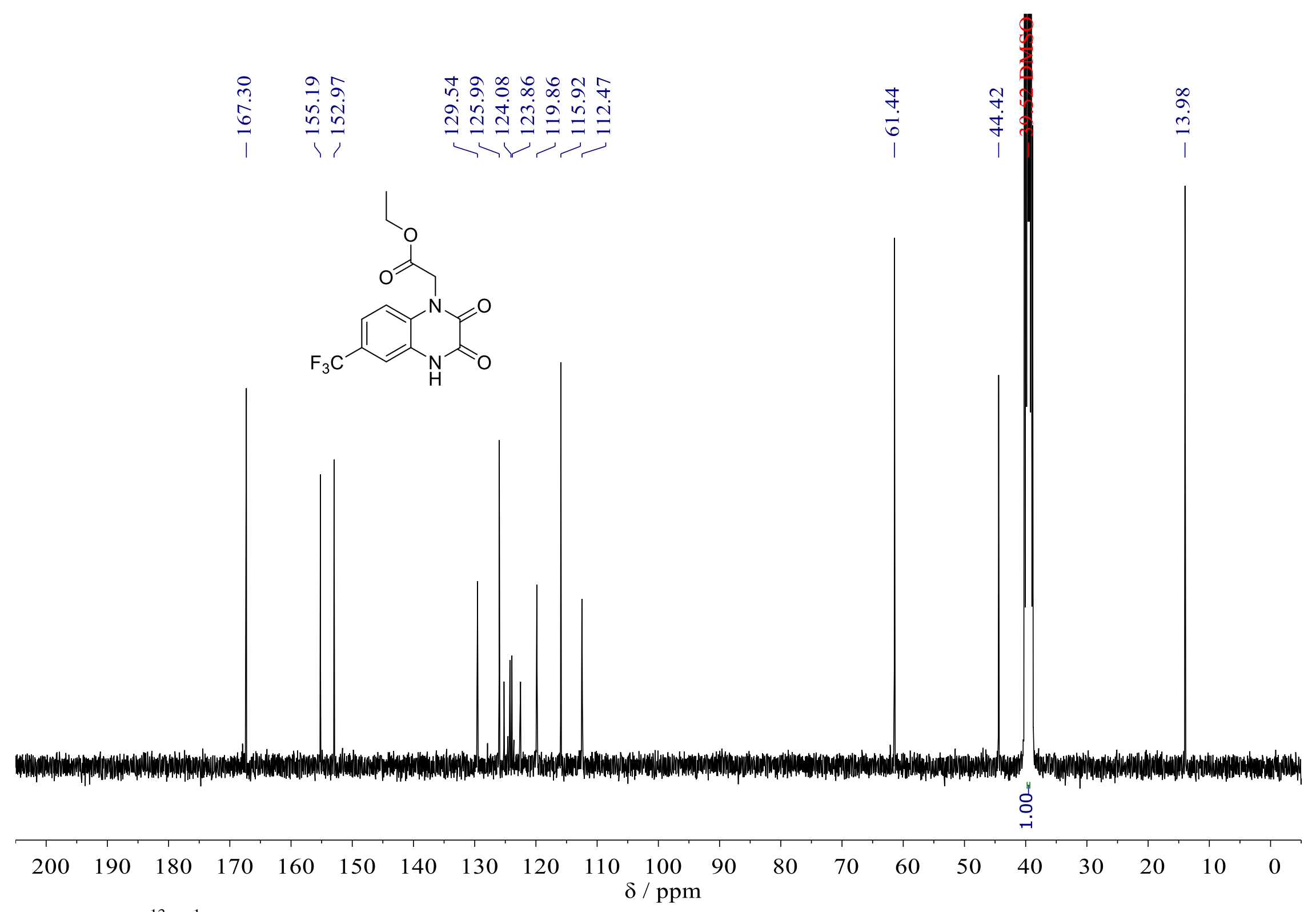

Figure S118. ${ }^{13} \mathrm{C}\left\{{ }^{1} \mathrm{H}\right\}$ NMR $\left(101 \mathrm{MHz}\right.$, DMSO- $\left.d_{6}\right)$ of compound $\mathbf{S 3 6}$. 


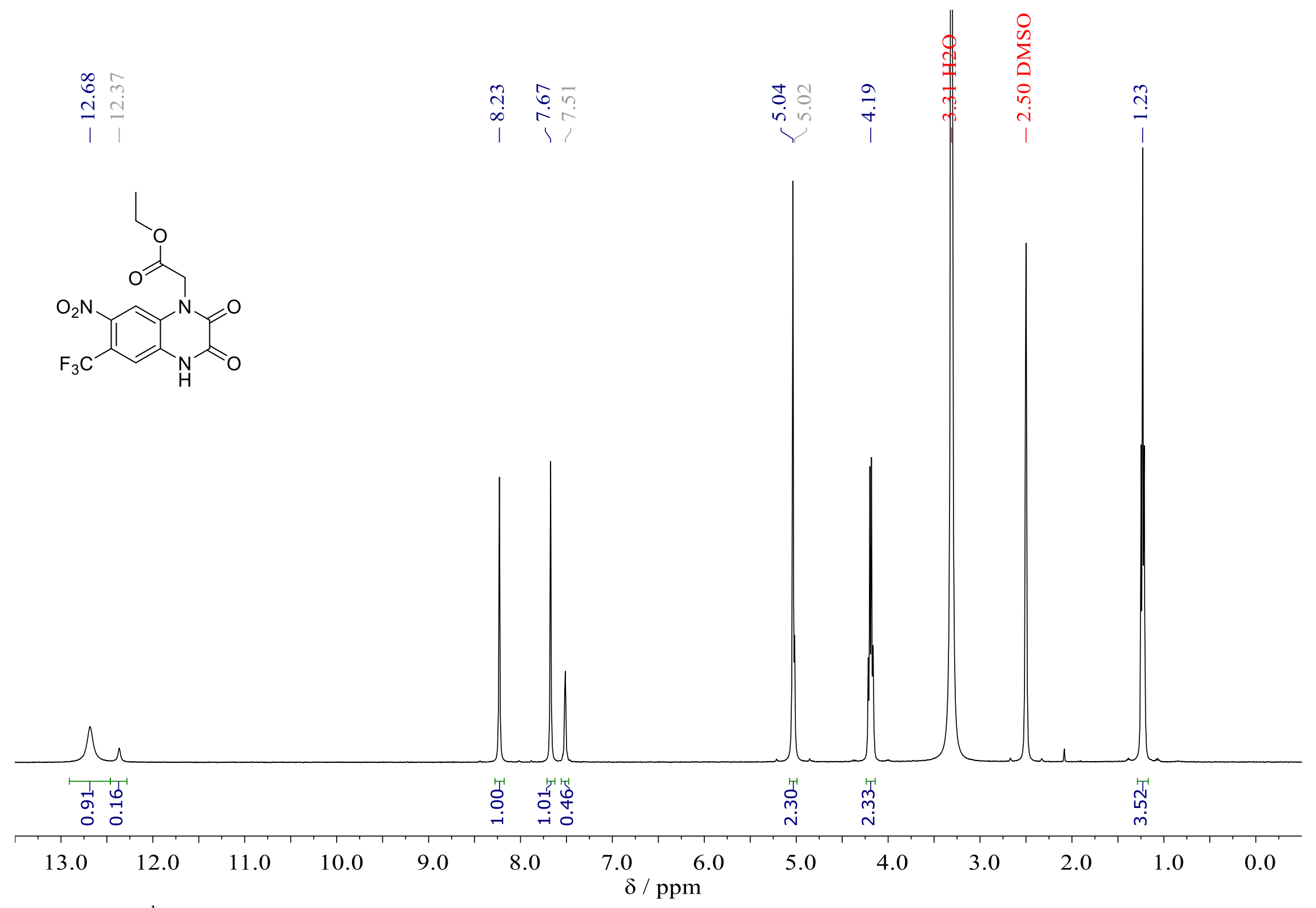

Figure S119. ${ }^{1} \mathrm{H}$ NMR (400 MHz, DMSO- $\left.d_{6}\right)$ of compound S37. 


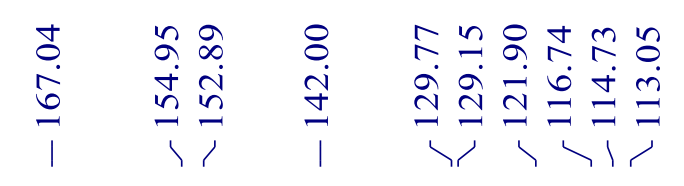
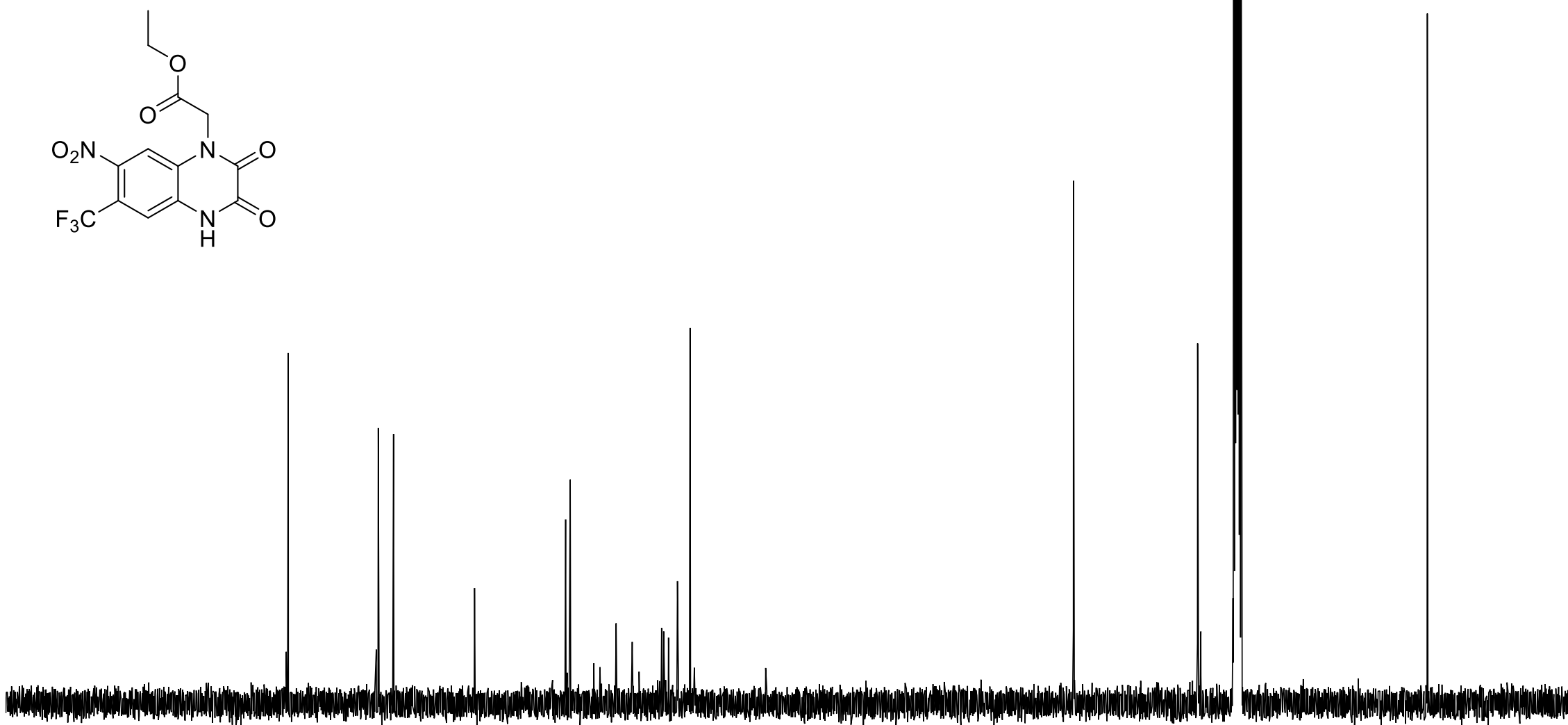

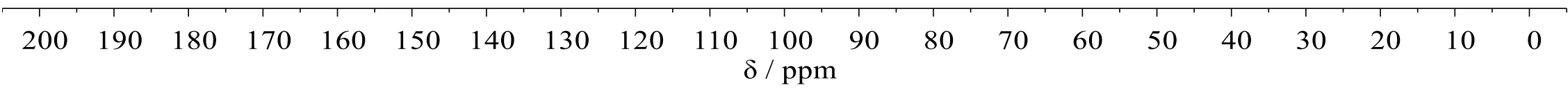

Figure S120. ${ }^{13} \mathrm{C}\left\{{ }^{1} \mathrm{H}\right\}$ NMR $(126 \mathrm{MHz}$, DMSO-d $)$ of compound $\mathbf{S 3 7}$. 


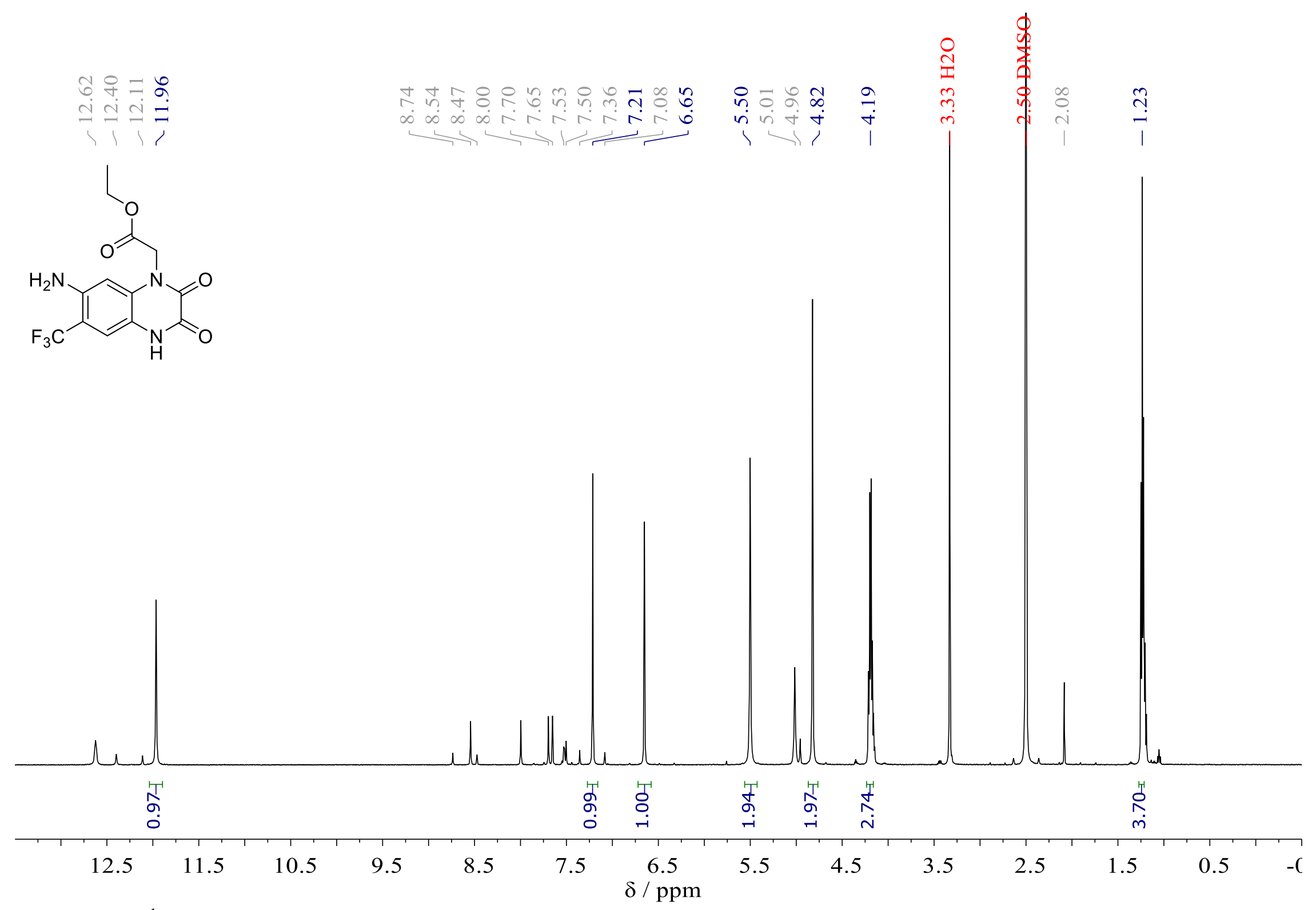

Figure S121. ${ }^{1} \mathrm{H}$ NMR $\left(500 \mathrm{MHz}, \mathrm{DMSO}-d_{6}\right)$ of compound S38. 


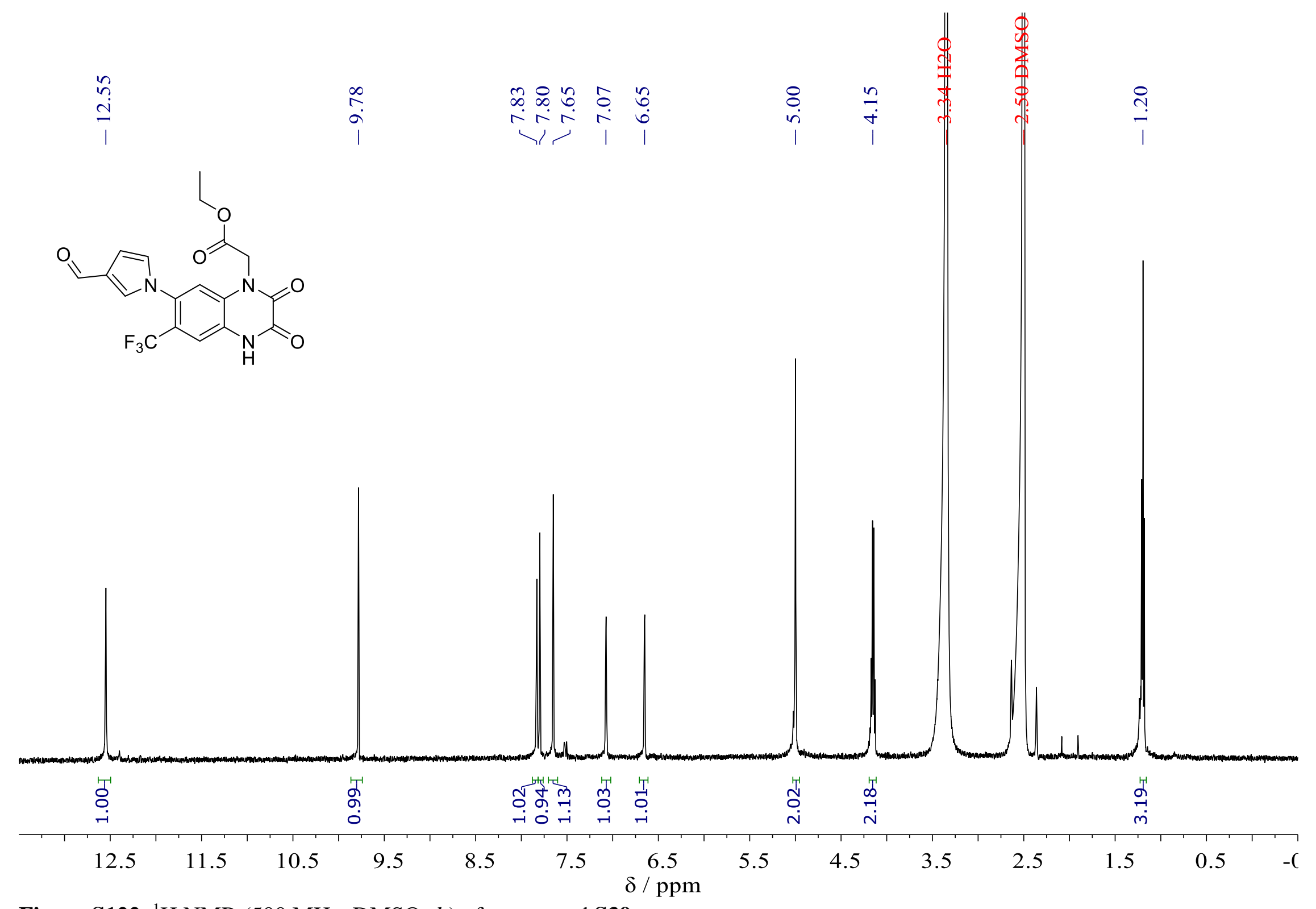

Figure S122. ${ }^{1} \mathrm{H}$ NMR $\left(500 \mathrm{MHz}, \mathrm{DMSO}-d_{6}\right)$ of compound S39. 


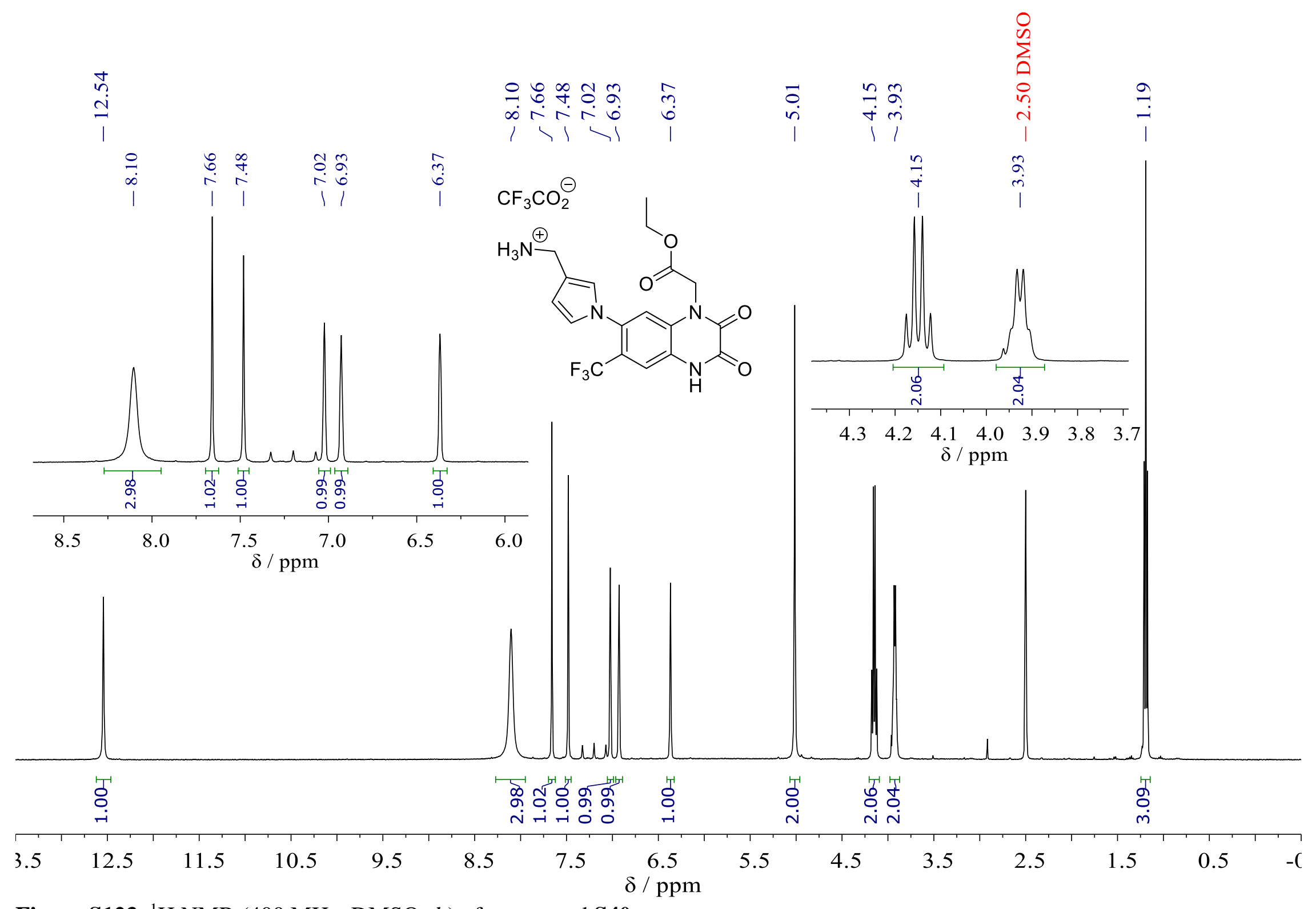

Figure S123. ${ }^{1} \mathrm{H}$ NMR (400 MHz, DMSO- $\left.d_{6}\right)$ of compound S40. 


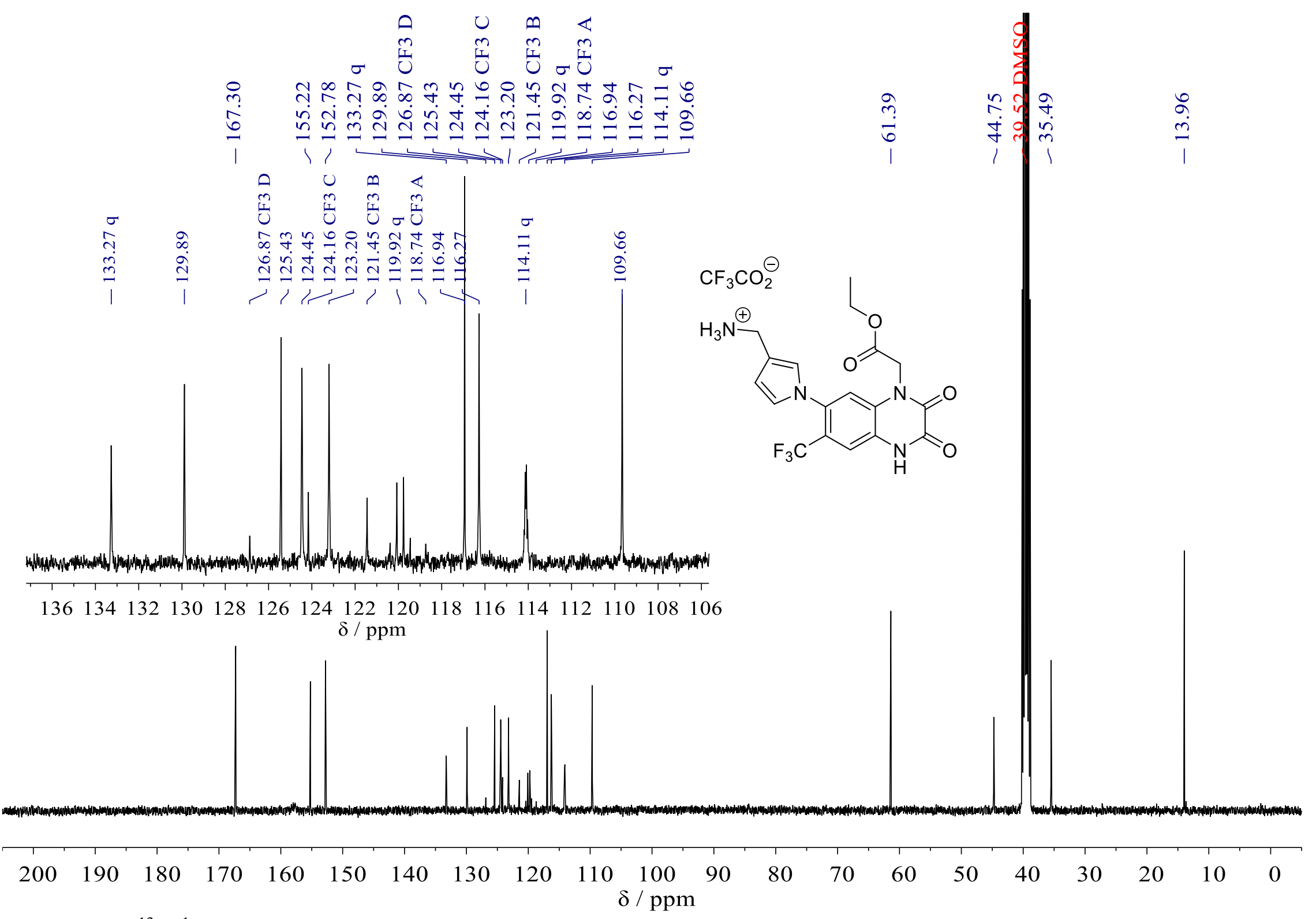

Figure S124. ${ }^{13} \mathrm{C}\left\{{ }^{1} \mathrm{H}\right\}$ NMR (101 MHz, DMSO- $\left.d_{6}\right)$ of compound $\mathbf{S 4 0}$. 


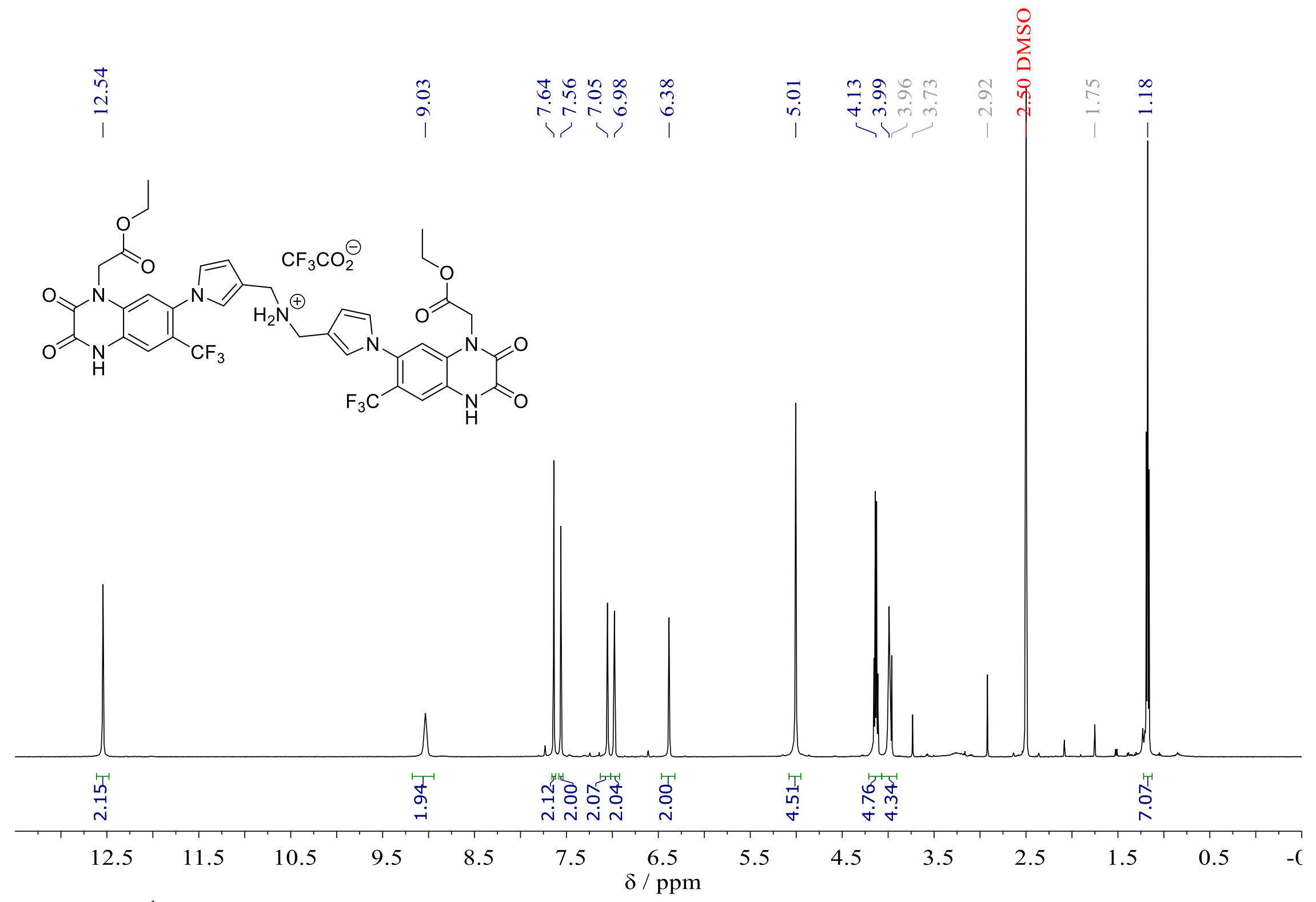

Figure S125. ${ }^{1} \mathrm{H}$ NMR $\left(500 \mathrm{MHz}, \mathrm{DMSO}-d_{6}\right)$ of compound $\mathbf{S 4 0 b}$. 


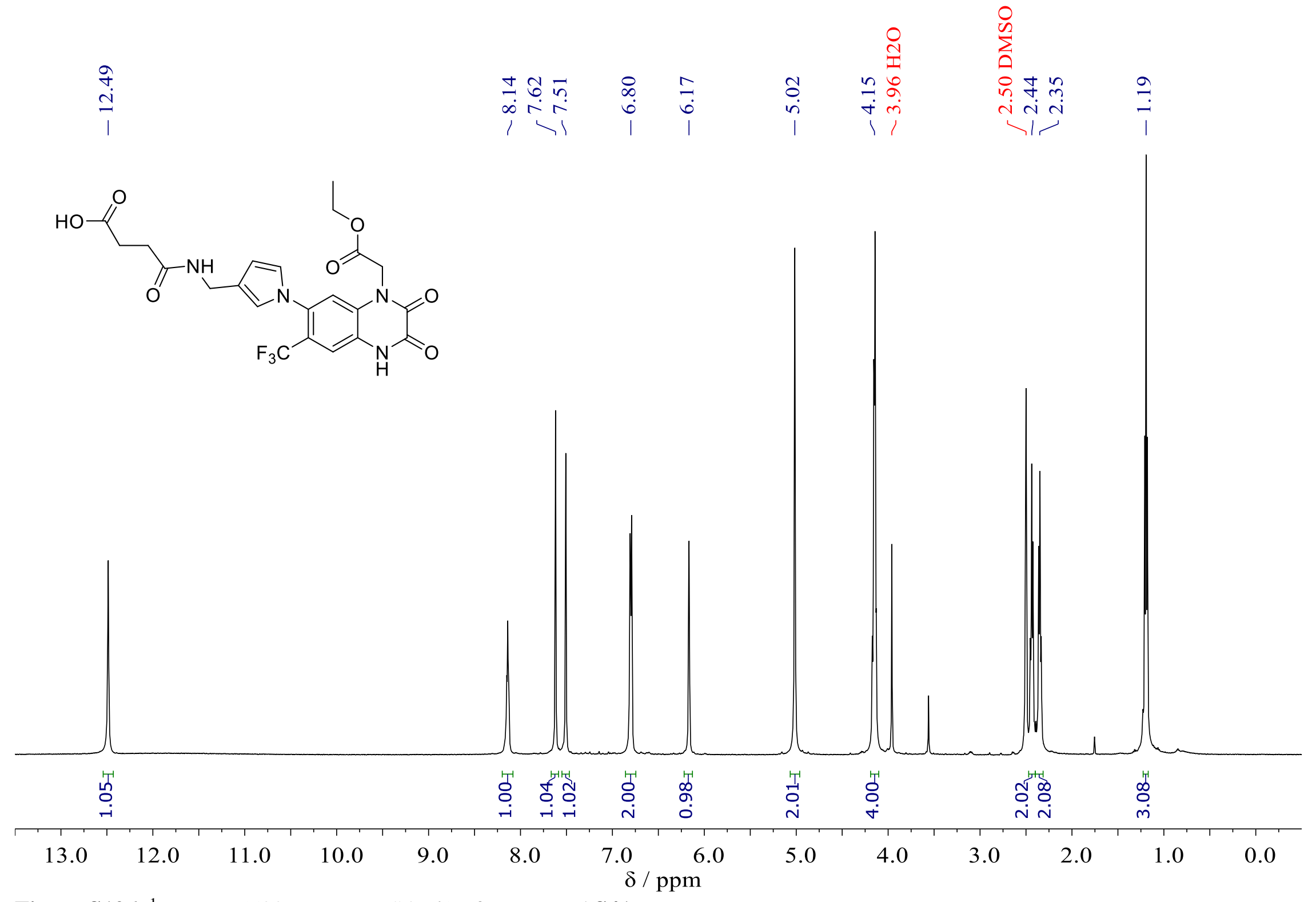

Figure S126. ${ }^{1} \mathrm{H}$ NMR $\left(500 \mathrm{MHz}, \mathrm{DMSO}-d_{6}\right)$ of compound $\mathbf{S 4 1}$. 

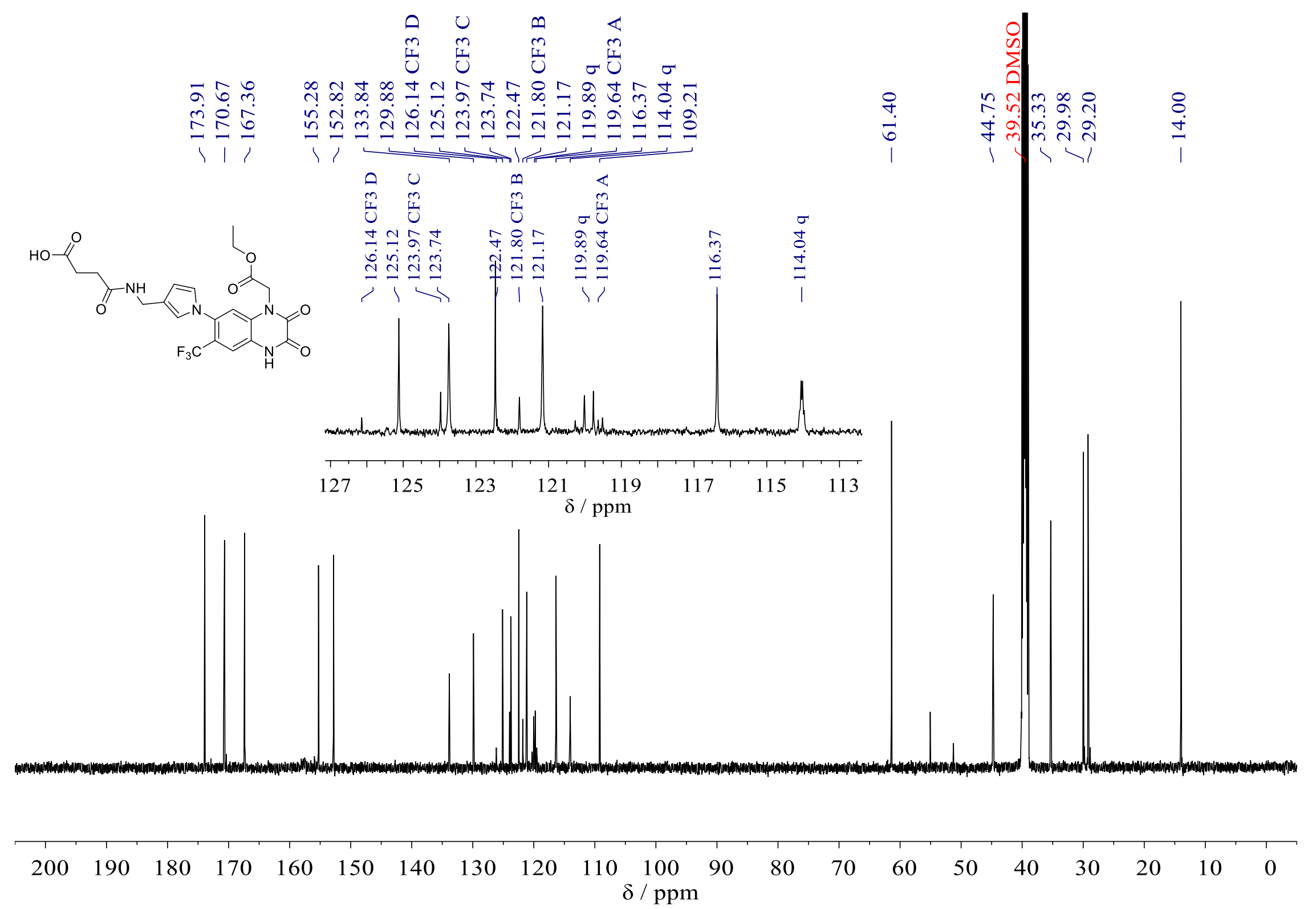

Figure S127. ${ }^{13} \mathrm{C}\left\{{ }^{1} \mathrm{H}\right\}$ NMR $\left(126 \mathrm{MHz}, \mathrm{DMSO}-d_{6}\right)$ of compound $\mathbf{S 4 1}$. 


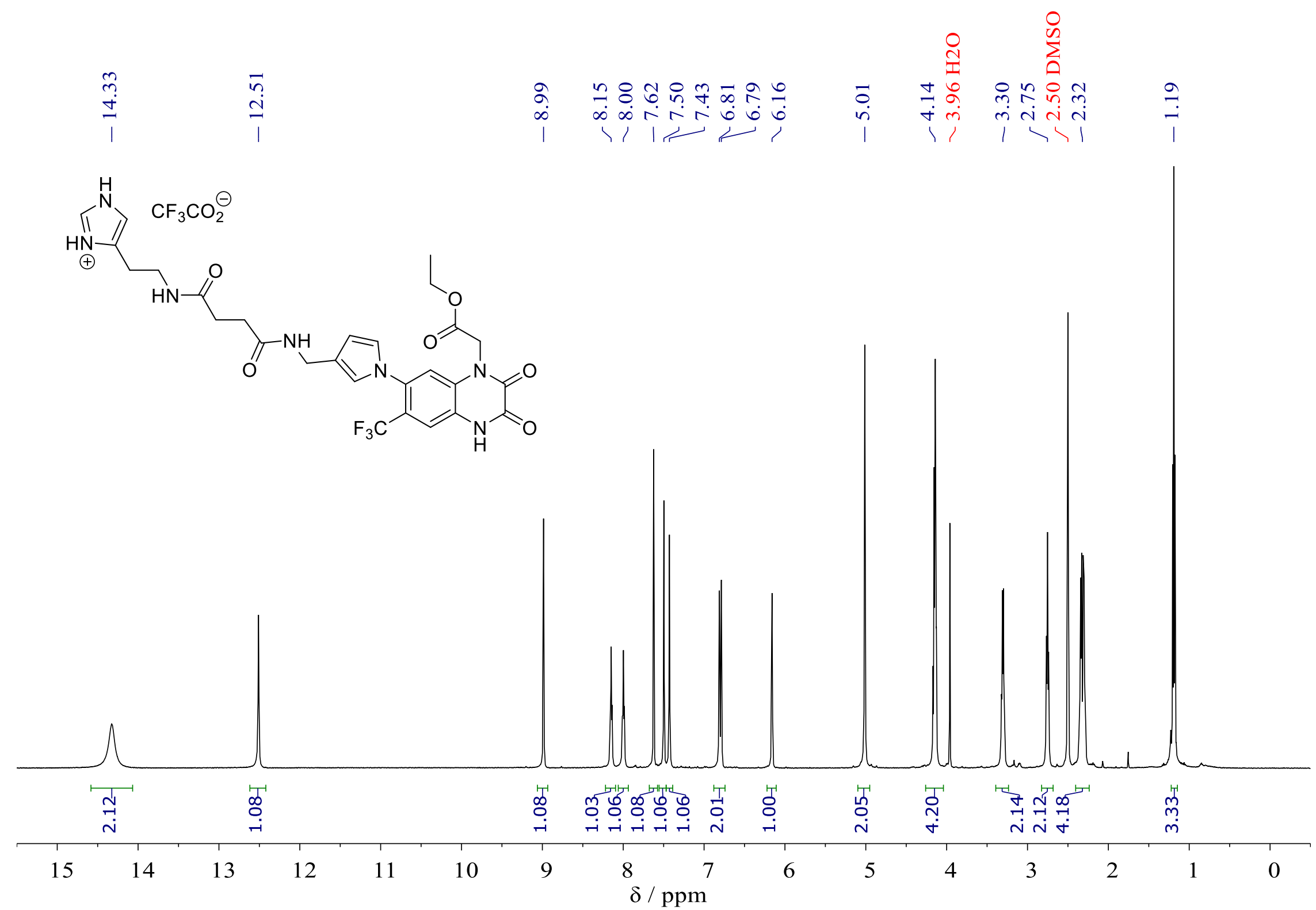

Figure S128. ${ }^{1} \mathrm{H}$ NMR (500 MHz, DMSO- $\left.d_{6}\right)$ of compound $\mathbf{S 4 2}$. 

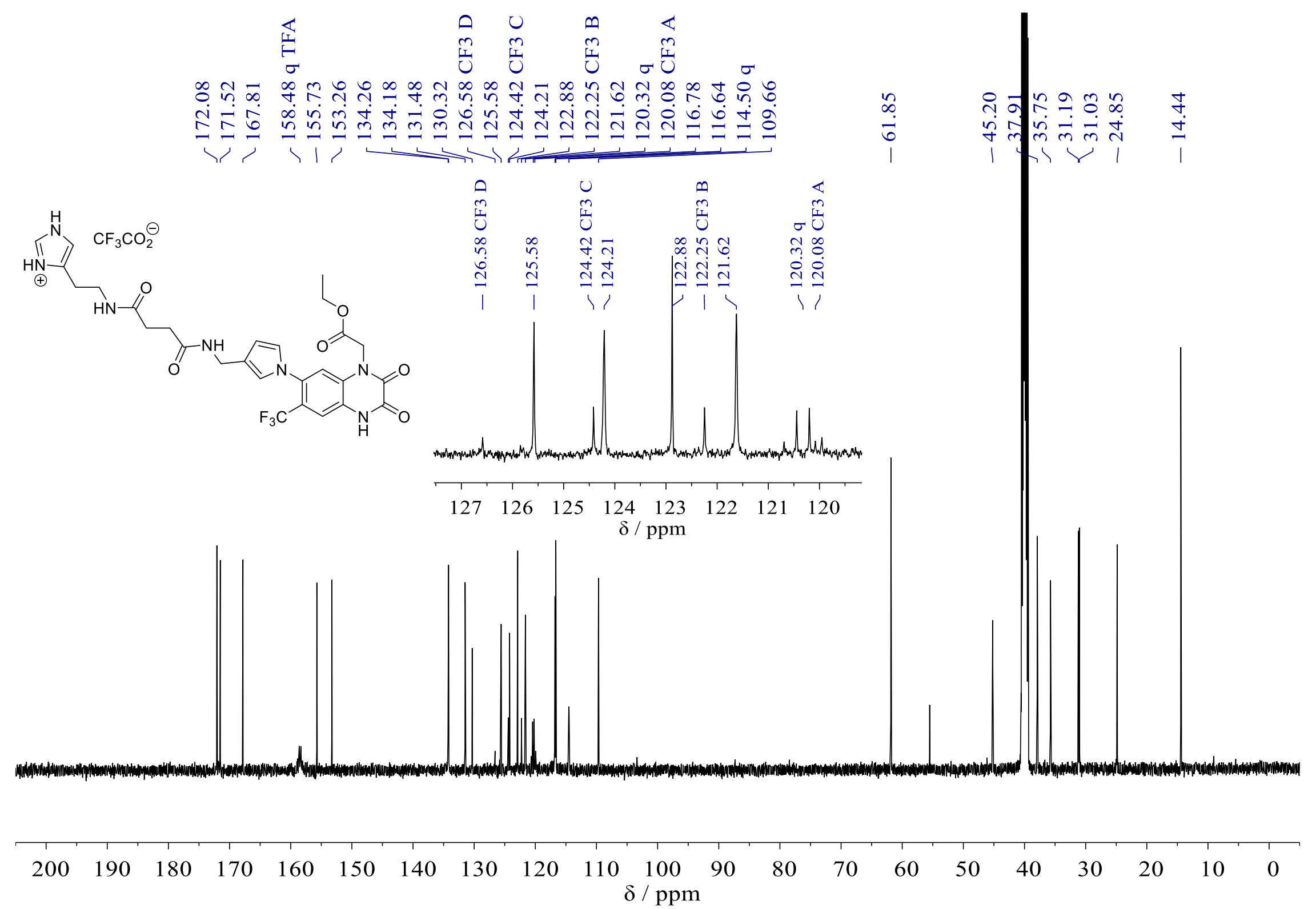

Figure S129. ${ }^{13} \mathrm{C}\left\{{ }^{1} \mathrm{H}\right\}$ NMR $\left(126 \mathrm{MHz}, \mathrm{DMSO}-d_{6}\right)$ of compound $\mathbf{S 4 2}$. 


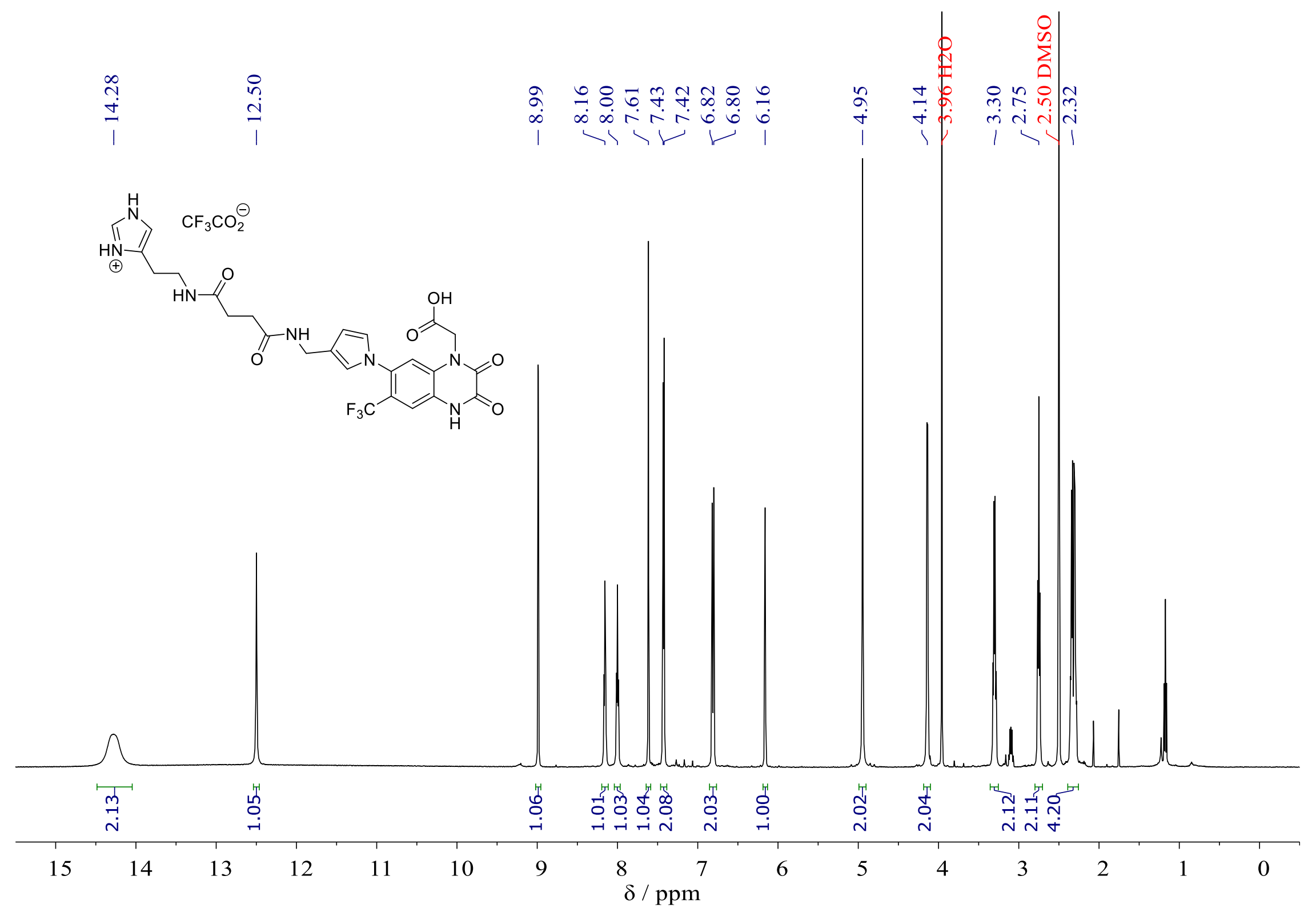

Figure S130. ${ }^{1} \mathrm{H}$ NMR (500 MHz, DMSO- $\left.d_{6}\right)$ of compound $\mathbf{S 2}\left(\mathrm{CF}_{3} \mathrm{CO}_{2}^{-}\right.$salt). 


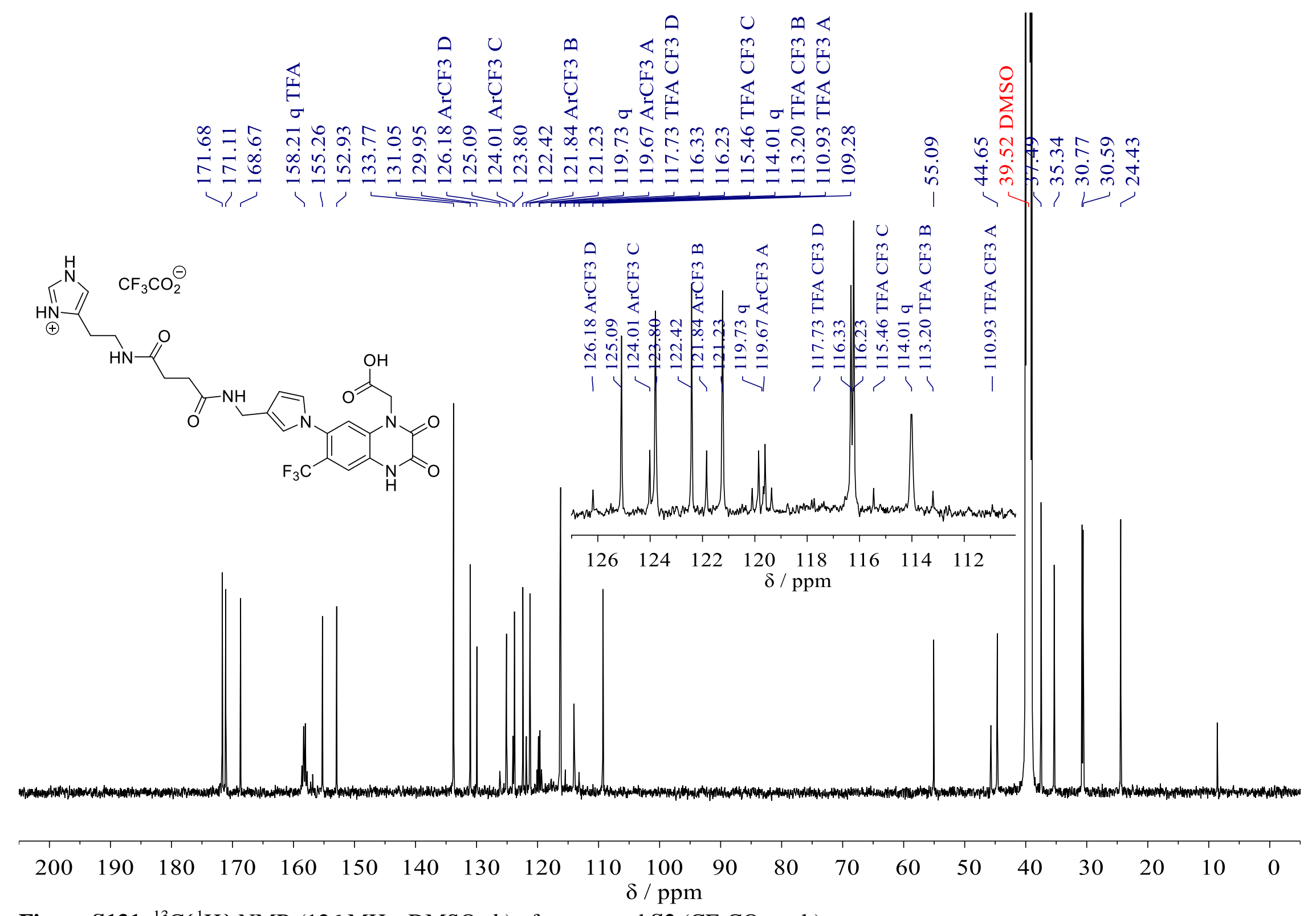

Figure S131. ${ }^{13} \mathrm{C}\left\{{ }^{1} \mathrm{H}\right\}$ NMR (126 MHz, DMSO- $\left.d_{6}\right)$ of compound $\mathbf{S 2}\left(\mathrm{CF}_{3} \mathrm{CO}_{2}^{-}\right.$salt). 


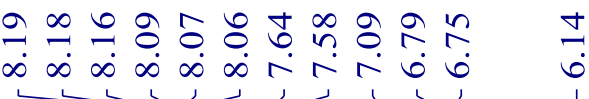

ก 쿼ำ ำ

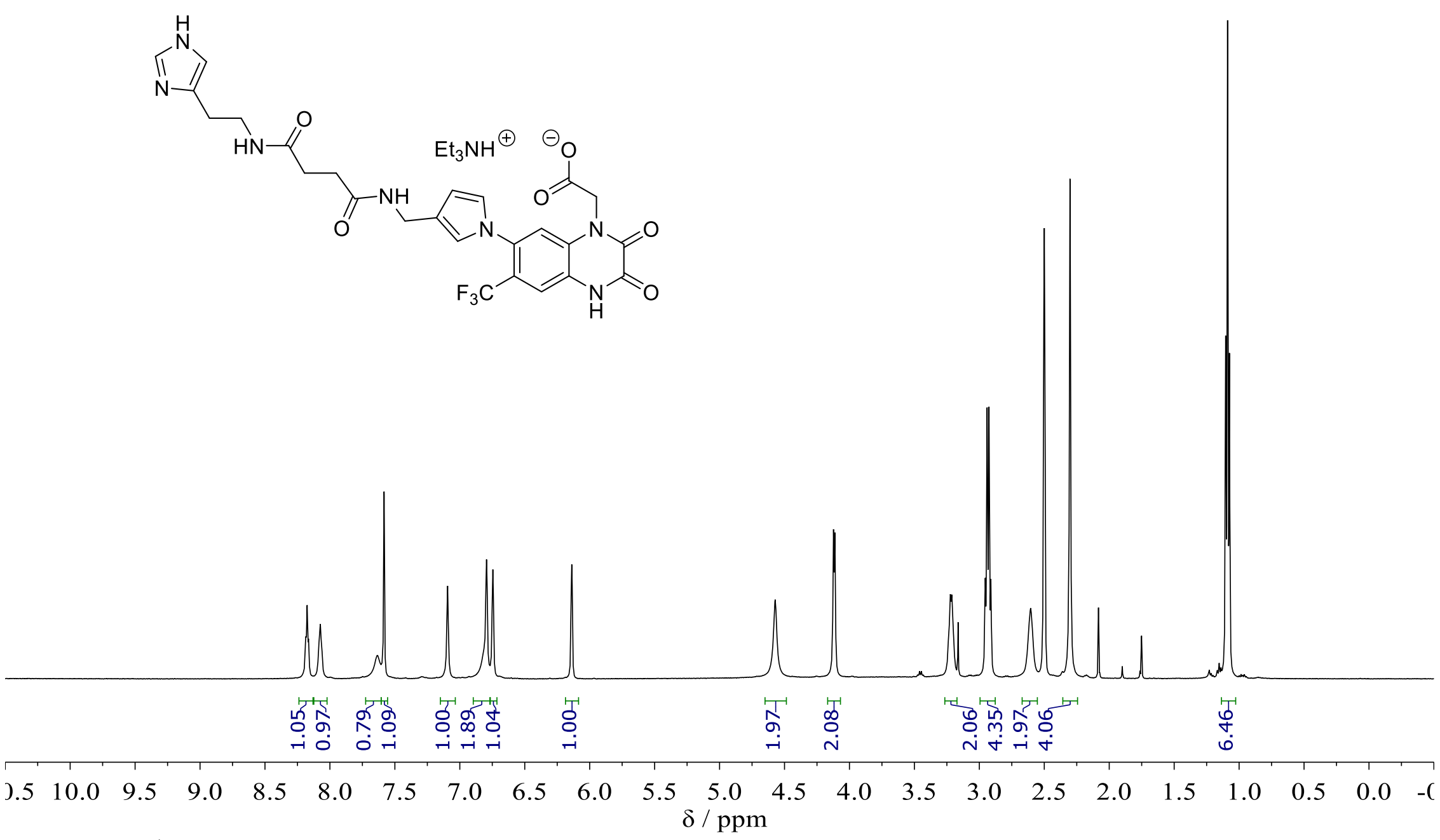

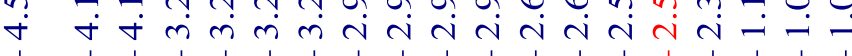

Figure S132. ${ }^{1} \mathrm{H}$ NMR $\left(500 \mathrm{MHz}, \mathrm{DMSO}-d_{6}\right)$ of compound $\mathbf{S 2}\left(\mathrm{Et}_{3} \mathrm{NH}^{+}\right.$salt $)$. 


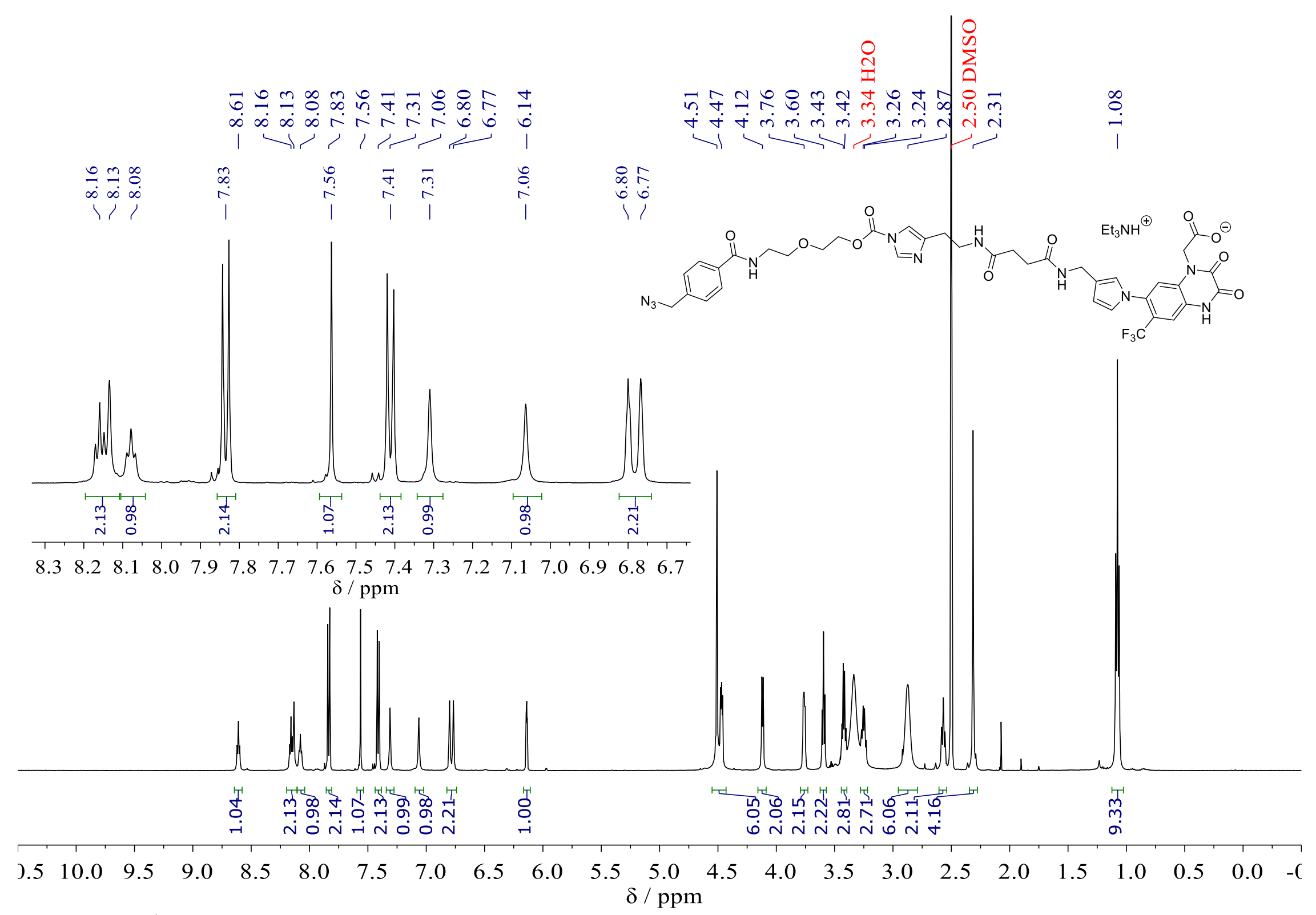

Figure S133. ${ }^{1} \mathrm{H}$ NMR $\left(500 \mathrm{MHz}, \mathrm{DMSO}-d_{6}\right)$ of compound 19. 


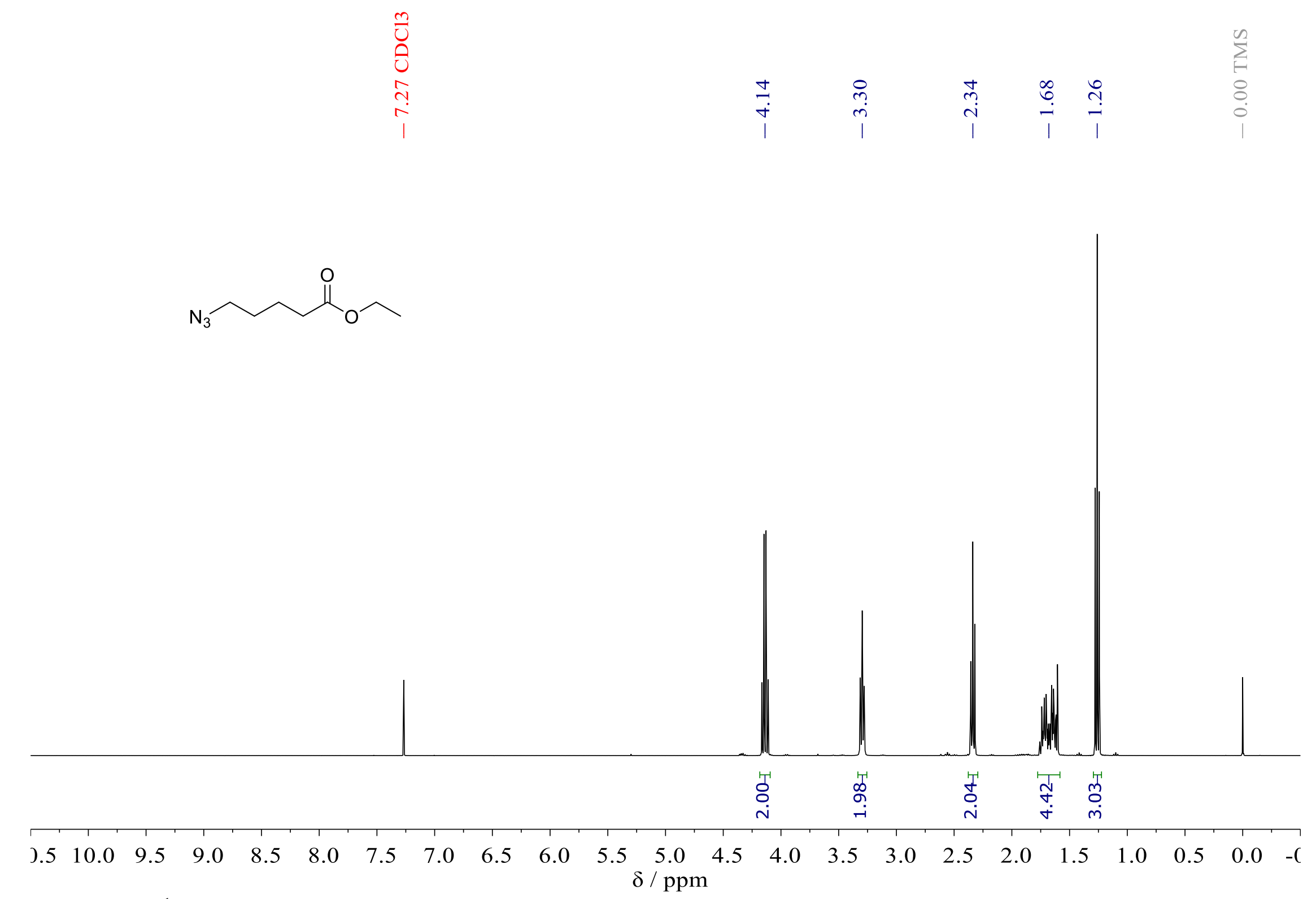

Figure S134. ${ }^{1} \mathrm{H}$ NMR $\left(400 \mathrm{MHz}, \mathrm{CDCl}_{3}\right)$ of ethyl 5-azidovalerate $(\mathbf{S 4 3})$. 

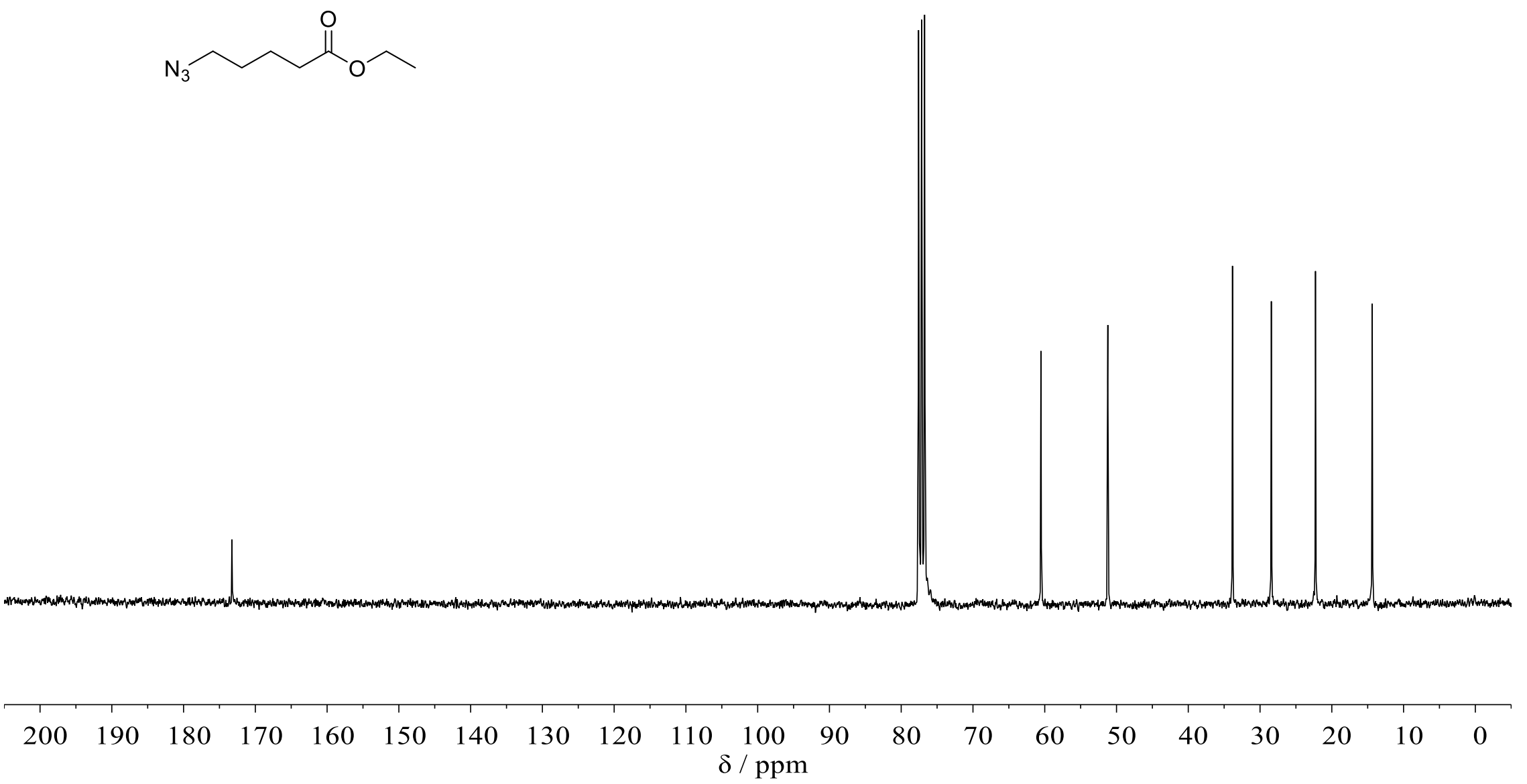

Figure S135. ${ }^{13} \mathrm{C}\left\{{ }^{1} \mathrm{H}\right\} \mathrm{NMR}\left(75 \mathrm{MHz}, \mathrm{CDCl}_{3}\right)$ of ethyl 5-azidovalerate (S43). 

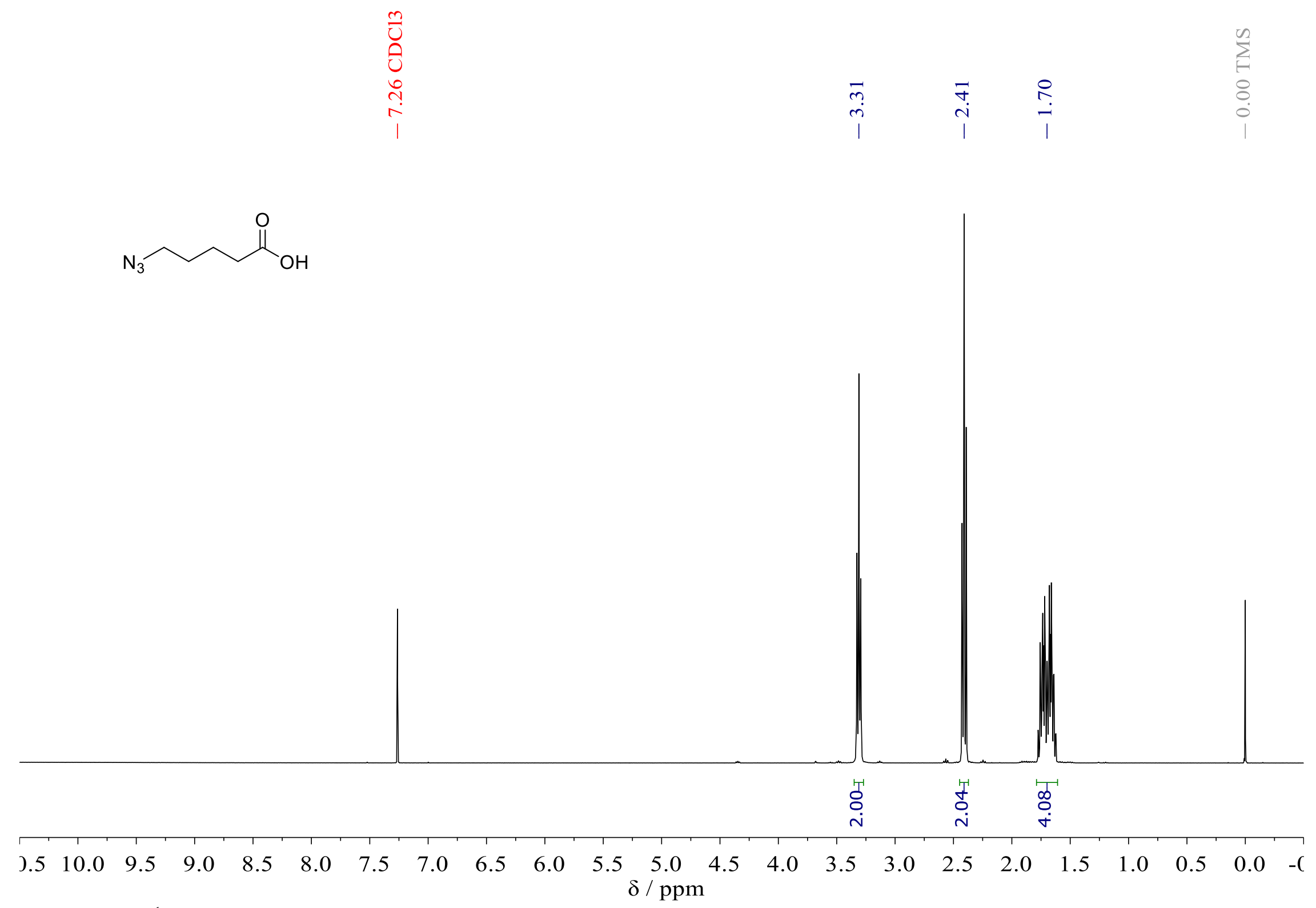

Figure S136. ${ }^{1} \mathrm{H}$ NMR (400 MHz, $\left.\mathrm{CDCl}_{3}\right)$ of 5-azidovaleric acid (S44). 

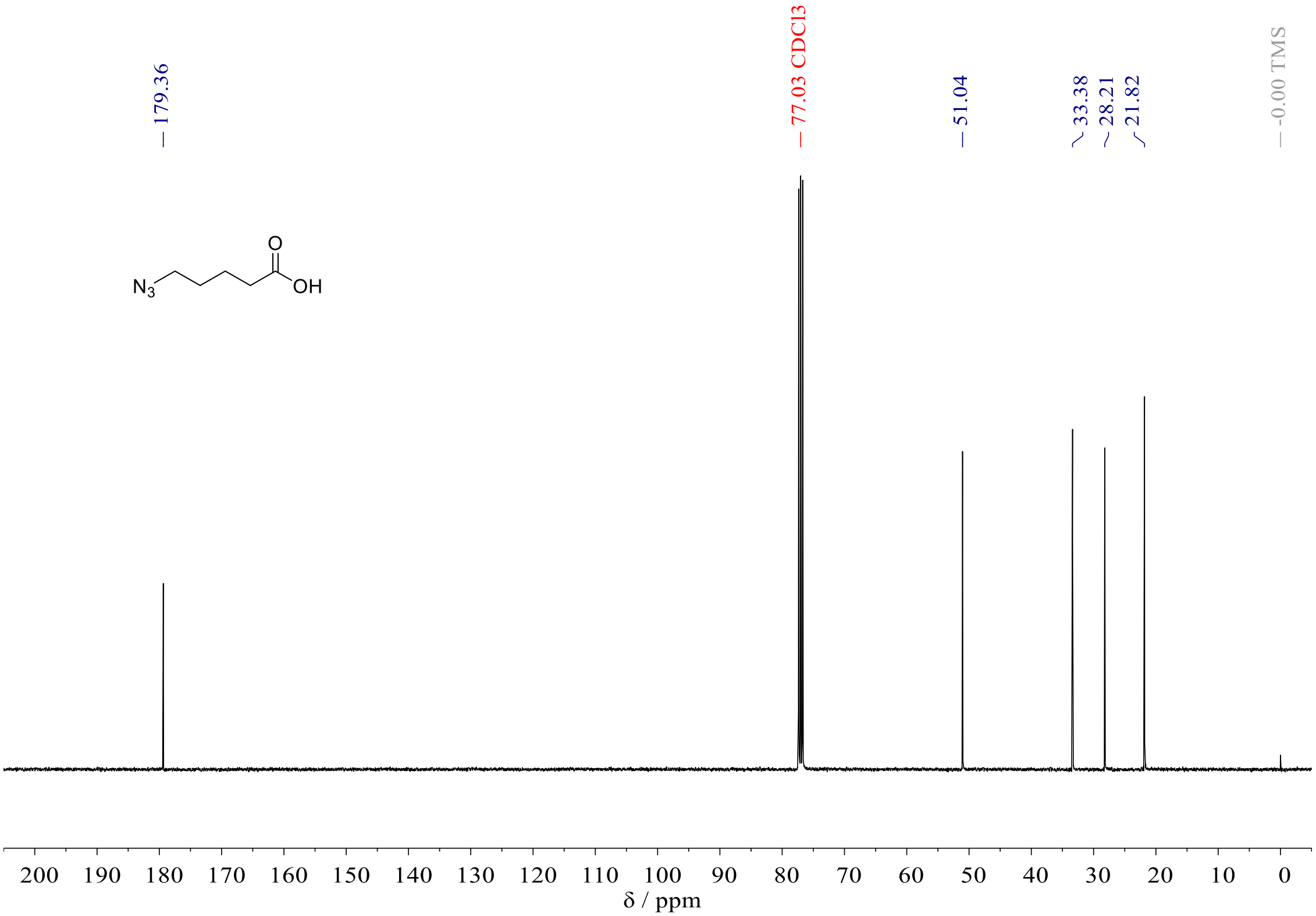

Figure S137. ${ }^{13} \mathrm{C}\left\{{ }^{1} \mathrm{H}\right\}$ NMR $\left(101 \mathrm{MHz}, \mathrm{CDCl}_{3}\right)$ of 5-azidovaleric acid (S44). 


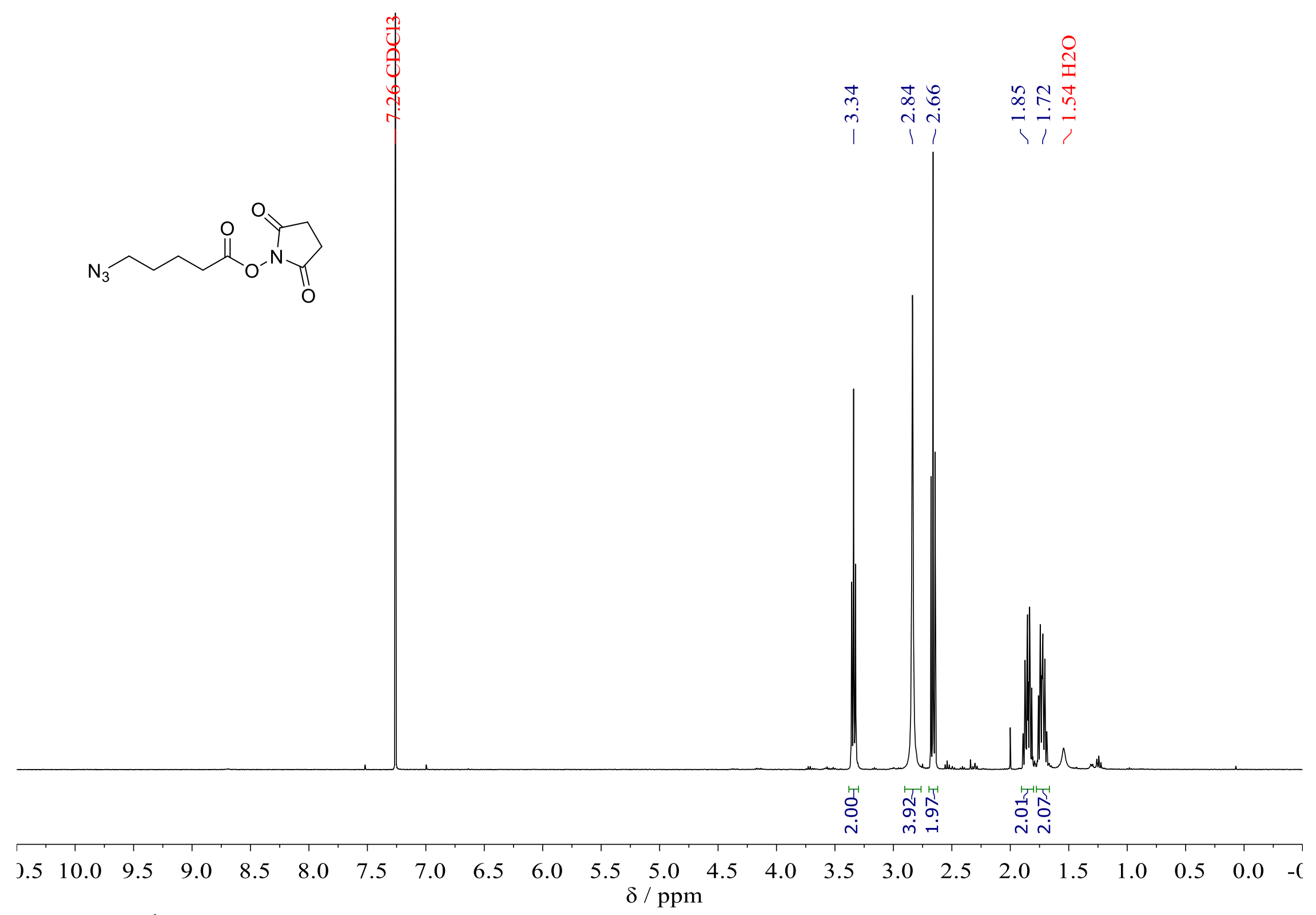

Figure S138. ${ }^{1} \mathrm{H}$ NMR (400 MHz, $\left.\mathrm{CDCl}_{3}\right)$ of 5-azidovaleric acid succinimidyl ester (S45). 


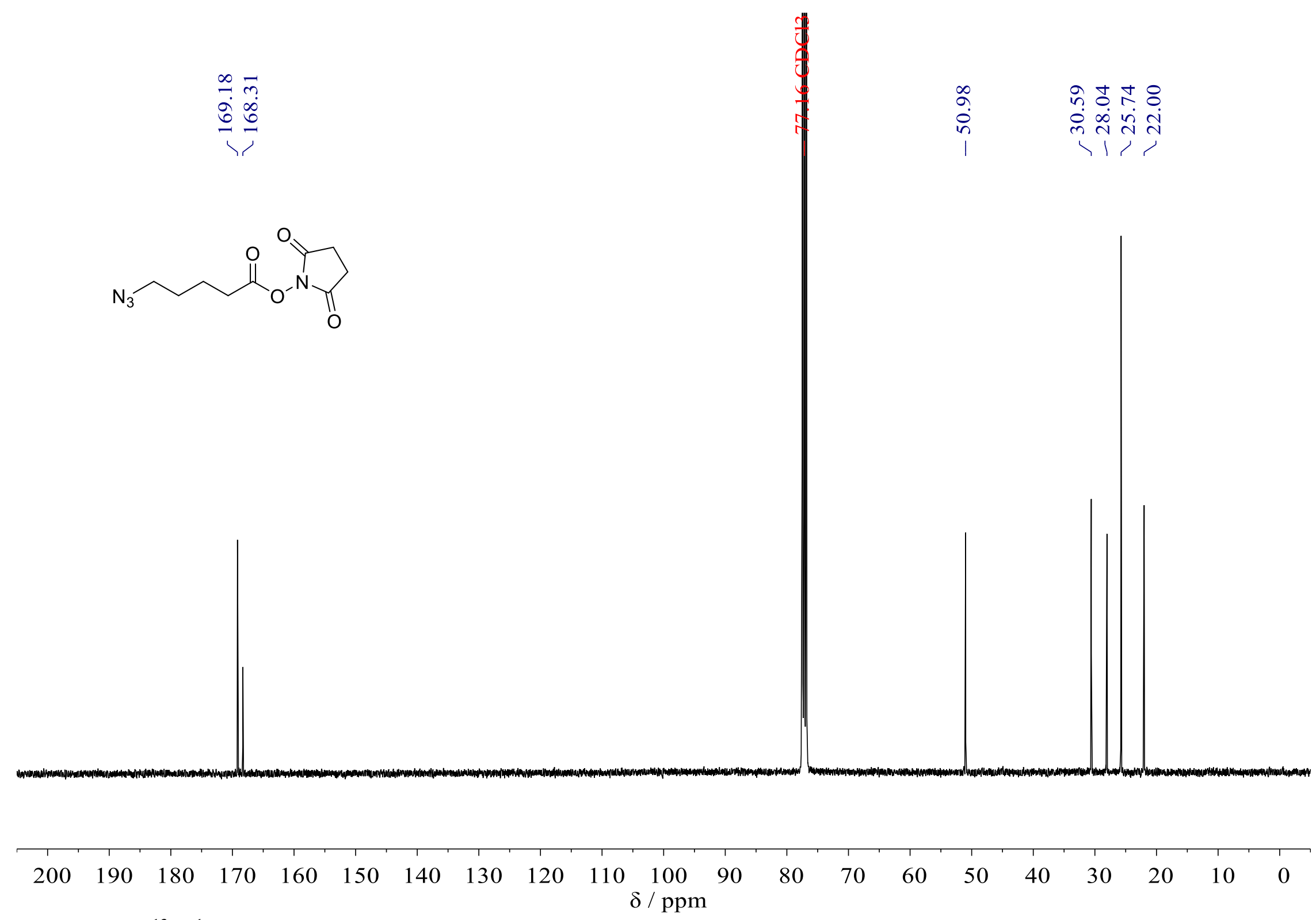

Figure S139. ${ }^{13} \mathrm{C}\left\{{ }^{1} \mathrm{H}\right\} \mathrm{NMR}\left(101 \mathrm{MHz}, \mathrm{CDCl}_{3}\right)$ of 5-azidovaleric acid succinimidyl ester (S45). 


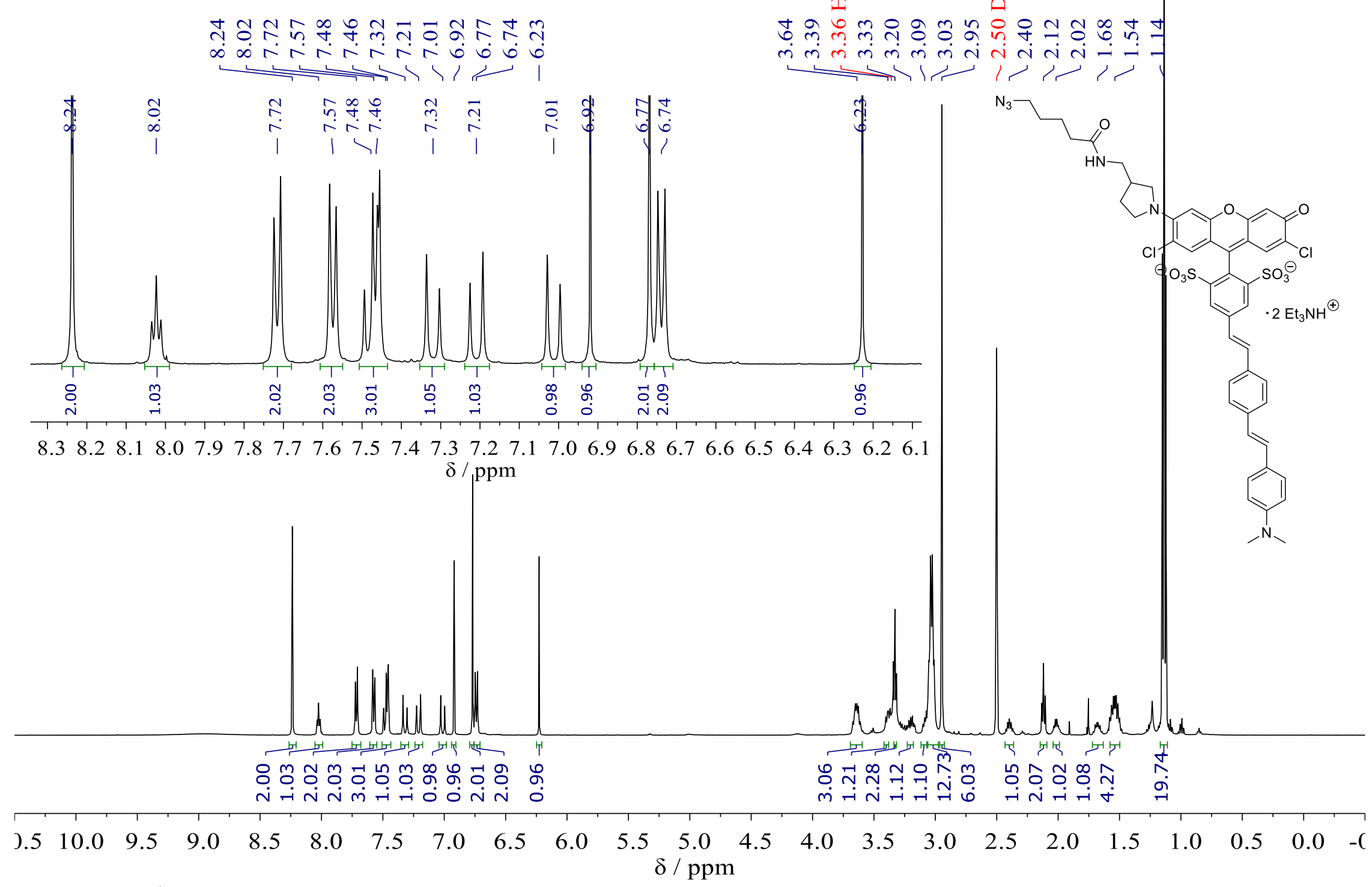

Figure S140. ${ }^{1} \mathrm{H}$ NMR $\left(500 \mathrm{MHz}, \mathrm{DMSO}-d_{6}\right)$ of dsRVF5-N ${ }_{3}(\mathbf{1 8})$. 

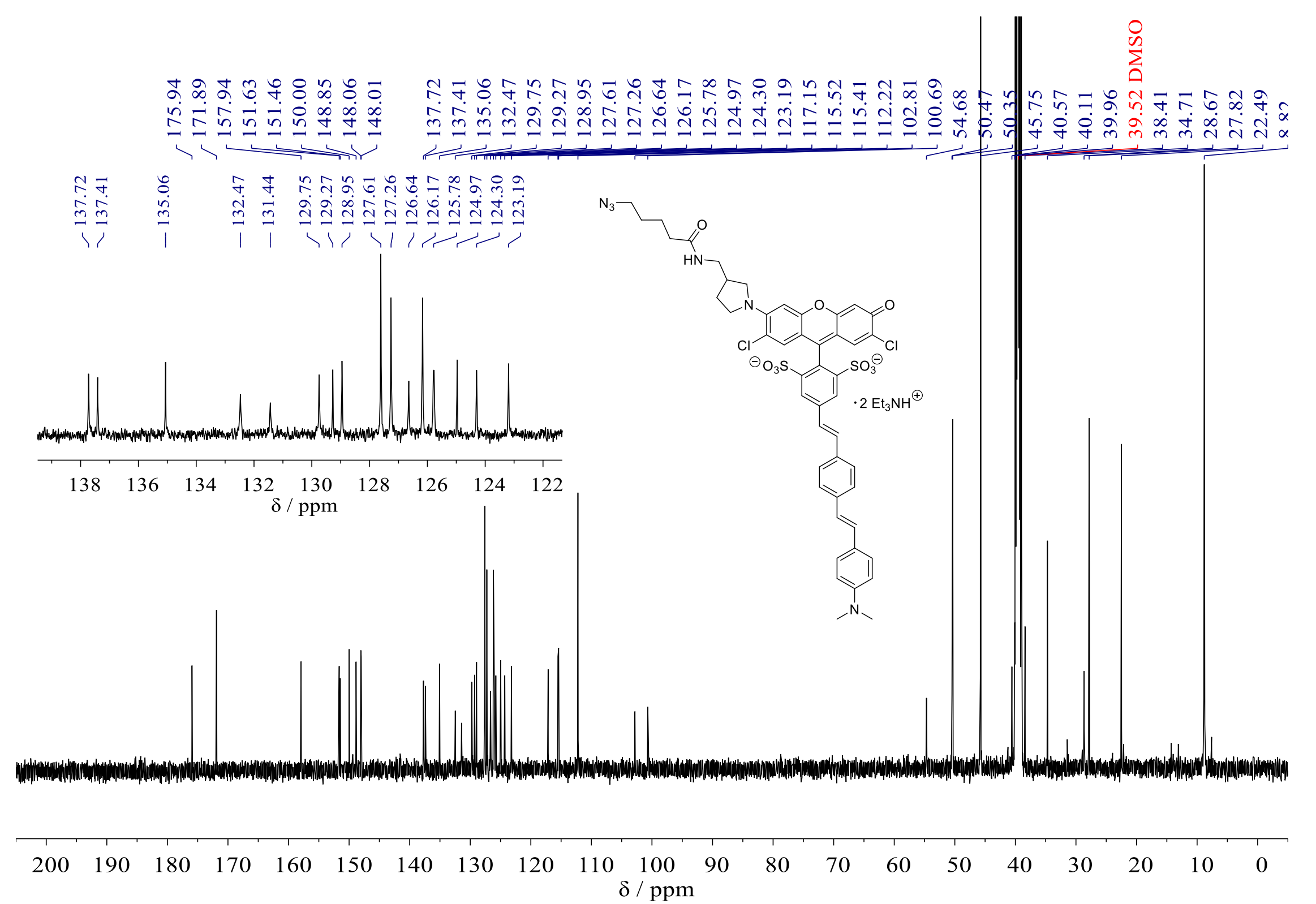

Figure S141. ${ }^{13} \mathrm{C}\left\{{ }^{1} \mathrm{H}\right\}$ NMR (126 MHz, DMSO- $\left.d_{6}\right)$ of dsRVF5-N ${ }_{3}(\mathbf{1 8})$. 


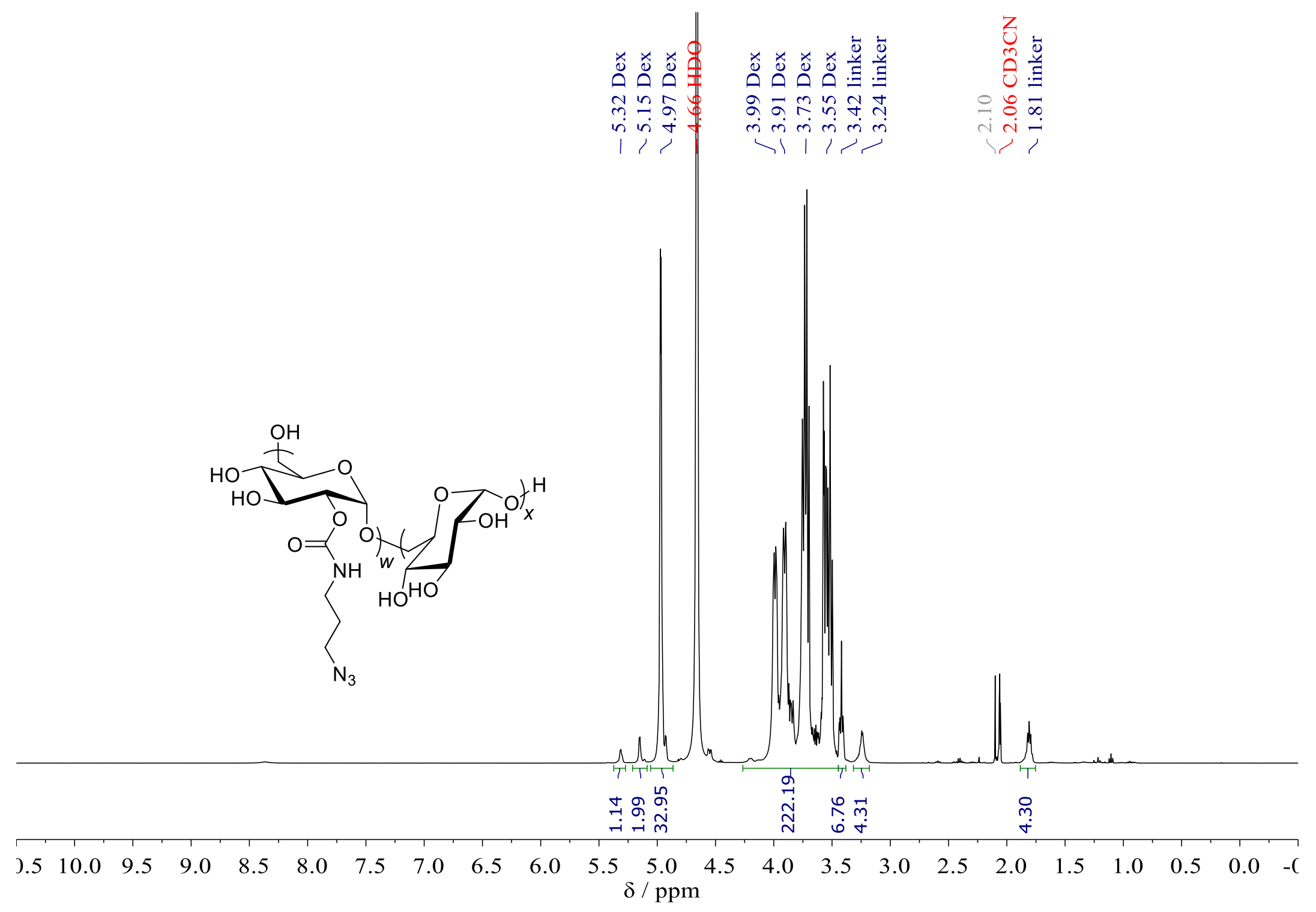

Figure S142. ${ }^{1} \mathrm{H}$ NMR $\left(500 \mathrm{MHz} ; \mathrm{D}_{2} \mathrm{O} / \mathrm{CD}_{3} \mathrm{CN} 5: 1, v / v\right)$ of azidopropyl-carbamate dextran(6000) (8-2.15). 


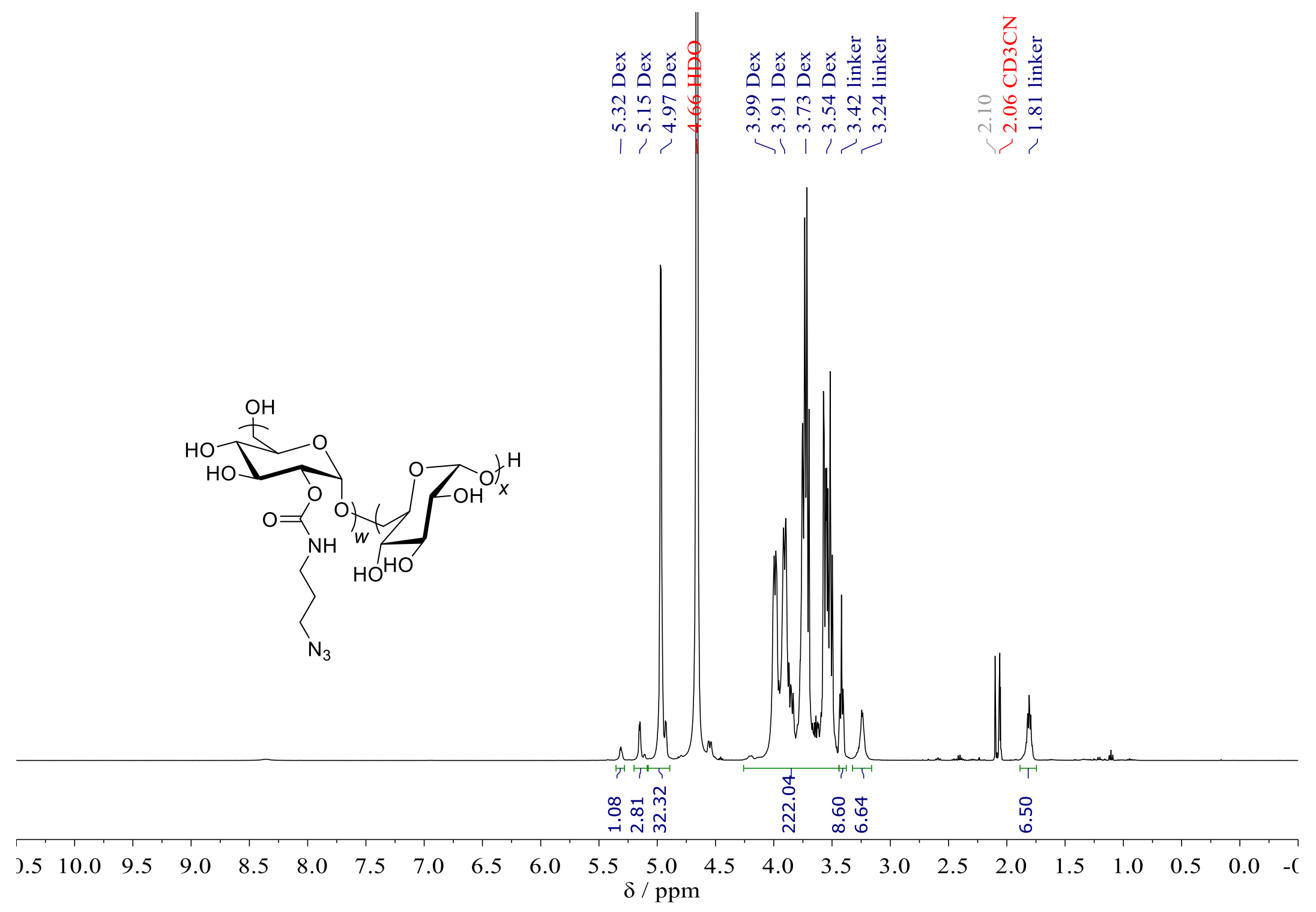

Figure S143. ${ }^{1} \mathrm{H}$ NMR (500 MHz; $\mathrm{D}_{2} \mathrm{O} / \mathrm{CD}_{3} \mathrm{CN}$ 5:1, v/v) of azidopropyl-carbamate dextran(6000) (8-3.25). 


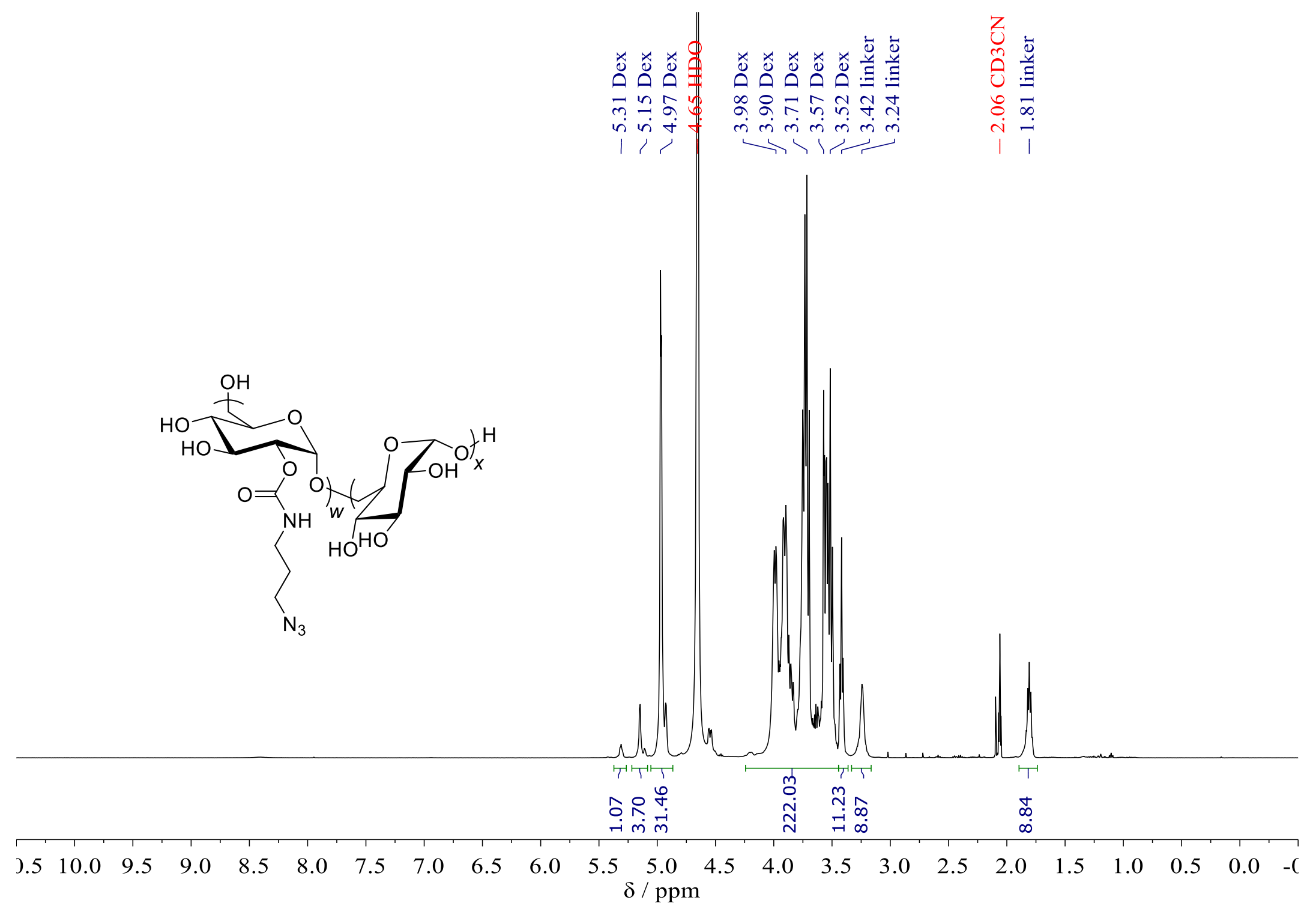

Figure S144. ${ }^{1} \mathrm{H}$ NMR $\left(500 \mathrm{MHz} ; \mathrm{D}_{2} \mathrm{O} / \mathrm{CD}_{3} \mathrm{CN} 5: 1, v / v\right)$ of azidopropyl-carbamate dextran(6000) (8-4.42). 


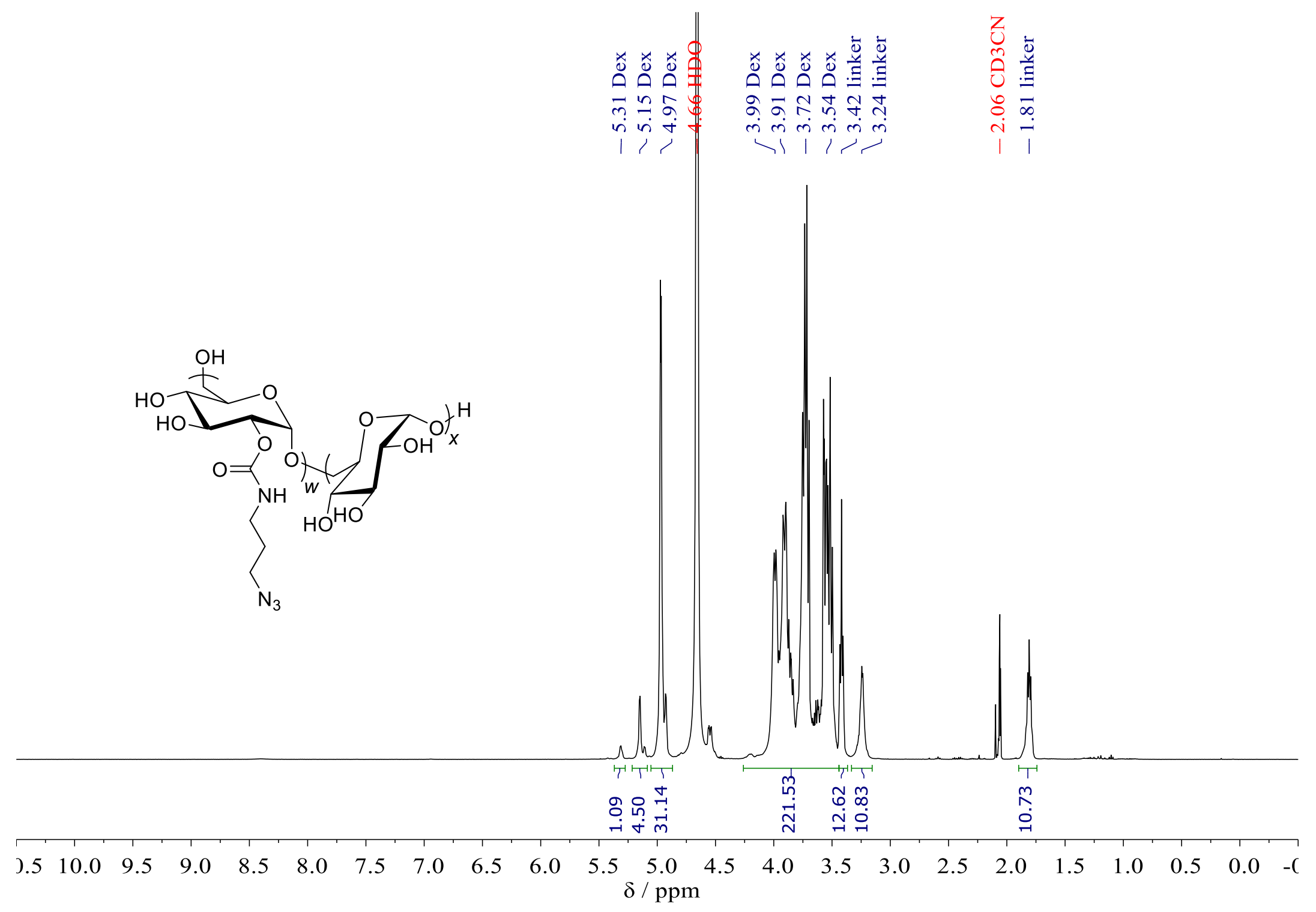

Figure S145. ${ }^{1} \mathrm{H}$ NMR $\left(500 \mathrm{MHz} ; \mathrm{D}_{2} \mathrm{O} / \mathrm{CD}_{3} \mathrm{CN} 5: 1, v / v\right)$ of azidopropyl-carbamate dextran(6000) (8-5.37). 


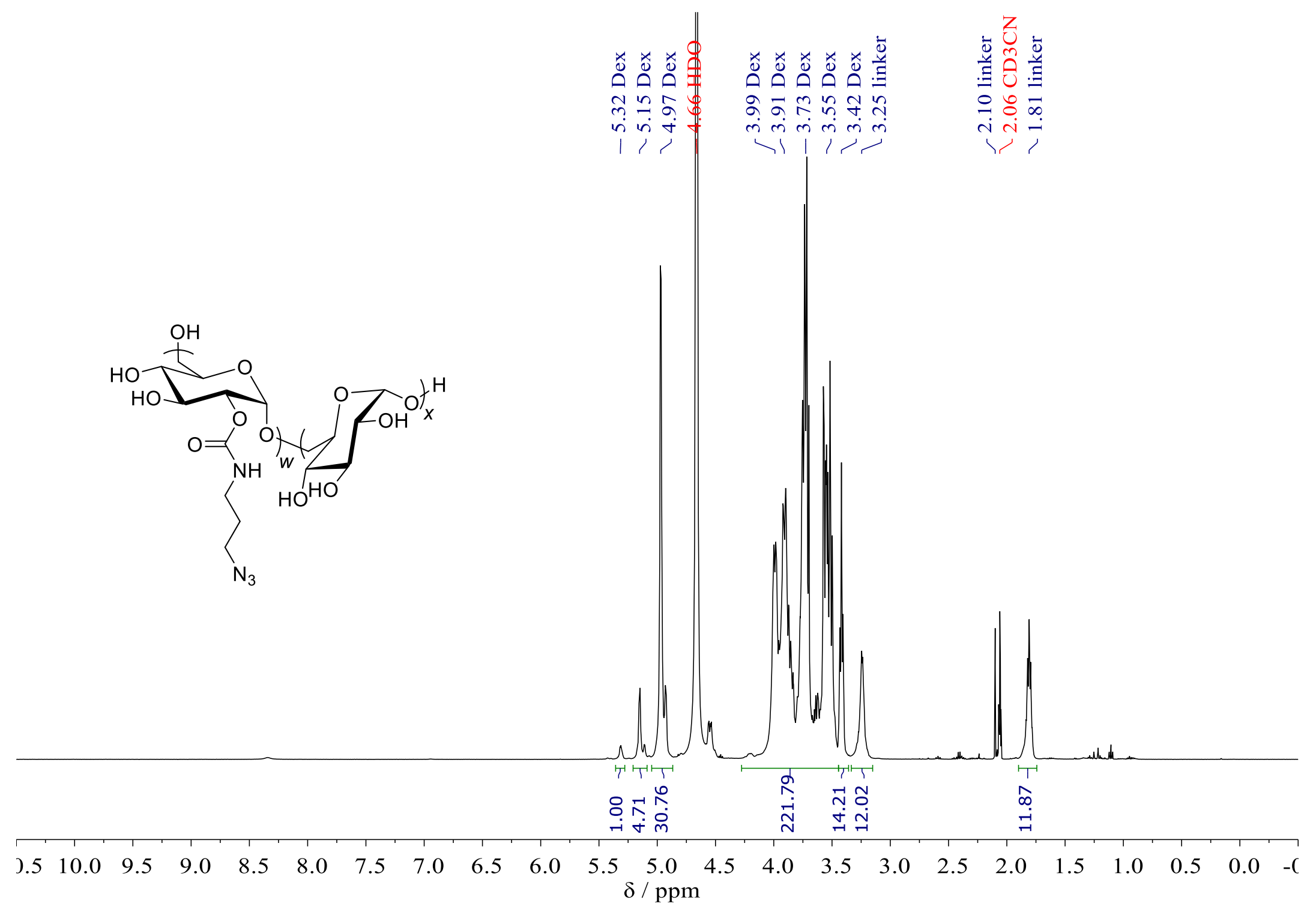

Figure S146. ${ }^{1} \mathrm{H}$ NMR $\left(500 \mathrm{MHz} ; \mathrm{D}_{2} \mathrm{O} / \mathrm{CD}_{3} \mathrm{CN}\right.$ 5:1, v/v) of azidopropyl-carbamate dextran(6000) (8-5.94). 


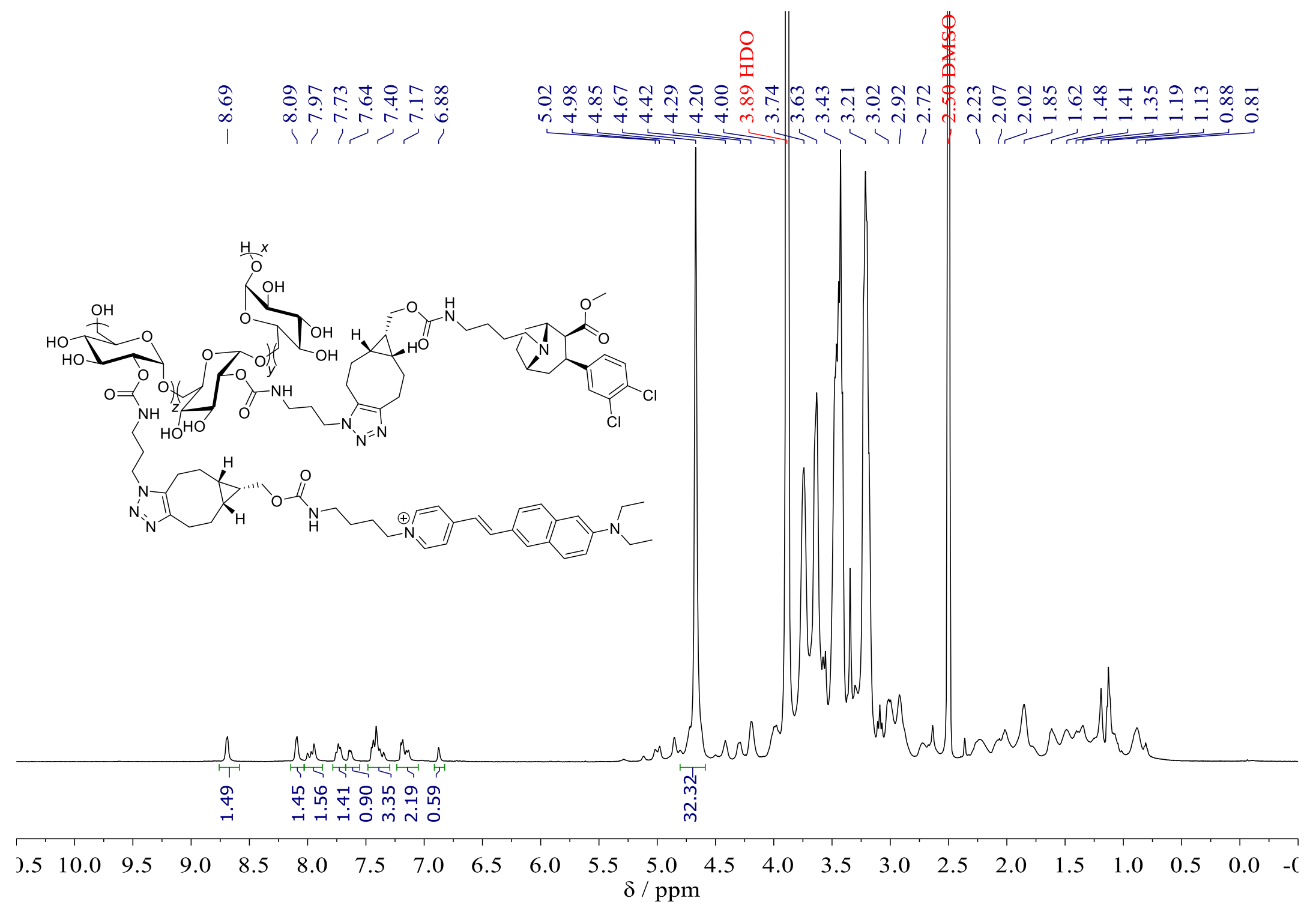

Figure S147. ${ }^{1} \mathrm{H}$ NMR (500 MHz; DMSO- $d_{6} / \mathrm{D}_{2} \mathrm{O}$ 5:1, v/v) of di-2-ANEP-VoLDeMo (12). 


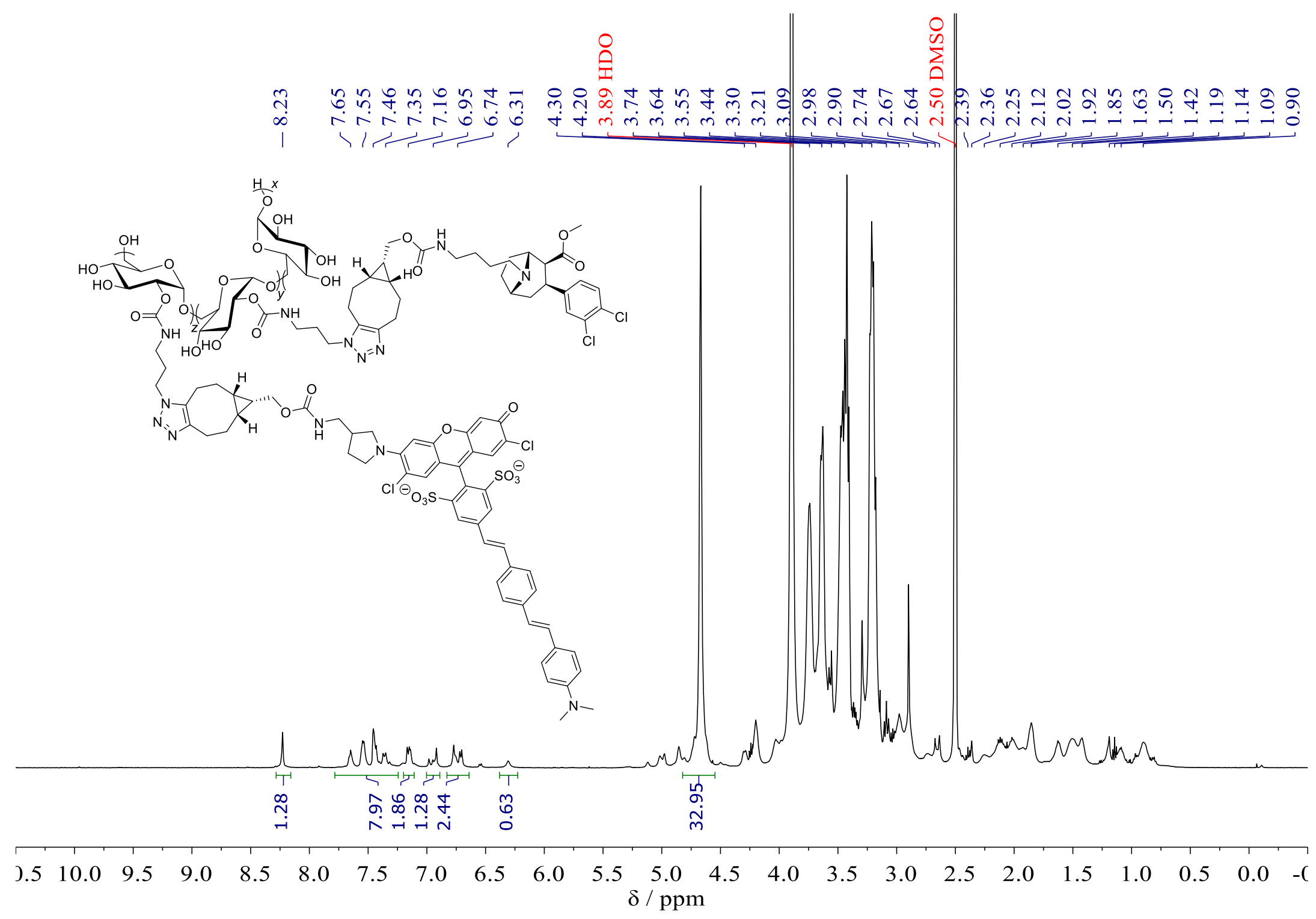

Figure S148. ${ }^{1} \mathrm{H}$ NMR (500 MHz; DMSO- $d_{6} / \mathrm{D}_{2} \mathrm{O}$ 5:1, v/v) of dsRVF5-VoLDeMo (13), sample 1. 


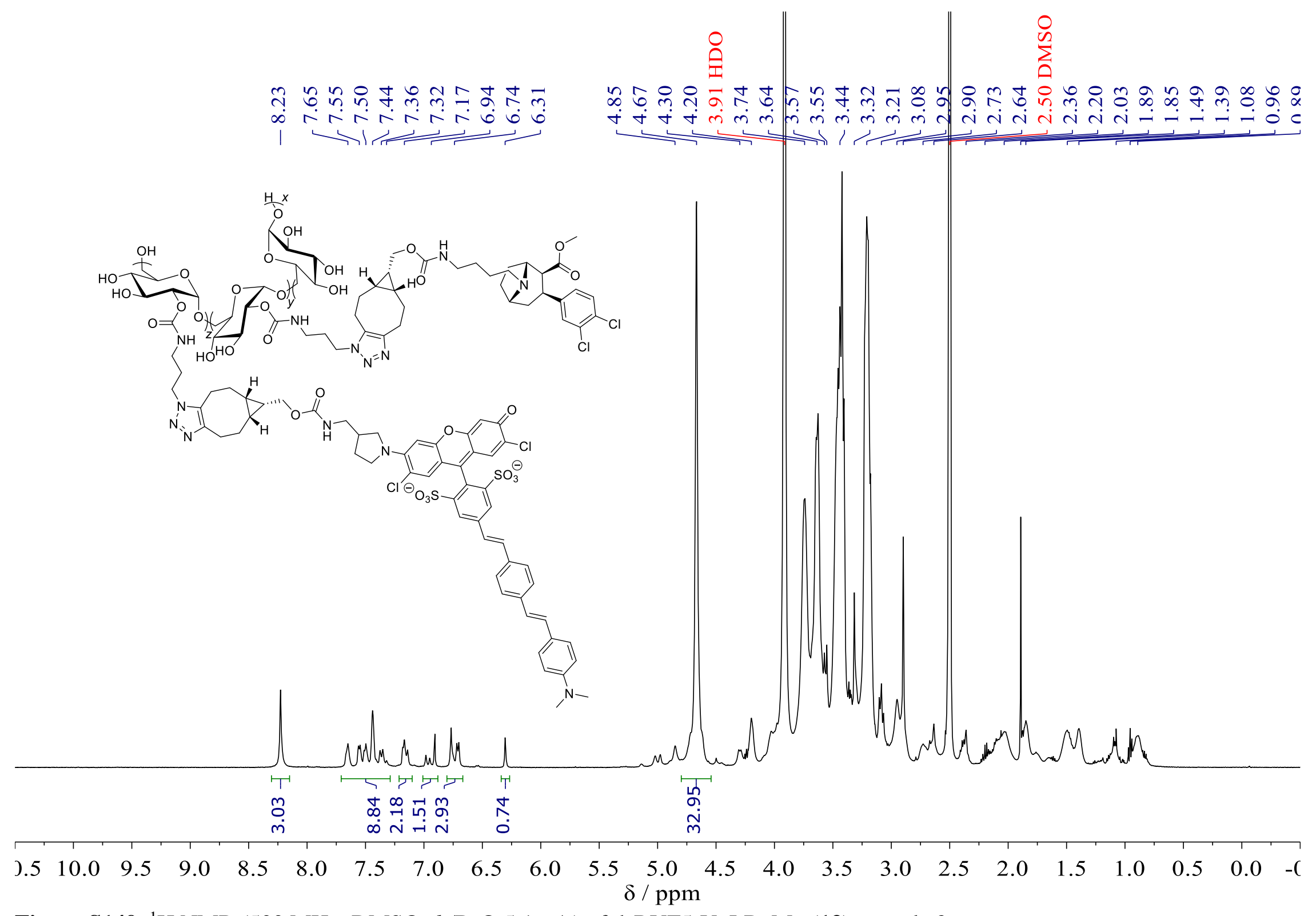

Figure S149. ${ }^{1} \mathrm{H}$ NMR (500 MHz; DMSO- $\left.d_{6} / \mathrm{D}_{2} \mathrm{O} 5: 1, v / v\right)$ of dsRVF5-VoLDeMo (13), sample 2. 


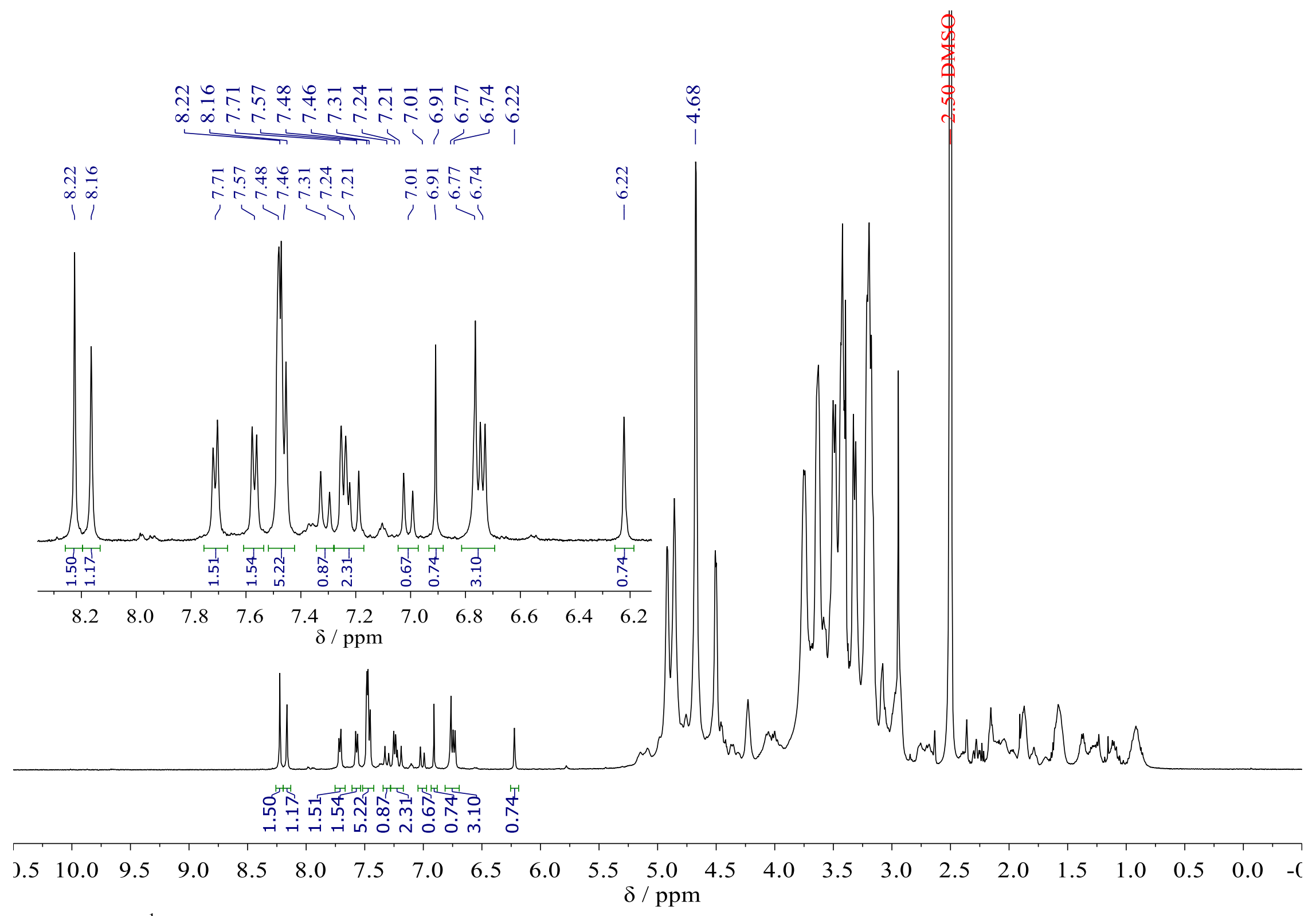

Figure S150. ${ }^{1} \mathrm{H}$ NMR (500 MHz; DMSO-d $)$ of dsRVF5-VoLDeMo (13), sample 2.

$\mathrm{S} 258$ 


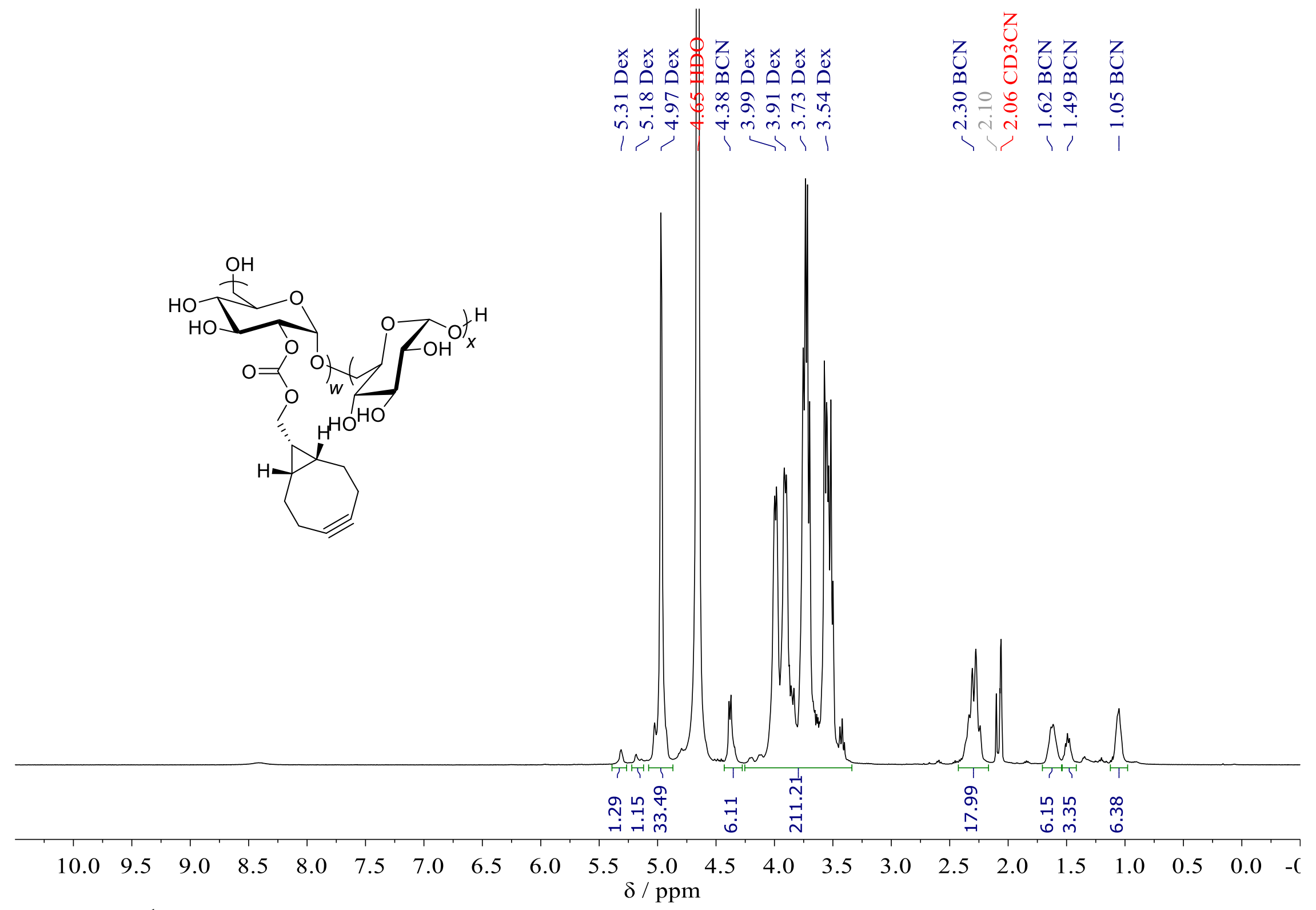

Figure S151. ${ }^{1} \mathrm{H}$ NMR $\left(500 \mathrm{MHz} ; \mathrm{D}_{2} \mathrm{O} / \mathrm{CD}_{3} \mathrm{CN} 5: 1, v / v\right)$ of $\mathrm{BCN}$-carbonate dextran(6000) (15-2.58). 


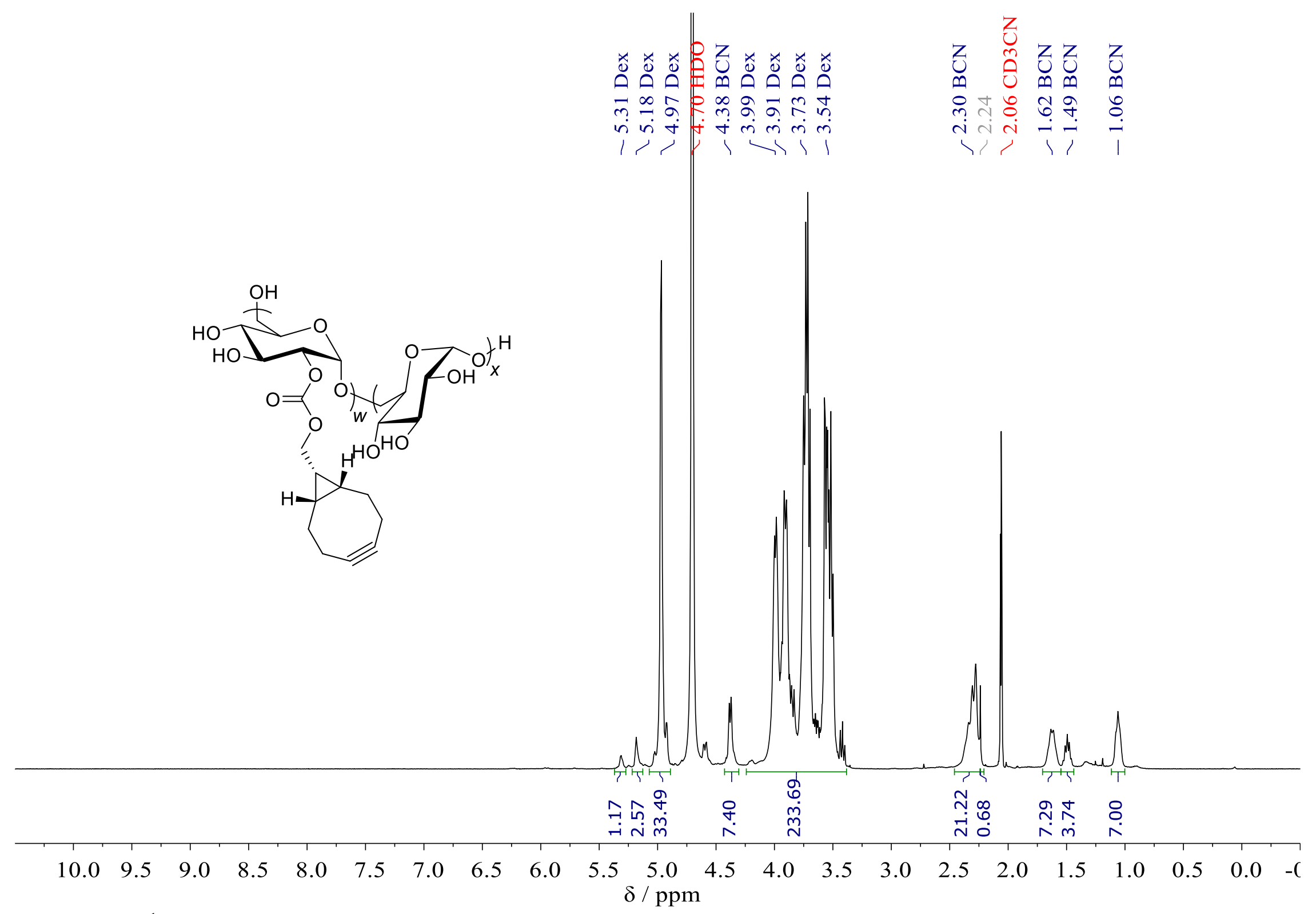

Figure S152. ${ }^{1} \mathrm{H}$ NMR $\left(500 \mathrm{MHz} ; \mathrm{D}_{2} \mathrm{O} / \mathrm{CD}_{3} \mathrm{CN} 5: 1, v / v\right)$ of BCN-carbonate dextran(6000) (15-2.95). 


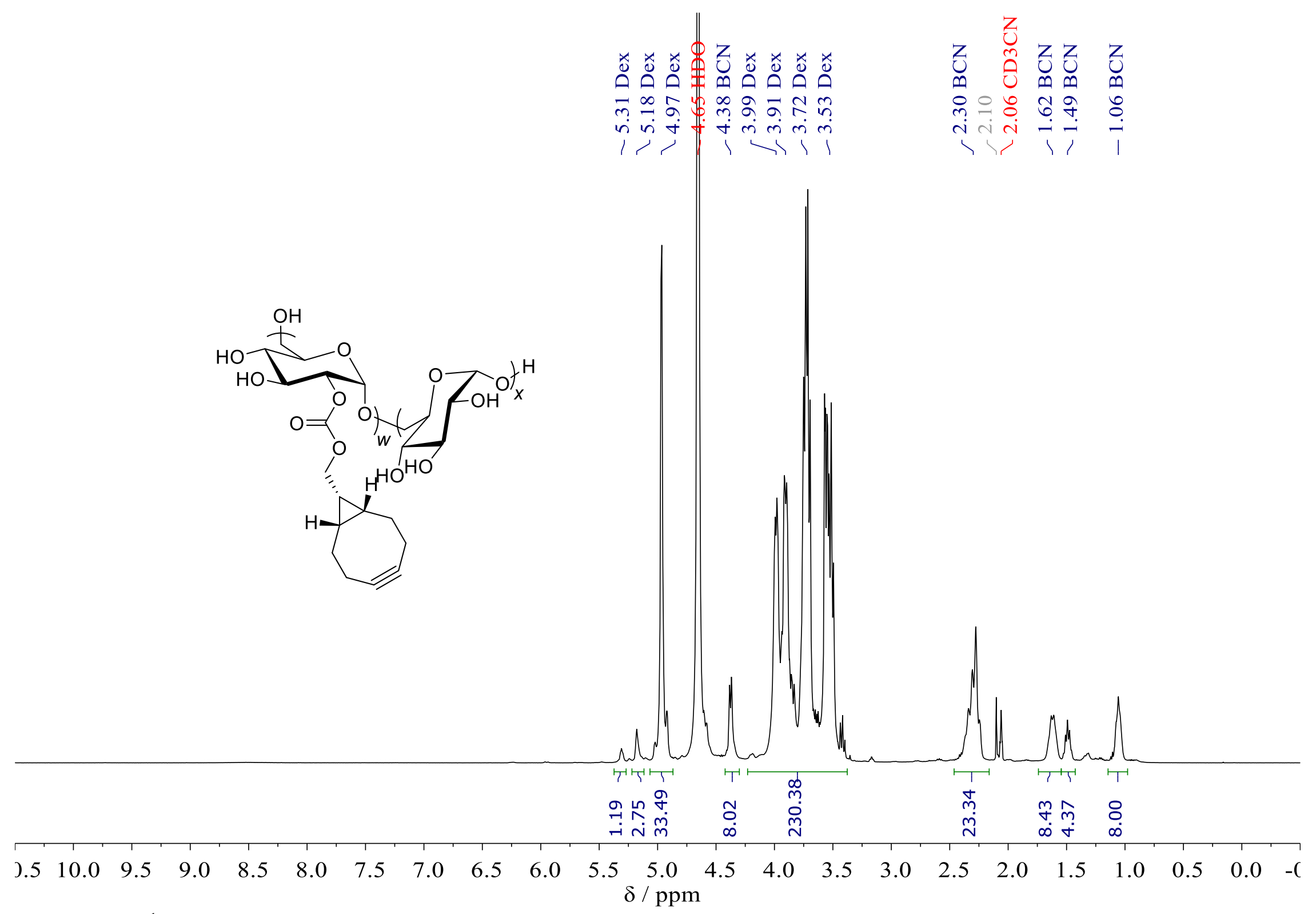

Figure S153. ${ }^{1} \mathrm{H}$ NMR $\left(500 \mathrm{MHz} ; \mathrm{D}_{2} \mathrm{O} / \mathrm{CD}_{3} \mathrm{CN} 5: 1, v / v\right)$ of $\mathrm{BCN}-$ carbonate dextran(6000) (15-3.28). 


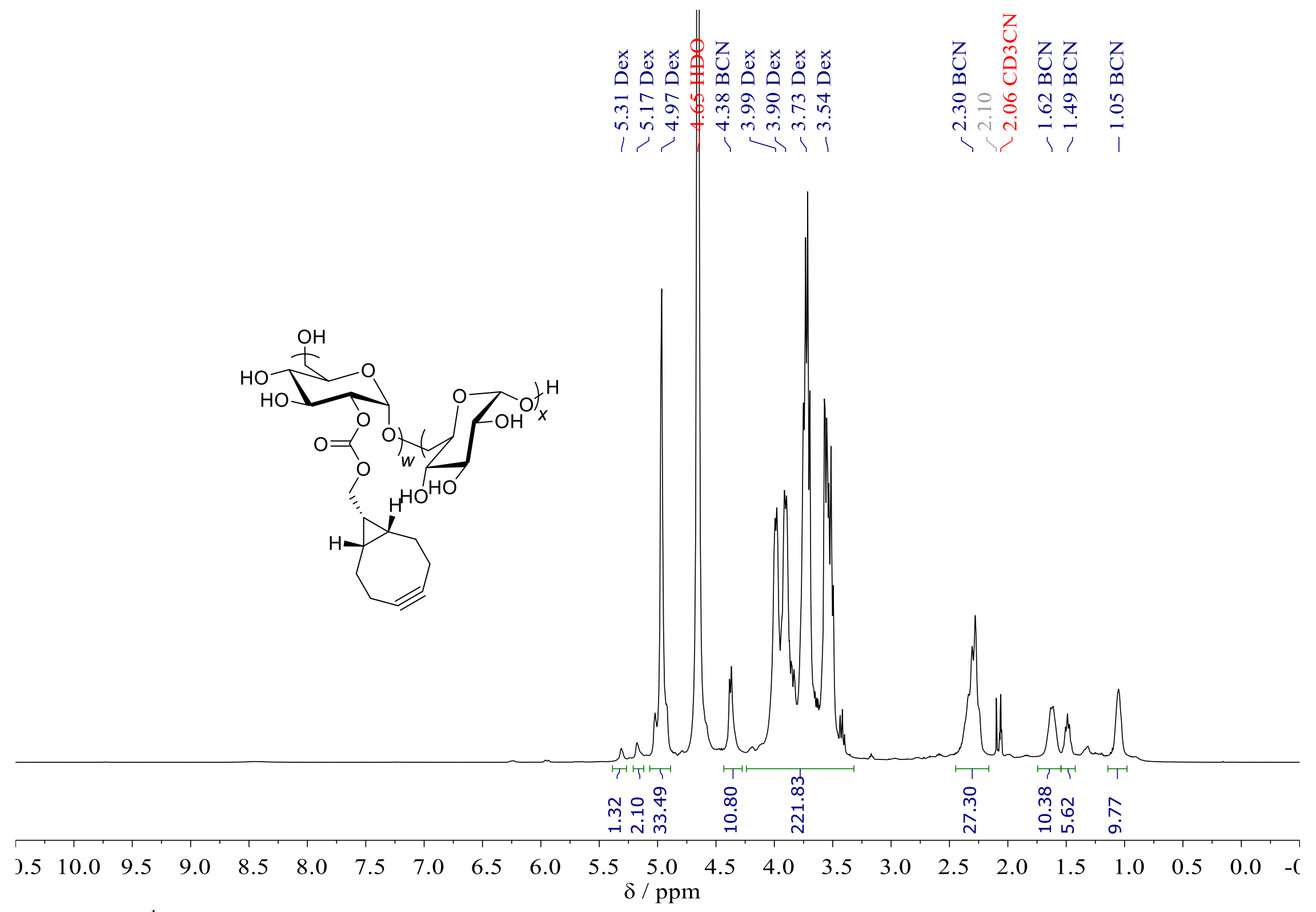

Figure S154. ${ }^{1} \mathrm{H}$ NMR $\left(500 \mathrm{MHz} ; \mathrm{D}_{2} \mathrm{O} / \mathrm{CD}_{3} \mathrm{CN} 5: 1, v / v\right)$ of $\mathrm{BCN}-$ carbonate dextran(6000) (15-3.54). 


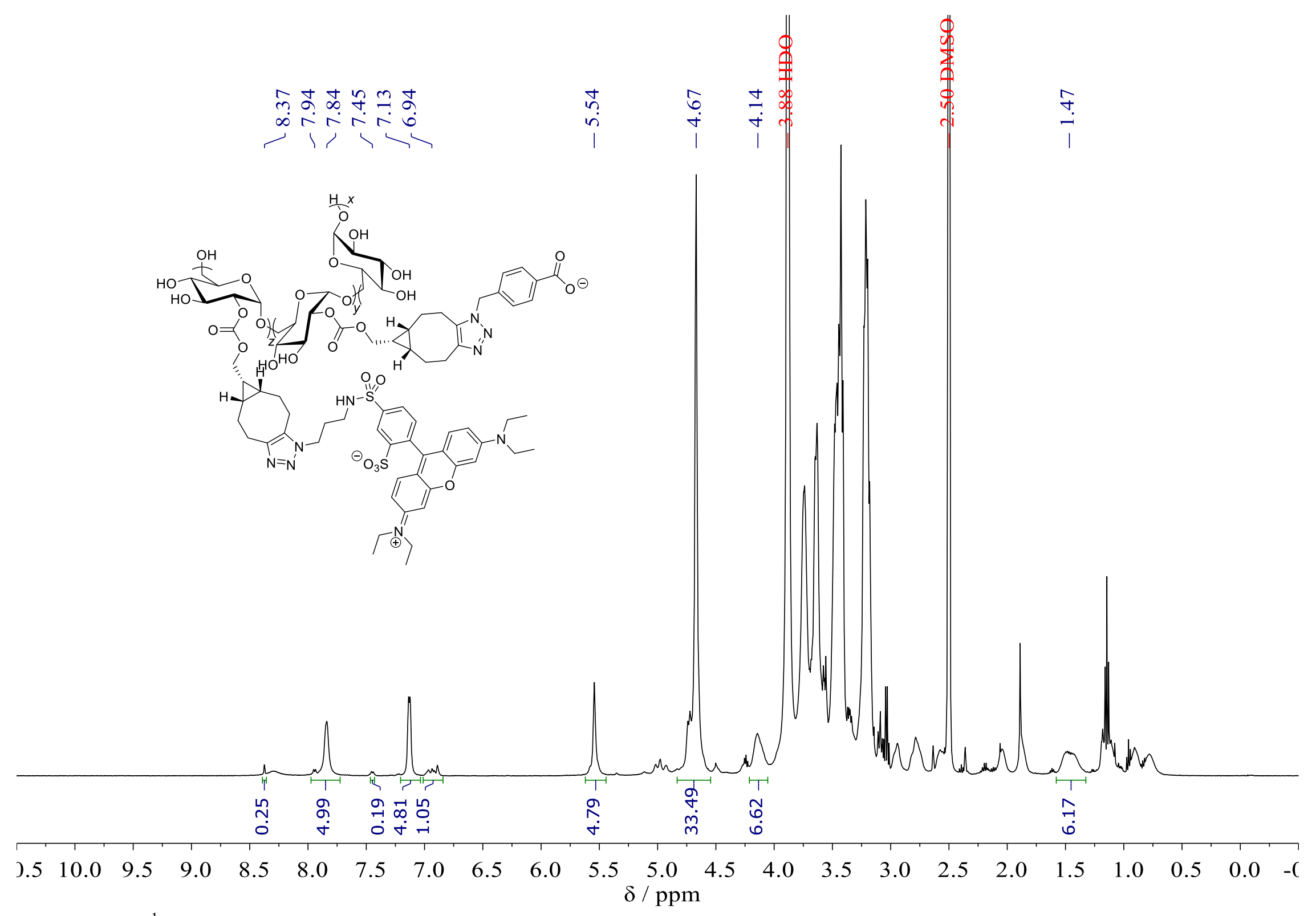

Figure S155. ${ }^{1} \mathrm{H}$ NMR $\left(500 \mathrm{MHz}\right.$; DMSO- $\left.d_{6} / \mathrm{D}_{2} \mathrm{O} 5: 1, v / v\right)$ of S48-2.58. 


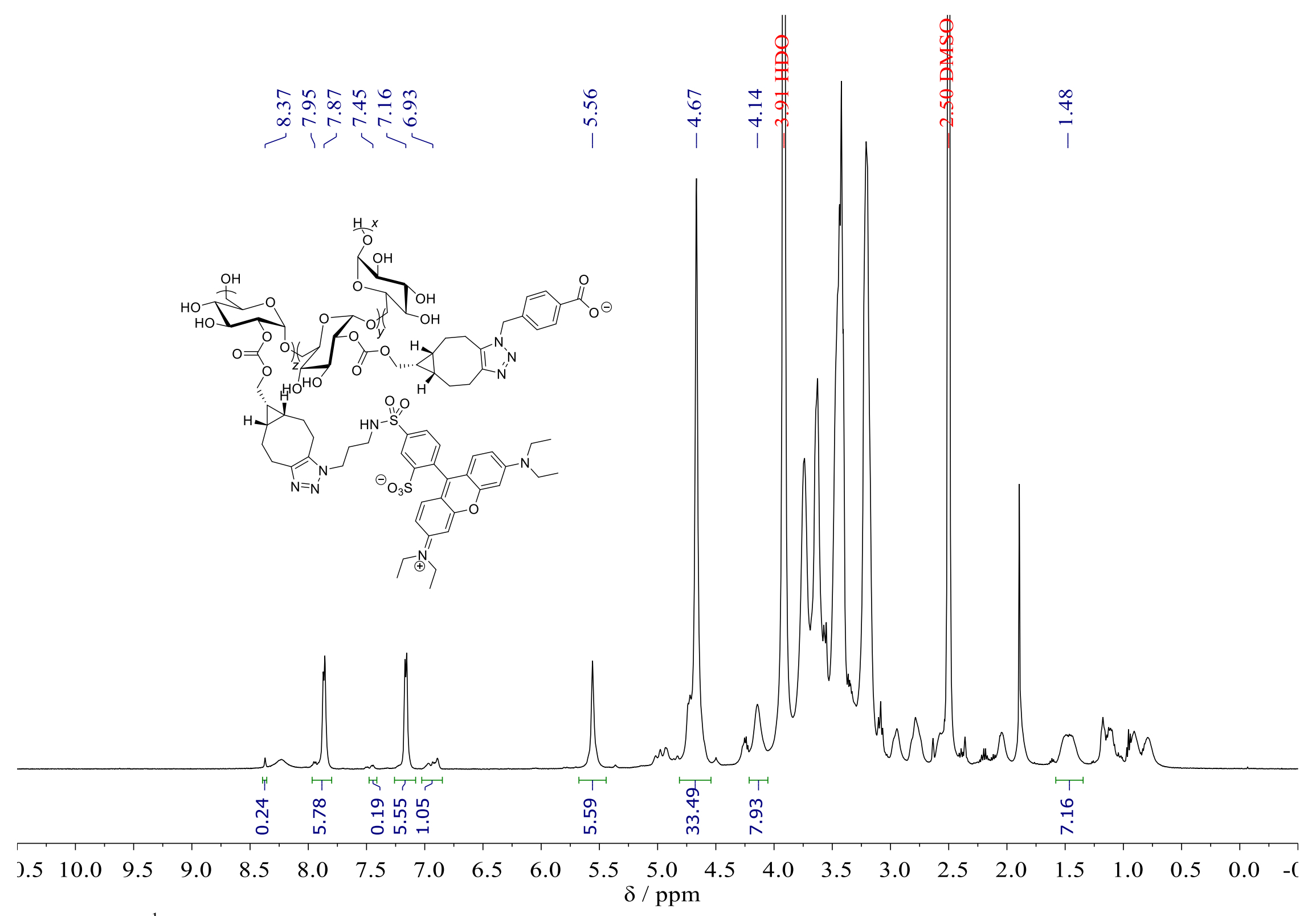

Figure S156. ${ }^{1} \mathrm{H}$ NMR (500 MHz; DMSO- $\left.d_{6} / \mathrm{D}_{2} \mathrm{O} 5: 1, v / v\right)$ of S48-2.95. 


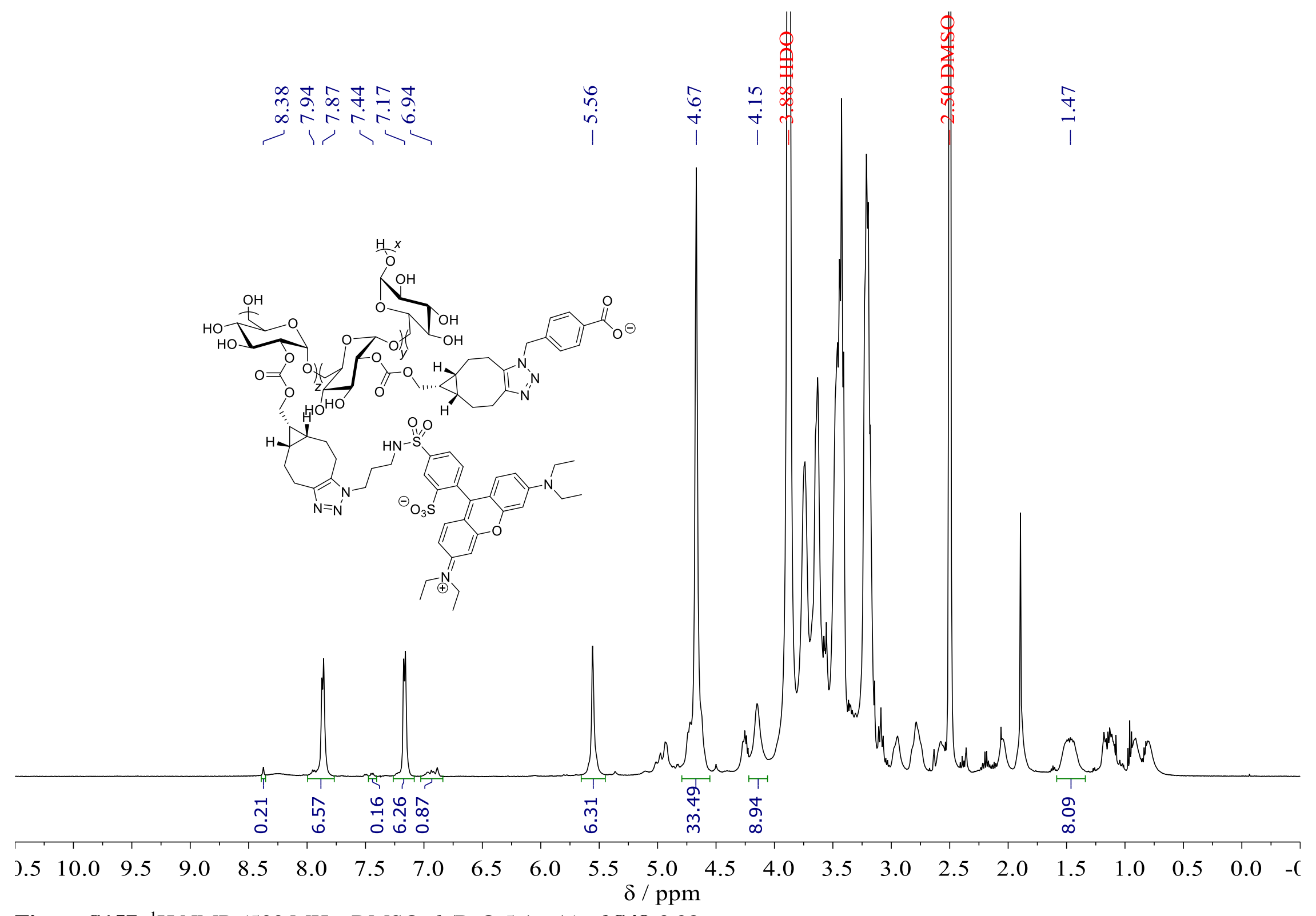

Figure S157. ${ }^{1} \mathrm{H}$ NMR $\left(500 \mathrm{MHz}\right.$; DMSO- $\left.d_{6} / \mathrm{D}_{2} \mathrm{O} 5: 1, v / v\right)$ of $\mathbf{S 4 8}-3.28$. 


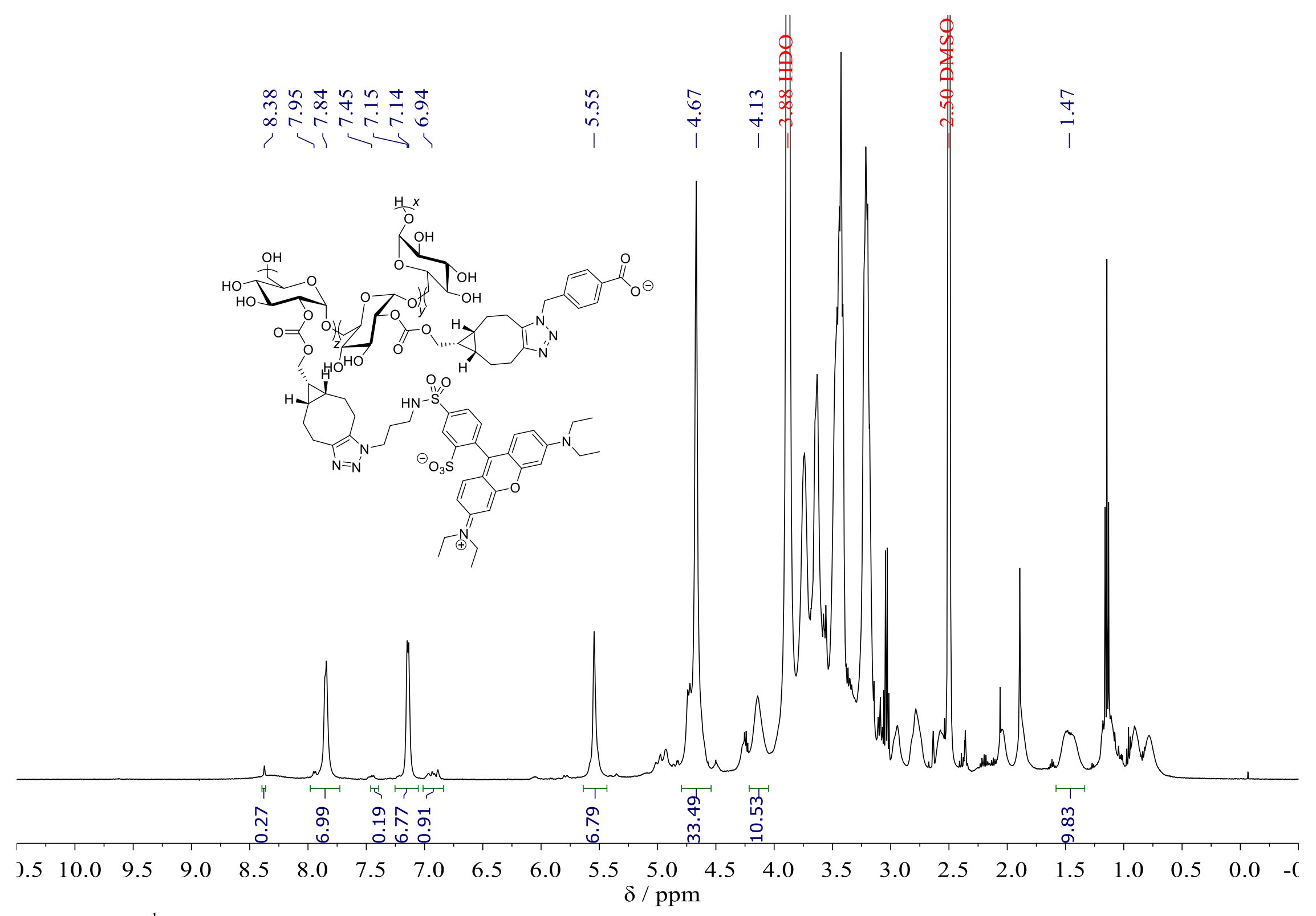

Figure S158. ${ }^{1} \mathrm{H}$ NMR (500 MHz; DMSO- $\left.d_{6} / \mathrm{D}_{2} \mathrm{O} 5: 1, v / v\right)$ of S48-3.54. 


\section{References}

(1) Brogden, R. N.; Heel, R. C.; Speight, T. M.; Avery, G. S. Nomifensine: A Review of Its Pharmacological Properties and Therapeutic Efficacy in Depressive Illness. Drugs 1979, 18 (1), 1-24. https://doi.org/10.2165/00003495-197918010-00001.

(2) Rodriguez, P. C.; Pereira, D. B.; Borgkvist, A.; Wong, M. Y.; Barnard, C.; Sonders, M. S.; Zhang, H.; Sames, D.; Sulzer, D. Fluorescent Dopamine Tracer Resolves Individual Dopaminergic Synapses and Their Activity in the Brain. Proc. Natl. Acad. Sci. 2013, 110 (3), 870-875. https://doi.org/10.1073/pnas.1213569110.

(3) Wakayama, S.; Kiyonaka, S.; Arai, I.; Kakegawa, W.; Matsuda, S.; Ibata, K.; Nemoto, Y. L.; Kusumi, A.; Yuzaki, M.; Hamachi, I. Chemical Labelling for Visualizing Native AMPA Receptors in Live Neurons. Nat. Commun. 2017, 8, 14850. https://doi.org/10.1038/ncomms 14850.

(4) Fulmer, G. R.; Miller, A. J. M.; Sherden, N. H.; Gottlieb, H. E.; Nudelman, A.; Stoltz, B. M.; Bercaw, J. E.; Goldberg, K. I. NMR Chemical Shifts of Trace Impurities: Common Laboratory Solvents, Organics, and Gases in Deuterated Solvents Relevant to the Organometallic Chemist. Organometallics 2010, 29 (9), 2176-2179. https://doi.org/10.1021/om100106e.

(5) Yan, P.; Acker, C. D.; Zhou, W.-L.; Lee, P.; Bollensdorff, C.; Negrean, A.; Lotti, J.; Sacconi, L.; Antic, S. D.; Kohl, P.; Mansvelder, H. D.; Pavone, F. S.; Loew, L. M. Palette of Fluorinated Voltage-Sensitive Hemicyanine Dyes. Proc. Natl. Acad. Sci. U. S. A. 2012, 109 (50), 20443-20448. https://doi.org/10.1073/pnas.1214850109.

(6) Kulkarni, R. U.; Kramer, D. J.; Pourmandi, N.; Karbasi, K.; Bateup, H. S.; Miller, E. W. Voltage-Sensitive Rhodol with Enhanced Two-Photon Brightness. Proc. Natl. Acad. Sci. 2017, 114 (11), 2813-2818. https://doi.org/10.1073/pnas.1610791114.

(7) Kulkarni, R. U.; Yin, H.; Pourmandi, N.; James, F.; Adil, M. M.; Schaffer, D. V.; Wang, Y.; Miller, E. W. A Rationally Designed, General Strategy for Membrane Orientation of Photoinduced Electron Transfer-Based Voltage-Sensitive Dyes. ACS Chem. Biol. 2017, 12 (2), 407-413. https://doi.org/10.1021/acschembio.6b00981.

(8) Lang, K.; Davis, L.; Wallace, S.; Mahesh, M.; Cox, D. J.; Blackman, M. L.; Fox, J. M.; Chin, J. W. Genetic Encoding of Bicyclononynes and Trans-Cyclooctenes for Site-Specific Protein Labeling in Vitro and in Live Mammalian Cells via Rapid Fluorogenic Diels-Alder Reactions. J. Am. Chem. Soc. 2012, 134 (25), 10317-10320. https://doi.org/10.1021/ja302832g.

(9) Zlatopolskiy, B. D.; Kandler, R.; Kobus, D.; Mottaghy, F. M.; Neumaier, B. Beyond AzideAlkyne Click Reaction: Easy Access to 18F-Labelled Compounds via Nitrile Oxide Cycloadditions. Chem. Commun. 2012, 48 (57), 7134-7136. https://doi.org/10.1039/C2CC31335A.

(10) Dommerholt, J.; Schmidt, S.; Temming, R.; Hendriks, L. J. A.; Rutjes, F. P. J. T.; van Hest, J. C. M.; Lefeber, D. J.; Friedl, P.; van Delft, F. L. Readily Accessible Bicyclononynes for Bioorthogonal Labeling and Three-Dimensional Imaging of Living Cells. Angew. Chem. Int. Ed. 2010, 49 (49), 9422-9425. https://doi.org/10.1002/anie.201003761.

(11) Miller, E. W.; Lin, J. Y.; Frady, E. P.; Steinbach, P. A.; Kristan, W. B.; Tsien, R. Y. Optically Monitoring Voltage in Neurons by Photo-Induced Electron Transfer through Molecular Wires. Proc. Natl. Acad. Sci. 2012, 109 (6), 2114-2119. https://doi.org/10.1073/pnas.1120694109. 
(12) Lubisch, W.; Behl, B.; Hofmann, H. P. Pyrrolylquinoxalinediones: A New Class of AMPA Receptor Antagonists. Bioorg. Med. Chem. Lett. 1996, 6 (23), 2887-2892. https://doi.org/10.1016/S0960-894X(96)00534-3.

(13) Shi, Y.; Pierce, J. G. Synthesis of the 5,6-Dihydroxymorpholin-3-One Fragment of $\begin{array}{lllllll}\text { Monanchocidin A. Org. } & \text { Lett. } & \text { 2015, } & 17 & \text { (4), }\end{array}$ https://doi.org/10.1021/acs.orglett.5b00069.

(14) Zhu, H.; Wickenden, J. G.; Campbell, N. E.; Leung, J. C. T.; Johnson, K. M.; Sammis, G. M. Construction of Carbo- and Heterocycles Using Radical Relay Cyclizations Initiated by Alkoxy Radicals. Org. Lett. 2009, 11 (9), 2019-2022. https://doi.org/10.1021/o1900481e.

(15) Simonin, J.; Vernekar, S. K. V.; Thompson, A. J.; Hothersall, J. D.; Connolly, C. N.; Lummis, S. C. R.; Lochner, M. High-Affinity Fluorescent Ligands for the 5-HT3 Receptor. Bioorg. Med. Chem. Lett. 2012, 22 (2), 1151-1155. https://doi.org/10.1016/j.bmcl.2011.11.097.

(16) Rao, A. S.; Kim, D.; Wang, T.; Kim, K. H.; Hwang, S.; Ahn, K. H. Reaction-Based TwoPhoton Probes for Mercury Ions: Fluorescence Imaging with Dual Optical Windows. Org. Lett. 2012, 14 (10), 2598-2601. https://doi.org/10.1021/ol3009057.

(17) Froemming, M. K.; Sames, D. Fluoromorphic Substrates for Fatty Acid Metabolism: Highly Sensitive Probes for Mammalian Medium-Chain Acyl-CoA Dehydrogenase. Angew. Chem. Int. Ed. 2006, 45 (4), 637-642. https://doi.org/10.1002/anie.200502675.

(18) Hassner, A.; Birnbaum, D.; Loew, L. M. Charge-Shift Probes of Membrane Potential. Synthesis. J. Org. Chem. 1984, 49 (14), 2546-2551. https://doi.org/10.1021/jo00188a006.

(19) Sánchez, M. I.; Vázquez, O.; Vázquez, M. E.; Mascareñas, J. L. Light-Controlled DNA Binding of Bisbenzamidines. Chem. Commun. 2011, 47 (39), 11107-11109. https://doi.org/10.1039/C1CC13355A.

(20) Carroll, F. I.; Coleman, M. L.; Lewin, A. H. Syntheses and Conformational Analyses of Isomeric Cocaines: A Proton and Carbon-13 NMR Study. J. Org. Chem. 1982, 47 (1), 1319. https://doi.org/10.1021/jo00340a004.

(21) Malmgren, H.; Cotton, H.; Frøstrup, B.; Jones, D. S.; Loke, M.-L.; Peters, D.; Schultz, S.; Sölver, E.; Thomsen, T.; Wennerberg, J. Stereoselective Synthesis of Monoamine Reuptake Inhibitor NS9544 Acetate. Org. Process Res. Dev. 2011, 15 (2), 408-412. https://doi.org/10.1021/op1003117.

(22) Kuznetsov, V. F. Method for Synthesizing 2-Carbomethoxytropinone. US7855296 (B1), December 21, 2010.

(23) Willstätter, R.; Wolfes, O.; Mäder, H. Synthese Des Natürlichen Cocains. Justus Liebigs Ann. Chem. 1923, 434 (1), 111-139. https://doi.org/10.1002/jlac.19234340106.

(24) Meltzer, P. C.; Liang, Anna. Y.; Madras, Bertha. K. The Discovery of an Unusually Selective and Novel Cocaine Analog: Difluoropine. Synthesis and Inhibition of Binding at Cocaine Recognition Sites. J. Med. Chem. 1994, 37 (13), 2001-2010. https://doi.org/10.1021/jm00039a014.

(25) Majewski, M.; Zheng, G.-Z. Stereoselective Deprotonation of Tropinone and Reactions of Tropinone Lithium Enolate. Can. J. Chem. 1992, 70 (10), 2618-2626. https://doi.org/10.1139/v92-330.

(26) Findlay, S. Concerning 2-Carbomethoxytropinone. J. Org. Chem. 1957, 22 (11), 13851394. https://doi.org/10.1021/jo01362a022. 
(27) Carroll, F. I.; Lewin, A. H.; Abraham, P.; Parham, K.; Boja, J. W.; Kuhar, M. J. Synthesis and Ligand Binding of Cocaine Isomers at the Cocaine Receptor. J. Med. Chem. 1991, 34 (3), 883-886. https://doi.org/10.1021/jm00107a003.

(28) Meltzer, P. C.; Liang, A. Y.; Blundell, P.; Gonzalez, M. D.; Chen, Z.; George, C.; Madras, B. K. 2-Carbomethoxy-3-Aryl-8-Oxabicyclo[3.2.1]Octanes: Potent Non-Nitrogen Inhibitors of Monoamine Transporters. J. Med. Chem. 1997, 40 (17), 2661-2673. https://doi.org/10.1021/jm9703045.

(29) Meltzer, P. C.; Blundell, P.; Huang, H.; Liu, S.; Yong, Y. F.; Madras, B. K. 3-Aryl-2Carbomethoxybicyclo[3.2.1]Oct-2-Enes Inhibit WIN 35,428 Binding Potently and Selectively at the Dopamine Transporter. Bioorg. Med. Chem. 2000, 8 (3), 581-590. https://doi.org/10.1016/S0968-0896(99)00322-3.

(30) Meltzer, P. C.; Blundell, P.; Zona, T.; Yang, L.; Huang, H.; Bonab, A. A.; Livni, E.; Fischman, A.; Madras, B. K. A Second-Generation 99mTechnetium Single Photon Emission Computed Tomography Agent That Provides in Vivo Images of the Dopamine Transporter in Primate Brain. J. Med. Chem. 2003, 46 (16), 3483-3496. https://doi.org/10.1021/jm0301484.

(31) Cha, J. H.; Zou, M.-F.; Adkins, E. M.; Rasmussen, S. G. F.; Loland, C. J.; Schoenenberger,

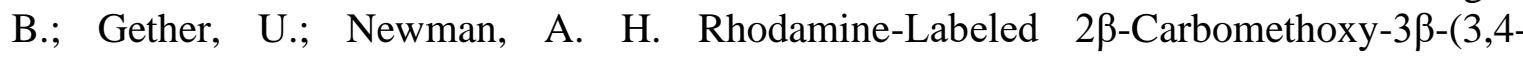
Dichlorophenyl)Tropane Analogues as High-Affinity Fluorescent Probes for the Dopamine Transporter. J. Med. Chem. 2005, 48 (24), 7513-7516. https://doi.org/10.1021/jm050431y.

(32) Cheng, H. N.; Neiss, T. G. Solution NMR Spectroscopy of Food Polysaccharides. Polym. Rev. 2012, 52 (2), 81-114. https://doi.org/10.1080/15583724.2012.668154.

(33) Dupayage, L.; Nouvel, C.; Six, J.-L. Protected versus Unprotected Dextran Macroinitiators for ATRP Synthesis of Dex-g-PMMA. J. Polym. Sci. Part Polym. Chem. 2011, 49 (1), 3546. https://doi.org/10.1002/pola.24409.

(34) Karpowicz, R. J.; Dunn, M.; Sulzer, D.; Sames, D. APP+, a Fluorescent Analogue of the Neurotoxin MPP+, Is a Marker of Catecholamine Neurons in Brain Tissue, but Not a Fluorescent False Neurotransmitter. ACS Chem. Neurosci. 2013, 4 (5), 858-869. https://doi.org/10.1021/cn400038u.

(35) Watson, B. O.; Yuste, R.; Packer, A. M. PackIO and EphysViewer: Software Tools for Acquisition and Analysis of Neuroscience Data. bioRxiv 2016, 054080. https://doi.org/10.1101/054080.

(36) Freyberg, Z.; Sonders, M. S.; Aguilar, J. I.; Hiranita, T.; Karam, C. S.; Flores, J.; Pizzo, A. B.; Zhang, Y.; Farino, Z. J.; Chen, A.; Martin, C. A.; Kopajtic, T. A.; Fei, H.; Hu, G.; Lin, Y.-Y.; Mosharov, E. V.; McCabe, B. D.; Freyberg, R.; Wimalasena, K.; Hsin, L.-W.; Sames, D.; Krantz, D. E.; Katz, J. L.; Sulzer, D.; Javitch, J. A. Mechanisms of Amphetamine Action Illuminated through Optical Monitoring of Dopamine Synaptic Vesicles in Drosophila Brain. Nat. Commun. 2016, 7, 10652. https://doi.org/10.1038/ncomms 10652.

(37) Aguilar, J. I.; Dunn, M.; Mingote, S.; Karam, C. S.; Farino, Z. J.; Sonders, M. S.; Choi, S. J.; Grygoruk, A.; Zhang, Y.; Cela, C.; Choi, B. J.; Flores, J.; Freyberg, R. J.; McCabe, B. D.; Mosharov, E. V.; Krantz, D. E.; Javitch, J. A.; Sulzer, D.; Sames, D.; Rayport, S.; Freyberg, Z. Neuronal Depolarization Drives Increased Dopamine Synaptic Vesicle Loading via VGLUT. Neuron 2017, 95 (5), 1074-1088.e7. https://doi.org/10.1016/j.neuron.2017.07.038. 
(38) Cao, G.; Platisa, J.; Pieribone, V. A.; Raccuglia, D.; Kunst, M.; Nitabach, M. N. Genetically Targeted Optical Electrophysiology in Intact Neural Circuits. Cell 2013, 154 (4), 904-913. https://doi.org/10.1016/j.cell.2013.07.027.

(39) Schoofs, A.; Hückesfeld, S.; Pankratz, M. J. Serotonergic Network in the Subesophageal Zone Modulates the Motor Pattern for Food Intake in Drosophila. J. Insect Physiol. 2018, 106, 36-46. https://doi.org/10.1016/j.jinsphys.2017.07.007.

(40) Pörzgen, P.; Park, S. K.; Hirsh, J.; Sonders, M. S.; Amara, S. G. The AntidepressantSensitive Dopamine Transporter InDrosophila Melanogaster: A Primordial Carrier for Catecholamines. Mol. Pharmacol. 2001, 59 (1), 83-95. https://doi.org/10.1124/mol.59.1.83.

(41) Friggi-Grelin, F.; Coulom, H.; Meller, M.; Gomez, D.; Hirsh, J.; Birman, S. Targeted Gene Expression in Drosophila Dopaminergic Cells Using Regulatory Sequences from Tyrosine Hydroxylase. J. Neurobiol. 2003, 54 (4), 618-627. https://doi.org/10.1002/neu.10185.

(42) Wang, J. W.; Wong, A. M.; Flores, J.; Vosshall, L. B.; Axel, R. Two-Photon Calcium Imaging Reveals an Odor-Evoked Map of Activity in the Fly Brain. Cell 2003, 112 (2), 271-282. https://doi.org/10.1016/S0092-8674(03)00004-7. 